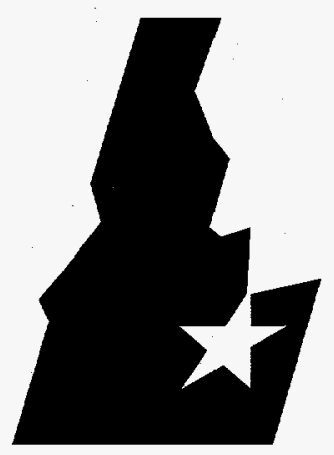

Idaho

National

Engineering

Laboratory
INEL-96/0273

January 1997

\section{RRCEIVED JUN 091999 \\ OSTI}

Charles Biagi - MK

William J. Quapp - Nuclear Metals

Tom Bechtold - LMITCO

Daryoush Bahar - MK

Blaine Brown - LMITCO

William Schwinkendorf - LMITCO

Ginger Swartz - Swartz \& Associates

Ben Teheranian - MK

Julia Vetromile - MK

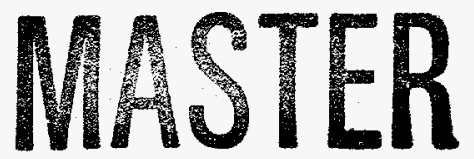

LOCKHEED MARTIN 
INEL-96/0273

\title{
Integrated Nonthermal Treatment System Study
}

\author{
Charles Biagi - MK \\ William J. Quapp - Nuclear Metals \\ Tom Bechtold - LMTTCO \\ Daryoush Bahar - MK \\ Blaine Brown - LMITCO \\ William Schwinkendorf - LMITCO \\ Ginger Swartz - Swartz \& Associates \\ Ben Teheranian - MK \\ Julia Vetromile - MK
}

January 1997

Idaho National Engineering Laboratory

Lockheed Martin Idaho Technologies Company

Idaho Falls, ID 83415

Prepared for the

U.S. Department of Energy

Idaho Operations Office

Under DOE Idaho Field Office

Contract No. DE-AC07-94D13223 


\section{DISCLAMMER}

Portions of this document may be illegible in electronic image products. Images are produced from the best available original document. 


\begin{abstract}
This report presents the results of a study of nonthermal treatment technologies. The study consisted of a systematic assessment of five nonthermal treatment alternatives. The treatment alternatives consist of widely varying technologies for safely destroying the hazardous organic components, reducing the volume, and preparing for final disposal of the contact-handled mixed low-level waste (MLLW) currently stored in the U.S. Department of Energy complex. The alternatives considered were innovative nonthermal treatments for organic liquids and sludges, process residue, soil and debris. Vacuum desorption or various washing approaches are considered for treatment of soil, residue and debris. Organic destruction methods include mediated electrochemical oxidation, catalytic wet oxidation, and acid digestion. Other methods studied included stabilization technologies and mercury separation of treatment residues.
\end{abstract}

This study is a companion to the integrated thermal treatment study which examined 19 alternatives for thermal treatment of MLLW waste.

The quantities and physical and chemical compositions of the input waste are based on the inventory database developed by the U.S. Department of Energy. The Integrated Nonthermal Treatment Systems (INTS) systems were evaluated using the same waste input (2,927 pounds per hour) as the Integrated Thermal Treatment Systems (ITTS). 


\section{ACKNOWLEDGEMENTS}

The authors would like to acknowledge the contributions of the following individuals in preparing, reviewing, and producing this report: Carl Cooley of the U.S. Department of Energy for his leadership and guidance; Gary Knight of Waste Policy Institute; William Emile Bernard of Sandia National Laboratory; Edward McNew of Lockheed Martin Idaho Technologies Company; and Angie Aylsworth, David Bean, Lisa Bishop, April Dixon, Narayanan Doraswamy, Brian Halton, Gina Morrison, Kathy Nakazawa, Cecilia Ransom, Conchita Rocillo, Dave Sonnier, Verne Trinoskey, and Gary Widlund of Morrison Knudsen Corporation.

The authors would like to acknowledge both the Tribal and Stakeholder Working Group and Technical Support Group members members for their guidance during the preparation and review of the document.

\section{Tribal \& Stakeholders Working Group Members}

Ross Vincent, Sierra Club

Richard Brusuelas, Bernalillo County Central Health Dept.

Anne Callison, Lowry Air Force Base Restoration Advisory Board

Dave Chambers, Sierra Club Legal Defense Fund

Terry Escarda, California EPA, Department of Toxic Substances Control

Karen Barkley-Fagg, MSE HKM, Inc.

Critz George, Brown and Root Environmental

Lorna Harrell, Butler County, Ohio Soil and Water Conservation District

Steve Juanico, Pueblos of Acoma

Dr. William Lawless, Savannah River Citizens Advisory Board

Momi Lovell, Kaho'olawe Island Reservation

Commission, Hawaii

Gary King, New Mexico State Legislature

Tom Marshall, Rocky Mountain Peace Center

Betsy McBride, League of Women Voters

Toby Michelena, Washington State Dept. of Ecology

Roy Mink, University of Idaho

Laura Olah, Citizens for Safe Water Around Badger

Terry Smith, Idaho Division of Environmental Quality

Stanley Sobczyk, Nez Perce Tribe

Edwin Tafoya, Eight North Indian Pueblos, San Juan Pueblo

Native American Technical Consultant:

Mary Jo Ondrechen, Northeastern University, Boston, Massachusetts

\section{Technical Support Group Members}

Leon Borduin, LANL

Carl Cooley, DOE, EM-50, Chair

Floyd DesChamps, DOE, EM-53

Steve Domotor, DOE, EM-332

Terry Dyches, DOE-SR

Dave Eaton, LMITCO

Dave Hutchins, DOE-OR

Don Musgrave, BCM Inc.

Jim Cummings, EPA

Steve Priebe, LMITCO

Michael Torbert, DOE, EM-34

Paul Strider, DOE, EM-44

Jack Watson, ORNL

\section{TSG Technical Stakeholders}

Gary King, New Mexico State Legislature

Ross Vincent, Sierra Club 


\section{CONTENTS}

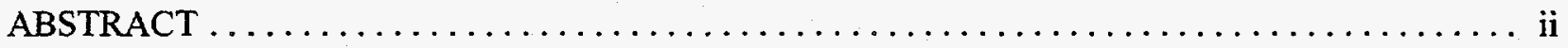

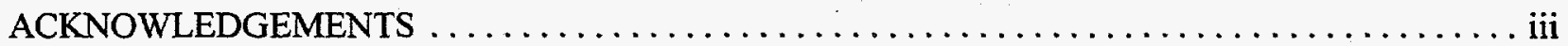

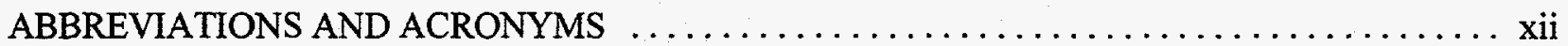

INTEGRATED NONTHERMAL TREATMENT STUDY

FINAL DRAFT

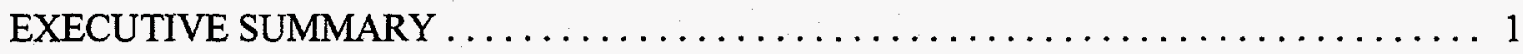

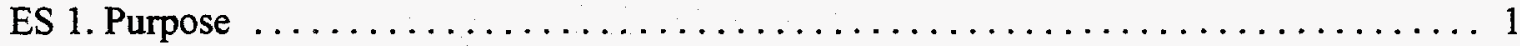

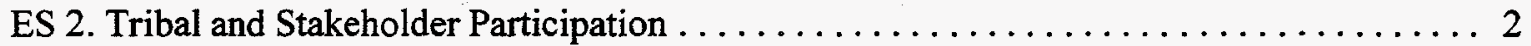

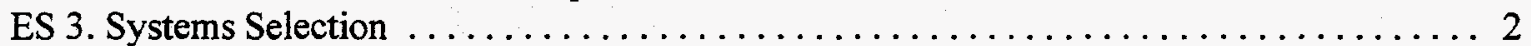

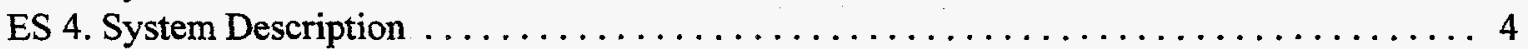

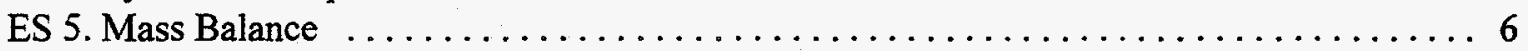

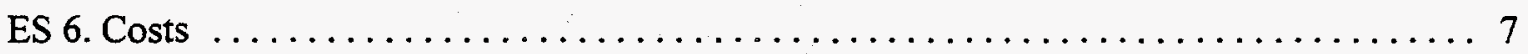

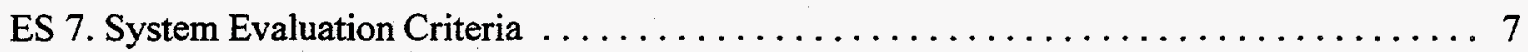

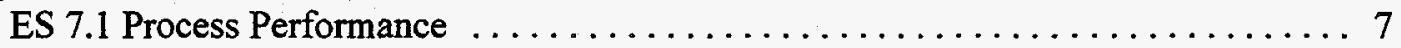

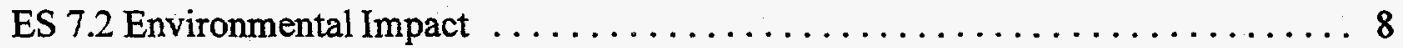

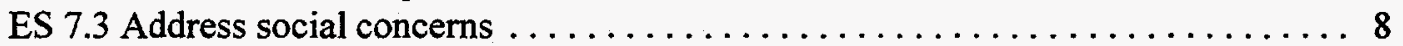

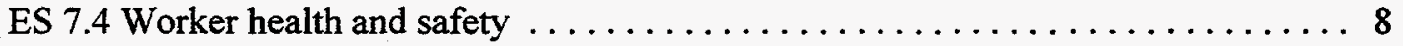

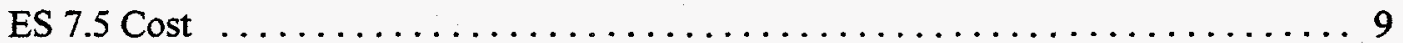

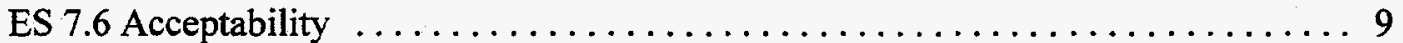

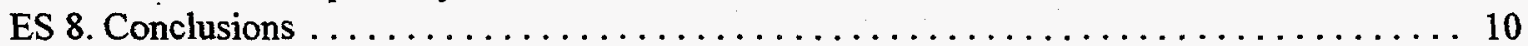

ES 9. Recommendations for Demonstration, Testing and Development $\ldots \ldots \ldots \ldots \ldots \ldots 10$

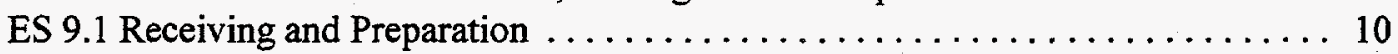

ES 9.2 Air Pollution Control Subsystem $\ldots \ldots \ldots \ldots \ldots \ldots \ldots \ldots \ldots \ldots \ldots \ldots \ldots \ldots$

ES 9.3 Aqueous Waste Treatment $\ldots \ldots \ldots \ldots \ldots \ldots \ldots \ldots \ldots \ldots \ldots \ldots \ldots \ldots \ldots$

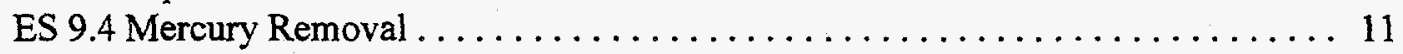

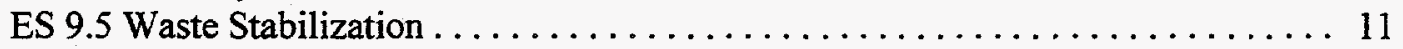

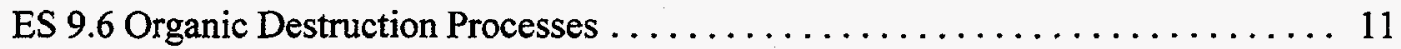

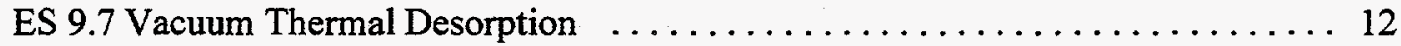

ES 9.8 Aqueous Washing and High Pressure Washing $\ldots \ldots \ldots \ldots \ldots \ldots \ldots$

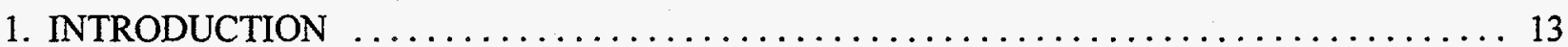

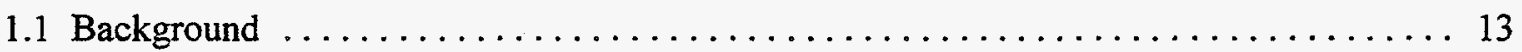

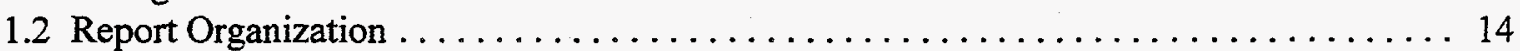

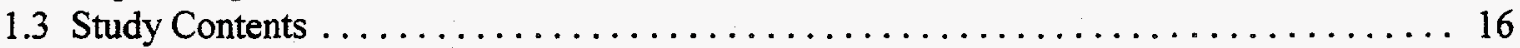

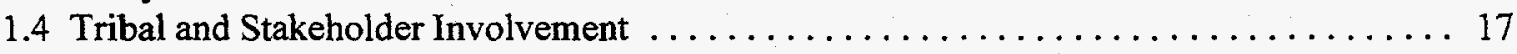

1.4.1 The Purpose of Tribal and Stakeholder Involvement in the INTS Study . . . . 17

1.4.2 Development of Tribal and Stakeholder Principles . . . . . . . . . . . 18

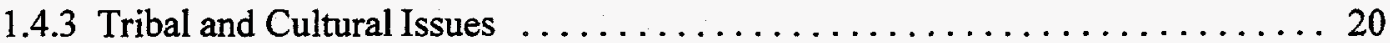

1.4.4 The Value of Tribal and Stakeholder Involvement in a Technical Exercise . . . 20

1.4.5 TSWG Comments on Systems Selection .................. 21

1.4.6 Presentation of Technical Data to the Nontechnical Reader . . . . . . . . . 22

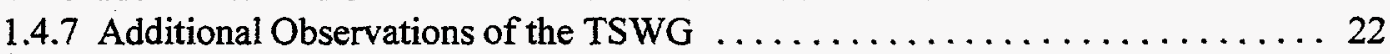

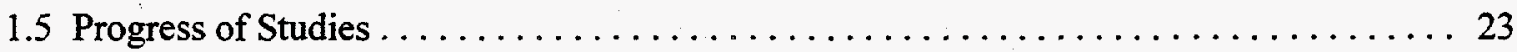

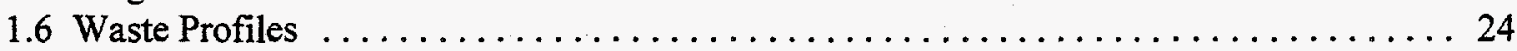




\section{CONTENTS (Continued)}

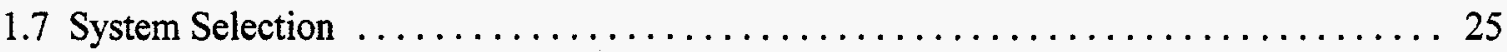

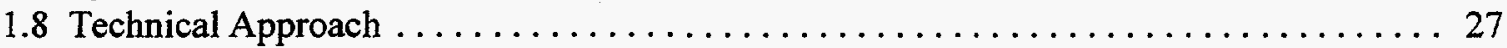

1.9 Report Limitations and Assumptions ................................ 29

1.9.1 Treatment Facility Design Assumption Summary . . . . . . . . . . . . . 29

1.9 .2 Operating Assumptions Summary $\ldots \ldots \ldots \ldots \ldots \ldots \ldots \ldots \ldots \ldots \ldots \ldots \ldots$

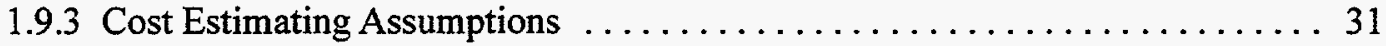

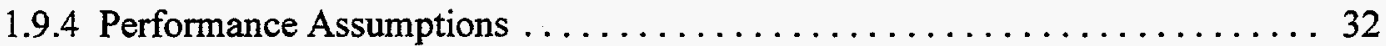

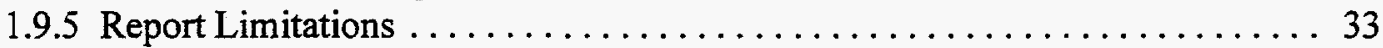

1.10 Summary of Regulations and Requirements $\ldots \ldots \ldots \ldots \ldots \ldots \ldots \ldots \ldots \ldots \ldots \ldots \ldots \ldots$

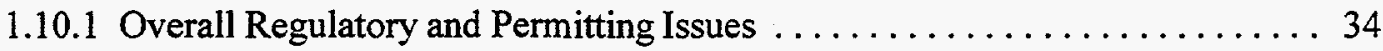

1.10.2 Department of Energy Requirements ....................... 34

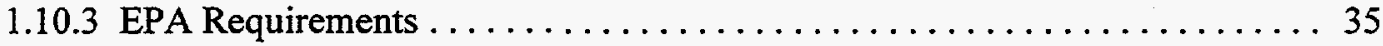

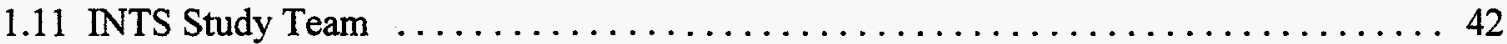

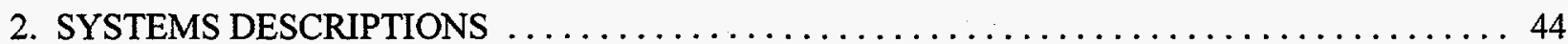

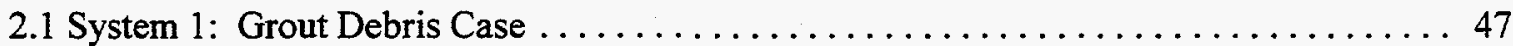

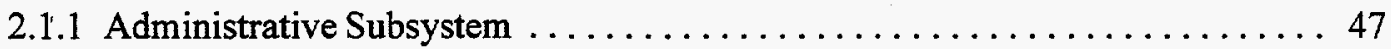

2.1.2 Receiving and Preparation Subsystem $\ldots \ldots \ldots \ldots \ldots \ldots \ldots \ldots \ldots \ldots, 48$

2.1.3 Aqueous Waste Treatment Subsystem ...................... 49

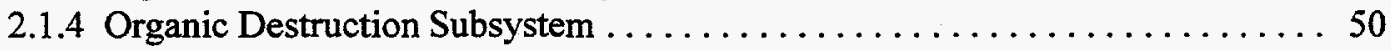

2.1.5 Process Residue and Inorganic Sludge Treatment Subsystem .......... 53

2.1.6 Bulk Soil Treatment Subsystem ........................... 54

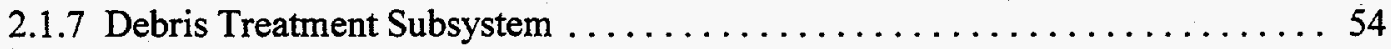

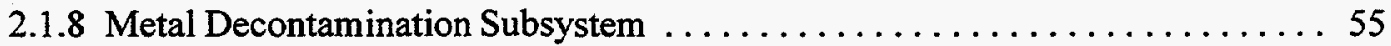

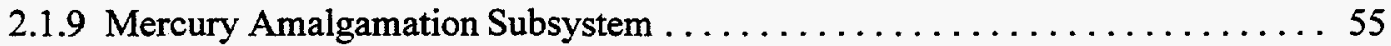

2.1 .10 Lead Recovery Subsystem $\ldots \ldots \ldots \ldots \ldots \ldots \ldots \ldots \ldots \ldots \ldots \ldots$

2.1.11 Special Waste Treatment Subsystem $\ldots \ldots \ldots \ldots \ldots \ldots \ldots \ldots \ldots \ldots \ldots$

2.1 .12 Polymer Stabilization Subsystem $\ldots \ldots \ldots \ldots \ldots \ldots \ldots \ldots \ldots \ldots \ldots$

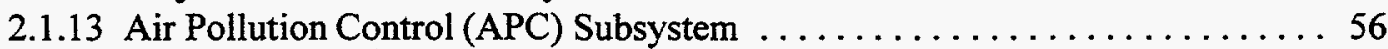

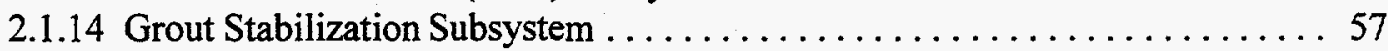

2.1 .15 Certification and Shipping Subsystem $\ldots \ldots \ldots \ldots \ldots \ldots \ldots \ldots \ldots \ldots$

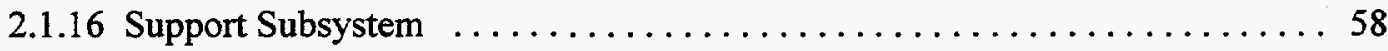

2.1.17 Disposal Subsystem .................................. 61

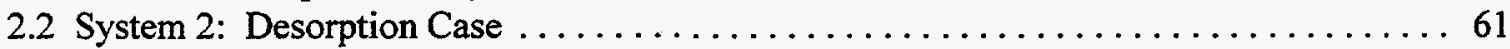

2.2.1 Catalytic Wet Oxidation Organic Destruction Subsystem ............ 61

2.2.2 Process Residue, Inorganic Sludge, Bulk Soil and Debris Treatment Subsystem ...........................................62 62

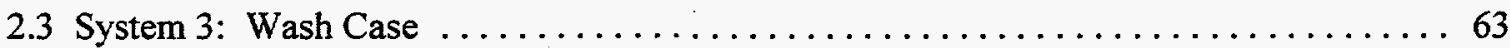

2.3.1 Process Residue, Inorganic Sludge and Bulk Soil Treatment Subsystem ..... 63

2.3.2 Soft, Open and Complex Debris Treatment Subsystem $\ldots \ldots \ldots \ldots \ldots \ldots 65$

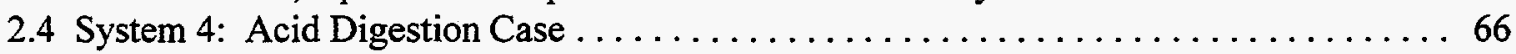

2.4.1 Acid Digestion Organic Destruction Subsystem $\ldots \ldots \ldots \ldots \ldots \ldots \ldots \ldots 67$

2.4.2 Aqueous Wash of Bulk Soil . ........................... 68

2.4.3 Acid Digestion Soft Debris Treatment Subsystem ................ 68

2.4.4 Open Debris Treatment Subsystem ........................ 68

2.4.5 Complex Debris Treatment Subsystem ...................... 69

2.4.6 Phosphate Bonded Ceramic Stabilization Subsystem ............... 69 


\section{CONTENTS (Continued)}

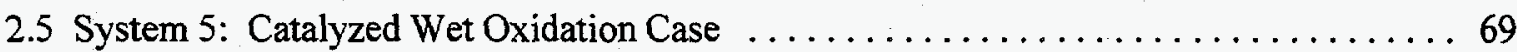

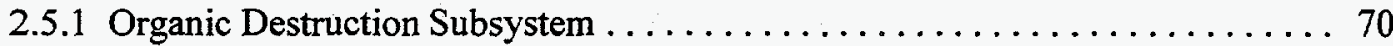

2.5.2 Bulk Soil Treatment Subsystem ......................... 70

2.5.3 Catalytic Wet Oxidation Soft Debris Treatment Subsystem ............ 70

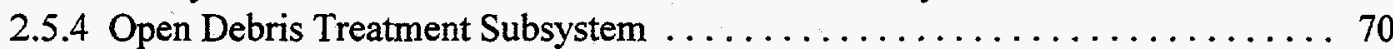

2.5.5 Complex Debris Treatment Subsystem .................... 70

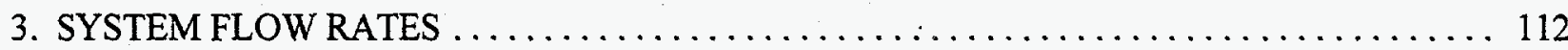

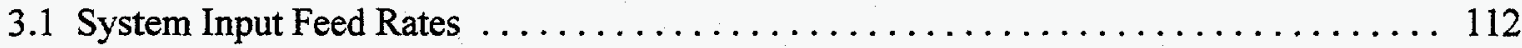

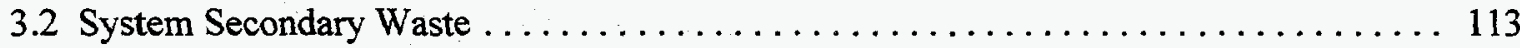

3.3 Key Assumptions for Mass Flow Calculations $\ldots \ldots \ldots \ldots \ldots \ldots \ldots \ldots \ldots \ldots \ldots \ldots \ldots \ldots$

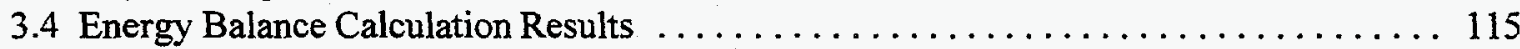

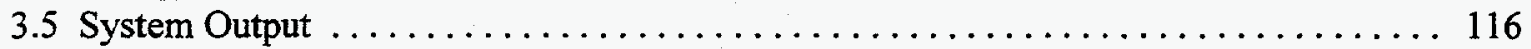

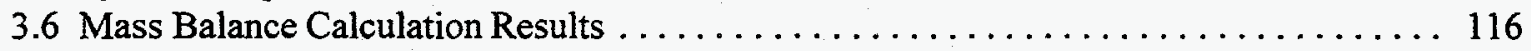

4. TOTAL LIFE CYCLE COST ESTIMATES AND SENSITIVITY ANALYSIS $\ldots \ldots \ldots \ldots \ldots 141$

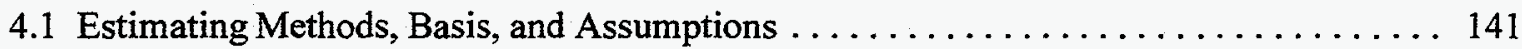

4.2 Treatment Facility TLCC Estimate (Without Disposal) Summaries .............. 142

4.2.1 Studies and Bench Scale Tests and Demonstration ................ 142

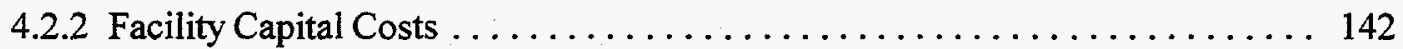

4.2.3 Preconstruction and Preoperational Activities .................. 144

4.2.4 Operations and Maintenance ............................ 144

4.2.5 Decontamination and Decommissioning $\ldots \ldots \ldots \ldots \ldots \ldots \ldots \ldots \ldots \ldots \ldots$

4.2.6 Total System Processing TLCC Estimates $\ldots \ldots \ldots \ldots \ldots \ldots \ldots \ldots \ldots$

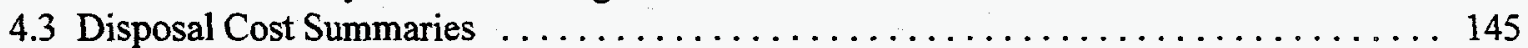

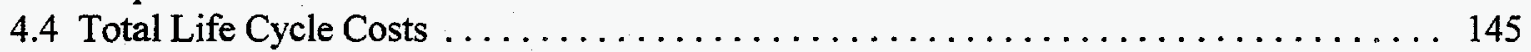

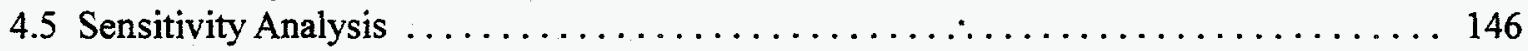

4.6 Sensitivity Analysis of the State of Technology Development $\ldots \ldots \ldots \ldots \ldots \ldots .147$

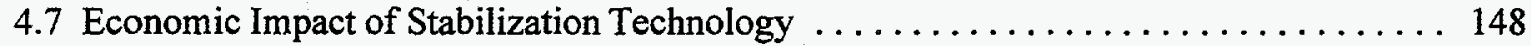

4.8 Economic Impact of Stabilizing Liquid Effluent $\ldots \ldots \ldots \ldots \ldots \ldots \ldots \ldots \ldots \ldots \ldots \ldots$

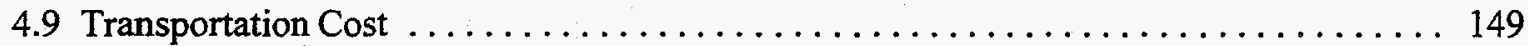

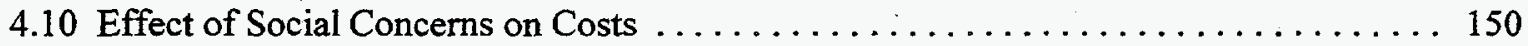

5. MATCHING TECHNICAL CRITERIA WITH TRIBAL AND STAKEHOLDER PRINCIPLES

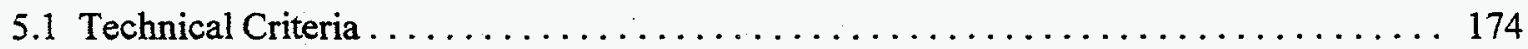

5.1.1 Process Performance Criteria ............................ 174

5.1.2 Environmental Impact Criteria ........................ 177

5.1 .3 Worker Health and Safety Criteria $\ldots \ldots \ldots \ldots \ldots \ldots \ldots \ldots \ldots \ldots \ldots$

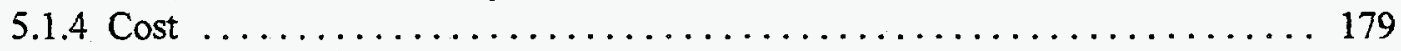

5.1 .5 Acceptability .................................... 179

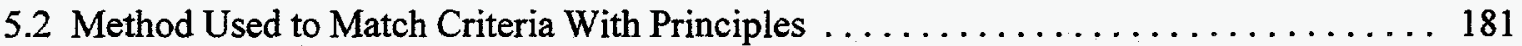

5.3 Areas Where Criteria and Principles Were Not Matched $\ldots \ldots \ldots \ldots \ldots \ldots \ldots . \ldots 182$

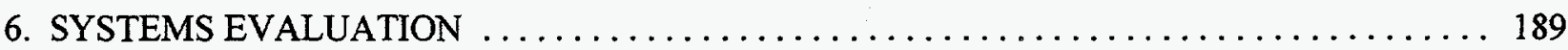

6.1 Evaluation Criteria ...................................... 189

6.2 Evaluation of System 1, Grout Debris $\ldots \ldots \ldots \ldots \ldots \ldots \ldots \ldots \ldots \ldots \ldots \ldots$ 


\section{CONTENTS (Continued)}

6.2.1 System Technology Uncertainties . . . . . . . . . . . . . . . . . . 190

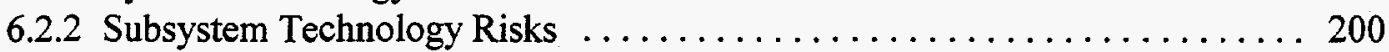

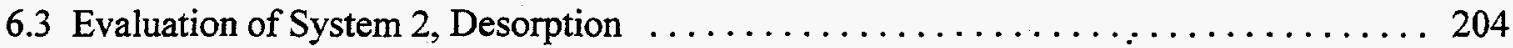

6.3.1 System Technology Risks ............................. 204

6.3 .2 Subsystems Technology Risks ........................ 208

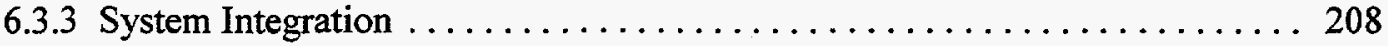

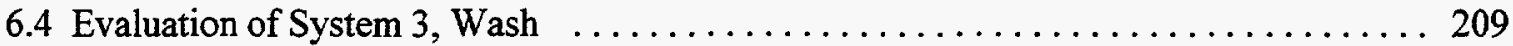

6.4.1 System Technology Risks ............................. 209

6.4 .2 Subsystems Technology Risks .......................... 212

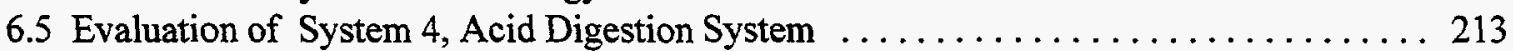

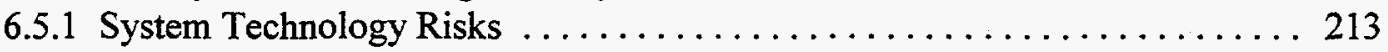

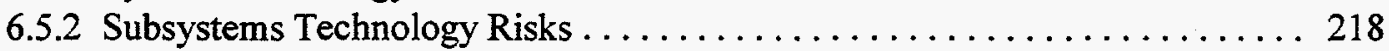

6.6 Evaluation of System 5 , CWO ................................ 219

6.6 .1 System Technology Risks ................................ 219

6.6 .2 Subsystems Technology Risks ........................... 222

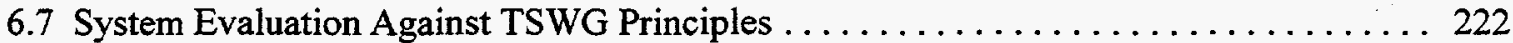

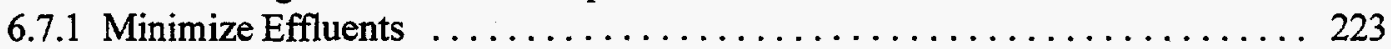

6.7.2 Minimize Effects on Human Health and the Environment ........... 224

6.7 .3 Minimize Waste Generation $\ldots \ldots \ldots \ldots \ldots \ldots \ldots \ldots \ldots \ldots \ldots . \ldots \ldots$

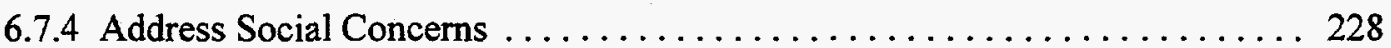

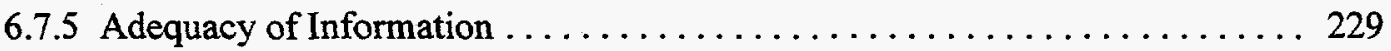

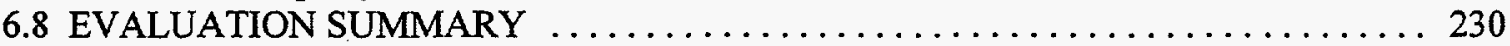

7. DEMONSTRATION, TESTING AND EVALUATION NEEDS $\ldots \ldots \ldots \ldots \ldots \ldots \ldots \ldots 245$

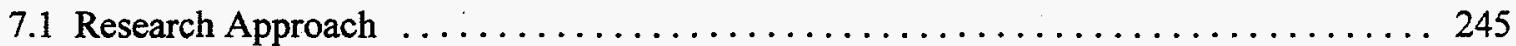

7.2 Development Objective .................................... 245

7.3 Suggested Program For Common Development Needs $\ldots \ldots \ldots \ldots \ldots \ldots \ldots \ldots 246$

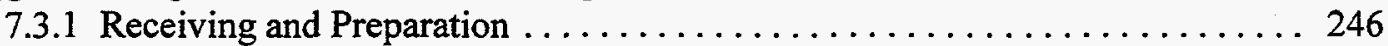

7.3.2 Air Pollution Control System ......................... 249

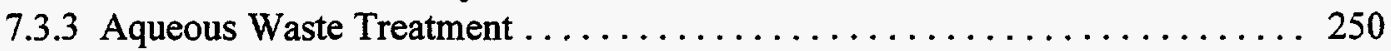

7.3.4 Mercury Removal ............................... 251

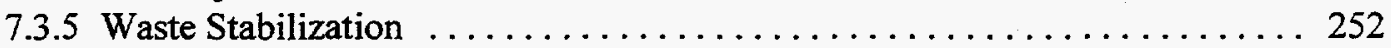

7.4 Development Needs for System 1: Grout Debris ..................... 253

7.4.1 Vacuum Thermal Desorption ........................... 253

7.4.2 Mediated Electrochemical Oxidation....................... 254

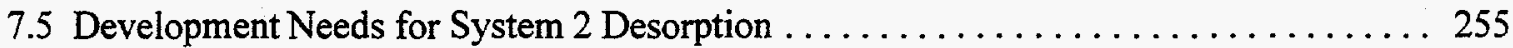

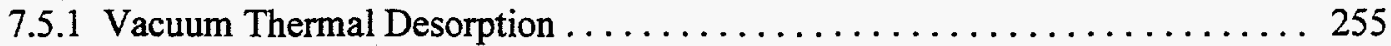

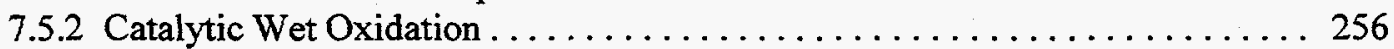

7.6 Development Needs for System 3: Washing ........................ 257

7.7 Development Needs for System 4: Acid Digestion ................... 258

7.8 Development Needs for System 5: Catalyzed Wet Oxidation .............. 260

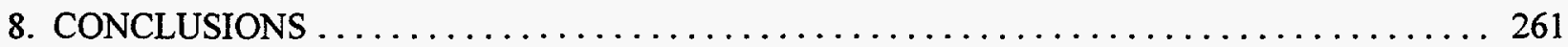

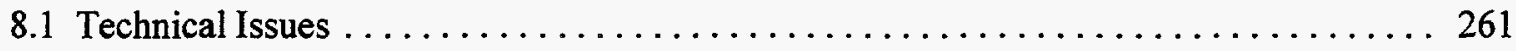

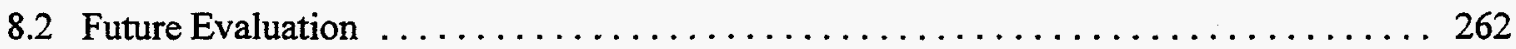

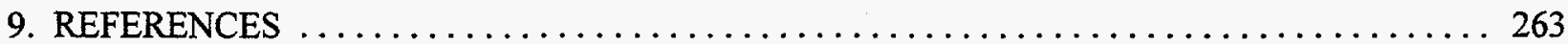




\section{CONTENTS (Continued)}

\section{APPENDICES}

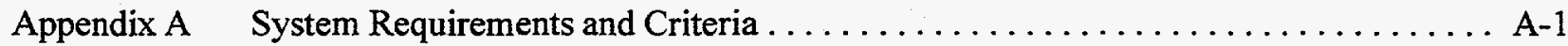

Appendix B Systems Preconceptual Design Figures $\ldots \ldots \ldots \ldots \ldots \ldots \ldots \ldots \ldots \ldots \ldots \ldots$ B-1

Appendix C $\quad$ INTS Study Mass Balances Modeling $\ldots \ldots \ldots \ldots \ldots \ldots \ldots \ldots \ldots \ldots \ldots \ldots \ldots$

Appendix D Technology and System Selection Process and Results $\ldots \ldots \ldots \ldots \ldots \ldots \ldots \ldots$ D- 1

Appendix E Tribal and Stakeholders Principles for Use in Evaluating Technology ......... E-1

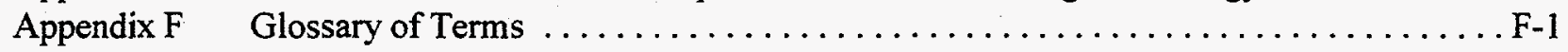

Appendix G Mercury Vapor Pressure Data Systems for Mixed Low Level Waste Treatment ... G-1

\section{TABLES}

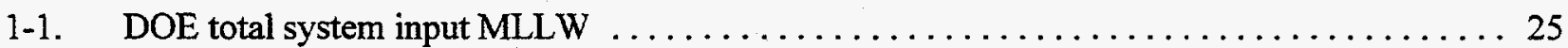

1-2. Systems included in the Integrated Nonthermal Treatment System Study. . . . . . . . 27

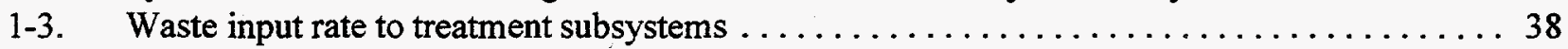

2-1. Comparison of sizes and concepts of the subsystems $\ldots \ldots \ldots \ldots \ldots \ldots \ldots \ldots \ldots \ldots$

3-1. Elemental composition of DOE contact-handled MLLW assigned directly to the aqueous waste

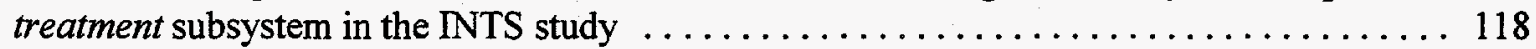

3-2. Elemental composition of DOE contact-handled MLLW assigned directly to the organic destruction treatment subsystem in the INTS study . . . . . . . . . . . . . . . . . . . . 119

3-3. Elemental composition of DOE contact-handled MLLW assigned directly to the process residue and inorganic sludge treatment subsystem in the NTS study $\ldots \ldots \ldots \ldots \ldots \ldots \ldots \ldots \ldots 120$

3-4. Elemental composition of DOE contact-handled MLLW assigned directly to the soil treatment

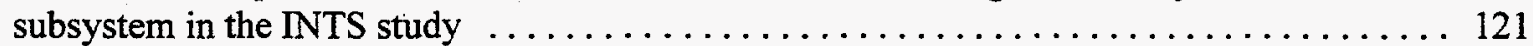

3-5. Elemental composition of DOE contact-handled MLLW assigned directly to the mercury

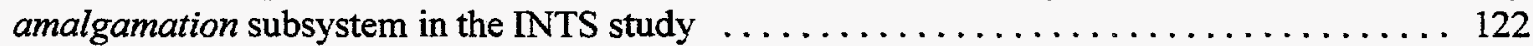

3-6. Elemental composition of DOE contact-handled MLLW assigned directly to the lead recovery

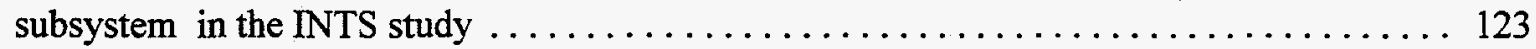

3-7. Elemental composition of DOE contact-handled MLLW assigned directly to the polymer stabilization subsystem in the INTS study . . . . . . . . . . . . . . . . . . 124

3-8. Elemental composition of DOE contact-handled MLLW assigned directly to the soft debris treatment

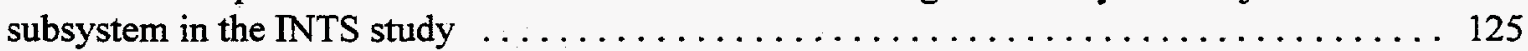

3-9. Elemental composition of DOE contact-handled MLLW assigned directly to the open debris

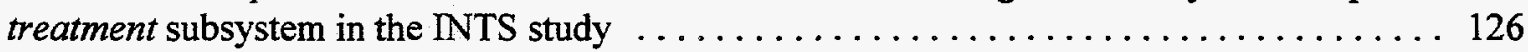

3-10. Elemental composition of DOE contact-handled MLLW assigned directly to the complex debris

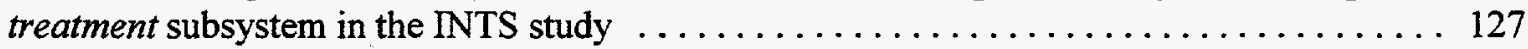

3-11. Elemental composition of DOE contact-handled MLLW assigned directly to the special waste

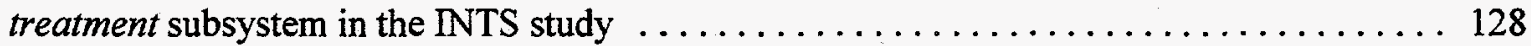

3-12. Elemental composition of DOE contact-handled LLMW assigned directly to the metal decontamination subsystem for the INTS study . . . . . . . . . . . . . . . . . 129

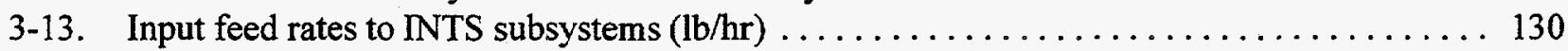

3-14. Energy requirements for the five INTS systems $(B T U / h r) \ldots \ldots \ldots \ldots \ldots \ldots \ldots \ldots \ldots 131$

$3-15$. Offgas, water and metal recycle outputs from the five INTS systems $(\mathrm{lb} / \mathrm{hr}) \ldots \ldots \ldots \ldots 132$

3-16. Waste volume for disposal for the five INTS systems $\left(\mathrm{ft}^{3} / \mathrm{hr}\right) \ldots \ldots \ldots \ldots \ldots \ldots \ldots \ldots$

4-1. Total life-cycle cost estimates (without disposal) for INTS (Costs in millions of dollars) . . 152 


\section{CONTENTS (Continued)}

4-2. Estimated facility administration staff for all INTS systems $\ldots \ldots \ldots \ldots \ldots \ldots \ldots \ldots \ldots$

4-3. Estimated facility operation staff for the INTS systems $\ldots \ldots \ldots \ldots \ldots \ldots \ldots \ldots \ldots \ldots \ldots \ldots$

4-4. Total system life cycle with disposal cost estimates ...................... 159

4-5. Total life cycle cost (with disposal) comparison of five INTS systems (in millions of dollars)

4-6. Normalized life cycle cost (without disposal) comparison of the five INTS systems (in millions of

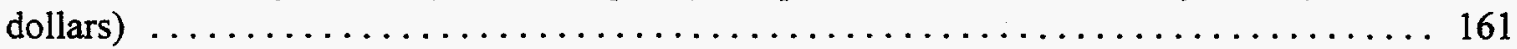

4-7. TLCC (without disposal) comparison by subsystem of the five INTS systems (in millions of dollars)

4-8. Cost sensitivity to contingency variations (in millions of dollars) $\ldots \ldots \ldots \ldots \ldots \ldots \ldots$

5-2. Technical criteria compared to TSG principles $\ldots \ldots \ldots \ldots \ldots \ldots \ldots \ldots \ldots \ldots \ldots \ldots \ldots \ldots \ldots$

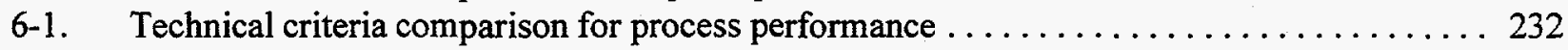

6-2. Technical criteria comparison for environmental impact ..................... 235

6-3. Technical criteria comparison for worker health and safety $\ldots \ldots \ldots \ldots \ldots \ldots \ldots \ldots \ldots \ldots \ldots$

6-4. Technical criteria comparison for cost (millions of dollars). . . . . . . . . . . . . . . 239

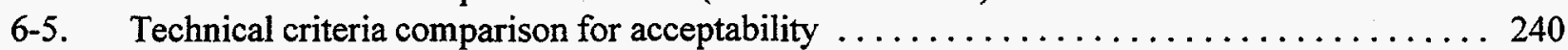

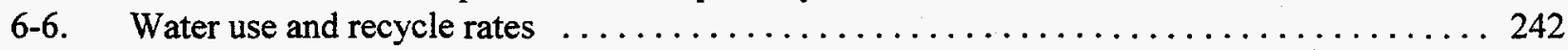

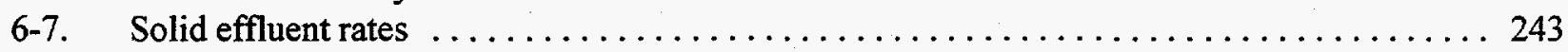

6-8. Estimated number of shipments of reagent per year $\ldots \ldots \ldots \ldots \ldots \ldots \ldots \ldots \ldots \ldots \ldots \ldots \ldots \ldots \ldots \ldots$

\section{FIGURES}

2-1. Grout Debris Case: PLAN View of Overall System (System 1) $\ldots \ldots \ldots \ldots \ldots \ldots \ldots 71$

2-2. Grout Debris Case: FAD for Receiving and Preparation Subsystem ............. 72

2-3. Grout Debris Case: PERSPECTIVE View of Receiving and Unloading Area of the Receiving and Preparation Subsystem ....................................... 73

2-4. Grout Debris Case: PERSPECTIVE View of Decap, Sort and Dump Cell of the Receiving and Preparation Subsystem ...................................... 74

2-5. Grout Debris Case: FAD for Aqueous Waste Treatment Subsystem .............. 75

2-6. Grout Debris Case: PERSPECTIVE View of Aqueous Waste Treatment Subsystem . . . . . . 76

2-7. Grout Debris Case: FAD for Organic Destruction Subsystem .................. 77

2-8. Grout Debris Case: PERSPECTIVE View of Organic Destruction Subsystem . . . . . . . 78

2-9. Grout Debris Case: FAD for Vacuum Desorption Portion of the Process Residue, Inorganic Sludge and Bulk Soil Treatment Subsystem .............................. 79

2-10. Grout Debris Case: FAD for Mercury Leaching Portion of the Process Residue, Inorganic Sludge and Bulk Soil Treatment Subsystem ............................ 80

2-11. Grout Debris Case: PERSPECTIVE View of Process Residue, Inorganic Sludge and Bulk Soil

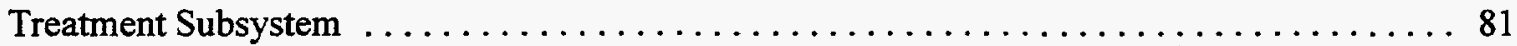

2-12. Grout Debris Case: FAD for Metal Decontamination Subsystem $\ldots \ldots \ldots \ldots \ldots \ldots \ldots 82$

2-13. Grout Debris Case: PERSPECTIVE View of Metal Decontamination Subsystem . . . . . . 83

2-14. Grout Debris Case: FAD for Mercury Amalgamation Subsystem ............... 84

2-15. Grout Debris Case: PERSPECTIVE View of Mercury Amalgamation and Lead Recovery

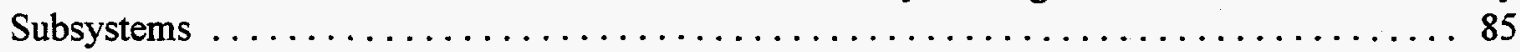

2-16. Grout Debris Case: FAD for Lead Recovery Subsystem $\ldots \ldots \ldots \ldots \ldots \ldots \ldots \ldots \ldots$

2-17. Grout Debris Case: FAD for Polymer Stabilization Subsystem .................. 87

2-18. Grout Debris Case: PERSPECTIVE View of Polymer Stabilization Subsystem ......... 88 


\section{CONTENTS (Continued)}

2-19. Grout Debris Case: FAD for Air Pollution Control Subsystem . . . . . . . . . . . . 89

2-20. Grout Debris Case: PERSPECTIVE View of Air Pollution Control Subsystem ......... 90

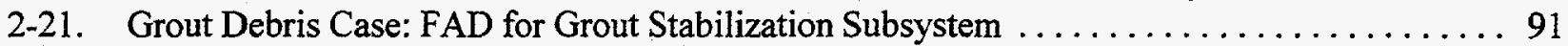

2-22. Grout Debris Case: PERSPECTIVE View of Grout Stabilization Subsystem . . . . . . . . 92

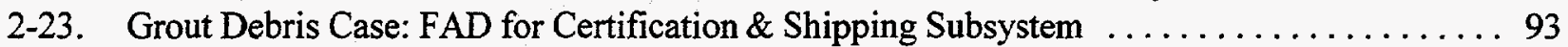

2-24. Grout Debris Case: PERSPECTIVE View of Certification \& Shipping Subsystem . . . . . . 94

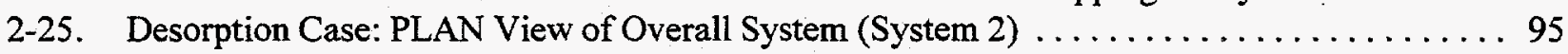

2-26. Desorption Case: FAD for Organic Destruction Subsystem $\ldots \ldots \ldots \ldots \ldots \ldots \ldots \ldots \ldots 96$

2-27. Desorption Case: PERSPECTIVE View of Organic Destruction Subsystem ......... 97

2-28. Wash Case: PLAN View of Overall System (System 3) $\ldots \ldots \ldots \ldots \ldots \ldots \ldots \ldots$

2-29. Wash Case: FAD for Process Residue, Inorganic Sludge and Bulk Soil Treatment Subsystems 99

2-30. Wash Case: PERSPECTIVE View of Process Residue, Inorganic Sludge and Bulk Soil Treatment

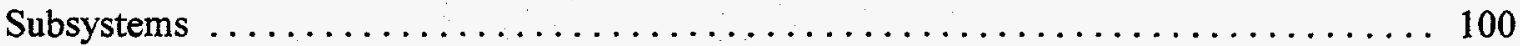

2-31. Wash Case: FAD for Soft, Open and Complex Debris Waste Treatment Subsystems . . . . 101

2-32. Wash Case: PERSPECTIVE View of Soft, Open and Complex Debris Waste Treatment

Subsystems . . . . . . . . . . . . . . . . . 102

2-33. Acid Digestion Case: PLAN View of Overall System (System 4) $\ldots \ldots \ldots \ldots \ldots \ldots \ldots$

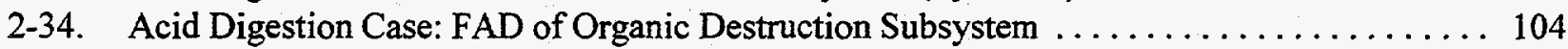

2-35. Acid Digestion Case: PERSPECTIVE View of Organic Destruction Subsystem ....... 105

2-36. Acid Digestion Case: PERSPECTIVE View of Bulk Soil Treatment Subsystem ....... 106

2-37. Acid Digestion Case: PERSPECTIVE View of Soft Debris Waste Treatment Subsystem . . 107

2-38. Acid Digestion Case; FAD for Phosphate Bonded Ceramic Stabilization Subsystem . . . . . . 108

2-39. Acid Digestion Case: PERSPECTIVE View of Phosphate Bonded Ceramic Stabilization

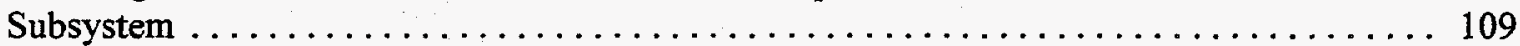

2-40. Catalytic Wet Oxidation Case: PLAN View of Overall System (System 5) $\ldots \ldots \ldots \ldots \ldots 110$

2-41. Catalytic Wet Oxidation Case: PERSPECTIVE View of Soft Debris Waste Treatment

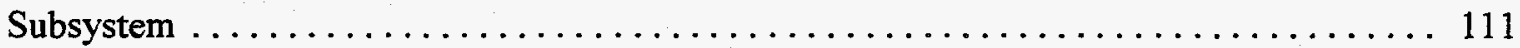

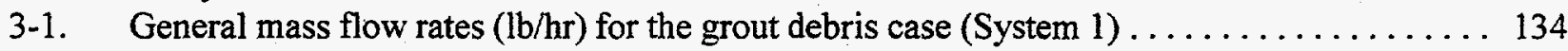

3-2. General mass flow rates $(\mathrm{lb} / \mathrm{hr})$ for the desorption case (System 2$) \ldots \ldots \ldots \ldots \ldots \ldots \ldots$

3-3. General mass flow rates $(\mathrm{lb} / \mathrm{hr})$ for the wash case (System 3$) \ldots \ldots \ldots \ldots \ldots \ldots \ldots$

3-4. General mass flow rates $(\mathrm{lb} / \mathrm{hr})$ for the acid digestion case (System 4$) \ldots \ldots \ldots \ldots \ldots$

3-5. General mass flow rates $(\mathrm{lb} / \mathrm{hr}$ ) for the CWO case (System 5$) \ldots \ldots \ldots \ldots \ldots \ldots \ldots \ldots$

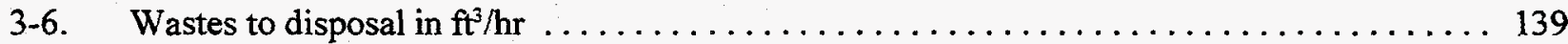

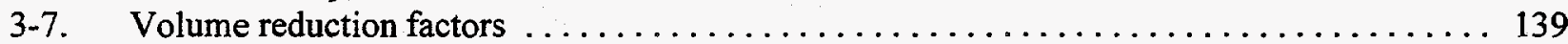

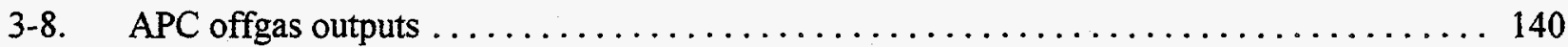

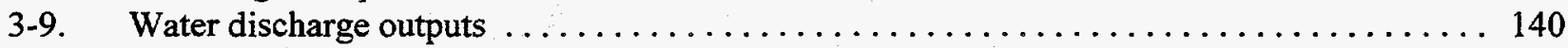

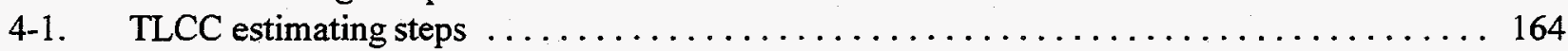

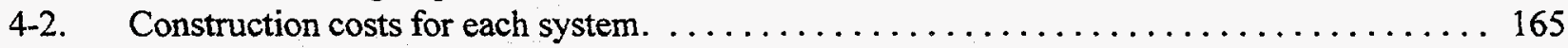

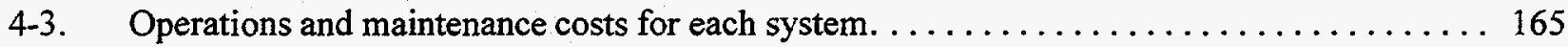

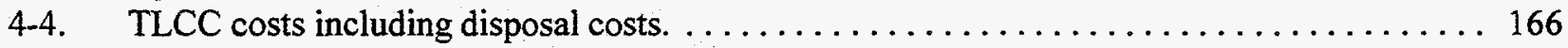

4-5. Unit costs in dollars/pound (including disposal costs) $\ldots \ldots \ldots \ldots \ldots \ldots \ldots \ldots \ldots \ldots$

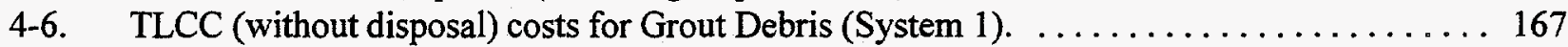

4-7. Unit costs (without disposal) in dollars/pound for Grout Debris (System 1). . . . . . . . 167

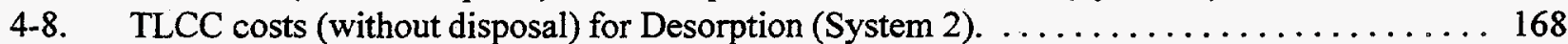

4-9. Unit costs (without disposal) in dollars/pound for Desorption (System 2) . . . . . . . 168

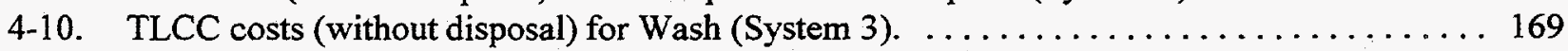

4-11. Unit costs (without disposal) in dollars/pound for Wash (System 3) . . . . . . . . 169 


\section{CONTENTS (Continued)}

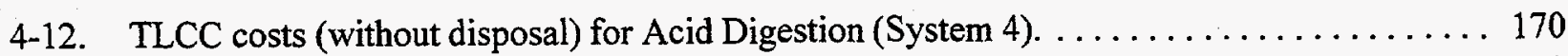

4-13. Unit costs (without disposal) in dollars/pound for Acid Digestion (System 4). . . . . . 170

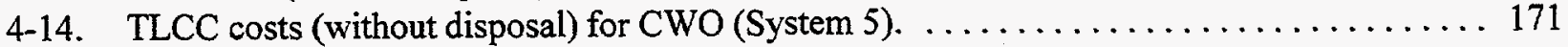

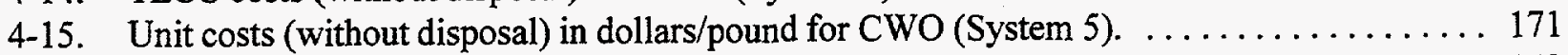

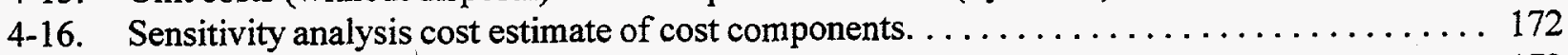

4-17. TLCC sensitivity to preoperation duration. $\ldots \ldots \ldots \ldots \ldots \ldots \ldots \ldots \ldots \ldots \ldots \ldots \ldots \ldots \ldots \ldots \ldots \ldots$ 


\section{ABBREVIATIONS AND ACRONYMS}

\begin{tabular}{|c|c|}
\hline ABS & American Bureau of Standards \\
\hline AEA & Atomic Energy Act \\
\hline $\mathrm{Ag}$ & silver \\
\hline $\mathrm{AgCl}$ & silver chloride \\
\hline $\mathrm{AgNO}_{3}$ & silver nitrate \\
\hline APC & air pollution control \\
\hline ANSI & American National Standards Institute \\
\hline ASME & American Society of Mechanical Engineers \\
\hline ASPEN & ASPEN PLUS computer model \\
\hline ASTM & American Society of Testing and Materials \\
\hline atm & atmosphere \\
\hline BDAT & best demonstrated available technology \\
\hline BIF & boiler and industrial furnace \\
\hline B.P. & boiling point \\
\hline BTEX & benzene, toluene, ethylbenzene, and xylenes \\
\hline Btu & British thermal unit \\
\hline $\mathrm{CA}$ & commercially available \\
\hline CAA & Clean Air Act \\
\hline $\mathrm{Cd}$ & Cadium \\
\hline $\mathrm{Ce}$ & Cerium \\
\hline CEM & continuous emissions monitoring \\
\hline CERCLA & Comprehensive Environmental Response, Compensation, and Liability Act \\
\hline CFC & chlorofluorocarbon \\
\hline CFR & Code of Federal Regulations \\
\hline $\operatorname{cfm}$ & cubic feet per minute \\
\hline $\mathrm{CH}$ & contact-handled \\
\hline $\mathrm{CM}$ & construction management \\
\hline $\mathrm{cm}$ & centimeter \\
\hline $\mathrm{CO}$ & carbon monoxide \\
\hline $\mathrm{CO}_{2}$ & carbon dioxide \\
\hline CPLM & cost-per-loaded mile \\
\hline CWO & catalytic wet oxidation \\
\hline $\mathrm{D} \& \mathrm{D}$ & decontamination and decommissioning \\
\hline DAF & dissolved air flotation \\
\hline DOD & U.S. Department of Defense \\
\hline DOE & U.S. Department of Energy \\
\hline DOT & U.S. Department of Transportation \\
\hline DRE & destruction removal efficiency \\
\hline dscm & dry standard cubic meters \\
\hline DTSC & $\begin{array}{l}\text { California Environmental Protection Agency, Department of Toxic Substances } \\
\text { Control }\end{array}$ \\
\hline e & electricity \\
\hline EDTA & ethylenediaminetetraacetic acid \\
\hline EM-50 & Environmental Management Office of Technology Development \\
\hline EPA & U.S. Environmental Protection Agency \\
\hline ER & environmental restoration \\
\hline ES\&H & environment, safety, and health \\
\hline
\end{tabular}




\section{ABBREVIATIONS AND ACRONYMS (Continued)}

\begin{tabular}{|c|c|}
\hline F\&ORs & functional and operational requirements \\
\hline FAR & Federal Acquisition Regulations \\
\hline FAD & functional allocation diagram \\
\hline $\mathrm{Fe}$ & iron \\
\hline FFCA & Federal Facility Compliance Act of 1992 \\
\hline FMEA & failure mode and effects analysis \\
\hline FOM & figure-of-merit \\
\hline FR & Federal Register \\
\hline F.P. & freezing point \\
\hline FRP & fiber reinforced plastic \\
\hline $\mathrm{ft}$ & feet \\
\hline FTE & full-time equivalent \\
\hline $\mathbf{g}$ & gram \\
\hline$g$ & gas \\
\hline GAC & granular activated carbon \\
\hline GC/MS & gas chromatography/mass spectroscopy \\
\hline GOCO & government owned and contractor operated \\
\hline GPCR & gas phase corona reactor \\
\hline gpm & gallons per minute \\
\hline $\mathrm{H}$ & hydrogen \\
\hline HAZOP & Hazard and Operability Study \\
\hline $\mathrm{Hcl}$ & hydrogen chloride \\
\hline HEPA & high-efficiency particulate air (filter) \\
\hline $\mathrm{HF}$ & hydrogen fluoride \\
\hline $\mathrm{Hg}$ & mercury \\
\hline $\mathrm{HNO}_{2}$ & nitrous acid \\
\hline $\mathrm{HNO}_{3}$ & nitric acid \\
\hline $\mathrm{hr}$ & hour \\
\hline HVAC & heating, ventilation, and air conditioning \\
\hline $\mathrm{H}_{2} \mathrm{O}$ & water \\
\hline $\mathrm{H}_{2} \mathrm{O}_{2}$ & hydrogen peroxide \\
\hline $\mathrm{I}_{2}$ & Iodine \\
\hline IAEA & International Atomic Energy Agency \\
\hline IATA & International Air Transport Association \\
\hline $\mathrm{IC}$ & ion chromatography \\
\hline ICP & inductively coupled plasma spectroscopy \\
\hline ICRP & International Commission on Radiological Protection \\
\hline IMWIR & Inerim Mixed Waste Inventory Report \\
\hline NEL & Idaho National Engineering Laboratory \\
\hline INTS & integrated nonthermal treatment system \\
\hline ISO & International Organization for Standardization \\
\hline ITTS & integrated thermal treatment system \\
\hline $\mathrm{kg}$ & kilogram \\
\hline $\mathrm{KI}$ & potassium iodine \\
\hline $\mathrm{kWh}$ & kilowatt per hour \\
\hline 1 & liter \\
\hline LANL & Los Alamos National Laboratory \\
\hline
\end{tabular}




\section{ABBREVIATIONS AND ACRONYMS (Continued)}

lb

LDR

LLNL

LLW

LMITCO

$\mathrm{M}$

$\mathrm{m}$

$\mathrm{M} \& \mathrm{O}$

$\mathrm{MCC}$

MEO

$\mathrm{mg}$

$\mathrm{MgO}$

MIOX

MK

$\mathrm{ml}$

mm

MLLW

MMBtu

Mpa

mrem

MSO

MWFA

MWIR

$\mathrm{NaCl}$

$\mathrm{NaOH}$

$\mathrm{nCi} / \mathrm{g}$

NCRP

ND

NEPA

NFPA

ng

NO

$\mathrm{NOCl}$

$\mathrm{No}_{\mathrm{x}}$

NPDES

NQA

NRC

$\mathrm{O}$

$\mathrm{O}_{3}$

$\mathrm{OH} \cdot$

ORNL

O\&M

OMB

OSHA

PAN

PCB

PEIS pound

land disposal restriction

Lawrence Livermore National Laboratory

low-level (radioactive) waste

Lockheed Martin Idaho Technology Company

molar

meter

management and operations

motor control center

mediated electrochemical oxidation

milligram

magnesium oxide

mixed oxidants

Morrison Knudsen Corporation

milliliter

millimeter

mixed low-level (radioactive) waste

million British thermal units

megaPascal

millirem

molten salt oxidation

Mixed Waste Focus Area

Mixed Waste Inventory Report

sodium chloride

sodium hydroxide

nanoCurie per gram

National Council on Radiation Protection and Measurements

needing development

National Environmental Policy Act

National Fire Association Protection

nanogram

nitrous oxide

nitrosyl chloride

nitrogen oxides

National Pollutant Discharge Elimination System

Nuclear Quality Assurance

Nuclear Regulatory Commission

oxygen

ozone

hydroxyl radical

Oak Ridge National Laboratory

operations and maintenance

Office Management and Budget

Occupational Safety \& Health Administration

passive/active neutron

polychlorinated biphenyls

Programmatic Environmental Impact Statement 


\section{ABBREVIATIONS AND ACRONYMS (Continued)}

PIC

PFD

PNL

POTW

PPE

ppm

ppmv

psi

psia

psig

PVC

RCRA

R\&D

RTR

RWMC

(s)

$s$

SAIC

SARA

SCC

scfm

SCWO

SEG

SEP

SGS

SI

SNR

SR

SSL

TCLP

TDS

TLCC

TOC

TRU

TSCA

TSD

TSG

TSWG

USC

UTS

UV

VOCs

WAC

WESP

WIPP

$w t \%$

$\mathrm{yr}$ product of incomplete combustion

process flow diagram

Pacific Northwest Laboratory

publically owned treatment works

personal protective equipment

parts per million

parts per million (by) volume

pounds per square inch

pounds per square inch absolute

pounds per square inch gauge

polyvinyl chloride

Resource Conservation and Recovery Act

research and development

real-time radiography

Radioactive Waste Management Complex

solid state

second

Science Applications International Company

Superfund Amendments Reauthorization Act

secondary combustion chamber

standard cubic feet per minute

supercritical water oxidation

Scientific Ecology Group

System Engineering Plan

segmented gamma scanning

system integration

sorting not required

sorting required

Scoping Study Layout

Toxicity Characteristic Leaching Procedure total dissolved solids

total life cycle cost

total organic carbon

transuranic

Toxic Substances Control Act

treatment, storage, and disposal

Technical Support Group

Tribal and Stakeholder Working Group

United States Code

Universal Treatment Standards

ultraviolet light

volatile organic compounds

waste acceptance criteria

Wet Electrostatic Precipitation

Waste Isolation Pilot Plant

percent by weight

year 
ABBREVIATIONS AND ACRONYMS (Continued)

$\begin{array}{ll}\mu \mathrm{Ci} & \text { microCurie } \\ \mu \mathrm{g} & \text { microgram } \\ \mu \mathrm{m} & \text { micrometer } \\ { }^{\circ} & \text { degree } \\ { }^{\circ} \mathrm{C} & \text { degree Centigrade } \\ { }^{\circ} \mathrm{F} & \text { degree Fahrenheit } \\ { }^{\circ} \mathrm{K} & \text { degree Kelvin } \\ \% & \text { percent } \\ \$ / \mathrm{lb} & \text { dollar per pound }\end{array}$




\section{INTEGRATED NONTHERMAL TREATMENT STUDY FINAL DRAFT EXECUTIVE SUMMARY}

\section{ES 1. Purpose}

The U.S. Department of Energy (DOE) has commissioned the Integrated Nonthermal Treatment System study (INTS) to evaluate alternative nonthermal treatment systems for mixed low level waste (MLLW). Previously, DOE commissioned a study focussing on thermal treatment, called the Integrated Thermal Treatment System Study (ITTS). The INTS study was performed in response to a recommendation by the panel that reviewed the ITTS study. The two studies share a common design basis, and both were developed to show systems that could be used to treat the variety of MLLW in the DOE complex. In the ITTS study, each alternative system was organized around a primary thermal treatment technology. In the INTS, treatment by nonthermal technologies necessitates several treatment technologies each of which is appropriate for a different waste type. Nonthermal technologies were arbitrarily defined as processes that operate below $660^{\circ}$ Fahrenheit $\left(350^{\circ}\right.$ Centigrade). Nonthermal technologies were combined in five alternative INTS systems which would be capable of treating all DOE MLLW waste. The purpose of this study is to evaluate the combination of technologies functioning as a system, and to compare the relative advantages of different systems.

The overall purpose of the INTS study is to assist DOE in the most effective use of their investment of resources, both in research and development and in facility development. Information about system performance and costs allows identification of research and development needs and the most efficient application of resources. One of the key values of the INTS and ITTS studies is the presentation of the multiple facets of handling DOE waste. Much more than simple technology assessments, these reports discuss the many aspects of finding an acceptable solution to the problem of treating DOE MLLW, from community acceptance through permitting, designing, constructing, operating, and decommissioning a treatment facility, to final disposal of treated waste.

DOE is evaluating nonthermal systems to address public concerns about thermal treatment technologies. A technical support group (TSG) was formed to assist and provide direction for the selection and evaluation of technologies. An additional purpose of the INTS study was to include Native American and stakeholder involvement in the technology assessment effort. A Tribal and Stakeholder Working Group (TSWG) was formed by DOE early in the INTS process. Public involvement at this early stage provided a record of non-site-specific public concerns to the DOE. To provide feedback to the tribal and stakeholder group, two technically trained members of the TSWG were also members of the TSG, and a Native American technical consultant was brought in to assist the TSWG. Stakeholder and Native American approaches to technology assessment add an additional dimension to engineering technical analysis. The inclusion of the evaluation principles developed by the TSWG is consistent with good engineering practice. 
Two other groups were brought into the study following the development of the second interim draft report. These were an independent peer review panel (IPRP) and the Mixed Waste Focus Area (MWFA) user's panel consisting of DOE site operations personnel.

\section{ES 2. Tribal and Stakeholder Participation}

The TSWG includes representatives of tribal and Native American ${ }^{2}$ governments, local governments, public schools, universities, regulatory agencies, public interest groups, environmental interest groups, business and industry, as well as interested members of the public. Their involvement provides a model for early and meaningful tribal and public participation.

The TSWG developed the tribal and stakeholder principles against which the technologies were to be selected and evaluated in this study. The key concerns expressed included the minimization of effluents, public and worker exposure, and waste generation. Social, cultural, and spiritual concerns were identified as important when implementing a system, and adequate information was to be made available to the TSWG during the study. The TSWG challenged the authors to include tribal and stakeholder considerations at this early stage, setting the groundwork for site specific solutions in the future.

\section{ES 3. Systems Selection}

About 210 technologies for treating MLLW were reviewed by the technical team for inclusion in the INTS. Most of these technologies were separation processes with very specific and narrow applications and were rejected because of their limitations to treat a range of waste types. For example, technologies that could treat sludge, soil and debris were favored over those that could treat only soil. Where there were variations to a general physical process, a representative technology was selected. The TSG initially reduced the number of technologies under consideration. Both the TSG and TSWG participated in further selection of the technologies to be considered as part of the five integrated systems. The treatment approaches included the basic steps of contaminant separation, organic destruction, and stabilization. Since treated solids would be stabilized, treatment did not include separation of inorganics, except mercury. Three primary organic destruction, three separation, and three stabilization technologies were selected for evaluation in integrated systems. These technologies are:

Organic Destruction: Mediated Electrochemical Oxidation

Acid Digestion

Catalytic Wet Oxidation

aIn this document the term "tribal" is used to include all Native American participants. 
Separation:

Vacuum Thermal Desorption, with leaching to remove mercury High or Low Pressure Debris Wash

Aqueous Wash

Stabilization:

\author{
Grout Stabilization \\ Phosphate Bonded Ceramic Stabilization. \\ Polymer Stabilization
}

As in ITTS, ancillary technologies are included in the systems for decontamination of metal and lead, stabilization of mercury wastes, air pollution control and several aqueous waste treatment methods. Destruction of organics in aqueous waste is provided by photo-oxidation, and treatment of gaseous process effluent utilizes a gas phase corona reactor. Also included are operations for (1) administration, (2) waste receiving and characterization, (3) sorting and size reduction of incoming waste, (4) support (facility operating equipment such as boilers, air compressors, and transformers), and (5) certification and shipping of treated waste. Except for the use of the gas phase corona reactor (GPCR) in the air pollution control subsystem, these ancillary technologies are the same as, or very similar, to those used in the Integrated Thermal Treatment Study.

The selected operations are integrated in alternative systems able to handle a range of DOE mixed waste streams. Major waste categories are:

- organic liquid wastes

- aqueous waste

- $\quad$ process residue and inorganic sludge

- bulk soil

- $\quad$ soft debris (plastics, paper, cardboard, wood, rags)

- $\quad$ open debris (drums and glass)

- complex debris (machinery, firebrick, molds)

- lead waste

- bulk mercury

- salt waste

- $\quad$ special waste (other hazardous metals, labpacks, cyanide, batteries)

Different subsystems were developed for treating organic liquid waste, inorganic sludge and process residue, soil, and debris. The sludge/residue stream is the largest percentage of the waste inventory, with over a third of the total mass. Debris is the next largest category, comprising nearly one quarter of the waste. Organic liquids represent a much smaller percentage of the incoming waste inventory, but the organic destruction process is key to meeting regulatory standards for the facility. 
The subsystems necessary for facility operation are integrated in systems. A total of five systems were developed to compare and contrast relative features. Each system contains the necessary subsystems to treat all DOE MLLW. The following subsystems are common to all systems:

$$
\begin{aligned}
& \text { - } \quad \text { receiving and preparation } \\
& \text { - } \quad \text { aqueous waste treatment } \\
& \text { - } \quad \text { metal decontamination } \\
& \text { - } \quad \text { mercury amalgamation } \\
& \text { - } \quad \text { special waste treatment } \\
& \text { - } \quad \text { air pollution control } \\
& \text { - } \quad \text { certification and shipping } \\
& \text { - } \quad \text { administration } \\
& \text { - } \quad \text { support }
\end{aligned}
$$

Subsystems which are not the same for all systems will be discussed in Section ES 4, System Description.

\section{ES 4. System Description}

System 1, the Grout Debris Case, was developed as the least complex while still meeting regulatory requirements. To take advantage of EPA's debris rule, System 1 does not include treatment of debris to remove organics; debris is only shredded and stabilized in grout. Thermal desorption, enhanced by vacuum to be effective at the low temperatures of INTS, provides removal of organics from soil and sludge. Vacuum operation allows desorption of high boiling point organics such as PCBs at $500^{\circ} \mathrm{F}$. The desorbed organics are collected as an offgas, condensed, and treated in the organic destruction subsystem. Organic destruction is accomplished by mediated electrochemical oxidation (MEO), a process that destroys organics in a nitric acid/silver nitrate solution by the presence of highly activated silver ions generated via an electrochemical cell. The subsystem is designed to recycle acid and silver. The distinctive subsystems of System 1 can be summarized as follows:

Separation:

Organic Destruction:

Stabilization:
Vacuum Thermal Desorption for treatment of process residue and soil with leaching to remove mercury.

Mediated Electrochemical Oxidation for treatment of organics and aqueous streams with greater than $1 \%$ organic concentrations.

Grout Stabilization for untreated debris and treated soil. Polymer Stabilization for treated process residue and salts. 
System 2, the Desorption Case, highlights vacuum thermal desorption for multiple waste streams. Vacuum thermal desorption has been developed as a batch process for the treatment of sludge, soil and debris. The organic destruction subsystem destroys organics under aggressive conditions, through the use of a hydrochloric acid, ferric chloride and catalyst mixture under pressures of 20 pounds per square inch at $300^{\circ} \mathrm{F}$ to $400^{\circ} \mathrm{F}$.

Separation:

Organic Destruction:

Stabilization:
Vacuum Thermal Desorption for treatment of process residue, soil and debris with leaching to remove mercury.

Catalytic Wet Oxidation for treatment of streams with greater than $1 \%$ organic concentrations.

Grout Stabilization for treated debris and soil.

Polymer Stabilization for treated process residue and salts.

System 3, the Wash Case, utilizes water and surfactants to wash residue and soil. Multiple recycling and separation processes allow heavy use of surfactant and effective removal of contaminants. Debris, in an agitated basket, is washed with water and surfactant using high pressure jets. Soft debris is washed with water and surfactant at lower pressure. Mediated electrochemical oxidation destroys organics.

Separation:

$$
\begin{aligned}
& \text { Aqueous Washing of soil and process residue. } \\
& \text { High Pressure Washing of complex and open debris. } \\
& \text { Low Pressure Washing of soft debris. }
\end{aligned}
$$

Organic Destruction:

Mediated Electrochemical Oxidation for treatment of organics and aqueous streams with greater than $1 \%$ organic concentrations.

Stabilization:
Grout Stabilization for treated debris and soil.

Polymer Stabilization for treated process residue and salts.

System 4, the Acid Digestion Case, uses a strong acid medium for organic destruction. In this process, the combination of highly reactive nitric acid in a solution of phosphoric acid effectively destroys organic compounds. Soil and debris are washed as in System 3, and sludges are desorbed. Treated soils and process residues are stabilized in a phosphate bonded ceramic which utilizes waste phosphoric acid from the organic destruction process. In other subsystems, multiple separation processes are employed to allow optimization of the process for specific waste streams. 
Separation:

Organic Destruction:

Stabilization:
Vacuum Thermal Desorption for treatment of process residue with leaching to remove mercury.

Aqueous Washing of soil.

High Pressure Washing of open debris.

Acid Digestion for treatment of streams with greater than $1 \%$ organic concentrations, and soft debris.

Grout Stabilization for treated debris.

Phosphate Bonded Ceramic Stabilization for treated soil and process residue.

Polymer Stabilization for salts.

System 5, the CWO Case, uses a combination of treatment subsystems and aggressive technologies. Systems 4 and 5 are analogous, using different subsystems for organic and soft debris destruction and for stabilization, but using the same subsystems for separation processes.

Separation:

Organic Destruction:

Stabilization:
Vacuum Thermal Desorption for treatment of process residue with leaching to remove mercury.

Aqueous Washing of soil.

High Pressure Washing of open debris.

Catalytic Wet Oxidation for treatment of streams with greater than $1 \%$ organic concentrations, and soft debris.

Grout Stabilization for treated debris and soil.

Polymer Stabilization for process residue and salts.

\section{ES 5. Mass Balance}

The INTS Study includes a detailed modeling of the transformations of the waste as it is treated and stabilized. The mass balance modeling is based on reported inventory of DOE MLLW, organized by waste type each of whose elemental composition and mass are described. With minor exceptions, the inventory was considered to represent all DOE MLLW. As in the ITTS, the study basis for the INTS assumed that the inventory of DOE waste would be treated at a single facility at a constant rate of $2927 \mathrm{lbs} / \mathrm{hr}$ over 20 years. This assumption forms a boundary condition for the size and complexity of a single DOE facility. The detailed mass balance, developed with ASPEN Plus software, provided quantification of the flow and

${ }^{2}$ The inventory is based on 1993 data (SAIC, 1993; Huebner, 1994) 
transformations of waste and additives through treatment to disposal. Tracking components through secondary treatment is necessary to track the fate of major contaminants such as organics.

\section{ES 6. Costs}

Total life cycle costs (TLCC) are an objective way to compare system performance. As in the ITTS, the approach is a total life cycle cost for waste treatment over a 20 year treatment period. The cost components are (1) studies and bench scale tests; (2) demonstration costs; (3) facility capital costs (design, inspection, project management, construction cost, and construction management); (4) preconstruction and preoperational activities (conceptual design, safety assurance, NEPA permitting, preparation for operations); (5) operations and maintenance (operating labor, utilities, consumable materials, maintenance parts and equipment, and maintenance labor costs); (6) decontamination and decommissioning; and (7) disposal cost.

Costs were developed for each subsystem and compiled to create a total system cost. Key elements are (1) equipment costs, developed from vendor data and sized based on the mass balance; (2) building costs, estimated by creating a layout for the equipment; and (3) operating labor, estimated by assessing the labor requirements for unit operations in a subsystem. Operations and maintenance are the most significant of all cost components at slightly over $50 \%$ of the TLCC for all INTS systems.

\section{ES 7. System Evaluation Criteria}

Criteria for system selection and evaluation were developed separately by both the TSG and the TSWG. To distinguish between them, they are referred to as the TSG technical criteria and the TSWG

principles. System evaluations were performed consistent with both the criteria and the principles. The criteria and principles overlap in many areas. The criteria and principles include the following elements: (1) process performance, (2) environmental impact, (3) social concerns, (4) worker health and safety, (5) cost, and (6) acceptability.

\section{ES 7.1 Process Performance}

System 1, which does not treat debris to remove organics, is considered to be less effective than the other systems. For all systems, the effectiveness of sludge treatment by either vacuum thermal desorption or aqueous washing is not demonstrated. Systems 3,4 , and 5 are considered more flexible and versatile than Systems 1 and 2 because more treatment methods are employed. System 1 is expected to have the highest availability (lowest mechanical failures). CWO and acid digestion are more corrosive and are expected to have less reliability and maintainability than MEO. Increased needs for size reduction are anticipated to adversely affect maintainability of Systems 3,4 , and 5. 
Hazardous waste generation is considered similar for all systems, although the wastes generated vary. All systems generate acids and salts. Systems 1 and 3 also generate waste silver.

\section{ES 7.2 Environmental Impact}

Systems 1, 2, and 3 result in more waste for disposal than Systems 4 and 5. Systems 4 and 5 break down soft debris to carbon dioxide, water and inerts, while the others treat and stabilize it. For the same reason, Systems 4 and 5 have more offgas generation. However, the offgas generated by nonthermal systems is more than an order of magnitude smaller than that of many thermal processes.

Environmental exposure from gaseous emissions is much less from nonthermal systems than from thermal systems, as volatilized metals and radionuclides are not anticipated from nonthermal processes. Mercury is the one exception, and control systems for its capture are included.

The environmental impact of contaminants leached from the disposed waste is a concern in all systems. The long term stability of organically contaminated debris in grout for System 1 may be a problem. Other systems have less contaminants in the solid waste. Polymer and phosphate bonded ceramic are perceived to be more stable than grout, an advantage for System 4. For all systems, disposal cells would be designed to minimize the potential of contaminants leaching from the waste.

\section{ES 7.3 Address social concerns}

Although several of the social concerns ultimately relate to a specific site, resources such as budget, staff, and schedule must be made available to the community prior to the site selection process. Respect for social, cultural, and spiritual values may affect siting, schedule, or requirements for community meetings. The community acceptance may be fostered through negotiations to achieve common goals. For all systems, evaluation of the acceptability by the community of a given site and processing facility would be necessary. Special site considerations would be considered when a site is selected. Similarly, intersite transportation would be addressed based on a specific site selection. Approximately one truck/day would be necessary to transport waste to the site. About two trucks/month would be sufficient for the transportation of reagents.

Land use for disposal or storage is higher for Systems 1,2 and 3. Retrievability of the treated waste from disposal could affect costs if a design optimizing for retrievability is more expensive. Although the cost relationship is not specifically calculated, clearly costs would be higher for Systems 1,2 and 3 due to the larger waste volume.

\section{ES 7.4 Worker health and safety}

Worker health and safety is preserved by proper facility design and operation. For processing alpha MLLW, several levels of confinement are required. The largest worker exposure risk for all systems is from 
sorting. Since System 1 has the least amount of sorting, worker exposure is minimized in this system. However, untreated debris in System 1 presents an additional exposure opportunity during grouting. Exposure risk is perceived to be somewhat higher for Systems 2, 4, and 5 with the aggressive processes of acid digestion and CWO. For all systems a detailed hazard and operability study must be performed to assess and continually update the safe operation of the facility.

\section{ES 7.5 Cost}

Research and development costs vary about $40 \%$ among the systems, but are minor compared to the total (an average of $\$ 160$ million out of $\$ 3.8$ billion). Results for the five INTS systems show that cost is not a significant discriminator between systems. The total life cycle cost, including disposal, ranged from $\$ 3.7$ billion to $\$ 3.9$ billion. This represents less than a five percent cost difference between the most expensive and the least expensive INTS alternative. Savings in treatment costs for Systems 1 and 2 are offset by increased disposal costs compared to Systems 4 and 5. The design, construction and operation of an MLLW treatment facility has many associated costs which are not a function of the type of treatment. The confinement zones are expensive to build and operate, and the use of robotics and overhead cranes to minimize worker exposure increase cost. The relative cost differences among the nonthermal systems is slight.

INTS costs are significantly higher than ITTS $(\$ 2.2$ to $\$ 2.6$ billion for cases with high temperature stabilization).

\section{ES 7.6 Acceptability}

Acceptability encompasses those aspects that would allow development of the system to proceed or would reduce the potential for success, such as technology maturity. The innovative nonthermal technologies for organic destruction, contaminant separation, and stabilization are not technologically mature. MEO is the most developed of the organic destruction subsystems, but is not a proven commercial technology. CWO is in the pilot stage, and acid digestion is only at the bench scale. The washing and desorption subsystems require demonstration, particularly performance with sludge. All stabilization technologies require additional development and demonstration. Phosphate bonded ceramic stabilization, particularly, would need significant development. The schedule for design and construction is affected by the technology status; System 1 could be built promptly while System 4 would be delayed pending further demonstration.

All systems are complex, but Systems 1 and 2 are less complex than the others. Systems 1 and 2 require the least building space. System 4 is the largest, because it requires polymer stabilization for soluble salts in addition to the phosphate bonded ceramic subsystem. Resource utilization is reflected in the utility costs, which are highest for System 5 and lowest for System 1. However, utility costs are less than $1 \%$ of the TLCC for all systems. Decontamination and decommissioning varies little for the five systems. Costs 
are slightly lower for the smaller sized buildings of Systems 1 and 2. Environmental restoration is expected to be similar for all systems.

\section{ES 8. Conclusions}

The INTS study developed functional requirements for systems to separate, destroy, or immobilize DOE MLLW. Technologies were evaluated and selected to be part of the study. Five alternative systems were developed and evaluated using criteria selected by both technical experts Native American and public stakeholders. The systems were found to have similar total life cycle costs, disposal volumes and gaseous effluent.

The results indicate the feasibility of treating DOE mixed waste using nonthermal technologies. The relative immaturity of these processes increases the schedule, cost risk, development time, and the permitting time required before the technologies could be put into operation. Volumes of waste for disposal are significantly higher for nonthermal systems than thermal systems. Regulatory compliance and environmental performance are less certain. Sorting and stabilization activities, worker health concerns, and land use requirements are the most significant drivers of the TLCC for nonthermal technologies.

\section{ES 9. Recommendations for Demonstration, Testing and Development}

A preliminary list of development needs was generated, based on the information gaps identified during the study. A development program is needed to provide the basis for the design and operation of production sized units. The program consists of an initial technology evaluation, bench scale tests, and selection of units for prototype demonstration. Most nonthermal technologies require further development at a sufficiently large pilot scale to validate laboratory and bench scale results, and to demonstrate performance at full scale. Pilot testing necessary for permitting these technologies must prove applicable to mixed waste matrices and contaminants.

\section{ES 9.1 Receiving and Preparation}

Improvements in front-end handling (receiving, characterizing, sorting, segregation and size reduction) would directly impact costs and worker exposure. Development of effective and efficient characterization methods and a waste characterization strategy for nonthermal processes would reduce costs. Improvements in material handling, sorting and segregation would have a direct impact on labor requirements and worker health and safety. Size reduction equipment can be unreliable, particularly with the small size requirements of the INTS processes. Further development is required. 


\section{ES 9.2 Air Pollution Control Subsystem}

The air pollution control subsystem requires further development to satisfy regulatory and system requirements. Requirements for the characterization of offgas need to be defined. Offgas treatment technologies need to be assessed for performance, reliability, maintainability, the effectiveness of organic destruction, and the potential for dioxin formation. The residual contaminants following treatment should be determined. Methods for removing mercury from air streams should be evaluated.

\section{ES 9.3 Aqueous Waste Treatment}

The aqueous waste treatment subsystem employs many common and well understood technologies. However, treatability studies on specific waste streams will be required. Operating and design requirements for various unit operations must be developed for DOE wastes. In cases where permitting requirements may limit aqueous discharge of some metals to very low levels, specific studies may be necessary.

\section{ES 9.4 Mercury Removal}

Thermal desorption or washing for removal of organics and mercury is performed in all systems. The effectiveness of mercury separation and the fate of mercury during processing should be demonstrated. The mercury leaching process requires further bench scale testing and pilot demonstration.

\section{ES 9.5 Waste Stabilization}

Phosphate bonded ceramic for the stabilization of various types of MLLW requires bench scale testing and pilot demonstration. Grout and polymer require optimization of waste form loadings and processing conditions which meet TCLP requirements. Optimizing stabilization can result in less leachable final waste forms and potentially lower costs.

\section{ES 9.6 Organic Destruction Processes}

For all of the organic destruction processes, further bench scale and pilot testing is necessary to optimize process design and evaluate operating conditions. Related recycling of acids and removal of inerts must be refined or developed. The flexibility of organic destruction with a range of feed types and particle sizes should be evaluated. The organic destruction effectiveness and the fate of metals and radionuclides must be assessed. The effect of process conditions on the offgas subsystem must be evaluated. Secondary reaction products must be evaluated and the potential to generate dioxins assessed. Process performance under upset conditions must be determined. 
Additionally, an evaluation of MEO technologies using silver, cobalt, and cerium might be performed. For CWO, pilot testing should be performed to evaluate the effect of corrosive reagents on equipment life and maintainability, and on construction materials. In the case of acid digestion, extensive bench scale and pilot testing will be necessary because of the immaturity of the technology. For acid digestion and $\mathrm{CWO}$, the effectiveness of reducing the volume of combustible debris, operating conditions for debris, and required size reduction must be determined.

\section{ES 9.7 Vacuum Thermal Desorption}

Bench scale tests and pilot tests will be required to demonstrate the feasibility of vaporizing organics from noncombustible waste. Process and operating optimization, and an evaluation of the fate of mercury, radionuclides, and organics in the desorber subsystem must be performed. Operating conditions for various feed types, including plastics and combustible debris, and the feed sorting required to optimize operation must be developed.

\section{ES 9.8 Aqueous Washing and High Pressure Washing}

Development work is needed to evaluate removal efficiencies for soil, inorganic sludges, and debris. Waste characterization necessary for surfactant selection must be determined. Optimization of processing conditions is needed. The fate of volatile components, metals, and radionuclides must be evaluated. 


\section{INTRODUCTION}

\subsection{Background}

The U.S. Department of Energy's (DOE) Environmental Management Office of Technology Development (EM-50) has commissioned an integrated nonthermal treatment system (INTS) study to assess alternative systems for treating contact-handled, alpha and non-alpha mixed low-level radioactive waste (MLLW). The MLLW in the DOE complex consists of a wide variety of organic and inorganic solids and liquids contaminated with radioactive substances. Treatment systems are needed to destroy organic material. Other operations are needed to stabilize the treatment residues, inorganic materials, and radionuclides prior to disposal in a MLLW disposal facility. Regulations promulgated by both DOE and the U.S. Environmental Protection Agency (EPA) govern the storage, treatment, and disposal of these wastes.

The purpose of the INTS study is to conduct a systematic engineering evaluation of a variety of MLLW treatment system alternatives. Because of public concern regarding emissions from thermal treatment facilities such as incinerators, DOE is evaluating nonthermal treatment technologies. This study is intended to inform the public, as well as DOE personnel, of nonthermal options for treatment of MLLW. Preconceptual designs (process flow diagrams, facility layouts, equipment lists, and material mass balances) are developed, and the relative merits and life cycle costs for each are identified. The study further identifies the associated research and development, demonstration, and testing and evaluation needed to ensure unit operability in the most promising system.

An additional goal of the INTS study is to include tribal and public participation in each stage of the technology assessment effort. Early in the INTS process, a Tribal and Stakeholder Working Group (TSWG) was convened for the purposes of assisting in determining the scope of the INTS study and demonstrating how technical and non-technical participants should work together in the early stages of technology planning. The primary focus of the TSWG has been to provide an opportunity for tribes and the general public to address the research and development phase of technology assessment by bringing them into the INTS process early enough to participate in "narrowing down" which nonthermal technology systems should be studied further.

In the selection of alternatives for this study, information was compiled from a variety of sources including the report "Overview of Nonthermal Mixed Waste Treatment Technologies" (DOE/EM-0281). Most often it came from equipment vendor catalogs. In cases where the central unit operation, e.g. vacuum thermal desorption, does not have published catalog data and prices, the appropriate vendors have been contacted to provide information for the preconceptual design of the system. The identification of a vendor in this study does not in any way constitute an endorsement of a particular product or item of equipment. Nor does the use of a piece of vendor equipment in a system necessarily constitute the vendor's endorsement that such use is the correct or preferred application. 
The authors have made every effort to use vendor information correctly, and to make accurate representations as to the performance of such equipment within the context of the total system. Most often the assessments are based on engineering judgment and not on actual equipment performance data. In no case has there been any willful intent to inaccurately describe the system performance. The opinions stated in this report are not a substitute for valid performance data or pilot testing. In addition, since there are virtually an infinite number of treatment system combinations, only a few can be evaluated in this scope of work.

The INTS study follows the two phase integrated thermal treatment system (ITTS) study (Feizollahi 1994, Feizollahi 1995). The ITTS study evaluated 19 thermal treatment systems based on incineration, plasma destruction (Phase 1), and innovative treatments (Phase 2). Thermal treatment is the most effective demonstrated technique for destroying toxic organic materials. However, to properly evaluate all technically feasible processes, this study has developed or proposed alternative nonthermal treatment concepts. This INTS report is an evaluation of five systems that are alternatives to thermal treatment of the DOE MLLW.

\subsection{Report Organization}

The draft INTS report is organized in nine sections with seven appendices. An executive summary is also provided.

Section 1, Introduction, provides the background for the INTS study, and discusses the volume of DOE MLLW and the treatment approach evaluated in the study. Section 1 includes a discussion of technical and regulatory requirements for treating this waste. Five treatment systems involving several nonthermal treatment technologies were selected for evaluation in the INTS study. The selection of the systems is discussed briefly in Section 1.7. DOE created two advisory groups for the study: the Technical Support Group (TSG) and the TSWG. The functions and involvement of these groups in this study, as well as the authors, is discussed in Section 1.4.

Section 2, System Descriptions, discusses the five systems selected for evaluation and each of the subsystems. The purpose of this Section is to explain the technologies and their integration in a complete treatment system.

${ }^{2}$ As defined by EPA in 40 CFR 260.10 , p. 10, "Thermal treatment means the treatment of hazardous waste in a device which uses elevated temperature as the primary means to change the chemical, physical, or biological character or composition of the hazardous waste. Examples of thermal treatment processes are incineration, molten salt, pyrolysis, calcination, wet air oxidation, and microwave discharge." 
Section 3, System Flow Rates, focuses on transformation of the waste at each processing step. The results of the detailed mass and energy balance for each of the five systems are presented in Section 3. The input waste and the output stabilized waste form are quantified, along with the gas and liquid effluents.

Section 4, Total Life Cycle Cost (TLCC) Estimates and Sensitivity Analysis, discusses the costs of the five INTS systems. The cost estimate basis is discussed and the cost estimate results are presented in graphic and tabular form. Sensitivity analysis is performed to indicate the relative effect of various factors, e.g. comparing construction cost variations with operating cost variations.

Section 5, Matching Technical Criteria with Tribal and Stakeholder Principles, discusses the evaluation criteria for the INTS study. Criteria were developed by the TSG and principles of evaluation were developed by the TSWG. This Section explains both, and provides a cross-reference for both approaches.

Section 6, Systems Evaluation, presents the detailed evaluation of the five systems with respect to the criteria and principles discussed in Section 5. Each system and subsystem is reviewed against the criteria, and concerns regarding system acceptability are discussed.

Section 7, Demonstration, Testing and Evaluation Needs, discusses the recommendations for further research and demonstration testing prior to design of the systems.

Section 8 , Conclusions, provides a brief analysis of the study results.

Section 9, References, lists references cited in this report.

Appendix A, System Requirements and Criteria, provides the detailed system requirements and criteria, supplementing the information provided in Section 1.

Appendix B, Systems Preconceptual Design Figures, includes a series of figures for the five INTS systems and their subsystems. Process flow diagrams (PFDs) show the basic flow path of waste through treatment. Functional Allocation Diagrams (FADs) illustrate the functions of the processing equipment in each subsystem. Perspective and plan drawings are three dimensional drawings showing a model layout of the equipment in each subsystem. Overall plan drawings show the total facility layout, including space allocation. Section 2 of the main report includes FADs and perspectives for the reader's convenience.

Appendix C, INTS Study Mass Balances Modeling, provides the detailed backup for the waste flow through the systems. It includes all input to the systems: waste, additives and reagents; all output from the systems: offgas, stabilized waste, treated water, recycled metals. Appendix $\mathrm{C}$ discusses the assumptions concerning the mass and energy balances made in Section 3. 
Appendix D, Technology and System Selection Process and Results, discusses in detail the evaluations performed in the selection of the five INTS systems and their subsystems. This complements the discussion in Section 1.

Appendix E, Tribal and Stakeholders Principles for use in Evaluating Technology Systems for Mixed Low Level Waste Treatment, presents the updated principles developed jointly by the TSWG and the Office of Science and Technology of the DOE.

Appendix F, Glossary of Terms, provides a glossary of technical terms to assist nontechnical personnel review this document.

Appendix G, Mercury Vapor Pressure Data, provides technical information regarding mercury and mercury compounds.

\subsection{Study Contents}

Each of the five alternative systems evaluated includes all subsystems necessary to perform waste receiving and inspection functions, waste treatment functions, and treated waste certification and shipping functions. The subsystems are the building blocks of the integrated nonthermal treatment systems.

Functional and operational requirements were developed for each subsystem. Conceptual designs were developed by using the functional and operational requirements as the design basis. Each conceptual design package includes the subsystem layout, equipment list, and a cost estimate. Individual subsystems were then integrated into a single nonthermal treatment system. The cost of treatment thus developed is added to the estimated disposal costs to provide the total life cycle cost of each nonthermal treatment alternative.

The various waste types identified for treatment in this study are described in the DOE Interim Mixed Waste Inventory Report (IMWIR) (DOE, 1993). Using the information contained in the IMWIR, a detailed estimate of the physical and chemical composition of the waste was developed for each DOE site. These data were combined to provide a typical composition of waste for the DOE complex (Science Applications International Company [SAIC], 1993). In addition, Section 3 provides detailed information on the specific composition of the wastes. The systems selected for evaluation were required to treat all types of waste stored in the DOE complex. This requirement created the need for several treatment lines to accommodate the range of MLLW encountered. 


\subsection{Tribal and Stakeholder Involvement}

The idea that tribes and the public should be given the opportunity to address policy issues before they are finalized is fundamental to the tribal and stakeholder involvement approach of the INTS study. Through various means, including participation in development of selection criteria and peer review of technical as well as non-technical findings, regulatory and technical representatives from tribal, as well as non-tribal, sources should participate equally in analysis of technologies and in decision-making related to the analysis. The idea of "public acceptability" should be viewed as a meaningful exercise in tribal and public cooperation in technology assessment.

In order to facilitate equal participation, DOE's Office of Science and Technology Development invited 24 representatives from a diverse range of interest areas to be members of the INTS TSWG. Membership includes participants from tribal governments, local governments, public schools, universities, regulatory agencies, public interest groups, environmental interest groups, business and industry, as well as citizens-at-large. Additionally, two members of the TSWG who have technical backgrounds were selected as liaisons from the TSWG to the TSG and participated in the TSG's technical analysis.

\subsubsection{The Purpose of Tribal and Stakeholder Involvement in the INTS Study}

Purposes and goals of the tribal and stakeholder involvement in the INTS study were first articulated at the August 8 and 9, 1995, TSWG meeting in Denver, Colorado. Goals outlined by the DOE varied to some degree from those presented by the working group members, but were generally agreed to be as follows:

- $\quad$ to involve the TSWG in a cooperative effort to determine the scope of the INTS study and to provide an opportunity for tribes and the public to address policy issues before they become final policy;

- to demonstrate that early and meaningful tribal and stakeholder involvement is beneficial in planning for technology development;

- to provide an example of how technical and non-technical, DOE and non-DOE, participants can work together in the early stages of technology planning;

- to give tribes and stakeholders the opportunity to participate directly and materially in the criteria selection for the INTS review;

- to provide opportunity for tribes and the public to participate in the research and development phase of technology assessment by bringing them into the INTS process early; and 
- to demonstrate a DOE commitment to a strong public education process focused on educating and informing the public so it can be capable of making informed decisions related to technology assessment issues.

TSWG members recognize that interested parties in the mixed waste treatment challenge have varying abilities to influence the treatment choice. One defined objective is the need to share their treatment choice selection with the diverse parties, while identifying technically feasible treatment options.

An additional goal of the study was identified by the TSWG as that of producing documents that identify the choices and the trade-offs within both the technical and the non-technical context. The operating assumption of this goal is that, when choices and trade-offs are clarified through a process seen as credible by all participants, the result will be a similar conclusion, or at least deliberate choices and trade-offs. Given this challenge, the outcome should be identified as a document or documents that explain the systematic consideration of the options with respect to the technical and non-technical criteria.

\subsubsection{Development of Tribal and Stakeholder Principles}

The TSWG drafted a list of non-technical criteria to be used in tandem with the technical criteria, already defined by the TSG as a baseline for down-selecting the $210+$ nonthermal technologies reviewed as part of the INTS study. The TSWG criteria became known as the "Tribal and Stakeholder Principles". The principles underwent multiple revisions over the course of four meetings. The final principles are presented in Appendix E. Due to time constraints, the original principles were used for evaluation criteria in this study. These are listed below:

- Minimize effluents that have the potential to carry hazardous materials or substances capable of causing adverse environmental consequences (i.e., minimize releases with a goal of zero releases);

- Minimize effects on human health and the environment, including worker health and safety. Minimize the potential for release of hazardous and radioactive materials from final disposal and storage products. Minimize the potential for accidents within the system.

- Minimize waste generation. Minimize the volume of final waste for final disposal and storage.

- Address tribal, social, cultural and spiritual concerns. Respect cultural values. Minimize land use for disposal and storage. Minimize transportation from one site to another. Reflect tribal and stakeholder considerations for specific sites. Dispose of wastes in a retrievable manner. 
- Provide complete information on health, safety and performance of technologies.

- Integrate or "bridge" the Integrated Thermal Treatment Systems Study with the Integrated Nonthermal Treatment Systems Study for overall comparison of systems;

- $\quad$ Consider the projected impact of future waste generation as well as of existing stored waste; and

- Clearly define the decision-making process and how tribes and the public can most effectively participate.

One of the primary concerns of TSWG participants has been the ability of the technical team to produce evidence that they have heard and valued tribal and stakeholder input.

In response to the TSWG, the INTS TSG created a matrix which matches its technical evaluation criteria with the TSWG Principles. It is presented in Section 5. To further identify the tradeoffs and choices presented by both the TSWG principles and the technical criteria, the systems are evaluated by both the principles and criteria. The technical criteria and the principles have common concepts. Indeed, major issues of the technical criteria and the principles overlap. These are minimizing effluent, minimizing waste generation, minimizing health/environmental effects and accidents, and minimizing the potential for release of harmful materials. In areas where there is no overlap, the principles and criteria are not in conflict but simply address different concerns. The major areas of difference are (1) the criteria include cost and schedule, and (2) the principles include social concerns that may be global or appropriate to a particular site.

The study results, discussed in Section 8 , indicate that cost is not a significant factor in system evaluation. Social considerations, such as respecting cultural values, are difficult to address in the absence of a particular site location. However, the cost impacts of social concerns can be assessed. These are discussed in Section 4.10. Likewise, time to address social considerations must be a factor in developing the project schedule. The inclusion of the social concerns at this stage of design provides critical input to DOE for future design development and project implementation. Overall, evaluation by either principles or criteria produces nearly identical results. The evaluations by both the principles and the criteria are presented in Section 6.

Other criteria-related issues raised by the TSWG include:

- Inclusion of regulatory compliance standards as part of the criteria screening process for the five systems

- Inclusion of material recycling and resource recovery as fundamental philosophic objectives of the study 
- Transportation impacts associated with the question of Regionally Centralized Treatment this might be effectively done by considering the transport of reagents to the facility and waste volume from the facility.

- Choice of nonthermal systems that can be economically and reliably used in capacities that might serve either local or regional treatment needs, thereby assuring that nonthermal systems would fit into any strategy considered in the Waste Management Environmental Impact Statement where national transportation issues are considered.

- Consideration of noise and visual impacts on public acceptability.

\subsubsection{Tribal and Cultural Issues}

Tribal governments place great importance on cultural issues related to environmental restoration and waste management activities on tribal lands, and believe that cultural resource discussions should take into account spiritual as well as cultural matters. To a tribal member, everything revolves around the environment and practices that might benefit the whole of humanity and future generations.

Because federal agencies have historically grouped tribes and stakeholders into the same category, these agencies have neglected to address tribal issues on a separate, nation-to-nation basis. Thus, tribal interests have been, knowingly or unknowingly, excluded from stakeholder discussions. In cases where agencies have consulted with tribes, it has most often been after the issuance of a decision, rather than before, thereby eliminating the opportunity for tribes to function in the decision making capacity to which they are entitled.

Additionally, the existence of tribal environmental quality departments and regulatory agencies has been regularly ignored, leaving tribes out of the realm of regulatory consultation. Tribal representatives on the TSWG strongly recommended that, in order to begin to incorporate cultural needs and values in the INTS, additional members drawn from tribal technical and regulatory offices should be added to the TSG, and should be consulted at the same level as current TSG members. This recommendation was offered as the strongest and most technically feasible action that the INTS study could accomplish to incorporate cultural values in the project. As a result of this recommendation, a tribal technical expert has participated in the INTS study since August 1996.

\subsubsection{The Value of Tribal and Stakeholder Involvement in a Technical Exercise}

In relation to the INTS review process, stakeholders have stated that they have gained a wider appreciation of the options available for lower temperature treatments and the comparative merits of both high and low temperature systems. Although some participants in the TSWG are concerned that biases in favor of thermal treatments are still pervasive, others indicate they have come to more fully understand the 
inherent trade-offs between the two approaches. For example, nonthermal systems supply the energy necessary to break organic molecular bonds through the use of aggressive chemical treatment of the organic contaminants and, therefore, use of chemical reagents to drive the conversion of hazardous organics is an unavoidable feature of nonthermal systems.

In terms of communication gains between tribes, stakeholders, and the DOE, both technical and non-technical participants in the INTS study have stated that they believe the direct interaction provided by the INTS stakeholder process (absent a community relations office) has provided increased understanding and credibility on all sides. TSWG members have gained an appreciation of the intense level of effort required to complete such an analysis. INTS TSG members have had the opportunity (some for the first time) to learn more about tribal and stakeholder issues and how they might apply to the technical tasks related to technology assessment and selection. Several TSWG members have recognized that, although it has been difficult to communicate certain technical concepts and tribal and stakeholder concerns, the majority of participants have exhibited a high level of patience and openness to the process.

Perhaps for the first time in the history of DOE stakeholder involvement, some TSWG members wondered if the public had been involved somewhat too early for effective input to occur. Several stakeholders expressed appreciation for the thorough and honest approach by the DOE in the area of tribal and public involvement.

\subsubsection{TSWG Comments on Systems Selection}

A variety of questions was raised by the TSWG regarding the systems selection process. The TSWG requested that the process of selecting technologies for inclusion in the study, as well as trade-offs and planning assumptions, be supported with explicit documentation. The process used to select five systems from more than 210 nonthermal technologies screened, as outlined in the preliminary version of the "Overview of Nonthermal Mixed Waste Treatment Technologies" Report (DOE/EM-0281), (1995), and presented to the TSWG at the January 1996 meeting in Phoenix, is described in Appendix D. This appendix is intended to provide an understandable description of decision-paths for the public.

TSWG members further commented that the logic for choosing the five systems should be constructed using the component subsystem objectives (e.g. separation of contaminants from the waste media or dissolution of the raw waste, and by what means). Because there has been a belief by some stakeholders that DOE will not consider nonthermal or low temperature systems fairly because of economic or other reasons, the logic by which the options were narrowed is described in detail.

Other comments included the recognition that, given the need for use of aggressive chemical treatments to break down organics, it will be important to avoid those that might carry significant risks of explosion, fire, toxic emissions, intractable effluents or prohibitive maintenance due to their particular reagents or operating conditions. Participants also suggested that direct attention to the use of the TSWG 
principles in screening chemical reagents to be used in treatment processes would provide convincing evidence of an effort to hear and value tribal and stakeholder input. An additional request was made to compare the systems in a matrix in order to clarify issues of worker risk, health risks to the general public, costs, volume reduction, stability of final waste forms, transportation impacts, present maturity of technologies, and environmental impacts, including land use.

\subsubsection{Presentation of Technical Data to the Nontechnical Reader}

It is clear that, in order to write a convincing public document, one must write clearly and carefully. This report is a technical document which uses technical terms. To facilitate public understanding, explanations of technical terms are provided in the glossary in commonly understood terms and commonly appreciated conceptual relationships (Appendix F).

The idea of how to present a final report that is accessible to the non-technical reader, and satisfies the needs of an audience more broadly oriented than the general cross-section of technical readers within DOE, was discussed at length by members of the TSWG. Some felt that writing separate reports for the general public and the technical community that must conduct the work would be a serious loss, and that two reports would leave the suspicion among some in the general public that the real decisions were communicated only to those on the inside. The continuing objective is to produce a report that increases the understanding of available choices.

The nonthermal systems report stands on its own, even though it elicits comparison with thermal treatment systems. The TSWG has requested that a bridge be created between the INTS and ITTS studies, with potential for some promising hybrids. This has been done in studies, entitled Comparison of Integrated Thermal Treatment Systems and Nonthermal Treatment Systems for Mixed Low Level Waste.

\subsubsection{Additional Observations of the TSWG}

In order to recognize and integrate the interests of tribal governments and communities in technology assessment efforts, in May 1995 DOE and the Western Governors' Association sponsored a forum to discuss and develop guidelines for working with tribes and communities on technology acceptance issues. This effort has come to be known as the Reno Forum.

Participants at the forum were tasked with (1) clearly defining the role of community in determining technology acceptance; and (2) developing formal guidance for federal and state agencies in working with tribes and communities on technology acceptance issues. The two-day forum was designed to produce a clear product: a draft technology assessment and selection guidance for federal and state agencies working with tribal governments and the public. 
Issues identified during INTS TSWG meetings have often echoed those raised at the Reno Forum. A number of INTS TSWG members were also participants in the Reno Forum. Both groups found the decision-making process for technology development to be unclear, with little perceived tribal and community impact on final decisions. What tribal and community involvement does occur is often late in the process, after many key decisions have already been made.

Furthermore, both groups believe that a site specific issue, such as acceptance, cannot be co-opted for broad or regional use. Participants in both groups agree that the concept of tribal and public cooperation in technology assessment should be used when characterizing community response, rather than approaching the issue only from the perspective of acceptance.

TSWG members who were also members of the Reno Forum have recommended to the TSG that the guidance resulting from the Forum be closely reviewed and incorporated, wherever possible, in INTS study activities. It is the hope of these TSWG members that efforts of the Reno Forum and the INTS project will be carried into future DOE technology assessment and will be adopted by DOE management. To support this goal, DOE invited a users panel of DOE site representatives to observe both the stakeholder process and products.

\subsection{Progress of Studies}

The ITTS Study was initiated in 1993 primarily to determine what research and development tasks would be required to support implementation of various mixed waste treatment technologies. Phase 1 of the study focused on using reasonably mature unit operations for the treatment system. After Phase I was completed, the study approach was found to be valuable for considering technology choices as part of a total system to treat MLLW. The initial study organized information on the relative life cycle costs, quantities of gaseous effluents, energy requirements, and volumes of solid residues from systems using different core technologies.

Phase 2 was subsequently initiated to evaluate a selection of new and innovative technologies being proposed by various companies or government laboratories for the treatment of MLLW. Phase 2 continued to focus primarily on thermally based technologies. However, two Phase 2 systems used chemical processes which operate at moderate temperatures.

The INTS study was initiated to evaluate nonthermal processes on a basis similar to that used for the 19 systems in Phases 1 and 2.

A report, titled "Comparison of Integrated Thermal Treatment Systems and Integrated Nonthermal Treatment Systems for Mixed Low-Level Waste" will evaluate the relative advantages and disadvantages 
of different alternatives. Another report will present enhanced nonthermal treatment studies, including hybrid thermal and nonthermal systems.

\subsection{Waste Profiles}

MLLW input as categorized for INTS is shown in Table 1-1. Several different categories of waste are described. For thermal treatment discussed in the ITTS Report, most waste was categorized as combustible or noncombustible, and effective treatments were discussed. Most nonthermal technologies evaluated, however, do not have the capability of processing a wide range of waste. For example, mediated electrochemical oxidation (MEO) can process organic liquid and sludge waste, but not other combustible waste. Aqueous washing techniques are appropriate for soil and possibly process residue, but may not treat all forms of debris.

Waste was categorized as follows:

- $\quad$ organic liquids or sludges

- inorganic sludges and process residues

- $\quad$ bulk soil

- $\quad$ soft debris (cardboard, plastic, wood, fiber reinforced plastic [FRP] boxes)

- $\quad$ open debris (drums, concrete, bricks, metal with surface contamination and glass)

- complex debris (bulk metals with entrained contamination, pipe, valves, pumps, ceramics)

- $\quad$ aqueous waste (contaminated with metals or $<1 \%$ organics)

- lead

- mercury

- $\quad$ special waste

The codes used within DOE based on the mixed waste inventory report (MWIR) (USDOE 1993) are listed in Appendix A (Table A-4). 
Table 1-1. DOE total system input MLLW

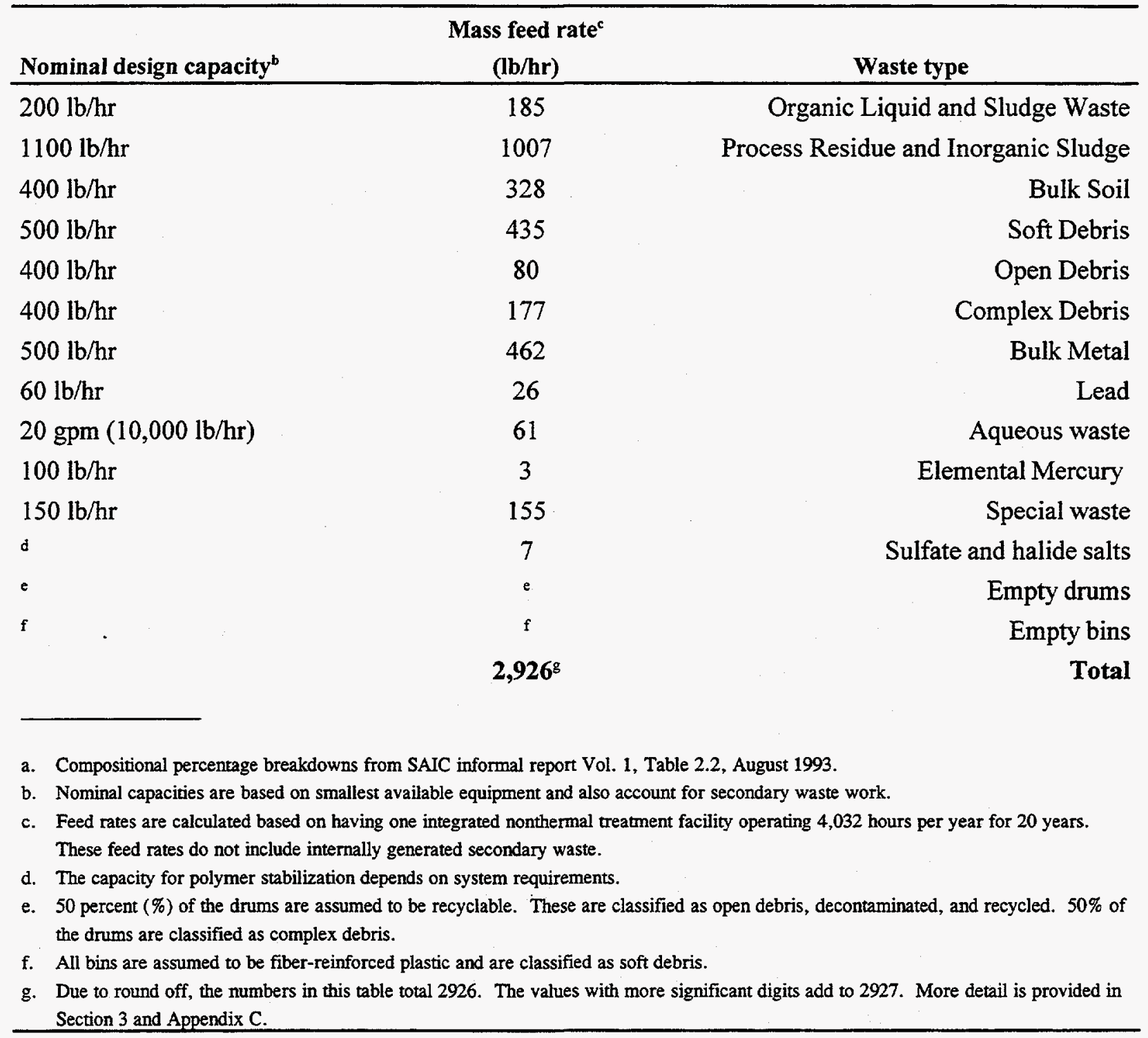

\subsection{System Selection}

Five systems were developed to showcase nonthermal technologies as part of a complete system to treat all of DOE's MLLW types. Technologies were selected based on their treatment effectiveness, implementability, nonthermal approach, minimal emissions, low environmental impact and their potential public acceptability. The selection criteria include the TSWG principles of minimizing effluent, waste generation, and effects on human health and the environment. Over 210 technologies were evaluated by the DOE prior to selection of technologies for the INTS study. "Overview of Nonthermal Mixed Waste Treatment Technologies", (DOE, 1995a). A more detailed description of the technologies comprising the five systems in this study can be found in the report "Description of Recommended Nonthermal Mixed Waste 
designed to demonstrate the effectiveness and applicability of each technology along with the combined effect of the technologies as a total system. Presentation of an overall system allows the consequences of a given technology's advantages and disadvantages to become apparent, e.g. the secondary waste processing costs. Table 1-2 provides a brief overview of the five systems.

The INTS systems are all based on nonthermal technologies, defined as processes that operate below $660^{\circ}$ Fahrenheit $\left({ }^{\circ} \mathrm{F}\right)\left(350^{\circ} \mathrm{Centigrade}\left[{ }^{\circ} \mathrm{C}\right]\right)$. This temperature is about the temperature of a commercial pizza oven $\left(600^{\circ} \mathrm{F}\right)$. The use of $660 \mathrm{~F}$ as the upper bound for the allowable temperature for process operations was somewhat arbitrary. At the time this study was initiated, it was thought that this temperature correlates with the generally agreed lower bound temperature threshold for the formation of dioxin by the recombination of chemical species in a combustion offgas system. Subsequently, it was determined that dioxin/furan formulation may occur in the presence of oxygen at temperatures ranging from $480^{\circ} \mathrm{F}$ to $930^{\circ} \mathrm{F}\left(250^{\circ} \mathrm{C}\right.$ to $500^{\circ} \mathrm{C}$ ). Some data indicate that $360^{\circ} \mathrm{F}\left(200^{\circ} \mathrm{C}\right)$ maybe a lower bound. Most INTS technologies selected operate at $\leq 480^{\circ} \mathrm{F}\left(250^{\circ} \mathrm{C}\right.$ ) except vacuum thermal desorption (up to $630^{\circ} \mathrm{F}\left[350^{\circ} \mathrm{C}\right.$ ], but with a nitrogen atmosphere). The effect of this maximum temperature bound is to limit the stabilization technologies and the metals processing technologies, eliminating vitrification and metal melting from the INTS systems. Appendix D details the technology selection process. Organic destruction technologies are based on chemical oxidation rather than thermal oxidation of organics. Processing of debris and residues is performed using physical and chemical processes rather than thermal treatment, including washing with detergents and high pressure sprays, decontamination by physical impact, vacuum desorption to remove organics, and acids to break down organics and combustible debris.

System 1, Grout Debris, was selected because it was thought to be the least complex and to have the lowest cost. The processes used were fairly well developed at the pilot scale. Organic destruction used MEO. A similar simple approach (vacuum thermal desorption) was used for soil and process residue to reduce equipment and operating costs. Secondary treatment subsystems were developed that would be applicable for other systems with only minor adjustments. These include stabilization, metals decontamination, mercury amalgamation, aqueous waste treatment, and air pollution control. For simplicity, and to take advantage of the EPA debris rule, debris was not treated prior to stabilization.

System 2, Vacuum Desorption, uses desorption as the major treatment process. Vacuum desorption of organics is employed for debris, soil and process residue. A catalytic wet oxidation (CWO) technology for organic destruction was substituted for the MEO selected in System 1. Other subsystems are identical to those of System 1. System 2 was selected to demonstrate improved treatment for debris and a grout form which has less risk of leaching.

System 3, Washing, is similar to System 1, but uses two washing technologies to process soil, residue, and soft, open and complex debris. This case highlights the advantages and disadvantages of washing as a treatment approach, especially when compared to vacuum thermal desorption. 
System 4, Acid Digestion, employs an innovative approach to organic destruction and treatment of soft debris: the use of a reactive hot acid environment to break down organics. Stabilization utilizes a new phosphate cement technology. A combination of vacuum desorption and washing subsystems used in Systems 2 and 3 are employed for treatment of soil, inorganic debris, and process residue. This system allows for evaluation of an aggressive chemical technology to destroy soft debris, and provides a more complete treatment process and reduced volume for disposal.

System 5, Catalytic Wet Oxidation, parallels the acid digestion case, but highlights a different, more aggressive technology for organic and debris treatment. Other subsystems are identical to those of System 4 except that polymer and grout are used for stabilization.

Table 1-2. Systems included in the Integrated Nonthermal Treatment System Study.

Designation System description

System 1 Vacuum thermal desorption of soil and inorganic sludge, silver MEO for organic

Debris Grout destruction, and grouting of untreated debris. Grout is the primary stabilization medium and polymer is the secondary stabilization process.

System 2 Vacuum thermal desorption of soil, inorganic sludges and debris, and the use of

Vacuum Desorption CWO for the destruction of organic liquids and sludges. Grout is the primary stabilization medium and polymer is the secondary stabilization process.

System 3 Washing of soil, sludges and debris with the use of silver MEO for the treatment of Washing System organic liquids and sludges. Grout is the primary stabilization medium and polymer is the secondary stabilization process.

System $4 \quad$ Acid digestion of soft debris and organic liquids and sludges, washing of open debris Acid Digestion and soil, grouting of complex debris, vacuum thermal desorption of inorganic sludges, and the use of phosphate bonded ceramic as a primary stabilization medium with polymer as a secondary stabilization process.

System 5 Catalytic wet oxidation of soft debris and organics, washing of open debris and soil, Catalytic grouting of complex debris, vacuum thermal desorption of inorganic sludges, and the Wet Oxidation use of grout for primary stabilization and polymer as a secondary stabilization process

\subsection{Technical Approach}

A key to successful evaluation of a treatment system is to use an integrated system engineering approach. The systems discussed in this report consist of all facilities, equipment, and methods needed for treating and 
disposing of MLLW currently stored at the DOE complex. This includes the complete process from waste receiving, characterization, sizing, treatment, air pollution control (APC), and secondary waste residue processing for eventual disposal. Systems that reduce volume during treatment might appear to cost more initially, but they can result in less volume for disposal. The systems engineering approach provides a uniform basis for comparing the merits of widely varying treatment alternatives. Table 1-2 provides a brief overview of the five systems.

The APC subsystem design which utilizes a gas phase corona reactor (GPCR), and the aqueous treatment system which uses photooxidation, are kept the same for all systems. The GPCR and photooxidation processes provide backup for destruction of organic materials in the gas stream and aqueous stream, respectively. Waste stabilization subsystem options included concrete, polymer, and phosphate bonded ceramic operations. Many more than five alternative systems could be configured. However, the five alternative systems evaluated in detail are judged sufficiently diverse to provide a range of performance and costs. The selection process preserved the ability to evaluate 1) the costs and benefits of nonthermal technologies and systems, and 2) the system effectiveness in treating the wastes to the required treatment standards within criteria established by tribal and stakeholder groups.

Disposal of hazardous solid residues, especially when radioactive, is a major public concern. Under the Resource Conservation and Recovery Act (RCRA), treatment alone will not render the wastes nonhazardous. The residues will contain hazardous wastes, as defined by RCRA, as well as radionuclides. Under EPA regulations, residues might have to be stabilized before disposal if leachability tests, using the Toxicity Characteristic Leaching Procedure (TCLP), are not met. To ensure RCRA compliance and to provide long-term isolation of residues, all systems provide residue stabilization by grout or polymer including treated solids. System 4 uses a phosphate bonded cement for residue stabilization. In this study, amalgamation was presumed to be the best stabilization process for mercury. Amalgamation is listed by the EPA as the best demonstrated available technology (BDAT) for radioactive contaminated mercury.

As part of the design process, functional and operational requirements, flowsheets, mass balances, and conceptual equipment layouts were developed for each system. Mass balances were performed to account for all materials treated or used in the processes. All secondary residues were processed in accordance with regulatory requirements, and the final volumes for disposal were estimated. Transportation and disposal cost estimates were applied to the disposal volume of each system as part of the PLCC estimate. Simplified system energy balances were conducted to ensure that no system was excessively energy intensive. Costs have been estimated assuming the system is a government owned and contractor operated (GOCO) facility. 


\subsection{Report Limitations and Assumptions}

This Section includes a brief summary of the major assumptions contained in this report, followed by a discussion of the uncertainties and limitations of this study. The assumptions summary is presented in bullet form, allowing this Section to serve as a quick reference for the reader.

\subsubsection{Treatment Facility Design Assumption Summary}

- Facility design must meet DOE order $6430.1 \mathrm{~A}$ for waste management facilities, and RCRA facility standards.

- Facilities are placed in seismic category 1.

- Waste is contact handled MLLW.

- The facility that houses the treatment systems will use the canyon concept where hot cells are separated from maintenance galleries and operating galleries.

- A system of airlocks and room ambient pressures are used to separate radiation zones.

- Hot cells are designed as alpha hot cells.

- The facility consists of four radiation zones that house the treatment subsystems and other support systems. These zones are as follows:

Low hazard areas: the zone that houses offices and packaged waste handling areas.

Moderate hazard areas: the zone that houses operating galleries and analytical laboratory.

Double confined alpha cells: the zone that houses maintenance galleries.

Triple confined alpha cells: the zone that houses alpha MLLW processing equipment.

- Inside hot cells material handling will be accomplished by overhead cranes, and self or remotely guided vehicles.

- Conveyor bins are used to transfer waste between subsystems. The conveyor bins employed are loaded from the top and unloaded from the bottom. 
- Filling and discharge stations are equipped with interlocking flanges that produce a seal between vessels.

- Solid waste treatment operations within each subsystem may be continuous processes. Transfer of solid wastes between subsystems are batch processes.

- Liquid and gaseous materials handling processes are continuous operations.

- Special design for radioactive criticality safety will not be required.

- The equipment must meet Nuclear Quality Assurance-1 (NQA-1) standards.

- The facility's units should be designed to handle at least $120 \%$ of the expected mass flow rates.

\subsubsection{Operating Assumptions Summary}

- The facility is designed to operate three shifts per day, seven days per week, 40 weeks per year at $60 \%$ operating efficiency for a total of 4,032 hours per year.

- $75 \%$ of all the waste received by the facility will require sorting to meet the Waste Acceptance Criteria (WAC) for the processes.

- $25 \%$ of all the waste received by the facility meets the WAC of the treatment processes and will not require further sorting.

- The treatment system receives incoming solid and sludge waste in drums, metal bins, and wooden or fiberglass boxes shipped to the treatment facility by onsite or offsite wheeled vehicles.

- Liquid waste is shipped to the system either by a pipeline, in tank trucks, or in containers placed on wheeled vehicles.

- Technologies selected are developed to an extent that is required to meet the system requirements for treating or otherwise handling MLLW.

- All debris waste will be identified.

- The treated solid waste output from the facility will meet all transportation and land disposal restrictions (LDR). 
- There is no radiological transformation of waste from one radiation category to another (i.e., alpha waste is not converted into transuranic waste through volume reduction and concentration of radionuclei to transuranic levels).

- The final waste form will meet disposal regulations as required by 40 Code of Federal Regulations (CFR) 268 and DOE Order 5820.2A.

- The final waste form will have alpha concentrations below 100 nanoCuries per gram (nCi/g).

- Air emissions will be continuously monitored and will meet discharge requirements.

- The process controls and instrumentation used are capable of providing real time process control data. No lag time is allowed.

\subsubsection{Cost Estimating Assumptions}

- Sorting costs also include costs associated with waste characterization.

- Technologies must be ready for pilot scale demonstration in 2 years, incorporation in a final design in 3 years, and construction in 5 years.

- Preoperations testing and startup will be accomplished in one year.

- The systems are designed for a 20-year operating life.

- PLCC estimates for facility construction are based on conditions at Idaho National Engineering Laboratory (INEL), in Idaho Falls, Idaho, including utilities, labor and related design, construction, operation, and management factors.

- The costs for each facility are divided into these six components: studies and bench scale tests, demonstration, production facility construction, preoperation costs, operating and maintenance (O\&M), and decontamination and decommissioning (D\&D). In addition, disposal costs are accounted for as part of the total PLCC.

- Planning studies and tests include three subcomponents: manpower, test equipment, and equipment installation.

- Demonstration costs consist of nine subcomponents: manpower during demonstration, building structure, equipment, design, inspection, project administration, indirect costs, construction management (CM), and contingency. 
- Equipment cost estimates are based on the use of stainless steel material for process vessels selected for ease of decontamination and maintenance, unless otherwise discussed.

- Whenever vendor quotes are based on off-the-shelf equipment, they are multiplied by an appropriate adjustment factor to allow for NQA-1 and other more complex requirements of the specific process.

- Design, inspection, project administration, indirect costs, construction management, and contingency cost subcomponents are developed using percentage guidelines provided by INEL.

- The allowance percentages are historical averages experienced by DOE contractors at INEL for the types of activities performed by waste management facilities.

- The maintenance cost subcomponent is divided into maintenance labor and maintenance replacement equipment cost. The annual maintenance equipment cost is $7 \%$ of the original equipment capital cost for most subsystems. For subsystems with corrosives, $10 \%$ of the equipment cost is estimated as the annual maintenance equipment cost. For subsystems with simple requirements, such as grout and metal decontamination, a factor of $4 \%$ is used. The annual maintenance labor cost is $250 \%$ of the maintenance equipment cost.

\subsubsection{Performance Assumptions}

- Vendor data are accurate and properly represent performance claims.

- Processes which destroy, separate or remove organics from waste will be sufficiently effective for the treated waste to meet RCRA's Universal Treatment Standards for disposal.

- Waste stabilized in the processes discussed (polymer, grout, phosphate bonded ceramic, and mercury amalgamation) will meet long term stability requirements such as TCLP and durability.

- The APC system will ensure air emissions meet the Clean Air Act. Continuous emission monitors will be available and reliable.

- The aqueous waste treatment subsystem will discharge water that meets federal and local discharge requirements.

- Decontamination processes will effectively remove radioactive components allowing the recycling of metals and lead within the DOE complex. 


\subsubsection{Report Limitations}

In a study such as INTS, the authors research available information, and evaluate that information using calculations, discussions with technology developers and independent experts, regulatory review, and engineering judgment. Throughout the process, assumptions based on current information and engineering practice must be made to provide the basis for evaluation. The summary of assumptions above indicates the range of assumptions necessary to perform a conceptual design study such as INTS.

Development of a system involves integrating process technology, material handling, process control and real time monitoring of the process. In the case of proven technologies, few technical assumptions are necessary because the basic process is well understood, and detailed information is available on multiple aspects of the technology, such as equipment, processing parameters (temperature, pressure, reactor residence time, etc.), space, maintenance required, chemical usage, energy requirements, and personnel requirements. For innovative technologies with limited or no operational data, the data that is available is considered and supplemented by assumptions and engineering judgment of the evaluators. The least technologically mature processes, with the least data for evaluation, have the greatest uncertainty. In the INTS study, the major technological components have high uncertainty.

Further uncertainty is introduced because of incomplete information about DOE waste. Contaminant concentrations are not known. Therefore, concentrations in the effluent streams (water, solid, air) can only be estimated generally.

To support reader recognition of technical maturity, graphics are used. In the FADs presented in Section 2, key equipment is identified in the legend as commercially available (CA), available but requiring system integration (SI), or needing development (ND). Commercially available equipment is supported by a firm vendor quote. Equipment requiring system integration is equipment that is available but not configured in the way described in the INTS system. Equipment needing development includes items where a concept exists but a commercial or proven application does not.

It should also be understood that the major treatment processes have not been fully demonstrated beyond the bench scale on the complex waste streams in the DOE complex. Thus, for the purposes of this study, the performance of these technologies and systems have been extended well beyond what has been demonstrated. The ability to treat wastes to meet the UTSs has been assumed.

\subsection{Summary of Regulations and Requirements}

This Section summarizes the regulatory assumptions and requirements used for developing the preconceptual design and PLCC estimates of the five systems. Detailed descriptions of specific regulatory assumptions and design bases and assumptions are presented in Appendix A. 


\subsubsection{Overall Regulatory and Permitting Issues}

It is assumed that a full environmental impact statement will be needed for the integrated nonthermal treatment facility. With a few exceptions, such as Rocky Flats pond waste and the Hanford Tank Wastes, it is assumed the facility will process all of the RCRA waste stored at all DOE installations. A facility permit will be required under RCRA for the treatment and storage of hazardous and radioactive waste. In addition, air emissions will be permitted under the Clean Air Act (CAA), and water discharges under either local regulations to a publicly owned treatment works (POTW) or the National Pollutant Discharge Elimination System (NPDES) to surface waters. Treatment units used to process polychlorinated biphenyls (PCBs) may require permitting under the Toxic Substances Control Act (TSCA).

It is assumed that listed and characteristic waste will be treated by the nonthermal treatment facility. Whether the waste is listed or not, the generator must determine if it exhibits any of the four characteristics (ignitability, corrosivity, reactivity, or extraction process toxicity) of hazardous waste by either (1) testing the waste, or (2) applying process engineering knowledge. Management of secondary waste will be in accordance with the EPA "derived from" rule. In this study, it is assumed that residue from treatment of all waste (except debris) is stabilized to meet the TCLP tests. Debris is stabilized in accordance with the debris rule.

\subsubsection{Department of Energy Requirements}

DOE policies governing design, construction, and/or operations are applicable to each of the five nonthermal treatment systems. Detailed design efforts will need to incorporate the directives of these referenced policies.

The magnitude of the compliance review activities will be related to how many systems are selected for further comparative analysis. Some of the DOE requirements focus primarily on a particular activity, e.g., hazardous materials transportation. Future design efforts must accommodate relevant procedures identified by these policies.

In addition to DOE policies, guidelines from government agencies, particularly those from the EPA, must also be reviewed to accomplish various permitting requirements. The EPA Guidance Document, Quality Assurance and Quality Control for Waste Containment Facilities, September 1993, is an example of such a guideline. Relevant regulatory guidance for selected technologies may be dependant on the results of development efforts. For example, disposal permitting will require information about the final waste form, using preliminary information on waste products developed from pilot tests or demonstration projects. The relevant DOE policies and guidelines are discussed in Appendix A. 


\subsubsection{EPA Requirements}

1.10.3.1 Clean Air Act. The CAA requires that all source categories, including nonthermal treatment units, be identified, and that technology-based emission standards be promulgated for each category. Because treatment systems are nonthermal, air emission volumes are small, less than 300 cubic feet per minute (cfm). Emission limits were developed for the ITTS and are listed in Appendix A of this report. Most of these limits are more stringent than required for nonthermal treatment. In any case, at the low temperatures of the INTS processes, metal volatilization and dioxin production are not anticipated. INTS includes appropriate technology to treat air pollutants and to monitor emissions.

1.10.3.2 RCRA. RCRA requires a facility permit for the treatment and storage of hazardous and mixed waste (40 CFR 264). The part B permit would be subject to a number of sections in 40 CFR 264 and 270. Permitting requirements for miscellaneous units and facilities (40 CFR 264.600 Subpart $X$ and 270.33) and for tank systems ( 40 CFR 264.190) are applicable to all INTS systems. Subpart X is particularly applicable to tank-like waste. For a Subpart X permit, steps similar to those for permitting an incinerator under Subpart O (40 CFR 264.340) apply. The major concern is migration of contaminants as emissions, to groundwater, etc. A risk assessment, groundwater modeling, and quantification of air emissions are necessary. For each treatment process, the method must be shown to be effective, as discussed in 40 CFR 270.23. The EPA permit writer would need to understand each treatment unit in the permitted facility. A Research Development and Demonstration (RD\&D) permit would be needed to prove the various treatment methods are effective. Under the RD\&D permit, treatment of expected waste types (actual waste or simulated) would be demonstrated for each type of waste that will be accepted.

Demonstrations of process effectiveness performed under the RD\&D permit could be used to support the Subpart X permit. These effectiveness demonstrations provide a "trial burn equivalent," reducing the time to get a Subpart X permit compared to a Subpart O permit. An EPA representative indicated that 2 to 3 years would be a reasonable expectation of permitting time for a Subpart $O$ permit. The total permitting time for Subpart $\mathrm{X}$, including the RD\&D permit, would likely be longer.

As part of the facility operation, Universal Treatment Standards (UTS) for land disposal and liquid effluents (40 CFR 168) will apply.

1.10.3.3 EPA Debris Rule. EPA defines debris and treatment standards for hazardous debris in 40 CFR Part 268.2. Debris is defined as solid material exceeding 60 millimeters ( $\mathrm{mm}$ ) in length that is intended for disposal, and is (1) a manufactured object, (2) plant or animal matter, or (3) natural geologic material. Process residuals, such as cemented sludge, or materials such as lead for which a specific treatment standard is listed in Subpart D Part 268.40 though 268.45, are not considered debris under this rule.

The alternative treatment standard for hazardous debris is as follows: (1) extraction (physical, chemical or thermal); (2) destruction (biodegradation, chemical oxidation, chemical reduction, or thermal destruction); 
and (3) immobilization (macroencapsulation, microencapsulation, or sealing). Wastes which are immobilized only must be considered hazardous and must be managed as mixed waste.

Nonthermal destruction technologies require an equivalency determination to demonstrate that the nonthermal technology is capable of achieving the contaminant level specified in thermal destruction technology performance standards or in the UTS. Physical extraction performance standards are based on removal of the contamination layer from the debris. Chemical extraction performance standards are based on the dissolution of the contaminants in the cleaning solution. Thermal extraction performance standards require an equivalency determination with other extraction technologies, e.g., clean to the BDAT levels.

1.10.3.4 Input Waste characterization. Each step of the nonthermal treatment process will require waste characterization, starting with the individual waste containers, and ending with the final waste form, offgas, and scrubber solids. Waste characterization determines the appropriate treatment subsystem and the appropriate treatment train within the subsystem for that waste.

$\mathrm{X}$-ray radiography can be useful in presorting waste before processing. Throughout normal operation, the owner/operator of a treatment unit must analyze a waste to verify that waste fed to the unit is within the physical and chemical limits specified in the permit.

The INTS will treat the waste types listed in Table 1-1. Table 1-1 lists the nominal capacities of the subsystem. Table 1-3 shows the waste input rates to INTS subsystems. The input waste assumed in the INTS study is contact-handled $(\mathrm{CH})$ mixed radioactive waste (as defined in the 1993 mixed waste inventory report by the DOE), including alpha-contaminated MLLW which contains a variety of hazardous materials.

Waste received by the facility is characterized in accordance with the facility waste analysis plan. This includes a review of the waste profile data sent by the generators, and sampling and analysis to characterize the waste for acceptance and treatment by the facility.

Characterization includes identification of chemical, radiological and physical properties. Chemical characterization may be performed to confirm RCRA waste codes and concentration of RCRA controlled substances. Physical characterization may include waste density and particle sizes, and hardness to determine treatment needs. Radiological characterization may include gross gamma, beta, and alpha analysis including surface dose determination.

Input waste is alpha and nonalpha MLLW. Alpha low-level waste has more than $10 \mathrm{nCi} / \mathrm{g}$ of TRU, but less than $100 \mathrm{nCi} / \mathrm{g}$. All waste considered in the INTS study is $\mathrm{CH}$ waste. If high-activity waste inside shielded containers is found, it will be rejected and returned to its point of origin. Consequently, the system will be designed with minimum shielding. Some shielding could be necessary in areas with concentrated TRU waste or fission-product radionuclides. However, shielding is not considered to be a factor in the 
relative performance of the different systems. No attempt has been made in this study to provide for shielding in any unit operation.

A direct radiological reading (alpha and beta-gamma) of each container and transport vehicle is taken using survey meters such as "Ludlum model 16 meter.with a sodium iodide crystal". Radiological characterization includes a dose rate survey to verify that the radiation dose rates are below U.S. Department of Transportation (DOT) limits. For enclosed vehicles the measured dose rate must be below 10 millirem (mrem) per hour beta-gamma at a distance of 2 meters from the trailer frame. The contact dose rate at the surface of the vehicle or trailer may not exceed 200 mrem per hour beta-gamma. The contact dose rate of any individual container may not exceed 200 mrem per hour beta-gamma. For containers registering above 200 mrem per hour, a non-conformance and corrective action report will be completed. Special handling may be required.

Liquids are analyzed for a number of parameters such as redox potential, organic content, metals content, total suspended solids, total dissolved solids (TDS). Redox potential is determined by measurement against a standard reference electrode such as the silver - silver chloride electrode. Test methods cited in the 9200 series of methods in EPA publication SW-846 include chemical oxidation demand (Standard Methods); ion chromatography (IC) (EPA Method 7199 for hexavalent chromium and Method 9056 for anions); and EPA Method 6020 for metals and their oxidation species by inductively coupled plasma spectroscopy (ICP).

EPA Methods 8240 and 8270 may be used to test for organics. Cations and anions may be tested by IC to determine if brine is carried over during the heating process using Standard Method 9056 for anions and Method 6010 (ICP) for cations.

Solids may be filtered from solutions and subjected to additional analysis using EPA Method 6010 by ICP. Spent carbon will require sampling to determine if the organic loading has triggered RCRA LDR for volatile or semi-volatile compounds, and polynuclear aromatics. A solid sample is scooped out of the carbon vessel and sent to the laboratory in a zero headspace container. Most analytes adsorbed to the carbon surface can be detected with high performance gas chromatography coupled with mass spectroscopy (GC/MS) using EPA Methods 8240 and $\mathbf{8 2 7 0}$. Other EPA methods include GC methods 8010 for chlorinated solvents, 8020 for benzene, toulene, ethylbenzene, and xylenes (BTEX), 8080 for chlorinated herbicides and PCBs, and the 8300 series for high performance liquid chromatography. EPA method 8240 is used to test the effluent after granular activated carbon (GAC) treatment. Liquid samples are collected in standard glass bottles and sent to the lab for analysis. 


\subsubsection{Waste Treatment.}

Waste will require pretreatment and primary treatment. Secondary wastes generated (e.g., gas and water streams) will also require treatment. The waste input to the various subsystems where treatment will be performed is listed in Table 1-3. Detailed waste breakdown is shown in Tables 3-1 through 3-12.

Table 1-3. Waste input rate to treatment subsystems.

\begin{tabular}{|c|c|c|}
\hline Waste Type & Treated by Subsystem & $\begin{array}{l}\text { Mass Input } \\
\text { Rate (Ib/hr) }\end{array}$ \\
\hline Organic Liquid and Sludge Waste & Organic Destruction & 185 \\
\hline Process Residue and Inorganic Sludge & Residue and Sludge Treatment & 1,007 \\
\hline Bulk Soil & Soil Treatment & 329 \\
\hline Soft Debris & Soft Debris Treatment & 435 \\
\hline Open Debris & Open Debris Treatment & 80 \\
\hline Complex Debris & Complex Debris Treatment & 177 \\
\hline Metallic Waste & Metal Decontamination & 462 \\
\hline Lead & Lead Recovery & 26 \\
\hline Elemental Mercury & Mercury Amalgamation & 3 \\
\hline Aqueous Waste & Aqueous Waste Treatment & 61 \\
\hline Special Waste & Special Waste Treatment & 154 \\
\hline Halide and Sulfate Salts & Polymer Stabilization & 7 \\
\hline Total & All Subsystems & 2,927 \\
\hline $\begin{array}{l}\text { Note: Waste input mass flow rates shown in t } \\
\text { Secondary wastes are included in Section } 3 \text {. I } \\
\text { total of } 2927 \text {. }\end{array}$ & $\begin{array}{l}\text { tinclude secondary wastes generat } \\
\text { ff, the values shown in this table to }\end{array}$ & $\begin{array}{l}\text { nt subsystems. } \\
\text { than the actual }\end{array}$ \\
\hline
\end{tabular}

Pretreatment. Waste pretreatment will be compatible with requirements of the primary waste treatment technology. Extensive sorting will be necessary to divide the waste into the appropriate treatment categories as listed in Table 1-3. Shredding and size reduction by cutting are provided as needed for specific waste streams. For example, bulk metal (e.g., ferrous metal and lead objects larger than about 4 inches or 10 centimeters $[\mathrm{cm}]$ in any dimension) will be segregated and sent to the appropriate treatment subsystem. Cemented sludges will be size reduced. Containers of potentially temperature-sensitive materials, such as pressurized gas cylinders, will be set aside and safely depressurized, emptied, or otherwise made safe for the next step of treatment. Debris which will be treated under EPA's debris rule will be segregated from other waste and processed separately. Parts of the system that process alpha-contaminated MLLW will operate at slightly negative pressure to avoid release of contamination outside the units. 
Organic destruction. Organic liquid and sludge waste will be treated to break organics into primary compounds carbon dioxide $\left(\mathrm{CO}_{2}\right)$ and water $\left(\mathrm{H}_{2} \mathrm{O}\right)$. Organic waste will be either oxidized or decomposed in an acid environment at temperatures below $660^{\circ} \mathrm{F}$ to achieve destruction of RCRA-controlled materials. Levels of substances in the offgas from organic treatment will meet requirements set forth in RCRA, CAA, and other regulations controlling air pollution.

Process residue, soil and debris. Residue, soil and debris materials, such as inorganic sludge contaminated with chlorinated solvents, will be treated to remove organic RCRA-controlled contaminants, if present. Treatments include one or more of the following, depending on the system: vacuum desorption, aqueous washing, agitation washing, high pressure spray washing, and organic destruction of contaminants in an acid medium. Organics separated by desorption or washing will be treated in the organic destruction subsystem. Offgas generated in these low temperature processes will be treated to meet regulatory Table requirements listed in RCRA, CAA and other air pollution regulations. Treated process residue will be stabilized in polymer, while treated soil and debris will be stabilized in grout.

Metals processing. Bulk ferrous metals with surface contamination will be treated by decontamination to free-release standards of DOE Order 5400.5. Metals meeting this standard will be recycled within the DOE complex. Bulk metals with entrained contamination are treated as complex debris which in all cases results in final grouting.

Lead recovery. Bulk lead such as lead brick will be treated by surface decontamination if it is reasonably determined to have no activation products. Other lead, such as blankets, wool, and activated lead, which cannot be effectively decontaminated, will be size reduced and grouted to EPA microencapsulation standards.

Mercury separation. Elemental mercury will be treated by amalgamation. Mercury contaminated debris will be separated from other debris and treated first by vacuum desorption, followed by mercury leaching or aqueous washing, followed by stabilization. In this study, treatment of mercury contaminated debris is provided for in all systems. The extent or concentrations of mercury contaminated debris are not available. Therefore treatment is provided, although in some cases, mercury is not specifically identified as a contaminant. Waste characterization and manifest information will be used by operations personnel to select the appropriate treatment. In the case of System 1, mercury contaminated debris will be treated in the process residue subsystem. Recovered elemental mercury will be treated by amalgamation.

Air Pollution Control. Gaseous streams will be treated in the air pollution control (APC) systems. Air systems will meet or exceed current and anticipated state and federal regulations. Performance specifications will be set to meet or exceed current regulations.

Aqueous Waste. Aqueous waste will be treated to destroy organics and to separate and immobilize the contaminants in the final waste forms. The treated water will be further treated to remove trace amounts of contaminants and recycled. Highly acidic or alkaline aqueous waste will be neutralized. Organic liquid waste 
will be treated, and the organic components destroyed. Cleaned water not recycled will be discharged in accordance with local regulations or an NPDES permit.

\subsubsection{Waste Output.}

Form of output waste. The characteristics of the system's polymer, ceramic and grout waste output must meet current and anticipated disposal regulations, such as 40 CFR 268, for substances regulated by RCRA and DOE Order 5820.2A. The desired output waste form is a stable, leach-resistant, high-integrity solid produced by one of three processes: solidification in polymer, phosphate bonded ceramic, or grout. Where possible, in accordance with the debris rule, the final waste form will be treated and stabilized to reduce the cost of disposal (Subtitle D versus Subtitle C). If the waste qualifies as TRU waste, then the final waste forms will comply with Waste Isolation Pilot Plant (WIPP) waste acceptance criteria and TRUPACT II packaging and shipping criteria. If waste is alpha MLLW, the chosen final waste form will be able to satisfy performance assessment requirements for each site for alpha concentrations below $100 \mathrm{nCi} / \mathrm{g}$.

Characterization of outgoing waste includes surface radiological surveys and physical surveys. Sampling and analysis for chemical and radiological tests, such as the toxicity characteristic leaching procedure (TCLP), will be performed at the treatment tanks.

Stabilized Waste. Process sampling is performed by taking a sample from filled drums after the process is complete and the waste has cured. The sample is subjected to extraction by the TCLP using EPA Method 1311. The extract is tested to determine if the treatment standard has been achieved. Testing for metals and organic components is required for characteristic RCRA waste. This will require the use of multiple EPA methods such as Method 6010 ICP for metal, GC/MS Methods 8240 and 8270 for organics, and other methods if pesticides are suspected.

Macro-Encapsulated Waste. This is generally used for debris that does not have a RCRA LDR, or is subject to a treatment variance. Analytical requirements revolve around alpha and beta surface wipe samples on the exterior of the stabilized mass. A radiological survey of the macro-encapsulated mass will be performed to confirm that the treatment process has successfully encapsulated the matrix. EPA Method 9310 will be used for gross alpha and beta testing, while EPA Method 9315 will be used for radium isotopes.

Surface Decontaminated Waste. Radiological survey of the decontaminated surfaces may be performed by wipe tests to confirm that the treatment process has been successful. EPA Method 9310 must be used to test for gross alpha and beta. EPA Method 9315 must be used to determine if radium isotopes are present.

Sampling Frequency. Statistical analysis must be performed to determine the sampling frequency and the number of samples to be collected. Sampling must ensure that at least $90 \%$ upper confidence limit for each control constituent is met for incoming and treated waste. 
Disposal. In this study costs for engineered shallow land disposal facilities have been used for all wastes. For purposes of calculating transportation and disposal costs, it is assumed that the output low-level waste (LLW), MLLW, alpha LLW, and alpha MLLW will be shipped to and disposed of at the Nevada Test Site. However, for purposes of selecting an output waste form, it is assumed that the Nevada Test Site might not be available. Therefore, the final waste form will be stable so that it can be accepted at surface disposal facilities at other major DOE installations, including Hanford Site, Washington; Idaho National Engineering Laboratory, Idaho; Oak Ridge Reservation, Tennessee; Los Alamos National Laboratory, New Mexico; and Savannah River Site, South Carolina.

All wastes from other sites will be transported to one of these sites for disposal. An average cost of disposal will be used for all shipments.

Packaging. The output waste will be packaged in 55-gallon drums meeting DOT requirements. Final waste forms will meet contact handling requirements with no additional shielding other than the shipping container.

\subsubsection{Operation and Design Considerations.}

Facility design. Facilities handling MLLW are classified as seismic category 1. Buildings that handle MLLW are classified as moderate-hazard facilities (Kennedy et al. 1992, p.41). The facilities will be designed in accordance with applicable local, state, and federal regulations, including general design criteria for DOE facilities contained in DOE Order 6430.1A. Specifically, RCRA places design constraints on storage and treatment facilities, and places performance specifications on the waste stabilization process. The facility's units will be designed to handle at least $120 \%$ of the expected mass flow rates shown on process flow diagrams (PFDs) for each system.

To ensure the safety of personnel, the system for treating alpha LLW and alpha MLLW should be designed to accommodate TRU waste with radioactive levels exceeding $100 \mathrm{nCi} / \mathrm{g}$. The primary design requirement is triple containment of alpha contaminated wastes.

Since the treatment systems are used for alpha and nonalpha waste, they will have a tertiary containment system for all process steps from waste sorting through waste stabilization. Only two levels of containment are required for other processes involving materials with a limited potential for becoming airborne.

Technology maturity. Acceptable technologies are those that have successfully completed the proof-of-principle phase, or those that have successful operating experience in other industrial applications. Technologies must be ready for pilot scale demonstration in two years, incorporation in a final design in three years, and construction in five years. Some of the innovative systems evaluated in INTS, particularly for organic destruction, may not be able to meet these criteria. 
Operating conditions. The preconceptual designs will be based on the assumption that MLLW inventories will be processed in treatment facilities, placed in storage facilities, and disposed of during a 20 -year operating period. During this period, the treatment facility will operate 24 hours per day, seven days per week, 40 weeks per year at $60 \%$ of capacity during operation. This is equivalent to operating 4,032 hours per year of operation. In unit operations with small capacities, where a three-shift operation is not justified, a single shift per day or part-time shifts are assumed.

\subsection{INTS Study Team}

The overall project was initiated and directed by Carl Cooley of the DOE EM-50. A team of employees of Lockheed Martin Idaho Technologies Company (LMITCO) and Morrison Knudsen Corporation's Environmental Group (MK) performed the engineering and analysis for the INTS study, with support from the Technical Support Group of INTS. The Technical Support Group includes DOE headquarters, DOE site, EPA, and consultants. The INTS study was also aided by the input of the TSWG. In addition to the authors, other individuals and organizations furnished their expertise and services for the INTS study. The chart below shows the project structure. 


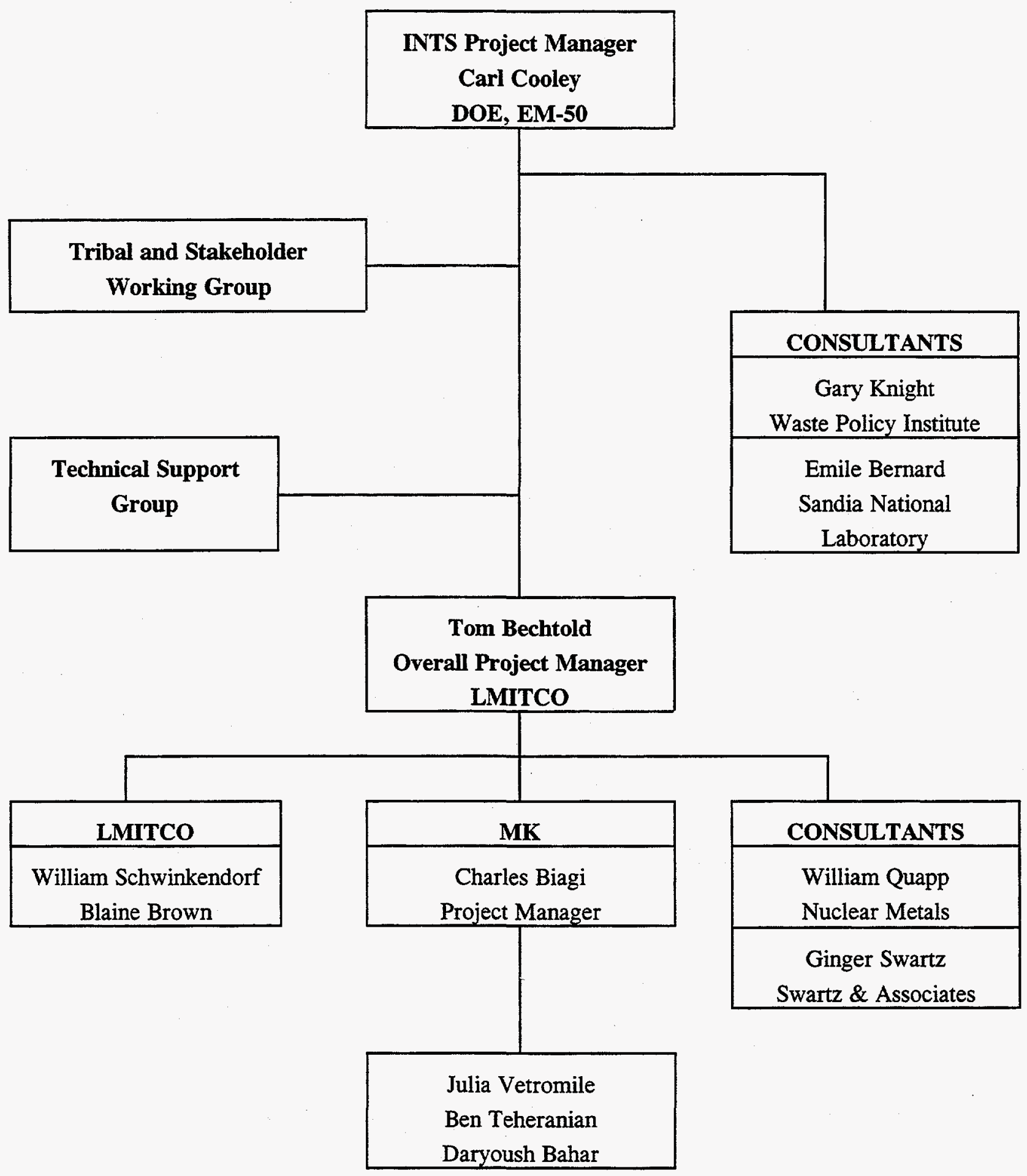




\section{SYSTEMS DESCRIPTIONS}

Five distinct nonthermal treatment systems were examined in the INTS study. All systems use extensive characterization and sorting, the gas phase corona reactor for destruction of any residual organic vapors in the offgas organic treatment, grout for debris stabilization, surface decontamination of bulk metals and recyclable drums, and photo-oxidation destruction of any residual organic materials in the aqueous treatment system.

Each system consists of several subsystems. Most of the subsystems are identical or only slightly modified for the different cases. The subsystems which are consistent for all systems are the following:

- administration

- receiving and preparation

- aqueous waste treatment

- metal decontamination

- mercury amalgamation

- lead decontamination and stabilization

- special waste treatment

- air pollution control

- grout stabilization

- polymer stabilization

- certification and shipping

- support

In the case of grout and polymer stabilization, the subsystems are functionally the same for all systems, although operation costs vary due to different throughputs.

Many of these subsystems are similar to those in ITTS, but thermal processes, such as metal melting, are not included in the scope of the INTS study. The APC subsystem is much smaller than the ITTS equivalent, and is based on dry filtration, wet offgas scrubbing, and organic destruction in the GPCR. The aqueous waste treatment subsystem includes photo-oxidation for organic destruction, along with the typical treatments of neutralization, precipitation, filtration and evaporation. Subsystems that are varied in the different systems studied include separation of organic materials from waste matrices, organic destruction, and the final stabilization processes.

The description of System 1, Grout Debris, includes detailed descriptions of all INTS subsystems. The descriptions of Systems 2 to 5 address only those subsystems that are different from those in System 1. 
The systems are listed in Table 2-1 and are briefly summarized below:

- System 1, Grout Debris, uses the following treatments: MEO for primary organic destruction, vacuum desorption for volatile organic separation of process residue and soil, followed by polymer stabilization of process residue and grout stabilization of soil. Polymer is used for stabilization of salt waste and grout is used to stabilize untreated debris.

- System 2, Desorption, uses CWO for primary organic destruction, vacuum desorption, followed by stabilization for process residue, soil, and treated debris waste.

- $\quad$ System 3, Washing, employs MEO for primary organic destruction; aqueous wash for soil, process residue, and inorganic sludge treatment, and high pressure wash for open and complex debris. The high pressure wash operation is modified to a lower pressure agitation wash for soft debris. Stabilization of secondary waste is accomplished through grout and polymer subsystems.

- System 4, Acid Digestion, uses phosphoric-nitric acid digestion for primary organic destruction and soft debris treatment, high pressure spray wash for open debris, vacuum thermal desorption for process residue and inorganic sludge, and aqueous wash for soil. Stabilization for treated debris waste is by grouting, and for soil and insoluble residues by phosphate bonded ceramic. Polymer stabilization is provided to stabilize soluble salts.

- System 5, Catalytic Wet Oxidation, includes CWO for primary organic destruction and soft debris treatment, high pressure spray wash for open debris, aqueous wash for soil, and vacuum thermal desorption for process residue and inorganic sludge. Stabilization for treated debris and soil is by grouting, and for process residues and salts by polymer.

Systems described in this Section are based on the requirements and assumptions given in Appendix A. Functional allocation diagrams (FADs) and perspective diagrams are included at the end of this Section. In addition, detailed PFDs, FADs, plan views, and perspective views of the systems and subsystems are included in Appendix B of this report. 
Table 2-1. Comparison of sizes and concepts of the subsystems.

\begin{tabular}{|c|c|c|c|c|c|}
\hline \multirow[b]{2}{*}{ SUBSYSTEMS } & \multicolumn{5}{|c|}{ SYSTEMS } \\
\hline & $\begin{array}{c}\text { Grout Debris } \\
\text { System } 1\end{array}$ & $\begin{array}{l}\text { Desorption } \\
\text { System } 2 \\
\end{array}$ & $\begin{array}{c}\text { Wash } \\
\text { System } 3\end{array}$ & $\begin{array}{c}\text { Acid Digestion } \\
\text { System } 4\end{array}$ & $\begin{array}{c}\text { CwO } \\
\text { System } 5\end{array}$ \\
\hline ADMINISTRATION & As needed & As needed & As needed & As needed & As needed \\
\hline RECEIVING AND PREPARATION & Extensive sorting & Extensive sorting & Extensive sorting & Extensive sorting & Extensive sorting \\
\hline AQUEOUS WASTE TREATMENT & Includes Photo-oxidation & Includes Photo-oxidation & Includes Photo-oxidation & Includes Photo-oxidation & Includes Photo-oxidation \\
\hline ORGANIC DESTRUCTION & $\begin{array}{l}\text { Mediated Electrochemical } \\
\text { Oxidation }\end{array}$ & Catalytic Wet Oxidation & $\begin{array}{l}\text { Mediated Electrochemical } \\
\text { Oxidation }\end{array}$ & Acid Digestion & Catalytic Wet Oxidation \\
\hline $\begin{array}{l}\text { PROCESS RESIDUE AND INORGANIC } \\
\text { SLUDGE TREATMENT }\end{array}$ & Vacuum Thermal Desorption & Vacuum Thermal Desorption & Aqueous Wash & Vacuum Thermal Desorption & Vacuum Thermal Desorption \\
\hline SOIL TREATMENT & Vacuum Thermal Desorption & Vacuum Thermal Desorption & Aqueous Wash & Aqueous Wash & Aqueous Wash \\
\hline SOFT DEBRIS & Grout & Vacuum Thermal Desorption & Low Pressure Wash & Acid Digestion & Catalytic Wet Oxidation \\
\hline OPEN DEBRIS & Grout & Vacuum Thermal Desorption & High Pressure Wash & High Pressure Wash & High Pressure Wash \\
\hline COMPLEX DEBRIS & Grout & Vacuum Thermal Desorption & High Pressure Wash & Grout & Grout \\
\hline BULK METAL & Decontamination & Decontamination & Decontamination & Decontamination & Decontamination \\
\hline MERCURY AMALGAMATION & Amalgamation & Amalgamation & Amalgamation & Amalgamation & Amalgamation \\
\hline LEAD RECOVERY & Decontamination & Decontamination & Decontamination & Decontamination & Decontamination \\
\hline SPECIAL WASTE PROCESSING & Portable Equipment & Portable Equipment & Portable Equipment & Portable Equipment & Portable Equipment \\
\hline POLYMER STABILIZATION & Polymer Stabilization & Polymer Stabilization & Polymer Stabilization & Polymer Stabilization & Polymer Stabilization \\
\hline $\begin{array}{l}\text { PHOSPHATE CERAMIC } \\
\text { STABILIZATION }\end{array}$ & Not Included & Not Included & Not Included & Phosphate Bonded Ceramic & Not included \\
\hline AIR POLLUTION CONTROL. & Gas Phase Corona Reactor & Gas Phase Corona Reactor & Gas Phase Corona Reactor & Gas Phase Corona Reactor & Gas Phase Corona Reactor \\
\hline GROUT STABILIZATION & Grout & Grout & Grout & Grout & Grout \\
\hline CERTIFICATION AND SHIPPING & Same for all systems & Same for all systems & Same for all systems & Same for all systems & Same for all systems \\
\hline SUPPORT & HVAC, MCC, etc & HVAC, MCC, etc & HVAC, MCC, etc & HVAC, MCC, etc & HVAC, MCC, etc \\
\hline DISPOSAL & Engineered Disposal & Engineered Disposal & Engineered Disposal & Engineered Disposal & Engineered Disposal \\
\hline
\end{tabular}




\subsection{System 1: Grout Debris Case}

System 1 is the grout debris case. The system consists of all structures, buildings, and equipment needed to accomplish the functional and operational requirements (F\&ORs). The organic destruction subsystem is mediated electrochemical oxidation. The residue and soil treatment subsystem is vacuum desorption to remove organics prior to stabilization. Debris is not treated for organic removal; it is sent directly to grout stabilization. The APC subsystem is based on dry filtration, wet offgas scrubbing and organic destruction in the GPCR. System 1 has seventeen subsystems described below. A plan view of the major components of the system is shown in Figure 2-1.

\subsubsection{Administrative Subsystem}

The administrative subsystem includes all administrative and analytical laboratory buildings required for waste management support functions. The administration subsystem is essentially the same for all five systems.

The administration subsystem incorporates all technical and administrative support functions needed to manage the operation of a waste management facility. These functions include:

- Security

- Access control including personnel decontamination (radioactive and hazardous)

- Maintenance of uncontaminated areas and equipment

- Health physics and radiation badges

- Sanitary facilities

- Work control and personnel support

- Internal and external (public relations) communications

- Spill or emergency response provisions (hazardous and radioactive)

- Analytical laboratory

- Environmental field sampling

- Environmental regulatory reporting

- Records management

The administrative subsystem maintains general interfaces with all treatment subsystems. O\&M consumables include analytical supplies, office supplies, sanitary supplies, and personal protective equipment. 


\subsubsection{Receiving and Preparation Subsystem}

Figure 2-2 shows a Functional Allocation Diagram (FAD) for the receiving and preparation subsystem. Figures 2-3 and 2-4 show perspective views of the two main areas of the subsystem. This subsystem is the same for all cases. This subsystem has cranes and forklift trucks to unload waste containers from incoming vehicles. The water is transferred to an assay and inspection area. The physical state of the waste in containers (liquid/solid/gas) is identified by a real-time radiography (RTR) unit. A passive/active neutron (PAN) assay unit determines the level of TRU contamination of the waste. A segmented gamma scanning (SGS) unit is used to assay beta and gamma radioactivity. A computer software and bar code scanning unit records and tracks the waste.

The wastes are classified either as those requiring sorting or not requiring sorting. Containers of wastes not requiring sorting, primarily liquids and wet waste, are distributed directly to the appropriate treatment subsystem. For purposes of this study, approximately $75 \%$ of the waste was assumed to require sorting. Waste containers requiring sorting are grouped into two categories: homogeneous waste (e.g. sludge) and heterogeneous waste (e.g. debris). For both categories, the container is manually opened or decapped by a saw mounted on a gantry robot. After decapping, homogeneous waste which does not require size reduction is dumped into transport hoppers. Heterogeneous waste containers are emptied on a sorting table equipped with master-slave and hydraulic manipulators used for segregating materials into treatment types including open debris, soft debris, complex debris, cemented process residue, soil, lead, and gas cylinders. Various sorting technologies, such as robotic assisted sort tables and vibratory screens, may be used in the sorting area. Operations personnel will select the appropriate unit operations based on the characterization of the waste and visual observation after opening the containers. Wastes with special packages, such as lab waste and waste in gas cylinders, may require sorting in a glove box. Operations personnel will control the sorting to ensure wastes are properly handled without mixing. The sorting tables will be equipped to collect fugitive liquids.

Waste identified by manifest or other documentation as contaminated with mercury compounds will be isolated from other debris. Waste classified as debris under the definition of debris by the EPA debris rule must be segregated from other wastes. This debris must be of a minimum size $(60 \mathrm{~mm})$ to be classified as debris. The sorted waste material is size reduced by saw cutting and/or mechanical auger shredders, as required, for the treatment subsystem. Debris waste which will be treated under the debris rule will not be size reduced below the minimum size accepted by regulation. Prepared sorted waste is placed in transfer bins and moved to the treatment subsystems. Nonmetallic containers are cut or shredded into smaller pieces, as required, for processing. Intact drums may be used for internal transfers of material or, if appropriate, final disposal containers. 


\subsubsection{Aqueous Waste Treatment Subsystem}

Figure 2-5 shows the FAD for the aqueous waste treatment subsystem and Figure 2-6 shows the perspective drawing. This subsystem collects and treats input aqueous waste, which could include wastewater with corrosive properties, dissolved solids, suspended solids, and a broad range of RCRAcontrolled toxic, heavy-metal, and organic contaminants. The subsystem includes processes designed to treat the wastes to meet the UTS in RCRA. In addition, the subsystem treats the system's secondary aqueous wastes, including the salt solutions from organic destruction, filtrate from the aqueous wash subsystem process residue and soil treatment, the aqueous waste from the APC subsystem condensate and scrubber blowdown, sludge from abrasive blasting in the metal decontamination subsystem, rinse water from container washdown, and water from equipment and floor drains.

The unit operations of the aqueous waste treatment subsystem are designed for maximum flexibility and can be used in series, in parallel, or as stand alone units. The selection of specific unit operations will be based on waste characterization from the receiving and preparation subsystem, as well as in-process evaluations. Operations staff will select the appropriate unit operations for characterized streams. Based on characterization, incoming waste will be segregated and stored in four batch tanks. For the purpose of discussing the treatment trains available, these tanks are classified as having high levels of total organic carbon (TOC), high total dissolved solids (TDS) levels, low TDS levels, or heavy metals/mercury containing liquids. In fact, characterized waste may require treatment for combinations of these contaminants. Where waste has multiple types of contamination, it will be processed through each relevant treatment train until it meets UTS for all contaminants.

For high-TOC waste, gross organic removal is accomplished in an oil/water separator or other flotation thickener. Organics removed in the separator are sent to the organic destruction subsystem. Suspended solid filtration is performed using back-flushable filters. Calcium removal prior to organic treatment is performed in a mixing reactor by the addition of sulfuric acid to achieve precipitation of calcium sulfate.

Dissolved organics destruction is accomplished in an ultraviolet (UV) photo-oxidation reactor. The destruction of organics by oxidation to carbon dioxide and water is accomplished by shining UV light on the aqueous solution to which hydrogen peroxide or ozone has been added. The UV light reacts with hydrogen peroxide $\left(\mathrm{H}_{2} \mathrm{O}_{2}\right)$ or ozone $\left(\mathrm{O}_{3}\right)$ to create hydroxyl radicals $(\mathrm{OH} \bullet)$ which attack and break down the organics. The reactor design allows the addition of both ozone and hydrogen peroxide at several points in the flow through the reactor. Waste characterization prior to transfer to the reactor allows optimizing the use of ozone, UV and hydrogen peroxide, because treatment effectiveness varies by chemical. Polishing of the treated water by carbon adsorption is available for further dissolved organic removal. Dissolved solids removal is accomplished in an ion exchange unit. 
For liquids with low levels of TDS, the same treatments as discussed for high TOC are appropriate, although gross organic removal may not be necessary. Again, waste characterization will dictate which unit operations are necessary for each batch tank.

For liquids with high levels of TDS or contaminated with metals, the treatment train involves additional precipitation and settling steps. If the stream is also high in TOC, gross organic removal may be required. Neutralization with appropriate chemicals such as sodium hydroxide is performed in an agitated tank. Depending on the waste characterization, metals are precipitated from the waste in a precipitation reactor. Precipitation converts the dissolved and particulate metals into insoluble salts. Precipitated solids are removed by a centrifuge. The sludge is collected in a sludge settling tank, and the clarified aqueous layer is transferred to suspended solids filtration, photo-oxidation in the UV reactor, and ion exchange. Ion exchange vessels are supplied with mercury selective resins. The sludge is concentrated in an evaporator and sent to the polymer stabilization subsystem.

In addition to the primary treatment trains, each waste type can be routed to or can bypass a given treatment. For example, neutralized scrubber liquor from the APC is typically transferred directly to the evaporation unit following characterization. The aqueous waste treatment subsystem concentrates all sludge waste produced by the various treatment trains and sends it to the stabilization subsystem. Spent ion exchange resin from treatment processes is dewatered and sent to the process residue treatment subsystem or to the organic destruction subsystem. Spent carbon is sent to the organic destruction subsystem.

The unit operations remove radionuclides, RCRA-regulated metal and trace organic concentrations to regulatory discharge levels, thus allowing the treated water to be either recycled or discharged.

\subsubsection{Organic Destruction Subsystem}

Figure 2-7 shows the organic destruction subsystem FAD, and Figure 2-8 shows a perspective view. Organic destruction is by silver MEO in the grout debris case. The organic destruction subsystem receives organic liquids and sludges from the receiving and preparation subsystem, and organic secondary wastes streams from the aqueous waste treatment subsystem, the APC and the process residue and soil treatment subsystems. The organic destruction subsystem consists of:

- feed characterization and batch tanks

- (anolyte) primary reactor

- catholyte tank

- mediated electrochemical oxidation reactors

- nitric acid conversion aerator

- offgas treatment

- $\quad$ silver recovery processing tanks

- centrifuges 
- evaporator

- fractionator

- condenser

- $\quad$ associated pumps, filters, and instruments

The liquid organic feed is sampled and analyzed at the characterization tank. Characterization provides information to optimize operation. Treatment processes are the same for all waste. Characterized feed is transferred to the batch tank. The batch tank contents are pumped through a grinding unit to eliminate oversized particles. The waste is then fed into the anolyte primary reactor where destruction of organics occurs. The primary reactor contains an agitator sufficient to keep all particulates in suspension. If appropriate, a basket device similar to a washing machine can be used to prevent larger sized particulates from leaving the reactor.

Large pumps transfer electrolyte and waste through the MEO electrochemical cells into the primary reactor. Electrolyte is recycled from the electrochemical reactors to the primary reactor. Nitric acid/silver nitrate electrolyte solutions are continuously, i.e. without break, supplied to the primary reactor. The waste feed rate is based on the amperage required for destruction of the organics. For typical organic streams, the ratio of input waste feed to recirculating electrolyte is approximately $1 \%$ by weight.

The MEO electrochemical process operates at $120^{\circ}$ to $140^{\circ} \mathrm{F}\left(50^{\circ}\right.$ to $\left.60^{\circ} \mathrm{C}\right)$ at atmospheric pressure. The electrochemical cells are packaged into modular units. The cells consist of a series of electrode compartments with Nafion membranes between the anode and the cathode compartments. Each module contains approximately 60 pairs of electrodes. An electrolyte solution of nitric acid and silver nitrate is recirculated through a series of cells in the MEO unit at rates of up to 100 gallons per minute (gpm). Electrical energy is supplied to the anode and cathode of each cell. The electrical energy transforms the silver ions $\mathrm{Ag}(\mathrm{I})$ to $\mathrm{Ag}(\mathrm{II})+\mathrm{e}-$. The oxidized metal mediator ( $\mathrm{Ag}[\mathrm{II}]$ ion) in the acid solution acts as the primary active oxidizer to destroy organics. The $\mathrm{Ag}(\mathrm{II})$ ion is reduced back to $\mathrm{Ag}(\mathrm{I})$ by reaction either directly with the organic species, or with water, to produce a reactive intermediate such as the hydroxyl radical $(\mathrm{OH} \bullet)$ that also reacts with the organic material. In this highly reactive environment, the organics are broken down into harmless constituents such as carbon dioxide and water. The reactions can occur in the cells and in the primary reactor. Offgas, primarily $\mathrm{CO}_{2}$, is generated at the anode as a result of the organic destruction reactions. The $\mathrm{CO}_{2}$ is removed from the headspace above the primary reactor.

The design of the MEO equipment is based on expected destruction removal efficiency (DRE) of $90 \%$ for each pass through the MEO cells. Electrolyte and feed mixture is pumped from the primary reactor past the cells at $100 \mathrm{gpm}$. Based on the 2200 gallon size reactor and the feed rate, the typical feed molecule will contact the MEO cells about 25 times. This is sufficient to degrade the original organics and subsequent breakdown products with a DRE well over $99.99 \%$. 
is passed through a turbo-aerator where the nitrous acid is forced into contact with oxygen in a highly turbulent environment favorable to quick conversion of the nitrous acid to nitric acid prior to the formation of undesirable nitrogen oxide $\left(\mathrm{NO}_{x}\right)$ byproducts. Oxygen concentration is maintained at about six times the stoichiometric requirement to convert all the nitrous acid. Some degradation of nitrous acid is expected, and a catalytic reactor has been placed downstream to convert $\mathrm{NO}_{\mathrm{x}}$ to nitrogen.

Remove Excess Water. Water formed in the reaction migrates to the catholyte tank, reducing the nitric acid concentration below 6 molar $(M)$. The nitric acid concentration at the cathode is maintained above $6 \mathrm{M}$ to avoid forming hydrogen. Below nitric acid concentrations of $2 \mathrm{M}$, hydrogen will be generated at the cathode. Water must be continuously removed to maintain the $6 \mathrm{M}$ nitric acid concentration in the electrolyte solution. Therefore, a slip-stream of the catholyte is continuously removed from the catholyte tank and processed by evaporation, silver recovery and acid recycling. These operations remove water and prevent build up of silver and small amounts of organics which can escape to the cathode side. This catholytic slip-stream is combined with the much smaller anolyte blowdown stream for the removal of salts and to prevent buildup of inerts. The electrolyte blowdown stream consists of water, nitric acid, silver nitrate, silver chloride, metal ions, unreacted waste and miscellaneous inorganic ions such as sulfate, nitrate and phosphate. If necessary, the entire electrolyte inventory can be removed, treated and replaced by fresh electrolyte on an occasional basis.

Recycle Silver. The electrolyte blowdown stream is processed for silver recovery, water removal and acid reclamation. To recover silver, the stream is reacted with hydrochloric acid to precipitate the silver as silver chloride. This solution with the suspended silver chloride is then passed to a continuously operated centrifuge to remove the insoluble silver chloride solids. These solids are transferred to the silver conversion mix tank, where hydrogen peroxide and sodium hydroxide are added causing the silver chloride to be reduced to silver and sodium chloride. The silver solids are removed from this mixture by centrifugation. The silver is then mixed with recycled nitric acid to form silver nitrate in the silver dissolving tank. Silver nitrate is recycled to the primary reactor.

Recycle Nitric Acid and Remove Metals and Inerts. The liquor from the centrifuge is evaporated to separate the nitric acid from water. The evaporator can be operated to produce nitric acid at an acceptable concentration for recycling to the catholyte tank. To improve the recovery of nitric acid, the evaporator is operated periodically so that water and nitric acid are removed from the top of the evaporator, and sludge, containing the inerts, is removed from the bottom of the evaporator. The acid and water are separated by a fractionator column, with the nitric acid being recycled either to the reactors or to the silver dissolving tank, and the acidic water to the aqueous waste treatment subsystem. The evaporator bottoms, which include salts, radionuclides and other metals, are filtered to remove water and acid, neutralized, and sent to polymer stabilization for microencapsulation. 
is operated periodically so that water and nitric acid are removed from the top of the evaporator, and sludge, containing the inerts, is removed from the bottom of the evaporator. The acid and water are separated by a fractionator column, with the nitric acid being recycled either to the reactors or to the silver dissolving tank, and the acidic water to the aqueous waste treatment subsystem. The evaporator bottoms, which include salts, radionuclides and other metals $s_{3}$ are filtered to remove water and acid, neutralized, and sent to polymer stabilization for microencapsulation.

\subsubsection{Process Residue and Inorganic Sludge Treatment Subsystem}

Figures 2-9 and 2-10 show the FAD for the combined process residue and inorganic sludge treatment subsystem. Figure 2-11 shows a perspective view. Wastes treated in the process residue and inorganic sludge treatment subsystem include (1) process residue and inorganic sludge from the receiving and preparation subsystem, and (2) process residue from other subsystems, including lead recovery. This subsystem employs an indirectly heated vacuum desorption dryer to remove volatile organics and some volatile inorganic compounds from the waste. Treated waste is then sent to leaching/washing operations for removal of mercury.

In the receiving and preparation subsystem, the waste is size reduced to less than one-inch particles. In the process residue subsystem, the shredded waste is characterized and blended (if appropriate) and staged in campaigns for treatment. The vacuum desorption unit, which is operated in the batch mode, separates organic contaminants from inert solids under low pressure at moderate temperatures. Organic chemicals are condensed and recovered as a liquid.

The waste is fed in batches at the top of the desorption unit through a vacuum valve. In the first step of the process, the waste stream is charged through an inlet flange located on top of the jacketed vacuum desorption vessel. The desorption vessel is sealed and nitrogen is used to purge it and gas equipment of air, removing the potential for combustion of waste material or desorbed gas. The purge stream also assists in the desorption of contaminated vapors.

The second step involves slowly applying heat to desorb the organic components from the waste. The vapors exit the vessel by passing through a chamber equipped with a filter which removes entrained particulate. Particulate is returned to the desorption unit and is removed with the processed waste solids.

The waste is also heated indirectly using a hot oil heating unit. The hot oil is circulated through a baffled external jacket which surrounds the desorption vessel. For homogeneous feed of soil or sludge, a top mounted agitator stirs the waste. The agitator shaft and impeller contain annular spaces into which the hot oil can be circulated. Heat is circulated through the waste for one to two hours, increasing the waste temperatures up to $500^{\circ} \mathrm{F}$. As the waste is heated, vacuum (as low as $25 \mathrm{~mm}$ mercury [Hg]) is applied for periods of from 15 minutes to several hours, depending on the feed. When treatment is complete the vacuum 
is released, the unit is opened, and the treated material is discharged from the bottom of the desorption vessel to a transportable hopper.

Desorber offgas passes through three condensers in series. The primary cooler/condenser reduces temperature to ambient conditions. Condensate which may include aqueous mercury solution, elemental mercury, as well as some organics, is collected and allowed to settle. The condensed aqueous mercury solution, the top layer in the collection vessel, is routed to the aqueous waste treatment subsystem. The elemental mercury, the dense lower layer, is sent to the mercury amalgamation subsystem. In the next condenser, chilled water reduces the temperature to near $45^{\circ} \mathrm{F}$. In the third condenser, chilled brine reduces the temperature to near $0^{\circ} \mathrm{F}$ for effective condensation of organics and water. The condensed organic liquid is captured and sent to the organic destruction subsystem. The remaining offgas from the dryer is routed to the air pollution control subsystem.

The bottom solids removed from the vacuum dryer are collected and evaluated for mercury. If solids contain mercury, they are processed for mercury removal by leaching. The solids are leached with a potassium iodide and iodine leaching solution in an agitated mixing tank. The leached solids are washed with fresh water, then rinsed with an acid and hydrogen peroxide solution. The wet solids are filtered, collected in hoppers, and routed to the polymer stabilization subsystem. The leaching solution and subsequent rinse water are collected and passed through a column containing steel wool for mercury removal. In the column, the mercury complex formed in the leaching tank is precipitated from solution and captured by the steel wool. The mercury contaminated steel wool is routed to mercury amalgamation. The mercury amalgamation process is sufficiently adaptable to handle this steel wool waste. The remaining solution is processed further in a metal precipitation/settling tank to remove dissolved metals. Metals and settled solids are sent to polymer stabilization. The aqueous phase from the precipitation is treated to recover the iodine by the use of a hydrogen peroxide and sulfuric acid mixture. The recovered iodine and the potassium iodide solutions are recycled to the leaching tank.

\subsubsection{Bulk Soil Treatment Subsystem}

This subsystem treats bulk soil from receiving and preparation. The vacuum desorption process described in Section 2.1.5 is sized and costed to treat the bulk soil as well as process residue. Soil and residue will be campaigned separately through the subsystem. Treated soil is sent to the grout stabilization subsystem, while treated process residue and inorganic sludge are sent to the polymer stabilization subsystem.

\subsubsection{Debris Treatment Subsystem}

In the grout debris case (System 1), all complex, open and soft debris is treated by grouting. Some size reduction of the debris may be performed. Segregation of debris into soft, open and complex debris classifications is not necessary. Section 2.1.14 discusses the grouting subsystem. For the other treatment cases (Systems 2 through 5), the debris is subjected to treatment prior to grouting. To enhance the treatment 
performed by these systems, the debris is segregated into open, soft and complex classifications, and the debris requiring treatment is shredded, as required, for the treatment process.

\subsubsection{Metal Decontamination Subsystem}

Figure 2-12 shows the FAD for the metal decontamination subsystem. Figure 2-13 shows the perspective view. The decontamination subsystem has size reduction tools (acetylene torch, saw and shears) and an inspection assay area. Decontamination is provided with water blasting booths designed to remove surface contamination. Decontaminated metal is assayed, and approved metals are recycled within the DOE complex. Contaminated liquid is separated from the grit and recycled within the subsystem. Contaminated grit sludge is sent to the aqueous treatment subsystem. Fugitive dust is captured in a prefilter and highefficiency particulate air (HEPA) filter.

\subsubsection{Mercury Amalgamation Subsystem}

Figure 2-14 shows the FAD for the mercury amalgamation subsystem and Figure 2-15 shows a perspective view of both the mercury amalgamation and lead recovery subsystems. The mercury amalgamation subsystem treats elemental mercury and steel wool impregnated with mercury from the leaching process in the process residue treatment system. The liquid mercury is transferred to an amalgamation operation where the mercury is combined with copper (or zinc) powder, steel shot (for proper mixing), and nitric acid. This combination is mixed in a pebble mill to form a copper-mercury amalgam, eliminating free mercury. The amalgam is packaged for assay and inspection to ensure that it meets TCLP standards.

\subsubsection{Lead Recovery Subsystem}

Figure 2-16 shows the FAD for the lead recovery subsystem. This subsystem has a decontamination unit for treating surface contamination. The subsystem contains a size reduction table with mechanical devices, including saws, shears and sanders to cut and remove metal cladding from lead. Decontamination of lead takes place in booths where a liquid abrasive solution is used to remove a thin layer of contamination. The liquid solution is recycled, and residual sludge is sent to the process residue and inorganic sludge treatment system. Lead which is effectively decontaminated can be recycled within the DOE complex. Lead containing radioactive contamination which cannot be decontaminated is grouted and disposed of. Drums containing cleaned material are washed and transferred to the certification and shipping subsystem. Lead gloves, aprons, etc. are grout stabilized without treatment under the debris rule. Regulatory acceptability of this approach is a prerequisite to this design. 


\subsubsection{Special Waste Treatment Subsystem}

It is assumed that there are special wastes that require capabilities not included in the basic system design concept. The treatment subsystem for special waste is located in a room equipped with a crane and all utilities needed for installing treatment equipment to process special wastes. Special treatment systems will be identified and provided on a case-by-case basis during facility operation. For all systems, the cost estimate includes the space in the building, but not processing equipment. Costs have not been identified for the special treatment processes because the treatment has not been defined.

\subsubsection{Polymer Stabilization Subsystem}

Figure 2-17 shows the FAD for the polymer stabilization subsystem. Figure 2-18 shows a perspective view. The polymer stabilization subsystem receives (1) salt waste from the receiving and preparation subsystem; (2) salts, fines and concentrated sludge waste from aqueous waste treatment and the air pollution control subsystems; and (3) treated process residue solids from the process residue and inorganic sludge treatment subsystem. The treatment of these waste streams is performed by the same process; however, the formula can be adjusted and operation can be optimized for specific waste streams.

Stabilization of wastes involves polymer encapsulation using polyethylene. The subsystem has a dryer that removes water from the incoming waste. Residue waste is shredded in the receiving and preparation subsystem. A tertiary shredder is available to size waste to a powder prior to an extrusion operation. The dried powder and polymer are metered into a mixer or extruder which heats and mixes the polymer with powdered waste in the mass ratio of one part polymer to one part waste by weight. The extruder feeds the mixture into a drum.

When filling is complete, the drum is capped automatically and sent to a wiping and decontamination station. A wipe sample is collected from the drum exterior. If surface contamination is detected, the container is washed by high-pressure water jets or blasts of dry ice. The inspected container is sent for assay, certification, and shipment to storage or disposal facilities.

\subsubsection{Air Pollution Control (APC) Subsystem}

Figures 2-19 and 2-20 show the FAD and a perspective view, respectively, for the APC subsystem. The APC subsystem receives offgas from all of the other processing subsystems that produce offgas requiring treatment. This offgas may include (1) gaseous reaction products from organic destruction (mainly carbon dioxide), (2) water and organics from the desorber, and (3) water and organics from the aqueous waste treatment subsystem. Several unit operations are provided in this subsystem.

The APC subsystem is composed of a dry filter, pre-filter, gas phase corona reactor (GPCR) unit, a wet gas scrubber, mist eliminator, activated carbon for trace organics removal, sulfur impregnated activated 
carbon for mercury vapor removal, and a system for continuous emissions monitoring (CEM). Treatment occurs near room temperature and pressure.

Gross particulate is removed from the gas stream by the dry filter. The gas is also passed through a HEPA filter to remove fine particulate. Once the particulates are removed from the gas stream, the gas enters the GPCR. Unlike carbon adsorption, the gas to the GPCR does not require any temperature reduction or moisture removal. The relative humidity of the inlet gas can be as low as $0 \%$ and as high as $90 \%$. The GPCR is a cylindrical quartz vessel with an inner high voltage electrode and a grounded electrode on its outer surface. The annulus of the vessel is filled with dielectric pellets. A strong alternating electric field placed across the packed bed of dielectric particles creates a corona that causes the gas to partially ionize and form a plasma in the voids between the particles. In the plasma, electrons are heated to extremely high temperatures while the bulk of the gas remains cool. The high-energy electrons in the plasma cause organic contaminant destruction through direct interaction with the contaminant molecules. Destruction also occurs through secondary ion and radical reactions.

The treated gas exits the GPCR and enters the acid gas scrubber. The gas scrubber includes a hydrosonic scrubber and a packed tower scrubber. The neutralized offgas passes through a mist eliminator. Final polishing is provided by granular activated carbon and the sulfur impregnated activated carbon for mercury vapor removal. The treated offgas is monitored continuously before release to the atmosphere.

\subsubsection{Grout Stabilization Subsystem}

Figure 2-21 shows the FAD for the grout stabilization subsystem. Figure 2-22 shows a perspective view. The grout stabilization subsystem receives (1) treated soil, (2) debris solids, (3) internally contaminated metal waste, (4) lead waste that cannot be decontaminated, and (5) other solid waste from the receiving and preparation subsystem. The stabilization process may be classified as macroencapsulation which meets EPA requirements for stabilizing solid wastes that fall under the debris rule. The debris rule does not require that a TCLP test be conducted when debris is stabilized. Waste such as soils, not classified as debris and grouted, must pass the TCLP test.

The subsystem has devices for the following:

- $\quad$ size reduction

- $\quad$ storing and feeding binder

- macroencapsulation of waste

- microencapsulation of waste

- container filling and capping, wiping

- decontamination 
A concrete core sampling unit is also provided for obtaining TCLP samples. Shredded waste and soil are microencapsulated. Unshredded waste, especially metal and lead, are macroencapsulated. For microencapsulation, a grout mixer blends grout with desorbed solids and other waste that has already been shredded to small particles by the receiving and preparation subsystem. Complex debris is size reduced by cutting, put into drums and grouted in place (macroencapsulation). The grout material for debris solids consists of cement, water, and sand. Approximately two parts of grout by weight are required for each part of waste. The exact formula may be varied depending on the waste form. The blended product is poured into containers and allowed to cure. Once the stabilized waste has solidified, containers are capped, wipe tested, washed if necessary, and moved to the certification and shipping subsystem.

\subsubsection{Certification and Shipping Subsystem}

Figure 2-23 shows the FAD for the certification and shipping subsystem. Figure 2-24 shows a perspective view. The certification and shipping subsystem characterizes the physical and radiological properties of the packaged waste to allow certification in accordance with transportation, storage, and disposal requirements. Equipment utilized is similar to that in the receiving and preparation subsystem. The containers of packaged waste for shipment are weighed. An RTR unit examines the container to ensure that the matrix is homogeneous and has no free water. If TRU or alpha contaminated waste is processed, the TRU concentration is measured by a PAN assay unit. An SGS unit is used to assay the beta and gamma radioactivity. After inspection, the waste is either sent to a temporary storage area or loaded onto a truck for offsite or onsite shipment.

\subsubsection{Support Subsystem}

The support subsystem includes the facilities necessary to ensure continuous functioning of the treatment facility. These include the auxiliary operating and maintenance galleries, retrieval and transfer areas, maintenance shop, mechanical equipment room, heating, ventilation and air conditioning (HVAC) equipment room, motor control center (MCC) and electrical rooms. Lighting, drainage, piping, fire protection, heating, and closed circuit television cameras are provided as part of this subsystem. All of this equipment incorporates proven technologies. Costs for maintenance and equipment galleries are included with each treatment subsystem.

A main control room is provided for the control of process operations. The control room contains monitoring and control instrumentation, remote video monitors, communications devices and other equipment used to operate the facility. Costs for monitors are included in each treatment subsystem.

For maintenance needs, the treatment facility provides large corridors or galleries next to each cell for equipment pull-out and maintenance. To minimize the consequences of equipment failure, the equipment layout is designed to allow the removal of equipment by crane. Equipment requiring repair is removed from its location and staged on a cart next to the maintenance gallery airlock. The equipment can then be 
transferred to the maintenance gallery. Repairs can be made in the maintenance gallery or, if necessary, removed to an offsite shop via the shipping or receiving subsystems. The gallery also has space to set up duplicate equipment outside the hot cell, and to assist in the remote repair of equipment using robotic or manipulator devices. Cell isolation minimizes the amount of process equipment that must be idled when a mechanical failure shuts down an operation.

The support subsystem includes the equipment to maintain the confinement zones necessary for facility operation. The zones can be described as follows:

- Zone 1 - primary confinement. This zone encompasses process enclosures.

- Zone 2 - secondary confinement. This zone encompasses process areas immediately surrounding Zone 1 areas, including maintenance galleries.

- Zone 3 - non-confinement areas. This zone encompasses shipping, receiving, transfer corridors, control rooms, and administration and support areas.

Zone 1 confinement is achieved by drawing air into process enclosures through adjustable dampers and openings from Zone 2. Zone 2 confinement is achieved by drawing air from non-confinement zone areas in Zone 3. Zone 1 is maintained at negative pressure with respect to Zone 2, while Zone 2 is maintained at negative pressure with respect to Zone 3. Air drawn from Zones 1 and 2 is passed through air handling units comprised of prefilter, HEPA filter and charcoal filter banks. Zone 3 is serviced by an air supply unit equipped with temperature control and air filtration devices that are used to provide fresh conditioned air to the facility from outside.

The confinement system consists of dust collection, process vent, and building ventilation and HEPA filtration units. These units ensure confinement of airborne radioactive and chemical contaminants within the equipment and process area boundaries. The system removes airborne contaminants from the process areas and filters the air before exhaust.

Dust control unit. Waste expected to generate dust will be handled in enclosures. Local vents and dust collection/filtration units are installed in areas where dust generation potential exists. After removal of dust, the filters exhaust the vent to the building ventilation and HEPA filter unit.

Process vent unit. Chemical vapors and fumes generated by processes are collected by a vapor collection system. Vented vapors are treated by filtration units designed to remove vapor, fumes and particulate before exhausting the vent system. Treated vapors are then sent to the building ventilation and HEPA filtration units prior to release to the atmosphere. 
Building ventilation and HEPA filtration system. This system ensures safe operating conditions by maintaining air flow from non-contaminated areas to areas of progressively higher potential contamination. A zoned approach is used, based on a cascade pressure differential principle. The building has been divided in three confinement zones.

The support subsystem includes the HVAC equipment. The functions of this equipment are (1) to provide heating and cooling requirements for the facility; (2) to provide air confinement barriers by removing air from the facility and recharging it with conditioned air from outside; (3) to remove airborne contaminants from facility and to treat them prior to discharging treated and conditioned air to the atmosphere; and (4) to monitor radiation activity of treated air released to the atmosphere.

Ventilation air will be introduced into the building after passing through $85 \%$ efficient pre-filters and nuclear grade HEPA filters. Air will be exhausted from Zone 1 by industrial class exhaust fans through a galvanized steel duct system. The exhaust fans will be located adjacent to the stack. Exhaust air will pass through the air treatment system. The air treatment system will consist of a dust collection system, a fume abatement system, vapor filters, charcoal filters and nuclear grade HEPA filters. Treated air is exhausted to the atmosphere through the stack. Intermediate sections will be provided with system redundancies to permit maintenance, testing or replacement of the abatement systems and filters.

The exhaust and makeup air systems operate continuously 24 hours a day, seven days a week. A redundant exhaust fan and filter train will be included to permit fan maintenance, filter change, and inspection of operational problems.

Air volume measuring stations located in the makeup and exhaust ducting will continuously monitor the air flow rates and send signals to a digital controller. The controller will adjust the variable frequency drive of the exhaust fans to maintain a constant exhaust rate. This feature will automatically compensate for fluctuations in flow rates across the HVAC filters.

Atmospheric and room static pressure probes will send pressure measurement signals to the digital controller. The controller will adjust the variable frequency drive of the makeup air fan to control the volume of air entering processing area zones, and thus will maintain the negative to atmosphere pressure relationships as required. A digital controller will be provided for all fans.

Air volume measuring stations will continuously monitor the exhaust air flow rate from each zone. A digital controller will adjust the position of a modulating damper located in the room exhaust ducts to maintain the desired air change rate for that zone. This feature will automatically compensate for pressure adjustment needs during events such as opening and closing doors of controlled spaces. 


\subsubsection{Disposal Subsystem}

This subsystem consists of engineered disposal units that are based on an earth mound and concrete cell concept (Morrison Knudsen/Chem Nuclear Services, 1991). In this concept, inspected drums are received from the certification and shipping subsystem and placed in concrete canisters. The canisters are stored in concrete vault cells which are equipped with leachate collection and monitoring equipment. When full, the cells are capped with an earth layer that is engineered to withstand long-term environmental and weathering effects. Engineered disposal for non-RCRA waste and RCRA waste are essentially the same, except that a RCRA disposal unit has a double leachate collection system to comply with the RCRA requirements.

\subsection{System 2: Desorption Case}

System 2 was developed to demonstrate improved debris treatment through vacuum thermal desorption. The key changes from the grout debris case are (1) an organic destruction subsystem based on catalytic wet oxidation and (2) debris treatment by vacuum thermal desorption. As in System 1, inorganic sludge, process residue and soil treatment is provided by vacuum thermal desorption and a mercury removal process. Table 2-1 shows the subsystems used in System 2. For aqueous waste treatment, air pollution control, metal decontamination, lead recovery, grout stabilization, and polymer stabilization subsystem descriptions, refer to the grout debris case presented in Section 2.1. A plan view of the major components of System 2 is shown in Figure 2-25.

\subsubsection{Catalytic Wet Oxidation Organic Destruction Subsystem}

Figure 2-26 shows the FAD for the organic destruction subsystem. Figure 2-27 shows a perspective view. This subsystem treats organic liquids and sludges from receiving and preparation, the APC, the aqueous waste treatment, and the vacuum desorption processes. The organic destruction subsystem is based on a catalytic wet oxidation process. This process uses an oxidant in a catalyzed solution to enhance the destruction of organic wastes. The waste feed is shredded and then added to the vessel continuously through a solid or liquid-feed assembly. Because organic destruction involves exothermic reactions, the waste is metered into the reactor to prevent a runaway reaction. Active cooling is provided for temperature control.

The catalytic wet oxidation process is a method of catalytically oxidizing waste organic compounds using iron oxidant which is regenerated by oxygen. Iron or $\mathrm{Fe}$ (III) in a hydrochloric acid solution oxidizes organics to $\mathrm{CO}_{2}$ and hydrogen $\left(\mathrm{H}^{+}\right)$. Oxygen $\left(\mathrm{O}_{2}\right)$ is added to reconvert the resulting $\mathrm{Fe}(\mathrm{II})$ to $\mathrm{Fe}(\mathrm{III})$. A set of homogeneous co-catalysts influences these reactions. Typically, these co-catalysts are copper ions and one or more of the ions platinum(IV), palladium(II), and ruthenium(III). Mercury, radioactive metals, and heavy metals are dissolved and concentrated in the acidic catalyst solution. The process conditions are expected to be pressures of 20 pounds per square inch gauge ( $\mathrm{psig}$ ) and temperatures of between $300^{\circ} \mathrm{F}$ and $400^{\circ} \mathrm{F}$ $\left(150^{\circ} \mathrm{C}\right.$ to $\left.200^{\circ} \mathrm{C}\right)$, depending on the waste form and composition. The 1000 gallon reactor can be operated 
at pressures of up to $200 \mathrm{psig}$. The standard solution consists of $60 \%$ by weight (wt $\%$ ) ferric chloride, approximately $3.6 \mathrm{wt} \%$ hydrogen chloride $(\mathrm{HCl})$, with the remainder being water. Catalyst concentration is approximately $0.001 \mathrm{M}$ (the number of moles of catalyst ion in 1 liter of solution). The reactor solution is agitated by recirculated headspace gases and by a mechanical agitator.

Oxidation of organics occurs principally at the surface of contact between the organics and the reagent solution. Carbon dioxide, generated by the oxidation reaction, is removed from the oxidation vessel with the headspace gases which are primarily water vapor, $\mathrm{CO}_{2}, \mathrm{O}_{2}$, and $\mathrm{HCl}$. Offgas is passed through a second reactor, to further break down organics. In this reactor, the same iron and hydrochloric acid solution is passed over the gas. Remaining liquids are recycled to the primary reactor. Offgas from the secondary reactor then flows to a condenser to remove most of the water vapor and $\mathrm{HCl}$. A portion of the condensed acidic water is recycled back to the reagent storage and feed tank, and the rest of the acid is neutralized using caustic. The neutralized salt solution is routed to the aqueous waste treatment subsystem for further treatment.

As the inorganic residues build up in the reaction vessel, a sidestream of reactor solution is removed from the bottom of the vessel and passed through a filter to remove residual solids. The acid filtrate is recycled to the reactor. Solids collected at the filter are rinsed in a wash tank with a mild hydrochloric solution to react chlorides, and are sent to the polymer stabilization subsystem.

Periodically, the concentration of dissolved metals and radionuclides builds up in the solution to a level that affects the working solutions' operational characteristics. The feed to the reactor is stopped, organics are allowed to react until gone, and then the reactor solution is cooled. In the precipitator, the solution is neutralized and the metals are precipitated from the reactor solution as hydroxides or oxides by the addition of caustic. Following filtration, the filtrate salt solution is transferred to the aqueous waste treatment subsystem and the metal precipitates are routed to the polymer stabilization subsystem.

\subsubsection{Process Residue, Inorganic Sludge, Bulk Soil and Debris Treatment Subsystem}

The treatment of process residue, inorganic sludge, bulk soil and debris is performed with vacuum desorption technology and a mercury leaching process. This approach is described in detail in Section 2.1.5. Figures 2-9 and 2-10 show the FAD and Figure 2-11 shows the perspective view, for the process residue, inorganic sludge, bulk soil and debris treatment subsystem. Debris waste is treated in the same vessel as process residue and sludge. Debris waste is segregated from soil and process residue because of EPA debris rule considerations. The debris waste operating conditions will vary based on the type of debris. Agitation of the waste may not be practical for some debris waste. Soft debris waste contains plastics and combustible waste such as paper, wood and cardboard. This waste is segregated and campaigned separately from complex and open debris. The soft debris will be desorbed at lower temperatures $\left(250^{\circ} \mathrm{F}\right)$ selected to avoid melting plastic and oxidizing combustibles. At these lower temperatures less volatile organics such as PCBs remain in the debris matrix. 


\subsection{System 3: Wash Case}

System 3 has several washing approaches for the separation of organic contents from process residue and sludge, soil and debris. System 3 is otherwise identical to the grout debris case. The wash case employs (1) MEO for organic destruction, (2) aqueous wash for residue, sludge and soil, (3) high pressure wash for open and complex debris, and (4) a modified operation of the pressure wash using lower pressure for soft debris. This section describes the washing subsystems of System 3. Table 2-1 lists all the subsystems of System 3. Refer to Section 2.1 for a discussion of MEO, and other subsystems that are identical to the grout debris case. A plan view of the major components of System 3 is shown in Figure 2-28.

\subsubsection{Process Residue, Inorganic Sludge and Bulk Soil Treatment Subsystem}

Figure 2-29 shows the FAD for the aqueous wash process residue, inorganic sludge and bulk soil treatment subsystem. Figure 2-30 shows a perspective view. Soil is treated in campaigns and kept separate from process residue and inorganic sludge. The process residue, inorganic sludge and bulk soil treatment subsystem is an aqueous wash technology which employs water and surfactant washing for contaminant removal, with subsequent reclaiming of the water and surfacants for recycling. Soil washing is a conventional process used in hazardous waste treatment, and is similar to soil processing operations in the mining industry. This subsystem is unusual because of the extensive recycling of water and surfactant. The aqueous wash subsystem is composed of:

- $\quad$ size reduction and screening equipment

- a wash water storage tank

- an agitated wash tank

- rotating screen and hydrocyclone separators

- a counter current solid/liquid rinse contactor vessel

- a solubility reduction process to separate surfactant/contaminant from wash water

- ultrafiltration

- soluble metals removal by precipitation followed by dissolved air flotation (DAF)

- surfactant/contaminant separation by steam stripping

- a surfactant recovery storage tank

The process is operated in a continuous mode. Costs and design are based on minimum size equipment requiring less than a three shift operation.

Several unit operations are required, but all utilize conventional equipment. Recycling occurs within most unit operations, minimizing the need for makeup water and surfactant, and reducing the final output volume. The wastes are first processed in an attrition mill to reduce the average particle size of the waste stream. Wastes are transferred to an agitated wash tank for primary organic removal. Wash water solution containing 
surfactants and other additives is continuously fed to the wash tank along with wastes. A $45^{\circ}$ pitch turbine agitator stirs the tank contents. For soil treatment, four tons of wash solution are used for each ton of soil. The water in the wash tank is maintained at between $140^{\circ}$ to $176^{\circ} \mathrm{F}\left(60^{\circ}\right.$ to $\left.80^{\circ} \mathrm{C}\right)$. Organics and metals will be removed from the soil or residue by the surfactant, additives, and agitation. A slurry of soil and wash water are continuously removed from the bottom of the wash tank and sent to the two step solid/liquid separation equipment. Vapors from the wash tank are vented to two heat exchangers in series. Air is released and the small volume of collected liquids, volatiles and water, are sent to the solubility reduction separator, which is discussed below.

The wash tank discharge passes through a two step solid/liquid separation system. The stream is passed over a rotating screen, which filters out solids. The filtrate is sent through one or more small bore hydrocyclones for further solids separation. The solids from the screening process, which include the contaminant, surfactants and water, are sent to a countercurrent soil/liquid contactor. The filtrate is sent to the solubility reduction separator.

Rinse water is added to the rinse contactor at a rate of 6 tons of water to one ton of soil or process residue. The contactor is essentially a trough-like vessel with paddles in which the soil is rinsed clean and the dirty rinse water contains the contaminants and surfactant. Following this step, the wet, treated waste is dried and transferred to stabilization. Soil is grouted, while process residue is stabilized with polymer. Both the contaminated contactor rinse water and the filtrate from the solid/liquid separation are sent to the solubility reduction separator described below.

The solubility reduction separator uses an approach that is proprietary to the vendor. In this step, temperature and $\mathrm{pH}$ effects are used to alter the solubility of the surfactant dramatically. The surfactant forms a dense lower phase, usually containing the contaminant. Colloidal matter such as humic components of soil also collect in the surfactant layer. The surfactant can be described as an adsorption agent, a "liquid active carbon", which holds the contaminant. The top layer of liquid, about $40 \%$ of the wash/rinse liquid, is recycled to the wash water storage tank for wash water. The remaining water layer is sent to ultrafiltration. The surfactant layer, containing the contaminant, is sent to a splitter process to separate the surfactant for reuse.

Ultrafiltration, also referred to as inertial separation, separates the incoming liquid into two liquid streams: the first containing a high level of particulate and the second, the filtrate containing essentially no particulate. The liquid feed is pumped through parallel porous metal tubes manifolded together. The feed stream, stored in a tank, is continuously recirculated through the ultrafiltration tubes at high speed. The principle of ultrafiltration is that particulate in a fast moving stream moving through a porous metal tube remains with the stream due to inertia. Clear water may pass through the porous metal. Thus, the filtrate is free of particulate and the particulate stream becomes more concentrated. Periodically, a back pulse of pressurized air is injected into the clear side of the tubes to remove particulate that may clog the pores of the tube. The 
concentrate from the ultrafiltration is returned to the solubility reduction stage. The concentrated particulate can also be removed as a sludge and sent directly to polymer stabilization.

The clear filtrate from ultra filtration is further treated to remove soluble metals. The dissolved metals are first precipitated then separated from the rinse water in a DAF unit. The collected waste is sent to the polymer stabilization subsystem. The rinse water is transferred to a storage tank at the solid/liquid contactor for reuse.

The surfactant and contaminant "complex" is sent to the splitter. Surfactant is separated by a proprietary process which includes steam stripping, followed by solvent extraction. The solvent is distilled and recycled. The extracted surfactant is recycled to the wash tank. Condensed organics from the stripping and nonvolatiles from the extraction process are sampled and sent to an appropriate subsystem for further treatment. If the waste is highly organic, it will be sent to organic destruction. If the waste contains high levels of mercury or other metals, it will be transferred to aqueous waste treatment.

Although this is a wash process, the only output to aqueous waste treatment is during shutdown or cleanup. All water is recycled, except the water which exits with the wet treated waste, the metal froth or the organic contaminants.

\subsubsection{Soft, Open and Complex Debris Treatment Subsystem}

Figures 2-31 and 2-32 show the FAD and a perspective view, respectively, for the pressure wash process used for soft, open and complex debris. This subsystem collects and treats open and complex debris having internal and external surfaces that may be contaminated with organics and heavy metals through the use of surfactants delivered by a high pressure wash. For soft debris, the same equipment is used, but with a low pressure spray and agitation. The debris treatment subsystem is composed of a shredder for soft debris, a spray/rinse chamber, a particulate filter, and a surfactant preparation tank. The subsystem is operated in batch mode.

The feed material is placed inside a 12 feet long, 7 feet wide, 8 feet deep spray/rinse chamber using a crane. Monolithic pieces (11 feet long, 7 feet wide, 7 feet high) and 55-gallon drums are placed in the chamber (Dosani, 1995). Smaller, loose pieces with a minimum size of 1 inch are placed in 4 feet diameter by 4 feet tall metal baskets which are then lowered into the chamber by crane. The size of the basket openings determines the size range of the loose pieces. For soft debris, a basket design with a closed top which can accommodate smaller diameter waste which may float is necessary. Each basket weighs 1,200 pounds and is capable of containing 1 to 2 tons of debris. The debris is placed in the cleaning chamber which is closed to prevent the release of fugitive volatile emissions (Taylor, 1994).

Three stages of high pressure wash occur in the cleaning chamber. In the first stage, the chamber is flooded with approximately 2,000 gallons of $160^{\circ} \mathrm{F}$ surfactant solution from the detergent holding tank. The 
surfactant is industrial-grade and biodegradable. The optimum cleaning solution concentration is $5 \%$, with the optimum solution temperature being $140^{\circ} \mathrm{F}$ (EPA, 1991).

A 600 gpm pump coupled with an eductor creates the turbulence for this first stage of washing. When baskets are used to hold the debris, the basket is rotated in the turbulent solution. Monolithic pieces are simply immersed in the turbulent solution. This cycle loosens surface contaminants and provides some superficial cleaning. After the first stage wash, the surfactant solution is pumped from the cleaning chamber into the detergent holding tank.

In the second wash stage, which achieves most of the cleaning, the detergent solution is delivered at a rate of $500 \mathrm{gpm}$ through spray nozzles generating 100 psi of pressure. Nozzles are located inside the basket and throughout the chamber sides, bottom, and inner surface of the cover. For soft debris, pilot work would determine an appropriate nozzle pressure. The surfactant solution is returned to the detergent holding tank after passing through an oil/water separator to remove oil and solids. The oil/water separator in the aqueous waste treatment system is used for this purpose. Reconditioning the surfactant solution reduces the volume of water consumed in this subsystem.

In the third wash stage, clean water is applied through the spray nozzles to remove residual contaminated liquid from the surfaces of the debris. The washed debris is then sent to the grout stabilization subsystem for further processing.

The following streams will be sent separately to the aqueous waste treatment subsystem: (1) aqueous waste with organic, dissolved and suspended solids and dissolved paper, (2) rinse water, and (3) the spent surfactant solution. The treated water from the aqueous waste treatment subsystem is recycled back to the water holding tank in this system.

\subsection{System 4: Acid Digestion Case}

The acid digestion case is a variation of the grout debris case. The treatment of organic wastes, bulk soil, soft and open debris, and secondary stabilization is handled differently in the acid digestion case. The acid digestion system has been developed to study the use of a strong acid medium to dissolve and subsequently destroy organic wastes and soft debris, and the use of phosphate bonded ceramic as a partial substitute for polymer and grout in the secondary stabilization subsystem. To help determine the cost differential of various treatment systems, the aqueous wash and high pressure wash systems for bulk soil and open debris, respectively, that are used in the wash case (System 3) were also included in this case. Inorganic sludges and process residues are thermally desorbed as in Systems 1 and 2. Complex debris is grouted without treatment as in system 1. A plan view of the major components of the system is shown in Figure 2-33. 
This system couples the acid digestion subsystem with 19 other subsystems to form the overall treatment train. As shown in Table 2-1, the subsystems are the same as those used in the grout debris system, the desorption system or the wash system. The subsystems that differ from previously described systems are (1) the acid digestion subsystem for organic wastes, (2) the acid digestion subsystem for soft debris, and (3) the phosphate bonded ceramic stabilization subsystem for soils, insoluble salts, and process residues and inorganic sludges. Details of these three subsystems are presented below.

\subsubsection{Acid Digestion Organic Destruction Subsystem}

Figure 2-34 shows the FAD for the organic destruction subsystem and Figure 2-35 shows a perspective view. In this subsystem organic wastes are subjected to acid digestion by a strong medium consisting of phosphoric and nitric acids, operating at a temperature of $392^{\circ} \mathrm{F}\left(200^{\circ} \mathrm{C}\right)$ and at pressures of up to 15 pounds per square inch gauge (psig) in order to oxidize the organic components to carbon dioxide, water and acid gases. Small quantities of palladium, which serves as a catalyst, reduce the generation of carbon monoxide to near zero. The actual destruction of the organic components is accomplished by the nitric acid. The phosphoric acid is used primarily to form a mixture that allows the nitric acid to remain in solution at elevated temperatures. The elevated temperatures accelerate the oxidization of the organic wastes. An additional benefit in using a phosphoric acid medium is the formation of very insoluble phosphate salts as byproducts of the destruction process. These phosphate salts are easily removed and become part of the phosphate bonded ceramic stabilization process for secondary waste streams. During the destruction process, the nitric acid used to oxidize the organic components is converted to nitrous acid and/or oxides of nitrogen. The nitrous acid and nitrogen oxides are captured and converted back to nitric acid for recycling.

Organic wastes obtained from the receiving and preparation subsystem typically arrive in 55-gallon drums. In the feed preparation step, these wastes are blended with organic wastes received from the other treatment subsystems to achieve as uniform a waste for treatment as possible. The wastes are charged to a holding vessel where they are periodically fed into an agitated glass-lined acid digestion reactor which contains a solution of $0.1 \mathrm{M}$ nitric acid, $14.8 \mathrm{M}$ phosphoric acid and $0.001 \mathrm{M}$ palladium ion. The vessel is heated to $392^{\circ} \mathrm{F}\left(200^{\circ} \mathrm{C}\right)$ and the pressure is increased up to $15 \mathrm{psig}$. The reaction is allowed to proceed with periodic removal of offgasses until all the organic components have been destroyed. During this reaction, organic components are converted to carbon dioxide, water, hydrogen chloride and sulfur dioxide with the conversion of nitric acid to nitrous acid and/or nitrogen oxides. Most inorganic cations are converted to insoluble phosphate salts by the phosphoric acid. The nitrous acid, nitrogen oxide and nitrogen dioxide which are formed during the oxidation of the organics are vented from the reactor and enter a nitric acid recovery vessel where they are converted back to nitric acid by reacting them with oxygen. The nitric acid is then recycled. Nitrous oxide, which can also be a byproduct of the oxidation reaction, is not convertible to nitric acid and exits the acid recovery vessel. The nitrous oxide, sulfur dioxide and hydrogen chloride gases exit the acid recovery vessel and enter a wet acid gas packed column scrubber which removes the sulfur dioxide and hydrogen chloride. The scrubber blowdown, a high TDS solution, is sent to aqueous waste treatment. The nitrous oxide, along with inert gasses, passes through this wet scrubbing system into an $\mathrm{NO}_{\mathrm{x}}$ conversion 
system where the nitrous oxide is reduced to nitrogen by a catalyzed reaction with ammonia. This nitrogen and the other inert gasses are then sent to the air pollution control subsystem.

Solids and metal phosphates formed in the reactor vessel are periodically removed from the acid media by centrifugal filtration. The removed solids are sent to the phosphate bonded ceramic stabilization subsystem.

\subsubsection{Aqueous Wash of Bulk Soil}

Bulk soil is treated in an aqueous washing subsystem that is the same as that described in Section 2.3.2. A perspective view of the bulk soil treatment subsystem is provided in Figure 2-36.

\subsubsection{Acid Digestion Soft Debris Treatment Subsystem}

Figure 2-37 shows a perspective view for the soft debris waste treatment subsystem. Figure 2-35, the FAD for the acid digestion case organic destruction subsystem, although not exactly the same, serves as an equivalent FAD for the soft debris waste treatment subsystem. Soft debris is received for treatment from the receiving and preparation subsystem. The debris is passed through a tertiary shredder to reduce the debris to pieces that are less than $1 / 2$-inch in size. The debris is then sent to an acid digestion subsystem similar to that used for organic destruction. The debris is fed into a stirred digestion reactor containing the same $0.1 \mathrm{M}$ nitric acid, 14.8 M phosphoric acid, and $0.001 \mathrm{M}$ palladium mixture as used in the acid digestion system for organic liquid destruction. Due to slower reaction time for the soft debris, as compared with that required for organic destruction, a larger vessel with a longer residence time is required. The soft debris reacts with the acid media at a temperature of $392^{\circ} \mathrm{F}\left(200^{\circ} \mathrm{C}\right)$ and pressures up to $15 \mathrm{psig}$ to form carbon dioxide, water, sulfur dioxide and hydrogen chloride gasses (Pierce, 1994). Materials such as polyethylene have been reported to require $15 \mathrm{psig}$, where other materials can be decomposed at lower pressures. These products

of destruction are formed by the reaction of the debris with nitric acid. During this reaction the nitric acid is reduced to nitrous acid and oxides of nitrogen. As with the organic destruction acid digestion subsystem, the nitrous acid and oxides of nitrogen are converted back to nitric acid by reacting them with oxygen in the acid recovery chamber. Most inorganic components in the soft debris are converted to insoluble phosphate salts which are removed from the acid mixture by filtration, and sent to the phosphate bonded ceramic stabilization subsystem.

\subsubsection{Open Debris Treatment Subsystem}

The treatment of open debris is done by the use of a high pressure aqueous spraying subsystem which is the same as that described in Section 2.3.2. 


\subsubsection{Complex Debris Treatment Subsystem}

Complex debris is treated by grouting, as described in Section 2.1.14.

\subsubsection{Phosphate Bonded Ceramic Stabilization Subsystem}

Figure 2-38 shows the FAD for the phosphate bonded ceramic stabilization subsystem and Figure 2-39 shows a perspective view. Process residues from the thermal desorber, the organic and soft debris acid digestion subsystems, and treated soil are received for stabilization. The chemically bonded phosphate waste forms are made by acid-base reactions in which the acid component is a water-based solution and the base is a soil oxide or hydroxide. Wastes which are solids, such as soil or dried residues, are combined with the base, and sludges or liquid wastes are combined with the acid component. The final product is a mixture of the acid and base. It is not required to have both solid and liquid waste to create a stable waste form. Solid waste is blended in a mixer with magnesium oxide $(\mathrm{MgO})$. Separately, sludges are thoroughly mixed with $50 \%$ phosphoric acid and a $15 \%$ solution of boric acid. The mixtures generated in the two steps are combined and thoroughly mixed to initiate the stabilization reaction. The resultant mixture is poured into drums or other containers for casting and allowed to settle and stabilize. The drums are then moved to an area where they are allowed to "cure" for approximately one week. Once the drummed, stabilized waste has cured, the drums are capped and sent to the certification and shipping subsystem. The final waste form could be as high as $70 \%$ treated waste with the remainder being the additives necessary to generate the stabilizing reaction. For this study it is assumed that the final waste form contains $50 \%$ by weight waste and $50 \%$ additives. Polymer is used to stabilize salts such as sodium chloride $(\mathrm{NaCl})$ because the ceramic is somewhat porous and soluble salt might leach out over time.

\subsection{System 5: Catalyzed Wet Oxidation Case}

System 5 is the catalytic wet oxidation case because CWO is used to treat both soft debris and organics. This case substitutes CWO for acid digestion in the treatment of organics and soft debris, which allows a direct cost and process comparison of the two organic destruction technologies.

The CWO case uses vacuum thermal desorption for process residue and inorganic sludge, aqueous wash for soil, high pressure wash for open debris, and grouting for complex debris. Treated open debris and soil are grouted, while salts, inorganic sludge and process residue are polymer stabilized. As in System 3, the polymer subsystem has a larger throughput than the grout debris case, primarily due to the processing of wet rather than dry solids as output from soil treatment subsystems. See Table 2-1 for a comparison of systems and a list of System 5 subsystems. Refer to Section 2.2.1 for a discussion of catalytic wet oxidation for organic destruction, Section 2.1.5 for a discussion of vacuum desorption for process residue, Section 2.4.2 for a discussion of aqueous wash for soil, and Section 2.3.2 for a discussion of high pressure wash for open debris. A plan view of the major components of System 5 is shown in Figure 2-40. 


\subsubsection{Organic Destruction Subsystem}

The organic destruction subsystem uses catalytic wet oxidation and is the same as that described in Section 2.2.1.

\subsubsection{Bulk Soil Treatment Subsystem}

The treatment of bulk soil is done in an aqueous washing subsystem which is the same as that described in Section 2.3.2. A perspective view of the bulk soil treatment subsystem is provided in Figure 2-36.

\subsubsection{Catalytic Wet Oxidation Soft Debris Treatment Subsystem}

Figure 2-41 shows the perspective drawing for the soft debris waste treatment subsystem. Figure 2-26 is the $\mathrm{FAD}$ of the CWO for the organic destruction in the desorption case, which is essentially equivalent to the CWO for this subsystem. Soft debris is received for treatment from the receiving and preparation subsystem. The debris is passed through a tertiary shredder to reduce the debris to pieces that are less than $1 / 2$-inch in all dimensions. The debris is then sent to a CWO subsystem. The debris is fed into a stirred reactor containing the same acid solution used in the CWO subsystem for organic liquid destruction. As with acid digestion, a slower reaction time is expected for soft debris compared with organic destruction and a larger vessel (2500 gallons) with a longer residence time is required. The soft debris reacts with the hot acid media to form water vapor, $\mathrm{CO}_{2}, \mathrm{O}_{2}$, and $\mathrm{HCl}$. Most inorganic components in the soft debris are converted to insoluble salts which are removed from the acid mixture by filtration as described in Section 2.2.1, and sent to the polymer stabilization subsystem.

\subsubsection{Open Debris Treatment Subsystem}

The treatment of open debris involves a high pressure wash subsystem which is the same as that described in Section 2.3.2.

\subsubsection{Complex Debris Treatment Subsystem}

The treatment for complex debris is grouting which is described in Section 2.1.14. 


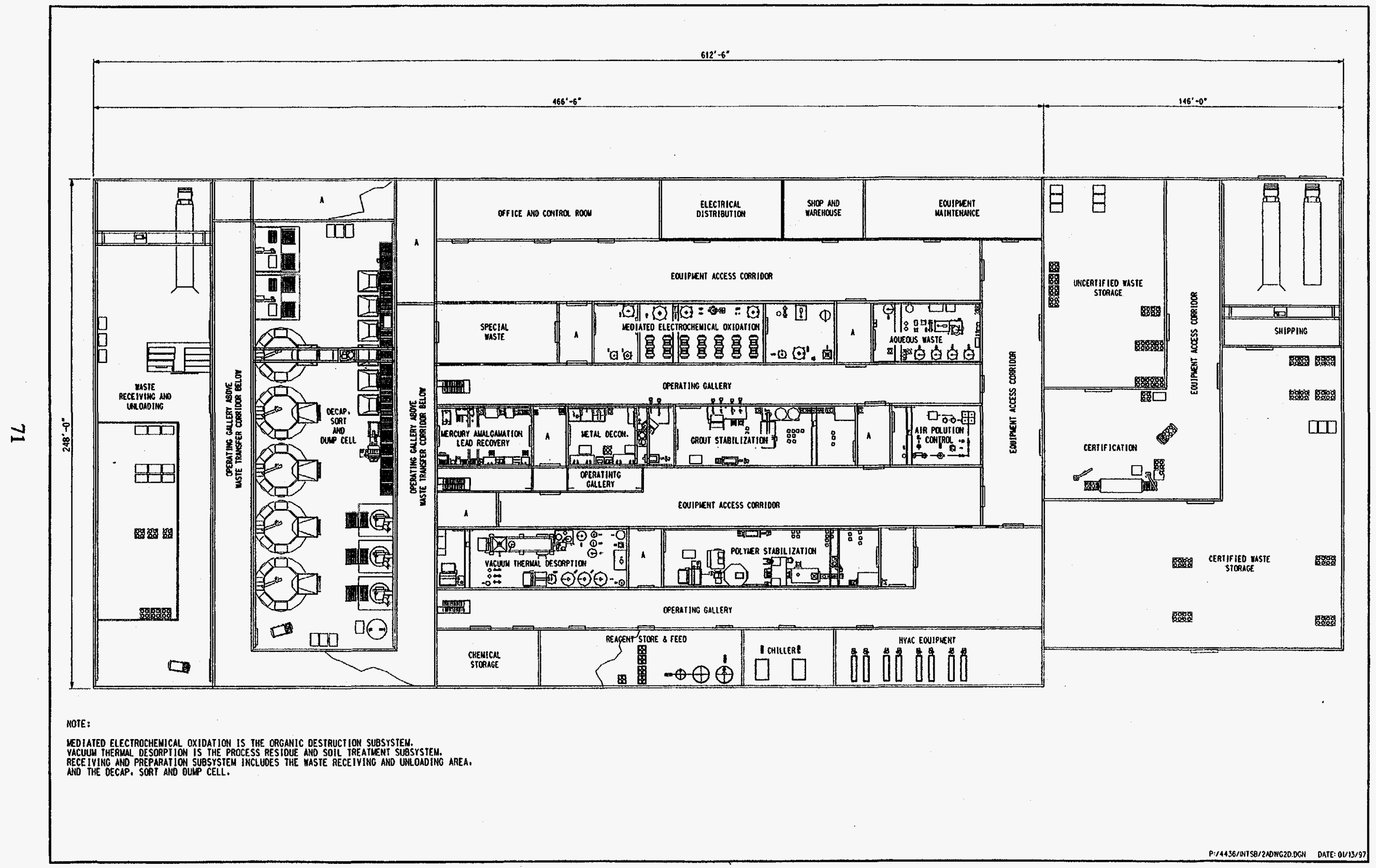

Figure 2-1. Grout Debris Case: PLAN View of Overall System (System 1). 

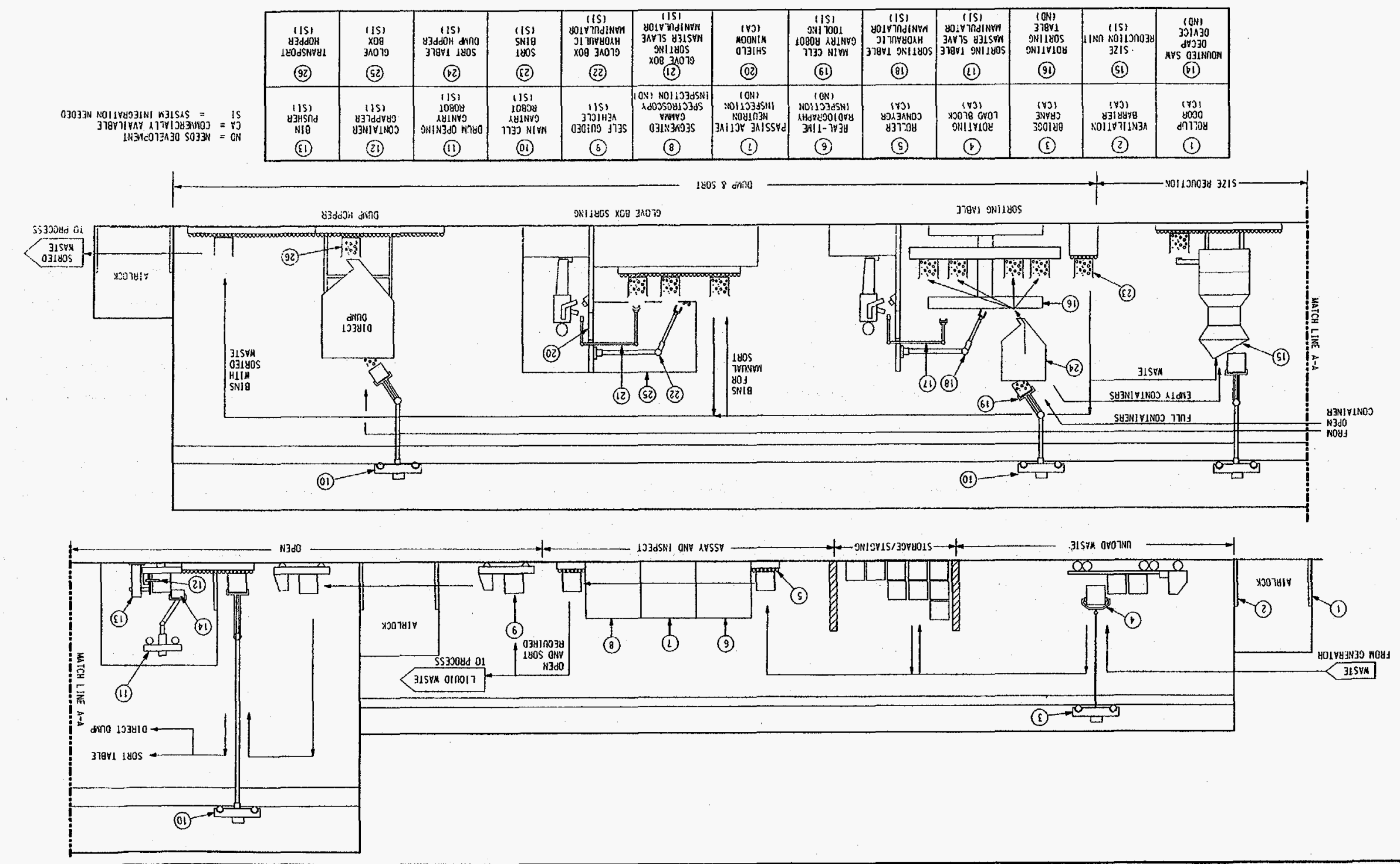


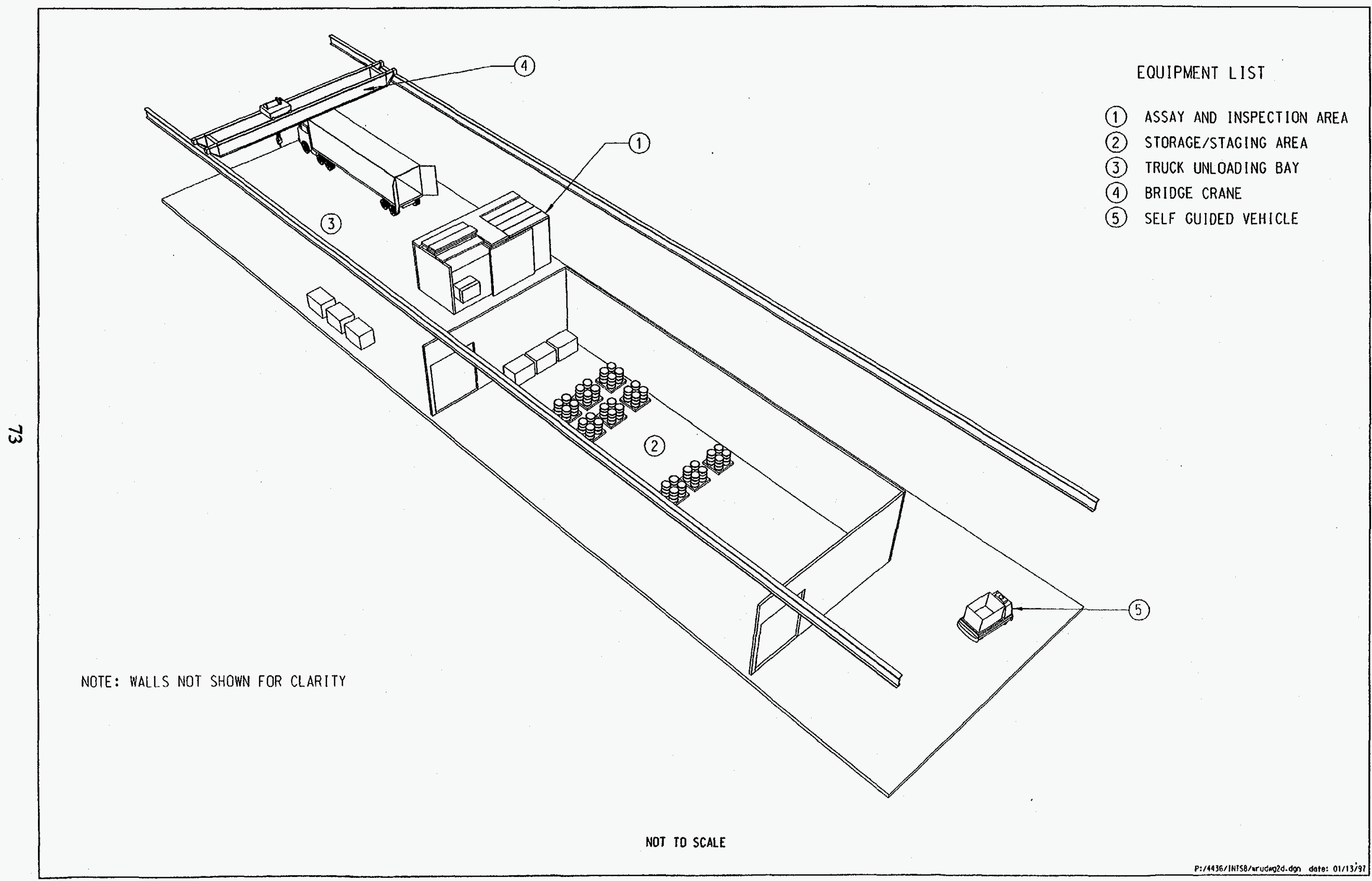

Figure 2-3. Grout Debris Case: PERSPECTIVE View of Receiving and Unloading Area of the Receiving and Preparation Subsystem. 


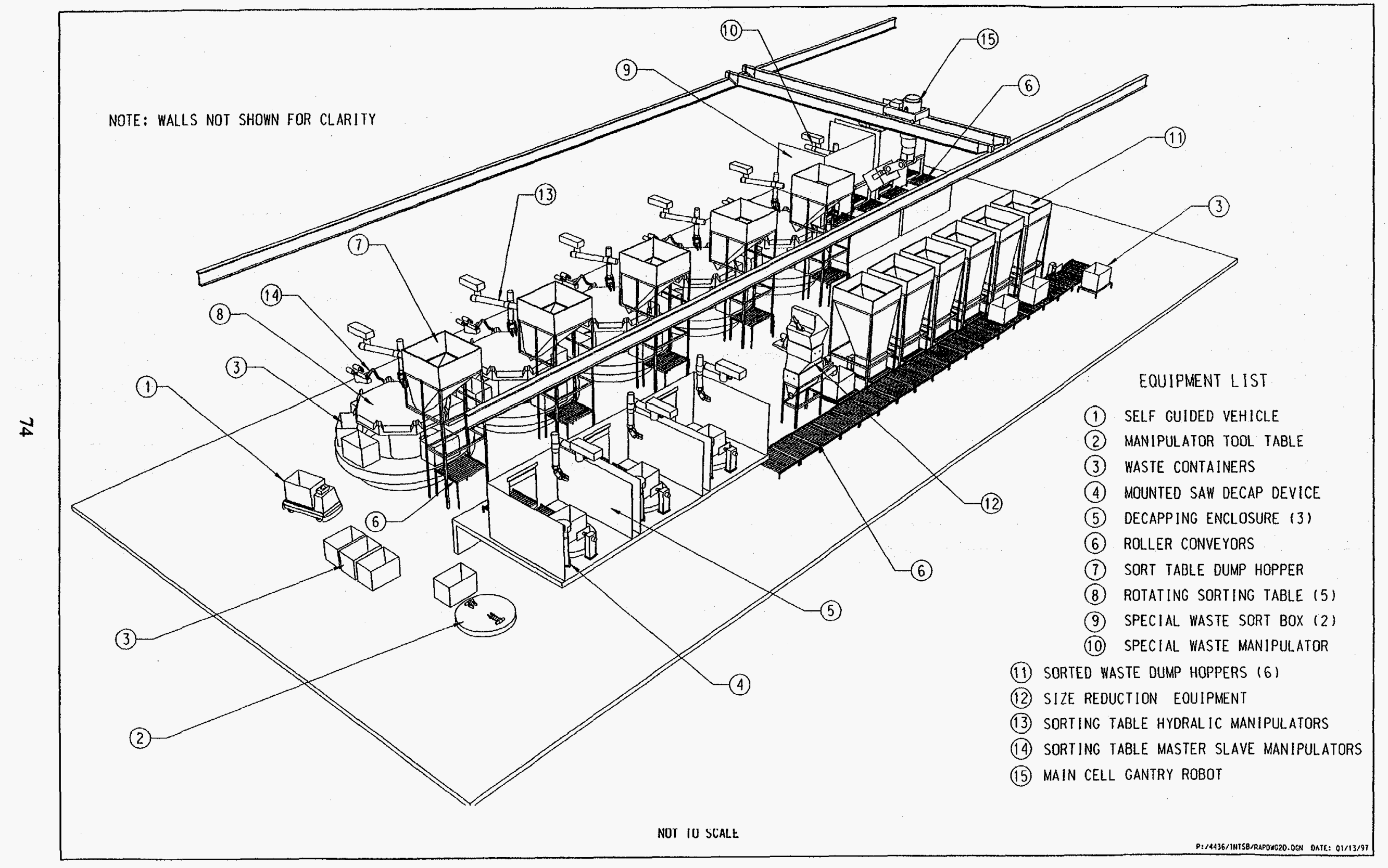

Figure 2-4. Grout Debris Case: PERSPECTIVE View of Decap, Sort and Dump Cell of the Receiving and Preparation Subsystem. 


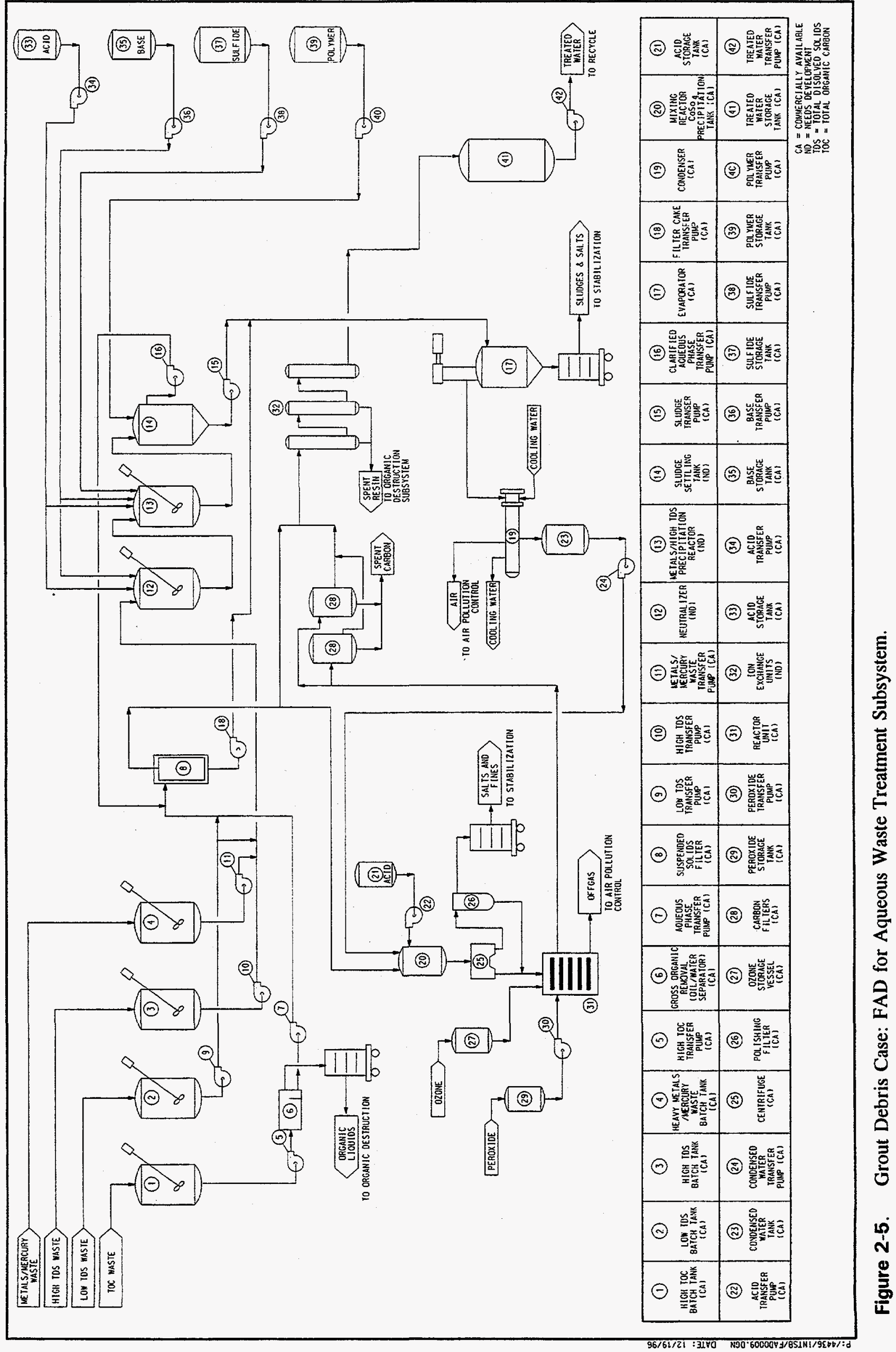




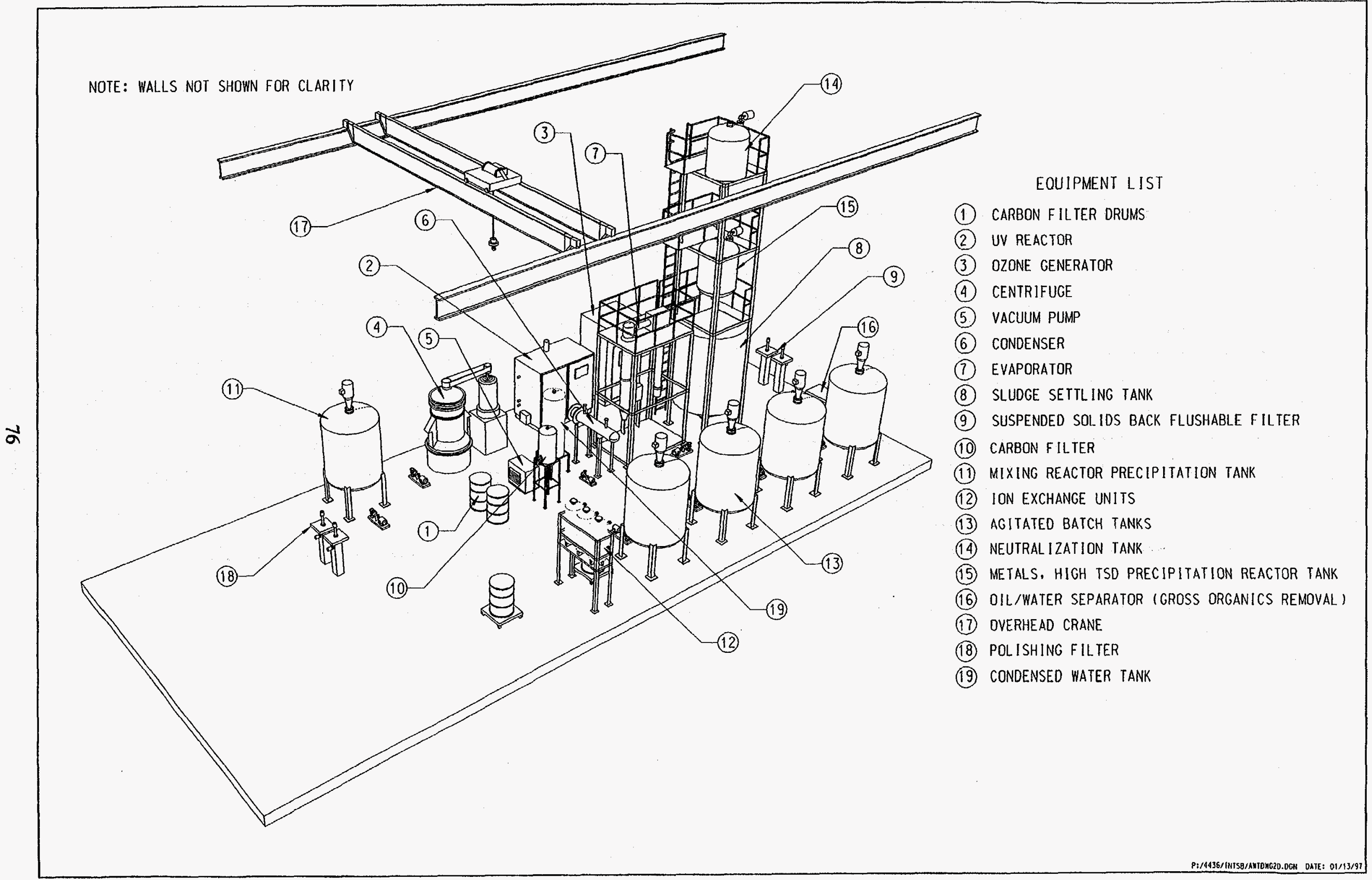

Figure 2-6. Grout Debris Case: PERSPECTIVE View of Aqueous Waste Treatment Subsystem. 


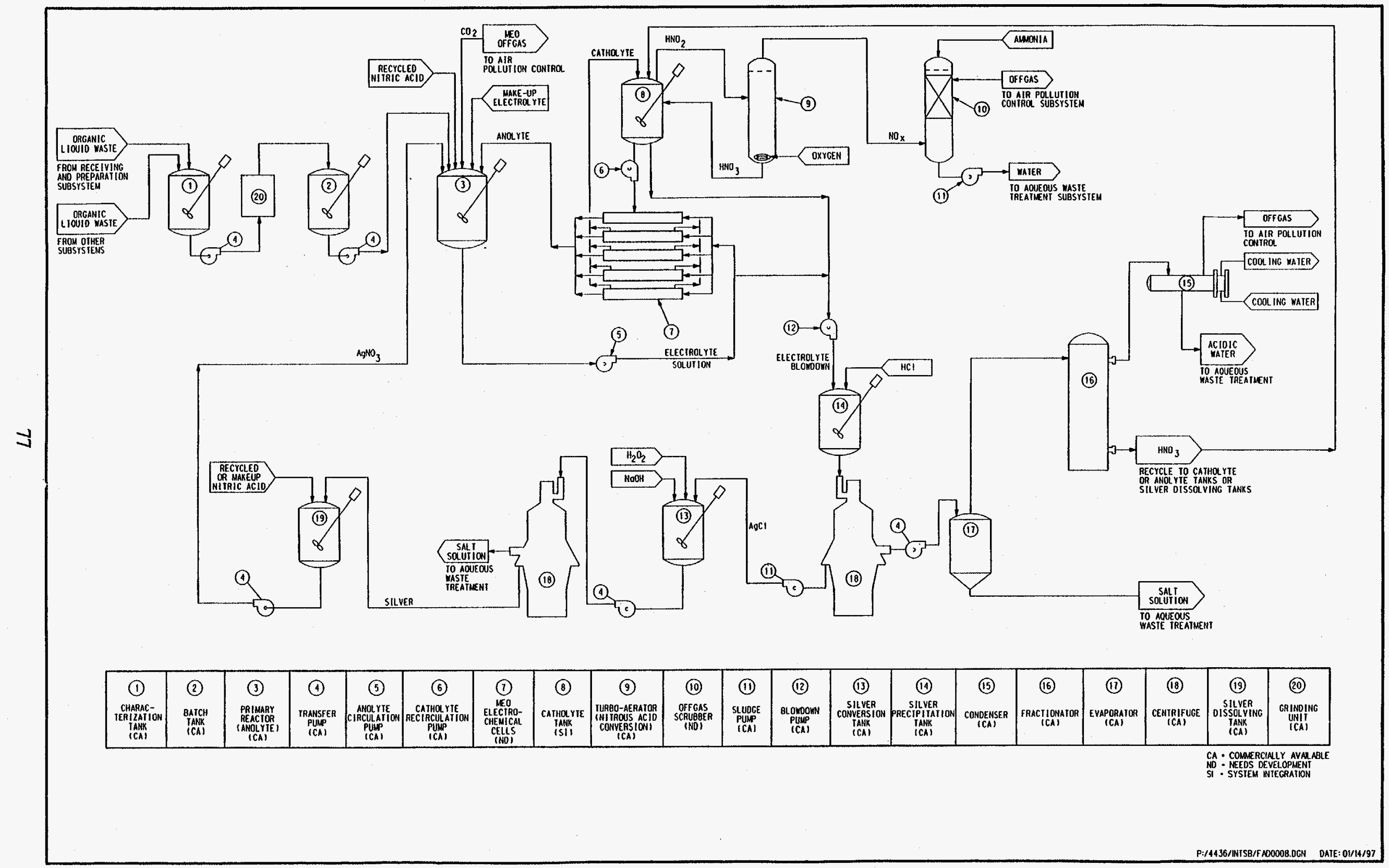

Figure 2-7. Grout Debris Case: FAD for Organic Destruction Subsystem. 


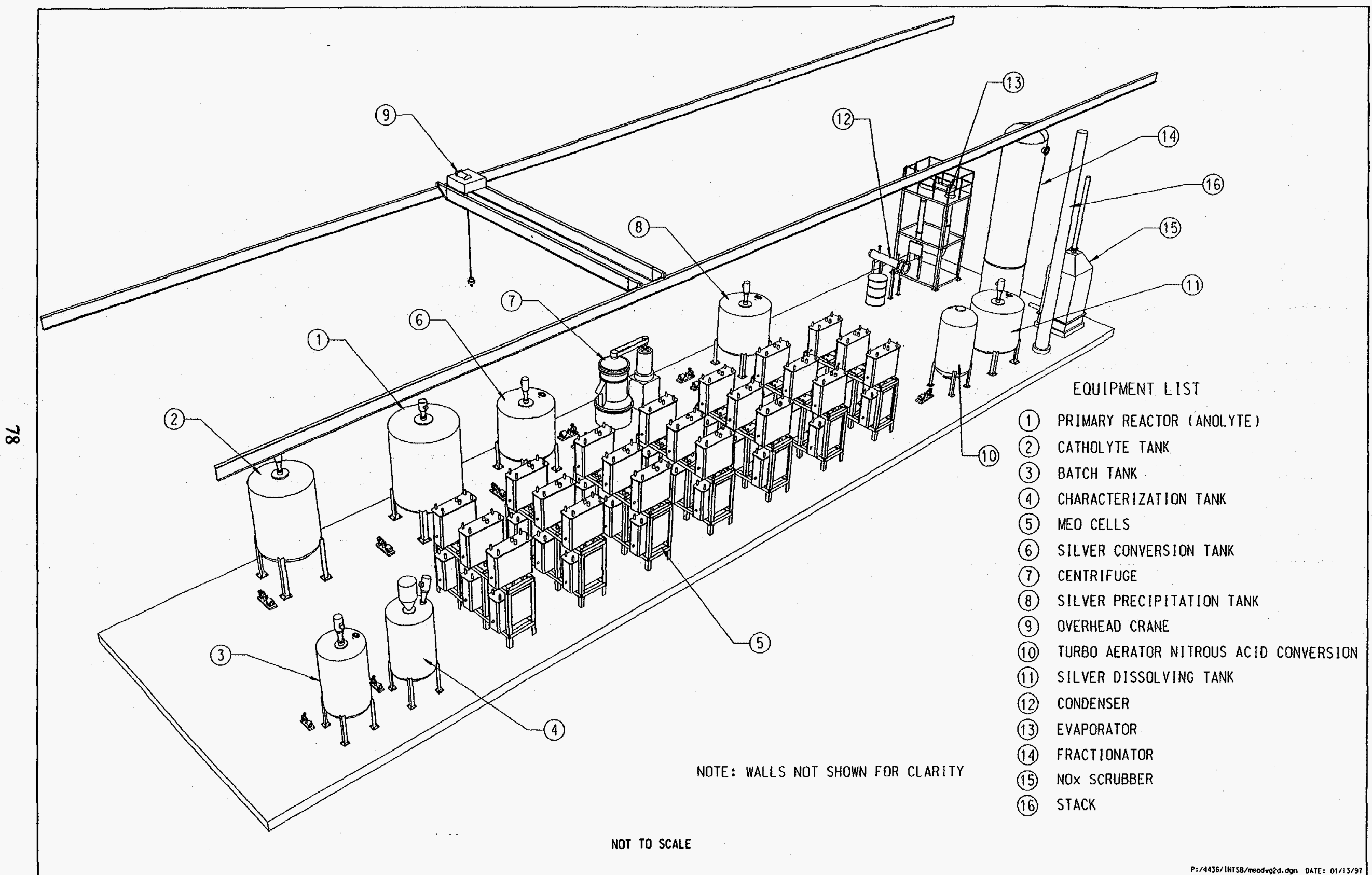

Figure 2-8. Grout Debris Case: PERSPECTIVE View of Organic Destruction Subsystem. 


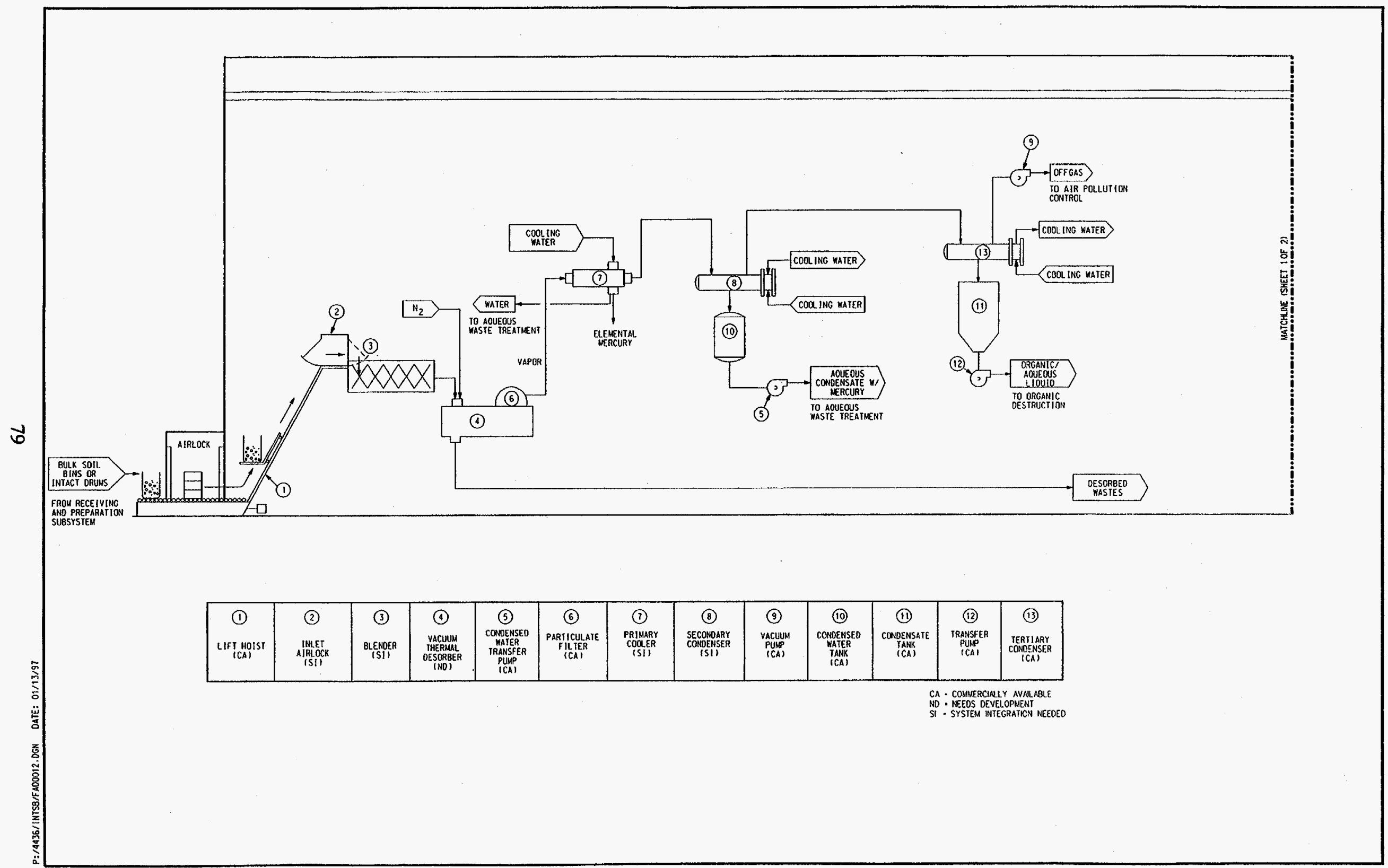

Figure 2-9. Grout Debris Case: FAD for Vacuum Desorption Portion of the Process Residue, Inorganic Sludge and Bulk Soil Treatment Subsystem. 


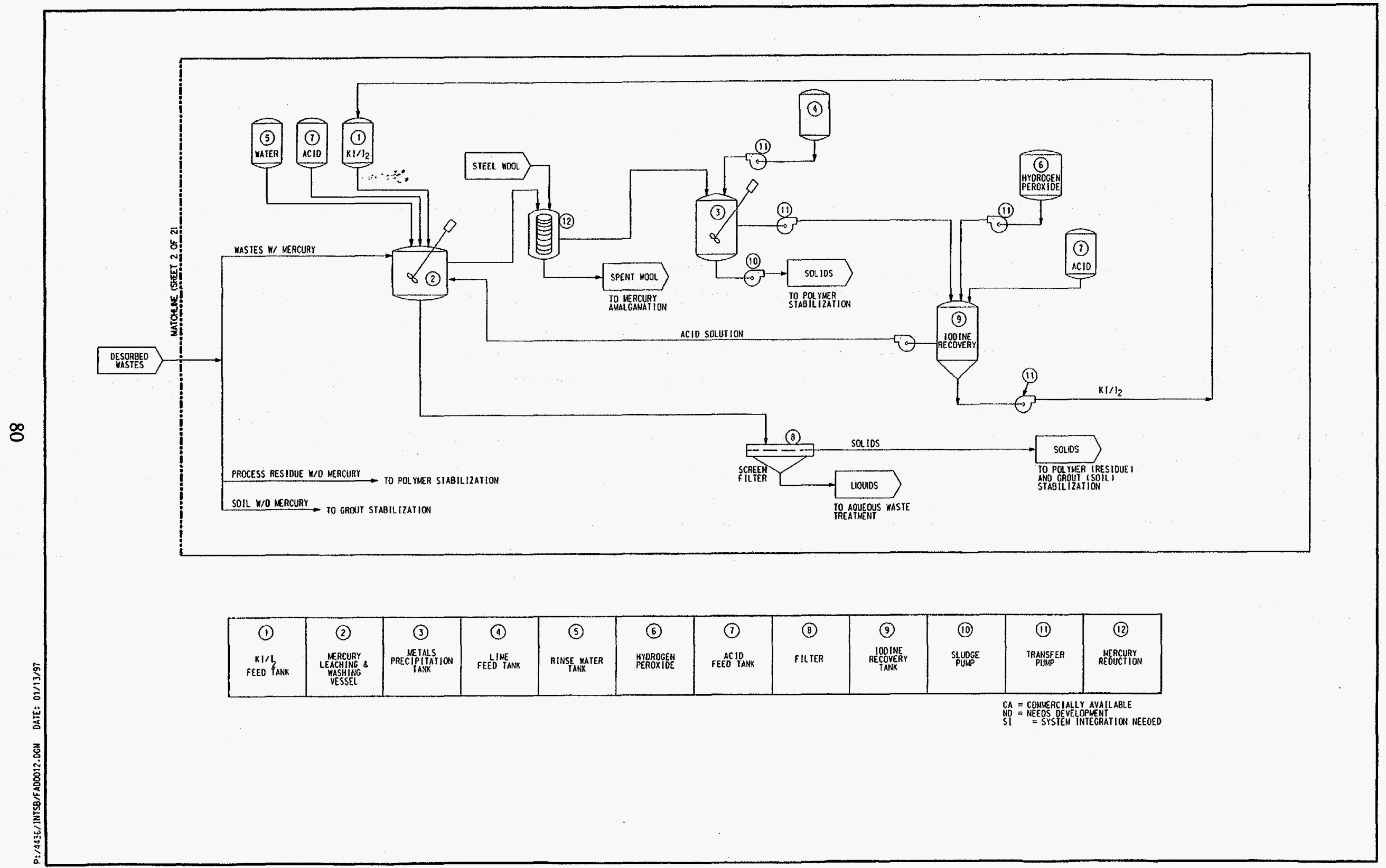

Figure 2-10. Grout Debris Case: FAD for Mercury Leaching Portion of the Process Residue, Inorganic Sludge and Bulk Soil Treatment Subsystem. 


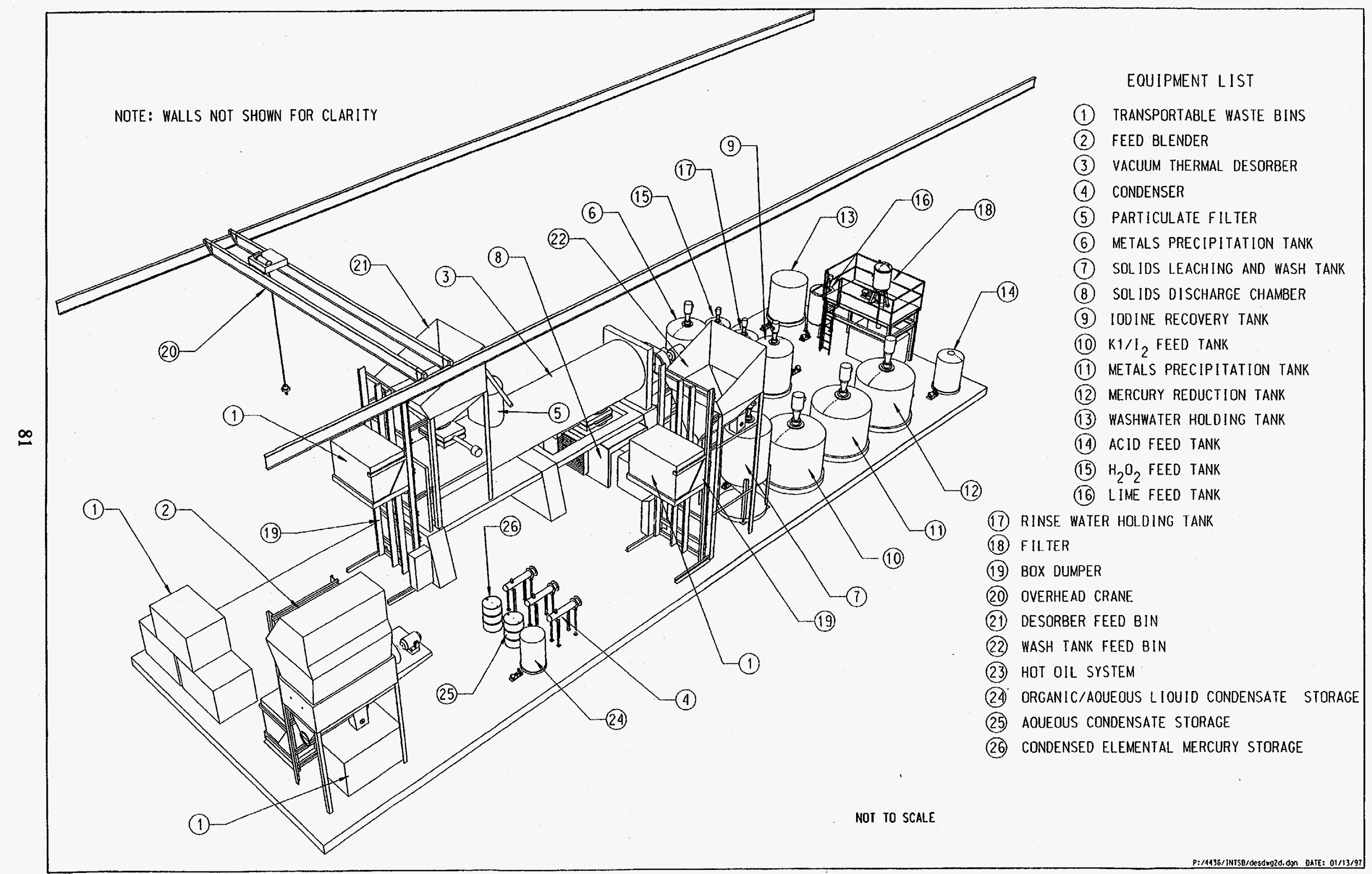

Figure 2-11. Grout Debris Case: PERSPECTIVE View of Process Residue, Inorganic Sludge and Bulk Soil Treatment Subsystem. 


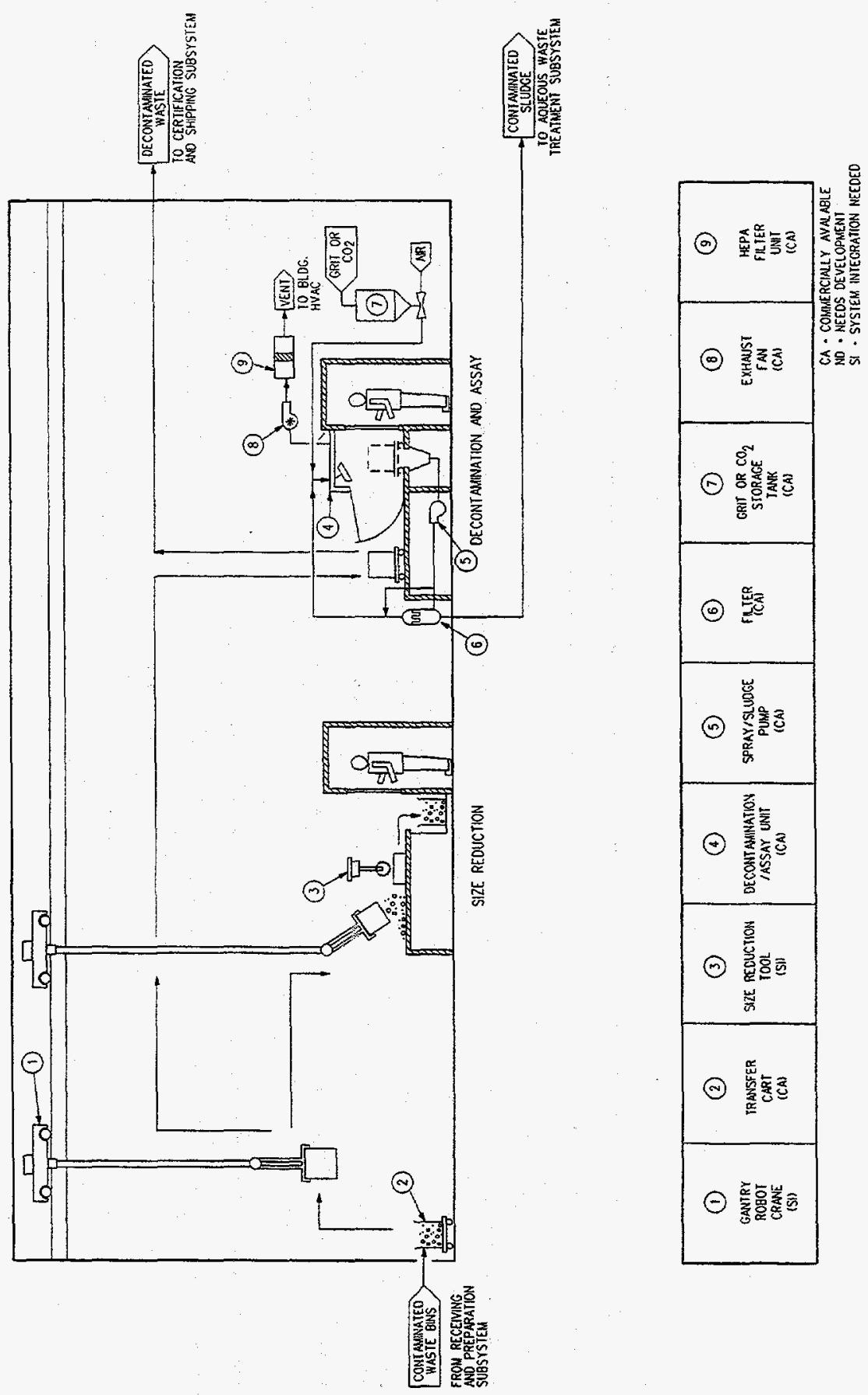

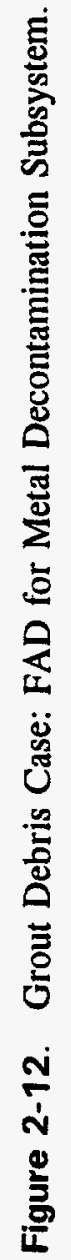




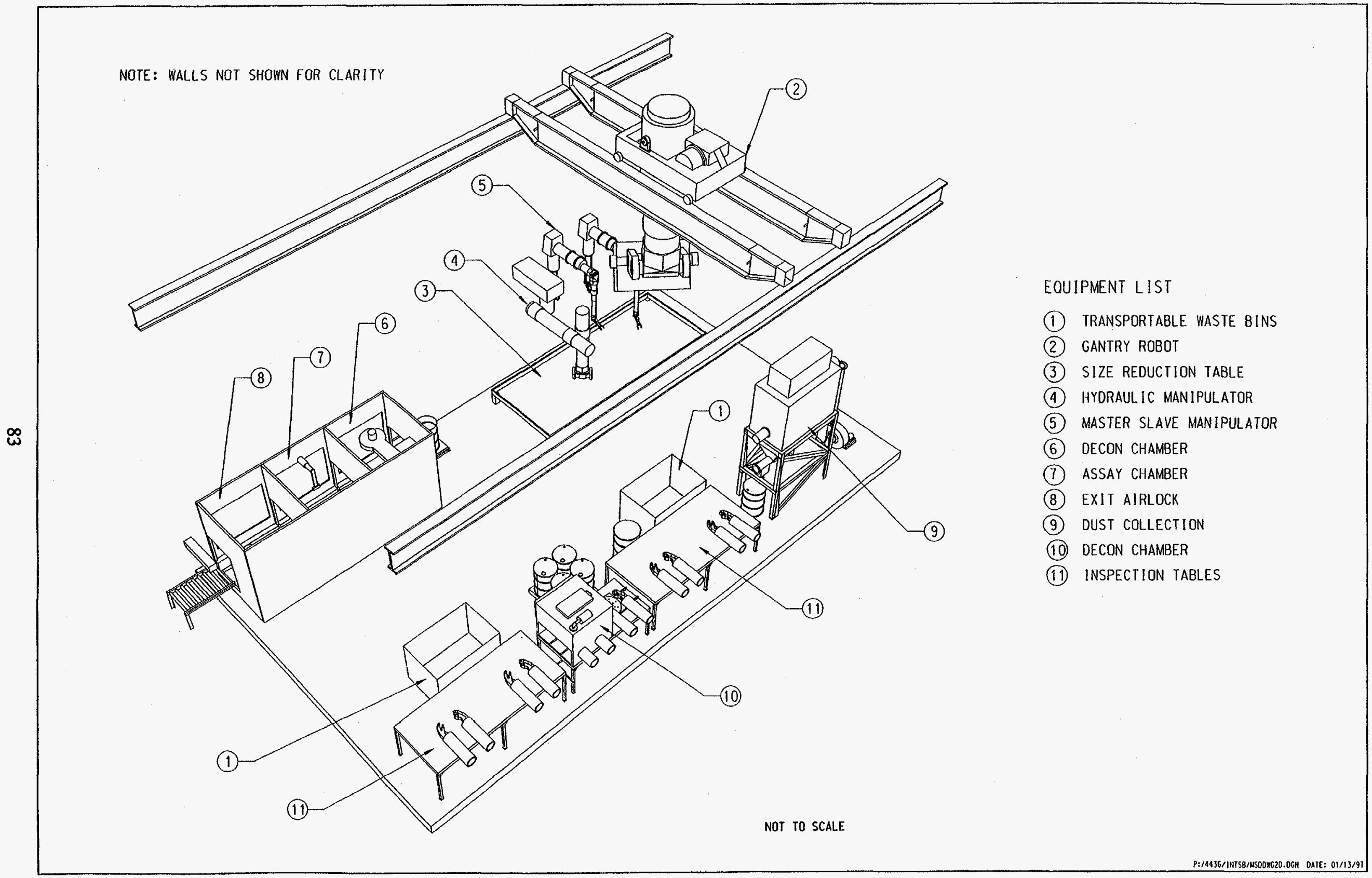

Figure 2-13. Grout Debris Case: PERSPECTIVE View of Metal Decontamination Subsystem. 


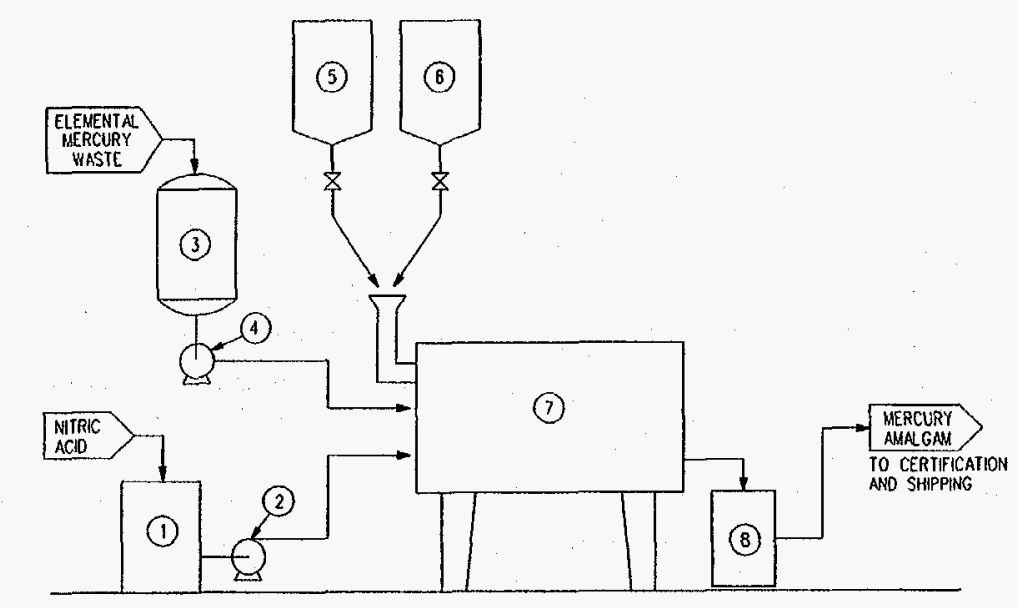

\begin{tabular}{|c|c|c|c|c|c|c|c|}
\hline 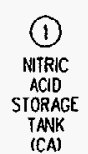 & 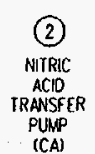 & 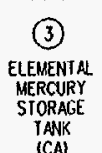 & $\begin{array}{l}\text { (4) } \\
\text { MERCURY } \\
\text { PUMP } \\
\text { (CA) }\end{array}$ & 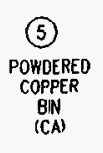 & 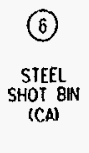 & 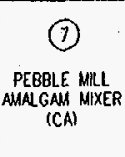 & $\begin{array}{c}\text { (8) } \\
\substack{\text { MANGAMA } \\
\text { PACKGANG } \\
\text { PCAA }}\end{array}$ \\
\hline
\end{tabular}

Figure 2-14. Grout Debris Case: FAD for Mercury Amalgamation Subsystem. 


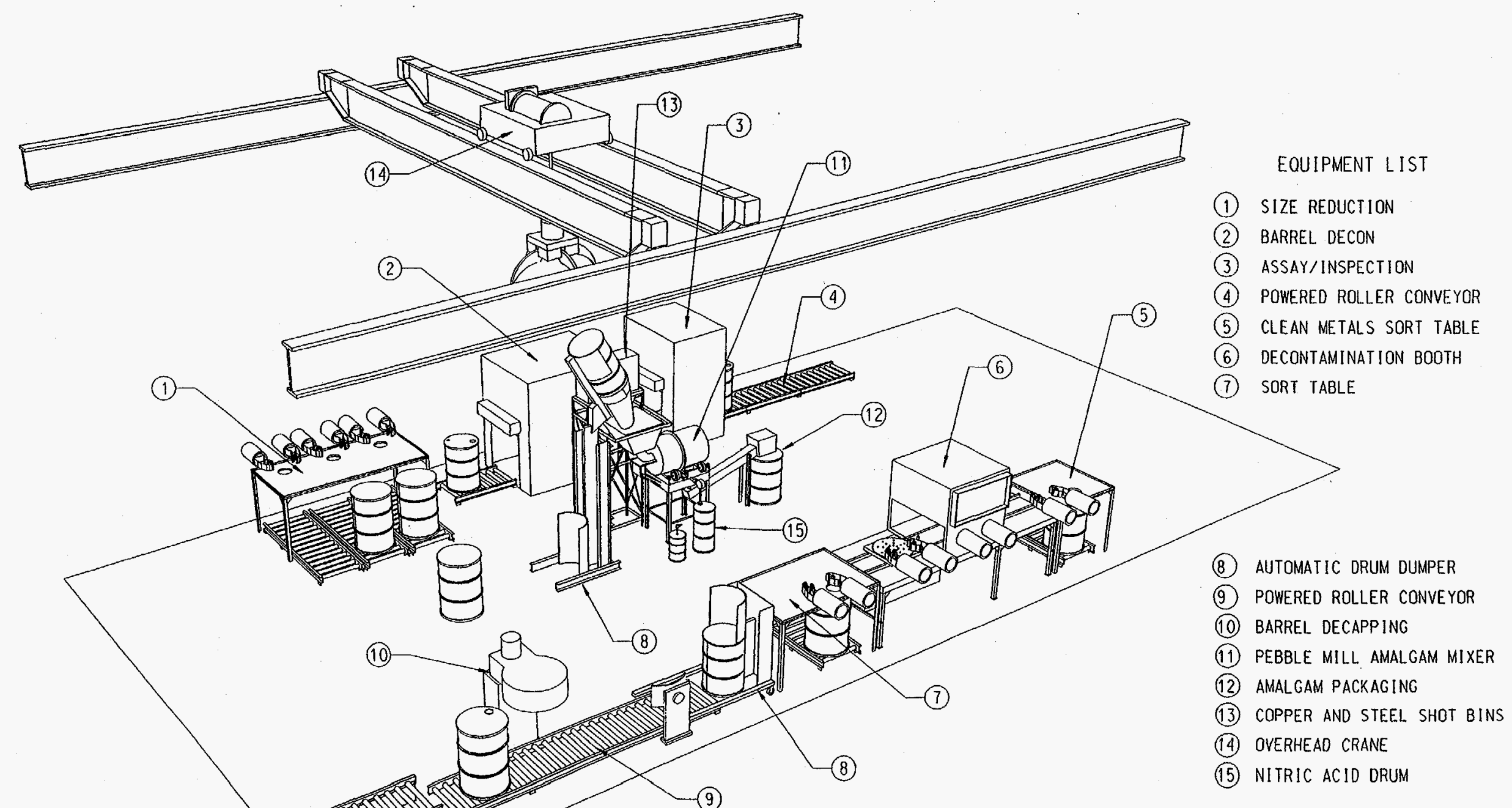

NOTE: WALLS NOT SHOWN FOR CLARITY

NOT TO SCALE

Figure 2-15. Grout Debris Case: PERSPECTIVE View of Mercury Amalgamation and Lead Recovery Subsystems. 


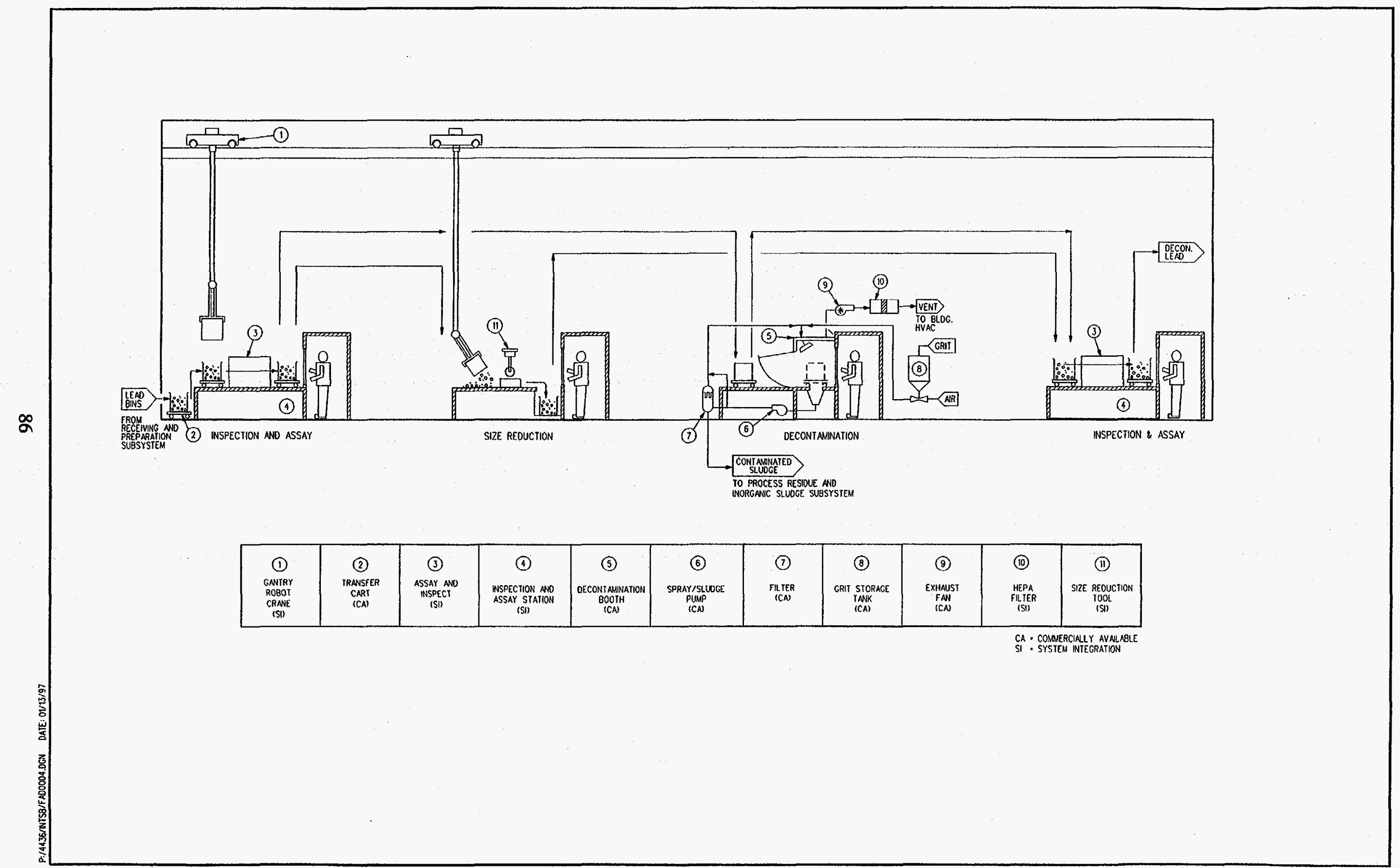

Figure 2-16. Grout Debris Case: FAD for Lead Recovery Subsystem. 


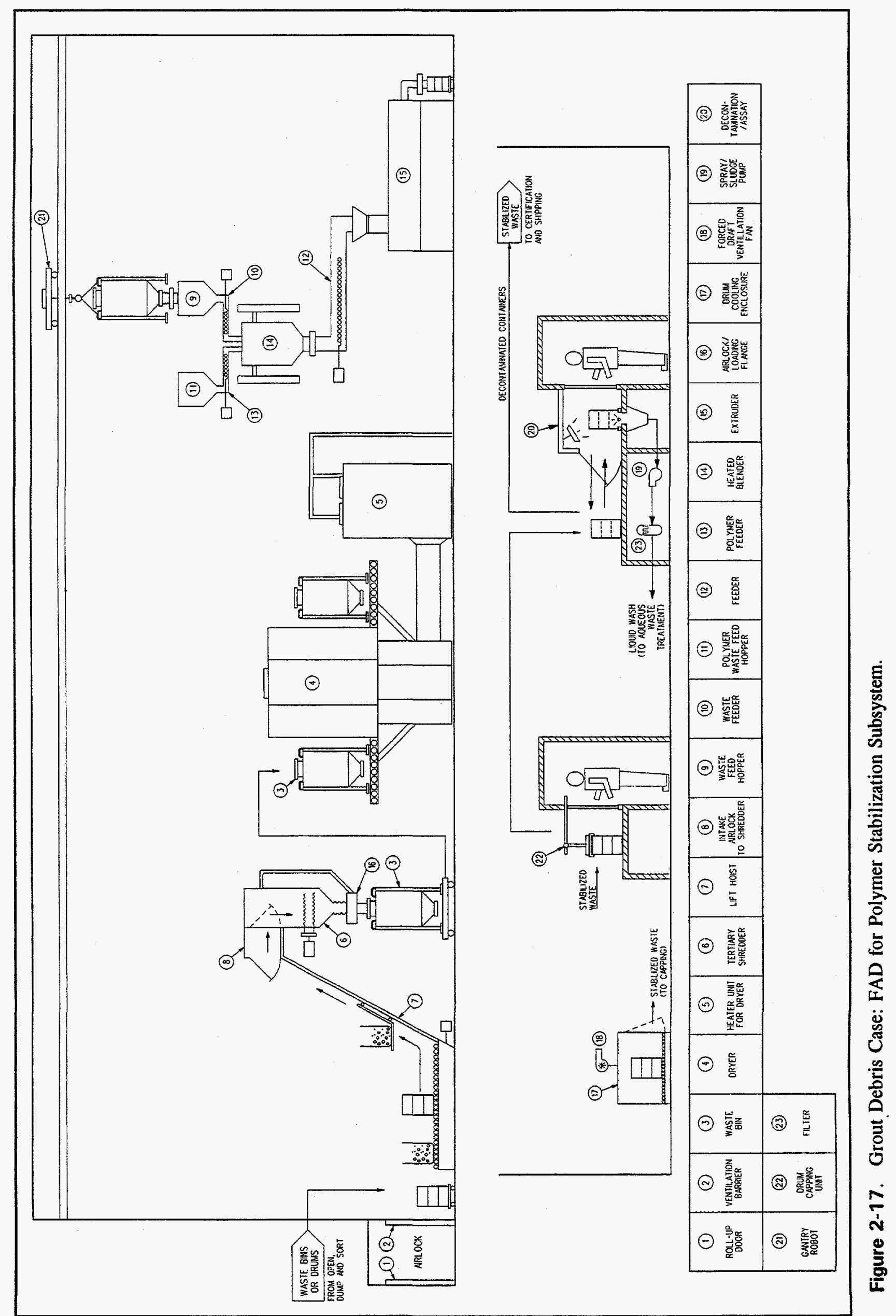




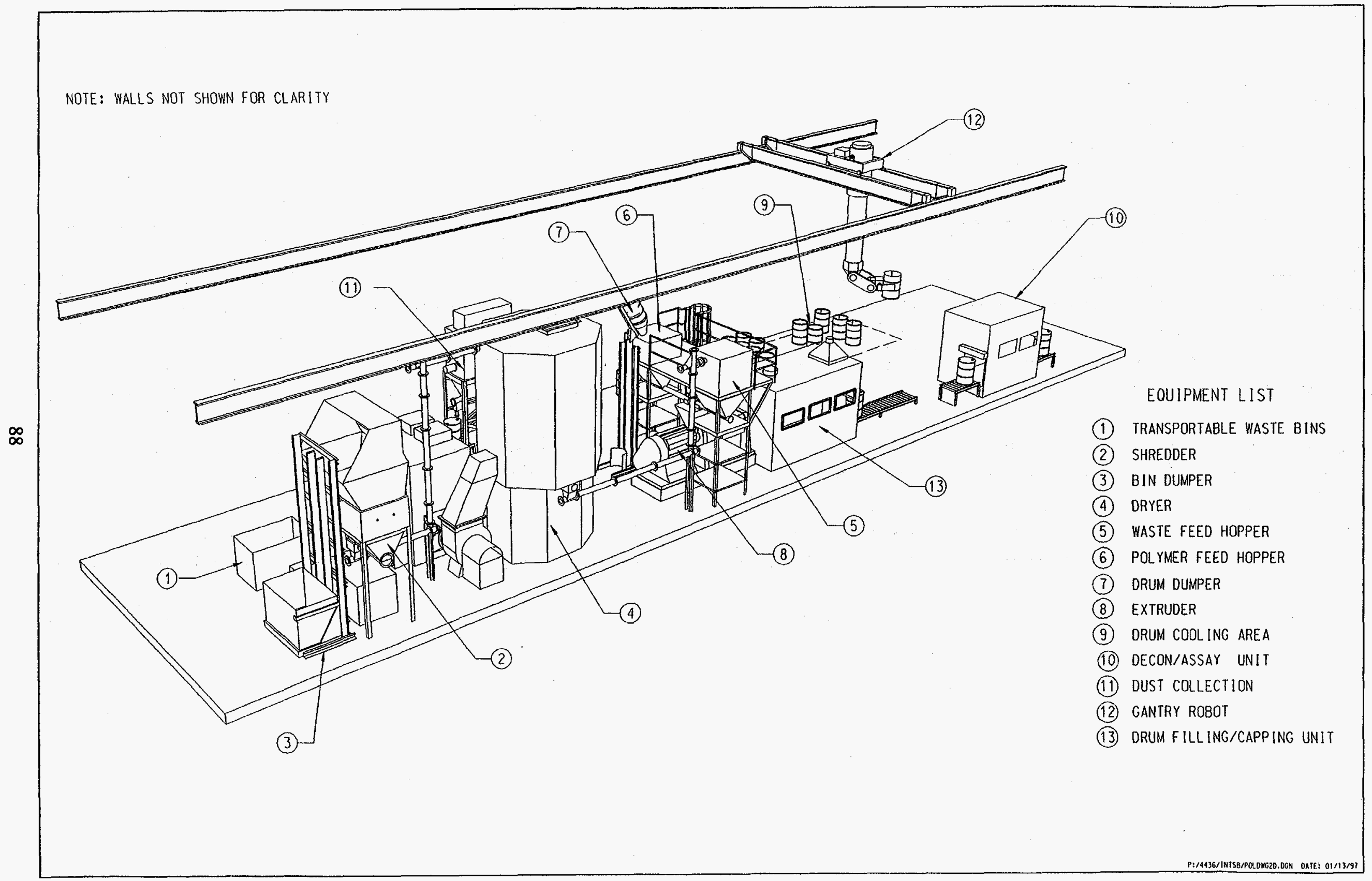

Figure 2-18. Grout Debris Case: PERSPECTIVE View of Polymer Stabilization Subsystem. 


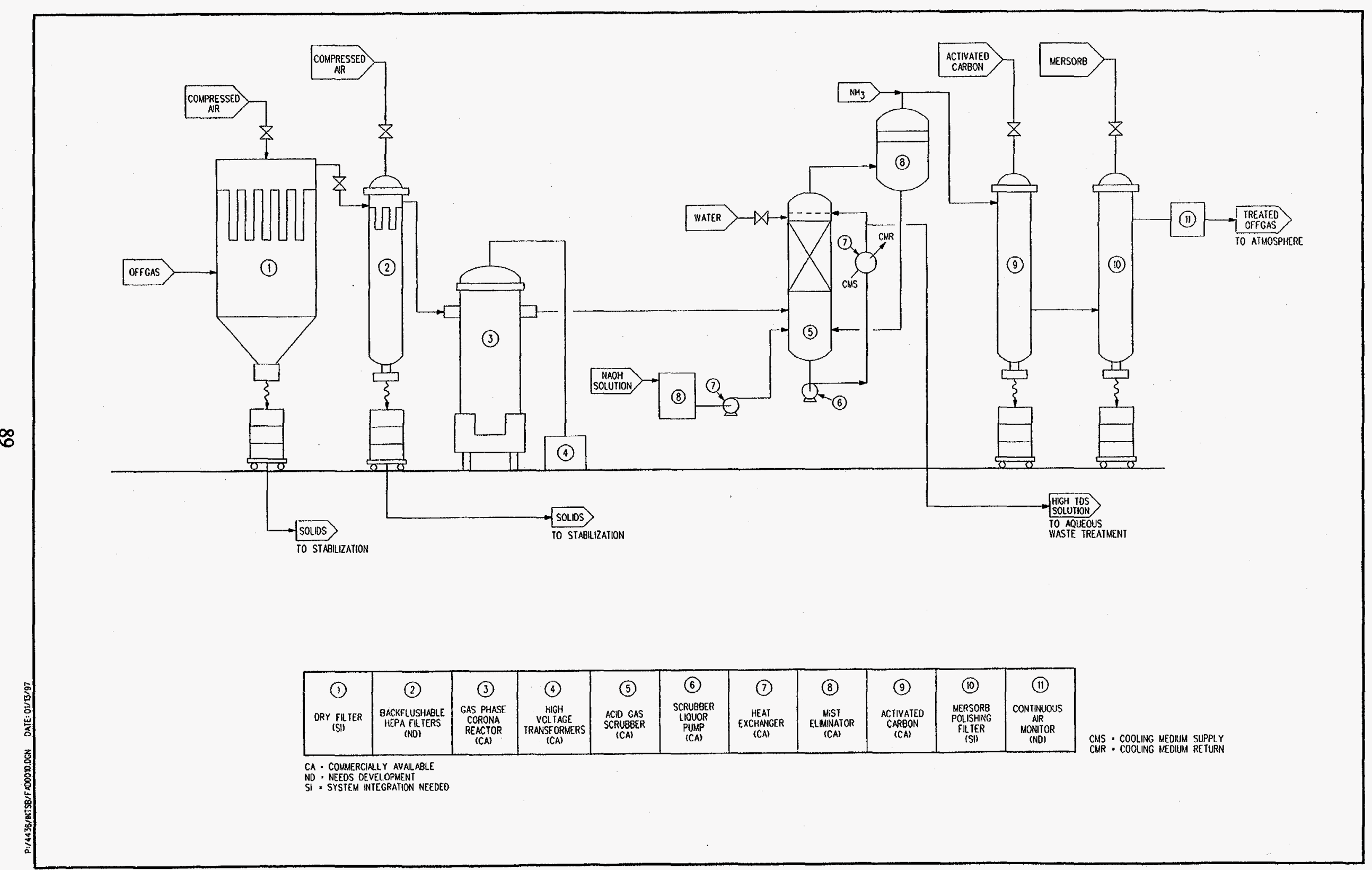

Figure 2-19. Grout Debris Case: FAD for Air Pollution Control Subsystem. 


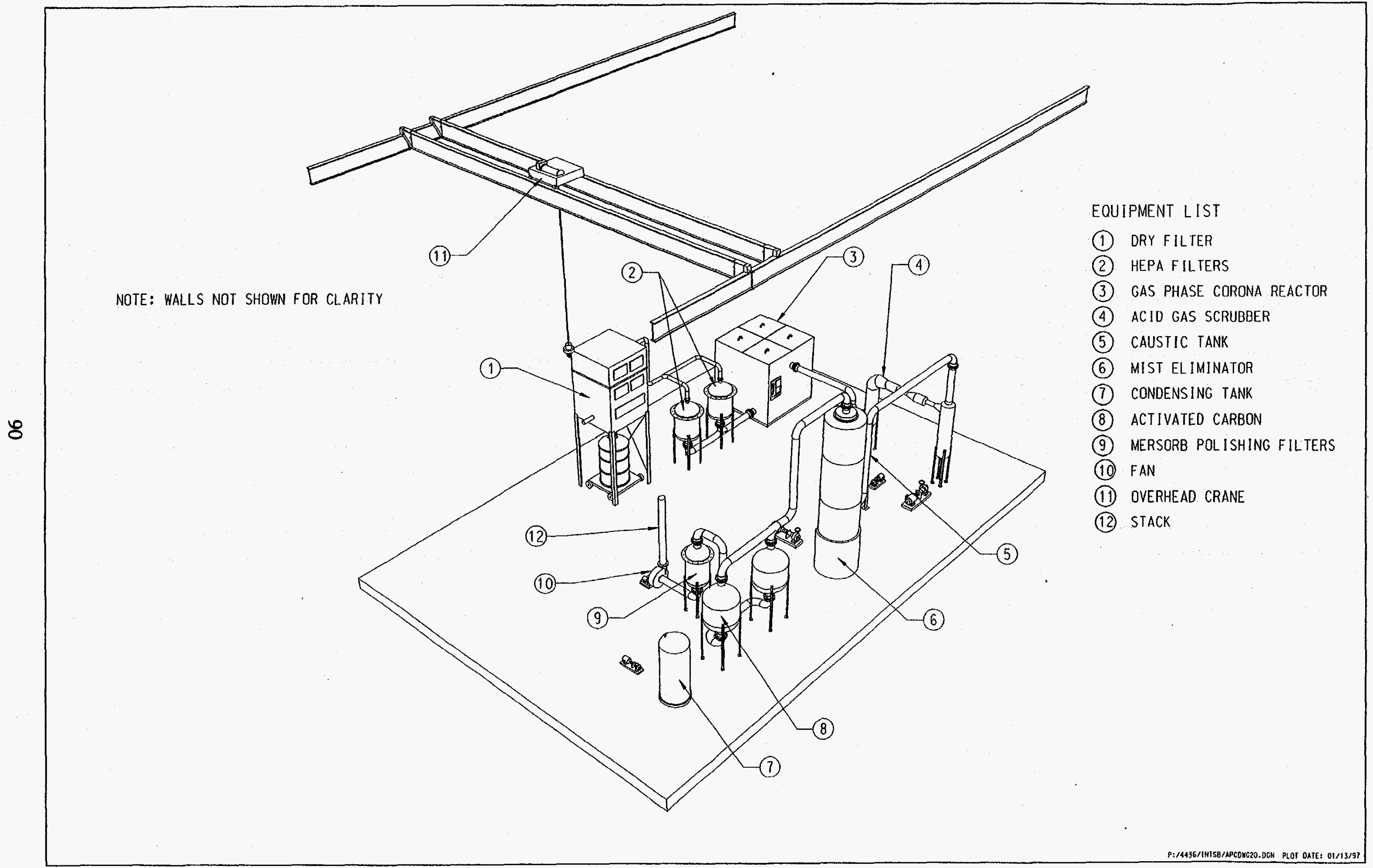

Figure 2-20. Grout Debris Case: PERSPECTIVE View of Air Pollution Control Subsystem. 

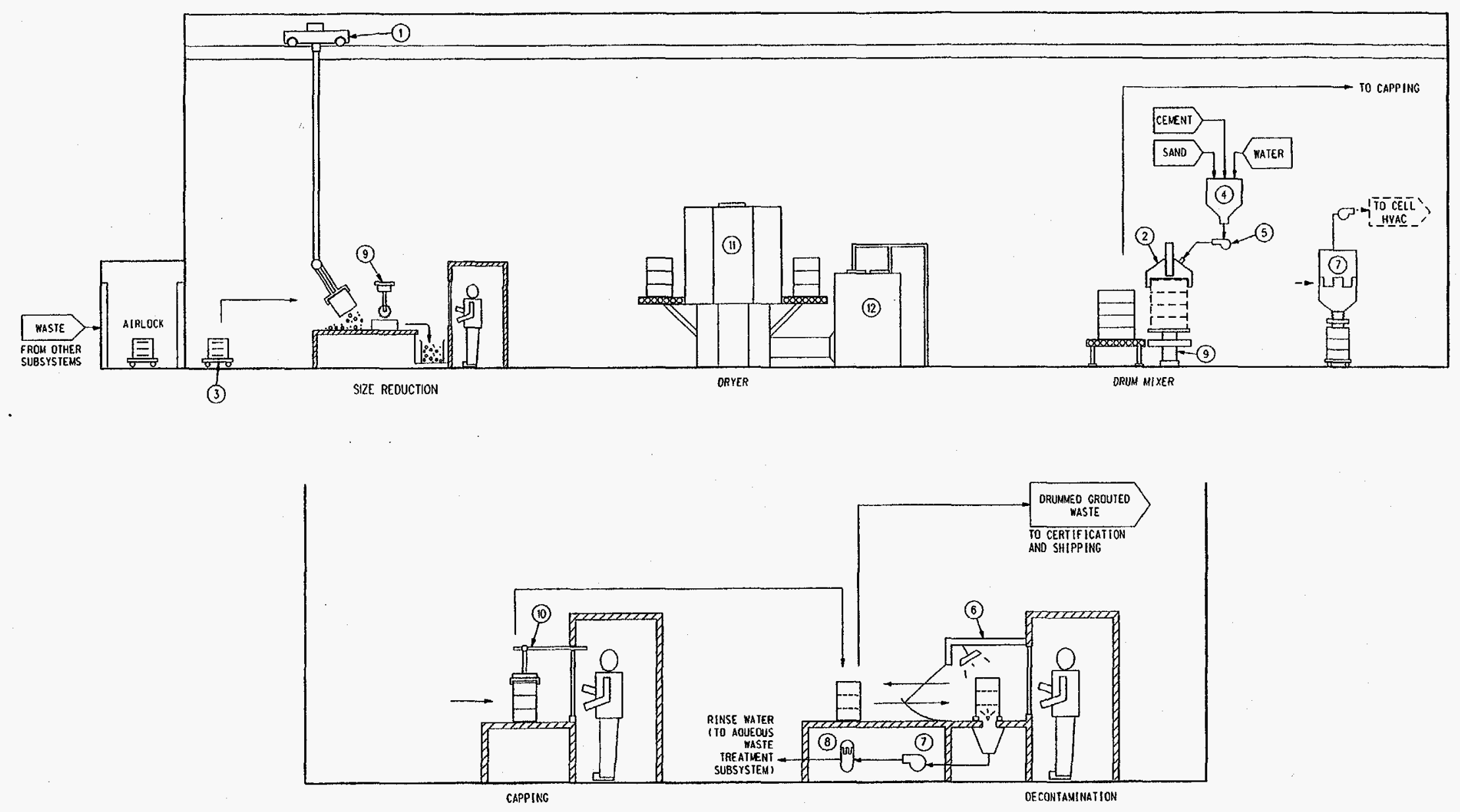

\begin{tabular}{|c|c|c|c|c|c|c|c|c|c|c|c|}
\hline 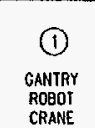 & 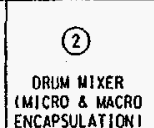 & 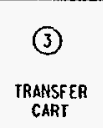 & $\underset{\substack{\text { MIXING } \\
\text { TANK }}}{(4)}$ & 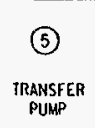 & 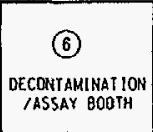 & $\begin{array}{c}\text { (7) } \\
\text { SPRAYYSLLUDGE } \\
\text { PUYP }\end{array}$ & $\begin{array}{c}\text { (8) } \\
\text { FILTER }\end{array}$ & 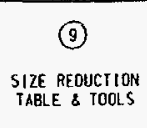 & 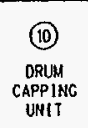 & $\begin{array}{l}\text { (11) } \\
\text { DRYER }\end{array}$ & 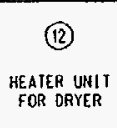 \\
\hline
\end{tabular}

Figure 2-21. Grout Debris Case: FAD for Grout Stabilization Subsystem.

P:/4436/NTSB/FADO007.0CN DAIE: 01/13/97 


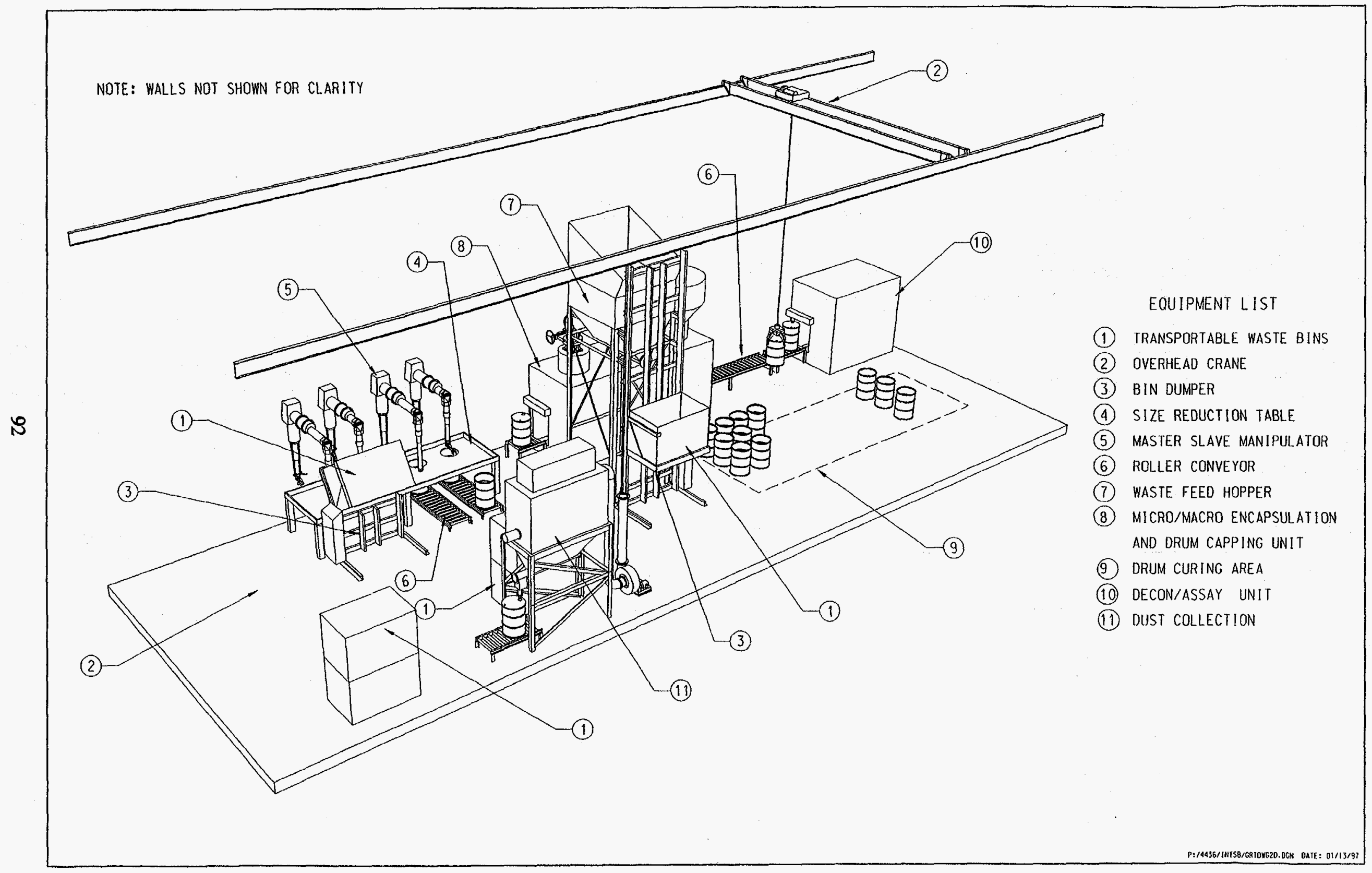

Figure 2-22. Grout Debris Case: PERSPECTIVE View of Grout Stabilization Subsystem. 


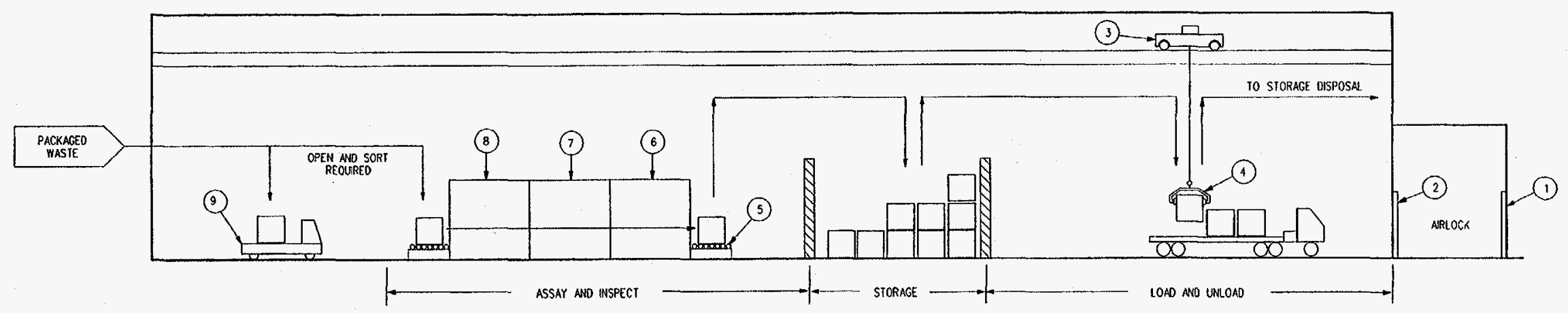

\begin{tabular}{|c|c|c|c|c|c|c|c|c|}
\hline $\begin{array}{c}\text { (1) } \\
\text { ROLLLUP } \\
\text { ofor } \\
\text { OCANA }\end{array}$ & (2) & 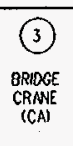 & 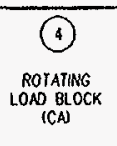 & 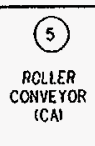 & 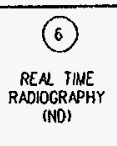 & 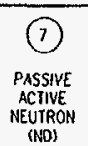 & 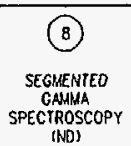 & $\underset{\substack{\text { SELF. GUDED } \\
\text { VEGCLIE } \\
\text { SIII }}}{(9)}$ \\
\hline
\end{tabular}

Figure 2-23. Grout Debris Case: FAD for Certification \& Shipping Subsystem. 


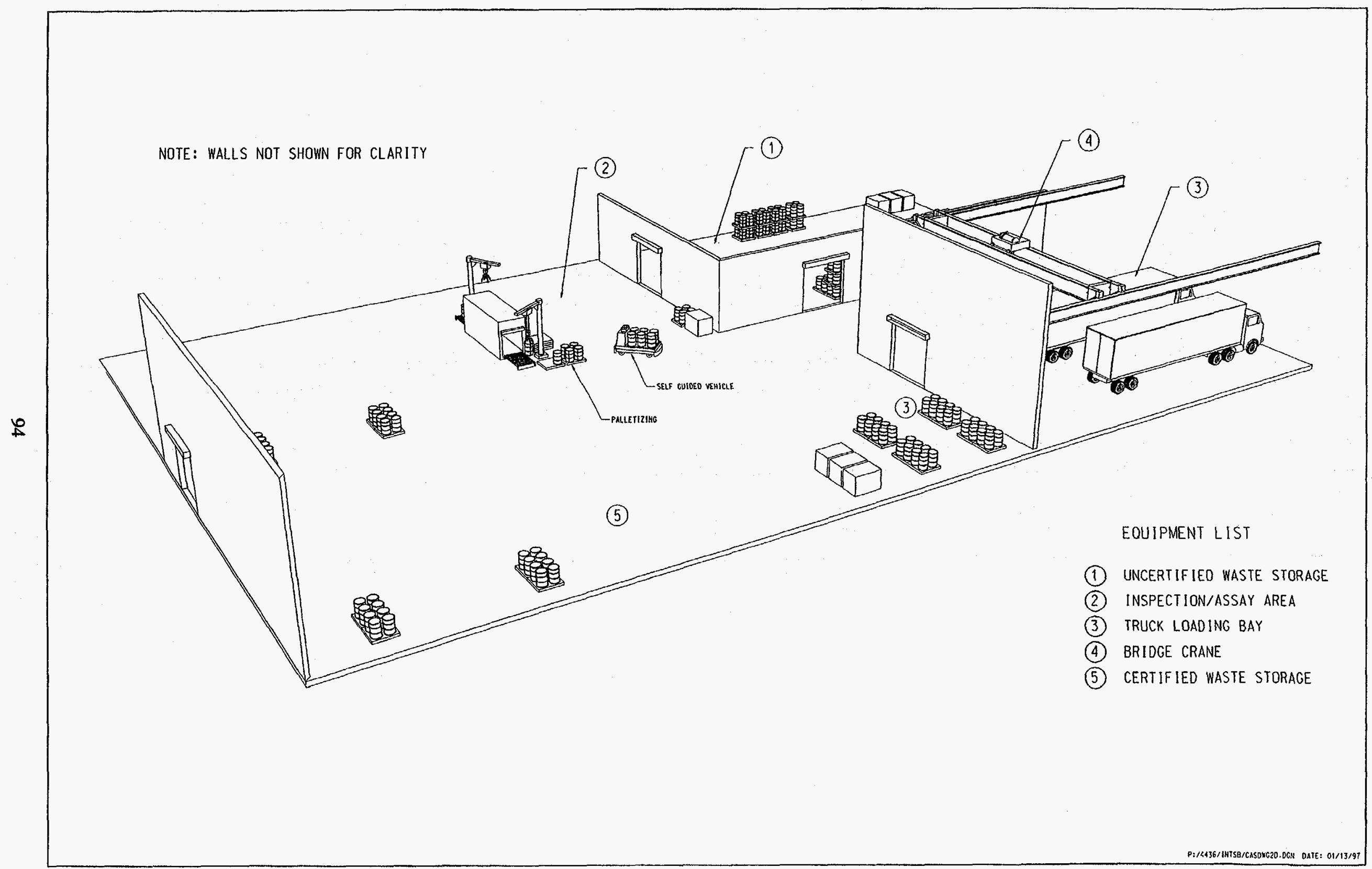

Figure 2-24. Grout Debris Case: PERSPECTIVE View of Certification \& Shipping Subsystem. 


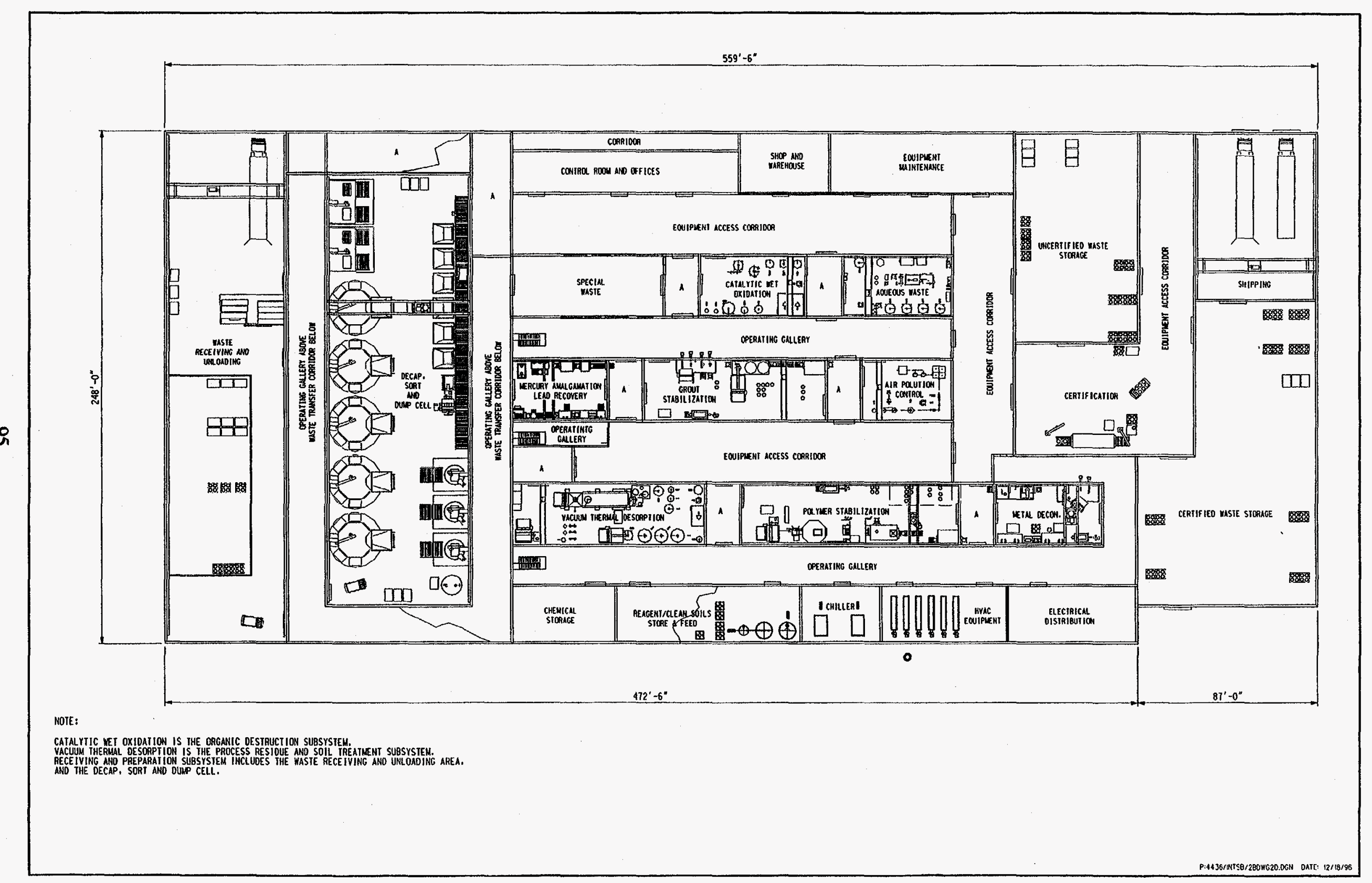

Figure 2-25. Desorption Case: PLAN View of Overall System (System 2). 


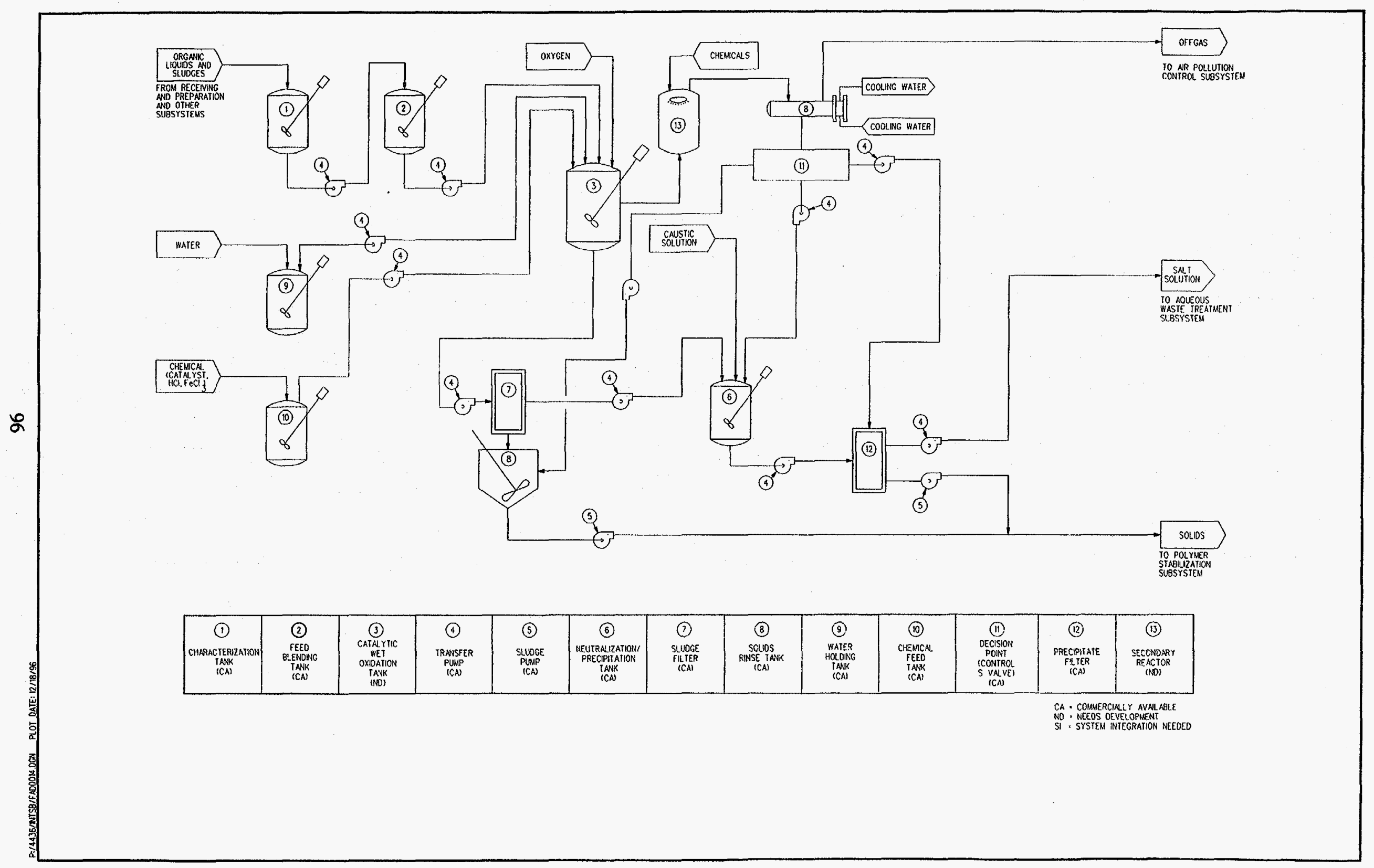

Figure 2-26. Desorption Case: FAD for Organic Destruction Subsystem. 


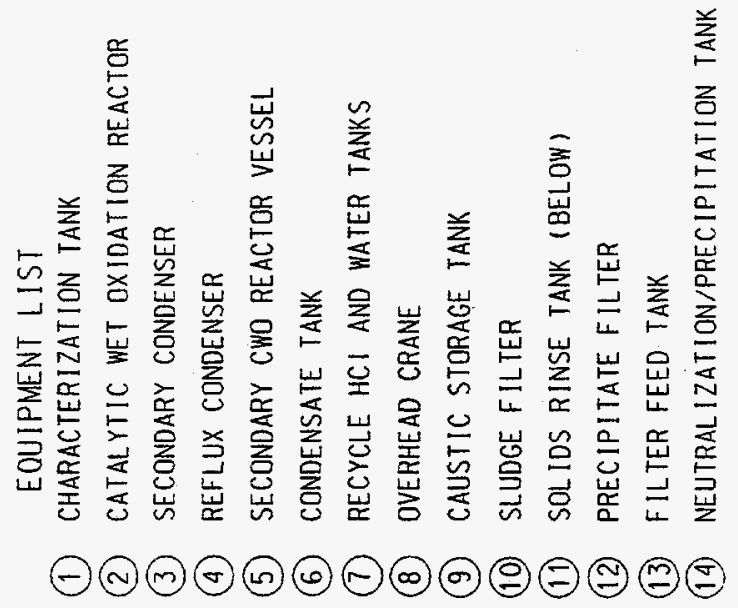

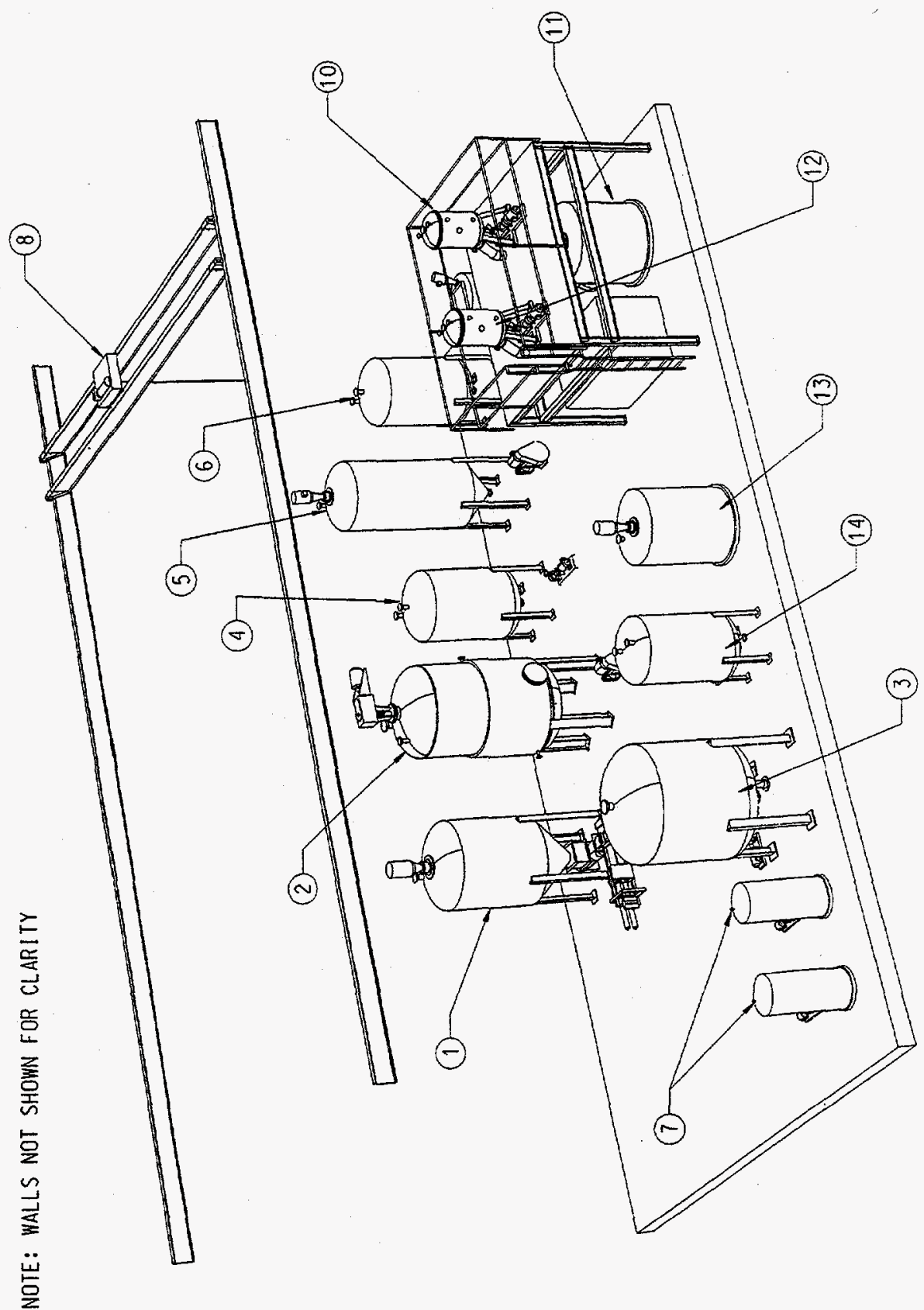

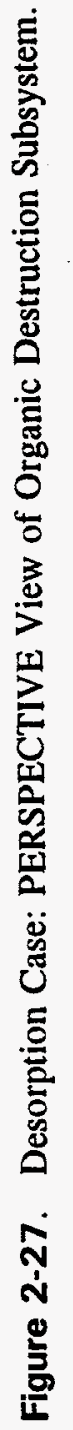




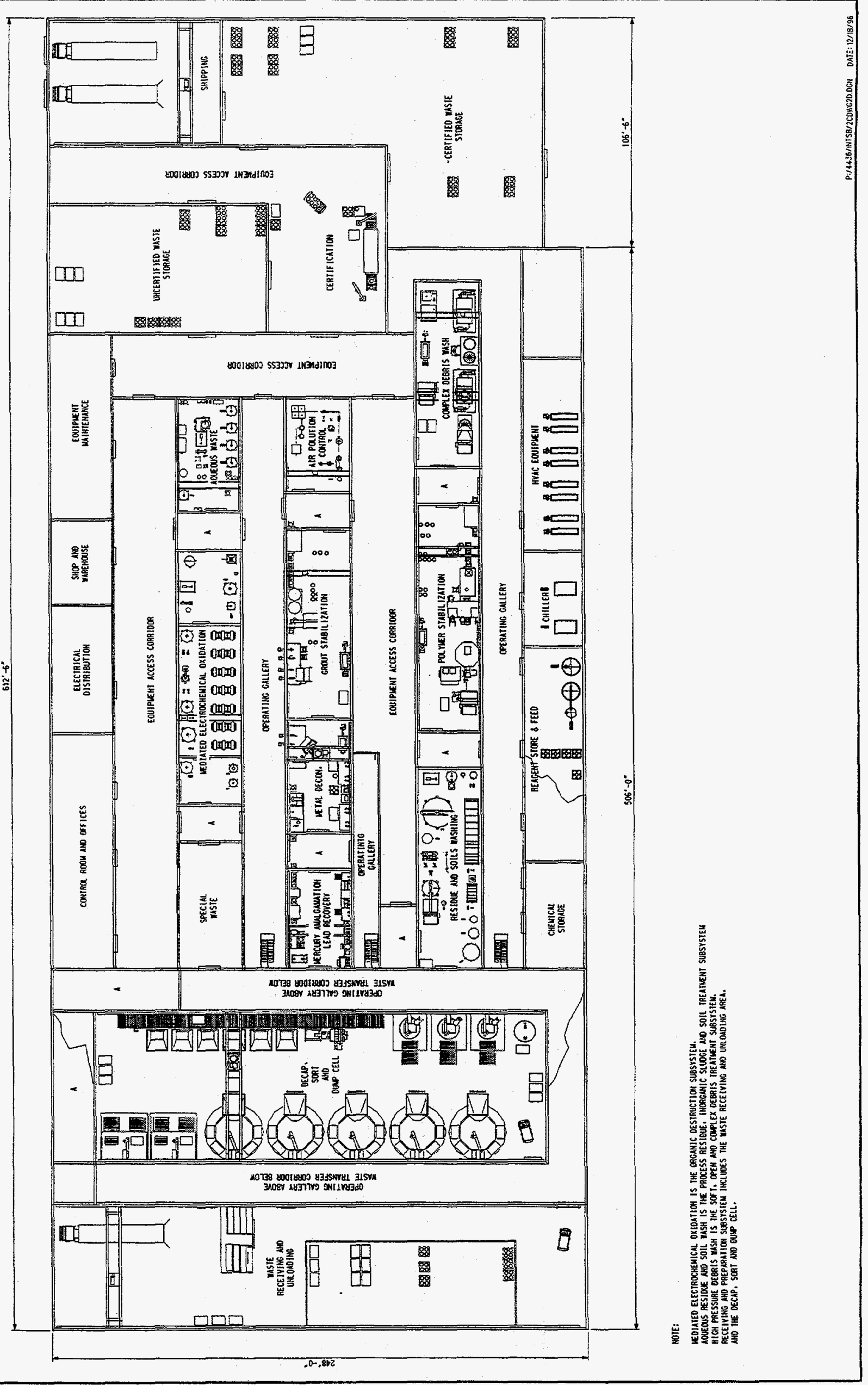

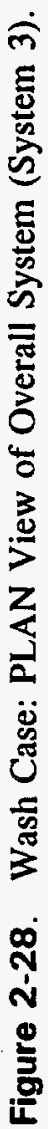




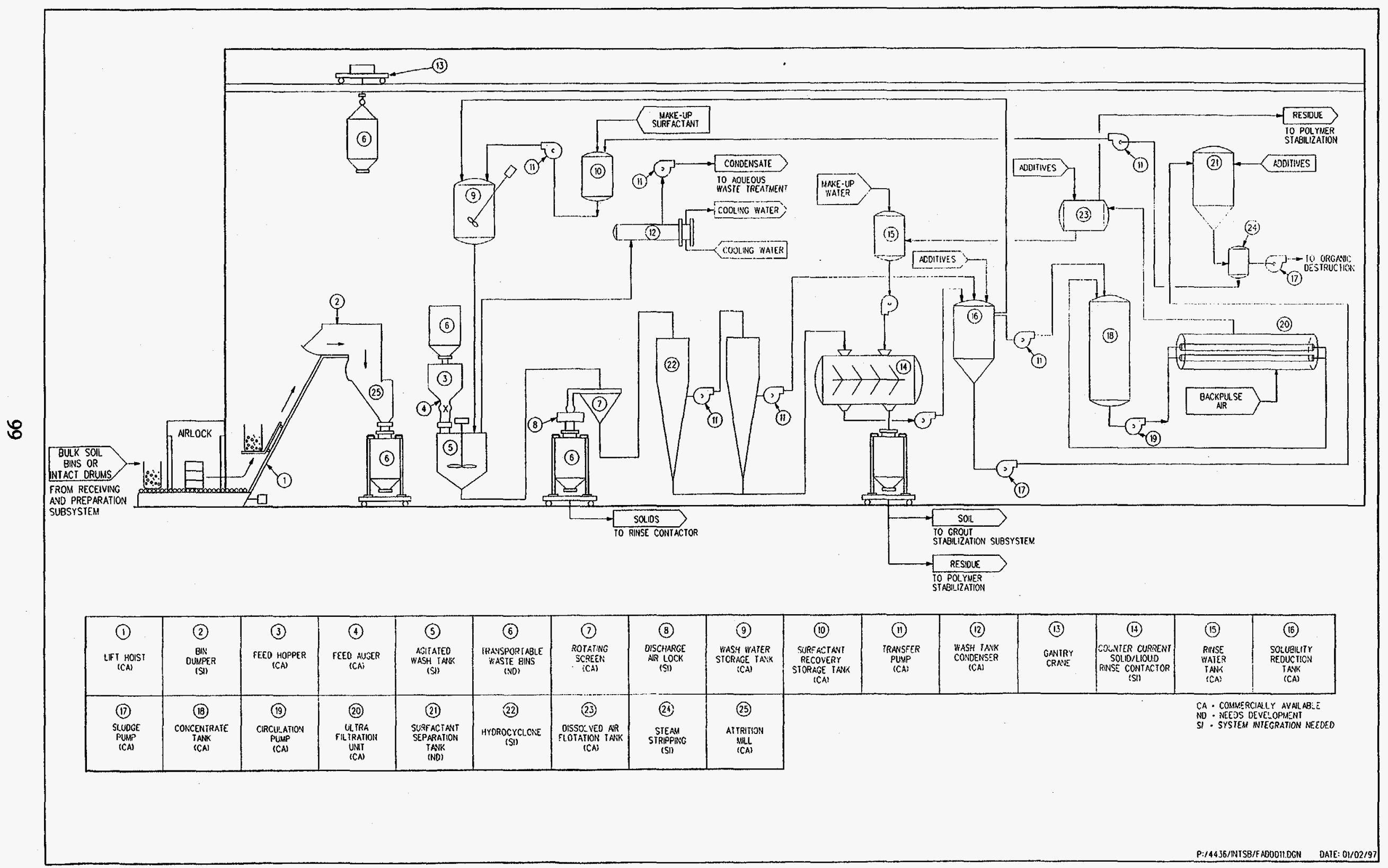

Figure 2-29. Wash Case: FAD for Process Residue, Inorganic Sludge and Bulk Soil Treatment Subsystems. 


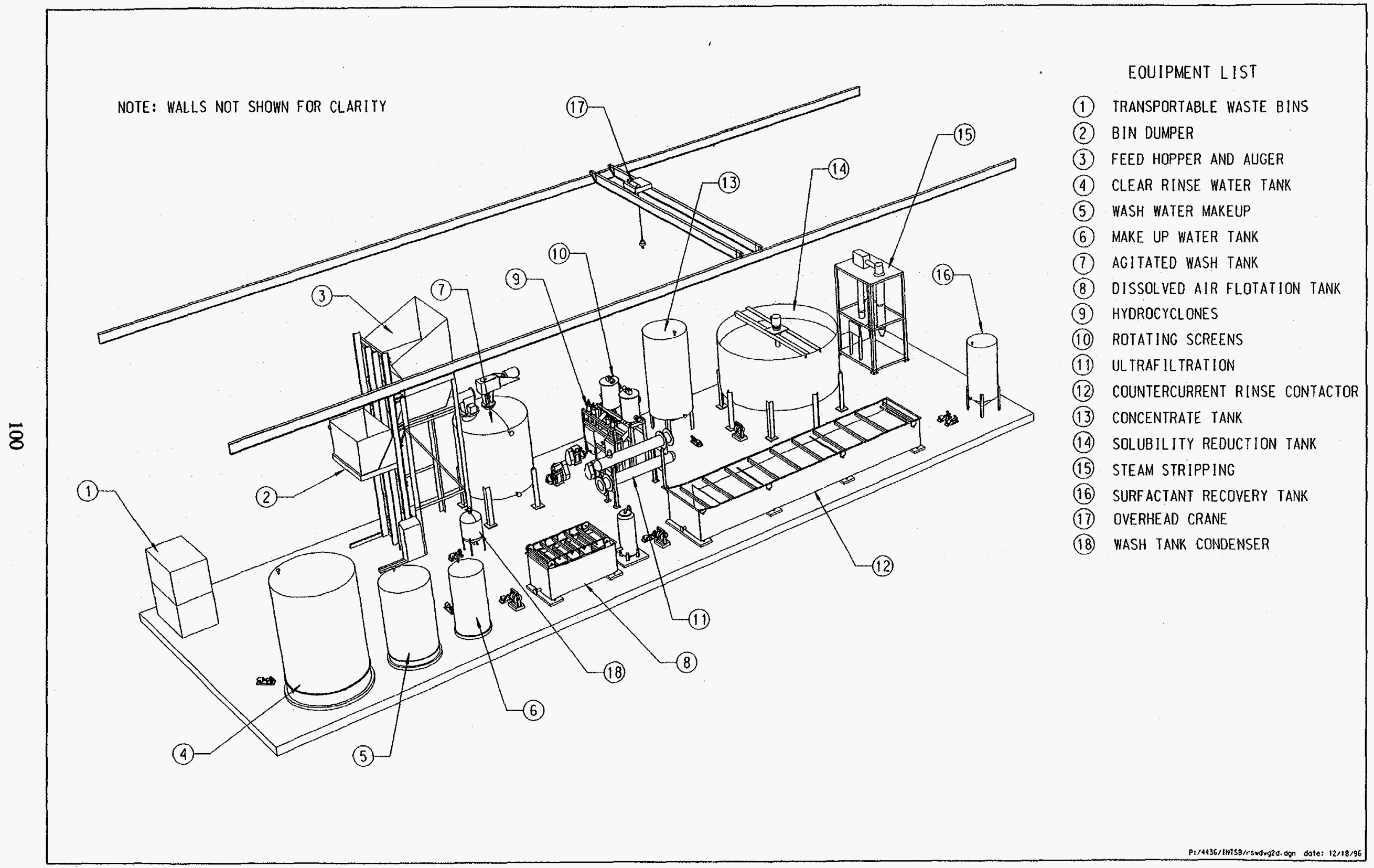

Figure 2-30. Wash Case: PERSPECTIVE View of Process Residue, Inorganic Sludge and Bulk Soil Treatment Subsystems. 


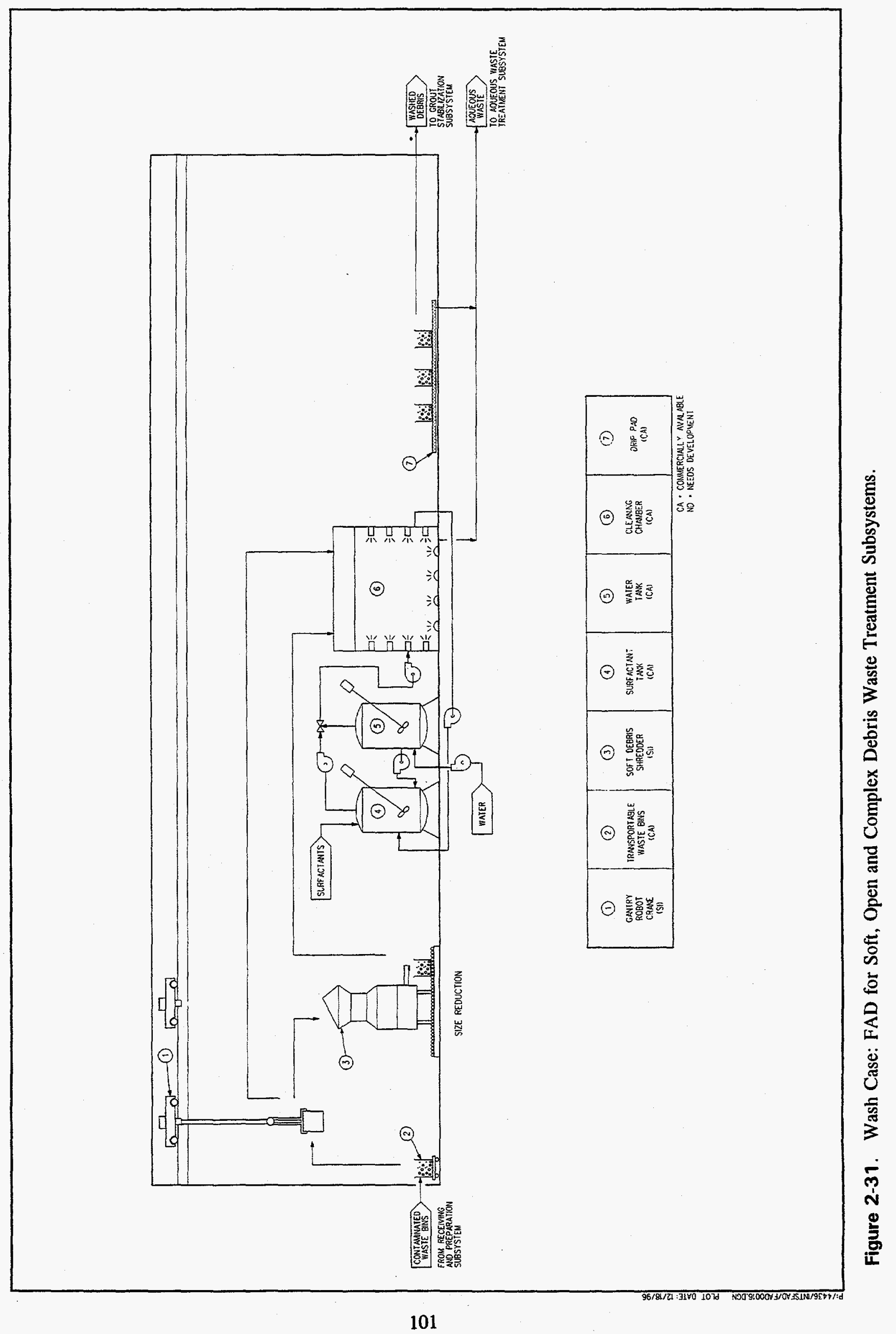




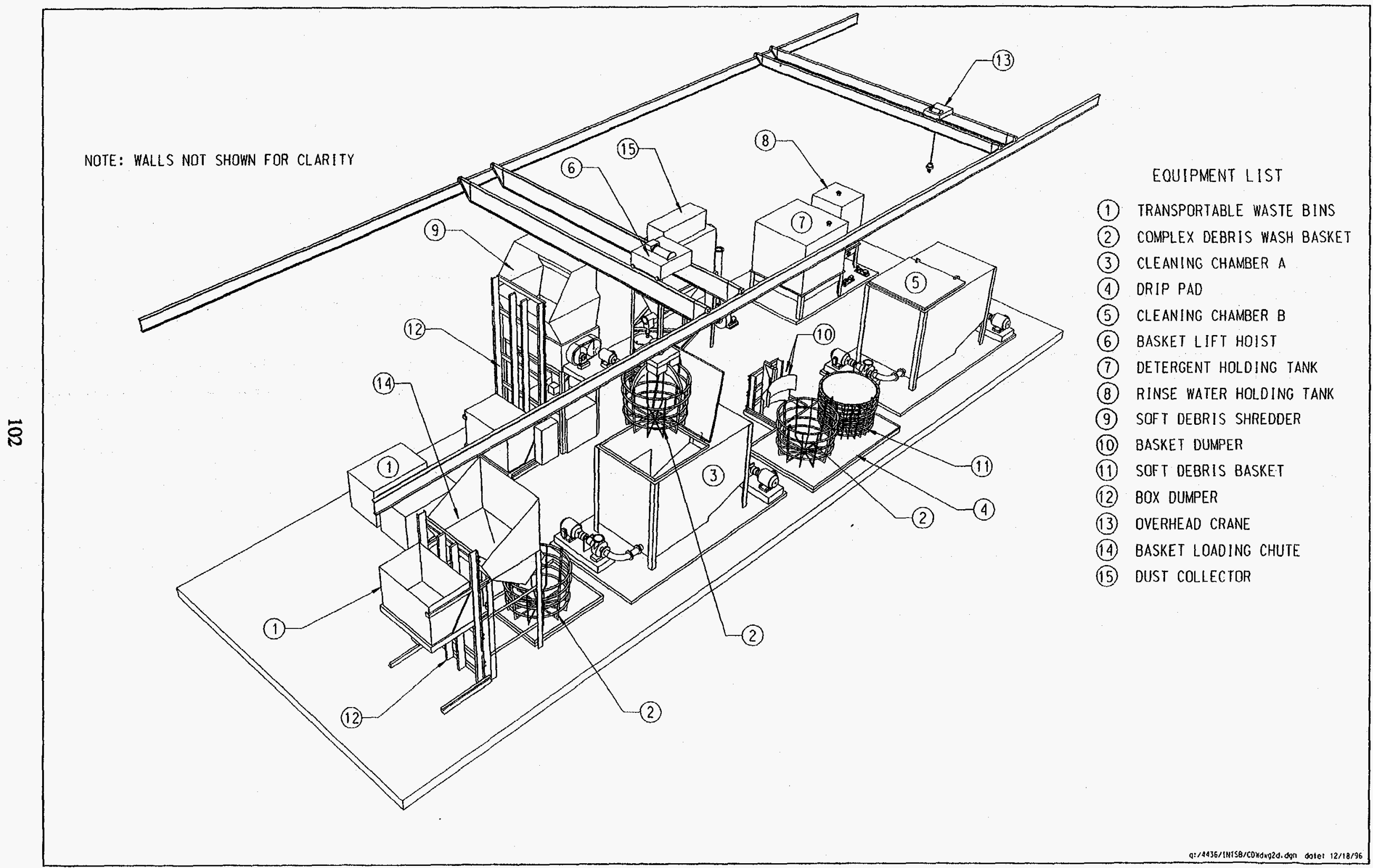

Figure 2-32. Wash Case: PERSPECTIVE View of Soft, Open and Complex Debris Waste Treatment Subsystems. 


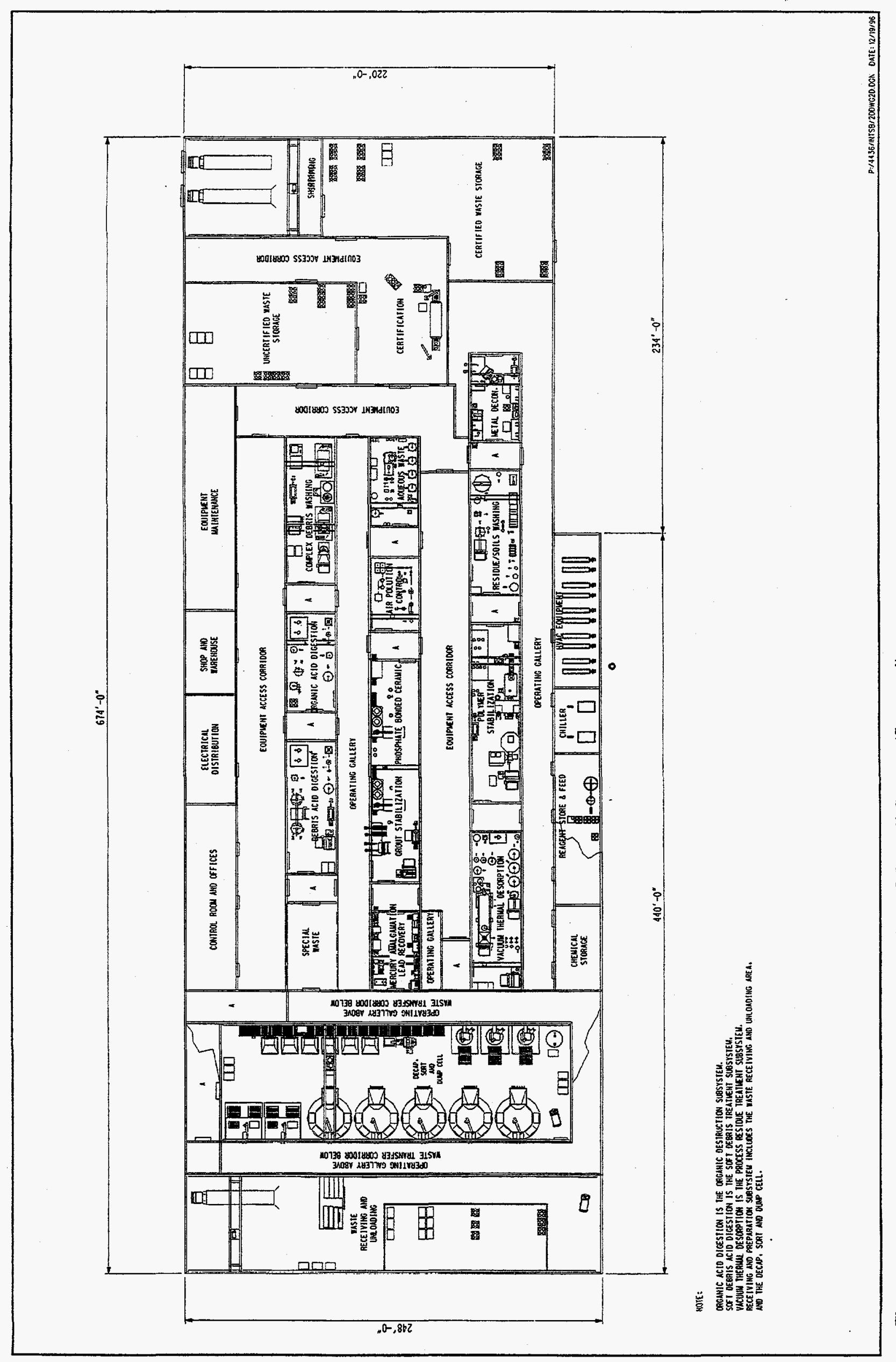

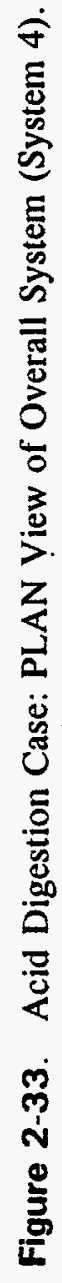




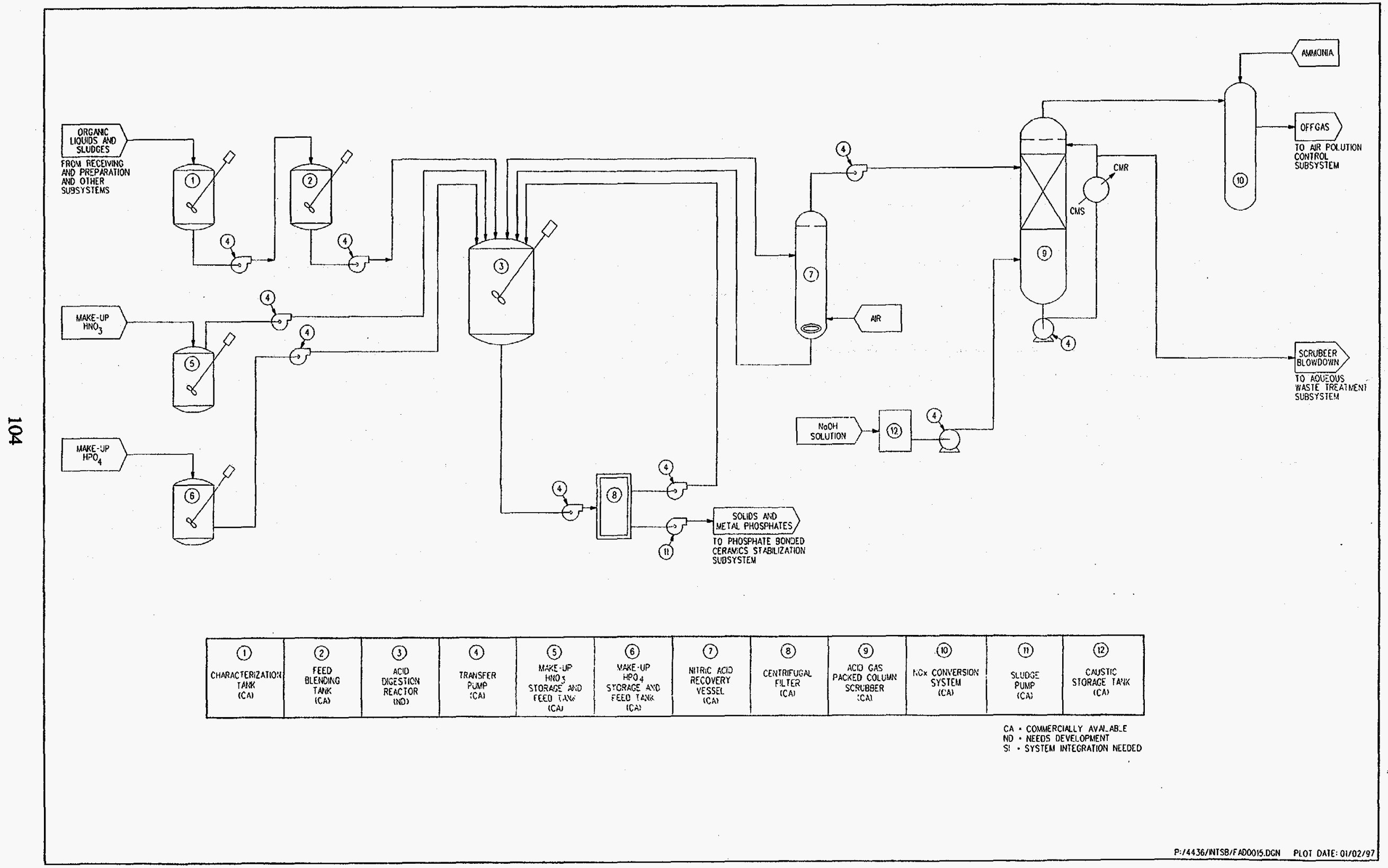

Figure 2-34. Acid Digestion Case: FAD of Organic Destruction Subsystem. 


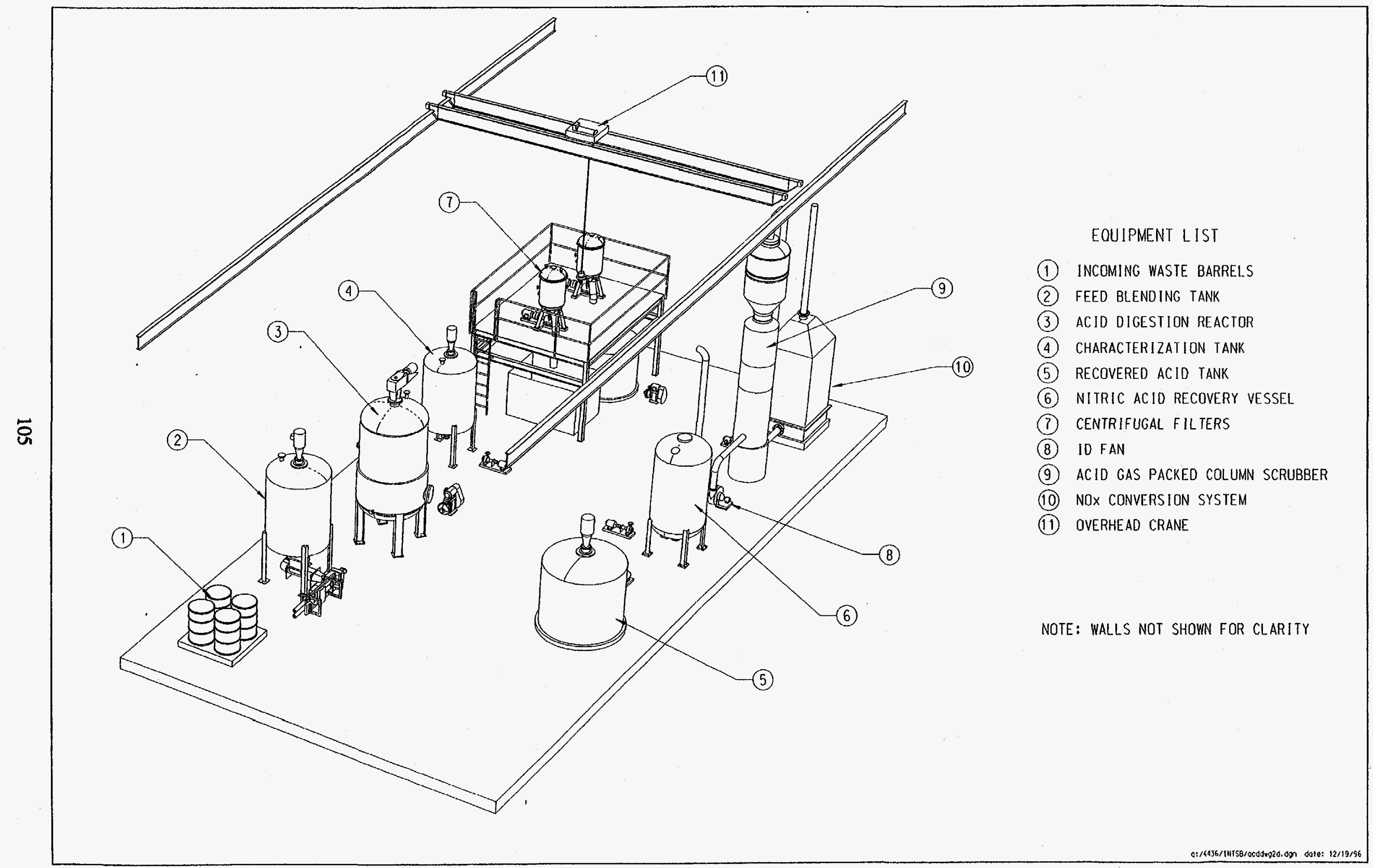

Figure 2-35. Acid Digestion Case: PERSPECTIVE View of Organic Destruction Subsystem. 


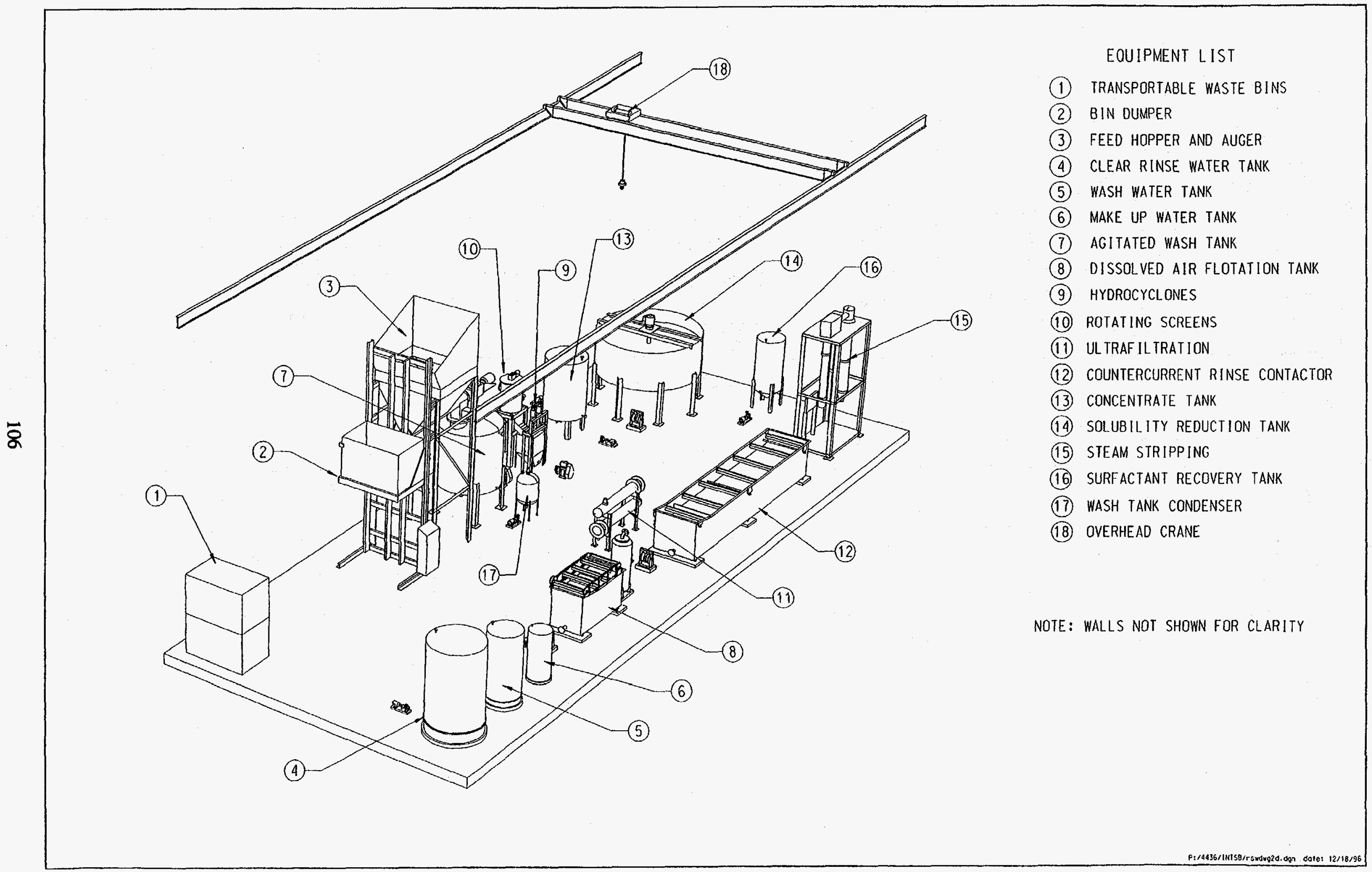

Figure 2-36. Acid Digestion Case: PERSPECTIVE View of Bulk Soil Treatment Subsystem. 


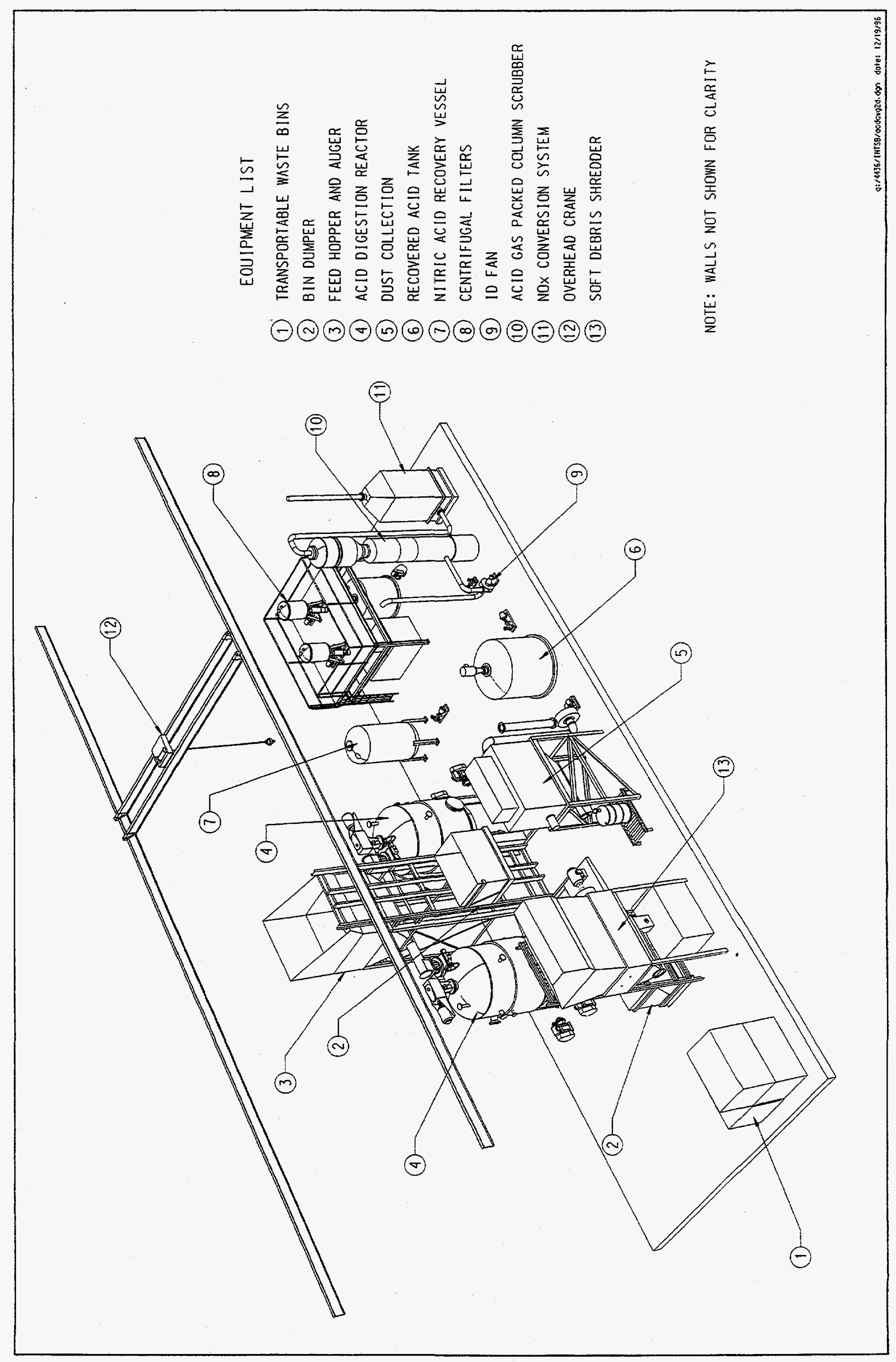

离 

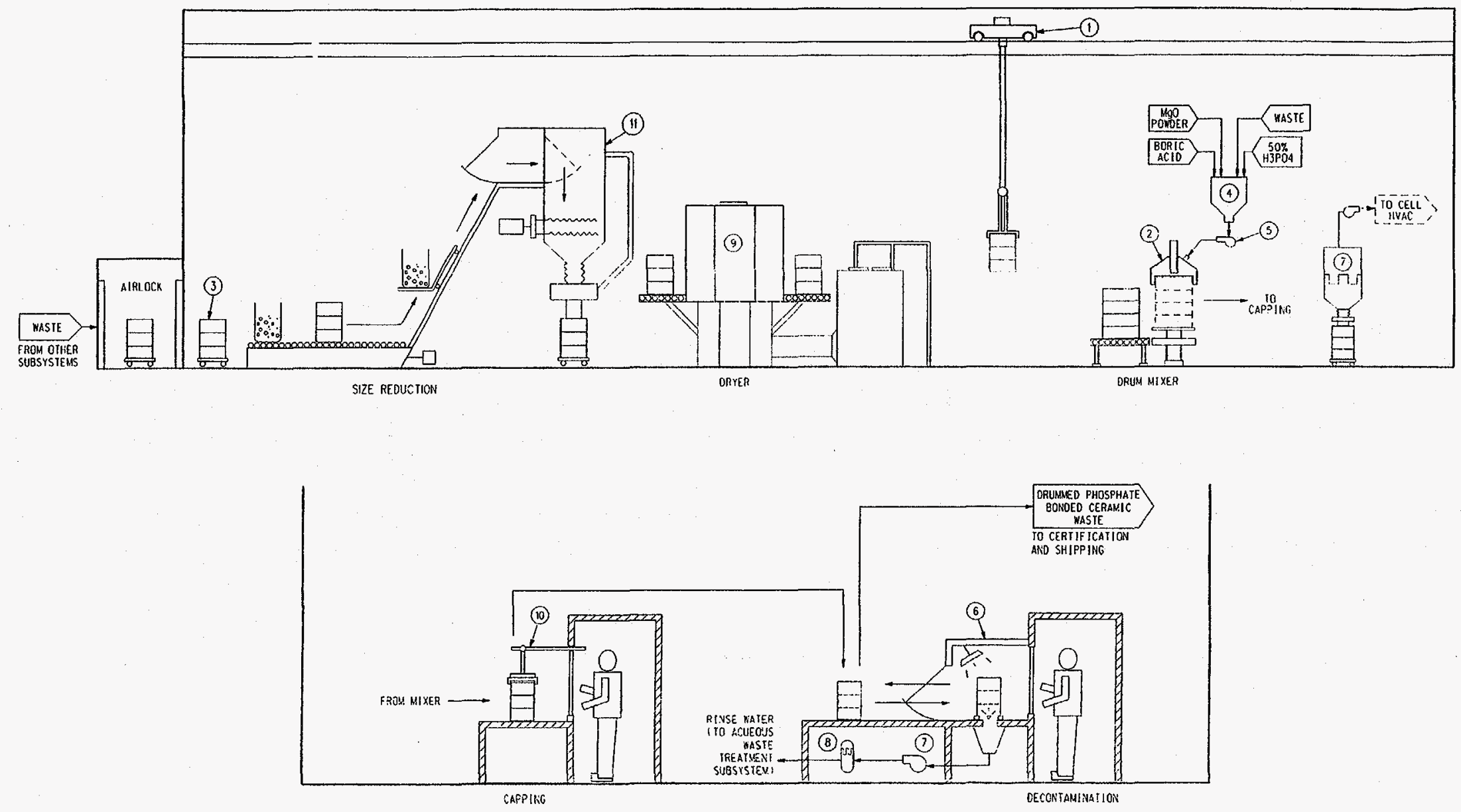

\begin{tabular}{|c|c|c|c|c|c|c|c|c|c|c|}
\hline 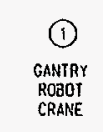 & $\begin{array}{c}\text { (2) } \\
\text { ORUM MIXER } \\
\text { MACRO \& AMACRO } \\
\text { EMCAPSULAT OOHA }\end{array}$ & 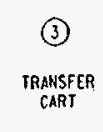 & $\underset{\substack{\text { MIXING } \\
\text { TANAK }}}{(4)}$ & $\begin{array}{c}\text { (5) } \\
\text { TRAASSER } \\
\text { PUSPP }\end{array}$ & $\begin{array}{c}\text { (5) } \\
\text { DECCOYYAMINATION } \\
\text { TASSAY BOOTH }\end{array}$ & $\underset{\substack{\text { SPRAYYSLUDOE } \\
\text { PUMPP }}}{(7)}$ & $\begin{array}{c}\text { (8) } \\
\text { FILIER }\end{array}$ & $\begin{array}{l}\text { (9) } \\
\text { ORYER }\end{array}$ & 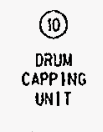 & $\begin{array}{c}\text { (11) } \\
\text { SHREDOER }\end{array}$ \\
\hline
\end{tabular}


NOTE: WALLS NOT SHOWN FOR CLARITY

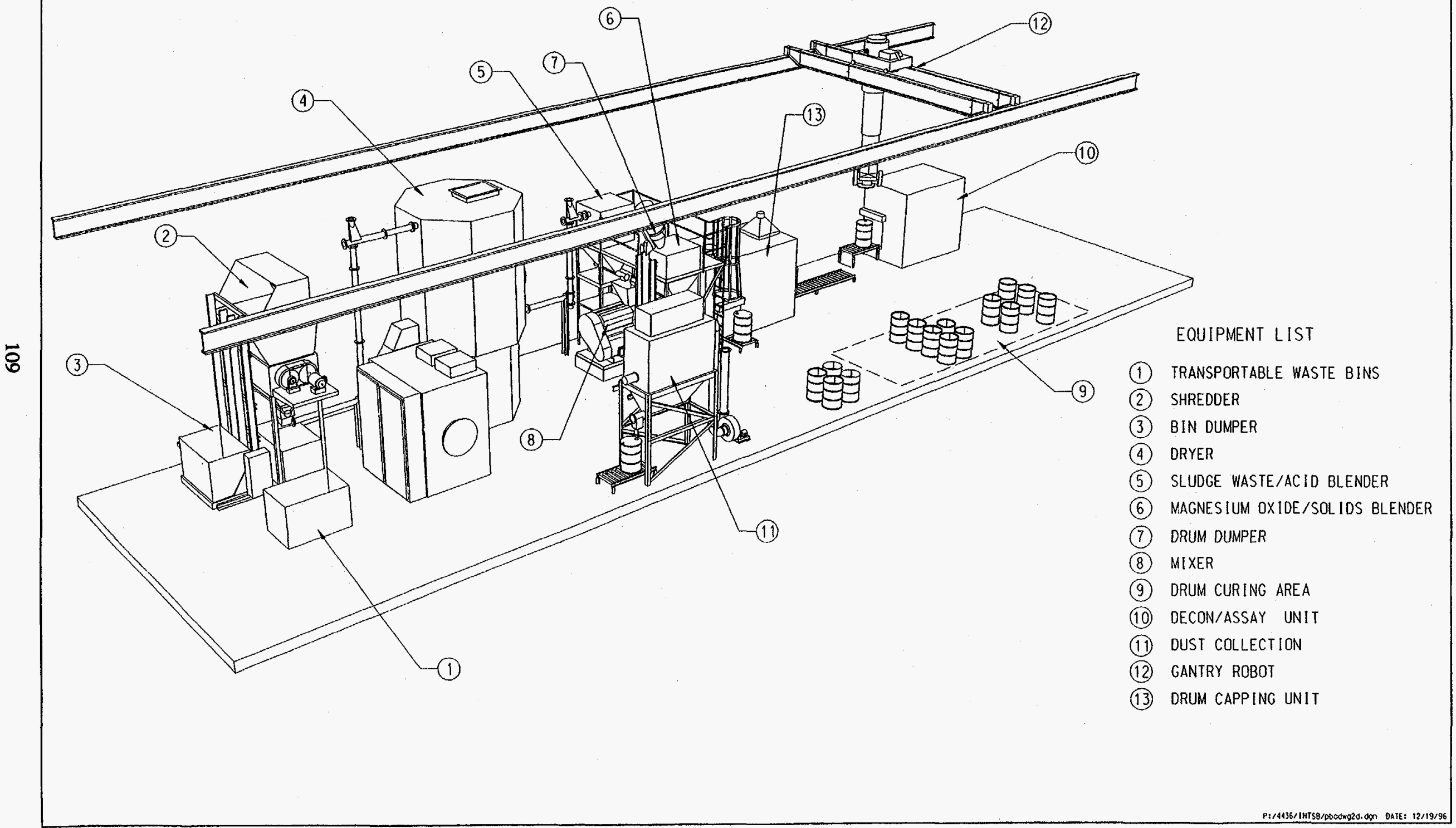

Figure 2-39. Acid Digestion Case: PERSPECTIVE View of Phosphate Bonded Ceramic Stabilization Subsystem. 


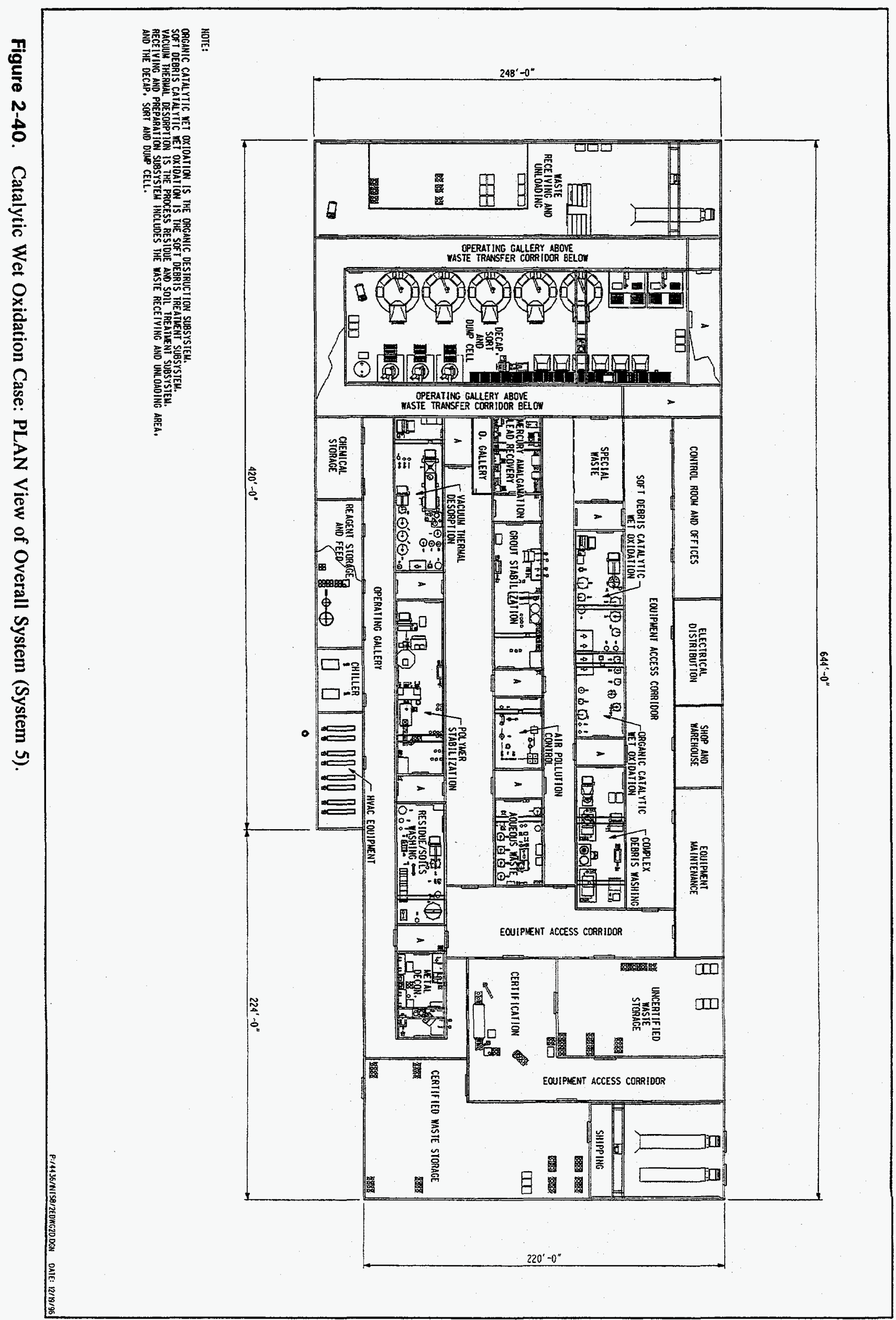




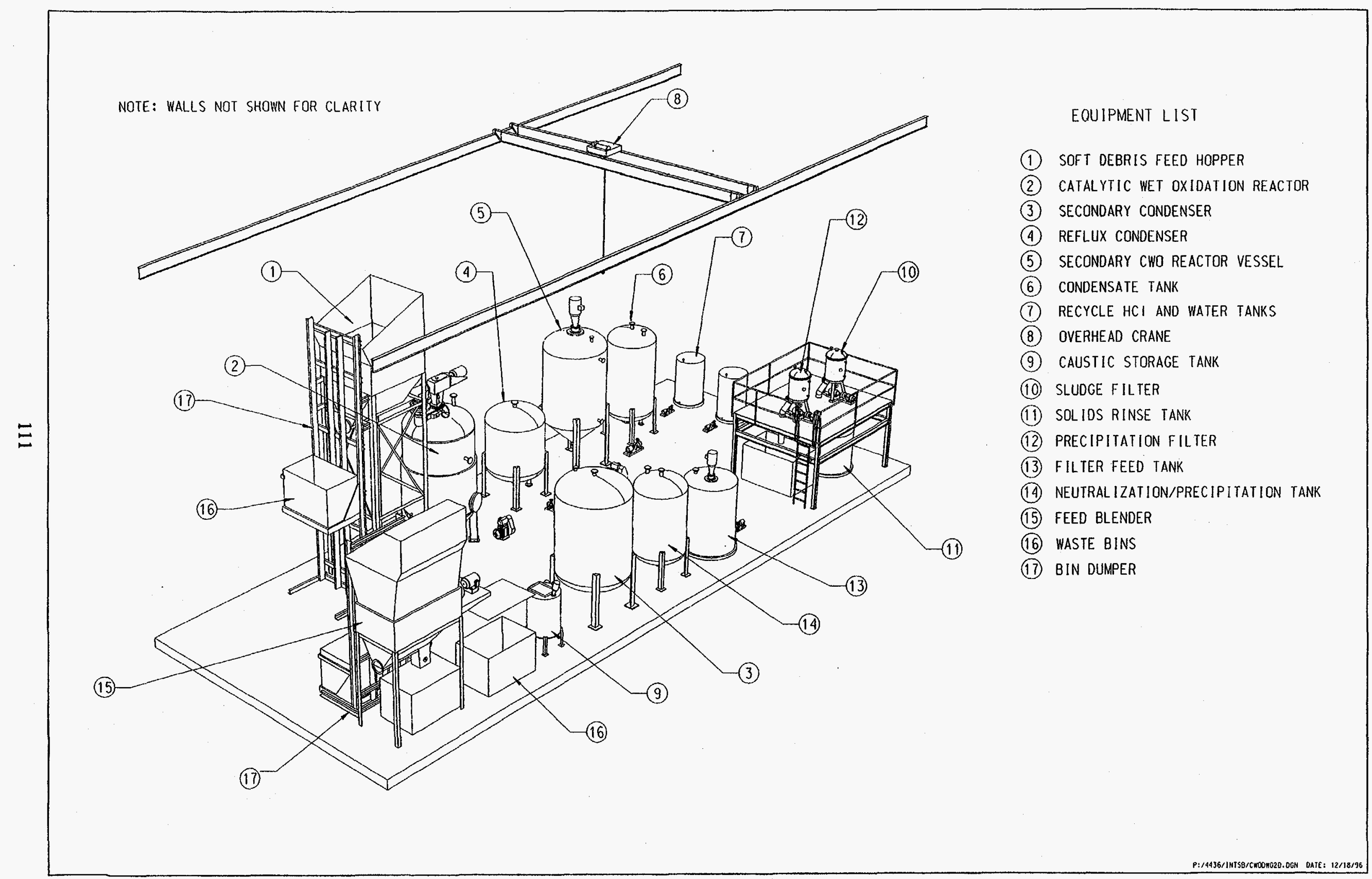

Figure 2-41. Catalytic Wet Oxidation Case: PERSPECTIVE View of Soft Debris Waste Treatment Subsystem. 


\section{SYSTEM FLOW RATES}

This section contains a summary of mass flow rates of the major inputs and outputs of the systems and the methods used to calculate these flow rates. The information is presented in Figures 3-1 through 3-9 in this section. Detailed information on mass flow rates is presented in the process flow diagrams in Appendix B and the mass balance modeling results in Appendix $\mathrm{C}$.

Tables 3-1 through 3-12 list the elemental composition of the input waste for each of the subsystems. Tables and figures are presented at the end of this section.

The mass flow rate data used in this preconceptual design study are based on many assumptions. These assumptions, discussed below, must be validated or revised before commencing more detailed design activities.

Mass flow rates for key subsystems of the five INTS flow sheets were calculated by modeling the flow sheets with ASPEN PLUS (ASPEN) (Aspen Technology, 1994). ASPEN was used to model the organic destruction, process residue and soil treatment, debris treatment, aqueous waste treatment, and the APC. ASPEN is a simulation program that models the steady-state performance of a chemical process. The process can be as simple as a single unit operation, or can include an entire industrial plant. ASPEN is used extensively in the chemical process industry and its utility is widely recognized. The capabilities of ASPEN include chemical thermodynamics, and mass and energy balances for individual unit operations, as well as for the entire process flow. ASPEN calculates the composition of individual flow streams, and it can also accept design specifications by which the performance of individual unit operations can be specified. The modeling capabilities of ASPEN make it ideally suited for use in calculating the mass and energy balances for the INTS flow sheets. However, ASPEN does not calculate reaction kinetics. Consequently, reaction rates and equipment sizes are based on data obtained from vendors and developers.

\subsection{System Input Feed Rates}

As in the ITTS (Feizollahi, et al. 1995b), the feed rates for the INTS systems are uniformly calculated based on 4,032 hours of operation per year for 20 years. The capacities of the modules are slightly greater than required by the waste inventory.

For the purpose of this conceptual study, the feed rate calculations are based on a total of 2,927 pounds per hour (lb/hr) to the system. The input to the various subsystems may vary, but the total feed rate to INTS and ITTS systems is always $2,927 \mathrm{lb} / \mathrm{hr}$. See Table 3-13 for input feed rates to the INTS subsystems, including secondary waste from other subsystems. 


\subsection{System Secondary Waste}

Waste input data in Table 3-13 show only primary and major secondary waste streams that have been calculated in the mass balance studies. Secondary wastes produced within the facility include APC scrubber salts, decontamination subsystem sludge, evaporator bottoms from the MEO process, and organic liquids from vacuum desorption condensed offgas. Final waste output resulting from treatment of other secondary waste streams (e.g., housekeeping trash, contaminated failed equipment, and parts generated during maintenance) is estimated to be the same for all systems. Hence, it is not expected to affect the outcome of the systems comparison. For cost estimating purposes, the final waste output from other secondary waste is assumed to be negligible. Final waste volume is from the following sources within the facility:

- housekeeping trash, disposable garments, and equipment maintenance waste

- $\quad$ spent baghouse, ventilation and HEPA filter elements

- accumulations in equipment drains and floor drains, and other liquids generated during routine flushing and cleanup of the systems, including floor wash-downs

- machinery oil and decontamination solutions generated during equipment replacement and maintenance

- $\quad$ spent activated carbon beds, ion-exchange resins, and other filter media used in the facility

\subsection{Key Assumptions for Mass Flow Calculations}

The detailed assumptions for mass flow calculations are presented in Appendix C. Appendix C describes the approach to the mass balance modelling for each subsystem. Densities, mercury content assumptions and stabilization formulas used in the modelling are as follows: 
- Density of waste input and output (pounds per cubic foot $\left.\left[\mathrm{lb} / \mathrm{ft}^{3}\right]\right)^{\mathrm{a}}$ :

- Input waste $=64 \mathrm{lb} / \mathrm{ft}^{3}$

- Metal $=480 \mathrm{lb} / \mathrm{ft}^{3}$

- $\quad$ Aqueous liquid $=62 \mathrm{lb} / \mathrm{ft}^{3}$

- $\quad$ Soil $=67 \mathrm{lb} / \mathrm{ft}^{3}$

- $\quad$ Polymer stabilized salts $=80 \mathrm{lb} / \mathrm{ft}^{3}$

- Polymer stabilized salts/residues/waste $=94 \mathrm{lb} / \mathrm{ft}^{3}$

- Phosphate ceramic stabilized waste $=94 \mathrm{lb} / \mathrm{ft}^{3}$

- $\quad$ Grout stabilized debris $=126.6 \mathrm{lb} / \mathrm{ft}^{3}$

- $\quad$ Stabilized mercury amalgam $=733 \mathrm{lb} / \mathrm{ft}^{3}$

- Density of reagents and additives:

- $\quad$ Caustic $(\mathrm{NaOH})=133 \mathrm{lb} / \mathrm{ft}^{3}$

- $\quad$ Acid solution $=62 \mathrm{lb} / \mathrm{ft}^{3}$

- $\quad$ Ammonia $=45 \mathrm{lb} / \mathrm{ft}^{3}$

- $\quad$ Grout $=126.6 \mathrm{lb} / \mathrm{ft}^{3}$

- $\quad$ Nitric acid $=94 \mathrm{lb} / \mathrm{ft}^{3}$

- $\quad$ Silver nitrate $=271 \mathrm{lb} / \mathrm{ft}^{3}$

- $\quad$ Hydrogen Peroxide $=91 \mathrm{lb} / \mathrm{ft}^{3}$

- $\quad$ Polyethylene $=80 \mathrm{lb} / \mathrm{ft}^{3}$

- $\quad$ Phosphate ceramic $=80 \mathrm{lb} / \mathrm{ft}^{3}$

- Copper for mercury amalgamation $=557 \mathrm{lb} / \mathrm{ft}^{3}$

- Mercury content:

- Elemental mercury $(100 \% \mathrm{Hg})$ comprises $5 \%$ of the waste designated as mercury-contaminated.

- Mercury concentration in the remaining $95 \%$ of the mercury-contaminated stream is $5 \%$.

${ }^{a}$ Values for waste density were taken from the Phase 1 report (Integrated Thermal Treatment System Study Phase 1 Results, EGG-MS-11211, Fred Feizollahi, William J. Quapp, Hugh G. Hempill, and Frank J. Groffie, July 1994). Other values for waste density (at the INEL) are found in "Identification of a Treatment Process for the Idaho Waste Processing Facility Project - A Preconceptual Design Study", EG\&G Idaho, Inc., Internal Technical Report, WM-PD-92-009, W.S. Roesener, N.R. Soelberg, and A.L. Ayers, Jr., July 1992. These values are approximately one-half of those used in the INTS study. It is recognized that waste density can be highly variable; however, density values affect only the calculations for volume reduction and not the overall mass balance.

${ }^{b}$ Average density of the waste was obtained by mass averaging the data for inidividual waste categories found in Table 3.3 of "Quantities and Characteristics of the Contact =Handled Low-Level Mixed Waste Streams for the DOE Complex", EGG-MS-11303, August 1994, T.L. Huebner, J.M. Wilson, A.H. Ruhter, and S.J. Bonney. 
- Stabilization formulas (all on a mass basis):

- $\quad$ Polymer $=$ One part polymer to one part waste.

- $\quad$ Phosphate bonded ceramic = One part ceramic additives to one part waste.

- $\quad$ Grout $=$ Two parts grout to one part waste.

\subsection{Energy Balance Calculation Results}

Calculations made with ASPEN provided overall energy requirements for all of the unit operations within the models for the five systems. A summary of these calculations is shown in Table 3-14. As shown in the table, some unit operations are common to all five INTS systems, while other unit operations are used in only one or two of the systems. In this analysis, the energy requirements were based on heating and cooling needed for the waste to be treated and organics broken down. For each unit operation, the energy required is the net energy; reactions that liberate heat provide energy; heating or reactions that require heat use energy. This analysis does not account for actual utility operation, such as the energy needed to operate pumps, agitators or conveyors. The utility costs provided in the cost estimates of Section 4 estimate actual utility costs. This approach is consistent with the ITTS approach.

The results are based on a waste feed of $2927 \mathrm{lb} / \mathrm{hr}$ to the reactor. For these calculations, no one energy type was specified for the ASPEN model. It was assumed that the energy requirements of each unit operation would be considered outside the model.

Table 3-14 lists the assumed energy type that would be used for each of the unit operations. Those unit operations using electricity include mercury amalgamation, GPCR, ozone generation, salt concentrator, and the MEO cells. Operations that utilize natural gas for process heat include the HEPA preheaters, vacuum thermal desorbers, and dryers. Cooling water is required for cooling all other unit operations, including acid gas scrubbers, OH-generators, photolytic-UV reactors, CWO reactors, gas equilibration reactors, acid digestion reactors, and $\mathrm{HNO}_{2}$ recovery reactors. Calculations for one process unit (dryer for polymer stabilization input) showed that this unit could require heating or cooling. If heating was required, it was included in the natural gas requirement. If the calculations showed that cooling was required, the cooling requirement was ignored on the assumption that the polymer stabilization unit could accept a warmer waste input.

Cost of the energy associated with each system was also calculated. A price of $\$ 0.05$ per kilowatt per hour $(\mathrm{kW} / \mathrm{hr}$ ) was used for electricity and $\$ 2.00$ per million British thermal units (MMBtu) per hour was used for natural gas. Cooling water costs were assumed to be negligible by comparison and were ignored in this simple analysis. The calculated costs for natural gas were somewhat variable, ranging from $\$ 0.37$ per hour for System 3 to $\$ 2.41$ per hour of operation for System 2 . 
Electricity costs were extremely variable, with a range of $\$ 0.38$ per hour for System 2 to $\$ 93.52$ per hour for System 3. The two systems with the highest energy costs were those using MEO for waste destruction.

As expected for nonthermal processes, overall energy costs in the INTS study are much lower than those of the ITTS study. Whereas calculated energy costs in the INTS study range from about $\$ 2$ per hour (systems 4 and 5) to $\$ 94$ per hour (System 3), calculated energy costs in the ITTS study (ITTS Table 3-10) ranged from $\$ 20$ to $\$ 410$ per hour.

\subsection{System Output}

Output calculations are presented for the five systems, allowing comparison of the offgas volumes and waste volume reduction for the different systems. Gaseous liquid effluents for the systems are shown in Table 3-15. The waste volume for disposal is shown in Table 3-16.

The solid waste volume output from the systems is presented as the sum of the output from subsystems sending waste to disposal. Wastes from subsystems which produce stabilized waste are included in this calculation (polymer stabilization, phosphate bonded stabilization, grout stabilization and special waste treatment). Decontaminated metal solids which are recyclable, including lead, are not included in the volume of waste output to disposal. The volume of recyclable metals is listed in Table 3-15. The volume reduction factor or input/output ratio is also presented.

Systems 4 and 5 have the highest volume reduction factors (lowest final volume) because of the breakdown of soft debris. System 1 has the lowest factor (highest volume) due to non-treatment of grouted debris waste.

\subsection{Mass Balance Calculation Results}

Simplified block diagrams showing the key system input and output products from the five systems are shown in Figures 3-1 through 3-5. The major input and output products of the subsystems are shown in the figures. These simplified figures do not include additives or all secondary streams, and therefore do not contain the complete numerical balance. Detailed mass balances are presented in the PFDs in Appendix B.

Waste input feed rates for all subsystems are shown in Table 3-13. Mass balance calculation details are provided in Appendix C. Figures 3-6 through 3-9 illustrate the mass balance results for the five systems.

The results of the mass balance calculations demonstrate some significant trends with regard to minimizing offgas. System 1 has the lowest volume of offgas, due to the least treatment of debris waste. Systems 2 and 3 have more offgas due to organic removal and subsequent destruction from debris in the 
vacuum desorption and washing systems. System 2 has more offgas due to volatilization of some organic matter that makes up the soft debris matrix. Systems 4 and 5 have similar offgas volumes due to the breakdown of soft debris. In all cases, offgas is less than 300 standard cubic feet per minute.

Water discharge rates, at less than $2 \mathrm{gpm}$, are very low for all systems due to extensive internal water recycling. 
Table 3-1. Elemental composition of DOE contact-handled combustible MLLW assigned to aqueous waste treatment subsystem in the INTS study.

\begin{tabular}{|c|c|c|}
\hline \multicolumn{2}{|l|}{ Waste component } & Mass \% \\
\hline & Carbon & 0.00 \\
\hline & Hydrogen & 0.13 \\
\hline & Oxygen & 6.09 \\
\hline & Nitrogen & 1.78 \\
\hline & Sulfur & 0.00 \\
\hline & Chlorine & 0.00 \\
\hline & Fluorine & 0.00 \\
\hline & No & 0.00 \\
\hline & $\mathrm{SO} 2$ & 0.00 \\
\hline & Water & 87.00 \\
\hline & Glass-forming inerts & 0.00 \\
\hline & Bulk nontoxic metal & 0.00 \\
\hline & Arsenic (As) & 0.20 \\
\hline & Barium (Ba) & 0.10 \\
\hline & Beryllium (Be) & 0.00 \\
\hline & Cadmium (Cd) & 1.50 \\
\hline & Chromium (Cr) & 2.00 \\
\hline & Lead $(\mathrm{Pb})$ & 0.50 \\
\hline & Mercury (Hg) & 0.50 \\
\hline & Selenium (Se) & 0.10 \\
\hline & Silver (Ag) & 0.10 \\
\hline & Tantalum (Ta) & 0.00 \\
\hline & Uranium (U) & 0.00 \\
\hline " & All inorganics & 92.00 \\
\hline All components & & 100.00 \\
\hline
\end{tabular}

Note: For all other subsystems, wastes are divided into the categories of organics and inorganics, based on elements anticipated to be present primarily in organic form. In this subsystem, organics are anticipated in trace quantities (less than $0.1 \%$ ) only.

Source of data: T.L. Heubner, J.M. Wilson, A.H. Ruhter, S.J. Bonney, Quantities and Characteristics of the Contact-Handled Low-Level Mixed Waste Streams for the DOE Complex, EGG-MS-11303, Idaho National Engineering Laboratory (August 1994). 
Table 3-2. Elemental composition of DOE contact-handled noncombustible MLLW assigned to organic destruction treatment subsystem in the INTS study.

Waste component

Mass \%

Organics

All organics and inorganics

\begin{tabular}{l|r}
\hline Carbon & 55.8 \\
Hydrogen & 9.0 \\
\hline Oxygen & 0.6 \\
\hline Nitrogen & 0.0 \\
\hline Sulfur & 0.1 \\
\hline Chlorine & 16.1 \\
\hline Fluorine & 0.0 \\
\hline NO & 0.0 \\
\hline SO2 & 0.0 \\
\hline All organics & 81.6 \\
\hline & \\
\hline Water & 12.0 \\
Glass-forming inerts & 2.0 \\
\hline Bulk nontoxic metal & 3.2 \\
\hline Arsenic (As) & 0.0 \\
\hline Barium (Ba) & 0.3 \\
\hline Beryllium (Be) & 0.0 \\
\hline Cadmium (Cd) & 0.3 \\
\hline Chromium (Cr) & 0.6 \\
\hline Lead (Pb) & 0.0 \\
\hline Mercury (Hg) & 0.0 \\
\hline Selenium (Se) & 0.0 \\
\hline Silver (Ag) & 0.0 \\
\hline Tantalum (Ta) & 0.0 \\
\hline Uranium (U) & 0.0 \\
\hline All inorganics & 18.4 \\
\hline & \\
\hline & 100.0 \\
\hline & \\
\hline & \\
\hline
\end{tabular}

Note: For all subsystems, wastes are divided into the categories of organics and inorganics, based on elements anticipated to be present primarily in organic form.

Source of data: T.L. Heubner, J.M. Wilson, A.H. Ruhter, S.J. Bonney, Quantities and Characteristics of the Contact-Handled Low-Level Mixed Waste Streams for the DOE Complex, EGG-MS-11303, Idaho National Engineering Laboratory (August 1994). 
Table 3-3. Elemental composition of DOE contact-handled MLLW assigned to process residue treatment subsystem in the INTS study.

Waste component

\begin{tabular}{|c|c|c|c|}
\hline \multicolumn{4}{|l|}{ Organics } \\
\hline & Carbon & & 2.9 \\
\hline & Hydrogen & s & 0.5 \\
\hline & Oxygen & & 0.0 \\
\hline & Nitrogen & & 0.0 \\
\hline & Sulfur & & 0.0 \\
\hline & Chlorine & & 0.7 \\
\hline & Fluorine & & 0.0 \\
\hline & $\mathrm{NO}$ & - & 0.0 \\
\hline & $\mathrm{SO} 2$ & & 0.0 \\
\hline & All organics & & 4.1 \\
\hline \multicolumn{4}{|l|}{ Inorganics } \\
\hline & Water & & 32.7 \\
\hline & Glass-forming inerts & & 62.1 \\
\hline & Bulk nontoxic metal & & 0.2 \\
\hline & Arsenic (As) & & 0.0 \\
\hline & Barium (Ba) & & 0.0 \\
\hline & Beryllium (Be) & & 0.0 \\
\hline & Cadmium (Cd) & & 0.3 \\
\hline & Chromium (Cr) & & 0.5 \\
\hline & Lead $(\mathrm{Pb})$ & & 0.0 \\
\hline & Mercury (Hg) & & 0.0 \\
\hline & Selenium (Se) & & 0.0 \\
\hline & Silver (Ag) & & 0.0 \\
\hline & Tantalum (Ta) & & 0.0 \\
\hline & Uranium (U) & & 0.0 \\
\hline & All inorganics & & 95.9 \\
\hline All organics and inorganics & & & 100.0 \\
\hline
\end{tabular}

Note: For all subsystems, wastes are divided into the categories of

organics and inorganics, based on elements anticipated to be present primarily in organic form.

Source of data: T.L. Heubner, J.M. Wilson, A.H. Ruhter, S.J. Bonney,

Quantities and Characteristics of the Contact-Handled Low-Level

Mixed Waste Streams for the DOE Complex, EGG-MS-11303,

Idaho National Engineering Laboratory (August 1994).

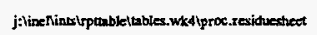


Table 3-4. Elemental composition of DOE contact-handled MLLW assigned to soil treatment subsystem in the INTS study.

\begin{tabular}{|c|c|c|}
\hline \multicolumn{2}{|l|}{ Waste component } & \multirow{2}{*}{ Mass \% } \\
\hline Organics & & \\
\hline & Carbon & 0.9 \\
\hline & Hydrogen & 0.1 \\
\hline & Oxygen & 0.0 \\
\hline & Nitrogen & 0.0 \\
\hline & Sulfur & 0.0 \\
\hline & Chlorine & 4.1 \\
\hline & Fluorine & 0.0 \\
\hline & $\mathrm{NO}$ & 0.0 \\
\hline & $\mathrm{SO} 2$ & 0.0 \\
\hline & All organics & 5.2 \\
\hline \multicolumn{3}{|l|}{ Inorganics } \\
\hline & Water & 8.2 \\
\hline & Glass-forming inerts & 86.7 \\
\hline & Bulk nontoxic metal & 0.0 \\
\hline & Arsenic (As) & 0.0 \\
\hline & Barium (Ba) & 0.0 \\
\hline & Beryllium (Be) & 0.0 \\
\hline & Cadmium (Cd) & 0.0 \\
\hline & Chromium (Cr) & 0.0 \\
\hline & Lead $(\mathrm{Pb})$ & 0.0 \\
\hline & Mercury (Hg) & 0.0 \\
\hline & Selenium (Se) & 0.0 \\
\hline & Silver (Ag) & 0.0 \\
\hline & Tantalum (Ta) & 0.0 \\
\hline & Uranium (U) & 0.0 \\
\hline & All inorganics & 94.8 \\
\hline All organics and inorganics & & 100.0 \\
\hline
\end{tabular}

Note: For all subsystems, wastes are divided into the categories of organics and inorganics, based on elements anticipated to be present primarily in organic form.

Source of data: T.L. Heubner, J.M. Wilson, A.H. Ruhter, S.J. Bonney, Quantities and Characteristics of the Contact-Handled Low-Level Mixed Waste Streams for the DOE Complex, EGG-MS-11303, Idaho National Engineering Laboratory (August 1994).

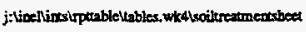


Table 3-5. Elemental composition of DOE contact-handled MLLW assigned to mercury amalgamation treatment subsystem in the INTS study.

Waste component

Mass \%

Organics

Inorganics

All organics and inorganics

\begin{tabular}{l|r}
\hline Carbon & 0.0 \\
Hydrogen & 0.0 \\
\hline Oxygen & 0.0 \\
\hline Nitrogen & 0.0 \\
\hline Sulfur & 0.0 \\
\hline Chlorine & 0.0 \\
\hline Fluorine & 0.0 \\
\hline NO & 0.0 \\
\hline SO2 & 0.0 \\
\hline All organics & 0.0 \\
\hline & \\
\hline Water & 0.0 \\
Glass-forming inerts & 0.0 \\
\hline Bulk nontoxic metal & 0.0 \\
\hline Arsenic (As) & 0.0 \\
\hline Barium (Ba) & 0.0 \\
\hline Beryllium (Be) & 0.0 \\
\hline Cadmium (Cd) & 0.0 \\
\hline Chromium (Cr) & 0.0 \\
\hline Lead (Pb) & 0.0 \\
\hline Mercury (Hg) & 100.0 \\
\hline Selenium (Se) & 0.0 \\
\hline Silver (Ag) & 0.0 \\
\hline Tantalum (Ta) & 0.0 \\
\hline Uranium (U) & 0.0 \\
\hline All inorganics & 100.0 \\
\hline & \\
\hline & 100.0 \\
& \\
\hline
\end{tabular}

Note: For all subsystems, wastes are divided into the categories of organics and inorganics, based on elements anticipated to be present primarily in organic form.

Source of data: T.L. Heubner, J.M. Wilson, A.H. Ruhter, S.J. Bonney, Quantities and Characteristics of the Contact-Handled Low-Level Mixed Waste Streams for the DOE Complex, EGG-MS-11303, Idaho National Engineering Laboratory (August 1994).

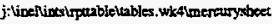


Table 3-6. Elemental composition of DOE contact-handled MLLW assigned to lead recovery treatment subsystem in the INTS study.

Waste component

Mass \%

\begin{tabular}{|c|c|c|}
\hline Organics & & \\
\hline & Carbon & 0.0 \\
\hline & Hydrogen & 0.0 \\
\hline & Oxygen & 0.0 \\
\hline & Nitrogen & 0.0 \\
\hline & Sulfur & 0.0 \\
\hline & Chlorine & 0.0 \\
\hline & Fluorine & 0.0 \\
\hline & $\mathrm{NO}$ & 0.0 \\
\hline & $\mathrm{SO} 2$ & 0.0 \\
\hline & All organics & 0.0 \\
\hline Inorganics & & \\
\hline & Water & 0.0 \\
\hline & Glass-forming inerts & 0.0 \\
\hline & Bulk nontoxic metal & 0.0 \\
\hline & Arsenic (As) & 0.0 \\
\hline & Barium (Ba) & 0.0 \\
\hline & Beryllium (Be) & 0.0 \\
\hline & Cadmium (Cd) & 0.0 \\
\hline & Chromium (Cr) & 0.0 \\
\hline & Lead $(\mathrm{Pb})$ & 100.0 \\
\hline & Mercury (Hg) & 0.0 \\
\hline & Selenium (Se) & 0.0 \\
\hline & Silver (Ag) & 0.0 \\
\hline & Tantalum (Ta) & 0.0 \\
\hline & Uranium (U) & 0.0 \\
\hline & All inorganics & 100.0 \\
\hline All organics and inorganics & & 100.0 \\
\hline
\end{tabular}

Note: For all subsystems, wastes are divided into the categories of organics and inorganics, based on elements anticipated to be present primarily in organic form.

Source of data: T.L. Heubner, J.M. Wilson, A.H. Ruhter, S.J. Bonney, Quantities and Characteristics of the Contact-Handled Low-Level Mixed Waste Streams for the DOE Complex, EGG-MS-11303, Idaho National Engineering Laboratory (August 1994). 
Table 3-7. Elemental composition of DOE contact-handled MLLW assigned to polymer stabilization treatment subsystem in the INTS study.

Waste component

\begin{tabular}{|c|c|c|}
\hline \multicolumn{3}{|l|}{ Organics } \\
\hline & Carbon & 0.0 \\
\hline & Hydrogen & 0.0 \\
\hline & Oxygen & 0.0 \\
\hline & Nitrogen & 0.0 \\
\hline & Sulfur & 0.0 \\
\hline & Chlorine & 26.8 \\
\hline & Fluorine & 0.0 \\
\hline & $\mathrm{NO}$ & 10.1 \\
\hline & $\mathrm{SO} 2$ & 12.8 \\
\hline & All organics & 49.7 \\
\hline \multicolumn{3}{|l|}{ Inorganics } \\
\hline & Water & 0.0 \\
\hline & Glass-forming inerts & 50.3 \\
\hline & Bulk nontoxic metal & 0.0 \\
\hline & Arsenic (As) & 0.0 \\
\hline & Barium (Ba) & 0.0 \\
\hline & Beryllium (Be) & 0.0 \\
\hline & Cadmium (Cd) & 0.0 \\
\hline & Chromium (Cr) & 0.0 \\
\hline & Lead $(\mathrm{Pb})$ & 0.0 \\
\hline & Mercury (Hg) & 0.0 \\
\hline & Selenium (Se) & 0.0 \\
\hline & Silver (Ag) & 0.0 \\
\hline & Tantalum (Ta) & 0.0 \\
\hline & Uranium (U) & 0.0 \\
\hline & All inorganics & 50.3 \\
\hline All organics and inorganics & & 100.0 \\
\hline
\end{tabular}

Note: For all subsystems, wastes are divided into the categories of organics and inorganics, based on elements anticipated to be present primarily in organic form.

Source of data: T.L. Heubner, J.M. Wilson, A.H. Ruhter, S.J. Bonney, Quantities and Characteristics of the Contact-Handled Low-Level Mixed Waste Streams for the DOE Complex, EGG-MS-11303, Idaho National Engineering Laboratory (August 1994).

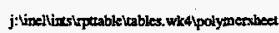


Table 3-8. Elemental composition of DOE contact-handled MLLW assigned to soft debris treatment subsystem in the INTS study.

\begin{tabular}{|c|c|c|}
\hline \multicolumn{2}{|l|}{ Waste component } & \multirow{2}{*}{ Mass \% } \\
\hline Organics & & \\
\hline & Carbon & 48.9 \\
\hline & Hydrogen & 7.5 \\
\hline & Oxygen & 23.3 \\
\hline & Nitrogen & 0.1 \\
\hline & Sulfur & 0.2 \\
\hline & Chlorine & 4.3 \\
\hline & Fluorine & 0.0 \\
\hline & $\mathrm{NO}$ & 0.0 \\
\hline & $\mathrm{SO} 2$ & 0.0 \\
\hline & All organics & 84.3 \\
\hline \multicolumn{3}{|l|}{ Inorganics } \\
\hline & Water & 2.8 \\
\hline & Glass-forming inerts & 9.9 \\
\hline & Bulk nontoxic metal & 0.7 \\
\hline & Arsenic (As) & 0.0 \\
\hline & Barium (Ba) & 0.0 \\
\hline & Beryllium (Be) & 0.0 \\
\hline & Cadmium (Cd) & 0.0 \\
\hline & Chromium (Cr) & 0.0 \\
\hline & Lead $(\mathrm{Pb})$ & 0.0 \\
\hline & Mercury (Hg) & 2.3 \\
\hline & Selenium (Se) & 0.0 \\
\hline & Silver (Ag) & 0.0 \\
\hline & Tantalum (Ta) & 0.0 \\
\hline & Uranium (U) & 0.0 \\
\hline & All inorganics & 15.7 \\
\hline All organics and inorganics & & 100.0 \\
\hline
\end{tabular}

Note: For all subsystems, wastes are divided into the categories of organics and inorganics, based on elements anticipated to be present primarily in organic form.

Source of data: T.L. Heubner, J.M. Wilson, A.H. Ruhter, S.J. Bonney,

Quantities and Characteristics of the Contact-Handled Low-Level

Mixed Waste Streams for the DOE Complex, EGG-MS-11303,

Idaho National Engineering Laboratory (August 1994).

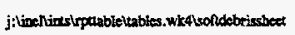


Table 3-9. Elemental composition of DOE contact-handled MLLW assigned to open debris treatment subsystem in the INTS study.

\begin{tabular}{|c|c|c|}
\hline \multicolumn{3}{|l|}{ Organics } \\
\hline & Carbon & 0.5 \\
\hline & Hydrogen & 0.1 \\
\hline & Oxygen & 0.4 \\
\hline & Nitrogen & 0.0 \\
\hline & Sulfur & 0.0 \\
\hline & Chlorine & 0.0 \\
\hline & Fluorine & 0.0 \\
\hline & $\mathrm{NO}$ & 0.0 \\
\hline & $\mathrm{SO} 2$ & 0.0 \\
\hline & All organics & 1.0 \\
\hline \multicolumn{3}{|l|}{ Inorganics } \\
\hline & Water & 0.0 \\
\hline & Glass-forming inerts & 99.0 \\
\hline & Bulk nontoxic metal & 0.0 \\
\hline & Arsenic (As) & 0.0 \\
\hline & Barium $(\mathrm{Ba})$ & 0.0 \\
\hline & Beryllium (Be) & 0.0 \\
\hline & Cadmium (Cd) & 0.0 \\
\hline & Chromium (Cr) & 0.0 \\
\hline & Lead $(\mathrm{Pb})$ & 0.0 \\
\hline & Mercury (Hg) & 0.0 \\
\hline & Selenium (Se) & 0.0 \\
\hline & Silver (Ag) & 0.0 \\
\hline & Tantalum (Ta) & 0.0 \\
\hline & Uranium (U) & 0.0 \\
\hline & All inorganics & 99.0 \\
\hline All organics and inorganics & & 100.0 \\
\hline
\end{tabular}

Note: For all subsystems, wastes are divided into the categories of organics and inorganics, based on elements anticipated to be present primarily in organic form.

Source of data: T.L. Heubner, J.M. Wilson, A.H. Ruhter, S.J. Bonney, Quantities and Characteristics of the Contact-Handled Low-Level Mixed Waste Streams for the DOE Complex, EGG-MS-11303, Idaho National Engineering Laboratory (August 1994). 
Table 3-10. Elemental composition of DOE contact-handled MLLW assigned to complex debris treatment subsystem in the INTS study.

Waste component

Mass \%

\begin{tabular}{|c|c|c|}
\hline Organics & & \\
\hline & Carbon & 9.5 \\
\hline & Hydrogen & 1.6 \\
\hline & Oxygen & 0.0 \\
\hline & Nitrogen & 0.0 \\
\hline & Sulfur & 0.0 \\
\hline & Chlorine & 0.0 \\
\hline & Fluorine & 0.0 \\
\hline & $\mathrm{NO}$ & 0.0 \\
\hline & $\mathrm{SO} 2$ & 0.0 \\
\hline & All organics & 11.1 \\
\hline Inorganics & & \\
\hline & Water & 0.1 \\
\hline & Glass-forming inerts & 3.2 \\
\hline & Bulk nontoxic metal & 78.9 \\
\hline & Arsenic (As) & 0.0 \\
\hline & Barium (Ba) & 0.0 \\
\hline & Beryllium (Be) & 0.0 \\
\hline & Cadmium (Cd) & 0.0 \\
\hline & Chromium (Cr) & 0.0 \\
\hline & Lead $(\mathrm{Pb})$ & 0.0 \\
\hline & Mercury (Hg) & 6.7 \\
\hline & Selenium (Se) & 0.0 \\
\hline & Silver (Ag) & 0.0 \\
\hline & Tantalum (Ta) & 0.0 \\
\hline & Uranium (U) & 0.0 \\
\hline & All inorganics & 88.9 \\
\hline All organics and inorganics & & 1000 \\
\hline
\end{tabular}

Note: For all subsystems, wastes are divided into the categories of organics and inorganics, based on elements anticipated to be present primarily in organic form.

Source of data: T.L. Heubner, J.M. Wilson, A.H. Ruhter, S.J. Bonney, Quantities and Characteristics of the Contact-Handled Low-Level Mixed Waste Streams for the DOE Complex, EGG-MS-11303, Idaho National Engineering Laboratory (August 1994).

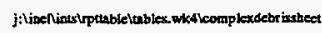


Table 3-11. Elemental composition of DOE contact-handled MLLW assigned to special waste treatment subsystem in the INTS study.

Waste component

Mass \%

Organics

All organics and inorganics

\begin{tabular}{l|r} 
& \\
\hline Carbon & 2.0 \\
Hydrogen & 0.2 \\
\hline Oxygen & 26.9 \\
\hline Nitrogen & 0.0 \\
\hline Sulfur & 0.0 \\
\hline Chlorine & 0.0 \\
\hline Fluorine & 0.0 \\
\hline NO & 0.0 \\
\hline SO2 & 0.0 \\
\hline All organics & 29.1 \\
\hline & \\
\hline Water & 3.0 \\
Glass-forming inerts & 17.0 \\
\hline Bulk nontoxic metal & 0.0 \\
\hline Arsenic (As) & 0.0 \\
\hline Barium (Ba) & 0.8 \\
\hline Beryllium (Be) & 11.4 \\
\hline Cadmium (Cd) & 0.2 \\
\hline Chromium (Cr) & 0.0 \\
\hline Lead (Pb) & 1.6 \\
\hline Mercury (Hg) & 0.0 \\
\hline Selenium (Se) & 0.0 \\
\hline Silver (Ag) & 0.0 \\
\hline Tantalum (Ta) & 36.6 \\
\hline Uranium (U) & 0.3 \\
\hline All inorganics & 70.9 \\
\hline & \\
\hline & 100.0 \\
\hline & \\
\hline & \\
\hline
\end{tabular}

Note: For all subsystems, wastes are divided into the categories of organics and inorganics, based on elements anticipated to be present primarily in organic form.

Source of data: T.L. Heubner, J.M. Wilson, A.H. Ruhter, S.J. Bonney,

Quantities and Characteristics of the Contact-Handled Low-Level

Mixed Waste Streams for the DOE Complex, EGG-MS-11303,

Idaho National Engineering Laboratory (August 1994).

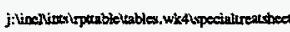


Table 3-12. Elemental composition of DOE contact-handled LLMW assigned to metal decontamination subsystem in the INTS study.

Waste component

Mass \%

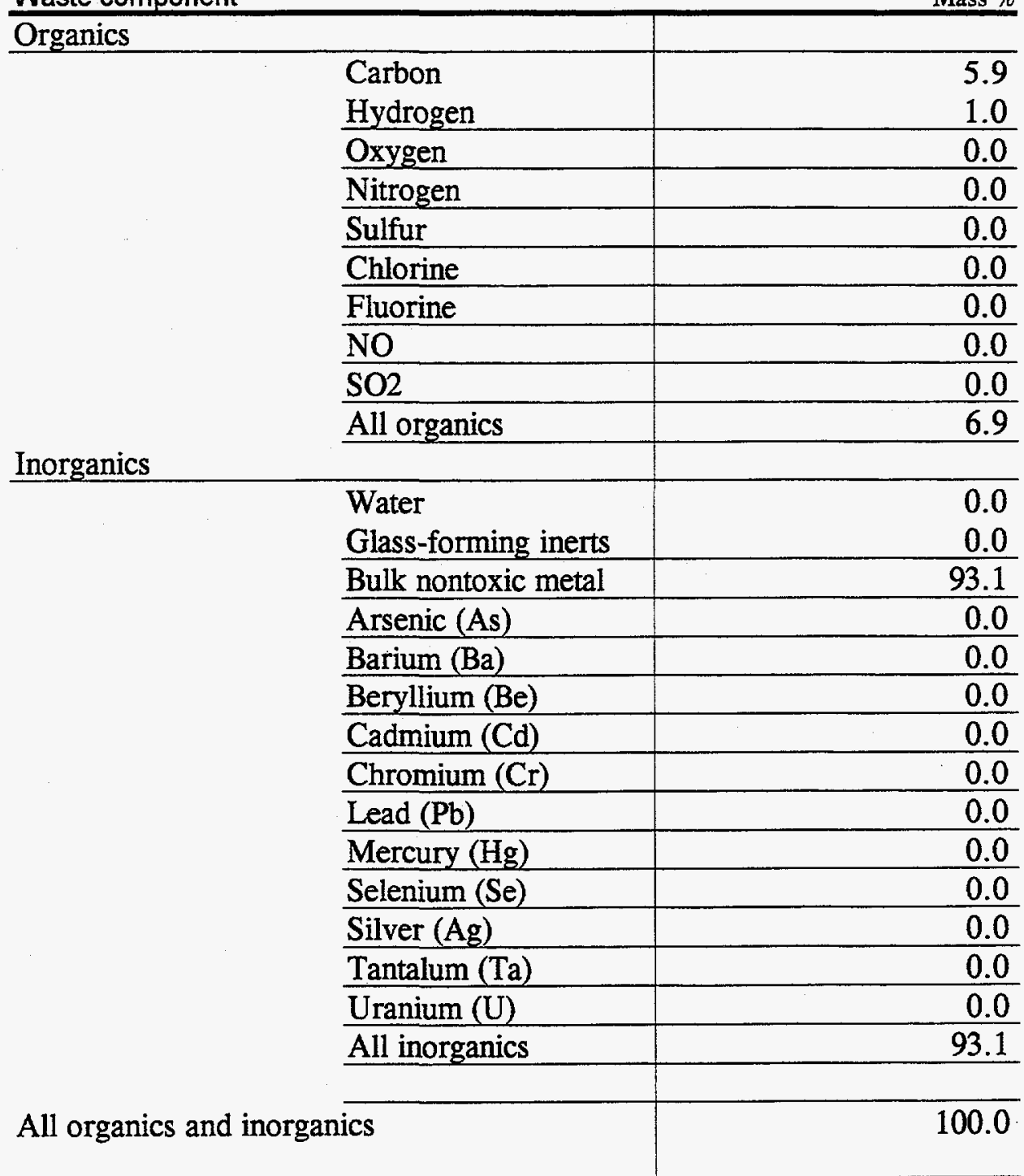

Note: For all subsystems, wastes are divided into the categories of

organics and inorganics, based on elements anticipated to be present primarily in organic form.

Source of data: T.L. Heubner, J.M. Wilson, A.H. Ruhter, S.J. Bonney, Quantities and Characteristics of the Contact-Handled Low-Level Mixed Waste Streams for the DOE Complex, EGG-MS-11303, Idaho National Engineering Laboratory (August 1994).

j: linel lints inptabtel tables. wk4 4 lmetaldeconsheet 
Table 3-13. Input feed rates to INTS subsystems (lb/hr).

\begin{tabular}{lrrrrr}
\hline & Grout Debris & Desorption & Wash & Acid Digestion & \multicolumn{1}{c}{ CWO } \\
Subsystem & System 1 & System 2 & System 3 & System 4 & System 5 \\
\hline Administration & 2,927 & 2,927 & 2,927 & 2,927 & 2,927 \\
Receiving and preparation & 2,927 & 2,927 & 2,927 & 2,927 & 2,927 \\
Aqueous waste treatment & 1,192 & 1,256 & 1,627 & 1,983 & 1,950 \\
Organic destruction & 237 & 268 & 326 & 256 & 256 \\
Process residue and & & & & & \\
inorganic sludge treatment & 1,020 & 1,020 & 1,020 & 1,020 & 1,020 \\
Bulk soil treatment & 329 & 329 & 329 & 329 & 329 \\
Debris treatment & 0 & 693 & 693 & 693 & 693 \\
Metal decontamination & 462 & 462 & 462 & 462 & 462 \\
Mercury amalgamation & 3 & 5 & 3 & 3 & 3 \\
Lead recovery & 26 & 26 & 26 & 26 & 26 \\
Polymer stabilization & 1,069 & 1,110 & 1,246 & 7 & 1,126 \\
Phosphate ceramic & & & & & \\
stabilization & & & & 2,946 & \\
Air pollution control & 695 & 823 & 827 & 1,396 & 1,433 \\
Grout stabilization & 1,082 & 1,228 & 958 & 265 & 650 \\
Special treatment & 155 & 155 & 155 & 155 & 155 \\
Certification and shipping & 5,126 & 5,067 & 4,919 & 4,115 & 4,270 \\
\hline
\end{tabular}

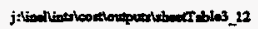

Note:

Input to all subsystems includes primary and secondary streams from other subsystems, but does not include additives. 
Table 3-14. Energy requirements for the five INTS systems (BTU/hr).

\begin{tabular}{|c|c|c|c|c|c|c|}
\hline Subsystem & Eneray Type & $\begin{array}{l}\text { Grout Debris } \\
\text { System } 1\end{array}$ & $\begin{array}{l}\text { Desorption } \\
\text { System } 2\end{array}$ & $\begin{array}{c}\text { Wash } \\
\text { System } 3\end{array}$ & $\begin{array}{l}\text { Acid Digestion } \\
\text { System } 4\end{array}$ & $\begin{array}{c}\text { CWO } \\
\text { System } 5\end{array}$ \\
\hline $\begin{array}{l}\text { APC } \\
\text { APC } \\
\text { APC }\end{array}$ & $\begin{array}{l}\text { cooling water } \\
\text { electricity } \\
\text { natural gas }\end{array}$ & $\begin{array}{r}(12,100) \\
20,600 \\
31,600 \\
\end{array}$ & $\begin{array}{c}(52,200) \\
24,400 \\
37,100 \\
\end{array}$ & $\begin{array}{c}(11,200) \\
22,900 \\
37,100 \\
\end{array}$ & $\begin{array}{r}(105,100) \\
40,300 \\
64,380 \\
\end{array}$ & $\begin{array}{r}(98,600) \\
39,100 \\
63,400 \\
\end{array}$ \\
\hline $\begin{array}{l}\text { Aqueous Waste Treatment } \\
\text { Aqueous Waste Treatment }\end{array}$ & $\begin{array}{l}\text { cooling water } \\
\text { electricity }\end{array}$ & $\begin{array}{c}(146,300) \\
1,110\end{array}$ & $\begin{array}{c}(98,100) \\
1,243\end{array}$ & $\begin{array}{c}(204,800) \\
2,711\end{array}$ & $\begin{array}{c}(38,700) \\
1,202\end{array}$ & $\begin{array}{c}(43,900) \\
1,312\end{array}$ \\
\hline Organic Destruction & $\begin{array}{l}\text { cooling water } \\
\text { electricity }\end{array}$ & $\begin{array}{c}(674,665) \\
4,619,800\end{array}$ & $\begin{array}{r}(4,071,300) \\
0\end{array}$ & $\begin{array}{c}(346,706) \\
6,360,000\end{array}$ & $\begin{array}{r}(4,119,800) \\
0\end{array}$ & $\begin{array}{r}(3,663,700) \\
0\end{array}$ \\
\hline Debris Treatment & natural gas & 0 & 518,600 & 0 & 0 & 0 \\
\hline Debris Treatment & cooling water & 0 & 0 & 0 & $(31,270,000)$ & $(4,987,494)$ \\
\hline Polymer Stabilization & natural gas & $(19,500)$ & 79,300 & 91,400 & 104,000 & 14,600 \\
\hline Process Residue & natural gas & 540,200 & 515,600 & 0 & 515,600 & 515,600 \\
\hline Soil Treatment & natural gas & 54,900 & 54,900 & 54,600 & 54,600 & 54,600 \\
\hline & $\begin{array}{l}\text { electricity } \\
\text { natural gas } \\
\text { cooling water }\end{array}$ & $\begin{array}{r}4,641,510 \\
626,700 \\
(833,065)\end{array}$ & $\begin{array}{r}25,643 \\
1,205,500 \\
(4,221,600)\end{array}$ & $\begin{array}{r}6,385,611 \\
183,100 \\
(562,706)\end{array}$ & $\begin{array}{r}41,502 \\
738,580 \\
(35,533,600)\end{array}$ & $\begin{array}{r}40,412 \\
648,200 \\
(8,793,694)\end{array}$ \\
\hline $\begin{array}{l}\text { Costs }(\$ / h r) \\
\text { Costs }(\$ / h r) \\
\text { Costs }(\$ / h r)\end{array}$ & $\begin{array}{l}\text { Electricity }(\$ 0.05 / \mathrm{kWh}) \\
\text { Natural Gas }(\$ 2.00 \mathrm{MMBtu}) \\
\text { Cooling Water }(\$ 0)\end{array}$ & $\begin{array}{r}\$ 67.98 \\
\$ 1.25 \\
\$ 0.00\end{array}$ & $\begin{array}{l}\$ 0.38 \\
\$ 2.41 \\
\$ 0.00\end{array}$ & $\begin{array}{r}\$ 93.52 \\
\$ 0.37 \\
\$ 0.00\end{array}$ & $\begin{array}{l}\$ 0.61 \\
\$ 1.48 \\
\$ 0.00\end{array}$ & $\begin{array}{l}\$ 0.59 \\
\$ 1.30 \\
\$ 0.00\end{array}$ \\
\hline \multicolumn{2}{|l|}{ Total Cost (\$/hr) } & $\$ 69.23$ & $\$ 2.79$ & $\$ 93.89$ & $\$ 2.08$ & $\$ 1.89$ \\
\hline
\end{tabular}

Notes:

a) This analysis is based on $2927 \mathrm{lb} / \mathrm{hr}$ waste feed.

b) The energy requirements shown on this table reflect required energy for heating, cooling, and heat of reaction. 
Table 3-15. Offgas, water and metal recycle outputs from the five INTS systems (lb/hr).

\begin{tabular}{|c|c|c|c|c|c|}
\hline Subsystem & $\begin{array}{c}\text { Grout Debris } \\
\text { System } 1\end{array}$ & $\begin{array}{l}\text { Desorption } \\
\text { System } 2\end{array}$ & $\begin{array}{c}\text { Wash } \\
\text { System } 3\end{array}$ & $\begin{array}{c}\text { Acid Digestion } \\
\text { System } 4\end{array}$ & $\begin{array}{c}\text { CWO } \\
\text { System } 5\end{array}$ \\
\hline Offgas from APC & 696 & 823 & 827 & 1,441 & 1,433 \\
\hline Water Discharge & 59 & 135 & 176 & 780 & 564 \\
\hline Recyclable metal/lead & 451 & 451 & 451 & 451 & 451 \\
\hline
\end{tabular}


Table 3-16. Waste volume for disposal for the five INTS systems $\left(\mathrm{ft}^{3} / \mathrm{hr}\right)$

\begin{tabular}{l|c|c|c|c|c}
\hline & $\begin{array}{c}\text { Grout Debris } \\
\text { System 1 }\end{array}$ & $\begin{array}{c}\text { Desorption } \\
\text { System 2 }\end{array}$ & $\begin{array}{c}\text { Wash } \\
\text { System 3 }\end{array}$ & $\begin{array}{c}\text { Acid Digestion } \\
\text { System 4 }\end{array}$ & $\begin{array}{c}\text { CWO } \\
\text { System 5 }\end{array}$ \\
\hline Polymer stabilization & 17.0 & 18.0 & 16.8 & 0.1 & 18.3 \\
\hline Grout stabilization & 23.0 & 21.8 & 21.6 & 6.2 & 15.3 \\
\hline $\begin{array}{l}\text { Mercury amalgamation } \\
\begin{array}{l}\text { Phosphate bonded } \\
\text { ceramic stabilization }\end{array}\end{array}$ Special waste $^{\text {Spll subsystems waste to }}$ & - & 0.0 & 0.0 & 0.0 & 0.0 \\
\hline \begin{tabular}{l} 
disposal \\
\hline $\begin{array}{l}\text { Volume reduction } \\
\text { factor (vol in/vol out) }\end{array}$
\end{tabular} & 1.0 & - & - & 28.8 & - \\
\hline
\end{tabular}

Notes:

1. The input waste volume is $45 \mathrm{ft}^{3}$.

2. The density of the grout stablization is $126.6 \mathrm{lb} / \mathrm{ft}^{3}$.

3. The density assumed for the polymerized salts and phosphate ceramic salts is

$80 \mathrm{lb} / \mathrm{ft}^{3}$. For polymer ash, debris, and salt mixture, the density assumed is $94 \mathrm{lb} / \mathrm{ft}^{3}$.

4. The data presented above is based on the October 22, 1996 Aspen mass balance 


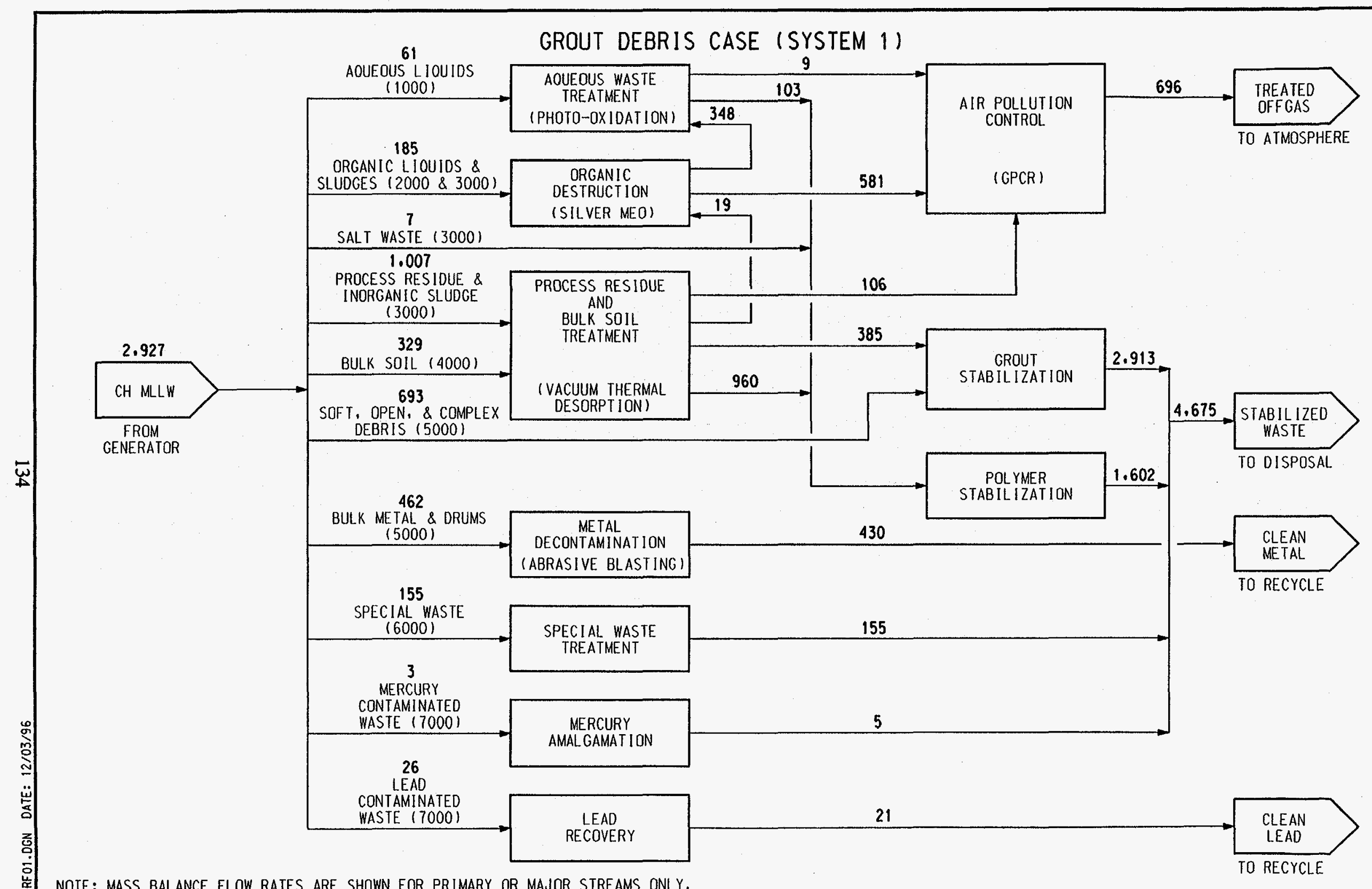

NOTE: MASS BALANCE FLOW RATES ARE SHOWN FOR PRIMARY OR MAJOR STREAMS ONLY. ADDITIVES AND RECYCLING STREAMS ARE NOT SHOWN FOR SIMPLICITY. DETAILED MASS BALANCE FIGURES ARE PRESENTED IN APPENDIX B.

Figure 3-1. General mass flow rates (lb/hr) for the grout debris case (System 1). 


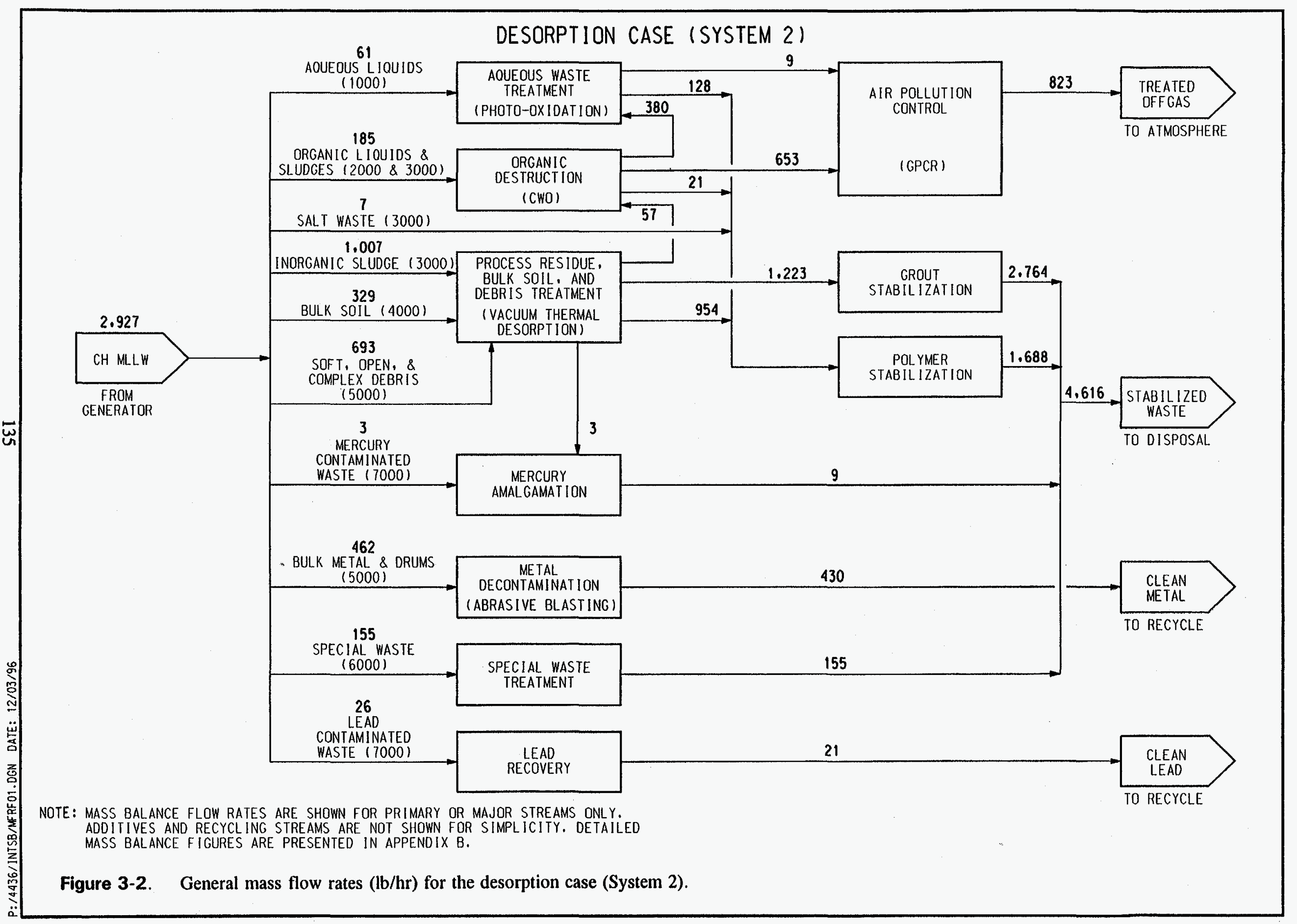




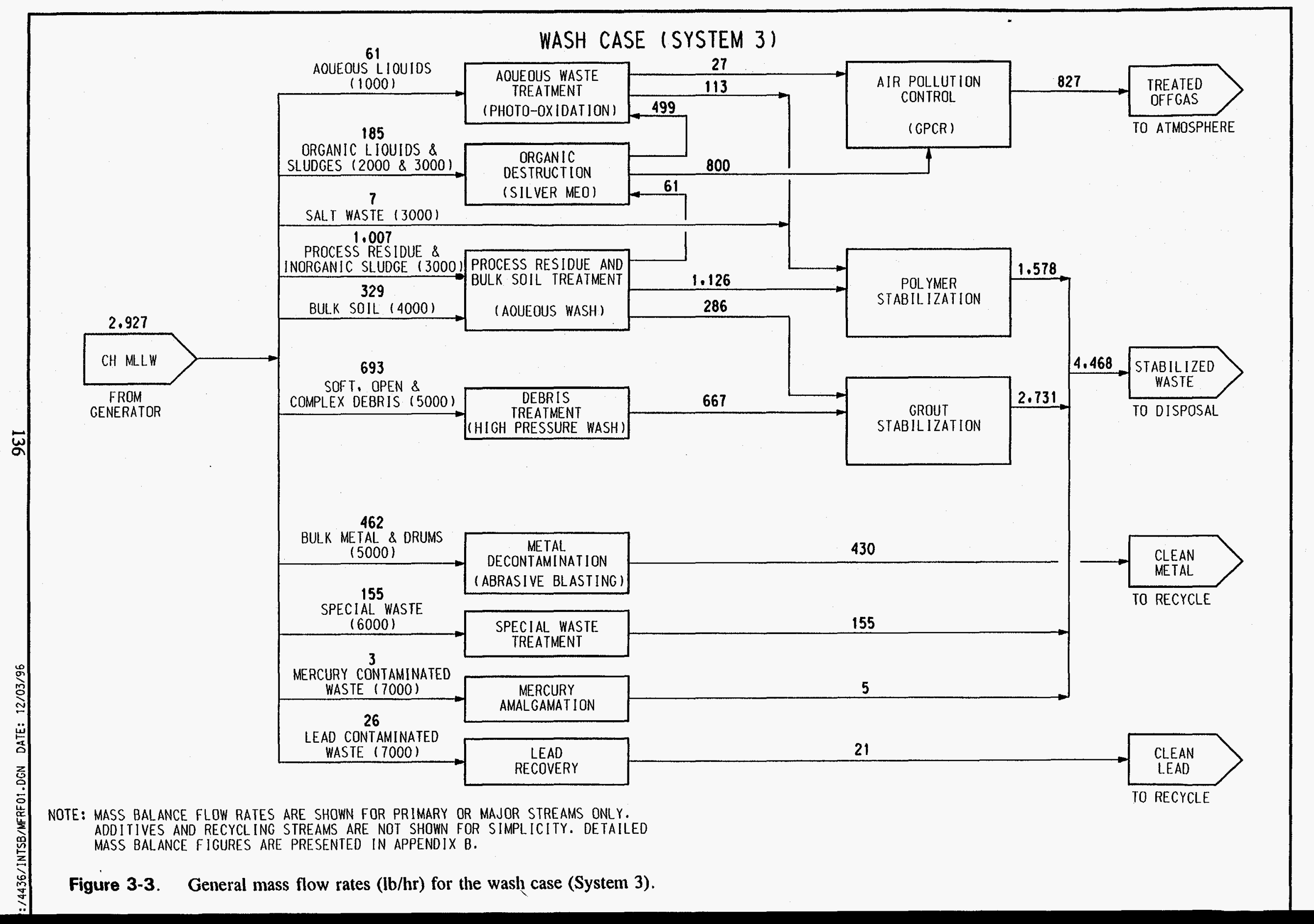




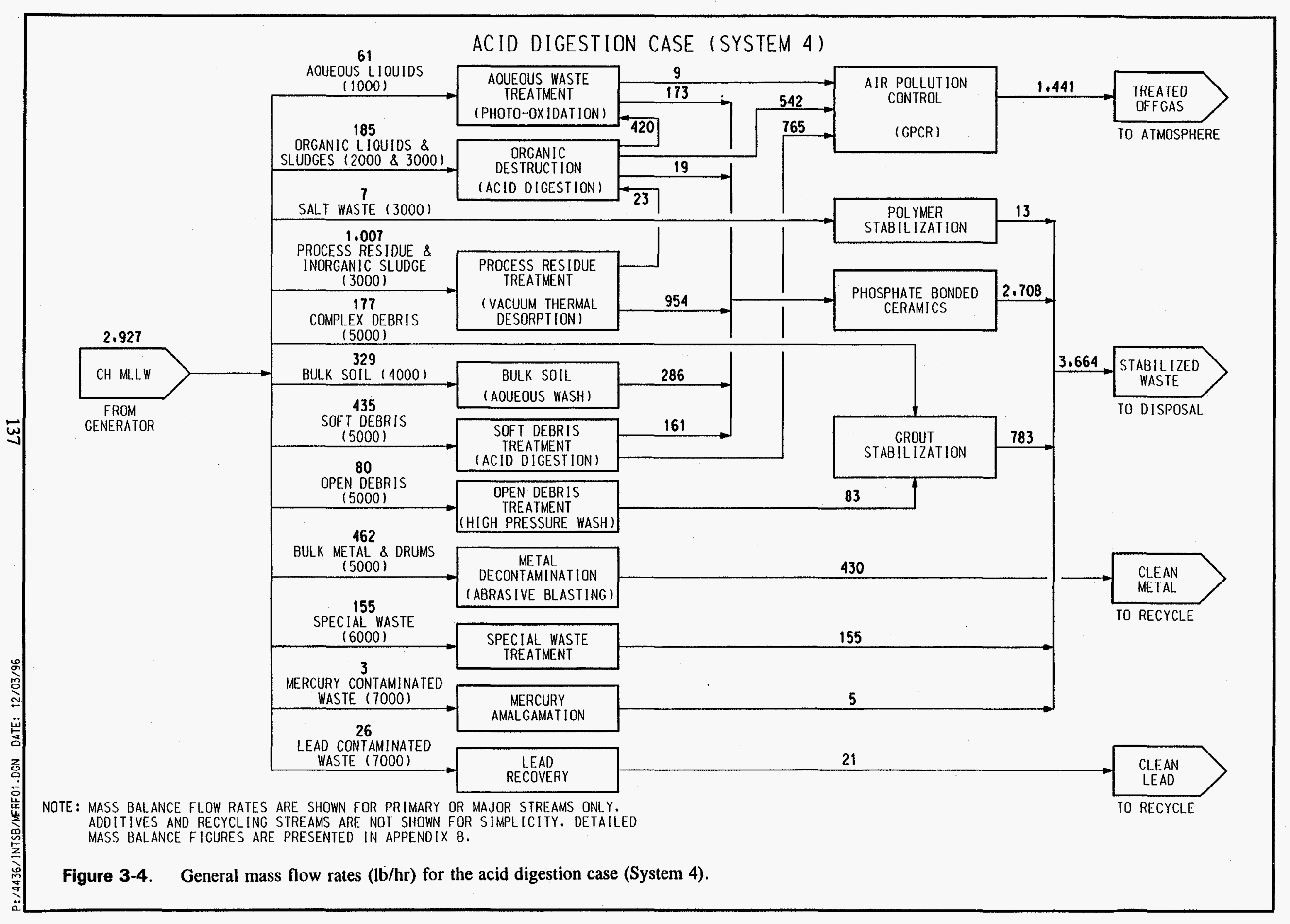




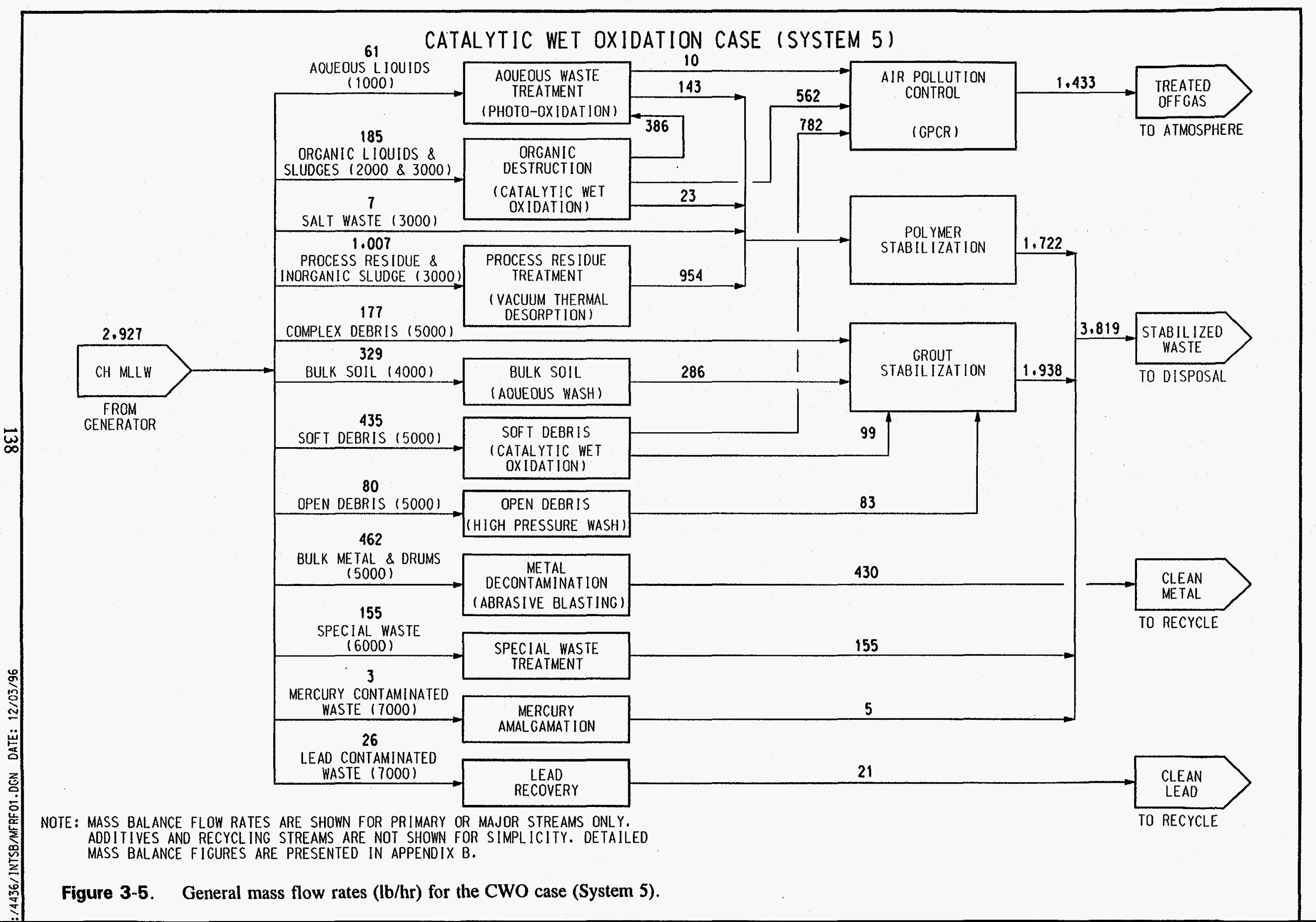




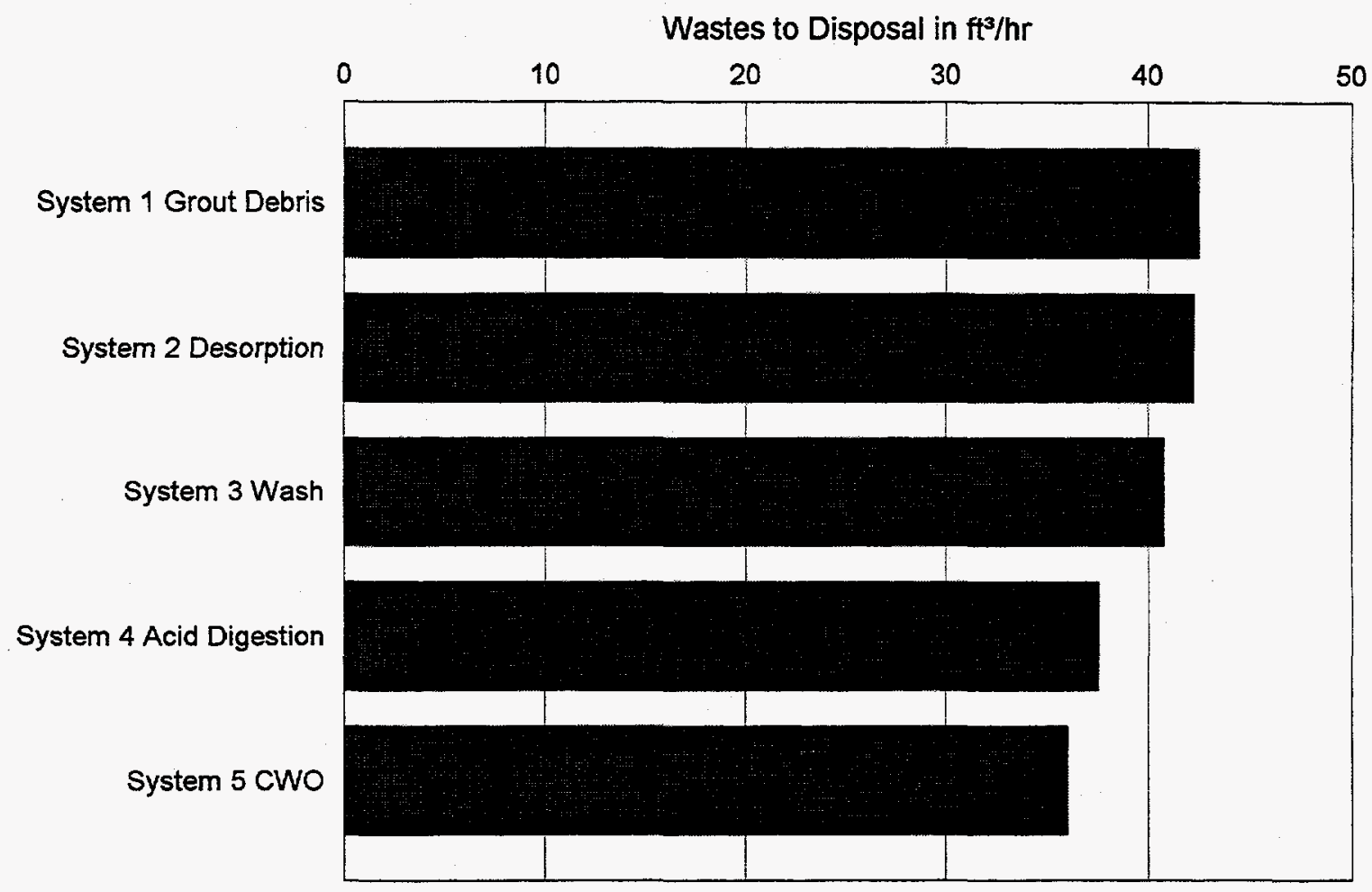

Figure 3-6. Wastes to disposal in $\mathrm{ft}^{3} / \mathrm{hr}$.

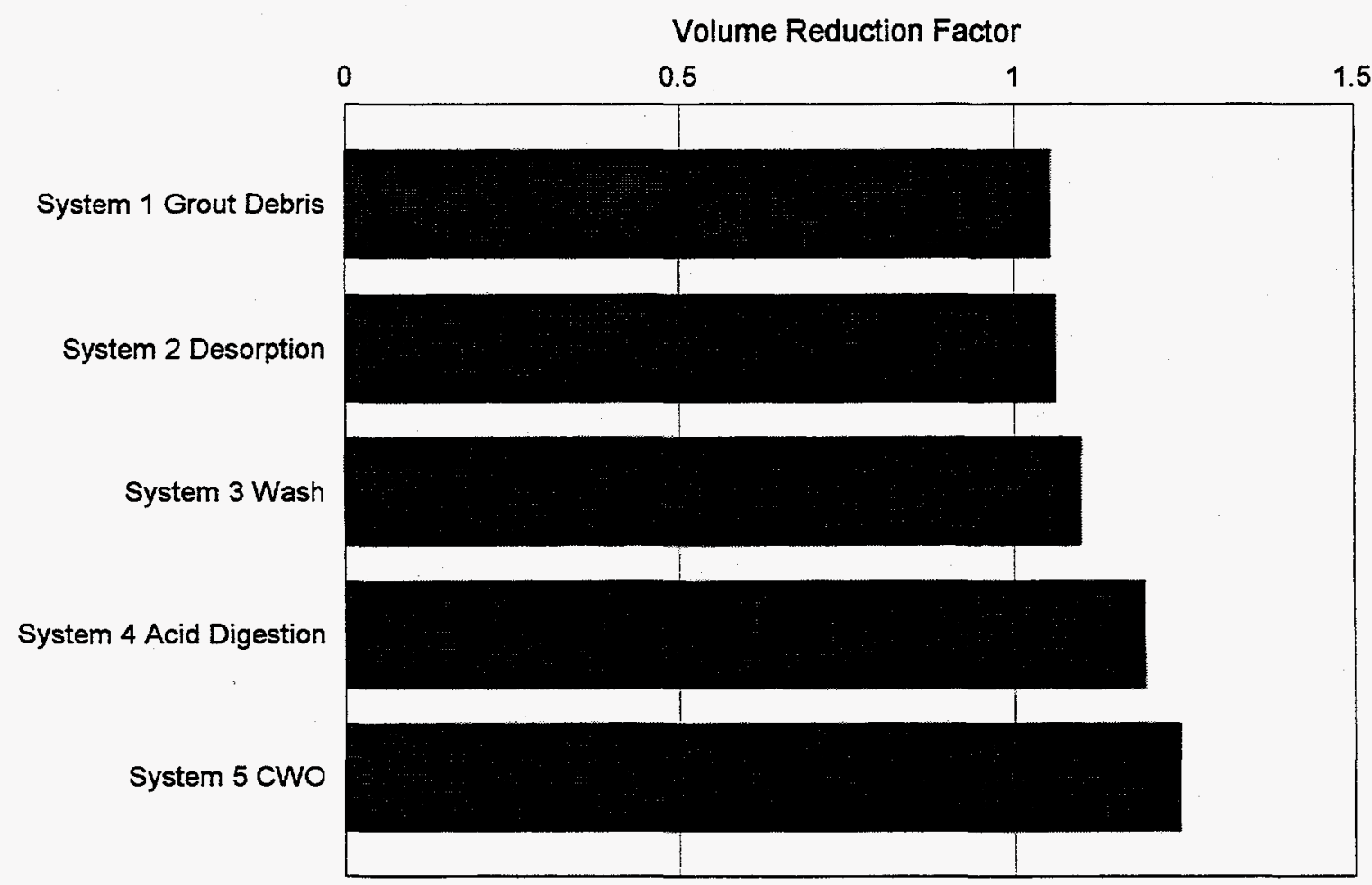

Figure 3-7. Volume reduction factor. 


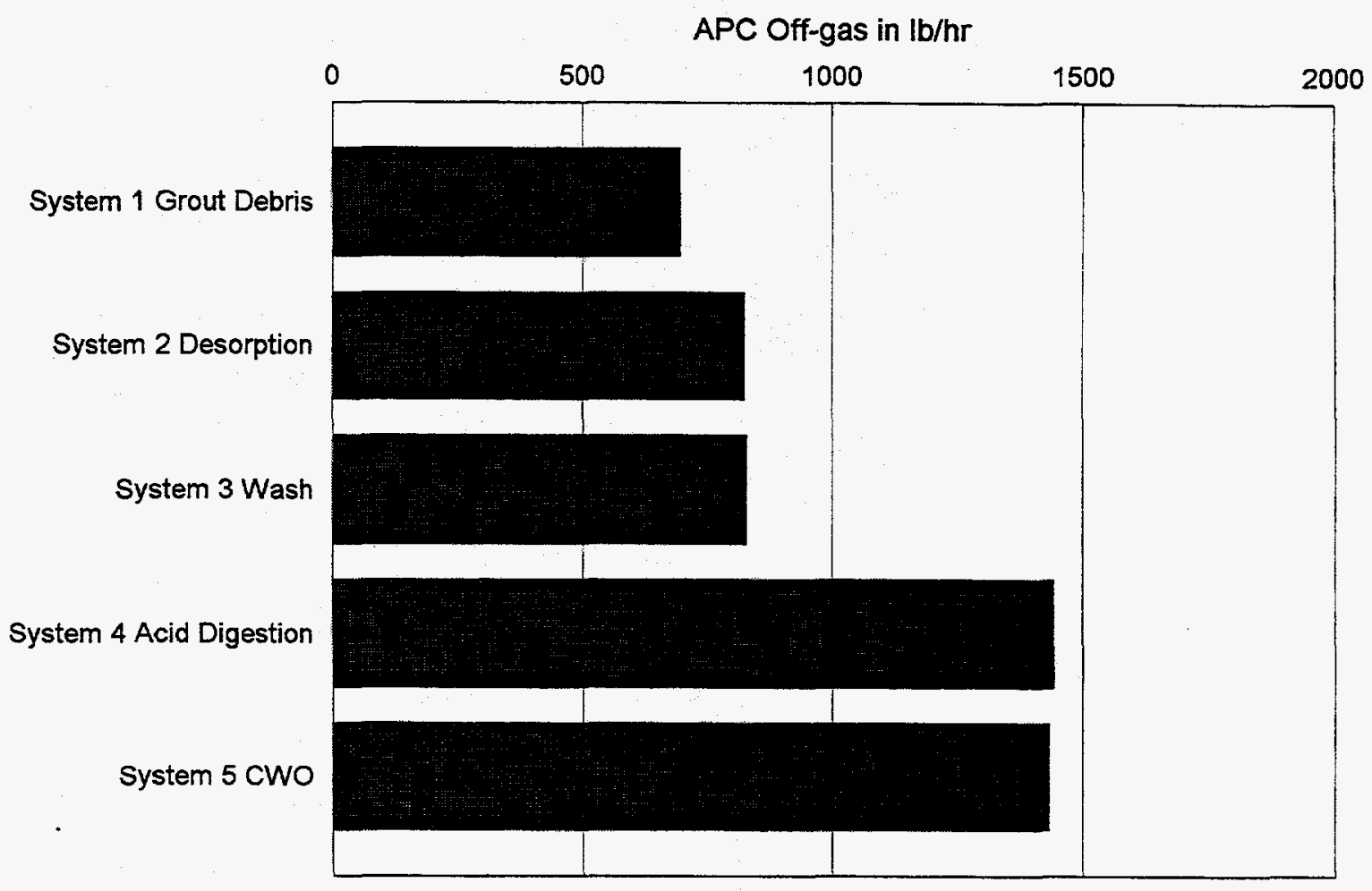

Figure 3-8. APC offgas outputs.

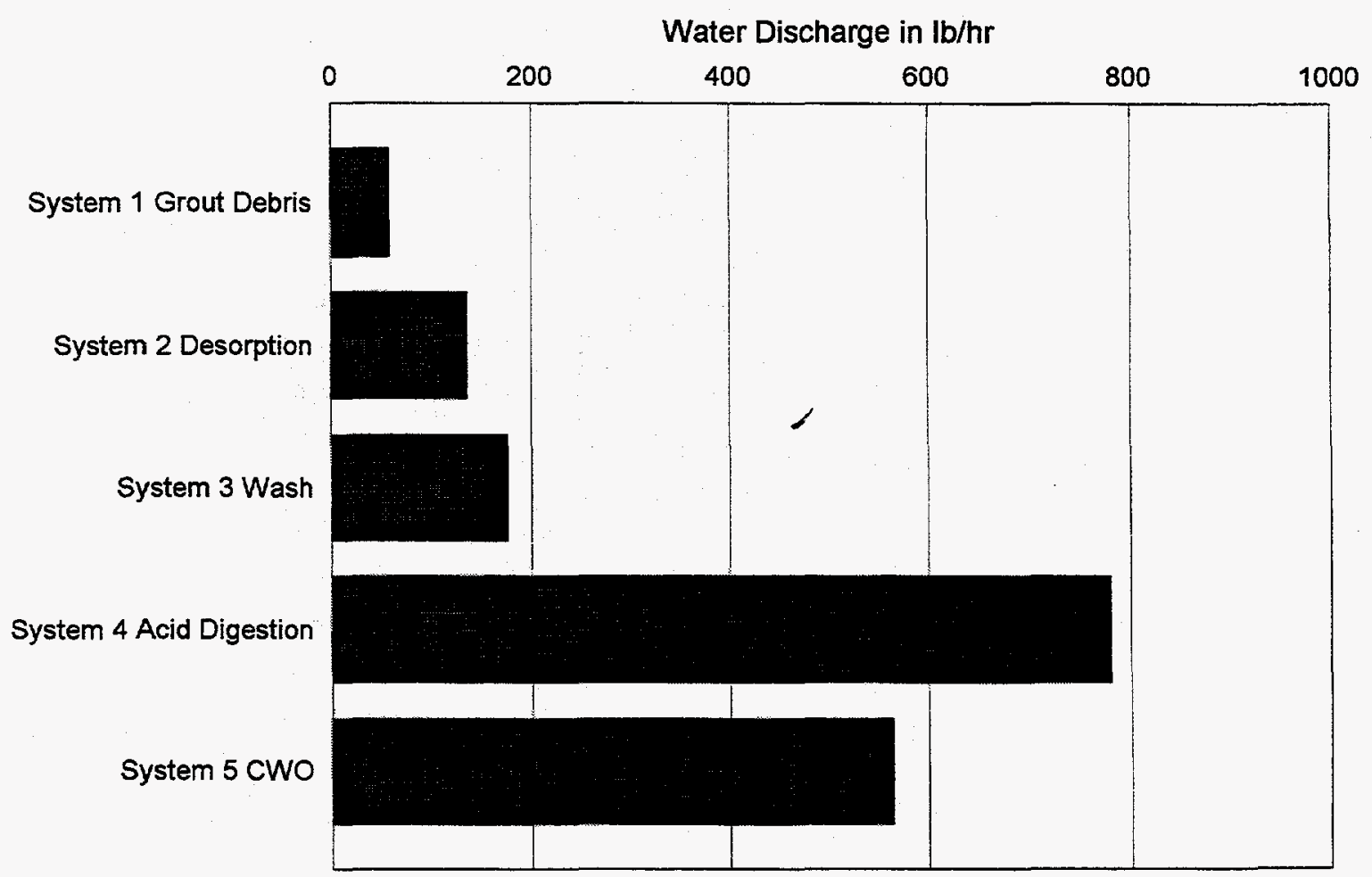

Figure 3-9. Water discharge outputs. 


\section{TOTAL LIFE CYCLE COST ESTIMATES AND SENSITIVITY ANALYSIS}

This section contains a summary of planning level total life cycle cost (TLCC) estimates for the five treatment systems. In this report, TLCC estimates will be expressed as either "with disposal". Transportation costs have been included on a generic basis in Section 4.8 but are not calculated as part of the TLCC. Cost studies have found that transportation costs are a small fraction (approximately 1\%) of the total life cycle costs.

Treatment systems presented in this report are sized at a nominal capacity (as described in Section 3) of $2,927 \mathrm{lb} / \mathrm{hr}$ to allow for direct comparison with the ITTS study (Feizollahi, 1995b). It is assumed that the facility will process both alpha and nonalpha MLLW. These systems will treat most of the stored alpha and nonalpha MLLW in a 20-year period. For smaller systems, the cost versus capacity curves from the Waste Management Facilities Cost Information Report (Feizollahi/Shropshire, 1995a) can be used to develop a reasonable cost versus size relationship.

Since only one facility is used for treating the average MLLW inventory, the facility is designed for alpha waste. TLCC estimates presented in this section are based on a facility that includes a stringent alpha radiation confinement design. The alternative is to provide two facilities, one for alpha and one for nonalpha MLLW.

\subsection{Estimating Methods, Basis, and Assumptions}

Figure 4-1 shows the steps used in the cost estimating approach. Cost information in this report was obtained during the second half of 1995 . The information is based on currently available information about waste processing requirements, technology availability, and cost data. The information may have to be updated when additional knowledge is gained in these areas. All facilities are assumed to be government owned and contractor operated. Thus, cost multipliers appropriate to this business relationship are used.

The TLCC estimates are based on the assumption that the operating life of the facility is 20 years, which is equal to 80,640 total operating hours. The treatment unit cost rates are obtained by dividing the total TLCC estimates (without disposal) by the total waste treated. The TLCC cost estimates (without disposal) represent one system handling a total of 236 million pounds of waste over 20 years $(2927 \mathrm{lb} / \mathrm{hr} \times 4032 \mathrm{hr} / \mathrm{yr} \times 20 \mathrm{yr})$. To get a rough estimate of the total costs for treating DOE waste now in inventory, the unit rates can be multiplied by the total mass in the inventory. 


\subsection{Treatment Facility TLCC Estimate (Without Disposal) Summaries}

The TLCC estimate (without disposal) for each facility is divided into six components. Each was estimated separately. A summary of the TLCC estimates for the five systems is presented in Table 4-1. Discussions of the six cost components are presented below.

\subsubsection{Studies and Bench Scale Tests and Demonstration}

Costs for the studies and bench scale tests and demonstration cost components are obtained by estimating the cost of research, labor, equipment, and facility needs.

\subsubsection{Facility Capital Costs}

The third cost component, production facility construction costs (also referred to as facility capital cost or "line-item" cost), consists of five key subcomponents which are described below. Construction costs for each system are shown in Figure 4-2.

Design. The design subcomponent includes Title 1, or preliminary design, and Title 2, or detailed design. Design is estimated at $25 \%$ of facility construction cost for an alpha facility.

Inspection. The inspection subcomponent includes Title 3, or engineering support during construction. Inspection is estimated at $7 \%$ of the facility construction cost.

Project management. The project management subcomponents include project management costs incurred both by DOE and the site management and operations (M\&O) contractor. Project management is estimated at $10 \%$ of facility construction cost.

Construction cost. Facility construction cost estimates are developed from a preconceptual design package developed for the subsystems and the integrated facilities. The preconceptual design packages include a PFD with mass flow rates, an FAD, a scoping study layout (SSL), and a summary of F\&ORs. Construction is divided into the following three parts:

- Building and structures. Building and structure costs are estimated by multiplying building unit costs by the space square footage allocated to each subsystem in the SSLs. Assumed unit rates are applied to several categories of buildings: $\$ 1,700$ per square foot for triple-confinement alpha cells (used for alpha waste processing areas); $\$ 800$ per square foot for double-confinement alpha cells (used for alpha equipment pull-out areas); $\$ 800$ per square foot for operating areas next to alpha cells; $\$ 420$ per square foot for packaged waste handling areas (truck bay or drum storage); $\$ 420$ per square foot for the process area for nonalpha waste; $\$ 420$ per square foot for the operator and 
packaged waste handling area for nonalpha waste; $\$ 180$ per square foot for office area; and $\$ 420$ per square foot for the analytical laboratory. Building unit rates include all material and labor needed for constructing the building shell including utilities, lighting, HVAC, and site development costs.

Site development costs assume that all utilities (power, sanitary and storm sewers, site communication and alarms) and access roads are available within 100 feet of the outer walls of the treatment facility. Special steel supports, foundations, and ventilation ducts and hoods required by the process components are not included in the standard building unit rates. These rates include costs for imposing stringent DOE health and safety standards on facility construction.

- Equipment. Cost estimates for equipment are developed by estimating the major equipment necessary for each unit operation. The major equipment costs are based on similar facility costs, on budgetary costs solicited from suppliers, or on engineering judgments. Vendor catalogs were used when available. Cost estimates for new technologies such as $\mathrm{MEO}, \mathrm{CWO}$, vacuum desorption, high pressure wash, and aqueous wash were obtained directly from the developers of the technologies. These cost estimates generally included peripheral as well as major equipment.

Cost estimates for technologies used in polymer stabilization, metal decontamination, lead recovery, air pollution control, grout stabilization, receiving and preparation, and certification and shipping were obtained from commercial vendors of the equipment needed.

Cost estimates for acid digestion and phosphate bonded ceramic equipment were based on published information for similar equipment, because these technologies are not commercially available.

For equipment that is not designed to NQA-1 standards, factors were used that represent the increased cost of adapting the equipment to these standards. Factors of three were used for equipment which is not food grade equivalent and would require major modifications. This factor was used, for example, for acid digestion equipment. Factors of two or 1.5 were applied based on an engineering judgment of the difference between the supplier cost and the NQA-1 standard.

For all processing subsystems, equipment is added for alpha containment, including shield windows and closed circuit television. Overhead cranes are a significant cost for most subsystems.

To estimate the additional cost of supporting equipment, allowances for electrical, instrumentation, and mechanical equipment costs such as piping are estimated as a percentage of the total equipment purchase costs.

For most subsystems which involve processing equipment, the allowance for electrical is $15 \%$, mechanical support is $30 \%$, and instrumentation is $30 \%$ of the equipment costs. For subsystems where material handling is the major processing activity, such as receiving and preparation, grout, 
polymer, metal decontamination, lead recovery, and certification and shipping, the allowances are $15 \%$ for electrical, $5 \%$ for mechanical, and $5 \%$ for instrumentation. In the case of acid digestion, where the process is highly immature technologically, a factor of 1.5 was applied in addition to the typical allowances to account for potential unidentified recycling, control, or other requirements.

For non-processing areas such as administration and support, the allowances are $1 \%$ for mechanical and instrumentation, and $15 \%$ for electrical.

In cases where the technology developer provided a total cost including electrical, instrumentation, mechanical, this estimate was used without additional allowances for their equipment. Allowances were applied to the additional equipment necessary for the subsystem.

- Indirect costs. Indirect costs include subcontractor overhead and fee. This is estimated at $29 \%$ of the total of building, structure, and equipment costs.

Construction management. The construction management subcomponent is estimated at $17 \%$ of construction costs, which is the sum of the equipment, building, and indirect costs. Construction management includes material and services procurement and control activities, which are usually handled by the site CM contractor.

Allowances for project scope change or management reserve is estimated at $10 \%$ of construction costs.

Because the costs are a planning-level estimate, a $25 \%$ contingency is included. The contingency is applied to the total of all components in the production facility construction cost.

\subsubsection{Preconstruction and Preoperational Activities}

The fourth cost component (operations budget funded activities) includes conceptual design, safety assurance, National Environmental Policy Act (NEPA) compliance efforts, and permitting, preparation for operation, and project management costs. Conceptual design is estimated to be $1.5 \%$ of line-item (production facility construction) cost. The cost for safety assurance (safety analysis reports) is estimated at $1 \%$. The costs for an environmental impact statement for NEPA compliance and for RCRA, TSCA, CAA, state, local and other permits are estimated at a flat $\$ 7$ million for all systems. All other subcomponents of the cost of the operations budget funded activities, including preoperation readiness reviews, facility start-up, operator hiring, and training costs are assumed to be equal to one year of total facility operating costs.

\subsubsection{Operations and Maintenance}

The fifth cost component, O\&M, consists of five subcomponents: operating labor, utilities, consumable materials, maintenance parts and equipment, and maintenance labor costs. The first three subcomponents 
are estimated by analyzing the requirements of each facility at the subsystem level (see Tables 4-2 and 4-3 for administration and operations staffs). Labor for the certification and shipping subsystem is based on a time and motion study relating labor to the volume of waste for disposal. The remaining two subcomponents are estimated as a percentage of the original equipment installed at the facility. Figure 4-3 shows O\&M costs for each system. The costs for annual maintenance, spare parts, and replacement equipment are estimated to be about $7 \%$ of the original equipment purchase cost. For subsystems subject to corrosion, $10 \%$ of equipment purchase cost is assumed. For subsystems utilizing equipment that is not complex, $4 \%$ is assumed. Maintenance labor is estimated to cost $250 \%$ of the cost of spare parts and replacement on an annual basis.

\subsubsection{Decontamination and Decommissioning}

The sixth cost component, decontamination and decommissioning (D\&D), is estimated by multiplying a D\&D unit rate of $\$ 450$ per square foot by the square footage of the total facility (Feizollahi, 1995a).

\subsubsection{Total System Processing TLCC Estimates}

TLCC cost estimates (without disposal) for all five systems are presented in Table 4-1. The TLCC cost estimate is the sum of the component costs developed. The components are presented in Sections 4.2.1 through 4.2.5.

\subsection{Disposal Cost Summaries}

Life cycle disposal costs of the five systems are listed in Table 4-4. The costs of disposal are based on a unit rate of $\$ 243 / \mathrm{ft}^{3}$ for burial in an engineered disposal facility. As shown, System 4 has the lowest disposal cost, while System 1 has the highest. Systems 4 and 5 have a lower disposal volume and cost than systems 1, 2, and 3 due to the oxidation of soft debris in Systems 4 and 5.

\subsection{Total Life Cycle Costs}

TLCC summary estimates (with disposal) are presented in Table 4-4. This table also presents the unit costs of treating and disposing of incoming wastes, calculated by dividing the TLCC cost by the input waste volume over 20 years of operation. Table 4-5 compares the TLCC costs of the five systems in three ways : with disposal, without disposal, and for disposal alone. Normalized life cycle cost breakdown by subsystem is presented in Table 4-6, where System 1 (grout debris) is used as the baseline for normalization. Several figures have been developed to graphically present the costs. Table 4-7 presents the subsystem costs for the five systems.

Figures 4-4 and 4-5 show the TLCC costs (with disposal) and the unit costs for all five systems. Figures 4-6 and 4-7 Show the TLCC and unit costs (without disposal) respectively for System 1 (Grout Debris). 
Figures 4-8 and 4-9 Show the TLCC and unit costs (without disposal) respectively for System 2 (Absorption). Figures 4-10 and 4-11 Show the TLCC and unit costs (without disposal) respectively for System 3 (Wash). Figures 4-12 and 4-13 Show the TLCC and unit costs (without disposal) respectively for System 4 (Acid Digestion). Figures 4-14 and 4-15 Show the TLCC and unit costs (without disposal) respectively for System 5 (CWO).

\subsection{Sensitivity Analysis}

The TLCC estimates (with disposal) have been analyzed for sensitivity against the system cost components and subcomponents. These factors include bench scale testing and demonstration, building, equipment, operations budget funded activities, O\&M, and D\&D costs. Results are presented in Figure 4-16 and are discussed below.

In the following discussion, sensitivity of the life cycle costs is calculated in terms of the cost per pound $(\$ / 1 b)$ of treating the input waste, and expressed as percent difference from the results in Table 4-1. The analysis was performed on the desorption case (System 2). The total cost for one facility is determined by multiplying the unit costs $(\$ / 1 b)$ by the assumed total quantity of the waste processed by the facility, which is 236 million pounds.

Bench scale testing and demonstration cost components. As shown in Figure 4-16, the TLCC estimate has a low sensitivity to the variation in bench scale testing and demonstration cost component. A doubling of this cost component will result in less than a $4 \%$ increase in the overall TLCC estimate.

Equipment cost subcomponent. Figure $4-16$ shows the TLCC estimate sensitivity to variations in the equipment cost. A doubling of the equipment costs subcomponent will result in an increase of less than $7 \%$ in the overall TLCC estimate.

Building cost subcomponent. Figure 4-16 shows variations in building costs versus the overall TLCC costs. Analyses indicate that a doubling of the building costs subcomponents will result in an increase of about $9 \%$ in the overall TLCC estimate.

Operating budget funded cost component. As shown in Figure 4-16, the TLCC estimate has a low sensitivity to the variation in the operations budget funded cost component. A doubling of this cost component will result in about a $2 \%$ increase in the overall TLCC estimate.

Operating and maintenance cost component. Figure 4-16 shows that the TLCC estimate is highly sensitive to the O\&M cost component. A $100 \%$ increase in the cost of operations and maintenance will result in a $50 \%$ increase in the overall TLCC estimate. 
Decontamination and decommissioning cost component. Figure 4-16 shows that the TLCC estimate has a low sensitivity to the D\&D cost component. A $100 \%$ increase in D\&D cost will result in an increase of less than $4 \%$ in the overall TLCC estimate.

Disposal costs. Figure 4-16 shows the sensitivity of the TLCC with respect to disposal costs. If the base estimate cost of $\$ 243 / \mathrm{ft}^{3}$ is doubled, the total life cycle cost will increase by about $23 \%$.

\subsection{Sensitivity Analysis of the State of Technology Development}

System cost risks for less developed technologies have been analyzed by considering the contingencies employed throughout the TLCC. Because disposal costs at a flat rate are assumed, contingency is not included. This sensitivity discussion focuses on TLCC without disposal. Risks are considered in the form of contingency allowances throughout the development of the TLCC. These contingencies are considered to be the same for all systems. However, some systems may have a higher level of uncertainty due to the state of development of the technology. System cost risks related to technology development have been analyzed in two ways. One is to look at the preoperations costs variability as a function of technology maturity. The second is to consider the uncertainties employed throughout the TLCC, particularly as expressed in contingency allowances assigned to aspects of the TLCC. Both these sensitivity analyses have been performed. Results are presented in Figures 4-16 and 4-17.

The first approach, varying preoperations costs, acknowledges the relative difficulty of the start-up phase following construction. In the TLCC estimates in this report, a one year period of preoperation is assigned for all systems. This assumption allows essentially one year of labor and maintenance before the system becomes operational. For commercially available systems, a wide experience base supports achieving quick resolution to problems. Startup problems will be primarily related to system integration and overall process engineering. For systems in a newer state of technology development, greater problems at startup should be expected. Process problems may arise for components for which the experience base is laboratory trial rather than years of operation.

Sensitivity analysis was performed to consider the effect of the state of technology development by varying the preoperation cost contingency. Figure 4-17 shows the results of this analysis. System sensitivities with respect to the preoperation costs were investigated by analyzing effects of preoperation duration on the TLCC for each system.

It was found that all systems were equally sensitive to the preoperations costs. An increase in preoperations duration from one year to four years will result in an increase of $12 \%$ in the TLCC (without disposal). 
Cost estimate uncertainties are indicated in the form of a variable contingency allowance. The base cost estimate presented in Table 4-1 employs a constant contingency of $25 \%$. This contingency is considered to be the same for all systems.

Sensitivity analysis is performed to consider the effect of technology development by varying the cost contingencies for certain subsystems. Table 4-8 shows the results of this analysis. For each system, those subsystems which are considered to have a higher cost uncertainty are given a $50 \%$ contingency. The affected subsystems are receiving and preparation, organic destruction (MEO, CWO and acid digestion), process residue (vacuum desorption, aqueous washing), soil treatment (aqueous washing) and debris treatment (high pressure washing, agitation washing, acid digestion, CWO). All other subsystems are costed with a $25 \%$ contingency. These contingencies are assigned to each cost component. The net adjustment to the contingency for each cost component of each system is shown in Table 4-8. The variation in contingency is found to have less than a $12 \%$ effect on the TLCC costs (without disposal).

\subsection{Economic Impact of Stabilization Technology}

Cost analyses are performed to determine sensitivity of the TLCC estimates to three different stabilization technologies: polymer solidification, cement solidification, and phosphate bonded ceramic stabilization. The results show that the three approaches are quite similar in cost, all between $\$ 14 / \mathrm{lb}$. and $\$ 15 / \mathrm{lb}$. The lowest life cycle unit cost is for polymer at just over $\$ 14 / \mathrm{lb}$. Phosphate bonded ceramic and grout are both close to $\$ 15 / \mathrm{lb}$. The economic impact does not take into effect the relative stability of the final waste form. Grout is considered to generate the least stable waste form, and polymer the most stable. A sensitivity analysis was performed to assess the cost of stabilizing all waste by polymer. For System 1 this would decrease disposal costs over 20 years by $6 \%$.

\subsection{Economic Impact of Stabilizing Liquid Effluent}

The INTS systems are designed to treat and recycle water within the facility. However, water is generated by the conversion of organics into carbon dioxide and water, and by the use of decontamination water and wash water. Water is recycled throughout the facility, but net generation of water occurs for all subsystems. The INTS study assumes that liquid water can be discharged. However, this may not be acceptable to the regulatory agencies. A sensitivity analysis was performed to illustrate the cost impact to INTS systems if the generated water cannot be discharged.

System 2, as shown in Table 3-15, generates excess water. If this water is grout stabilized, the grout stabilization facility would be enlarged only slightly with an insignificant cost increase. The disposal cost for the additional waste is about $\$ 50$ million over 20 years. 


\subsection{Transportation Cost}

The INTS study addresses transportation costs. However, due to the following assumptions, these costs have not been included as a component of TLCC:

- The cost of transporting the waste to the treatment facility is independent of the treatment technology used.

- The final waste output from the treatment process is disposed of at the same location as the treatment facility and the cost is assumed to be negligible.

In addition, cost estimates indicate that transportation is a small fraction of TLCC, $1 \%$ to $3 \%$ depending on disposal volume.

The following methodology (Feizollahi, 1995a) may be used to estimate transportation costs for truck shipment of treated waste from the treatment site to the disposal site if the disposal site is not co-located with the treatment facility.

The transportation cost estimating must be based on transportation unit rates in a cost-per-loaded-mile (CPLM) format. The transportation cost is divided into two components: variable costs and fixed costs. Variable costs consist of carrier cost and hardware cost. These are calculated by developing a CPLM unit rate, a variable dependent on the distance traveled.

Carrier cost covers the variable costs associated with the cargo carrier. The carrier is the entity that takes title to the waste from the owner during transportation, i.e., the trucking company. The carrier costs include tractor, fuel, labor (drivers), insurance, security escort, taxes, tools, permit fees, and related costs incurred while the waste is in transport.

Hardware cost covers the variable costs associated with procuring and maintaining the special hardware used during the transportation of waste. Special hardware consists mainly of trailers equipped with special tight-sealing enclosures or shielded casks.

Fixed costs generally consist of demurrage costs of the carrier and the hardware used in the shipment, which are independent of the distance traveled. Fixed costs are incurred during loading and unloading operations.

The cost of labor during loading and unloading operations (e.g. crane operators and health physics technicians) is part of the facility operating and maintenance costs rather than transportation costs. 
Transportation costs for contact-handled LLW, MLLW, alpha LLW and alpha MLLW are estimated based on the assumption that unshielded 48-ft-long truck trailers are used, yielding a total of $44,000 \mathrm{lbs}$ per shipment. The waste will be packaged to maximize shipment volume and weight. The total transportation costs can be calculated using the following steps:

a. Divide the total mass of treated waste (in pounds) by $44,000 \mathrm{lbs} /$ shipment to obtain the number of shipments.

b. Multiply the CPLM unit rates listed below by the one-way distance (in miles) and the number of shipments (from a. above) to obtain the CPLM cost:

- $\quad$ Below 30 miles: $\quad \$ 5.94 /$ mile

- $\quad 30$ to 200 miles: $\quad \$ 4.98 /$ mile

- $\quad$ Over 200 miles: $\$ 4.00 /$ mile.

c. Multiply the number of shipments (from a. above) by $\$ 880$ per shipment to obtain the fixed transportation costs.

d. Add the CPLM cost (from b. above) to the fixed cost (from c. above) to obtain the total transportation cost.

\subsection{Effect of Social Concerns on Costs}

The INTS study develops costs related to the construction and operation of a facility to treat DOE's mixed low level waste. The social costs of an operating facility are typically not included in this type of study. However, it is appropriate to quantify social costs that have been identified. The areas of expected cost include the following:

- Community meetings and presentations

- Newsletters, reports or other communication mechanism to the community

- Community liaison personnel, including office support

- Weekly emissions sampling by an outside laboratory supplementing plant sampling

- Traveling educational center, with staff and materials

- Technical editors to assure that documents are readable to the public. 
These costs are estimated to be $\$ 1.5$ million per year, or approximately $\$ 30$ million over the facility's life. For the INTS systems, the social costs are estimated at nearly $1 \%$ of the total life-cycle cost. 
Table 4-1. Total life-cycle cost estimates (without disposal) for INTS (Costs in millions of dollars). (Sheet 1 of 5.)

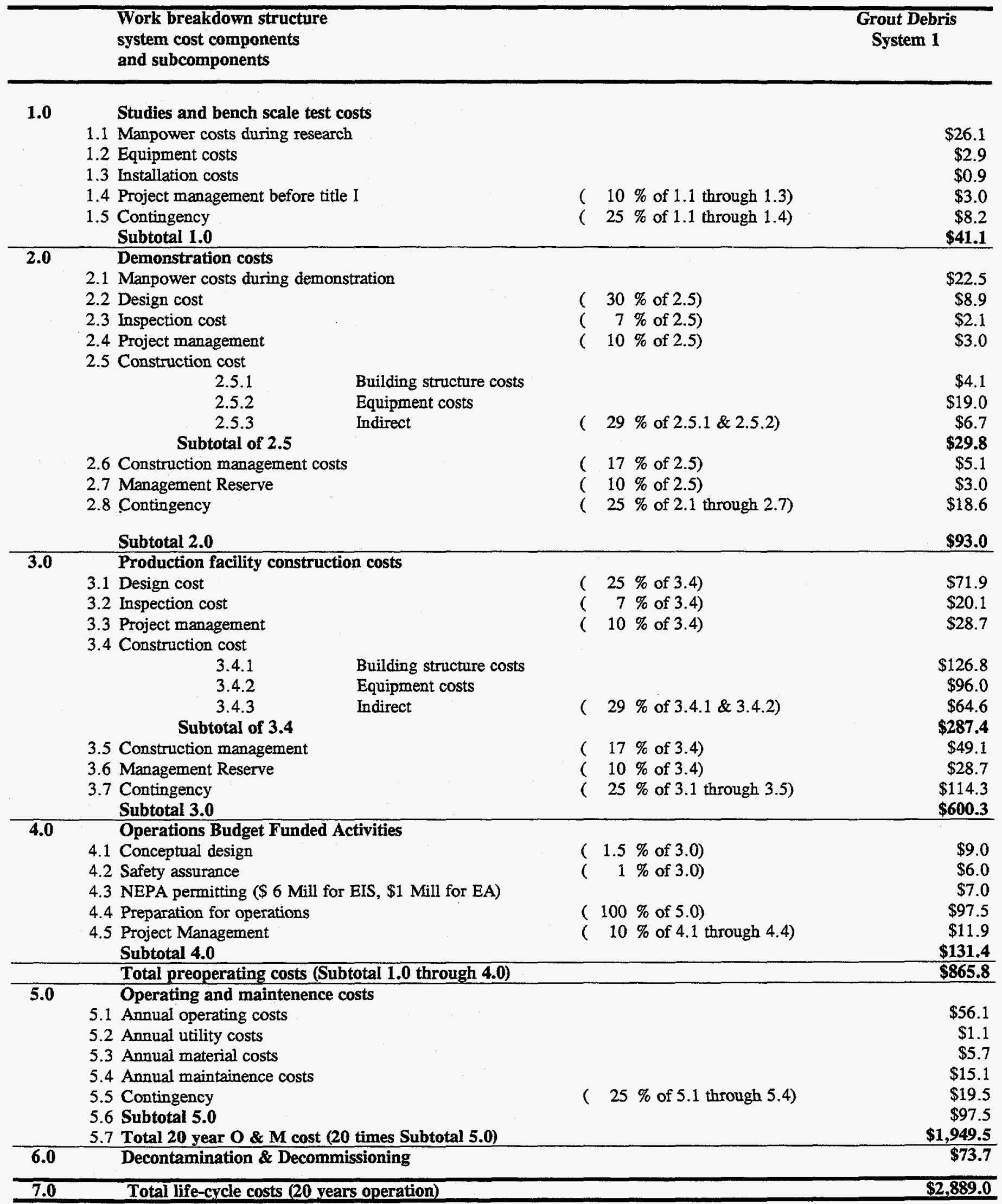


Table 4-1. Total life-cycle cost estimates (without disposal) for INTS (Costs in millions of dollars). (Sheet 2 of 5. )

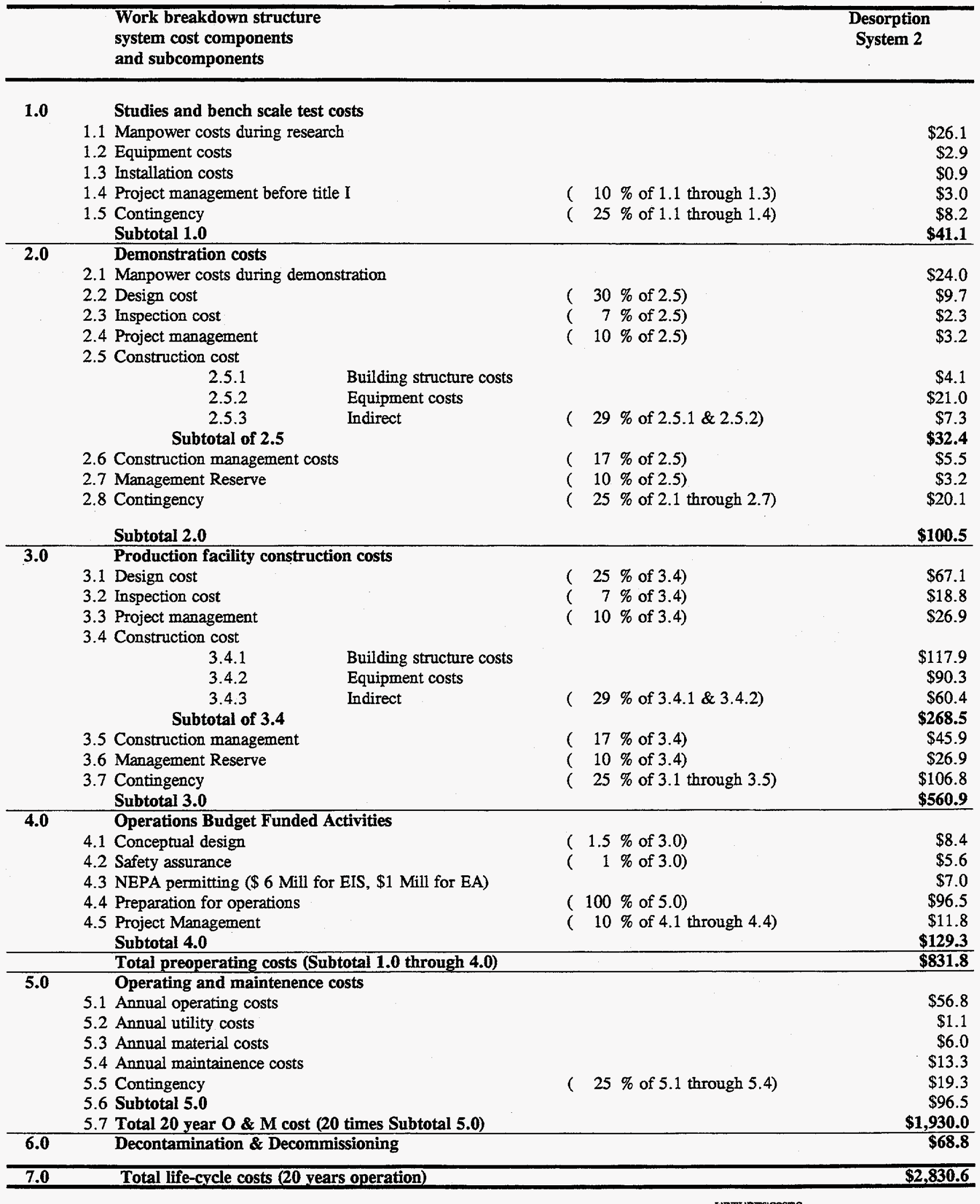


Table 4-1. Total life-cycle cost estimates (without disposal) for INTS (Costs in millions of dollars). (Sheet 3 of 5. )

\begin{tabular}{|c|c|c|c|c|c|}
\hline & & $\begin{array}{l}\text { Work breakdown structure } \\
\text { system cost components } \\
\text { and subcomponents }\end{array}$ & & & $\begin{array}{c}\text { Wash } \\
\text { System } 3\end{array}$ \\
\hline \multirow[t]{7}{*}{1.0} & & \multicolumn{2}{|c|}{ Studies and bench scale test costs } & & \\
\hline & 1.1 & \multicolumn{2}{|c|}{ Manpower costs during research } & & $\$ 26.6$ \\
\hline & 1.2 & \multirow{2}{*}{\multicolumn{2}{|c|}{$\begin{array}{l}\text { Equipment costs } \\
\text { Installation costs }\end{array}$}} & & $\$ 2.7$ \\
\hline & 1.3 & & & & $\$ 1.0$ \\
\hline & 1.4 & \multicolumn{2}{|c|}{ Project management before title I } & ( $10 \%$ of 1.1 through 1.3 ) & $\$ 3.0$ \\
\hline & 1.5 & \multicolumn{2}{|l|}{ Contingency } & ( $25 \%$ of 1.1 through 1.4 ) & $\$ 8.3$ \\
\hline & & \multicolumn{2}{|l|}{ Subtotal 1.0} & & $\$ 41.6$ \\
\hline \multirow[t]{14}{*}{2.0} & & \multicolumn{2}{|c|}{ Demonstration costs } & & \\
\hline & 2.1 & \multicolumn{2}{|c|}{ Manpower costs during demonstration } & & $\$ 24.0$ \\
\hline & 2.2 & \multicolumn{2}{|c|}{ Design cost } & $(30 \%$ of 2.5$)$ & $\$ 9.8$ \\
\hline & 2.3 & \multicolumn{2}{|l|}{ Inspection cost } & ( $7 \%$ of 2.5$)$ & $\$ 2.3$ \\
\hline & 2.4 & \multirow{2}{*}{\multicolumn{2}{|c|}{$\begin{array}{l}\text { Project management } \\
\text { Construction cost }\end{array}$}} & $(10 \%$ of 2.5$)$ & $\$ 3.3$ \\
\hline & 2.5 & & & & \\
\hline & & 2.5 .1 & Building structure costs & & $\$ 4.8$ \\
\hline & & 2.5 .2 & Equipment costs & & $\$ 20.5$ \\
\hline & & 2.5 .3 & Indirect & $(29 \%$ of $2.5 .1 \& 2.5 .2)$ & $\$ 7.3$ \\
\hline & & Subtotal of $\mathbf{2 . 5}$ & & & $\$ 32.6$ \\
\hline & 2.6 & Construction management cost & & ( $17 \%$ of 2.5$)$ & $\$ 5.6$ \\
\hline & 2.7 & Management Reserve & & ( $10 \%$ of 2.5$)$ & $\$ 3.3$ \\
\hline & 2.8 & Contingency & & ( $25 \%$ of 2.1 through 2.7 ) & $\$ 20.2$ \\
\hline & & Subtotal 2.0 & & & $\$ 101.0$ \\
\hline \multirow[t]{13}{*}{3.0} & & \multicolumn{3}{|c|}{ Production facility construction costs } & \\
\hline & 3.1 & Design cost & & $(25 \%$ of 3.4$)$ & $\$ 75.7$ \\
\hline & 3.2 & Inspection cost & & ( $7 \%$ of 3.4$)$ & $\$ 21.2$ \\
\hline & 3.3 & Project management & & ( $10 \%$ of 3.4$)$ & $\$ 30.3$ \\
\hline & 3.4 & Construction cost & & & \\
\hline & & $\quad 3.4 .1$ & Building structure costs & & $\$ 135.4$ \\
\hline & & 3.4 .2 & Equipment costs & & $\$ 99.1$ \\
\hline & & 3.4 .3 & Indirect & $(29 \%$ of $3.4 .1 \& 3.4 .2)$ & $\$ 68.0$ \\
\hline & & Subtotal of $\mathbf{3 . 4}$ & & & $\$ 302.6$ \\
\hline & 3.5 & Construction management & & $(17 \%$ of 3.4$)$ & $\$ 51.7$ \\
\hline & 3.6 & Management Reserve & & $(10 \%$ of 3.4$)$ & $\$ 30.3$ \\
\hline & 3.7 & Contingency & & ( $25 \%$ of 3.1 through 3.5 ) & $\$ 120.4$ \\
\hline & & Subtotal 3.0 & & & $\$ 632.1$ \\
\hline \multirow[t]{8}{*}{4.0} & & \multicolumn{2}{|c|}{ Operations Budget Funded Activities } & & \\
\hline & 4.1 & \multirow{2}{*}{\multicolumn{2}{|c|}{$\begin{array}{l}\text { Conceptual design } \\
\text { Safety assurance }\end{array}$}} & $(1.5 \%$ of 3.0$)$ & $\$ 9.5$ \\
\hline & 4.2 & & & ( $1 \%$ of 3.0$)$ & $\$ 6.3$ \\
\hline & 4.3 & \multicolumn{2}{|c|}{ NEPA permitting ( $\$ 6$ Mill for EIS, \$1 Mill for EA) } & & $\$ 7.0$ \\
\hline & 4.4 & \multicolumn{2}{|c|}{ Preparation for operations } & $(100 \%$ of 5.0$)$ & $\$ 100.2$ \\
\hline & 4.5 & Project Management & & ( $10 \%$ of 4.1 through 4.4$)$ & $\$ 12.3$ \\
\hline & & Subtotal 4.0 & & & $\$ 135.3$ \\
\hline & & Total preoperating costs (Sub & total 1.0 through 4.0 ) & & $\$ 910.1$ \\
\hline 5.0 & & Operating and maintenence & osts & & \\
\hline & 5.1 & Annual operating costs & & & $\$ 58.2$ \\
\hline & 5.2 & Annual utility costs & & & $\$ 1.2$ \\
\hline & 5.3 & Annual material costs & & & $\$ 5.9$ \\
\hline & 5.4 & Annual maintainence costs & & & $\$ 14.9$ \\
\hline & 5.5 & Contingency & & ( $25 \%$ of 5.1 through 5.4 ) & $\$ 20.0$ \\
\hline & 5.6 & Subtotal 5.0 & & & $\$ 100.2$ \\
\hline & 5.7 & Total 20 year $O \&$ M cost (2 & times Subtotal 5.0) & & $\$ 2,004.8$ \\
\hline 6.0 & & Decontamination \& Decomm & issioning & & $\$ 77.0$ \\
\hline
\end{tabular}


Table 4-1. Total life-cycle cost estimates (without disposal) for INTS (Costs in millions of dollars). (Sheet 4 of 5.)

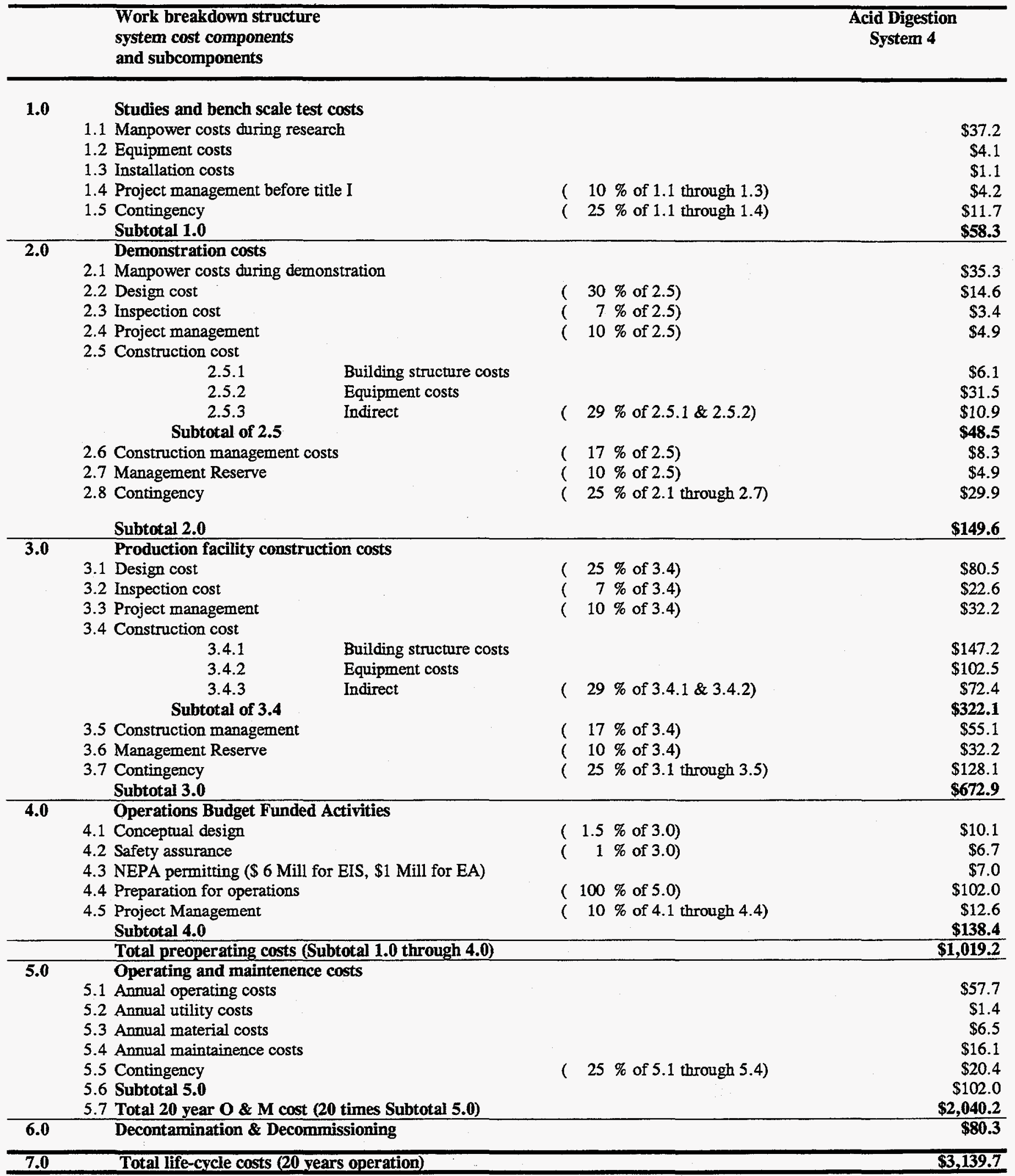


Table 4-1. Total life-cycle cost estimates for (without disposal) INTS (Costs in millions of dollars). (Sheet 5 of 5 .)

\begin{tabular}{|c|c|c|c|c|c|}
\hline & & $\begin{array}{l}\text { Work breakdown structure } \\
\text { system cost components } \\
\text { and subcomponents }\end{array}$ & & & $\begin{array}{c}\text { CWO } \\
\text { System } 5\end{array}$ \\
\hline \multirow[t]{7}{*}{1.0} & \multicolumn{3}{|c|}{ Studies and bench scale test costs } & & \\
\hline & 1.1 & \multicolumn{2}{|c|}{ Manpower costs during research } & & $\$ 30.3$ \\
\hline & 1.2 & \multirow{2}{*}{\multicolumn{2}{|c|}{$\begin{array}{l}\text { Equipment costs } \\
\text { Installation costs }\end{array}$}} & & $\$ 3.2$ \\
\hline & 1.3 & & & & $\$ 1.2$ \\
\hline & 1.4 & \multicolumn{2}{|c|}{ Project management before title I } & ( $10 \%$ of 1.1 through 1.3 ) & $\$ 3.5$ \\
\hline & 1.5 & \multirow{2}{*}{\multicolumn{2}{|c|}{$\begin{array}{l}\text { Contingency } \\
\text { Subtotal 1.0 }\end{array}$}} & ( $25 \%$ of 1.1 through 1.4 ) & $\$ 9.5$ \\
\hline & & & & & $\$ 47.7$ \\
\hline \multirow[t]{14}{*}{2.0} & \multicolumn{3}{|c|}{ Demonstration costs } & & \\
\hline & \multicolumn{3}{|c|}{ 2.1 Manpower costs during demonstration } & & $\$ 28.5$ \\
\hline & 2.2 & \multicolumn{2}{|c|}{ Design cost } & $(30 \%$ of 2.5$)$ & $\$ 11.5$ \\
\hline & 2.3 & \multicolumn{2}{|l|}{ Inspection cost } & ( $7 \%$ of 2.5$)$ & $\$ 2.7$ \\
\hline & 2.4 & \multirow{2}{*}{\multicolumn{2}{|c|}{ Project management }} & ( $10 \%$ of 2.5$)$ & $\$ 3.8$ \\
\hline & 2.5 & & & & \\
\hline & & 2.5 .1 & Building structure costs & & $\$ 5.3$ \\
\hline & & 2.5 .2 & Equipment costs & & $\$ 24.5$ \\
\hline & & 2.5 .3 & Indirect & $(29 \%$ of $2.5 .1 \& 2.5 .2)$ & $\$ 8.6$ \\
\hline & \multicolumn{3}{|c|}{ Subtotal of 2.5} & & $\$ 38.4$ \\
\hline & \multicolumn{3}{|c|}{ 2.6 Construction management costs } & $(17 \%$ of 2.5$)$ & $\$ 6.6$ \\
\hline & \multicolumn{3}{|c|}{ 2.7 Management Reserve } & $(10 \%$ of 2.5$)$ & $\$ 3.8$ \\
\hline & \multicolumn{3}{|c|}{2.8 Contingency } & ( $25 \%$ of 2.1 through 2.7 ) & $\$ 23.9$ \\
\hline & \multicolumn{3}{|c|}{ Subtotal 2.0} & & $\$ 119.3$ \\
\hline \multirow[t]{13}{*}{3.0} & \multicolumn{3}{|c|}{ Production facility construction costs } & & \\
\hline & 3.1 & \multicolumn{2}{|c|}{ Design cost } & $(25 \%$ of 3.4$)$ & $\$ 79.9$ \\
\hline & 3.2 & Inspection cost & & ( $7 \%$ of 3.4 ) & $\$ 22.4$ \\
\hline & 3.3 & Project management & & $(10 \%$ of 3.4$)$ & $\$ 32.0$ \\
\hline & 3.4 & Construction cost & & & \\
\hline & & 3.4 .1 & Building structure costs & & $\$ 141.2$ \\
\hline & & 3.4 .2 & Equipment costs & & $\$ 106.7$ \\
\hline & & 3.4 .3 & Indirect & $(29 \%$ of $3.4 .1 \& 3.4 .2)$ & $\$ 71.9$ \\
\hline & & Subtotal of 3.4 & & & $\$ 319.7$ \\
\hline & 3.5 & Construction management & & $17 \%$ of 3.4$)$ & $\$ 54.7$ \\
\hline & 3.6 & Management Reserve & & $(10 \%$ of 3.4$)$ & $\$ 32.0$ \\
\hline & 3.7 & Contingency & & $25 \%$ of 3.1 through 3.5 ) & $\$ 127.2$ \\
\hline & & Subtotal 3.0 & & & $\$ 667.8$ \\
\hline 4.0 & & Operations Budget Funded $A$ & ctivities & & \\
\hline & 4.1 & Conceptual design & & $(1.5 \%$ of 3.0$)$ & $\$ 10.0$ \\
\hline & 4.2 & Safety assurance & & $(1 \%$ of 3.0$)$ & $\$ 6.7$ \\
\hline & 4.3 & NEPA permitting ( $\$ 6$ Mill for & EIS, \$1 Mill for EA) & & $\$ 7.0$ \\
\hline & 4.4 & Preparation for operations & & $(100 \%$ of 5.0$)$ & $\$ 102.9$ \\
\hline & 4.5 & Project Management & & ( $10 \%$ of 4.1 through 4.4 ) & $\$ 12.7$ \\
\hline & & Subtotal 4.0 & & & $\$ 139.3$ \\
\hline & & Total preoperating costs (Sub & total 1.0 through 4.0 ) & & $\$ 974.1$ \\
\hline 5.0 & & Operating and maintenence $c$ & osts & & \\
\hline & 5.1 & Annual operating costs & & & $\$ 56.6$ \\
\hline & 5.2 & Annual utility costs & & & $\$ 1.9$ \\
\hline & 5.3 & Annual material costs & & & $\$ 6.8$ \\
\hline & 5.4 & Annual maintainence costs & & & $\$ 17.0$ \\
\hline & & Contingency & & $(25 \%$ of 5.1 through 5.4$)$ & $\$ 20.6$ \\
\hline & 5.6 & Subtotal 5.0 & & & $\$ 102.9$ \\
\hline & 5.7 & Total 20 year $O \& M$ cost $(2$ & times Subtotal 5.0) & & $\$ 2,058.1$ \\
\hline 6.0 & & Decontamination \& Decomm & ssioning & & $\$ 77.6$ \\
\hline 7.0 & & Total life-cycle costs ( 20 yea & s operation) & & $\$ 3,109.7$ \\
\hline
\end{tabular}


Table 4-2. Estimated facility administration staff for all INTS systems.

\begin{tabular}{l} 
Job \\
\hline Accountant \\
Secretary and clerk \\
Document control \\
Manager-day shifts \\
Technical personnel \\
Communications
\end{tabular}


Table 4-3. Estimated facility operation staff for the INTS systems.

Full Time Equivalent Workers

\begin{tabular}{|c|c|c|c|c|c|}
\hline Subsystem & $\begin{array}{c}\text { Grout Debris } \\
\text { System } 1 \\
\end{array}$ & $\begin{array}{c}\text { Desorption } \\
\text { System } 2 \\
\end{array}$ & $\begin{array}{c}\text { Wash } \\
\text { System } 3\end{array}$ & $\begin{array}{c}\text { Acid Digestion } \\
\text { System } 4 \\
\end{array}$ & $\begin{array}{c}\text { CWO } \\
\text { System } 5\end{array}$ \\
\hline Administration Subsystem & 32 & 32 & 32 & 32 & 32 \\
\hline Receiving \& Inspection Subsystem & 170 & 170 & 170 & 170 & 170 \\
\hline Metal Decontamination Subsystem & 4 & 4 & 4 & 4 & 4 \\
\hline Lead Recovery Subsystem & 3 & 3 & 3 & 3 & 3 \\
\hline Organic Destruction Treatment & 12 & 12 & 12 & 12 & 12 \\
\hline Process Residue Treatment & 10 & 16 & 3 & 8 & 8 \\
\hline Soil Treatment & 0 & 0 & & 2 & 2 \\
\hline Open and Complex Debris Treatment & & & 16 & 8 & 8 \\
\hline Soft Debris Treatment & & & & 18 & 14 \\
\hline Air Pollution Control Subsystem & 4 & 4 & 4 & 4 & 4 \\
\hline Aqueous Waste Treatment Subsystem & 5 & 5 & 5 & 5 & 5 \\
\hline Polymer Stabilization Subsystem & 38 & 38 & 40 & 5 & 38 \\
\hline Phosphate Cement Stabilization & & & & 40 & \\
\hline Mercury Amalgamation Subsystem & 1 & 1 & 1 & 1 & 1 \\
\hline Certification \& Shipping Subsystem & 106 & 105 & 110 & 87 & 88 \\
\hline Grout Stabilization Subsystem & 8 & 8 & 8 & 5 & 8 \\
\hline Support Subsystem & 5 & 5 & 5 & 5 & 5 \\
\hline TOTAL & 401 & 406 & 416 & 412 & 405 \\
\hline
\end{tabular}


Table 4-4. Total system life cycle with disposal cost estimates.

\begin{tabular}{|c|c|c|c|c|c|c|}
\hline & units & $\begin{array}{c}\text { Grout Debris } \\
\text { System } 1 \\
\end{array}$ & $\begin{array}{r}\text { Desorption } \\
\text { System } 2 \\
\end{array}$ & $\begin{array}{c}\text { Wash } \\
\text { System } 3 \\
\end{array}$ & $\begin{array}{c}\text { Acid Digestion } \\
\text { System } 4 \\
\end{array}$ & $\begin{array}{c}\text { CWo } \\
\text { System } 5 \\
\end{array}$ \\
\hline Treatment Cost & $\$ / \mathrm{b}$ & $\$ 12$ & $\$ 12$ & $\$ 13$ & $\$ 13$ & $\$ 13$ \\
\hline Normalized Treatment Cost & System I $=1.0$ & 1.00 & 0.98 & 1.04 & 1.09 & 1.08 \\
\hline Output Volumetric Flow Rate & $\mathrm{ft} 3 / \mathrm{hr}$ & 42.46 & 42.20 & 40.77 & 37.55 & 36.04 \\
\hline Total Treatment Cost & $(\$ 000,000)$ & $\$ 2,889$ & $\$ 2,831$ & $\$ 2,992$ & $\$ 3,140$ & $\$ 3,110$ \\
\hline Unit Treatment Cost (b) & $\$ / l b$ & $\$ 12.24$ & $\$ 11.99$ & $\$ 12.68$ & $\$ 13.30$ & $\$ 13.17$ \\
\hline Final Waste Volume (c) & $\mathrm{ft} 3$ & $3,423,974$ & $3,403,008$ & $3,287,693$ & $3,028,032$ & $2,906,266$ \\
\hline Disposal Cost & $(\$ 000,000)$ & $\$ 832$ & $\$ 827$ & $\$ 799$ & $\$ 736$ & $\$ 706$ \\
\hline Disposal Unit Cos! & $\$ / 1 b$ & $\$ 3.53$ & $\$ 3.50$ & $\$ 3.38$ & $\$ 3.12$ & $\$ 2.99$ \\
\hline Total Life Cycle Cost & $(\$ 000,000)$ & $\$ 3,721$ & $\$ 3,657$ & $\$ 3,79 !$ & $\$ 3,876$ & $\$ 3,816$ \\
\hline Total Life Cycle Unit Cost (d) & $\$ / \mathrm{lb}$ & $\$ 15.76$ & $\$ 15.50$ & $\$ 16.06$ & $\$ 16.42$ & $\$ 16.17$ \\
\hline
\end{tabular}

\section{Notes:}

(a) Costs do not include transportation costs.

(b) The treatment cost unit rate is calculated by dividing the treatment cost by the total waste input to the INTS facility for 20 years of operation (236 million pounds).

(c) The final waste volume is the total waste disposed over 20 years, or the waste flow rate times $80,640 \mathrm{hrs}$.

(d) The total life cycle cost unit rate is calculated by dividing the total lifecycle cost (treatment + disposal) by the total waste input to the INTS facility for 20 years of operation (236 million pounds). 
Table 4-5. Total life cycle cost (with disposal) comparison of five INTS systems (in millions of dollars).

\begin{tabular}{|c|c|c|c|c|c|}
\hline WBS Element Costs (a) & $\begin{array}{c}\text { Grout Debris } \\
\text { System } 1 \\
\end{array}$ & $\begin{array}{c}\text { Desorption } \\
\text { System } 2 \\
\end{array}$ & $\begin{array}{c}\text { Wash } \\
\text { System } 3 \\
\end{array}$ & $\begin{array}{c}\text { Acid Digestion } \\
\text { System } 4 \\
\end{array}$ & $\begin{array}{c}\text { CWO } \\
\text { System } 5 \\
\end{array}$ \\
\hline \multicolumn{6}{|l|}{ TREATMENT } \\
\hline Item $1.0,2.0$, and 4.0 & $\$ 265$ & $\$ 271$ & $\$ 278$ & $\$ 346$ & $\$ 306$ \\
\hline Item 3.0 & $\$ 600$ & $\$ 561$ & $\$ 632$ & $\$ 673$ & $\$ 668$ \\
\hline Item 5.0 & $\$ 1,949$ & $\$ 1,930$ & $\$ 2,005$ & $\$ 2,040$ & $\$ 2,058$ \\
\hline Item 6.0 & $\$ 74$ & $\$ 69$ & $\$ 77$ & $\$ 80$ & $\$ 78$ \\
\hline Total Treatment TLCC & $\$ 2,889$ & $\$ 2,831$ & $\$ 2,992$ & $\$ 3,140$ & $\$ 3,110$ \\
\hline \multicolumn{6}{|l|}{ DISPOSAL (b) } \\
\hline Disposal TLCC & $\$ 832$ & $\$ 827$ & $\$ 799$ & $\$ 736$ & $\$ 706$ \\
\hline \multicolumn{6}{|c|}{ TOTAL TREATMENT \& DISPOSAL } \\
\hline TLCC & $\$ 3,721$ & $\$ 3,657$ & $\$ 3,791$ & $\$ 3,876$ & $\$ 3,816$ \\
\hline
\end{tabular}

(a) Work breakdown structure elements used in developing treatment TLCC are:
1 Studies and Bench Scale Tests
2 Demonstration Costs
4 Operating Budget Funded Activities
6 Decontamination \& Decommisioning Costs

(b) The disposal cost has been calculated using a unit disposal cost rate of $\$ 243 / \mathrm{ft}^{3}$.

(c) Transportation costs are excluded. 
Table 4-6. Normalized life cycle cost (without disposal) comparison of the five INTS systems (in millions of dollars).

Cost Component

1.0 Studies and bench scale test costs

2.0 Demonstration costs

3.0 Production facility construction costs

4.0 Operations Budget Funded Activities

5.0 Operating and maintenance costs

6.0 Decontamination \& Decommissioning

7.0 Planning life cycle costs (20 years operation)

Notes:

1. System 1 costs are taken as the normalizing cost basis.

2. Actual Costs are in millions of dollars.

2. Actual Costs are in millions of dollars. 
Table 4-7. TLCC (without disposal) comparison by subsystem of the five INTS systems (in millions of dollars).

\begin{tabular}{|c|c|c|c|c|c|}
\hline SUBSYSTEM & $\begin{array}{l}\text { Grout Debris } \\
\text { System } 1\end{array}$ & $\begin{array}{c}\text { Desorption } \\
\text { System } 2 \\
\end{array}$ & $\begin{array}{c}\text { Wash } \\
\text { System } 3 \\
\end{array}$ & $\begin{array}{c}\text { Acid Digestion } \\
\text { System } 4 \\
\end{array}$ & $\begin{array}{c}\text { CWO } \\
\text { System } 5 \\
\end{array}$ \\
\hline Administration & $\$ 169.9$ & $\$ 169.9$ & $\$ 169.9$ & $\$ 169.9$ & $\$ 169.9$ \\
\hline Receiving \& Preparation & $\$ 940.5$ & $\$ 940.5$ & $\$ 940.5$ & $\$ 940.5$ & $\$ 940.5$ \\
\hline Aqueous Waste Treatment & $\$ 149.7$ & $\$ 149.7$ & $\$ 149.7$ & $\$ 149.7$ & $\$ 149.7$ \\
\hline Organic Destruction & $\$ 245.7$ & $\$ 177.0$ & $\$ 245.7$ & $\$ 181.1$ & $\$ 177.0$ \\
\hline Process Residue \& Sludge & $\$ 134.3$ & $\$ 156.7$ & $\$ 78.1$ & $\$ 126.7$ & $\$ 126.7$ \\
\hline Bulk Soil Treatment & & & & $\$ 54.8$ & $\$ 54.8$ \\
\hline Phosphate Bonded Ceramic Stabilization Subsystem & & & & $\$ 273.2$ & \\
\hline Soft Debris Treatment & & & & $\$ 209.4$ & $\$ 219.3$ \\
\hline Complex Debris Treatment & & & $\$ 136.2$ & & \\
\hline Open Debris Treatment & & & & $\$ 98.5$ & $\$ 98.5$ \\
\hline Metal Decontamination & $\$ 64.2$ & $\$ 64.2$ & $\$ 64.2$ & $\$ 64.2$ & $\$ 64.2$ \\
\hline Special Waste Treatment & $\$ 45.9$ & $\$ 45.9$ & $\$ 45.9$ & $\$ 45.9$ & $\$ 45.9$ \\
\hline Mercury Amalgamation & $\$ 34.7$ & $\$ 34.7$ & $\$ 34.7$ & $\$ 34.7$ & $\$ 34.7$ \\
\hline Lead Recovery & $\$ 41.1$ & $\$ 41.1$ & $\$ 41 . !$ & $\$ 41.1$ & $\$ 41.1$ \\
\hline Air Pollution Control & $\$ 70.4$ & $\$ 70.4$ & $\$ 70.4$ & $\$ 70.4$ & $\$ 70.4$ \\
\hline Polymer Stabilization & $\$ 289.2$ & $\$ 289.2$ & $\$ 297.4$ & $\$ 97.1$ & $\$ 289.2$ \\
\hline Grout Stabilization & $\$ 120.1$ & $\$ 120.1$ & $\$ 120.1$ & $\$ 76.0$ & $\$ 120.1$ \\
\hline Certification and Shipping & $\$ 498.9$ & $\$ 491.0$ & $\$ 513.7$ & $\$ 420.3$ & $\$ 423.9$ \\
\hline Support & $\$ 84.4$ & $\$ 80.3$ & $\$ 84.4$ & $\$ 86.2$ & $\$ 83.8$ \\
\hline TOTAL & $\$ 2,889.0$ & $\$ 2,830.6$ & $\$ 2,991,9$ & $\$ 3,139.7$ & $\$ 3,109.7$ \\
\hline
\end{tabular}


Table 4-8. Cost sensitivity to contingency variations (in millions of dollars).

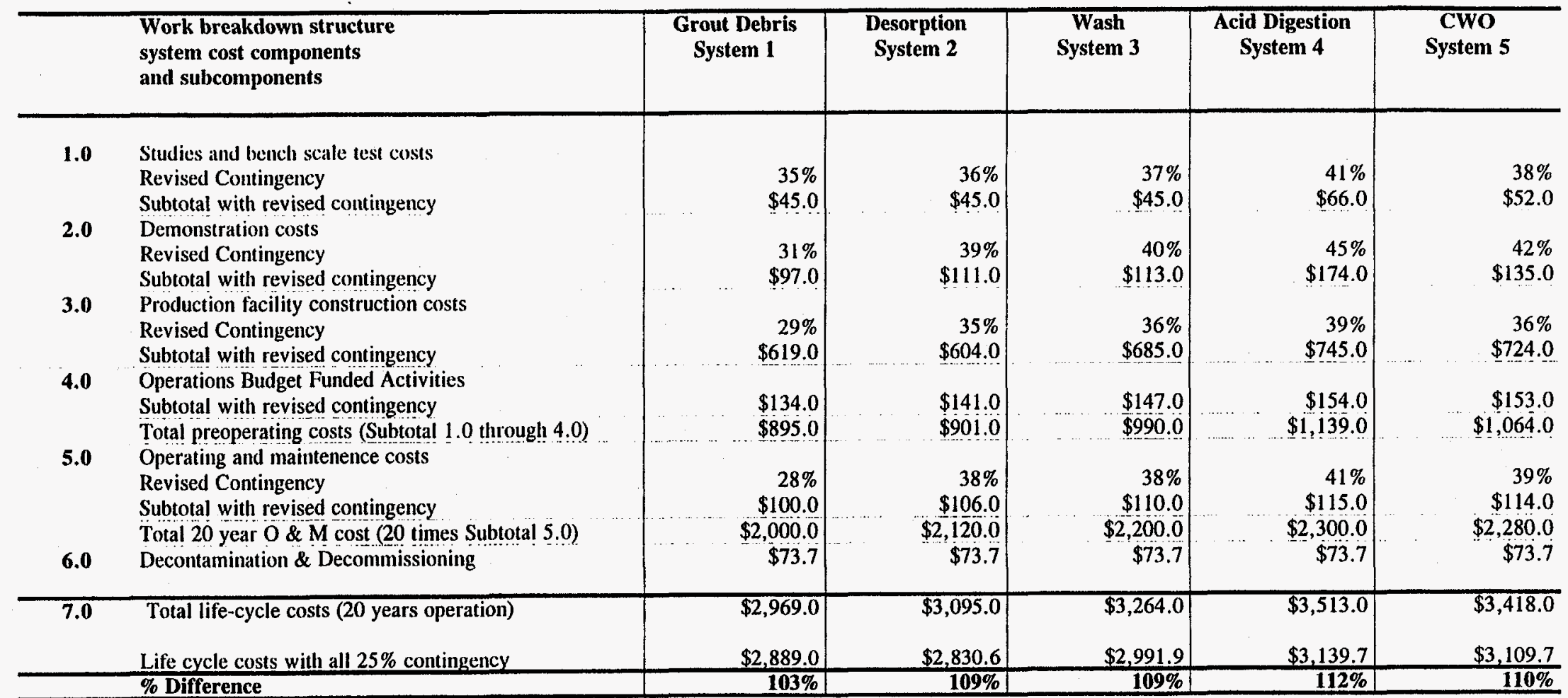

Notes:

1. This table presents the effect of increasing contingencies for certain subsystems within each system.

2. The percentages shown reflect the effect of increasing contingencies to $50 \%$ for subsystems perceived to have higher cost risk as follows:

a. Grout debris case: receiving and preparation, organic destruction, process residue

b. Desorption case: receiving and preparation, organic destruction, process residue.

c. Wash case: receiving and preparation, organic destruction, process residue, soft debris and complex debris.

d. Acid digestion case: receiving and preparation, organic destruction, process residue, soil, soft debris and open debris.

e. CWO case: receiving and preparation, organic destruction, process residue,soil, soft debris and open debris.

3. All other subsystems are assumed to have $25 \%$ contingency. 


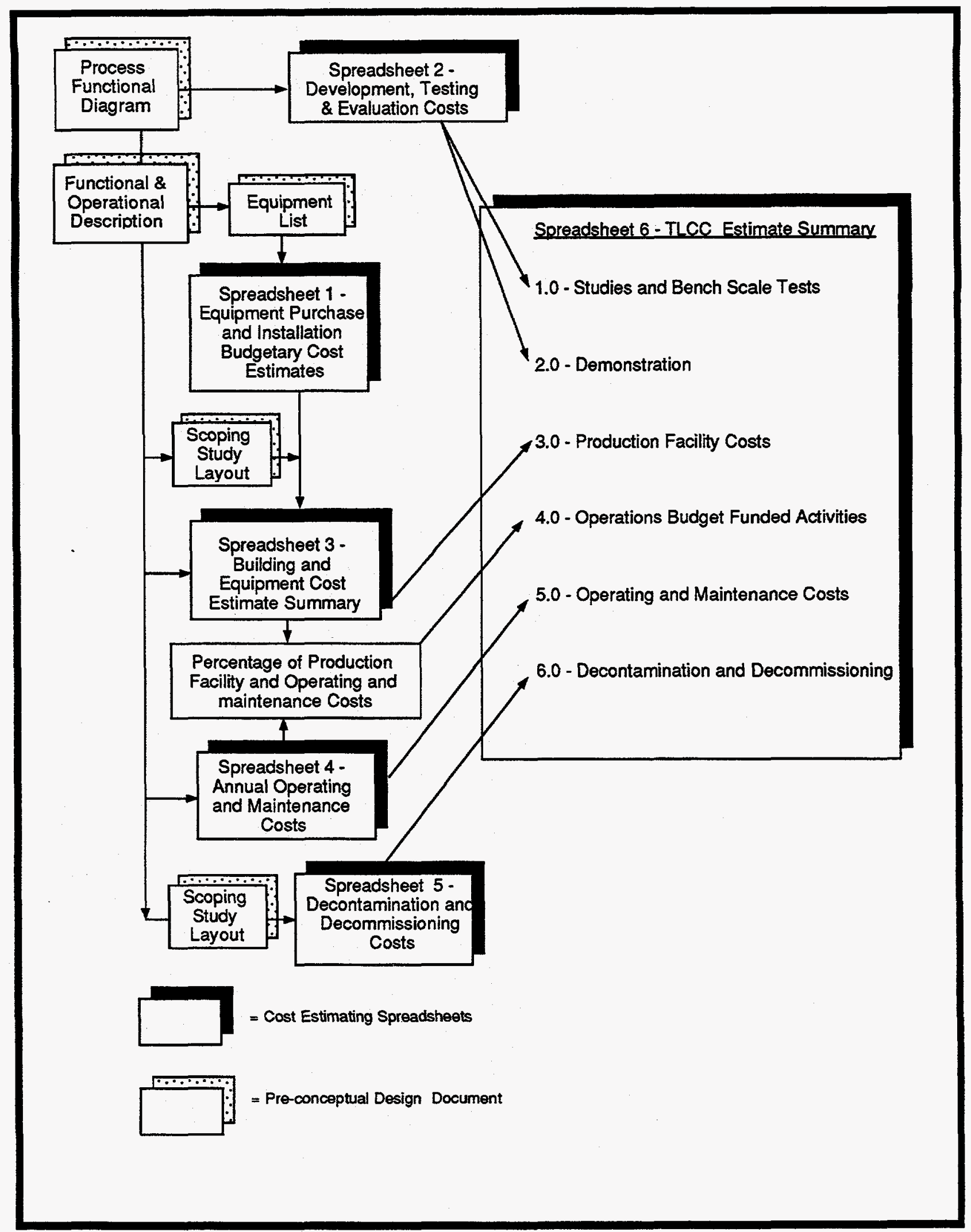

Figure 4-1. TLCC estimating steps. 


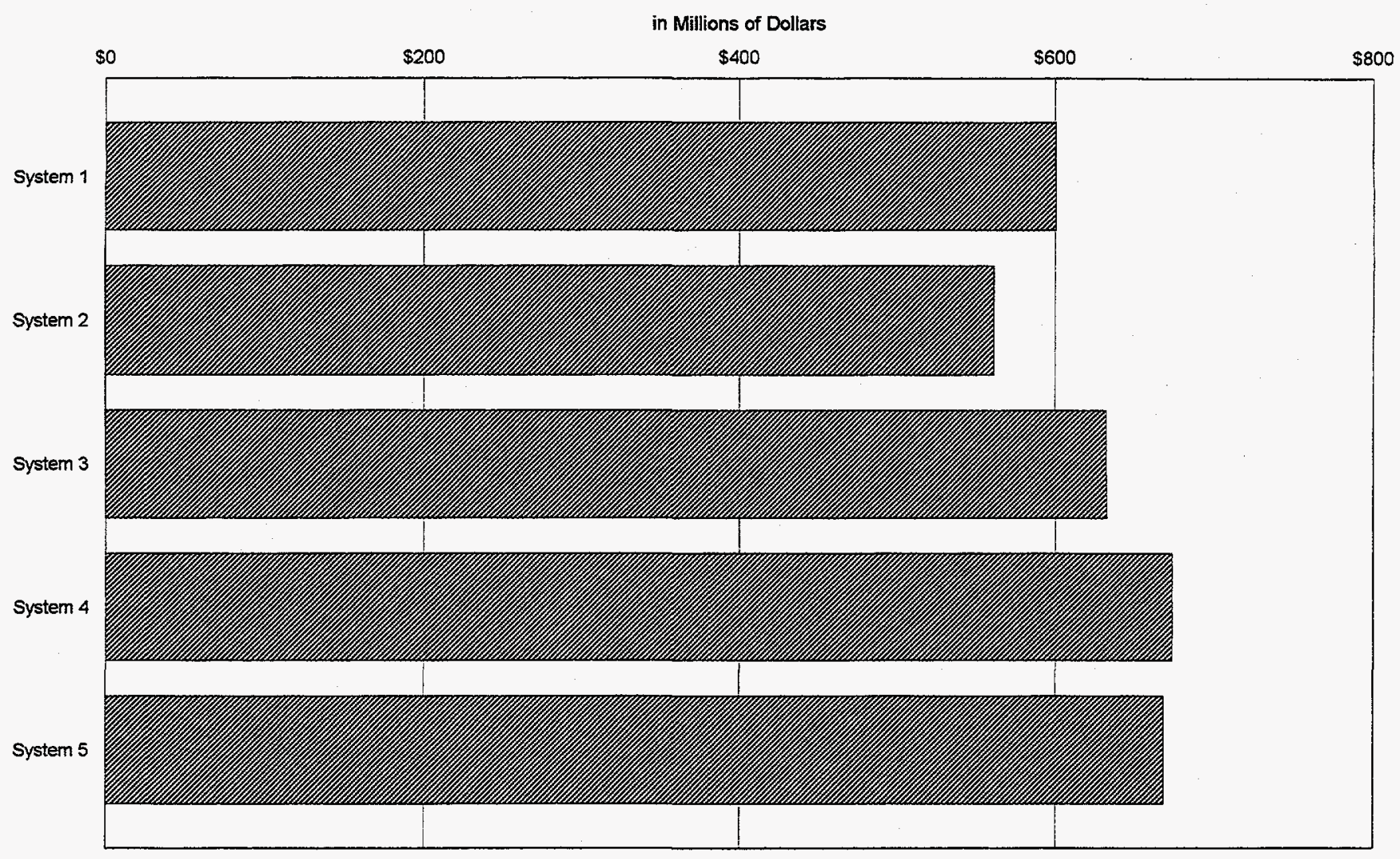

Figure 4-2. Construction costs for each system.

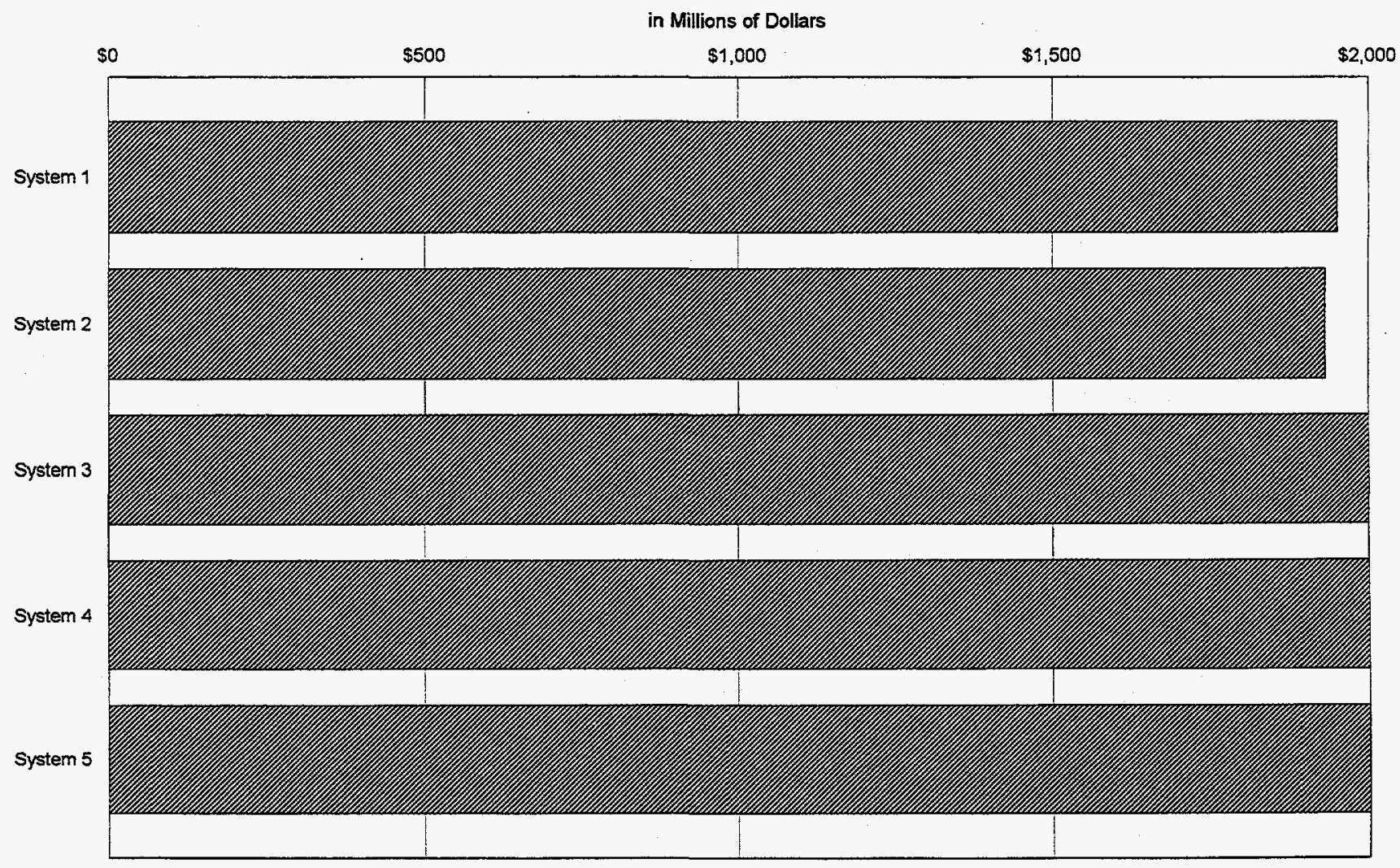

Figure 4-3. Operations and maintenance costs for each system. 


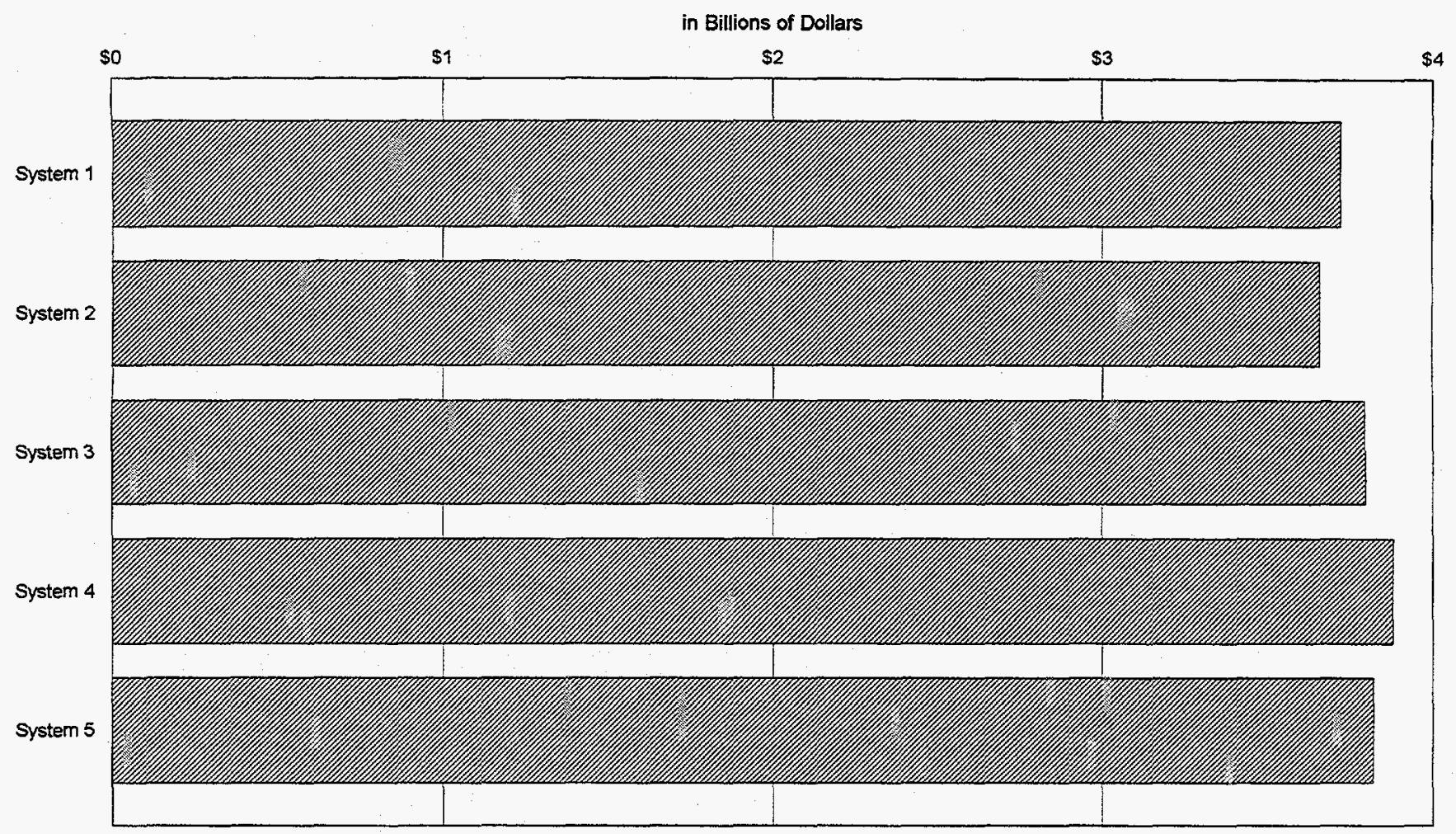

Figure 4-4. TLCC costs including disposal costs.

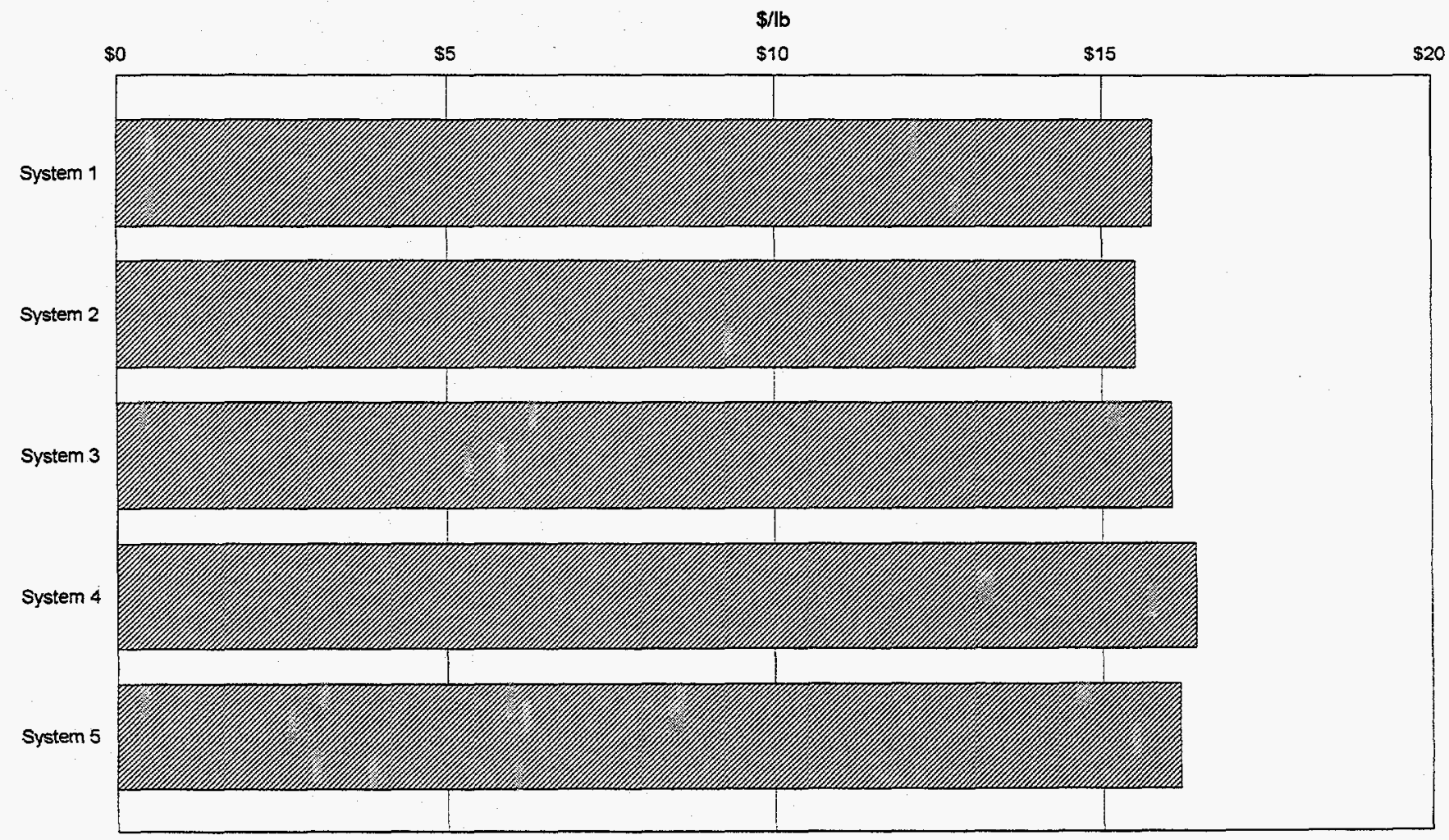

Figure 4-5. Unit costs in dollars/pound (including disposal costs). 


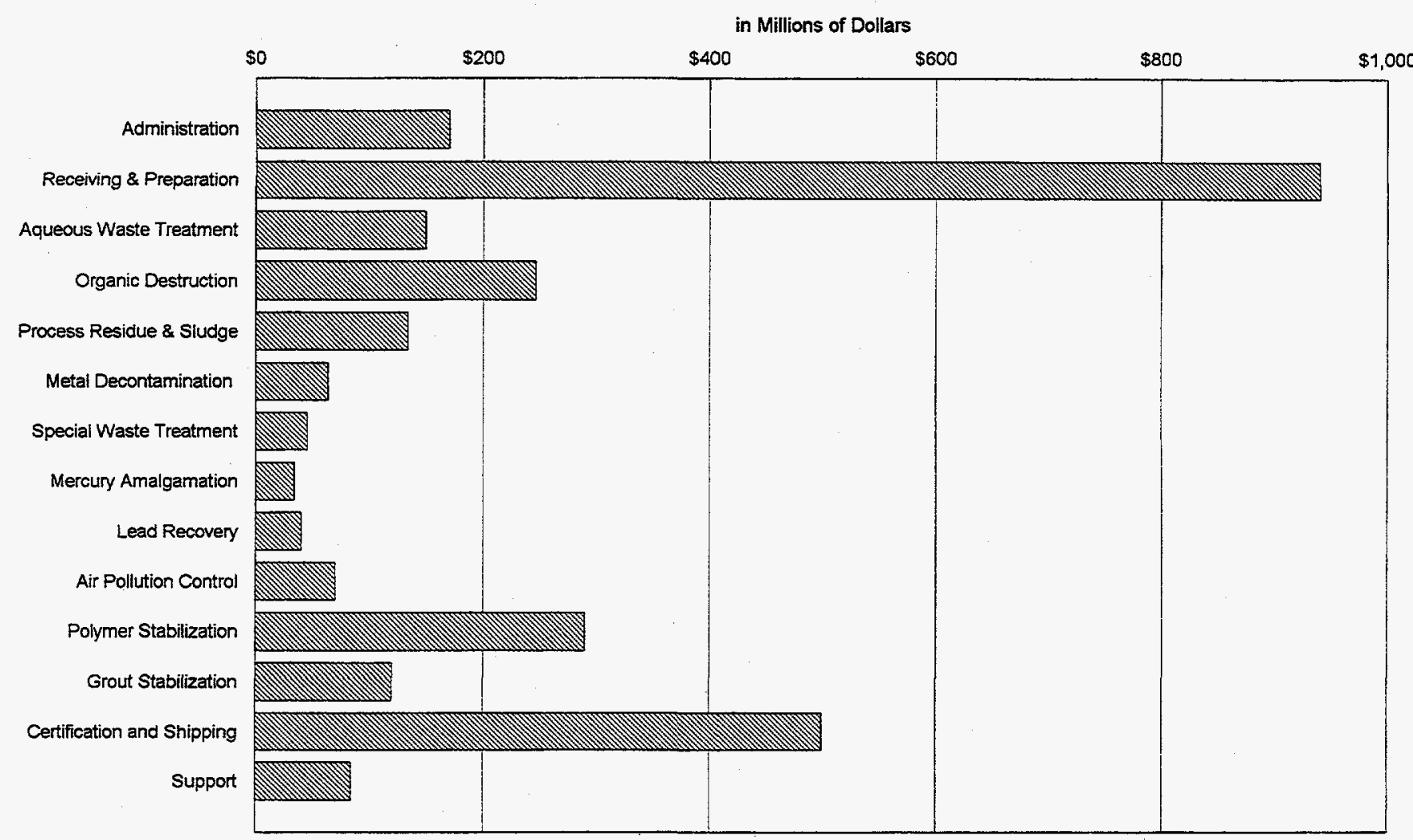

Figure 4-6. TLCC (without disposal) costs for Grout Debris (System 1).

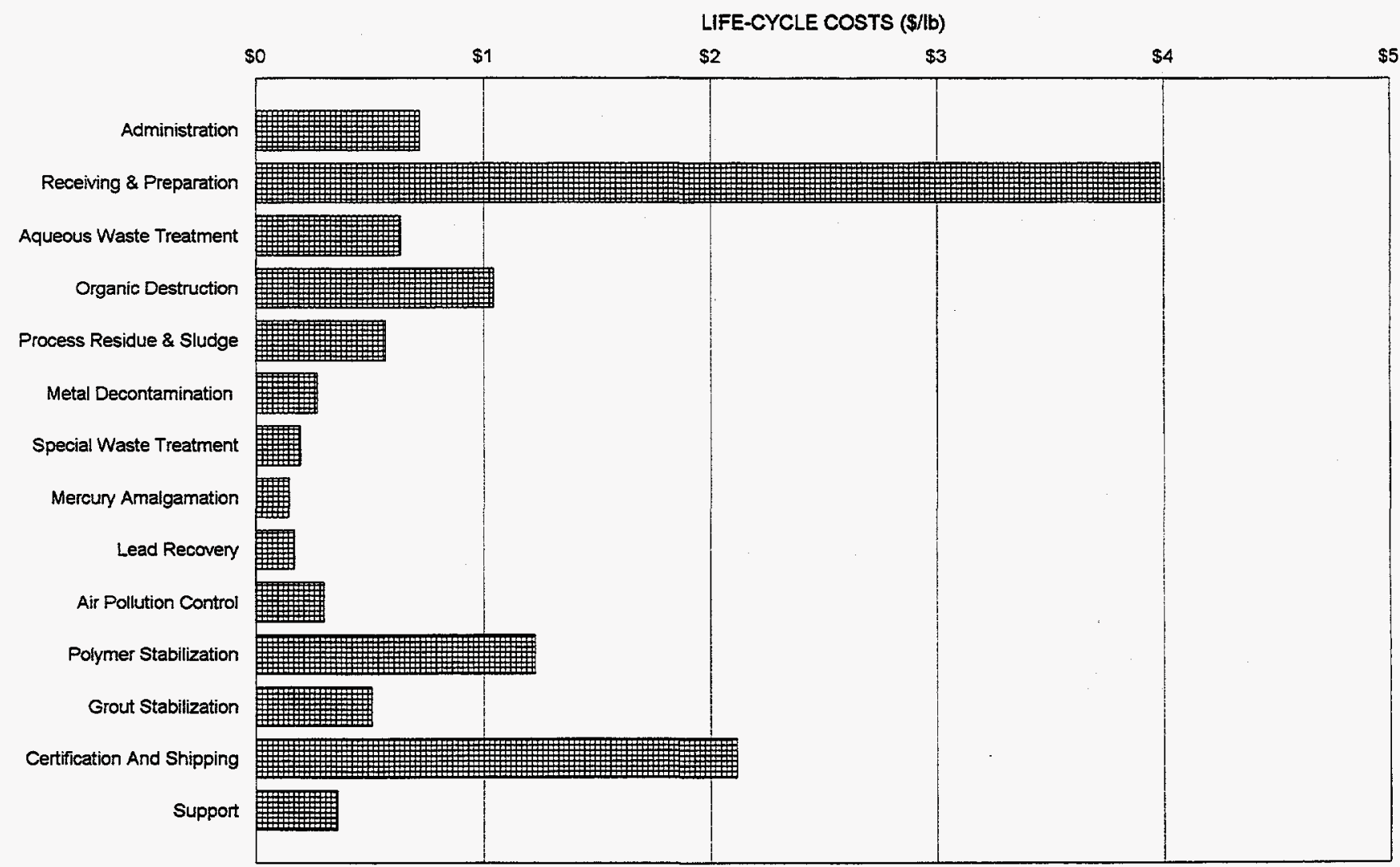

Figure 4-7. Unit costs (without disposal) in dollars/pound for Grout Debris (System 1). 


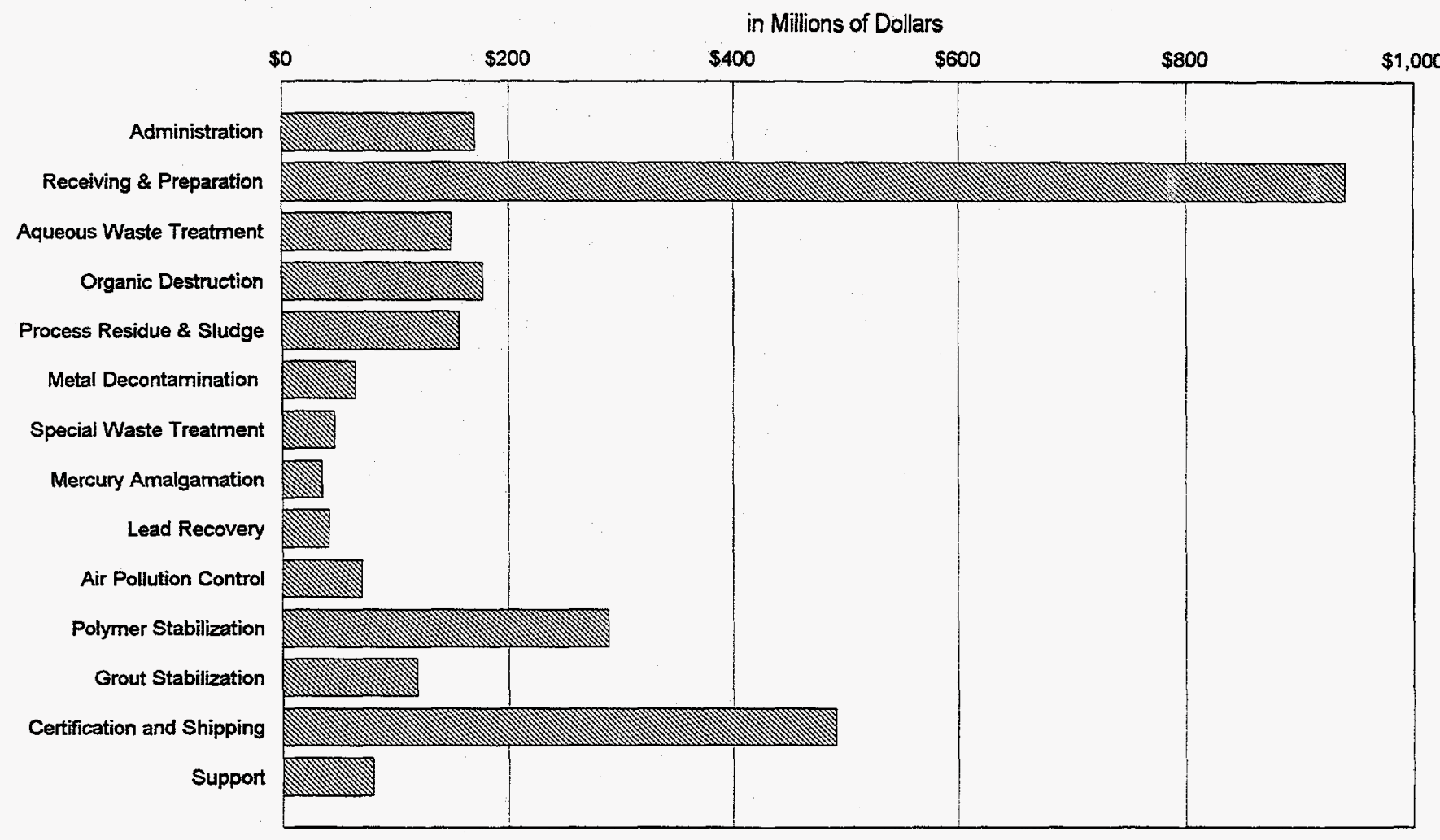

Figure 4-8. TLCC costs (without disposal) for Desorption (System 2).

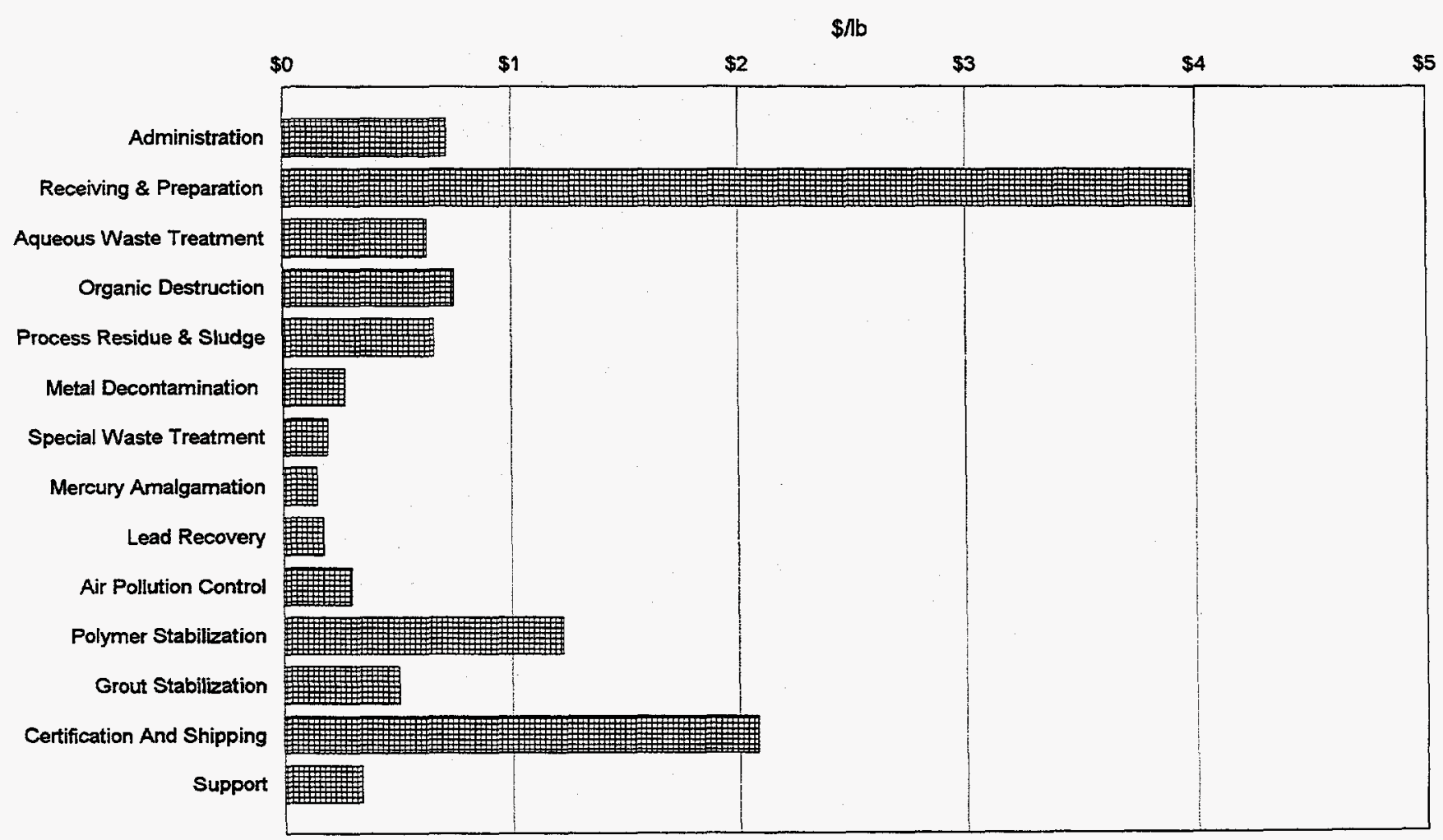

Figure 4-9. Unit costs (without disposal) in dollars/pound for Desorption (System 2). 


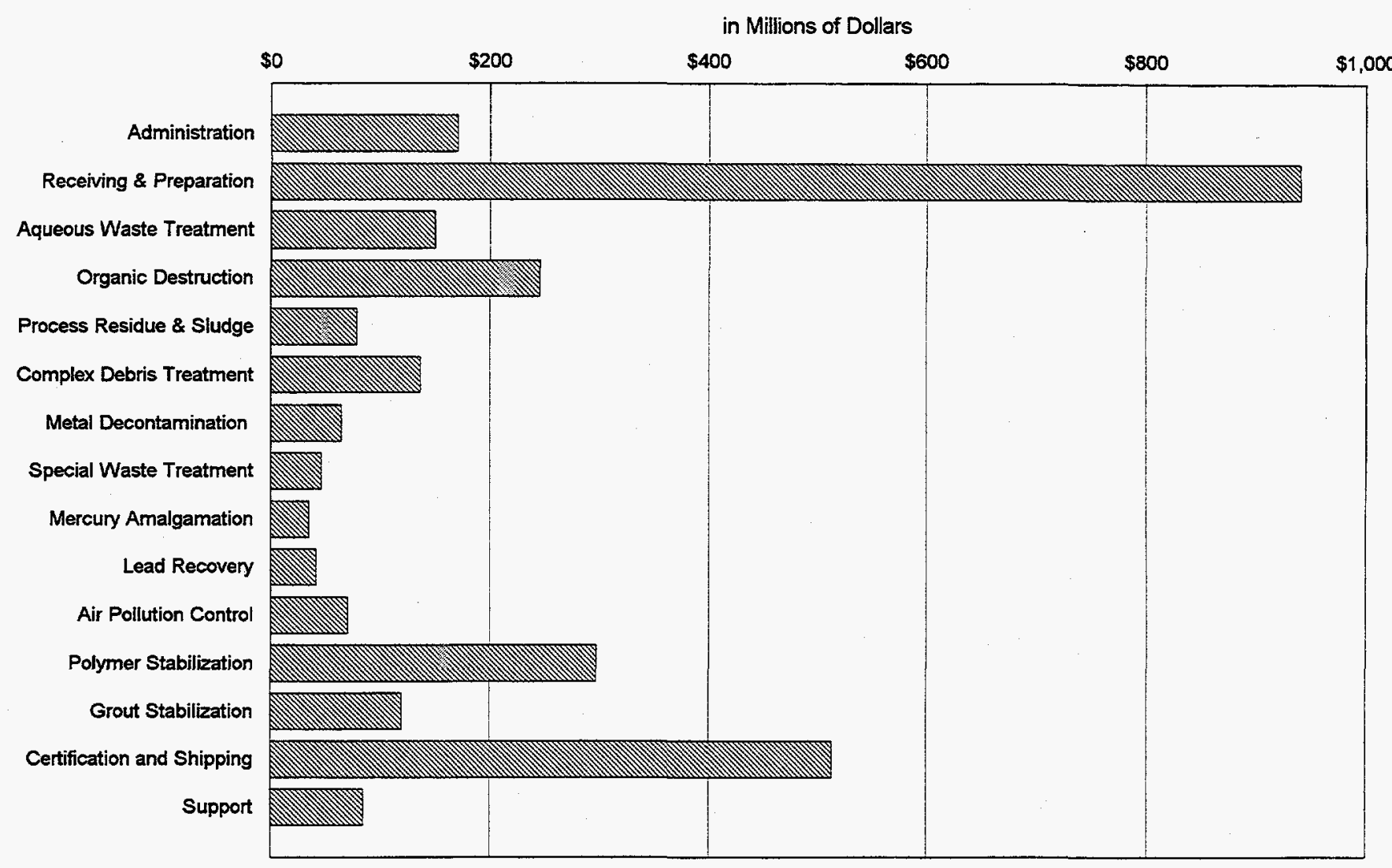

Figure 4-10. TLCC costs (without disposal) for Wash (System 3).

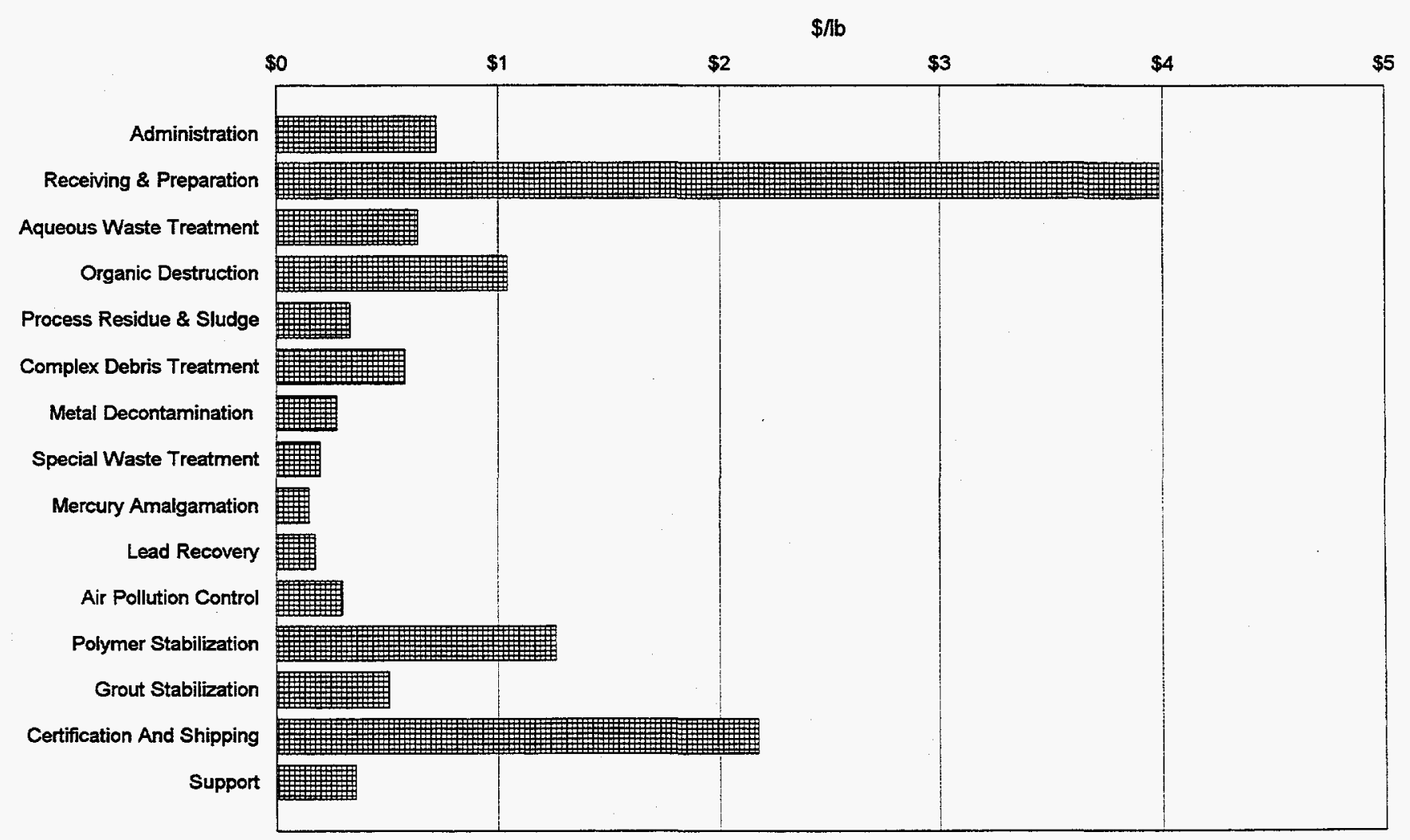

Figure 4-11.Unit costs (without disposal) in dollars/pound for Wash (System 3). 


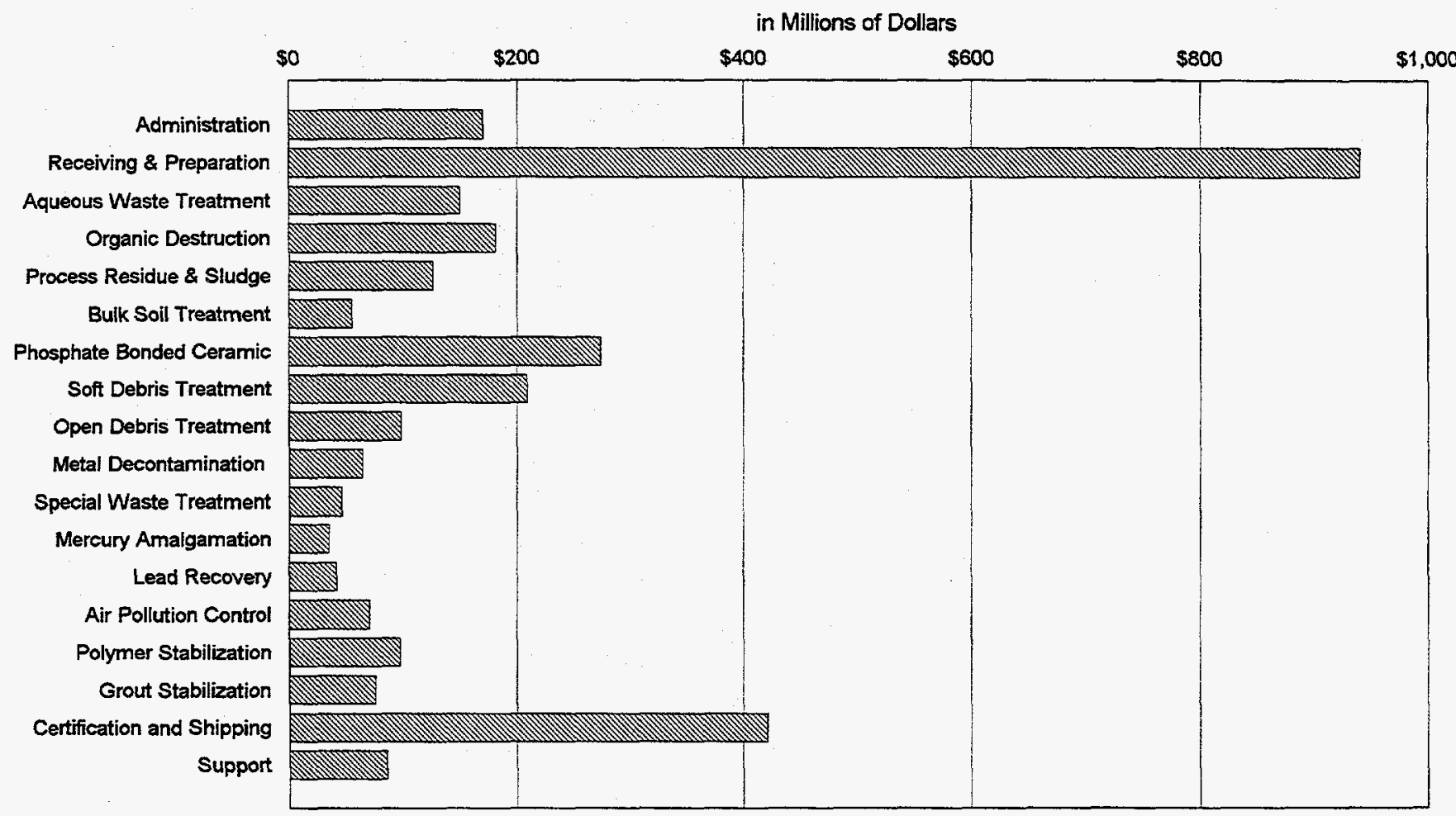

Figure 4-12. TLCC costs (without disposal) for Acid Digestion (System 4).

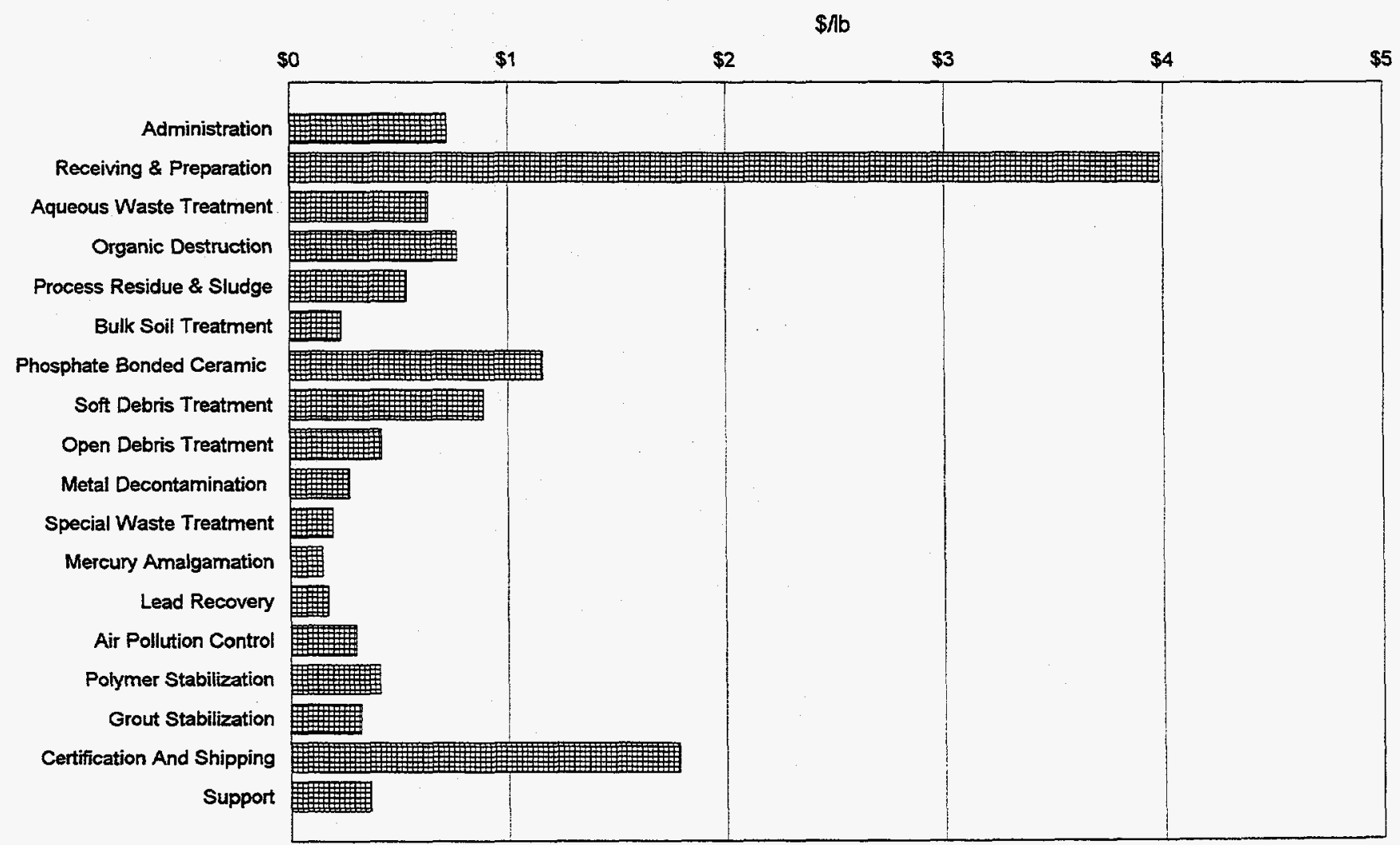

Figure 4-13. Unit costs (without disposal) in dollars/pound for Acid Digestion (System 4). 


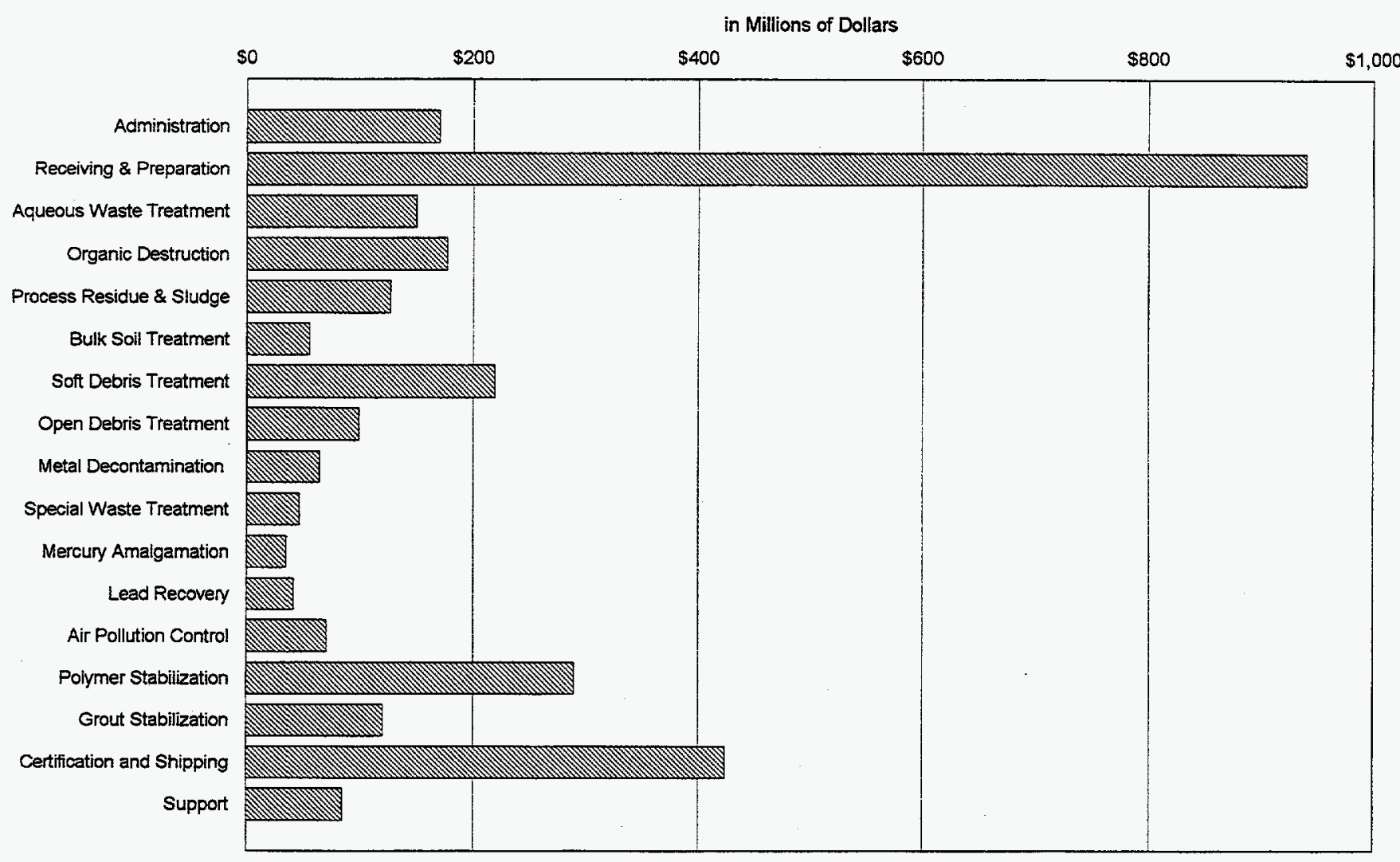

Figure 4-14. TLCC costs (without disposal) for CWO (System 5).

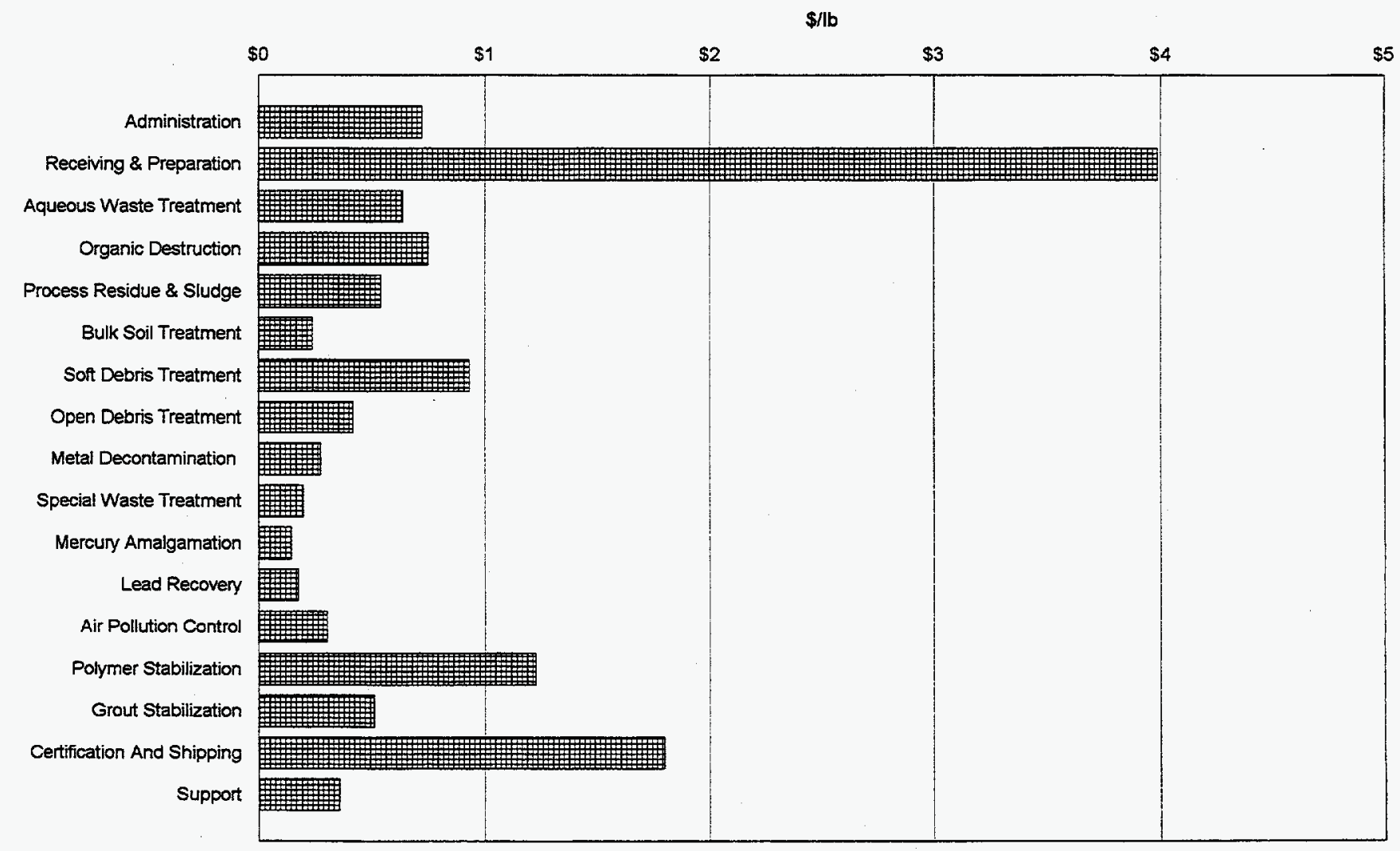

Figure 4-15. Unit costs (without disposal) in dollars/pound for CWO (System 5). 


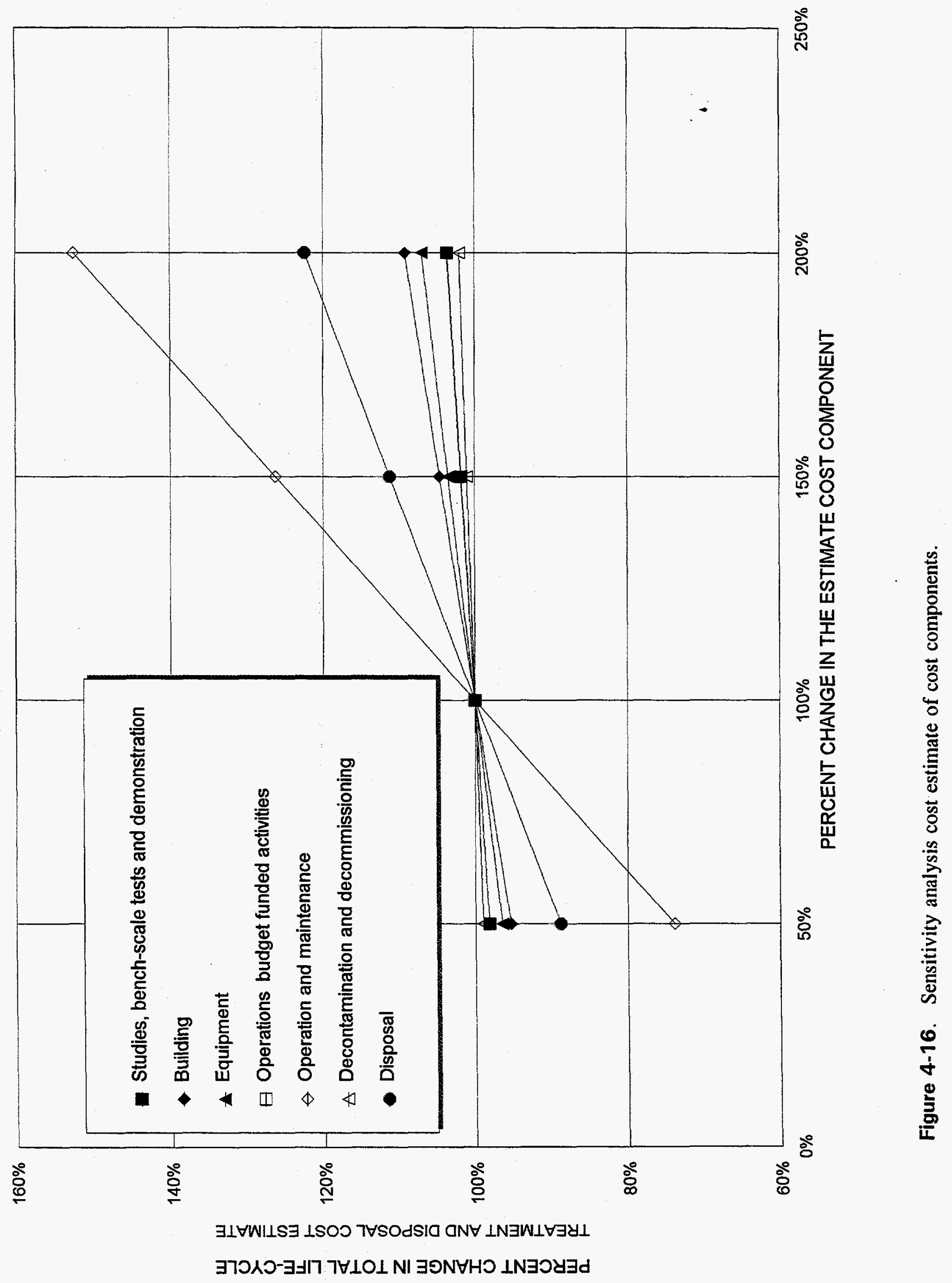




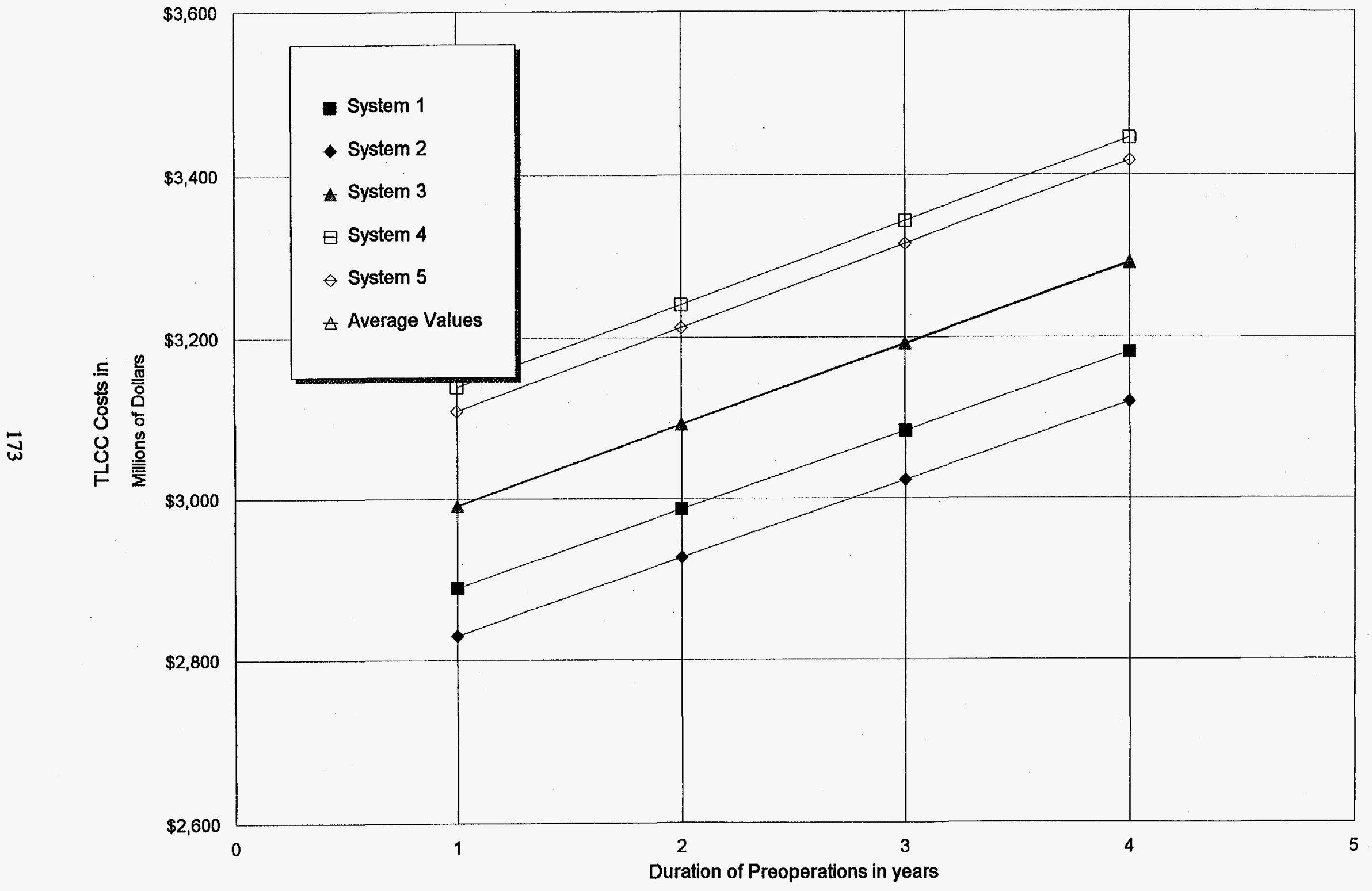

Figure 4-17. TLCC sensitivity to preoperation duration. 


\section{MATCHING TECHNICAL CRITERIA WITH TRIBAL AND STAKEHOLDER PRINCIPLES}

This section explains the technical criteria developed by the TSG for the evaluation of the INTS systems. The TSWG also developed principles for evaluation. These are discussed in Section 1.4. The technical criteria and TSWG principles have many issues in common. This section includes a match-up of the TSG technical criteria and the TSWG principles. Although the match-up is not exact, the principles and criteria are not in conflict, but simply address different concerns. Section 6.7 provides an evaluation of the five systems from the perspective of the TSWG principles.

\subsection{Technical Criteria}

The technical criteria developed to select technologies for inclusion in the INTS study and to evaluate technologies selected is presented in Table 5-1. These criteria include standard concepts for technology evaluation. Quantification of these criteria requires detailed analysis, testing of technologies on specific waste streams, or production experience. Because this data is not available, these criteria are not applied quantitatively. During the system selection process in particular, acquiring quantitative data for the broad range of nonthermal technologies available was not possible. Thus, the criteria were applied qualitatively to select the most representative technologies for effective treatment and removal of contaminants, and stabilization of DOE MLLW. Appendix D discusses the selection evaluation process in detail.

Following system selection, the same technical criteria were employed to evaluate the five systems relative to each other and relative to their performance to meet DOE's requirements as discussed in Appendix A. In a subsequent study, the INTS systems will be evaluated relative to thermal systems. In many cases the five systems perform similarly, and discrimination between the systems is not sharp. The relative technological immaturity of key technologies in the systems contributes to the lack of clear definition between systems. Most of the quantifiable criteria, such as cost, final waste form volume and contaminants, and offgas volume, show only minor variations well within the error bounds of the analysis. Nonthermal systems costs, for example, reflect the extensive sorting and stabilization of the waste required in a nonthermal system. These costs are virtually identical for all systems.

Descriptions of these criteria are provided in the following sections along with some general comments regarding the application of the criteria to the nonthermal systems. Detailed application of these criteria to the nonthermal systems is discussed in Section 6.

\subsubsection{Process Performance Criteria}

Process performance combines two concepts: process effectiveness and the potential to maintain normal operation of the process. The first concern is how effectively the process performs its assigned function, e.g. 
contaminant removal or stabilization. Or more simply, does the process work? The second evaluates how the technology will actually work in a normal operation. This criterion considers whether the process can operate consistently and reliably.

Effectiveness. This criterion defines how well the technology or a system performs its required function. For organic removal or destruction technologies this means how well the technology removes or separates contaminants from the waste matrices. A common indicator for organic contaminant destruction, the DRE is a quantifiable indicator of process effectiveness. Vendor data and engineering judgment regarding technology and overall system effectiveness were used in the initial selection of technologies for the nonthermal systems. Based on available vendor data, all systems were assumed capable of treating the organic contaminants to the EPA UTS. However, this requires validation through testing on actual DOE mixed waste. Vendor data was used to determine reaction rates and the time required to achieve UTS, both of which dictate the number and/or size of the reaction of vessels required to treat the given throughput. The number and size of vessels, which are included in the construction cost estimate, are based on reaction rates and residence time necessary to achieve the required contaminant removal or other process function.

Flexibility. This criterion estimates the range of waste throughput and composition variations that the process can tolerate. Flexibility identifies the need for waste pretreatment, waste characterization, and waste feed controls to maintain process stability. These needs were factors in determining the system costs. This study did not evaluate variations in waste throughput or concentrations, but developed the systems to treat the wastes at the given throughput. In a normal operation, waste throughput and concentration will vary. Flexibility indicates how well the technology or system will perform when concentrations of components or throughput change. For example, it is believed the potential for upset will increase with increasing aggressiveness of the reagents in the chemical destruction process. More robust designs and safety measures will be required to mitigate the consequences of unplanned increases in concentration or throughput.

Versatility. This criterion identifies the fraction of the waste inventory that can be treated by the technology or system. Versatile technologies, such as vacuum desorption, can treat multiple waste streams. During the selection of technologies, this criterion was used to eliminate those technologies that could not treat an entire waste stream, or that required significant pretreatment or posttreatment of the waste. By combining the selected technologies, it was considered that the nonthermal systems could treat all the waste in the waste profile used in this study. This waste profile represents approximately $99 \%$ of the stored mixed waste in the DOE complex.

Availability. This criterion identifies the amount of time the technology or system is available for operation and treatment of waste. A technology is considered not available when equipment fails and/or maintenance must be performed. Evaluation of this criterion for this preconceptual study is highly qualitative. A detailed system design combined with a detailed analysis of the reliability of each component in the system would be necessary to quantify availability. The alternative, long term operational experience, is not available. 
Reliability. This criterion assesses the expected stability of system operations and the potential for system upsets, breakdowns, and off-normal operations. Just as for availability, quantitative evaluation of reliability requires a detailed system design beyond the preconceptual level used in this study and a detailed analysis of the reliability of each component. Long term operational experience is not available.

Maintainability. This criterion assesses the ease or complexity of scheduled or unscheduled maintenance, and the levels of safety, time, frequency, economy, and expertise required. Although some qualitative engineering judgments may be made, detailed analysis of maintainability of these technologies or systems at this stage of design is beyond the scope of this study.

Hazardous waste generation. This criterion identifies, characterizes and quantifies secondary hazardous waste streams generated by the process. Technologies were selected and systems designed to treat any hazardous materials generated within the system. The design basis of the study requires that hazardous waste produced within these systems is either converted to a nonhazardous compound, or captured before being discharged to the environment and stabilized in a leach resistant final form. Volumes of gaseous, liquid and solid effluent have been estimated. However, details of potential contaminant carryover in the effluent streams cannot be determined. To determine the types and concentrations of contaminants that may be carried over in the effluent requires a detailed knowledge and testing of the waste stream constituents under specific operating conditions.

Byproducts and residuals. This criterion identifies, characterizes and quantifies nonhazardous byproducts and residuals generated by the process. Using mass balance analyses, byproducts from the systems have been estimated. These byproducts are estimated in calculations of the various gaseous, aqueous, and solid effluent streams. Byproducts from individual technologies within the systems either end up as effluent from the system, or are recovered and recycled within the system. During technology selection, potential byproducts were evaluated, and methods for recovery and reuse, or subsequent treatment, were identified.

Volume reduction. This criterion quantifies the volumes of waste inputs and outputs, and the relationship of output waste to input waste. Volume reduction has been estimated for the nonthermal systems using the mass balance analysis. Volume reduction is less than one for systems which have less waste going out than coming in.

Final waste form volume and contaminant loading. This criterion identifies the volume of the final waste form sent to disposal and the contaminant loading associated with the waste form volume. Waste form volume has been estimated for the nonthermal systems using the mass balance analysis. Waste form volume could not be identified prior to system selection, design and analysis. Waste loadings in the stabilization media were assumed to be the same as for the ITTS study. 


\subsubsection{Environmental Impact Criteria}

The environmental impact criteria address the controlled and uncontrolled (off-normal) releases that affect the health of the public and non-operational workers. They also address the qualitative aspects of environmental contamination.

Airborne releases. This criterion determines the volume of gaseous effluent and airborne hazardous metals and organics released. Gaseous effluent has been estimated in this study. However, determination of contaminant carryover requires testing of specific waste streams under specific operating conditions. Technologies that were thought to minimize gaseous effluent were selected.

Wastewater releases. This criterion assesses wastewater discharge volume and concentration of hazardous materials in the wastewater discharge. Wastewater discharge volume has been estimated in this study. However, determination of contaminant carryover requires testing of specific waste streams under specific operating conditions. The systems were designed to recycle wastewater where possible.

Final waste form performance. This criterion determines waste form stability and identifies long term repository requirements. To quantify this criterion, tests of specific wastes and waste loadings are required. Long term stability under specified disposal site conditions must be estimated. The design basis of the study requires waste forms that meet TCLP leach tests required by EPA. Developer data appear to verify that the waste forms selected can meet EPA criteria. Actual waste form stability will be a function of many operational factors, including proper waste loadings, mixing, and curing.

Ecological effects. This criterion addresses the extent of soil, groundwater and facility contamination resulting from the treatment and disposal of the final waste form. This requires testing and analysis of the waste form performance, and a detailed performance analysis of the disposal site, both of which are beyond the scope of the current INTS study. However, for the cost analysis engineered disposal facilities were assumed to provide the maximum environmental protection available. Several levels of containment are assumed to prevent release of radionuclides or hazardous material to the environment.

\subsubsection{Worker Health and Safety Criteria}

These criteria address worker exposures due to normal and off-normal operations. They identify upset and accident conditions, the probability of their occurrence, their consequences to workers, and the potential for release of radiological and hazardous materials to the environment.

Hazardous reagents. This criterion characterizes and quantifies hazardous reagents used in the process. All nonthermal systems use hazardous reagents (typically acids) to oxidize organic materials. The amount may vary depending on the aggressiveness of the acid solution, the type and volume of waste, and the 
system's ability to recover and recycle the acids. Selection of technologies was based on the least hazardous materials that would still perform the required functions.

Hazardous operating conditions. This criterion establishes the radiological and hazardous exposure levels in the normal and off-normal modes of operation. The front-end handling area where the waste is received, sorted and size reduced exposes workers the most during normal operation. This area is common to all the nonthermal systems. Determination of off-normal modes of operation, their effect, and the potential for exposure, requires a more detailed system design, more thorough knowledge of the technologies and the rates of the chemical reactions, and more sophisticated analysis of the system operation and control.

Hazardous process equipment. This criterion identifies potential hazards to workers from the normal ranges of pressure, temperature, and electrical and kinetic energy requirements for the process. These characteristics of the technologies were considered in the selection, and are discussed in Section 2. Technologies and equipment that operate under extremely hazardous conditions, such as high pressures and with the use of flammable materials, were eliminated from consideration. The equipment used in the nonthermal systems is no more hazardous than that used in standard chemical processes except for its application to radioactive material. Some system components use more corrosive reagents and operate at higher temperatures and pressures than others and therefore may be considered more hazardous.

Process controls. This criterion identifies process monitoring and control features and their capability to (1) verify that operations remain within specification, (2) detect and respond to off-normal trends, and (3) avert or mitigate upset and accident conditions. The process controls assumed for the nonthermal systems are standard. It is believed the waste is metered into the reactors and that the nonthermal reactions occur at a sufficiently slow rate that the controls can detect and respond to off-normal trends to prevent upset and/or accident conditions. Controls for the high temperature, high pressure, and organic destruction processes may need to be more sophisticated than the controls for the less aggressive processes to prevent a runaway reaction if excess organic material is introduced into the process.

Maintenance worker exposure. This criterion identifies potential worker exposure during routine and non-routine maintenance. Details regarding the maintenance requirements for the nonthermal technologies are unknown due to the early state of development and because the potential for worker exposure is undefined. However, it is believed that the maintenance requirements for all systems will be similar so that this is not a significant discriminator between systems.

Off-normal conditions and their probabilities and consequences. This criterion estimates worker exposure levels resulting from off-normal conditions, determines the probability of exposure, and identifies the radiological and hazardous material that may be released to the environment. As with the other criteria discussed above, technologies that operate under unnecessarily hazardous conditions were not selected in the nonthermal systems. To quantify this criterion requires a detailed system design and a risk or safety analysis of the system to determine probabilities and consequences. Therefore, the evaluation is qualitative. 
Upset and accident conditions and their probabilities and consequences. This criterion addresses (1) potential accident scenarios and conditions, (2) the probability that these upset conditions and accidents will occur, (3) the resulting exposures to workers, and (4) the radiological and hazardous material that may be released to the environment. It is believed that the nonthermal reactions are sufficiently slow, and that the waste is metered into the reactors at a sufficiently slow rate that upset conditions will be rare. Reaction rate data for the selected technologies are required in order to determine upset conditions. A detailed system design and safety analysis is required to determine how upset conditions might occur, and to quantify probabilities and consequences.

Process containment. This criterion addresses the need for engineered safety features to avoid adverse exposures and releases during normal operations, and to mitigate the consequences of an upset or accident condition. Very high pressure technologies were not included in the nonthermal systems. Double or triple containment for the nonthermal systems studied is standard for systems treating radioactive waste and is not a significant discriminator between systems. Some system components use more corrosive reagents and operate at higher temperatures and pressures than others, and therefore cost more to contain.

\subsubsection{Cost}

These criteria define the cost of needed research and development of emerging or existing technologies to be implemented for the treatment of DOE mixed waste. Total life cycle costs for complete treatment systems are also estimated.

Research and development (R\&D). This criterion addresses the cost and time required to complete the demonstration, testing, evaluation and implementation of proposed technologies. This cost element was not evaluated prior to technology selection or system design. Analysis indicates that research and development costs are small relative to implementation costs.

Total life cycle. This criterion determines the total life cycle cost. This includes $R \& D$, pre-operational activities, production facilities and equipment, $O \& M$ activities, $D \& D$, and disposal. These costs were quantified as part of the INTS study at a preconceptual design level with uncertainties in the range of $+75 \%$ to $-30 \%$. The maximum difference in cost for the five systems evaluated is $9 \%$ which is not considered significant.

\subsubsection{Acceptability}

These criteria provide information on decisions to implement the proposed systems.

Complexity and simplicity. This criterion assesses the mechanical, chemical, and operational complexity of the processes and the systems, and evaluates the number of processes required to meet the treatment 
standards. This criterion was used to evaluate the initial set of technologies. The simplest systems that would meet the treatment requirements were selected.

Technology development status. This criterion identifies the current level of development of the proposed technologies, i.e., laboratory or bench scale, pilot scale, production or full scale, commercial, etc. The criterion was also used to evaluate the initial set of technologies to determine if sufficient data were available to perform a systems analysis, and to determine if the technologies were past the conceptual stage.

Site expanse. This criterion describes the fenced area required to accommodate operating facilities, including the main facility, peripheral facilities (holding tanks, settling ponds, staging areas, etc.), buffer areas, and other features needed for safe and effective operations. This criterion was not used for initial system selection because the system sizes could not be determined prior to analysis. Subsequent analysis and sizing of the selected systems indicate that they are all approximately the same size. Thus, this criterion will not discriminate between systems.

Resource utilization. This criterion evaluates the requirements for energy (electrical or thermal in the form of hydrocarbons), chemical reagents, and personnel for effective and safe operation. This criterion was not used for initial system selection because resource utilization could not be determined prior to analysis. Subsequently, the labor intensive O\&M costs and the chemical reagents required were determined for each of the five systems analyzed. Energy costs are small compared to labor and cannot be used to differentiate between systems. Energy costs were calculated as part of the O\&M costs.

Schedule. This criterion estimates the time required for implementation and includes required R\&D, demonstrations, engineering design, permitting, construction and installation, and start-up. This criterion is determined by level and status of technology development. Most of the technologies used in these systems are at the bench scale or pilot scale level of development. The systems studied are estimated to require 7 to 10 years of development, demonstration, construction and permitting before they can be implemented for treatment of DOE mixed waste.

Decontamination and decommissioning. This criterion assesses D\&D requirements including hazards, expected effectiveness, residual contamination levels, and the potential for turning over the facility for alternative uses. All five nonthermal systems will require approximately the same amount of D\&D. At this stage of design this criterion cannot discriminate between systems. Specific requirements and results of D\&D efforts have not been determined.

Environmental restoration. This criterion assesses the extent of environmental restoration required because of facility operation, including the need for soil, groundwater and vegetation restoration. It also estimates the residual contamination after site restoration. Nonthermal systems will require approximately the same amount of site restoration. At this stage of design this criterion cannot discriminate between systems. Specific requirements for restoration have not yet been determined. 


\subsection{Method Used to Match Criteria With Principles}

The tribal and stakeholders principles (referred to hereafter as the principles) discussed in Section 1, and the TSG technical criteria (referred to hereafter as the criteria) discussed above, provide two sets of guidelines for evaluation. In fact, the overlap of these two sets of guidelines is quite extensive. Because the TSG criteria and the TSWG principles are not in conflict, the resulting effect is that the principles add more criteria for evaluation. In Section 6 the systems are first evaluated against the TSG criteria and then against the TSWG principles.

This section provides a match-up of the criteria and the principles. This allows the reader to recognize when the evaluation by criteria is essentially the same as the evaluation by the principles. The TSWG has written the principles clearly, including a high level of detail within the sub-principles. Therefore, this section does not include a detailed description of each principle.

To match the technical criteria with the principles deemed most important by the tribes and stakeholders, relationships between the principles and criteria were defined at the subcriterion and subprinciple level. For example, the TSWG subprinciple "Minimize effluent that has the potential to carry hazardous materials or substances capable of causing adverse environmental consequences (i.e. minimize releases with the goal of zero releases of pollutants) can be cross-referenced to eight TSG subcriteria. These include the following:

- Two process performance subcriteria: byproducts and residuals, and final waste form volume and contaminant loadings.

- Four environmental impact subcriteria: airborne releases, wastewater releases, final waste form performance, and ecological effects.

- Two worker health and safety subcriteria: off-normal conditions and their probabilities and consequences, and upset and accident conditions and their probabilities and consequences.

The "Minimize effluent..." subprinciple can be assessed by examining the qualitative evaluations of the eight cross-referenced subcriteria.

In most cases two or more subcriteria that can be matched with a subprinciple. In several cases a particular subcriterion can be matched with more than one subprinciple. In some cases the principles cannot be related to any criteria because the concerns expressed by the principles are not conducive to quantitative analysis and must be addressed separately from the technical issues.

Cross-references of subprinciples and subcriteria are presented in Table 5-2 for each of the five principles. This allows the quantitative and qualitative evaluation of the subcriteria resulting from the INTS study to be 
compared with the subprinciples important to the tribes and stakeholders, and allows the tribes and stakeholders to assess the thermal and nonthermal system options in terms of their principles.

\subsection{Areas Where Criteria and Principles Were Not Matched}

Several siting, social, cultural, and communication principles were not matched with technical criteria. This is because these principles relate to nontechnical issues or are outside the scope of the current study, although several of the issues identified in the subprinciples may be addressed in subsequent studies.

The premise for this study, as with the ITTS study, was that a single facility will treat all the existing DOE mixed waste in 20 years (excluding the Hanford tank wastes and the Rocky Flats pondcrete on the assumption that facilities will be constructed at these sites specifically to treat these wastes). This facility is used as an example to evaluate treatment costs and to estimate effluents. Issues regarding single versus multiple facility options, and transportation of waste to such facilities, were not considered in this study. Rather, they are addressed in the draft DOE Programmatic Environmental Impact Statement (PEIS). To eliminate duplication of effort, such site issues were considered beyond the scope of this study. However, transportation risk associated with thermal and nonthermal systems will be considered in a subsequent task, as will portable treatment systems.

Several cultural and social values could not be compared with specific technical criteria, although many of these issues ( e.g. principles 1,2, and 3) were cast in technical terms that could be evaluated. It is incumbent on the tribes and stakeholders to use the results of these studies to make decisions regarding options which most reflect their values and mitigate their concerns.

Many of the communication principles are being addressed through inclusion of the TSWG in the study. Communication issues regarding information desired by the tribes and stakeholder representatives is included in this report, or will be addressed in subsequent studies.

Cost was not identified in the TSWG principles and therefore was not part of the comparison. 
Table 5-1. Technical Criteria.

\section{Process Performance}

Effectiveness
Flexibility
Versatility
Availability
Reliability

Maintainability

Hazardous Waste Generation

Byproducts and Residuals

Volume Reduction

Final Waste Form Volume and Containment Loading

\section{Environmental Impact}

Airborne Releases

Final Waste Form Performance

Wastewater Releases

Ecological Effects

\section{Worker Health and Safety}

Hazardous Reagents
Hazardous Operating Conditions

Hazardous Process Equipment

Process Controls
Maintenance Exposure

Off-Normal Conditions and their Probabilities and

Consequences

Upset and Accident Conditions and their Probabilities and Consequences

Process Containment

\section{Cost}

Research and Development

Total Life Cycle

\section{Acceptability}

Complexity/Simplicity

Technology Development Status

Site Expanse

Resource Utilization

\section{Schedule}

Decontamination and Decommissioning

Environmental Restoration 
Table 5-2. Technical criteria compared to TSG principles

Tribal and Stakeholder Principles

Principle

Subprinciple

1. Minimize Effluents
- Minimize effluents that can carry hazardous materials that cause adverse environmental consequences (i.e. minimize releases with goal of zero releases of pollutants)

\section{Technical Criteria}

\section{Byproducts and Residuals}

Final Waste Form Volume and Contaminant Loadings

Airborne Releases

Wastewater Releases

Final Waste Form Performance

Ecological Effects

Off-Normal Conditions and their

Probabilities and Consequences

Upset/Accident Conditions and their

Probabilities and Consequences 
Table 5-2. Continued

Tribal and Stakeholder Principles

Principle

Subprinciple

Technical Criteria

2. Minimize Effects on Human Health and the Environment (includes workers)
- Minimize exposure now and in the future

Final Waste Form Volume and Contaminant Loadings

Airborne Releases

Wastewater Releases

Final Waste Form Performance

Ecological Effects

Hazardous Reagents

Hazardous Operating Conditions

Hazardous Exposures

Process Containment

Process Controls

Off-Normal Conditions and their

Probabilities and Consequences

Upset/Accident Conditions and their

Probabilities and Consequences

Complexity/Simplicity

Decontamination and Decommissioning

Environmental Restoration

- Minimize potential for release of hazardous materials from final storage/disposal products

Final Waste Form Volume and Contaminant Loadings

Final Waste Form Performance

- Minimize potential for accidents in the system
Flexibility

Maintainability

Process Controls

Upset/Accident Conditions and their

Probabilities and Consequences

Complexity/Simplicity

Technology Development Status 
Table 5-2. Continued

Tribal and Stakeholder Principles

Principle

3. Minimize Waste

Generation
Subprinciple

Technical Criteria

- Minimize creation of new hazardous materials by treatment

Byproducts and Residuals

Volume Reduction

Ecological Effects

Hazardous Reagents

- Minimize final waste volume/amount for storage disposal
Maintainability

Hazardous Waste Generation

Byproducts and Residuals

Volume Reduction

Final Waste Form Volume and Contaminant Loadings

Ecological Effects

Hazardous Reagents

Decontamination and Decommissioning

Environmental Restoration 
Table 5-2. Continued

Tribal and Stakeholder Principles

$\begin{array}{lll}\text { Principle Subprinciple } & \text { Technical Criteria }\end{array}$

4. Address Social Concerns

- Minimize land use for storage and disposal

Hazardous Waste Generation

Byproducts and Residuals

Volume Reduction

Final Waste Form Volume and Contaminant

Loadings

- Waste should be disposed of in a

Final Waste Form Volume and Contaminant retrievable manner

Loadings

Final Waste Form Performance

- Stable waste forms for long term

Final Waste Form Volume and Contaminant storage and disposal

Loadings

Final Waste Form Performance

- Reflect special site considerations

Site Expanse

Resource Utilization

- Minimize intersite transportation

This is a siting matter regarding the number and location of treatment facilities and the wastes at each site. This is studied and discussed in the DOE PEIS. A subsequent study will evaluate portable treatment systems.

- Reflect cultural values Independent assessments of cultural impacts should be made by the tribes and stakeholders using the information developed during this study 
Tribal and Stakeholder Principles

Principle

Subprinciple

Technical Criteria

5. Adequacy of Information

- Provide better information on health

All Worker Health and Safety Criteria and safety

- Provide better information on

All Process Performance Criteria performance

All Environmental Impact Criteria

- Focus on existing stored waste. Add

Flexibility

projected impact of future waste

generation

Versatility

Impact of future waste will be the subject of

a subsequent study

- Communicate implementation schedule Technology Development Status

Schedule

- Criteria for evaluating systems should

Information will be provided for be flexibly applied

independent evaluations.

- Integrate ITTS and INTS for system

This is the subject of a subsequent study. comparisons

- Bridge ITTS and INTS to use the best This is the subject of a subsequent study. elements of each

- Identify planned documentation Not related to technical criteria ${ }^{\mathbf{a}}$.

- End use of study should be known Not related to technical criterial.

- Identify decision-making process and Not related to technical criteria ${ }^{a}$. participants

- Inform TSWG of responses and $\quad$ Not related to technical criteria ${ }^{a}$ suggestions

- Keep TSWG involved throughout study Not related to technical criteria.

a This information has been provided to the TSWG as part of the ongoing study. The INTS report and selected documents will also be available on the DOE website. 


\section{SYSTEMS EVALUATION}

Evaluations are conducted at both the system and subsystem level. Evaluation at the system level includes reviewing the overall system concept against the TSG criteria and TSWG principles discussed in Section 5.

The goal of evaluating the systems is to qualitatively assess technology uncertainties, including system maturity and developmental work needed to prepare the system for detailed design. Key issues related to system performance are identified and discussed. Subsystems are evaluated against technology uncertainties in the same manner as for the systems. The systems and their subsystems are compared with each other.

In the evaluations presented in this section, systems for processing either alpha or nonalpha waste are considered. The discussions apply to both waste categories except when a specific category is mentioned.

\subsection{Evaluation Criteria}

In broad terms, evaluation against technology uncertainty assesses the performance of systems or subsystems against the TSG criteria. The TSG criteria listed below are described in detail in Section 5.

The criteria are:

$\begin{array}{ll}\text { - } & \text { Process Performance } \\ \text { - } & \text { Environmental Impact } \\ \text { - } & \text { Worker Health and Safety } \\ \text { - } & \text { Acceptability }\end{array}$

Tables 6-1 through 6-5 summarize the results of applying the technical subcriteria discussed in Section 5 to the TSG criteria listed above for each of the five nonthermal systems.

\subsection{Evaluation of System 1, Grout Debris}

The grout debris system is designed to use the most developed of the nonthermal treatment technologies evaluated. The system has twelve major subsystems.

The receiving and preparation subsystem uses SGS and PAN assay units, an RTR inspection unit, gantry robots, hydraulic and master-slave manipulators, sorting tables, and remotely operated vehicles. Extensive sorting is performed in order to send specific waste to the proper treatment subsystem. 
Organic destruction subsystem uses silver catalyzed MEO.

Process residue, inorganic sludge, and the bulk soil subsystems are treated by vacuum desorption followed by stabilization.

Debris, except mercury contaminated debris, is grouted without treatment for organic removal. The use of grouting avoids more expensive polymer stabilization and takes advantage of the EPA debris rule. This EPA rule allows minimal processing and stabilization of a certain category of MLLW debris (trash-like material). Debris waste must be kept separate from process wastes. Mercury contaminated debris may not be disposed of without treatment. Therefore, this waste is treated on a campaign basis utilizing the process residue vacuum desorption subsystem.

The offgas generated by processes in System 1 is a much smaller volume than would be generated by thermal subsystems. In general, thermal systems release 10 to 20 times more treated offgas than nonthermal systems. Particulate removal from the offgas is performed with a sintered metal filter and HEPA filters. Entrained organics are destroyed by a GPCR. Acid gases are neutralized in a packed tower scrubber utilizing lime or caustic.

The various metal treatment subsystems utilize nonthermal technologies. The lead recovery subsystem uses mechanical cutting tools and decontamination booths. The mercury amalgamation subsystem uses an amalgamation reactor. The metal decontamination subsystem uses an abrasive blasting booth.

The aqueous waste treatment subsystem uses commercially available precipitators, solids filters, ion-exchange vessels, carbon filters, distillation columns, and evaporators. Photooxidation with $\mathrm{H}_{2} \mathrm{O}_{2}$ and $\mathrm{O}_{3}$ is used to oxidize dissolved organic contaminants.

The grout stabilization subsystem uses commercially available equipment to mix shredded waste with grout.

The polymer stabilization subsystem uses commercially available dryers and extruders to dry the incoming waste prior to mixing it with polymer.

The certification and shipping subsystem uses RTR and PAN assay units to inspect the waste prior to shipping.

\subsubsection{System Technology Uncertainties}

The evaluation of system and subsystem technology uncertainties is most detailed for System 1 . Evaluation of other systems will reference the System 1 discussion for components or aspects which are common to multiple systems. 


\subsubsection{Process Performance.}

Effectiveness. Process performance issues are summarized in Table 6-1. The grout debris system treats organic waste, aqueous waste, soil and residue to reduce organic and metal content of the waste by processes designed to meet regulatory discharge requirements. Several of these processes are not technically mature, and this study does not attempt to specifically evaluate the ability of the processes discussed to meet regulatory requirements. Data from vendors and developers were used to indicate the effectiveness of the process. Prior to final system design, technology demonstration will be necessary to ensure the processes selected will treat waste to meet regulatory standards. The expected effectiveness of these subsystems is discussed in this section. The treatment of non-mercury contaminated debris, which is grouted without pretreatment, does not remove the organics or metals prior to stabilization. This may not be sufficient to meet future regulatory requirements. Management of mercury contaminated debris, the grouting of other debris, waste characterization, and the effect of inerts in organic destruction processes are discussed further below.

Soundness of the concept for mercury management. The concept for managing mercury in the system is untested. Three key parameters, (1) the level of mercury contamination in the feedstock, (2) the removal efficiency of activated carbon filters for volatile mercury, and (3) the effectiveness of the mercury debris leaching process are not known at this time.

Current DOE waste inventory data indicate that a large part of the inventory has mercury contamination, but little is known about the concentrations or chemical form. This introduces a high level of uncertainty regarding the quantity and nature of mercury contamination. For this study, it is assumed that contaminated organic sludges may include cutting and lubrication oils contaminated with mercuric acetates. Inorganic sludges may include those generated from acid leaching, thermal treatment, and mercury sulfide precipitates. Solids may include mercury-specific ion-exchange resin (e.g. Ionac SR-5), rags, wipes, and personal protective equipment.

The system strategy for managing mercury is to segregate all elemental bulk mercury or bulk mercury compounds. These are sent to the mercury amalgamation subsystem. Treatment of other mercury containing waste is provided depending on the waste type. Mercury contaminated liquids will be sent to the aqueous waste treatment subsystem which is equipped with mercury precipitation and ion exchange units. Process residue and soil containing mercury will be treated by a leaching process. Offgas from all processes is treated for mercury using sulfur impregnated activated carbon filters.

In June 1990 EPA promulgated treatment standards for radioactive mixed wastes containing elemental mercury and mercury-containing hydraulic oil contaminated with radioactive materials. These wastes cannot be treated under the debris rule treatment standards, but, rather, must be treated to meet waste-specific mercury standards (TCLP of 0.025 milligrams 
per liter [mg/l]). If debris wastes are frequently contaminated with elemental mercury, the approach to treating debris waste by stabilization will not be acceptable. Therefore, debris contaminated with mercury will be treated similar to process residues.

Debris treatment by grouting alone. The effectiveness of debris stabilization in preventing organics, RCRA metals and radionuclides from migrating out of grout-stabilized debris must be established for a wide range of DOE waste. The fate of other contaminants must be evaluated.

Waste characterization. The feedstock characterization unit proposed for the receiving and preparation subsystem needs engineering development. The receiving and preparation subsystem includes primary and secondary shredding capability. Depending on the requirements of the treatment subsystem, most waste will be shredded. An approach has been developed to characterize the waste in conjunction with the shredding. Two specific sets of components support the characterization. The first set consists of a remotely operated on-line grab sampler that is mounted at the shredder discharge port. The sample obtained by the grab sampler is remotely sealed and sent to an analytical laboratory by a pneumatic tube conveyor. The second set of components includes a number of transportable hoppers for transport or storage of the waste after it has been characterized. Each hopper has a fill port on top and a dump port in the bottom. The hoppers collect waste from the shredder and a crane transports the filled hopper to a storage area or to the treatment subsystem. The on-line sampler provides several grab samples before waste is discharged from the shredder to each hopper.

The characteristics of waste in each hopper are determined by analytical testing of the samples. Once the waste characteristics are known, the operator will be able to organize the waste for shipment to treatment subsystems. Treatment subsystem operators can process waste immediately, or store the waste so that it can be worked off in campaigns of similar waste, or blended. The waste hoppers would connect directly to the feeding system of the treatment subsystem. This characterization and processing concept, which has not yet been tested, will require further engineering development and demonstration before integration in the overall system design.

Effect of inerts in organic destruction treatments. For all organic destruction processes, the issue of effective inert residual handling must be addressed. Organic processes have been developed to treat organic waste. Non-organic compounds present in DOE wastes may affect the performance, reliability and availability of the organic destruction subsystems.

Flexibility and versatility. The system concept of the grout debris system is flexible and allows adjustment for variations in feed. The sorting requirements for this system are less than those required by the 
other systems because debris will not be separated into open, soft or complex categories. Only metals will be removed. In accordance with the debris rule, debris is treated separately from other wastes and not allowed to mix with residues or soil. Because debris is not treated, this system is less versatile if debris does not meet treatment standards. The MEO technology used for organic destruction to treat soft debris has not been developed, making it less versatile than acid digestion and CWO.

Availability, reliability and maintainability. The evaluation of these criteria must be based on production experience. However, in general, industrial experience does not exist. Therefore, these evaluations are highly subjective, based on the authors' experience with similar processing operations. The grout debris system is expected to have excellent availability, reliability, and maintainability. Organic destruction by MEO utilizes banks of cells operated in parallel. If maintenance is required, specific banks of cells can be taken off-line without shutting down the entire process. The nitrous oxide turbo aerator converter is a pilot scale process not proven outside the laboratory. Reliability of this equipment, which must operate whenever waste is processed through the MEO cells, affects the availability of the MEO subsystem. The acid and silver recovery processes generally use commercial equipment and simple unit operations. However, the silver recovery process is only at bench scale development. Process control will be a key factor in attaining acceptable availability and reliability. Temporary shutdown of the acid and silver recovery operations will not require shutdown of the MEO waste destruction process. The thermal vacuum desorption unit is larger than required, increasing the availability of the system. Although thermal desorption is a common commercial technology, the vacuum desorption process has not yet achieved commercial reliability. Availability therefore cannot be effectively estimated.

Overall, System 1 should have the greatest availability. The effect of radioactive contamination on worker safety could reduce system availability due to exposure limitations.

Hazardous waste generation. MEO treatment generates a hazardous waste in the form of silver chloride. Developers claim a recovery of $99.9 \%$ of the silver, but have achieved this efficiency only on a laboratory scale. For this report, $99 \%$ recovery is estimated. Any unrecovered silver chloride is sent to polymer stabilization. As with CWO and acid digestion, MEO generates corrosive waste which is neutralized, and salts which are stabilized.

Byproducts and residuals. System 1 generates the least amount of byproducts and residuals. MEO has multiple streams for acid and silver recovery, but about the same amount of residuals (inorganics and metals) as the other organic destruction processes. Since debris is not treated, organic and sludge residuals are not generated from a treatment process. Vacuum desorption does not produce residuals other than the organics removed during treatment. Mercury removal through the leaching process generates steel wool contaminated with mercury. This steel wool is stabilized in the mercury amalgamation subsystem. All systems generate water and sludge contaminated with various components. These byproduct streams are sent to aqueous waste treatment. 
Volume reduction. Table 6-1, shows volume reduction for all systems. Organic destruction, vacuum desorption and aqueous treatment reduce the volume prior to stabilization, but volume increases occur in grout and polymer stabilization. Overall, the mass increases in all INTS systems, but, due to density increases, an overall volume reduction occurs. The greatest reduction occurs with Systems $4 \& 5$ where soft debris is oxidized.

Final waste form volume and contaminant loading. System 1 has a comparatively large disposal volume due to the grouting of debris without treatment. System 1 debris has the highest contaminant loadings because organics are not removed from the debris.

\subsubsection{Environmental Impact.}

Airborne releases. Table 6-2 presents the comparison of systems with respect to the environmental impacts criteria. Table 6-1 lists the mass rate of gaseous emissions to the atmosphere. System 1 has the lowest gas emissions of any INTS system. Sources are desorber offgas, MEO reaction product gas, and aqueous waste treatment process vents. With the exception of mercury and some mercury compounds, volatilized metals, actinides and other radionuclides are not released into the offgas streams from nonthermal treatment. It is anticipated that air emissions can be easily controlled. Radionuclide concentrations in offgas from nonthermal processes should be nondetectable. Formation of dioxins in the offgas is not anticipated because the higher temperature conditions required for their formation are not present in the nonthermal treatment systems. Oxides of nitrogen are formed from the use of nitric acid in MEO. These are removed by scrubbers and catalyzed reactor chambers within the MEO subsystem. The air pollution control subsystem is designed to remove organics, acids, volatile mercury and water from offgas streams in the facility. The system is highly redundant for removal of organics and acid gases. Other than the GPCR, the other technologies in the APC train are commercially available, proven, and widely used. Volatile mercury is not expected to pass the condensers in the APC subsystem. However, the APC subsystem includes sulfur impregnated carbon absorption for mercury removal as a backup measure. Release of carbon dioxide will be minimal, being generated only from the destruction of organics and operation of a standard industrial boiler.

Wastewater releases. Treated water will be recycled, with the excess, if any, discharged from the facility. Table 6-1 shows the amount of water discharged. Wastewaters generated in various treatment subsystems will be processed in the aqueous waste treatment subsystem and most of the water will be recycled. However, water will be generated from drying and from reaction of incoming wastes with oxidizers. Water contaminants include dissolved and suspended metals, organics, and particulate. Commercially proven processes are used to reduce contaminants to UTS and other regulatory requirements prior to discharge. Aqueous solutions may include radionuclides, but removal by precipitation and ion exchange is generally effective. Continuous effluent testing is part of the design for this facility. 
Final waste form performance. The final waste form is designed to comply with LDR. Land disposal restrictions are designed to limit leaching of hazardous contaminants from the disposal facility to groundwater and other environmental media. Salt generated as process residue will be stabilized by polymer to meet LDR for specific contaminants. Contaminants of concern include mercury, radionuclides, heavy metals, PCBs and organics. Treatment technologies will not remove all of the contaminants. Debris will be grouted in compliance with the debris rule, but treatment is not provided. Further demonstration and testing must be conducted to determine the effectiveness of grout to limit the mobility of contaminants and in the case of nondebris waste, to meet TCLP requirements. Following stabilization, waste will be performance tested as required by regulation.

Ecological effects. Hazardous wastes which are released from a facility may create an exposure risk to wildlife, plants and ecosystems. Treated stabilized wastes are disposed of by placement in engineered structures. These structures are secured facilities which prevent contact with animals and minimize contact with plants. Releases of radioactivity from the stabilized waste are minimized by use of the disposal cell design described in Section 2.1.17. The potential for exposure to the stabilized waste is low. Releases of treated offgas or wastewater are strictly controlled by continuous emission monitoring. Unplanned releases of solids, liquids or gases to the environment are minimized by the confinement zone concept of the facility design as discussed in Section 2.1.16.

Other potential ecological effects, such as global warming and ozone depletion, are not anticipated to be significant. Carbon dioxide emissions from all systems are low because the combustion of fuel for heating in nonthermal systems is low. The systems do not use significant amounts of ozone depleting chemicals such as chlorofluorocarbons (CFCs). Site specific concerns, such as the effect on endangered species, cannot be evaluated in this study, since a facility location has not been defined.

System 1 has the lowest carbon dioxide emissions of the five systems and it generates less aqueous waste than the other systems. However, the stabilized waste has the most contaminants and there is considerable uncertainty about its leaching performance.

Permitting. The facility will require a full Part B permit as a RCRA treatment, storage and disposal facility. As discussed in Section 1.10.3.3, these systems would be permitted under 40 CFR 264.600 (Subpart $\mathrm{X}$ ). A risk assessment, groundwater modeling and emissions studies are required. Overall permitting will be based on demonstrating that the required performance standards for treatment specific wastes and debris are met by the treatment technologies and specific methods used in the facility. Nonthermal treatment does not require extensive trial burn demonstrations as would an incinerator. However, the demonstration of technology effectiveness will be required for each treatment technology for each waste stream. Pilot work permitted under RD\&D permits will be used to demonstrate the effectiveness of innovative processing methods such as MEO. The total time to permit a nonthermal system is expected to be more lengthy than to permit an incinerator. For innovative thermal treatment, process technology demonstration would also be required under either Subpart O (40CFR 264.340), which is designed specifically for incineration, or Subpart 
$\mathrm{X}$, cannot be effectively compared for miscellaneous units. Permitting schedules for innovative technologies cannot be effectively compared as agencies have limited experience with Subpart X permitting.

The system is designed to meet the TSCA performance standards established for PCBs. TSCA requires that the effectiveness of $\mathrm{PCB}$ destruction, using alternative treatment to incineration, be demonstrated to the EPA Regional Administrator. Debris contaminated with PCBs would be segregated. Liquids would be removed from containers and sent to the Organic Destruction subsystem. Nonliquid PCB contaminated debris above the regulatory threshold will be sent to the special waste subsystem for treatment. Further design efforts are required to develop specific concepts for such wastes.

Grouting produces a less stabilized waste form and a larger volume than would occur using a process such as incineration followed by vitrification. The waste form developed from the grout stabilization should be evaluated for acceptability under regulatory standards (EPA and DOE). The final waste form must be shown to meet $L D R$ requirements.

\subsubsection{Worker Health and Safety.}

Hazardous reagents. Table 6-3 presents the technical criteria comparison for worker health and safety. System 1 uses concentrated nitric acid, silver nitrate and iodine, all hazardous reagents. Other milder acids and bases are used in MEO for acid and silver recycling, including caustic, hydrochloric acid and hydrogen peroxide. The reagants used in MEO are the least aggressive of the chemicals used for the destruction of organics. Reagents are maintained at low temperatures and transferred in closed equipment. Operator contact with reagents during operations would be limited to sample collection.

Worker exposure. The facility design concept is expected to ensure that the worker health and safety objectives will be met. The INTS systems are expected to comply with a majority of the design requirements described in Appendix A. A major area of concern is the design to ensure alpha confinement. This design is discussed in Section 2.1.16. When the system is used to process alpha MLLW, further studies will be needed to refine facility layout concepts and confinement levels in the process, maintenance and operating areas. Two or three levels of confinement are needed when processing alpha MLLW. The concept used in the preconceptual system layouts is based on placing equipment in air-tight cells that have three levels of confinement. Personnel access to the cells is through airlock doors. Personnel entering alpha cells must wear Level A protective equipment, including self-contained breathing equipment. Large corridors are provided next to each cell for equipment pull-out and maintenance. In this corridor, major equipment is disassembled, decontaminated (if required), and sent to the maintenance shop for repair. This confinement approach requires further evaluation.

For nonalpha MLLW, the worker health and safety objectives can be met by reducing confinement to one or two levels, depending on the potential for release of radioactivity to the environment. 
For System 1, a low temperature and pressure environment, maintenance exposures should be minimal. Exposures from MEO are less than in systems where organic destruction occurs in a single reactor. Individual MEO cells can be easily removed for repair with minimal disturbance to operations. The equipment necessary to recover silver and nitric acid can be taken off-line for repairs without halting organic destruction operations. This flexibility allows repairs to be performed while minimizing exposure of workers.

The largest exposure risk to workers from radioactivity is in the sorting operations where waste is dumped out of containers. This risk is slightly less in System 1 where sorting of debris involves only metal removal. The INTS assumption is that $75 \%$ of incoming waste will require sorting.

Process Equipment and Controls. Process equipment hazards are primarily mechanical and chemical in nature, and require only standard industrial practice controls (improved for radioactive work), such as "lock out and tag out" procedures, guarding of gears and drives, vehicle operator training, and procedures for safe operating, startup, shutdown and maintenance activities. Operations which occur at temperatures above ambient require worker caution. These include $\operatorname{MEO}\left(120^{\circ} \mathrm{F}\right)$, evaporation, acid fractionation, and vacuum desorption $\left(500^{\circ} \mathrm{F}\right)$.

Process controls for System 1 are the simplest of the INTS systems. MEO processing requires controls to maintain anolyte temperature, feed rate, nitrous acid conversion rate, and catholyte concentration. Pilot work indicates process controls are not difficult. The MEO process can be easily monitored by noting the distinctive color changes of silver nitrate solution (clear) and $\mathrm{Ag}(\mathrm{II})$ solution (dark). When there is too much organic material, the $\mathrm{Ag}(\mathrm{II})$ solution becomes clear, indicating a reduction in the $\mathrm{Ag}$ (II) concentration. Process control of other aspects of the subsystem is not integral to the performance of MEO.

Process control of the GPCR reactor, which uses electricity to supply reaction energy, is maintained with electrical controls. Process controls for other subsystems, including vacuum desorption (a batch process), are similar to typical industrial operations.

Off-normal, upset or accident conditions. Off-normal, upset, or accident conditions are properly assessed in failure mode effects analysis (FMEA), or in hazard and operability (HAZOP) studies. Off-normal conditions could occur if waste is inappropriately sorted. For System 1, this type of failure would have negligible consequences. Sludge residues would be returned to process residue treatment for reprocessing. Debris would be identified and returned to receiving and inspection for later reprocessing. However, if debris waste is inappropriately mixed with process waste, it could not be treated under the EPA debris rule criteria. If alpha waste were improperly monitored or identified, worker exposure could increase. However, each subsystem performs some characterization prior to beginning treatment. This serves as a safeguard against processing incorrectly identified wastes.

System 1 subsystems are expected to respond with self correction to off-normal conditions. For example, in the MEO organic destruction subsystem, if the anolyte reactor begins to overheat, the reaction rate of 
organic destruction will increase. As waste reacts, water is formed, cooling the reactor. In the case of a process upset, shutdown of the MEO process can be virtually instantaneous. Reactions stop when the electrical power is turned off. The process residue and soil treatment subsystem, which treats by vacuum desorption, would be designed to shut down in an overheat condition. If the vacuum malfunctions, the treatment would be less effective, but would not cause an accident.

The major consequences of off-normal or upset conditions are ineffective processing and increased cost to reprocess waste. Since most equipment in System 1 is electrically operated (as opposed to heated), power failures would lead to inefficiency and possible mechanical problems, but not hazards. The GPCR reactor, for example, would contain waste in the event of power loss. Use of cranes to transfer wastes in bins also minimizes exposure due to equipment failure by eliminating other transfer equipment, such as conveyors, as a source. Other occurrences generated outside the plant (hurricane, earthquake, plane crash) are accommodated for by the building design and the facility containment requirements, described in Section 1 and Appendix A.

Process containment. Containment features are standard design for facilities handling radioactive material. Process control of MEO, aqueous waste treatment, APC, and vacuum desorption is expected to be straightforward. Process control of othersubsystems, e.g., metal decontamination, should be simple. Shutdown of equipment following offnormal or upset conditions is not likely to lead to waste releases such as major spills, fires or other conditions where process containment is critical. Process containment is enhanced by the facility containment design which limits the risk of contact outside a cell.

\subsubsection{Cost.}

Research and development costs. R\&D costs for bench scale operations are the least for System 1 and increase as the systems become more complex and/or employ less developed technology. These costs are shown in Table 6-4. System 1 uses fewer treatment processes, so less research is necessary. System 1 has lower demonstration costs projected than the other systems because MEO has had extensive pilot testing at Lawrence Livermore National Laboratory (LLNL), Pacific Northwest Laboratory (PNL) and two commercial firms. Silver recovery has not been pilot tested and will require demonstration.

Lifecycle costs. Lifecycle costs are listed in Table 6-4. The variation in total costs among the five systems is less than $15 \%$. The cost of the grout debris system, which has the least amount of processing, is not the lowest of the systems, because MEO is an expensive technology. As with all INTS systems, many components are not technically mature. In general, the cost risk is high because of lack of proven commercial experience. However, the cost risk of the grout debris system is judged to be lower than other INTS systems due to the use of slightly more reliable technology. The risk of cost overruns increases significantly when the system is applied to alpha MLLW rather than for nonalpha MLLW. The reason for this is because most system components require further development to allow decontamination and maintenance in an alpha cell environment. Maintenance costs for an alpha environment will also increase significantly. 
At this preconceptual design level, both $R \& D$ and total life cycle costs are quantified at an estimated $-30 \%$ to $+75 \%$.

\subsubsection{Acceptability.}

Complexity/simplicity. System 1 is the simplest of the five INTS systems. MEO is more complicated than the other organic destruction subsystems, but System 1 does not include debris treatment. The remaining subsystems involve the same processes used in most of the other systems and are either the same or simpler than the subsystems used in the other systems. Acceptability issues are listed in Table 6-5.

Technology development status. MEO technology cannot be considered to have advanced to the commercial stage, although two vendors offer such processes. A number of prototype organic destruction processes using commercial electrochemical cells have been built. The technology is being developed in conjunction with work being performed at LLNL. Pilot tests have been performed at LLNL and the Rocky Flats Environmental Technology Site in Colorado. The relative simplicity of the individual process components suggests that the process can be commercialized once the operating parameters have been established. MEO silver and acid recovery will require development.

Vacuum desorption modifies existing commercial technology for waste processing. This process is at the pilot stage. The simplicity of the process concept should minimize the time required for technology development.

The GPCR reactor used in the APC has not reached the commercial stage. Detailed design and construction of a commercial prototype is currently in progress as part of a Cooperative Research and Development Agreement between PNL and a commercial partner.

Site expanse and resource utilization. The overall facility size is the same for Systems 1 and 2 . These require the smallest building size of the five INTS systems. Of all the organic destruction processes, the resource utilization is highest for the MEO subsystem because of the electrical energy expended. Table 6-5 shows the building costs and utility costs for each system. The cost of these line items gives an approximation of site expanse and resource utilization. The site plans provided as Figures 2-1, 2-25, 2-28, 2-33 and 2-40 also give a direct indication of site exposure for all systems.

Schedule. The grout debris system employs conventional technology except as noted above. Schedule may be affected by development needs for MEO. These are expected to be less than for acid digestion due to extensive pilot work already completed. Significant development problems resulting in long delays are not expected. Markets for the MEO and vacuum desorption technology exist commercially as well as within DOE. 
Decontamination and decommissioning. Requirements for decontamination and decommissioning are based on the amount of equipment and building size. System 1, with one of the smallest buildings and equipment, has the second lowest D\&D costs, as shown in Table 6-5.

Environmental restoration. All systems are designed to minimize impact to the surrounding environment and hence to minimize environmental restoration requirements. Facility operations are designed to minimize releases to the environment. At this preconceptual phase, determination of environmental restoration differences by subsystem is not distinguishable.

\subsubsection{Subsystem Technology Risks}

6.2.2.1 Receiving and Preparation Subsystem. The receiving and preparation subsystem has three major functions: (1) waste container receiving and unloading from the incoming vehicles; (2) assay and non-destructive examination of the incoming containers; and (3) decapping, dumping, and sorting of the waste. The latter two functions require extensive system concept development, component development, hardware engineering, and system integration. Consequently, there are technical, worker health and safety, cost, and schedule risks associated with this subsystem.

The first function, receiving and unloading, can be accomplished by well-known designs and commercially available equipment. The remaining two functions, assay and non-destructive examination, and decapping, dumping, and sorting will require further development, particularly when the facility is used for alpha MLLW'.

Systems engineering and integration and hardware development are needed. The sorting operation will require extensive development. The preconceptual design presented in this study is based on robotic sorting, which has not been tested. Manual sorting and automated sorting devices (e.g., trommels, air classifiers, eddy current devices, magnetic separators) need examination and evaluation. The treatment of alpha contaminated water greatly complicates this subsystem.

If this preconceptual design is to be successful in achieving the intended goals, the hardware listed below must be developed and tested for use in the intended system environment.

Real-time-radiography. State-of-the-art RTR has been in use on an experimental basis at the Radioactive Waste Management Complex (RWMC) at INEL. Suggested development needs are the enhancement of the RTR design and assessment of the data interpretations based on the experience gained at NNEL.

\footnotetext{
${ }^{2}$ The Hanford waste receiving and packaging facility and the INEL waste characterization facilities projects, which are in Title I and II design stages, have already done engineering development work on most of the components needed for accomplishing the functions of this subsystem.
} 
Alpha-radiation assay. Alpha radiation detectors based on PAN assay devices are the current technology base for DOE facilities. The PAN device, while suitable for TRU waste $(>100 \mathrm{nCi} / \mathrm{g})$, has questionable accuracy at lower ranges of alpha contamination.

Container decapping device. Remote decapping of the containers holding alpha MLLW is desirable. Manual decapping could become labor intensive since the operators would have to wear personal protective equipment with self-contained breathing equipment. To develop a remote decapping system, engineering review of various options and selection of a workable concept for the intended application is needed. The decapping step is a crucial rate limiting step. The availability of this device will have a major effect on the transfer of all waste to subsequent processes.

Sorting table. The INTS concept hinges on extensive and effective sorting of waste into treatability groups. A rotary table concept suitable for manipulator sorting (for alpha MLLW) or hand sorting (for nonalpha LLW) is proposed. The manipulator sorting concept, which has not been used or tested before on wastes with the anticipated levels of contamination, will require engineering development. Process residues are anticipated to be homogeneous and not to require sorting.

Solids separation. The current design concept for the subsystem is to accomplish solids separation at the sorting tables. Additional sorting equipment, such as eddy current separators to segregate metals, or screening devices to separate soil from debris, may increase efficiency in sorting. Screening equipment is included to separate process residue liquids from solids.

Shredders and other feed preparation equipment. Shredding equipment is provided in the receiving and preparation subsystem. A dual-stage low-speed shredder can provide the required preparation function for most treatment subsystems. The polymer stabilization subsystem includes a tertiary shredder. However, large shredders are mechanically complex and will require frequent maintenance. This presents some problems for the subsystem when it is used for alpha MLLW. Another concern is the potential for fire in the shredder, which might require use of a blanket of inert gas inside the shredder housing. Inert gas in the shredder will increase the total system offgas. Hence, development work to improve equipment safety, reliability, and maintainability is needed.

\subsubsection{Silver MEO Organic Destruction Subsystem.}

MEO treats organic liquids and sludges from the receiving and preparation subsystem. The ability of the MEO cell reactors to handle multicomponent mixtures and feed variations must be assessed. The potential to generate dioxins from precursors in the feed should be assessed. The frequency of electrolyte replacement and potential for regeneration of the electrolyte must be developed. Process control for the multistep silver and acid recovery processes must be optimized. The long term potential of MEO to treat soft debris should be evaluated to increase system flexibility and reduce waste volume. The effect of mercury on MEO should be investigated. The production of secondary waste needs to be quantified. 
6.2.2.3 APC Subsystem. Most of the equipment (except the GPCR and monitoring equipment) used in this subsystem is well developed and widely used in industry. However, the subsystem will require extensive development for system integration. The subsystem is designed for highly efficient removal of pollutants. This is provided by using state-of-the-art air pollution control equipment. The performance of the design should be verified and enhanced through full scale demonstration units.

The APC subsystem has to remove three major types of pollutants from the treatment subsystems' offgas: particulates, trace organics, and acid gas. The APC must ensure that offgas released to the environment has minimal pollution. The subsystem will also be designed for mercury removal, despite the fact that little mercury is expected in the offgas. Although the proposed design is believed to be state-of-the-art for high efficiency contaminant removal, several areas will need further studies and development before the final system design can be finalized.

GPCR. The GPCR reactor used to treat organics must be evaluated for the processing of offgas from these nonthermal treatment processes. Current studies indicate GPCR can achieve organic destruction efficiencies over $99.9 \%$. The destruction efficiency increases with residence time.

Stack discharge monitoring equipment. A real-time monitoring system must be developed to measure both RCRA metals and radioactive species leaving the stack. As stated previously, the public and regulatory agencies put great emphasis on this feature of the system. Real-time monitoring allows system shut-down in the event of malfunction, thus preventing uncontrolled release of pollutants or subsystem malfunction.

6.2.2.4 Lead Recovery Subsystem. Most of the equipment used in the lead recovery subsystem is well developed. The lead recovery subsystem involves surface decontamination only. Lead with entrained radiation cannot be decontaminated and must be stabilized and disposed of. The overall technology risk for this subsystem is judged to be low. Figure 2-16 lists components that are commercially available but require engineering development and demonstration on a system integration level.

6.2.2.5 Mercury Amalgamation Subsystem. Mercury amalgamation is a commercially available process. The equipment used for this application is well developed and widely used in industry. Hence, the overall technology risk for this subsystem is judged to be low. The subsystem uses amalgamation of elemental mercury and highly contaminated mercury debris waste, such as the steel wool used in the mercury leaching process of the process residue treatment subsystem. Bench scale work at $\mathrm{NEL}$ indicates that the steel wool can be amalgamated with the mercury and copper (Tyson, 1996). Figure 2-14 lists components that are commercially available but require engineering development and demonstration on a system integration level.

6.2.2.6 Metal Decontamination Subsystem. The metal decontamination subsystem uses an abrasive blasting booth to remove surface contamination. This technology is well developed and many units are currently in use in the nuclear industry. Hence, the technology risk associated with this subsystem is low. 
Only some of the equipment in the subsystem requires development in terms of system integration (see Figure 2-12).

6.2.2.7 Special Waste Treatment Subsystem. Equipment used in the special waste treatment subsystem will be designed and developed on a case-by-case basis. A detailed listing of the waste types must be obtained, and potential process application must be studied as part of future tasks. One potential waste stream to be treated by this subsystem is reactive metal wastes such as sodium-contaminated debris. Another potential wastestream is lead battery waste.

6.2.2.8 Aqueous Waste Treatment Subsystem. The aqueous waste treatment subsystem uses commercially available processes and equipment. Development needs for this subsystem are minor. As a whole, the technology risk for this subsystem is judged to be low.

Photolytic oxidation. The optimum mix of oxidants and UV must be determined for the types of organics typically present. Process control may require adjustments to minimize operations and maintenance expenses, and to reduce downstream concentrations of hydrogen peroxide. Excess hydrogen peroxide reduces carbon filter life.

Mercury removal strategy. The pre-conceptual design strategy for mercury removal for this subsystem is precipitation and filtration followed by removal of trace mercury by ion-exchange resins. This approach must be verified on the basis of more accurate knowledge of input waste properties.

6.2.2.9 Process Residue and Soil Treatment Subsystem. This subsystem uses vacuum desorption followed by leaching to treat wastes. This treatment removes organics and volatile mercury, but leaves heavy metals and radionuclides on desorbed soils and sludges. Vacuum desorption is a batch process. The key aspects include loading and removing residues from the desorber and maintaining effective vacuum. These operations may require frequent maintenance. The appropriate batch length for a given waste must be determined. Pilot scale work is needed to evaluate the removal efficiency for separating contaminants from soils and sludges, and to evaluate performance compared to the UTS. The effectiveness of mercury removal by leaching wastes with iodine and potassium iodide needs further development. This process is bench scale only at this time. The flexibility of this technology for different types of waste must be established.

6.2.2.10 Grout Stabilization Subsystem. The grout stabilization subsystem encases debris waste. Operational parameters, including process control, availability and reliability, should be excellent. Organics and mercury have the potential to leach from the grouted waste. The use of grouting as a stabilization technology is a higher risk approach than vitrification. Disposal costs of grouted waste are higher than polymer stabilized or vitrified waste due to the larger volume. Grouted waste is twice the volume of vitrified waste and $112 \%$ of the volume of polymer stabilized waste. 
6.2.2.11 Polymer Stabilization Subsystem. The polymer stabilization subsystem uses a polymer agent for stabilizing salts and treated process residues. Development needs include selecting a suitable polymer agent that can stabilize the waste while meeting the EPA-specified TCLP tests. The subsystem uses commercially available equipment. Hence, the technology risk associated with this subsystem is judged to be low.

6.2.2.12 Certification and Shipping Subsystem. The certification and shipping subsystem uses commercially available equipment except for the assay unit. The development need for the assay unit is the same as that for the assay unit in the receiving and preparation subsystem. The technology risk associated with this subsystem is judged to be low.

6.2.2.13 System integration. A series of figures lists components that are commercially available but require engineering development and demonstration on a system integration level for each subsystem. These FADs are Figures 2-2, 2-5, 2-7, 2-9, 2-10, 2-12, 2-14, 2-16, 2-17, 2-19, 2-21, and 2-23.

\subsection{Evaluation of System 2, Desorption}

System 2 combines CWO for organic destruction with vacuum desorption for treatment of process residue, debris and soil. The use of one approach to the treatment of three waste types makes this the least expensive system. The other subsystems are identical to System 1.

\subsubsection{System Technology Risks}

System 2 has a similar technology risk to System 1, the grout debris system, aside from some unique risks associated with CWO. The treatment of debris for both organics and mercury reduces the overall technology risk to meet regulatory requirements.

\subsubsection{Process Performance.}

Effectiveness. Treatment effectiveness for System 2 is an improvement over System 1. Debris is treated by vacuum desorption for organic removal and by leaching for mercury removal prior to stabilization. The major differences in effectiveness between System 2 and System 1 relate to CWO and debris treatment. The issues of mercury management and waste characterization discussed for System 1 are relevant to System 2 as well. Bench scale tests indicate CWO organic destruction at efficiencies of $99.9 \%$, with some variation depending on the feed and feed rate. As in System 1, the leaching technology for mercury must be demonstrated for debris and other wastes. The size reduction requirements of debris for effective treatment by both vacuum desorption and leaching must be established. Overall, System 2 is more effective than System 1 because debris is treated for organics and mercury. The vacuum desorption technology does not 
remove heavy and radioactive metals. Stabilization of these materials in grout in the presence of debris has not been demonstrated.

Flexibility and versatility. System 2 is more flexible and versatile than System 1 because of the former's ability to treat debris. Sorting requirements are more strenuous than System 1 because debris must be separated. However, System 2 uses vacuum desorption followed by leaching for treatment of multiple waste types. The versatility of this equipment is an advantage, but the dependency on a single piece of equipment minimizes flexibility. CWO appears to be a versatile technology with the potential for treating soft debris as well as organics.

Availability, reliability and maintainability. System 2 has many unknowns regarding these characteristics because of a lack of commercial experience. CWO is a new technology with significant scaleup issues. Operation at elevated pressures and temperatures in an aggressively corrosive atmosphere increases maintenance requirements, and will likely affect availability and maintainability. Corrosion of wetted parts is typically a major cause of downtime. The CWO technology is dependent on a single reactor with extensive process controls, and reliability for such an approach must be demonstrated. Because of potential worker exposure, redundant process controls and safety controls are necessary. These redundant controls may reduce availability. The availability for CWO is reduced by use of only one reactor (as opposed to MEO's multiple cells). Use of multiple reactors would increase availability. Availability for vacuum desorption is critical because this technology is used for multiple waste streams. Availability can also be increased by installing multiple units. Key areas for maintainability for vacuum desorption are the feed equipment, the vacuum seals, and the offgas removal.

Hazardous waste generation. As with MEO and acid digestion, CWO generates corrosive waste which is neutralized, and salts which are stabilized. The neutralized waste is no longer hazardous, but will be slightly radioactive.

Byproducts and residuals. Residuals generated include sludges from CWO and aqueous waste and metal decontamination treatment, and offgas from these processes. Sludges and inorganic solids are treated by vacuum desorption and stabilized in polymer.

Volume reduction. System 2 volume reduction factor is the same as System 1, as shown in Table 6-1. Volume reduction occurs in organic destruction, vacuum desorption, and aqueous treatment, but volume increases occur in stabilization.

Final waste volume and contaminant loadings. Final waste volume, as shown in Table 6-1, is very slightly less for System 2 than System 1, but more than Systems 4 and 5. It is assumed that a small portion of the soft debris volatilizes and/or decomposes in the thermal vacuum desorption, creating offgas but reducing total waste volume. Removal of organics from the debris wastes also reduces volume. Contaminants are reduced in the debris treated by System 2 compared to System 1 . The lack of treatment 
for heavy metals and radioactive metals in debris, soil and residue results in these contaminants being in the final waste form. Metals removed during treatment are stabilized by polymer.

\subsubsection{Environmental Impact.}

Airborne releases. As shown in Table 6-1, the APC discharge is greater in System 2. This results directly from the destruction of a portion of the soft debris and the destruction of the organics removed by the vacuum desorption. Processing temperatures are higher for System 2 than System 1, but below the temperatures generally assumed necessary for dioxin formation. Organics remaining in offgas from treatment processes are subject to further treatment in the APC subsystem.

Wastewater releases. Wastewater discharges are slightly higher for System 2, as shown in Table 6-1. Water generation is about the same, but System 2 grouts less waste, using less water than System 1.

Final waste form performance. As with System 1, waste is treated, stabilized and tested in conformance with the EPA regulations. System 2 debris waste has fewer contaminants due to treatment, as compared to System 1. System 2 debris waste is treated only for organics and volatiles, leaving potentially more contaminants than systems which wash debris. The relative effectiveness of contaminant removal from desorption compared to washing has not been established.

Ecological effects. System 2 is similar to System 1 in potential ecological effects. More refrigerants are required for System 2, but non-ozone depleting chemicals can be used. System 2 has slightly more carbon dioxide emissions than System 1. The actual volume is small (Table 6-2). System 2 has fewer contaminants in the grout stabilized waste than System 1.

Permitting. Permitting issues should be similar to System 1.

\subsubsection{Worker Health and Safety.}

Hazardous reagents. CWO uses hydrochloric acid, ferric chloride and metal catalysts. Mercury removal from process residue, soil and debris wastes requires iodine.

Worker exposure. CWO requires above ambient temperature and pressure combined with the use of aggressive corrosives. This combination is much more hazardous than MEO. The operation of the CWO reactor under these conditions, as well as the potential for a runaway reaction, presents some risks to workers. These risks can be mitigated by effective process controls. Maintenance worker exposure is expected to be higher for CWO than for MEO because of the predicted increase in mechanical/electrical/processing problems in the highly corrosive conditions. 
Other worker exposure issues are similar to System 1. Worker exposure in the grout stabilization subsystem will be reduced because of debris treatment in System 2.

Process equipment and controls. Process controls include built-in redundancy controls, hardwired emergency switches, and secondary containment for various pumps and pipes. Process controls for the reactor are designed to control temperature and, to some extent, reaction rate. The vendor states that the most critical engineering aspect for CWO is to identify suitable materials of construction for areas of the CWO unit which are exposed to hot process solution and vapors. The reaction vessel combines a highly corrosive oxidizing environment in the presence of the process liquid, with a highly corrosive reducing environment in the reaction vessel headspace. Tantalum is the design construction material for the reaction vessel. Effective process controls and a corrosion management program are critical to safe operation of CWO.

Off-normal, upset, and accident conditions. These conditions are the same as for System 4, with the exceptions of the risks associated with CWO as opposed to acid digestion with the exception of the risks associated with CWO as compared to MEO. Because of the increased pressure and temperature and exothermic reactions, the CWO process has a greater risk for incurring an upset condition than the other methods of organic destruction. A thorough FMEA or HAZOP study by technical experts knowledgeable in corrosive and radioactive conditions must be performed to adequately assess the consequences of offnormal or accident conditions, and to institute mitigative measures.

Process containment. As discussed for System 1, containment features are standard design for facilities handling radioactive material. Process control of $\mathrm{CWO}$ will require robust safety features which will include additional features such as double containment for piping to provide adequate process containment.

6.3.1.4 Cost. As shown in Table 6-4, the cost of System 2 is the lowest of the five systems. The use of the vacuum desorption process to treat multiple waste types is cost-effective. $R \& D$ costs are estimated to be the same for Systems 1 and 2. CWO has been bench scale tested extensively but not pilot tested. Table 6-3 lists bench scale, demonstration, and total lifecycle costs for all systems.

\subsubsection{Acceptability.}

Complexity and simplicity. System 2 is only slightly more complex than System 1. CWO is conceptually simpler and has fewer unit operations than MEO. Debris treatment does add to the complexity, because it requires sorting and campaigning of the waste. However, the use of the same equipment for residue and soil treatment is simple. The remaining subsystems involve processes very similar to those in System 1 .

Technology development status. Except for CWO, the technology status is the same as for System 1. CWO has had several years of bench scale tests with a variety of feeds. A 25 kilograms per hour $(\mathrm{kg} / \mathrm{hr})$ 
demonstration unit is currently being fabricated. The unit will be demonstrated on dry organics. Demonstrations are planned at the Savannah River Site and at Rocky Flats Environmental Technology Site.

Site expanse and resource utilization. The overall facility size is slightly less than for System 1 and requires the smallest building size. Resource utilization for System 2 is the same as System 1, as indicated by the utility costs shown in Table 6-5.

Schedule. The desorption system employs conventional technology except as noted above. Schedule may be affected by development needs for CWO. Due to extensive bench scale work, these are expected to be less than for acid digestion. Significant development problems resulting in long delays could occur if construction material and process control issues are not resolved. Markets for the vacuum desorption technology exist commercially as well as within DOE.

Decontamination and decommissioning. Requirements for decontamination and decommissioning are based on the amount of equipment and building sizes. System 2 with a small building size and less equipment has the lowest D\&D costs, as shown in Table 6-5.

Environmental restoration. Considerations are the same as for System 1.

\subsubsection{Subsystems Technology Risks}

Technology risks associated with the subsystems are similar to those of the grout debris system, except for organic destruction. Technology uncertainties for CWO have been extensively discussed in the above sections.

System components that are commercially available, but require engineering development and demonstration on a system integration level, are listed in Figure 2-26. The key area requiring engineering development is the CWO reactor and wetted parts in contact with the reaction fluids. Proper development of reactor design, including construction materials, is critical. Corrosion risks must be assessed. Removal and filtration of inorganic waste is another area for further design and demonstration.

\subsubsection{System Integration}

Figure 2-26 lists components that are commercially available, that require system integration and that need development for CWO. 


\subsection{Evaluation of System 3, Wash}

The wash system is similar to System 1, except for treatment of process residues, soil and debris. Two wash subsystems are used to treat these wastes. The washing approach provides greater contaminant removal and uses more conventional equipment. Additional sorting of debris is required to separate the debris into open, soft and complex streams.

\subsubsection{System Technology Risks}

The technology risk is less than for the grout debris system because debris treatment is included, and washing can remove soluble metals. Mercury removal, however, is not specifically developed for these subsystems. Mercury removal could be performed with the leaching process included in other systems, if the wash process does not separate out mercury compounds.

\subsubsection{Process Performance.}

Effectiveness. Process effectiveness for MEO and other subsystems which are the same as System 1 is discussed above. Pilot work using the aqueous wash subsystem has demonstrated organic removal of $99.99 \%$ and greater, and metals removal of over $96 \%$, for soil (EPA, 1994b). Process effectiveness for sludge and residue is not known. High pressure wash treatment for debris found similar results, with bench scale tests demonstrating a 98\% removal of PCBs, organics and lead (EPA, 1991). Commercial scale tests are not available.

The removal efficiency for DOE MLLW organic and metal contaminants must be demonstrated. Mercury removal is not specifically provided for in the wash system. Mercury removal from debris, soil and process residue via the wash water is assumed. Subsequent treatment of the wash water, which includes metals precipitation and dissolved air flotation, are appropriate for mercury treatment. The effectiveness of the wash subsystems treatment for mercury must be evaluated.

Flexibility and versatility. System 3 is quite flexible because of the multiple treatment approaches available: $\mathrm{MEO}$ and two wash subsystems. Like thermal desorption, washing is not very specific to waste type. The effectiveness of washing is a function of contaminant, matrix and the characteristics of the contaminated substrate.

Availability, reliability and maintainability. Washing has been commercially employed in the hazardous waste field. Availability and reliability should be excellent with this system, and similar to System 1. Maintainability for MEO is discussed above in System 1. For wash subsystems, the equipment consists of commercially available tanks and rinsing vessels. The adaptation for agitation should not require extensive maintenance. 
Hazardous waste generation. As in System 1, silver and acids are used in the MEO process.

Byproducts and residuals. Wash subsystems generate wet residue which requires treatment. Since MEO is designed to treat wet organics, organic residuals and waste surfactant are easily processed. Precipitated metal waste is collected and sent to polymer stabilization.

Volume reduction. System 3 has similar volume reduction to Systems 1 and 2 (Table 6-1). Washing provides removal of contaminants from debris, but destruction of soft debris waste does not occur.

Final waste form volume and contaminant loadings. The final form volume is similar to Systems 1 \& 2 (Table 6-1). Surfactant and additive wastes add to the final volume, and soft debris is not decomposed. Contaminant removal is good, with treatment to remove both organics and soluble metals. Demonstration of the level of contaminant removal for sludges must be performed.

\subsubsection{Environmental Impact.}

Airborne releases. The treated offgas volume is only slightly higher than for System 1 (Table 6-1). The generated offgas is composed almost entirely of carbon dioxide. The larger volume relates to the increased load to MEO from treating the organics removed in the washing process.

Wastewater releases. The wash subsystems use extensive water recycling and little water is discharged.

Final waste form performance. The final waste form is designed to meet LDR requirements. Effective separation of contaminants in the wash subsystems results in contaminant destruction, or encapsulation in polymer which is more stable than grout.

Ecological effects. System 3 is similar to Systems 1 and 2 in potential ecological effects. Because of the effectiveness of this system, ecological effects are expected to be low. System 3 has similar carbon dioxide emissions to Systems 1 and 2, but less than the other INTS systems. Actual discharge is shown in Table 6-2. System 3 has fewer contaminants in the grout stabilized waste than Systems 1 or 2, but soft debris is not destroyed as in System 4 or 5 .

Permitting. Permitting issues should be similar to System 1.

\subsubsection{Worker Health \& Safety.}

Hazardous reagents. System 3 uses the MEO reagents nitric acid, silver nitrate, caustic and hydrochloric acid for recovery. The wash subsystems use surfacant and additives, which, according to the vendors, are not known toxics (Gotlieb, 1993). 
Worker exposure. System 3 is judged to be the safest system. Temperatures and pressures are close to ambient. Hazardous reagents are less aggressive than in other systems. Maintenance related exposures should be the least of any system because commercial components are used for both wash subsystems and MEO.

Process equipment and controls. Overall operating hazards are primarily mechanical risks. Process controls, although complex because of extensive recycling, are standard industrial equipment, except as upgraded for the radioactive environment.

Off-normal, upset, and accident conditions. As with all INTS systems, a detailed FMEA or HAZOP study is required to evaluate these conditions. System 3 is expected to have the least serious consequences as the result of upsets or accidents. Both the wash subsystems and MEO, as discussed above, shut down the process and reactions upon electrical shutdown. Loss of cooling, heat, or other reagents would cause processing problems, but not significant accident conditions.

Process containment. The System 1 discussion is appropriate for System 3. System 3 wash subsystems are easily contained.

6.4.1.4 Cost. System 3 is more expensive than System 1 (Table 6-4). MEO is more expensive than the other organic destruction subsystems, and washing is more expensive than vacuum desorption. Costs are higher for wash subsystems because of larger building space, water recycling equipment, and additives. Research and development costs are more than for Systems 1 and 2 because of the need to evaluate the two separate wash subsystems.

6.4.1.5 Acceptability. System 3 should have the highest acceptability of the INTS systems.

Complexity and simplicity. System 3 is complex, with two more subsystems than System 1 . The wash subsystems have several unit operations to effectively recycle water and additives. However, System 3 is perceived as simple to operate. The operations are predictable, controllable and use commercial equipment.

Technology development status. MEO is discussed above with System 1. The aqueous and high pressure wash subsystems are not fully proven, although nearly all the equipment components are commercially available. Development work necessary for scale-up would be required, and pilot testing of DOE waste should be performed. Mercury removal effectiveness must be demonstrated in addition to the effectiveness for treating sludges.

Site expanse and resource utilization. The overall facility size is larger than Systems 1 and 2 (Table 6-5). Resource utilization, reflecting washing costs, is slightly higher than for System 1, as indicated by the utility costs shown in Table 6-5. 
Schedule. Schedule issues are similar to System 1. The wash subsystems development should be less than that of MEO. Schedules are not anticipated to be adversely affected by significant development problems.

Decontamination and decommissioning. Requirements for D\&D are based on the building size and the amount of equipment. System 3 with a larger building size has D\&D costs higher than System 1, as shown in Table 6-5.

Environmental Restoration. Considerations are the same as for System 1.

\subsubsection{Subsystems Technology Risks}

6.4.2.1 Aqueous Wash Process Residue and Soil Treatment Subsystem. The aqueous wash subsystem is based on a design that is not yet proven. The process uses a high level of surfactant and proprietary surfactant separation technology to recycle the surfactant. The approach uses commonly available commercial equipment, and successful tests have been performed with soil. The treatment of process residue has not been extensively tested, and problems related to residue treatment may require resolution. This subsystem may be able to treat soft debris after appropriate size reduction, and this option should be explored.

The aqueous wash uses multiple additives to effect the separation of contaminant and surfactant in several unit operations. The effective partitioning of a surfactant/contaminant phase from the aqueous phase in the solubility reduction process is necessary for both surfactant recycling and contaminant removal from the washing process. Pilot studies would be required to optimize additive use and to confirm that contaminants in DOE waste can be effectively partitioned out of the aqueous phase. In particular, removal rates for insoluble metals and other inorganics must be demonstrated. The fate of soluble metals depends on the effectiveness of metal precipitation and dissolved air flotation steps, which are proven technologies. The metal precipitation step prior to the DAF may require optimization for DOE metals.

Surfactant separation from contaminants requires further demonstration. This separation uses a proprietary process based on steam or solvent stripping. Removal of organics can be accomplished with such a process, but removal of inerts may not be, depending on the details of this process. If inerts remain in the surfactant, the ability to recycle the surfactant will be reduced. Should more surfactant remain with the waste, costs to treat the surfactant by organic destruction or to dispose of the surfactant will increase. As a separate sensitivity analysis, the costs to remove and treat the total volume of surfactant were estimated. Treatment would increase the cost to the MEO organic destruction subsystem by approximately $50 \%$, or $\$ 100$ million over the life of the facility. Purchase of fresh surfactant over the life of the facility would add less than $\$ 1$ million. 
Another concern for both aqueous and high pressure wash is the fate of mercury and mercury compounds. This analysis assumes mercury will partition with the aqueous phase. For aqueous wash, the mercury is anticipated to be removed from waste at the rinse contactor step. Mercury would then be removed either at the precipitation/DAF steps or would remain with the surfactant.

\subsubsection{High Pressure Wash Soft Open and Complex Debris Treatment Subsystem. This} debris decontamination subsystem can accept both small and large size open and complex debris using two wash/rinse tanks for the different size ranges. For soft debris, the same process equipment is used as the high pressure wash subsystem, with the substitution of low pressure nozzles for high pressure ones. The feed system will require development for radioactive waste usage and for a range of debris types. Optimum feed size requirements should be developed. For the wash, a new basket or an insert designed for the shredded soft debris is needed. The reliability and maintainability of the basket agitation system should be established for all debris types. The potential for paper to solubilize in the wash water, and the treatment of the wash water by MEO or aqueous waste treatment, should be evaluated.

Figures show key areas regarding engineering development and demonstration on a system integration level.

\subsection{Evaluation of System 4, Acid Digestion System}

The acid digestion system utilizes an innovative approach to organic destruction and soft debris treatment. The acid digestion treatment destroys organics in the oxidizing atmosphere of hot acid. The remaining subsystems are a combination of other previously discussed technologies, including vacuum desorption for process residue treatment, aqueous wash for soil treatment, high pressure wash for open debris, and grouting without treatment for complex debris.

\subsubsection{System Technology Risks}

Overall, the implementation risks must be judged higher than in the grout debris system, based on the lack of pilot or commercial experience with acid digestion.

\subsubsection{Process Performance.}

Effectiveness. The acid digestion process uses hot nitric acid in a phosphoric acid solution to oxidize organic waste. Currently the subsystem development is at the bench scale stage. Ethylene diamine tetra acetic acid (EDTA) and cellulose were destroyed with a destruction efficiency of $99.5 \%$ within an accuracy of $\pm 2 \%$. While clearly the acid can destroy organics, the response of the process to complex feed streams is not apparent from the limited test data. Feed rate, feeding mechanisms, removal of inorganics, the effect of inorganic buildup on the rate of organic destruction, reactor scale-up, process control, and many other 
issues have not been investigated. The process is highly promising at this stage, but determining its effectiveness for treating DOE wastes requires significant demonstration.

System 4 also includes an innovative treatment approach to stabilization, phosphate bonded ceramic, which utilizes waste phosphoric acid from acid digestion. This technology is at the bench scale stage and requires further development to assess effectiveness. As in the other systems, treated debris and soil waste is grouted.

System 4 uses a combination of treatments for treating soil, residue and debris which may achieve excellent effectiveness. Vacuum desorption as process residue treatment appears appropriate but needs demonstration. However, vacuum desorption does not treat the waste for heavy metals and radionuclides. Soil treatment by aqueous washing and debris washing with high pressure also utilize treatment methods specifically designed for these waste types. These wash subsystems are designed around soil and are not optimized for residue.

Flexibility and versatility. Overall, System 4 is both flexible and versatile. Three available treatment subsystems for process residue, soil, and debris provide good flexibility. Acid digestion, although at an early stage of development, appears not to be affected by feed type. The actual flexibility, particularly regarding feed rate and mixtures of feed types, requires demonstration. The acid digestion approach is combined with a phosphate bonded ceramic stabilization process. System 4 also has a small polymer stabilization subsystem for treatment of water soluble salts and wastes deemed not appropriate for phosphate bonded ceramic. This approach is highly flexible, with two processes available to treat process residues and salts.

Availability, reliability and maintainability. Acid digestion using nitric acid and phosphoric acid is a very immature technology. The process has been investigated for treatment of DOE waste at the bench scale only. Estimates for availability, reliability, and maintainability are sketchy at best. Like CWO, acid digestion uses aggressive reagents in a heated reaction vessel. Selection of appropriate construction materials and precision manufacturing will be critical to avoid corrosion problems and the poor reliability associated with corrosion. Separate reactors are included for processing organic waste and soft debris. This adds to overall availability and flexibility.

Maintainability depends on corrosion control. The relatively simple process outside the reactor itself helps maintainability, because there isn't much equipment to keep operational. Phosphate bonded ceramic stabilization is a bench scale process. Several parameters have been found to affect the final waste product (Wagh, 1995). These include the rate of mixing, temperature control during setting, the amount of cure retardant, and water content of the setting product. The curing process of the waste is critical. Process control of the phosphate ceramic stabilization process is expected to be considerably more important compared to other stabilization processes. Failure rate of the waste may occur, with subsequent reprocessing required. 
Availability may be affected if longer periods of curing than anticipated are required. The remainder of the system should have good availability. The wash subsystems, as discussed with System 3, use commercial equipment. The vacuum desorber should be less reliable or maintainable than a wash subsystem because it is not yet a proven technology. However, it is a much simpler operation than the wash subsystems.

Hazardous waste generation. System 4 generates waste phosphoric acid which is incorporated in the phosphate bonded ceramic.

Byproducts and residuals. System 4 generates phosphoric acid as a treatable byproduct. Wash subsystems generate wet residue requiring treatment in the process residue subsystem. Vacuum desorption generates organic waste which is treated by acid digestion and the APC subsystem. Byproducts and residuals from other subsystems are the same as for System 1.

Volume reduction. System 4 has the best volume reduction of the INTS systems. This is based on the breakdown of soft debris to carbon dioxide and water, and stabilization of both soil and process residue waste in the phosphate bonded ceramic. Other systems use grout for stabilization of soil, which increases final waste volumes. Phosphate bonded ceramic, like polymer, has an assumed mass increase of twice the initial waste compared to three times the mass for grout. As in other systems, treated debris is grouted.

Final waste form volume and contaminant loadings. System 4 has the lowest waste volume of the INTS systems (Table 6-1). Contaminant loadings with phosphate bonded ceramic are similar to those of other systems. The wash subsystems are designed to remove organics and metals. The desorption process removes organics and mercury.

\subsubsection{Environmental Impact.}

Airborne releases. The treated offgas volume is higher than Systems 1, 2 and 3 and similar to System 5 (Table 6-1). The offgas volume combines offgas from the acid digestion of organic destruction and soft debris, plus offgas from aqueous waste treatment and thermal desorption. The offgas is composed almost entirely of carbon dioxide. In the acid digestion processes, chlorine is released as nitrosyl chloride (NOCl), and $\mathrm{NO}_{\mathrm{x}}$ is formed from nitric acid. These products are treated in the APC and removed or reduced to acceptable levels.

Wastewater releases. System 4 discharges more water than Systems 1 and 3. Because phosphate bonded ceramic requires less water than grout, System 4 does not use much water. However, the organic destruction reactions generate water and the excess water is discharged. In most systems, water is consumed in the grout subsystem but acid digestion uses little grout for stabilization so there is excess water in the system. 
Final waste form performance. The final waste form is designed to meet LDR requirements. Phosphate bonded ceramic is used to stabilize waste. In this study it is assumed that the stability will be similar to polymer and better than grout. However, this remains to be demonstrated.

Ecological effects. System 4 is similar to the other INTS systems in potential ecological effects. However, System 4 employs innovative technologies whose true effectiveness and waste form performance are not known. System 4 has more carbon dioxide emissions than Systems 1,2 and 3.

Permitting. Permitting issues should be similar to System 1. Demonstration that acid digestion is an effective treatment technology for debris treatment would be necessary.

\subsubsection{Worker Health and Safety.}

Hazardous reagents. Acid digestion uses concentrated nitric and phosphoric acid. Extracting mercury from residue after thermal desorption requires iodine.

Worker exposure. Acid digestion requires above ambient temperature and pressure combined with the use of aggressive corrosives. This combination is more hazardous than MEO, but perhaps less hazardous than the elevated pressures of CWO. Maintenance worker exposure is expected to be similar to CWO, with potential mechanical/electrical/processing problems in the highly corrosive conditions.

Other worker exposure issues are similar to System 1. Worker exposure in the grout stabilization is reduced compared to System 1 because of debris treatment in System 4.

Process equipment and controls. Because acid digestion is not developed at the pilot scale, process controls have not been developed. Redundant process controls, hardwired emergency switches and secondary containment for various pumps and pipes must be included in the acid digestion subsystem. One advantage of acid digestion is the formation of water as the reaction proceeds. The water evaporates quickly, but the evaporation also cools the mixture and dampens the reaction. Water that does not evaporate dilutes the nitric acid, reducing the oxidizing effect and thus the reaction. Thus the risk of a runaway reaction is less than with CWO, which is less dependent on acid concentration.

As with CWO, suitable materials of construction are critical for areas of the acid digestion unit which are exposed to hot process solution and the hot vapors from that solution. Tantalum or glass-lined steel is the design construction material for the reaction vessel. Effective process controls and a corrosion management program are critical to safe operation of acid digestion.

Off-normal, upset, and accident conditions. These conditions are the same as System 1, except when comparing acid digestion to MEO. Acid digestion has a greater risk of off-normal or upset conditions because of its technological immaturity and the absence of practical experience even at the pilot plant level. 
Elevated temperature and aggressive reagents increase the risk of upset compared to MEO. A thorough FMEA or HAZOP study by technical experts knowledgeable in corrosive and radioactive conditions must be performed to adequately assess the consequences of off-normal or accident conditions, and in order to institute mitigative measures.

Process containment. As discussed for System 1, containment features are standard design for facilities handling radioactive material. Process control of acid digestion requires robust safety features. Additional features such as double containment for piping and reactor are recommended to provide adequate process containment.

6.5.1.4 Cost. System 4 costs are similar to those of System 5. Acid digestion is similar in cost to CWO. Vendor costs were not available for this bench scale process, and the costs were developed using commercial equipment and anticipated utility needs. The cost risk of this estimate is highest of the INTS systems because of the many unknowns concerning acid digestion such as the need for additional secondary treatment, or the actual operation requirements. R\&D costs are estimated to be the highest of all INTS systems. Unlike MEO and CWO, acid digestion has not been pilot tested. Table 6-4 lists bench scale, demonstration and total lifecycle costs for all systems.

\subsubsection{Acceptability.}

Complexity and simplicity. System 4, with multiple subsystems for debris, residue and soil treatment, is considerably more complex than System 1. Acid digestion, similar to CWO, is conceptually simpler and has fewer unit operations than MEO. Actual process experience is necessary to fully evaluate the complexity of the acid digestion operation. Similar to System 3, multiple subsystems for the treatment of residue, soil, open debris and soft debris add to the system complexity. Unknowns with phosphate bonded ceramic prevent a clear evaluation of the subsystem simplicity. The remaining subsystems involve the same processes as System 1 .

Technology development status. System 4 has immature technology for organic destruction, soft debris treatment and stabilization. Both acid digestion and phosphate bonded ceramic stabilization are bench scale processes with significant development required. The technology status of these processes makes the overall evaluation of this system more qualitative than other systems.

Site expanse and resource utilization. The overall facility size is the largest of all systems but very similar to System 5. Resource utilization is higher than all systems except System 5 , as indicated by the utility costs shown in Table 6-5.

Schedule. Schedule is affected by development needs for acid digestion and phosphate bonded ceramic. Acid digestion and phosphate bonded ceramic require extensive bench scale and pilot work. Development problems which are not resolved could result in long delays. 
Decontamination and decommissioning. Requirements for $D \& D$ are based on the building sizes and the amount of equipment. System 4 , with a medium building size and less equipment, has D\&D costs similar to Systems 3 and 5, as shown in Table 6-5.

Environmental restoration. Considerations are the same as for System 1.

\subsubsection{Subsystems Technology Risks}

6.5.2.1. Acid Digestion Organic Destruction Subsystem. There are many issues associated with this subsystem that require resolution. The ability to treat different waste types is one of the advantages of acid digestion. However, data must be developed to fully assess the flexibility of the subsystem for contaminants in the waste. The effectiveness in treating volatile organics is an unknown. There is a risk that volatiles could vaporize prior to destruction. Bulk metals treatment with acid digestion is not advisable. Iron forms iron phosphate precipitates which build up as a residue. Nickel should be removed from the wastestreams because it forms a hydrated phosphate which tends to set up almost like a gel.

The reactor design requires development. A fluidized bed design that would provide easier removal of residuals is under consideration. The shape of the reactor would be based on the feed type processed.

Residual inorganics build up in the acid media. Periodically the acid is transferred from the reactor to remove impurities. The frequency of removal depends on the amount of metals and/or the amount of radionuclides in the media. Early tests on resins indicate replacement of the acid media is necessary only every three months. For debris or waste with a significant inorganic content, changeout may be necessary as often as weekly. Residual treatment is an area where significant development is required.

6.5.2.2. Acid Digestion Soft Debris Treatment. In addition to the issues discussed above for organic destruction using acid digestion, soft debris treatment raises further concerns. For example, the bench scale process does not provide data for developing an adequate feed preparation system for the acid digestion reactor. Shredded waste may be preferable because of increased surface area for reaction. Acceptable waste feed types need further evaluation. The current targets are organic wastes, including combustible waste, plastics and cellulose based waste.

The ability of acid digestion to treat plastics by complete decomposition is one of its advantages. However, different plastics require different optimum temperatures. In general, as the temperature increases, the oxidation increases. But at high temperatures, plastics melt and may fuse together as a semi-solid mass rather than dissolve. Reaction occurs following dissolution. If the feed added contains a new plastic, it is not known if this will depress the reaction of already dissolved plastics. Alternatively, the reaction of plastics in the media could delay the dissolution of added plastic. Finding an appropriate temperature for mixed plastic waste presents a challenge. 
6.5.2.3. Phosphate Bonded Ceramic Stabilization Subsystem. In this subsystem, a two step stabilization process is used. First, soils are blended with magnesium oxide. Then process residues are thoroughly mixed with phosphoric acid, and boric acid as a second step. The mixtures generated in the two steps are combined and thoroughly mixed to initiate the stabilization reaction. Any waste phosphoric acid would be used as an ingredient in the ceramic stabilization process. This removes the inorganics and allows stabilization of radionuclides in the ceramic. However, this approach has not been tested.

Pretreatment of the phosphate waste may be necessary or the stabilization process may not work with waste ingredients. Significant additional evaluation is required to demonstrate the phosphate bonded ceramic subsystem effectiveness, implementability, scale-up potential, cost, final waste form performance, and many other factors.

6.5.2.4. System Integration. Figure 2-34 lists components that are commercially available but require engineering development and demonstration on a system integration level.

\subsection{Evaluation of System 5, CWO}

System 5 combines CWO for organic destruction and soft debris treatment with (1) vacuum desorption for process residue treatment, (2) aqueous wash for soil treatment, and (3) high pressure spray for open debris treatment. System 5 is similar to System 4, the only differences being (1) the substitution of CWO for acid digestion for organic destruction and soft debris treatment, and (2) polymer and grout stabilization instead of phosphate bonded ceramic. Soft debris treatment by CWO is the only subsystem which is unique to System 5 .

\subsubsection{System Technology Risks}

\subsubsection{Process Performance.}

Effectiveness. System 5 uses the same combination of treatments for treating soil, residue and debris as System 4. These treatments are expected to be very effective. The organic destruction efficiencies of CWO have been found to be $99.99 \%$ for organics. Tests performed on soil rinsed with CWO solution indicated metal removal to nondetect levels $(0.02$ milligram per kilogram [mg $/ \mathrm{kg}$ ] or lower for typical metals).

Flexibility and versatility. Overall, System 5 is both flexible and versatile. Use of three available treatment processes for process residue, soil, and debris provides maximum flexibility. CWO appears to be versatile, with the potential for treating soft debris and organic liquids. 
Availability, reliability and maintainability. As discussed for System 2, CWO availability, reliability and maintainability information is not available due to lack of proven industrial experience. The elevated temperature, pressure, and aggressive corrosive reagents reduce availability. Separate reactors are included for processing organic waste and soft debris. This adds to overall availability and flexibility. Maintainability depends on corrosion control. The relatively simple process outside the reactor itself helps maintainability because there is not much equipment to be kept operational. The remainder of the system should have good availability. The wash subsystems, as discussed for System 3, use commercial equipment. The vacuum desorber should be less reliable or maintainable than a wash subsystem because it is not yet a proven technology. However, it is a simple process.

Hazardous waste generation. System 5 generates corrosive waste which is neutralized, and salts which are stabilized. The neutralized waste is no longer hazardous, but remains radioactive.

Byproducts and residuals. As in System 2, residuals generated include organics from vacuum desorption, sludges from $\mathrm{CWO}$, aqueous waste, sludge from aqueous washing, high pressure washing and metal decontamination treatment, and offgas from these processes. Sludges and inorganic solids are treated by vacuum desorption and stabilized in polymer. Organics are treated by CWO, photolytic oxidation (low concentration aqueous waste) or GPCR (vapors). Byproducts and residuals from other subsystems are the same as System 2.

Volume reduction. System 5 has the second best volume reduction factor of the five INTS systems. The complete destruction of soft debris contributes to the high volume reduction. Volume reduction is less than for System 4 because grout is used to stabilize soil.

Final waste form volume and contaminant loadings. System 5 has the second lowest waste volume of the INTS systems (Table 6-1), with volume and contaminant results similar to System 4.

\subsubsection{Environmental Impact.}

Airborne releases. The treated offgas volume is higher than that for Systems 1,2 and 3, and is similar to System 4 (Table 6-1). The offgas volume combines offgas from CWO treatment of organic destruction and soft debris with offgas from aqueous waste treatment and thermal desorption. The offgas is composed almost entirely of carbon dioxide.

Wastewater releases. System 5, like System 4, discharges more water than Systems 1, 2 and 3. System 5 discharges the water created in the organic destruction reaction. Some recycled water is used in the grout process. 
Final waste form performance. The final waste form is expected to meet LDR requirements. Final waste form performance to meet LDR requirements must be demonstrated, especially for stabilized residue from CWO treatment.

Ecological effects. System 5 is similar to the other INTS systems in potential ecological effects. System 5 has carbon dioxide emissions similar to System 4 and greater than Systems 1,2 and 3.

Permitting. Permitting issues should be similar to System 1. Demonstration that CWO is an effective treatment technology for debris treatment would be necessary.

\subsubsection{Worker Health \& Safety.}

Hazardous reagents. CWO uses hydrochloric acid, ferric chloride and metal catalysts. Extracting mercury from residue after thermal desorption requires iodine.

Worker exposure. Worker exposure issues are similar to those for System 4, except for CWO which is discussed with System 2. Two CWO subsystems increase risks to maintenance and other workers.

Process equipment and controls. As discussed for System 2, CWO requires above ambient temperature and pressure combined with the use of aggressive corrosives. System 5 requires two CWO subsystems, thus increasing potential worker exposure. Rigorous safety and process control are included for both subsystems. Suitable construction materials are critical for both organic destruction and soft debris treatment.

Off-normal, upset, and accident conditions. These conditions are the same as for System 4, with the exception of the risks associated with CWO as opposed to acid digestion. Risks for CWO are considered to be higher due to elevated pressure and temperature, process control issues and corrosion concerns. A thorough FMEA or HAZOP study is required.

Process containment. As discussed for System 1, containment features are standard design for facilities handling radioactive material. Process control of $\mathrm{CWO}$ will require robust safety features and will include additional features such as double containment piping to provide adequate process containment.

6.6.1.4 Cost. System 5 is the second most expensive system with costs very similar to System 4 . Extensive R\&D is necessary for CWO, although it should be less than for acid digestion. Overall costs are increased by the use of multiple subsystems to treat several waste streams, rather than a larger sized simpler approach (e.g. System 2). 


\subsubsection{Acceptability.}

Complexity and simplicity. System 5, with multiple subsystems for debris, residue and soil treatment, is very similar to the acid digestion system. CWO is conceptually simpler and has fewer unit operations than MEO. Process controls for CWO are complex. Similar to Systems 3 and 4, multiple subsystems for the treatment of residue, soil, open debris and soft debris add to the system's complexity. The remaining subsystems involve the same processes as, or very similar to, System 1.

Technology development status. System 5 technology status is very similar to System 2 , with the addition of washing processes. CWO is a young technology ready for demonstration testing. Both aqueous wash and pressure wash technologies are new to DOE MLLW.

Site expanse and resource utilization. The facility size for System 5 is the second largest. CWO requires similar space as acid digestion. As in System 4, the inclusion of several subsystems for residue, soil and debris treatment increases the building size over Systems 1,2 and 3. Resource utilization is the highest of the INTS subsystems, as indicated by the utility costs shown in Table 6-5.

Schedule. Schedule is affected by development needs for CWO organic and soft debris treatment. Pilot work and scale-up tests are necessary and must be included in the schedule. Development problems which are not resolved could result in long delays.

Decontamination and decommissioning. Requirements for $D \& D$ are based on building sizes and the amount of equipment. System 5 with a large building size and high equipment costs has D\&D costs similar to System 4, as shown in Table 6-5.

Environmental restoration. Considerations are the same as for System 1.

\subsubsection{Subsystems Technology Risks}

6.6.2.1 CWO Soft Debris Treatment Subsystem. Treatment of soft debris requires additional demonstrations. Feed system requirements and optimum particle size need development. The handling of inert residuals must be established for DOE MLLW. The effect of inerts on reaction rate and corrosion properties needs evaluation.

\subsection{System Evaluation Against TSWG Principles}

In earlier subsections of Section 6, nonthermal systems were evaluated against the technical criteria described in Section 5. In this subsection, the systems are evaluated with respect to the TSWG principles 
presented in Section 1. Many of the TSWG principles are similar to the TSG technical criteria, but this section was developed to allow the reader a direct comparison with the TSWG principles.

\subsubsection{Minimize Effluents}

This principle evaluates the amount of potentially hazardous effluents. There are three categories of effluent that may carry hazardous material into the environment: (1) offgases from the APC; (2) discharged wastewater; and (3) stabilized solids sent to disposal.

Offgas. The volume of offgas from the nonthermal systems ranges from approximately $700 \mathrm{lbs} /$ hour for System 1 to $1400 \mathrm{lbs} /$ hour for System 5 (Table 6-1). The major portion of this offgas is carbon dioxide (from $75 \%$ for System 2 to $92 \%$ for System 5). Carbon dioxide is produced when organic wastes are decomposed (oxidized). System 1 has the least amount of offgas because debris waste is not treated, but, rather, sent directly to grout stabilization. Systems 2 and 3 have more offgas due to removal of organic contaminants from debris and subsequent oxidation. Systems 4 and 5 generate the most offgas because of the additional oxidation of soft debris. Thus, the consequence of oxidizing soft (or combustible) debris in Systems 4 and 5 is higher offgas volumes.

In all cases the APC subsystem contains all the necessary components to treat the offgas and prevent release of contaminants. This includes condensers, dry filters and scrubbers to remove volatilized organics and metals, particulate and acid gases. A GPCR oxidizes remaining trace organics. Polishing steps are provided to remove any remaining trace contaminants. These steps include activated carbon filters to remove trace organics, sulfur impregnated carbon filters to remove trace levels of mercury, and HEPA filters to remove radioactive and heavy metal particulates. Because the offgas is at a low temperature, the potential for contaminants such as dioxins, volatilized metals and radionuclides in the offgas, is significantly reduced relative to thermal systems.

Wastewater. In all systems the treated water leaving the aqueous treatment subsystem is recycled to minimize consumption. However, more water is produced by the oxidation of organic materials than is required by the system for operation. Thus, there is some discharge which varies from about $60 \mathrm{lb} / \mathrm{hr}(0.1$ $\mathrm{gpm}$ ) for System 1 to about $800 \mathrm{lb} / \mathrm{hr}$ (1.6 gpm) for System 4 (Table 6-1). The system requirements for water, the total water leaving aqueous waste treatment, and the percentage of water recycled are shown in Table 6-6.

Instead of being discharged as a liquid, excess water could be evaporated and discharged as a vapor. However, this would increase the offgas rate, place a larger burden on the APC subsystem, and increase energy usage. As with the APC subsystem, the aqueous waste treatment subsystem contains all the unit operations required to meet discharge requirements. 
Solid Effluent. Solid effluent from the nonthermal systems is in two forms: (1) stabilized waste and (2) decontaminated metal which is stored for recycling within the DOE complex. Waste is stabilized in several media: grout, polymer and, in System 4, phosphate bonded ceramic. Mercury is stabilized in an amalgam. The mass flow rates of these wastes are shown in Table 6-7, and the volume flow rates are shown in Table 6-1. These waste quantities are fairly high, compared to wastes from thermal treatment. The nonthermal stabilization processes require significant use of additives. Polymer or phosphate bonded ceramic require one pound of polymer or ceramic additives for every pound of waste stabilized. Grout stabilization requires two pounds of grout mix for every pound of waste stabilized. The largest amount of solid waste generated is from Systems 1, 2 and 3 that do not oxidize soft debris.

The fate of mercury is a major issue in the INTS study. Yet the amount of waste containing mercury and the mercury concentrations are undefined. For the study, it was assumed that trace concentrations of mercury, too small to affect the mass balance, are present. Treatment processes are developed to treat mercury contaminated waste, although the mass balance shows little waste undergoing treatment. In the case of System 1, a procedure for treated mercury contaminated debris is developed, but the mass balance does not indicate any debris treated by that process. For all systems, $2.5 \mathrm{lbs} / \mathrm{hr}$ of $\mathrm{Hg}$ is sent directly from receiving and preparation to the mercury amalgamation subsystem. In System 2, an additional $2.5 \mathrm{lbs}$ of $\mathrm{Hg}$ is recovered from the desorption of debris and treated by mercury amalgamation. In other systems, mercury is assumed to be removed from the debris in wash water. The water is treated by precipitation and the resulting mercury precipitate is stabilized in the polymer stabilization subsystem.

\subsubsection{Minimize Effects on Human Health and the Environment}

This principle has three components defined in Section 1 and listed in Table 5-2.

\subsubsection{Minimize Present and Future Exposure.}

Worker Exposure. Worker exposure is minimized by proper system and facility design and operation. The INTS designs comply with the design requirement described in Appendix A. When processing alpha MLLW, two or three levels of confinement are required. The concept used in these preconceptual system designs is based on placing equipment in air-tight cells that have three levels of confinement. Personnel access is through airlock doors. Personnel entering alpha cells must wear appropriate personal protective equipment (PPE). For non-alpha MLLW, worker health and safety objectives can be met by reducing confinement to one or two levels depending on the potential for release of radioactivity.

The largest exposure risk for all the systems is in the sorting operations area where the waste is dumped out of the containers. Exposure risk is reduced with the MEO systems because the reagents are less aggressive, the systems have a small throughput of waste, and the process is at atmospheric pressure and only

slightly above room temperature. Exposure risk is higher for the more aggressive processes such as acid 
digestion and CWO which operate at higher temperatures and pressures than MEO. However, proper design and control procedures will minimize the potential for accidents and exposure.

Issues relative to worker health and safety are discussed in more detail for each of the systems in subsections 6.2.1.3, 6.3.1.3, 6.4.1.3, 6.5.1.3 and 6.6.1.3.

Environmental Exposure. Short term environmental exposure may result from gaseous emissions and aqueous discharges from the treatment facility. As indicated in Section 6.7.1, these emissions are small and control equipment has been included which will remove potential contaminants prior to release.

With the possible exception of mercury and some mercury compounds, volatilized metals and radionuclides are not released into the air from nonthermal treatment processes. Formation of dioxins in the offgas is not anticipated because the higher temperatures required for their formation are not present. Oxides of nitrogen are formed by the use of nitric acid as the oxidizing agent in the MEO and acid digestion processes. However, the $\mathrm{NO}_{\mathrm{x}}$ is recovered as nitric acid or converted to nitrogen by a catalyzed reaction with ammonia. Other acid gases, such as $\mathrm{HCl}$, are removed in the condenser or scrubber and neutralized. Mercury that is volatilized in the thermal desorbers is not expected to pass the condensers. As a backup, the APC subsystem includes sulfur impregnated carbon filters for mercury removal.

Wastewater from storage or generated within the various subsystems is treated in the aqueous waste treatment subsystem. Untreated water may contain dissolved metals or radionuclides, suspended solids, or organics. Various unit operations have been included in the aqueous treatment subsystem design to remove these contaminants prior to recycling or discharge. Treated water is either recycled or discharged in accordance with regulatory requirements.

Long term environmental exposure may occur due to leaching of contaminants from the final waste form at the disposal site. It is assumed that the stabilized waste will meet all regulatory requirements. However, there is a higher risk of release from the untreated, grout stabilized debris from System 1 . The long term stability of grout, and the ability of grout to stabilize organically contaminated debris, needs to be verified. In addition, the thermally desorbed solid matrices will retain the inorganic contaminants which will be stabilized with the treated solids. Some of these contaminants may be in the form of soluble salts that could be subject to long term leaching. Phosphate bonded ceramic and polyethylene are thought to have longer term stability than grout, but this needs to be verified.

The stabilized wastes are disposed of in an engineered structure that is secured to prevent contact with the surrounding biota. Releases of radionuclides or heavy metals from the stabilized waste are minimized by the disposal cell design described in Section 2.1.17.

\subsubsection{Minimize the Potential for Release of Hazardous and Radioactive Materials from} Final Disposal/Storage Products. Waste is stabilized to minimize the potential of releases which could 
harm the environment. Different stabilization approaches are selected to provide effective stabilization of a certain waste matrix. Polymer has been found to be best for soluble salts and all systems employ polymer stabilization for this waste. The EPA debris rule recommends grout for debris, which is the method selected for all systems. Soils are also grouted in all systems except System 4. Treated sludge and secondary wastes including soluble salts, are stabilized in polymer. In System 4, treated sludge, soils and secondary wastes, such as insoluble salts and oxides, are stabilized in phosphate bonded ceramic. This is a new approach which requires further evaluation to determine the potential for leaching hazardous or radioactive compounds.

In all cases, the INTS study includes design and operational features to meet DOE requirements. All waste forms must be acceptable to EPA, and all non-debris waste must pass TCLP tests. Waste failing TCLP tests will be reprocessed and restabilized. The long term stability of the waste forms chosen for this study must be verified for specific wastes and waste loadings.

As indicated above, System 1 has the maximum release potential because (1) untreated debris is stabilized in grout, and (2) heavy metals and radionuclides remain on the desorbed soils and sludges. Washing, as used in System 3, removes organics and soluble compounds of heavy metals and radionuclides. Thus, the potential for release is reduced. Chemical destruction of soft debris, as in Systems 4 and 5, decreases the disposal volume: In addition, the acids solubilize inorganic contaminants which are then precipitated as insoluble salts or oxides and removed, thereby decreasing the release potential. The removed inorganic compounds are stabilized in polymer or ceramic.

6.7.2.3 Minimize the Potential for Accidents in the System. All systems are designed to minimize the potential for accidents, and to mitigate the consequences if an accident occurs. More robust designs are required to prevent accidents with high temperature and pressure processes, and highly aggressive subsystems such as acid digestion and CWO. Labor requirements include sufficient personnel to adopt a buddy system approach. Accident conditions are assessed through an FMEA or a HAZOP study which require significantly more design and operational detail than is available at the preconceptual level of design used for this study. However, some general comments regarding upset or accident conditions for each of the systems is provided in subsections 6.2.1.3, 6.3.1.3, 6.4.1.3, 6.5.1.3 and 6.6.1.3.

\subsubsection{Minimize Waste Generation}

This principle has two components described in Section 1.

6.7.3.1 Minimize Creation of New Hazardous Material by Treatments. The INTS processes use chemicals to effect separations, contaminant removal, and organic destruction. In a thermal system, heat is the primary cause of organic destruction and volatile contaminant removal. In the INTS systems, the use of hazardous chemical reagents is minimized by extensive recycling of reagents and additives. Acids are used in the treatment processes to decompose the organic contaminants, and hydrochloric acid is generated during the treatment of chlorinated hydrocarbons. Although most of the acids are recovered and recycled, 
excess acids are neutralized with a caustic reagent (sodium hydroxide $[\mathrm{NaOH}]$ ). These excess acids occur from acid gas recovery in the APC subsystem, and from spent acids in the organic destruction processes.

During the oxidation processes some nitric acid decomposes to nitrous acid and $\mathrm{NO}_{\mathrm{x}}$, most of which are recovered as nitric acid and recycled. Heavy metals and radionuclides originally in the waste are precipitated from solutions and stabilized in polymer or phosphate bonded ceramic.

In System 1, the MEO process uses concentrated nitric acid, silver nitrate and iodine, all hazardous reagents. Other milder acids and bases are used to recover silver, including $\mathrm{NaOH}$ and hydrogen peroxide $\left(\mathrm{H}_{2} \mathrm{O}_{2}\right)$. The nitric acid and iodine are recovered and recycled. Silver, which is a RCRA metal, is used in the MEO process to oxidize organic contaminants. When treating chlorinated hydrocarbons, silver forms silver chloride $(\mathrm{AgCl})$, a very insoluble salt which precipitates from the solution. Developers claim that up to $99.9 \%$ of this silver may be recovered as silver nitrate $\left(\mathrm{AgNO}_{3}\right)$ from the $\mathrm{AgCl}$ precipitate. However, this has only been demonstrated in bench scale tests. Thus, a small amount of silver in the form of $\mathrm{AgCl}$ will leave the subsystem and require stabilization.

The CWO process in System 2 uses hydrochloric acid, ferric chloride, and metal catalysts to decompose the organic contaminants. Iodine is required for the process in which mercury is removed from soils, sludges and debris.

System 3 uses the same MEO reagents as System 1, and the washing processes use surfactants and additives which the developers claim are not known toxics. The surfactants are recovered and recycled, and spent surfactant is sent to organic destruction.

System 4 uses nitric and phosphoric acids as the organic destruction medium. Spent phosphoric acid is a result of acid digestion; it can be incorporated in the phosphate bonded ceramic. Any excess phosphoric acid is neutralized and the resulting salts stabilized prior to disposal. The washing processes generate spent surfactants as in System 3. Iodine is used to recover mercury after thermal desorption as in Systems 1 and 2.

System 5 uses CWO in place of acid digestion so the secondary waste resulting from this system is similar to that generated in Systems 2 and 4.

6.7.3.2 Minimize Volume or Amounts of Final Waste for Disposal or Storage. All systems recycle as much as possible to minimize waste which must be disposed of. Technologies which generate secondary waste are avoided. For example, activated carbon is used only as a backup to remove trace organics from offgases and wastewater. Organic destruction processes instead of carbon are used to treat both offgas and wastewater. This minimizes the generation of secondary waste. 
Solid, stabilized waste results from the waste treatment. As shown in Table 6-1, the waste volume reduction ranges from 5\% (System 1) to $24 \%$ (System 4). Lower waste volumes are produced by the systems that destroy soft debris (Systems 4 \& 5). Vitrification of waste would produce lower final volumes than do the nonthermal stabilization methods.

\subsubsection{Address Social Concerns}

This principle has six components.

6.7.4.1 Minimize Land Use for Disposal or Storage. This concern is addressed above in Section 6.7.3.2, minimizing the final volume of waste sent to disposal or storage.

6.7.4.2 Waste should be Disposed of in a Retrievable Manner. Retrievability depends on the configuration of the final waste form and the design of the disposal facility. The concept used in this study is to package the final waste forms in steel drums which are placed in concrete canisters. The canisters are stored in concrete vault cells which, when full, are capped with an earth layer that is engineered to withstand long-term environmental and weathering effects. Determination of the ability to retrieve the waste from such a disposal facility is beyond the scope of this study.

6.7.4.3 Stable Waste Forms for Long Term Storage and Disposal. This is consistent with the need to minimize the potential for release of hazardous and radioactive materials from final disposal or storage products discussed in Section 6.7.2.2.

6.7.4.4 Reflect Special Site Considerations. This study addresses a centralized facility at a generic, unspecified, site. Complex wide issues rather than site-specific issues are addressed.

6.7.4.5 Minimize Intersite Transportation. This study addresses a centralized facility at an undefined site. Transportation of reagents and waste to the facility from across the complex will be required. The INTS study assumption is that wastes will be treated on-site and disposed of on-site. With respect to transportation to the treatment facility, the number of tanker trucks transporting reagents to the facility per year is estimated in Table 6-8. The frequency of waste delivery to the site, assuming 44,000 pounds per truck, is 268 trucks per year.

6.7.4.6 Reflect Cultural Values. This is an engineering study. It is not intended as a means of establishing policy or site-specific decision making processes. Independent assessments of the cultural impact of treating wastes by the methods described in this study should be made by the tribes and stakeholders using the information provided in this report. 


\subsubsection{Adequacy of Information}

This principle has 12 components. Most of these components are not related to specific technical issues but are related to the process used to involve the TSWG in the study and to keep its members informed.

6.7.5.1 Provide Better Information on Health and Safety. Information on health and safety was developed during the course of this study and during the evaluation of the technologies used in the system designs. Health and safety issues are discussed in earlier sections, and a risk analysis of these systems is planned for a subsequent study.

6.7.5.2 Provide Better Information on Performance. Process performance has been discussed previously. The performance of the technologies for the treatment of DOE mixed waste is uncertain because many of the technologies are at the early pilot scale or bench scale level of development. When available, performance data has been used in the systems analysis. One of the purposes of this report is to provide an evaluation of the available information. Most of the nonthermal technologies have not been thoroughly tested on the variety of mixed wastes in the DOE complex.

\subsubsection{Focus on Existing Stored Waste; Add Projected Impact of Future Waste} Generation. This study focused on existing waste. However, the technologies and systems are also applicable to wastes that will be generated in the future.

6.7.5.4 Communicate Implementation Schedule. Technology needs were identified as part of this study and are discussed in Section 7. The times required for development of the technologies to the point where they are ready for implementation depends on the current technology maturity. This factor varies significantly for the various technologies used in these systems. A typical time frame for development, testing and demonstration, construction, and permitting for subsequent implementation is estimated at 7 to 10 years.

6.7.5.5 Criteria for Evaluating Systems Should be Flexibly Applied. The technical criteria had to be applied in a qualitative way for many aspects of the INTS systems. Qualitative analysis tends to be more flexible. In the system selection process, criteria were applied both qualitatively and, when data was available, quantitatively. Systems were selected to be representative of effective nonthermal systems. Flexibility did not extend to technologies that were not perceived to be effective.

6.7.5.6 Integrate ITTS and INTS for System Comparisons. Comparison of thermal systems evaluated in the ITTS study and nonthermal systems evaluated in the INTS study will be made in a subsequent study and presented in a separate report. 
6.7.5.7 Bridge ITTS and INTS to Use the Best Elements of Each. Hybrid systems that combine the best elements of thermal and nonthermal technologies will be the subject of a subsequent study.

6.7.5.8 Identify Planned Documentation. The preliminary plans for systems studies and associated documentation have been presented to the TSWG. More detailed plans will be presented at future TSWG meetings.

6.7.5.9 End Use of INTS and ITTS Studies Should be Known. These systems studies provide information for evaluation of technologies and treatment options by the DOE's Office of Environmental Restoration and Office of Waste Management managers. The results have not been available for a sufficient period of time to make an impact. At the completion of the INTS study considerably more data will be available to DOE decision makers at the sites and to their tribal, state, and local stakeholders.

6.7.5.10 Jdentify Decision Making Process and Participants. The INTS is an engineering study which compares performance and cost of nonthermal systems, but with no planned demonstration of hardware or treatment of waste. Decisions on the technologies and systems to be studied were made by the TSG, which included two technical members of the TSWG, and were presented to the full TSWG for its concurrence.

6.7.5.11 Inform TSWG of Responses to Suggestion. As part of the process of involving the TSWG in the study, updates to the study progress are sent periodically to the TSWG members. Meetings were held to present and discuss progress and results, and to respond to suggestions and comments.

6.7.5.12 Keep the TSWG Involved Throughout the Study. The TSWG continued to be involved in the ITTS and INTS studies until completion. Periodic progress reports were issued and meetings were held to present and discuss progress and results.

\subsection{EVALUATION SUMMARY}

All systems provide varying degrees of compliance with both the TSG technical criteria and the TSWG principles. The more aggressive treatments have the ability to decrease waste volumes by destroying more of the waste. Waste capable of complete breakdown include organic liquids, organic sludges, and soft or combustible debris. The consequences of oxidizing more of the organic waste is higher offgas volumes. Also, the more aggressive technologies have less performance risk but require more robust and costly designs to mitigate safety risk.

For all systems, worker and environmental risk, as well as the potential of contaminant release in the effluent, is minimized by proper system design and operation. 
The long term stability of the waste forms is dependent on effective stabilizing operation and proper loading factors of stabilizing agents. Long term stability must be measured after wastes are produced. At this stage of design, this information is not available. 
Table 6-1. Technical criteria comparison for process performance (Sheet 1 of 3 ).

\begin{tabular}{|c|c|c|c|c|c|}
\hline Criteria & $\begin{array}{c}\text { Grout Debris } \\
\text { System } 1\end{array}$ & $\begin{array}{l}\text { Desorption } \\
\text { System } 2 \\
\end{array}$ & $\begin{array}{c}\text { Wash } \\
\text { System } 3\end{array}$ & $\begin{array}{c}\text { Acid Digestion } \\
\text { System } 4\end{array}$ & $\begin{array}{c}\text { CWO } \\
\text { System } 5\end{array}$ \\
\hline Effectiveness & $\begin{array}{l}\text { If significant debris } \\
\text { wastes are } \\
\text { contaminated with } \mathrm{Hg} \text {, } \\
\text { stabilization will not be } \\
\text { acceptable. The } \\
\text { effectiveness of debris } \\
\text { stabilization in grout is } \\
\text { unknown. }\end{array}$ & $\begin{array}{l}\text { Better than System } 1 \\
\text { because debris is } \\
\text { treated for organics and } \\
\mathrm{Hg} \text {. CWO has } \\
\text { reported } 99.9 \% \\
\text { destruction efficiency. } \\
\text { Ability to treat sludge } \\
\text { is unknown. }\end{array}$ & $\begin{array}{l}\text { Organic removal by } \\
\text { washing reported as } \\
99.99 \% \text {, metals } \\
\text { removal of } 96 \% \text { for } \\
\text { soils. Ability to treat } \\
\text { sludge is unknown. }\end{array}$ & $\begin{array}{l}\text { Acid digestion } \\
\text { destruction efficiency } \\
\text { of } 99.5 \% \text { on some } \\
\text { wastes. Response to } \\
\text { complex feeds is } \\
\text { unknown. } \\
\text { Combinations of } \\
\text { technologies should } \\
\text { provide high } \\
\text { effectiveness. }\end{array}$ & $\begin{array}{l}\text { CWO has reported } \\
99.9 \% \text { destruction } \\
\text { efficiency. } \\
\text { Combinations of } \\
\text { technologies should } \\
\text { provide high } \\
\text { effectiveness. }\end{array}$ \\
\hline Flexibility & $\begin{array}{l}\text { Allows variations in } \\
\text { feed; sorting debris is } \\
\text { not required. }\end{array}$ & $\begin{array}{l}\text { Similar to System 1; } \\
\text { debris is treated but } \\
\text { separation of plastics is } \\
\text { required. Sorting } \\
\text { requirements less than } \\
\text { Systems } 3,4 \& 5 \text {. }\end{array}$ & $\begin{array}{l}\text { Very flexible because } \\
\text { of multiple approaches } \\
\text { - MEO and two } \\
\text { washing subsystems. }\end{array}$ & $\begin{array}{l}\text { Highly flexible due to } \\
\text { various treatment } \\
\text { subsystems. Variations } \\
\text { in contaminants can be } \\
\text { dealt with but acid } \\
\text { digestion needs } \\
\text { demonstration. }\end{array}$ & $\begin{array}{l}\text { Highly flexible with } \\
\text { various treatment } \\
\text { subsystems. Variations } \\
\text { in contaminants can be } \\
\text { dealt with. } \\
\text { Concentration increases } \\
\text { may require decreased } \\
\text { feed rates. }\end{array}$ \\
\hline Versatility & $\begin{array}{l}\text { Less versatile if debris } \\
\text { does not meet treatment } \\
\text { standards. MEO } \\
\text { cannot treat soft debris. }\end{array}$ & $\begin{array}{l}\text { More versatile than } \\
\text { System } 1 \text { because } \\
\text { debris is treated. CWO } \\
\text { can treat soft debris; } \\
\text { this is more versatile } \\
\text { than MEO. }\end{array}$ & $\begin{array}{l}\text { High versatility because } \\
\text { washing is less specific } \\
\text { to the waste type than } \\
\text { other treatment } \\
\text { methods. Ability to } \\
\text { treat process residues is } \\
\text { unknown. }\end{array}$ & $\begin{array}{l}\text { Combinations of } \\
\text { treatment subsystems } \\
\text { are designed to treat all } \\
\text { entering waste. }\end{array}$ & $\begin{array}{l}\text { Combinations of } \\
\text { treatment subsystems } \\
\text { are designed to treat all } \\
\text { entering waste. CWO } \\
\text { can treat organic liquids } \\
\text { and solids. }\end{array}$ \\
\hline
\end{tabular}


Table 6-1. Technical criteria comparison for process performance (Sheet 2 of 3).

\begin{tabular}{|c|c|c|c|c|c|}
\hline Criteria & $\begin{array}{c}\text { Grout Debris } \\
\text { System } 1\end{array}$ & $\begin{array}{l}\text { Desorption } \\
\text { System } 2\end{array}$ & $\begin{array}{c}\text { Wash } \\
\text { System } 3\end{array}$ & $\begin{array}{c}\text { Acid Digestion } \\
\text { System } 4\end{array}$ & $\begin{array}{c}\text { CWO } \\
\text { System } 5\end{array}$ \\
\hline Availability & $\begin{array}{l}\text { Expected to be the } \\
\text { highest. MEO cells in } \\
\text { parallel so shutdown } \\
\text { not required for } \\
\text { maintenance. Desorber } \\
\text { larger than required. }\end{array}$ & $\begin{array}{l}\text { Somewhat less than for } \\
\text { System } 1 \text { due to single } \\
\text { CWO reactor, more } \\
\text { downtime due to highly } \\
\text { corrosive reagents, and } \\
\text { a single desorber for } \\
\text { multiple waste streams. }\end{array}$ & $\begin{array}{l}\text { Excellent because } \\
\text { washing is a proven } \\
\text { approach and consists } \\
\text { of commercial } \\
\text { equipment. Similar to } \\
\text { System 1. }\end{array}$ & $\begin{array}{l}\text { Acid digestion is very } \\
\text { immature, more } \\
\text { downtime due to } \\
\text { corrosive reagents, but } \\
\text { separate reactors for } \\
\text { organics and soft debris } \\
\text { increase availability. }\end{array}$ & $\begin{array}{l}\text { Availability reduced } \\
\text { due to high temperature } \\
\text { and pressure, and } \\
\text { highly corrosive } \\
\text { reagents. Separate } \\
\text { reactors for organics } \\
\text { and soft debris increase } \\
\text { availability. }\end{array}$ \\
\hline Reliability & $\begin{array}{l}\text { Similar to chemical } \\
\text { processing facility and } \\
\text { expected to be } \\
\text { excellent. }\end{array}$ & $\begin{array}{l}\text { Single reactor with } \\
\text { extensive process } \\
\text { controls - must be } \\
\text { demonstrated. }\end{array}$ & $\begin{array}{l}\text { Excellent because } \\
\text { washing is a proven } \\
\text { approach and consists } \\
\text { of commercial } \\
\text { equipment. Similar to } \\
\text { System } 1 \text {. }\end{array}$ & $\begin{array}{l}\text { Separate reactors for } \\
\text { organics and soft debris } \\
\text { increase reliability. } \\
\text { Wash systems are } \\
\text { commercial and } \\
\text { desorption is simple. }\end{array}$ & $\begin{array}{l}\text { Separate reactors for } \\
\text { organics and soft debris } \\
\text { increase reliability. } \\
\text { Wash systems are } \\
\text { commercial and } \\
\text { desorption is simple. }\end{array}$ \\
\hline Maintainability* & $\begin{array}{l}\text { Less maintenance than } \\
\text { other systems due to } \\
\text { less size reduction } \\
\text { needs for debris. MEO } \\
\text { cells maintained } \\
\text { off-line. }\end{array}$ & $\begin{array}{l}\text { More maintenance due } \\
\text { to high temperature and } \\
\text { pressure, corrosive } \\
\text { reagents, and increased } \\
\text { size reduction needs for } \\
\text { debris. }\end{array}$ & $\begin{array}{l}\text { MEO cells maintained } \\
\text { off line. Washing uses } \\
\text { commercial equipment } \\
\text { which is easily } \\
\text { maintained. }\end{array}$ & $\begin{array}{l}\text { Depends on corrosion } \\
\text { control for acid } \\
\text { digestion. Increased } \\
\text { size reduction needs for } \\
\text { debris. }\end{array}$ & $\begin{array}{l}\text { Depends on corrosion } \\
\text { control for CWO. } \\
\text { Increased size reduction } \\
\text { needs for debris. }\end{array}$ \\
\hline $\begin{array}{l}\text { Hazardous Waste } \\
\text { Generation }\end{array}$ & $\begin{array}{l}\text { Contaminated debris is } \\
\text { stabilized; } \\
\text { approximately } 0.1 \% \text { of } \\
\text { the silver is stabilized } \\
\text { and disposed of. }\end{array}$ & $\begin{array}{l}\text { Acids are neutralized } \\
\text { and the salts containing } \\
\text { heavy metais and } \\
\text { radionuclides are } \\
\text { stabilized. }\end{array}$ & $\begin{array}{l}\text { Approximately } 0.1 \% \\
\text { of the silver from MEO } \\
\text { is stabilized and } \\
\text { disposed of. }\end{array}$ & $\begin{array}{l}\text { Phosphoric acid is } \\
\text { generated - excess acid } \\
\text { is neutralized and the } \\
\text { salts stabilized and } \\
\text { disposed of. }\end{array}$ & $\begin{array}{l}\text { Acids are neutralized } \\
\text { and the salts containing } \\
\text { heavy metals and } \\
\text { radionuclides are } \\
\text { stabilized. }\end{array}$ \\
\hline
\end{tabular}


Table 6-1. Technical criteria comparison for process performance (Sheet 3 of 3).

\begin{tabular}{|c|c|c|c|c|c|}
\hline Criteria & $\begin{array}{c}\text { Grout Debris } \\
\text { System } 1\end{array}$ & $\begin{array}{l}\text { Desorption } \\
\text { System } 2\end{array}$ & $\begin{array}{c}\text { Wash } \\
\text { System } 3\end{array}$ & $\begin{array}{l}\text { Acid Digestion } \\
\text { System } 4\end{array}$ & $\begin{array}{c}\text { CWO } \\
\text { System } 5\end{array}$ \\
\hline $\begin{array}{l}\text { Byproducts and } \\
\text { Residuals }\end{array}$ & $\begin{array}{l}\text { Lowest except for } \\
\text { stabilized solids. }\end{array}$ & $\begin{array}{l}\text { Precipitated metals and } \\
\text { neutralized acid. }\end{array}$ & $\begin{array}{l}\text { Precipitated metals and } \\
\text { waste surfactant. }\end{array}$ & $\begin{array}{l}\text { Precipitated metals and } \\
\text { waste surfactant. }\end{array}$ & $\begin{array}{l}\text { Precipitated metals and } \\
\text { waste surfactant. }\end{array}$ \\
\hline gas residuals $(\mathrm{lb} / \mathrm{hr})$ & & 823 & 827 & 1,441 & 1,433 \\
\hline water residuals & 59 & 135 & 176 & 780 & 564 \\
\hline $\begin{array}{r}\text { solid residuals } \\
(\mathrm{b} / \mathrm{hr})\end{array}$ & 4,675 & 4,616 & 4,468 & 3,664 & 3,819 \\
\hline (vol in/vol out) & $\begin{array}{l}\text { Slight volume } \\
\text { reduction. } \\
1.05\end{array}$ & $\begin{array}{r}\text { Slight volume } \\
\text { reduction. } \\
1.06 \\
\end{array}$ & $\begin{array}{l}\text { Slight volume } \\
\text { reduction. } \\
1.10\end{array}$ & $\begin{array}{l}\text { Significant reduction } \\
\text { due to soft debris } \\
\text { treatment. } \\
\qquad 1.19\end{array}$ & $\begin{array}{l}\text { Significant reduction } \\
\text { due to soft debris } \\
\text { treatment. } \\
\qquad 1.24\end{array}$ \\
\hline $\begin{array}{l}\text { Final Waste Form } \\
\text { Volume }\left(\mathrm{ft}^{3} / \mathrm{hr}\right) \\
\text { \& Contaminant } \\
\text { Loading }\end{array}$ & $\begin{array}{l}\qquad 42.46 \\
\text { Large disposal volume } \\
\text { and highest } \\
\text { contaminant loading on } \\
\text { untreated debris. }\end{array}$ & $\begin{array}{l}\quad 42.2 \\
\text { Soft debris volatilizes } \\
\text { in the desorber } \\
\text { generating more offgas } \\
\text { but less solids. Heavy } \\
\text { metals and } \\
\text { radionuclides remain } \\
\text { with the waste. }\end{array}$ & $\begin{array}{l}\qquad 40.77 \\
\text { Surfactants add to the } \\
\text { waste, but soluble } \\
\text { metals and } \\
\text { radionuclides are } \\
\text { removed, precipitated, } \\
\text { and sent to polymer. }\end{array}$ & $\begin{array}{l}\qquad 37.55 \\
\text { Low waste volume due } \\
\text { to destruction of soft } \\
\text { debris. Contaminant } \\
\text { loadings similar to } \\
\text { System 3. }\end{array}$ & $\begin{array}{l}\qquad 36.04 \\
\text { Low waste volume due } \\
\text { to destruction of soft } \\
\text { debris. Contaminant } \\
\text { loadings similar to } \\
\text { System 3. }\end{array}$ \\
\hline
\end{tabular}

J: linellints lcostloutputsitable6_1sheet

*Note: All systems require maintenance on UV lamps and windows of the photooxidation processors. 
Table 6-2. Technical criteria comparison for environmental impact.

\begin{tabular}{|c|c|c|c|c|c|}
\hline Criteria & $\begin{array}{c}\text { Grout Debris } \\
\text { System } 1 \\
\end{array}$ & $\begin{array}{c}\text { Desorption } \\
\text { System } 2 \\
\end{array}$ & $\begin{array}{c}\text { Wash } \\
\text { System } 3\end{array}$ & $\begin{array}{c}\text { Acid Digestion } \\
\text { System } 4 \\
\end{array}$ & $\begin{array}{c}\text { CWO } \\
\text { System } 5\end{array}$ \\
\hline $\begin{array}{l}\text { Airborne releases } \\
\text { (lb/hr) }\end{array}$ & 696 & 823 & 827 & 1,441 & 1,433 \\
\hline $\begin{array}{l}\text { Wastewater releases } \\
\text { (lb/hr) }\end{array}$ & 59 & 135 & 176 & 780 & 564 \\
\hline $\begin{array}{l}\text { Final waste form } \\
\text { performance }\end{array}$ & $\begin{array}{l}\text { Long term stability of } \\
\text { organically } \\
\text { contaminated debris in } \\
\text { grout may be a } \\
\text { problem. }\end{array}$ & $\begin{array}{l}\text { Fewer contaminants in } \\
\text { stabilized waste } \\
\text { compared to } \\
\text { System } 1 .\end{array}$ & $\begin{array}{l}\text { Separation of inorganic } \\
\text { contaminants which are } \\
\text { stabilized in polymer, } \\
\text { a more stable waste } \\
\text { form than grout. } \\
\text { Treated soil is grouted. }\end{array}$ & $\begin{array}{l}\text { Ceramic is perceived to } \\
\text { have improved } \\
\text { performance over } \\
\text { grout. }\end{array}$ & $\begin{array}{l}\text { Grout is perceived to } \\
\text { be less stable than } \\
\text { polymer or ceramic. }\end{array}$ \\
\hline Ecological effects & $\begin{array}{l}\text { Lowest gaseous and } \\
\text { aqueous emissions. } \\
\text { Solids disposed of in an } \\
\text { engineered structure to } \\
\text { prevent release. }\end{array}$ & $\begin{array}{l}\text { Gaseous discharge } \\
\text { higher due to } \\
\text { volatization of soft } \\
\text { debris and destruction } \\
\text { of desorbed organics. }\end{array}$ & $\begin{array}{l}\text { Similar to Systems } \\
1 \& 2 \text {. Extensive } \\
\text { recycling of water } \\
\text { required for washing. }\end{array}$ & $\begin{array}{l}\text { Similar to other } \\
\text { systems. Higher offgas } \\
\text { due to soft debris } \\
\text { destruction. Organic } \\
\text { destruction generates } \\
\text { more water than } \\
\text { required. }\end{array}$ & $\begin{array}{l}\text { Similar to other } \\
\text { systems. Higher offgas } \\
\text { due to soft debris } \\
\text { destruction. Organic } \\
\text { destruction generates } \\
\text { more water than } \\
\text { required. }\end{array}$ \\
\hline
\end{tabular}

Note: All final waste forms are expected to conform to LDR's; this requires verification. 
Table 6-3. Technical criteria comparison for worker health and safety (Sheet 1 of 3).

\begin{tabular}{|c|c|c|c|c|c|}
\hline Criteria & $\begin{array}{c}\text { Grout Debris } \\
\text { System } 1 \\
\end{array}$ & $\begin{array}{c}\text { Desorption } \\
\text { System } 2 \\
\end{array}$ & $\begin{array}{c}\text { Wash } \\
\text { System } 3 \\
\end{array}$ & $\begin{array}{c}\text { Acid Digestion } \\
\text { System } 4 \\
\end{array}$ & $\begin{array}{c}\text { CWO } \\
\text { System } 5 \\
\end{array}$ \\
\hline $\begin{array}{l}\text { Hazardous } \\
\text { Reagents }\end{array}$ & $\begin{array}{c}\text { Concentrated nitric acid } \\
\text { and silver nitrate, } \\
\text { iodine, sodium } \\
\text { hydroxide, and } \\
\text { hydrogen peroxide. }\end{array}$ & $\begin{array}{l}\text { Hydrochloric acid, } \\
\text { iodine, sodium } \\
\text { hydroxide, and } \\
\text { hydrogen peroxide. }\end{array}$ & $\begin{array}{c}\text { Concentrated nitric acid } \\
\text { and silver nitrate, } \\
\text { sodium hydroxide, and } \\
\text { hydrogen peroxide. }\end{array}$ & $\begin{array}{c}\text { Concentrated nitric and } \\
\text { phosphoric acids, } \\
\text { iodine, sodium } \\
\text { hydroxide, and } \\
\text { hydrogen peroxide. }\end{array}$ & $\begin{array}{l}\text { Hydrochloric acid, } \\
\text { iodine, sodium } \\
\text { hydroxide, and } \\
\text { hydrogen peroxide. }\end{array}$ \\
\hline $\begin{array}{l}\text { Hazardous } \\
\text { Operating } \\
\text { Conditions }\end{array}$ & $\begin{array}{c}\text { Ambient temperature } \\
\text { and atmospheric } \\
\text { pressure MEO process. } \\
\text { Slightly less than for } \\
\text { other systems because of } \\
\text { less requirements for } \\
\text { debris sorting. }\end{array}$ & $\begin{array}{l}\text { Highest temperature and } \\
\text { pressure CWO process } \\
\text { and aggressive } \\
\text { corrosives. More } \\
\text { hazardous than MEO. } \\
\text { More debris sorting but } \\
\text { less exposure than } \\
\text { System } 1 \text { in grout } \\
\text { subsystem. }\end{array}$ & $\begin{array}{c}\text { Safest system. Low } \\
\text { temperature and } \\
\text { pressure, less aggressive } \\
\text { reagents. More debris } \\
\text { sorting but less. exposure } \\
\text { than System } 1 \text { in grout } \\
\text { subsystem. }\end{array}$ & $\begin{array}{l}\text { High temperature and } \\
\text { medium pressure and } \\
\text { aggressive corrosives. } \\
\text { More hazardous than } \\
\text { MEO but possibly less } \\
\text { hazardous than CWO. } \\
\text { Less exposure in grout } \\
\text { subsystem due to debris } \\
\text { treatment. More debris } \\
\text { sorting. }\end{array}$ & $\begin{array}{l}\text { Highest temperature and } \\
\text { pressure and most } \\
\text { aggressive reagents in } \\
\text { CWO process. More } \\
\text { hazardous than MEO. } \\
\text { More debris sorting. } \\
\text { Less exposure than } \\
\text { System } 1 \text { in grout } \\
\text { subsystem. }\end{array}$ \\
\hline $\begin{array}{l}\text { Hazardous } \\
\text { Process } \\
\text { Equipment }\end{array}$ & $\begin{array}{c}\text { Shredder, electrolytic } \\
\text { cells, and high voltage } \\
\text { GPCR. }\end{array}$ & $\begin{array}{l}\text { Shredder, highly } \\
\text { corrosive reagents and } \\
\text { high voltage GPCR. }\end{array}$ & $\begin{array}{c}\text { Shredder, electrolytic } \\
\text { cells, and high voltage } \\
\text { GPCR. }\end{array}$ & $\begin{array}{c}\text { Shredder, highly } \\
\text { corrosive reagents and } \\
\text { high voltage GPCR. }\end{array}$ & $\begin{array}{l}\text { Shredder, highly } \\
\text { corrosive reagents and } \\
\text { high voltage GPCR. }\end{array}$ \\
\hline Process controls & $\begin{array}{c}\text { Standard industrial } \\
\text { practice improved for } \\
\text { radioactive service. } \\
\text { Simplest of all systems. }\end{array}$ & $\begin{array}{c}\text { Standard industrial } \\
\text { practice improved for } \\
\text { radioactive service, but } \\
\text { more sophisticated than } \\
\text { System } 1 .\end{array}$ & $\begin{array}{l}\text { Complex due to } \\
\text { extensive recycling, but } \\
\text { standard industrial } \\
\text { practice improved for } \\
\text { radioactive service. }\end{array}$ & $\begin{array}{c}\text { Standard industrial } \\
\text { practice improved for } \\
\text { radioactive service - } \\
\text { similar to System } 2 \text { but } \\
\text { with two acid digesters. }\end{array}$ & $\begin{array}{l}\text { Standard industrial } \\
\text { practice improved for } \\
\text { radioactive service - } \\
\text { similar to System } 2 \text { but } \\
\text { with two CWO reactors. }\end{array}$ \\
\hline
\end{tabular}


Table 6-3. Technical criteria comparison for worker health and safety (Sheet 2 of 3).

\begin{tabular}{|c|c|c|c|c|c|}
\hline Criteria & $\begin{array}{c}\text { Grout Debris } \\
\text { System } 1 \\
\end{array}$ & $\begin{array}{c}\text { Desorption } \\
\text { System } 2 \\
\end{array}$ & $\begin{array}{c}\text { Wash } \\
\text { System } 3 \\
\end{array}$ & $\begin{array}{c}\text { Acid Digestion } \\
\text { System } 4 \\
\end{array}$ & $\begin{array}{c}\text { CWO } \\
\text { System } 5 \\
\end{array}$ \\
\hline $\begin{array}{l}\text { Off-normal } \\
\text { Conditions, } \\
\text { Probabilities \& } \\
\text { Consequences* }\end{array}$ & \begin{tabular}{|} 
Sudden high organic \\
input may cause increase \\
in offgas, MEO \\
temperature \& reaction \\
rate. Low \\
consequences involving \\
less effective treatment - \\
self correcting.
\end{tabular} & $\begin{array}{l}\text { Sudden high organic } \\
\text { input may cause increase } \\
\text { in offgas, CWO } \\
\text { temperature \& reaction } \\
\text { rate - higher potential } \\
\text { for runaway reaction. } \\
\end{array}$ & \begin{tabular}{|c|} 
Fewest consequences. \\
Sudden high organic \\
input may cause increase \\
in offgas, MEO \\
temperature \& reaction \\
rate. Low consequences \\
involving less effective \\
treatment - self \\
correcting.
\end{tabular} & \begin{tabular}{|c} 
Sudden high organic \\
input may cause increase \\
in offgas, acid \\
temperature \& reaction \\
rate - lower potential of \\
runaway reaction than \\
CWO due to self \\
correcting processes.
\end{tabular} & $\begin{array}{l}\text { Sudden high organic } \\
\text { input may cause increase } \\
\text { in offgas, CWO } \\
\text { temperature \& reaction } \\
\text { rate - higher potential } \\
\text { for runaway reaction. }\end{array}$ \\
\hline $\begin{array}{l}\text { Upset and } \\
\text { Accident } \\
\text { Conditions, } \\
\text { Probabilities \& } \\
\text { Consequences* }\end{array}$ & $\begin{array}{l}\text { Sudden high organic } \\
\text { input may cause } \\
\text { temperature and } \\
\text { pressure excursions - } \\
\text { low consequences and } \\
\text { self correcting. }\end{array}$ & $\begin{array}{l}\text { Sudden high organic } \\
\text { input may cause } \\
\text { temperature and } \\
\text { pressure excursions - } \\
\text { may be more prevalent } \\
\text { in aggressive CWO } \\
\text { process. }\end{array}$ & $\begin{array}{l}\text { Sudden high organic } \\
\text { input may cause } \\
\text { temperature and } \\
\text { pressure excursions - } \\
\text { low consequences and } \\
\text { self correcting. }\end{array}$ & $\begin{array}{l}\text { Sudden high organic } \\
\text { input may cause } \\
\text { temperature and } \\
\text { pressure excursions - } \\
\text { lower potential of } \\
\text { runaway reaction than } \\
\text { CWO due to self } \\
\text { correcting processes. }\end{array}$ & $\begin{array}{l}\text { Sudden high organic } \\
\text { input may cause } \\
\text { temperature and } \\
\text { pressure excursions - } \\
\text { may be more prevalent } \\
\text { in aggressive CWO } \\
\text { process. }\end{array}$ \\
\hline $\begin{array}{l}\text { Process } \\
\text { Containment** }\end{array}$ & $\begin{array}{l}\text { Low pressure MEO and } \\
\text { all other subsystems are } \\
\text { easy to contain. }\end{array}$ & \begin{tabular}{|} 
High pressure and \\
corrosive CWO - most \\
difficult to achieve. \\
More robust safety \\
features needed than for \\
System 1 including \\
double containment.
\end{tabular} & $\begin{array}{c}\text { Low pressure MEO and } \\
\text { all other subsystems are } \\
\text { easy to contain. }\end{array}$ & $\begin{array}{l}\text { Medium pressure and } \\
\text { corrosive acid digestion } \\
\text { - more difficult to } \\
\text { achieve than MEO. } \\
\text { More robust safety } \\
\text { features needed than for } \\
\text { System } 1 \text { including } \\
\text { double containment. }\end{array}$ & $\begin{array}{l}\text { High pressure and } \\
\text { corrosive CWO - most } \\
\text { difficult to achieve. } \\
\text { More robust safety } \\
\text { features needed than for } \\
\text { System } 1 \text { including } \\
\text { double containment. }\end{array}$ \\
\hline
\end{tabular}


Table 6-3. Technical criteria comparison for worker health and safety (Sheet 3 of 3).

\begin{tabular}{|c|c|c|c|c|c|}
\hline Criteria & $\begin{array}{c}\text { Grout Debris } \\
\text { System 1 }\end{array}$ & $\begin{array}{l}\text { Desorption } \\
\text { System } 2 \\
\end{array}$ & $\begin{array}{c}\text { Wash } \\
\text { System } 3\end{array}$ & $\begin{array}{c}\text { Acid Digestion } \\
\text { System } 4\end{array}$ & $\begin{array}{c}\text { CWO } \\
\text { System } 5\end{array}$ \\
\hline $\begin{array}{l}\text { Maintenance } \\
\text { Exposure }\end{array}$ & $\begin{array}{l}\text { Smaller than other } \\
\text { systems. Replace } \\
\text { electrolytic cells and } \\
\text { repair other MEO } \\
\text { equipment off line to } \\
\text { minimize exposure, } \\
\text { replace UV lamps, } \\
\text { unjam the shredder, } \\
\text { Higher exposure in } \\
\text { grouting subsystem. }\end{array}$ & $\begin{array}{l}\text { Higher than System } 1 \\
\text { due to corrosive } \\
\text { conditions, replace UV } \\
\text { lamps, unjam the } \\
\text { shredder. Lower } \\
\text { exposure than System } 1 \\
\text { in grout subsystem. }\end{array}$ & $\begin{array}{l}\text { Least of any system - } \\
\text { commercial components } \\
\text { are used. Replace } \\
\text { electrolytic cells, replace } \\
\text { UV lamps, unjam the } \\
\text { shredder. Lower } \\
\text { exposure than System } 1 \\
\text { in grout subsystem. }\end{array}$ & $\begin{array}{l}\text { Higher than System } 1 \\
\text { due to corrosive } \\
\text { conditions, replace UV } \\
\text { lamps, unjam the } \\
\text { shredder. Lower } \\
\text { exposure than System } 1 \\
\text { in grout subsystem. } \\
\text { More maintenance } \\
\text { because of multiple } \\
\text { subsystems. }\end{array}$ & $\begin{array}{c}\text { Similar to Systems } 2 \text { and } \\
\text { 4. Two CWO } \\
\text { subsystems increase risk } \\
\text { to workers. }\end{array}$ \\
\hline
\end{tabular}

Notes: $\quad$ * Probabilities and consequences have not been determined for these systems; more detailed design and an FMEA or HAZOP study is required.

** Systems have triple, double, or single containment depending on the class of radionuclides being processed. 
Table 6-4. Technical criteria comparison for cost.

\begin{tabular}{l|r|r|r|r|r}
\hline Criteria & $\begin{array}{l}\text { Grout Debris } \\
\text { System 1 }\end{array}$ & $\begin{array}{c}\text { Desorption } \\
\text { System 2 }\end{array}$ & $\begin{array}{c}\text { Wash } \\
\text { System 3 }\end{array}$ & $\begin{array}{c}\text { Acid Digestion } \\
\text { System 4 }\end{array}$ & $\begin{array}{c}\text { CWO } \\
\text { System 5 }\end{array}$ \\
\hline & & & & & \\
$\begin{array}{l}\text { Research and } \\
\text { Development Cost }\end{array}$ & $\$ 134$ & $\$ 142$ & $\$ 143$ & $\$ 208$ & $\$ 167$ \\
\hline Total TLCC & $\$ 3,721$ & $\$ 3,657$ & J:Vinellintslcostlcompare.wk31table6_3sheet & $\$ 3,876$ & $\$ 3,816$ \\
\hline
\end{tabular}


Table 6-5. Technical criteria comparison for acceptability (Sheet 1 of 2).

\begin{tabular}{|c|c|c|c|c|c|}
\hline Criteria & $\begin{array}{l}\text { Grout Debris } \\
\text { System } 1\end{array}$ & $\begin{array}{l}\text { Desorption } \\
\text { System } 2 \\
\end{array}$ & $\begin{array}{c}\text { Wash } \\
\text { System } 3\end{array}$ & $\begin{array}{c}\text { Acid Digestion } \\
\text { System } 4 \\
\end{array}$ & $\begin{array}{c}\text { CWO } \\
\text { System } 5\end{array}$ \\
\hline Complexity & $\begin{array}{l}\text { Considered least complex } \\
\text { with minimum shredding } \\
\text { although MEO is more } \\
\text { complex than other organic } \\
\text { destruction processes. }\end{array}$ & $\begin{array}{l}\text { Only slightly more complex than } \\
\text { System 1. CWO is conceptually } \\
\text { simpler than MEO with fewer } \\
\text { operations. Other subsystems } \\
\text { are similar to those in System } 1 \text {. }\end{array}$ & $\begin{array}{l}\text { More complex than System } 2 \\
\text { with } 3 \text { more subsystems than } \\
\text { System } 1 \text {. More extensive } \\
\text { water treatment and } \\
\text { recycling. However, simple } \\
\text { to operate. }\end{array}$ & $\begin{array}{l}\text { With many subsystems, } \\
\text { this is considerably more } \\
\text { complex than System } 1 \text {. } \\
\text { Acid digestion is } \\
\text { conceptually simpler than } \\
\text { MEO with fewer } \\
\text { operations. }\end{array}$ & $\begin{array}{l}\text { Complexity similar to } \\
\text { System } 4 \text {. }\end{array}$ \\
\hline $\begin{array}{l}\text { Technology } \\
\text { Development Status }\end{array}$ & $\begin{array}{l}\text { Parts of MEO are near } \\
\text { commercial stage; silver } \\
\text { recovery is at laboratory } \\
\text { scale; GPCR, desorber and } \\
\text { polymer at pilot scale; } \\
\text { photooxidation is proven } \\
\text { and grout is well known. }\end{array}$ & $\begin{array}{l}\text { Except for CWO, this is the } \\
\text { same as for System } 1 \text {. CWO } \\
\text { has had extensive bench scale } \\
\text { testing, and a pilot system is } \\
\text { designed and planned for } \\
\text { demonstration. }\end{array}$ & $\begin{array}{l}\text { MEO and other subsystems } \\
\text { were discussed with System } \\
\text { 1. Wash subsystems use } \\
\text { commercial equipment, but } \\
\text { development work is } \\
\text { necessary for scale-up and } \\
\text { demonstration. }\end{array}$ & $\begin{array}{l}\text { Very immature technolgy } \\
\text { for organic and soft debris } \\
\text { treatment and stabilization. } \\
\text { Significant development } \\
\text { required. }\end{array}$ & $\begin{array}{l}\text { Similar to System } 2 \text { with } \\
\text { addition of washing. } \\
\text { CWO is ready for pilot } \\
\text { scale demonstration, but } \\
\text { washing is new and } \\
\text { requires scale-up and } \\
\text { demonstration. }\end{array}$ \\
\hline Site Expanse & $\begin{array}{l}\text { Systems } 1 \text { and } 2 \text { have } \\
\text { smallest building size. } \\
\text { Building cost is } \$ 126.77 \\
\text { million. }\end{array}$ & $\begin{array}{l}\text { Building size is about the same } \\
\text { as System } 1 \text { with a cost of } \\
\$ 117.91 \text { million. }\end{array}$ & $\begin{array}{l}\text { Facility is larger than for } \\
\text { Systems } 1 \text { or } 2 \text {. Building } \\
\text { cost is } \$ 135.45 \text { million. }\end{array}$ & $\begin{array}{l}\text { Building cost is larger than } \\
\text { systems } 1,2 \text {, and } 3 \text {. } \\
\text { Building cost is } \$ 147.18 \\
\text { million. }\end{array}$ & $\begin{array}{l}\text { Building cost is larger than } \\
\text { systems } 1,2 \text {, and } 3 \text {. } \\
\text { Building cost is } \$ 141.16 \\
\text { million. }\end{array}$ \\
\hline Resource Utilization & $\begin{array}{l}\text { Highest for the MEO } \\
\text { system due to electrical } \\
\text { energy expended. Overall } \\
\text { utility costs are less than } \\
\text { for most other systems at } \\
\$ 1.07 \text { million. }\end{array}$ & $\begin{array}{l}\text { Utility Cost is slightly higher } \\
\text { than for System } 1 \text {. Utility cost } \\
\text { is } \$ 1.13 \text { million. }\end{array}$ & $\begin{array}{l}\text { Resource utilization is } \\
\text { slightly higher due to } \\
\text { additives for washing. } \\
\text { Utility cost is } \$ 1.21 \text { million. }\end{array}$ & $\begin{array}{l}\text { Resource utilization is } \\
\text { higher than the previous } \\
\text { systems. Utility cost is } \\
\$ 1.37 \text { million. }\end{array}$ & $\begin{array}{l}\text { Resource utilization is } \\
\text { highest of all systems. } \\
\text { Utility cost is } \$ 1.87 \\
\text { million. }\end{array}$ \\
\hline
\end{tabular}


Table 6-5. Technical criteria comparison for acceptability (Sheet 2 of 2 ).

\begin{tabular}{|c|c|c|c|c|c|}
\hline Criteria & $\begin{array}{c}\text { Grout Debris } \\
\text { System } 1\end{array}$ & $\begin{array}{l}\text { Desorption } \\
\text { System } 2 \\
\end{array}$ & $\begin{array}{c}\text { Wash } \\
\text { System } 3\end{array}$ & $\begin{array}{c}\text { Acid Digestion } \\
\text { System } 4 \\
\end{array}$ & $\begin{array}{c}\text { CWO } \\
\text { System } 5\end{array}$ \\
\hline Schedule & $\begin{array}{l}\text { Shorter than for other } \\
\text { systems due to extensive } \\
\text { pilot and bench scale work } \\
\text { on most subsystem } \\
\text { components. }\end{array}$ & $\begin{array}{l}\text { Similar to System } 1 \text { except for } \\
\text { CWO which requires extensive } \\
\text { pilot testing and demonstration, } \\
\text { but less development than that } \\
\text { required for acid digestion. }\end{array}$ & $\begin{array}{l}\text { Similar to System 1, but } \\
\text { development and } \\
\text { demonstration work on the } \\
\text { washing systems at the pilot } \\
\text { scale is required. }\end{array}$ & $\begin{array}{l}\text { Affected by development } \\
\text { needs of acid digestion and } \\
\text { phosphate bonded ceramic. } \\
\text { Extensive bench and pilot } \\
\text { scale work required. }\end{array}$ & $\begin{array}{l}\text { Affected by development } \\
\text { needs for CWO organic } \\
\text { and soft debris treatment. }\end{array}$ \\
\hline $\begin{array}{l}\text { Decontamination } \\
\text { and } \\
\text { Decommissioning }\end{array}$ & $\begin{array}{l}\text { Least } D \& D \text { required. D\& } \\
D \text { costs are } \$ 73.70 \text { million. }\end{array}$ & $\begin{array}{l}\text { Similar to System } 1 . \text { D\&D costs } \\
\text { are } \$ 68.80 \text { million. }\end{array}$ & $\begin{array}{l}\text { Because of higher building } \\
\text { and equipment costs, higher } \\
\text { than System 1.D\&D costs } \\
\text { are } \$ 77.01 \text { million. }\end{array}$ & $\begin{array}{l}\text { Similar to System 3. D\&D } \\
\text { costs are } \$ 80.30 \text { million. }\end{array}$ & $\begin{array}{l}\text { Similar to System 3. D\&D } \\
\text { costs are } \$ 77.56 \text { million. }\end{array}$ \\
\hline $\begin{array}{l}\text { Environmental } \\
\text { Restoration }\end{array}$ & $\begin{array}{l}\text { No perceived differences } \\
\text { between systems. }\end{array}$ & $\begin{array}{l}\text { No perceived differences } \\
\text { between systems. }\end{array}$ & $\begin{array}{l}\text { No perceived differences } \\
\text { between systems. }\end{array}$ & $\begin{array}{l}\text { No perceived differences } \\
\text { between systems. }\end{array}$ & $\begin{array}{l}\text { No perceived differences } \\
\text { between systems. }\end{array}$ \\
\hline
\end{tabular}


Table 6-6. Water use and recycle rates.

\begin{tabular}{l|c|c|c|c|c}
\hline & $\begin{array}{c}\text { Grout Debris } \\
\text { System 1 }\end{array}$ & $\begin{array}{c}\text { Desorption } \\
\text { System 2 }\end{array}$ & System 3 & $\begin{array}{c}\text { Acid } \\
\text { Digestion } \\
\text { System 4 }\end{array}$ & System 5 \\
\hline $\begin{array}{l}\text { Water Leaving } \\
\text { Treatment } \\
(\mathrm{lb} / \mathrm{hr})\end{array}$ & 1,101 & 1,186 & 1,424 & 1,790 & 1,795 \\
\hline $\begin{array}{l}\text { Water required } \\
(\mathrm{lb} / \mathrm{hr})\end{array}$ & 1,041 & 1,051 & 1,248 & 1,010 & 1,231 \\
\hline $\begin{array}{l}\text { Water } \\
\text { Discharged } \\
(\mathrm{lb} / \mathrm{hr})\end{array}$ & 59 & 135 & 176 & 780 & 564 \\
\hline $\begin{array}{l}\text { Percent } \\
\text { Recycled }\end{array}$ & $95 \%$ & $89 \%$ & $88 \%$ & $56 \%$ & $69 \%$ \\
\hline
\end{tabular}

j: inellintsicosthoutputs.mkatbibie6_6sheet 
Table 6-7. Solid effluent rates.

\begin{tabular}{l|c|c|c|c|c}
\hline $\begin{array}{c}\text { Solid Effluent } \\
\text { Ibs/hr }\end{array}$ & $\begin{array}{c}\text { Grout Debris } \\
\text { System 1 }\end{array}$ & $\begin{array}{c}\text { Desorption } \\
\text { System 2 }\end{array}$ & $\begin{array}{c}\text { Wash } \\
\text { System 3 }\end{array}$ & $\begin{array}{c}\text { Acrd } \\
\text { Digestion } \\
\text { System 4 }\end{array}$ & $\begin{array}{c}\text { CWO } \\
\text { System 5 }\end{array}$ \\
\hline Grout & 2,913 & 2,764 & 2,731 & 783 & 1,938 \\
\hline Polymer & 1,602 & 1,688 & 1,578 & 13 & 1,722 \\
\hline $\begin{array}{l}\text { Phosphate Bonded } \\
\text { Ceramic }\end{array}$ & 0 & 0 & 0 & 2,708 & 0 \\
\hline $\begin{array}{l}\text { Mercury } \\
\text { Amalgam }\end{array}$ & 5 & 9 & 4 & 5 & 5 \\
\hline $\begin{array}{l}\text { Total Stabilized } \\
\text { Waste }\end{array}$ & 4,520 & 4,461 & 4,313 & 3,509 & 3,664 \\
\hline $\begin{array}{l}\text { Special Waste* } \\
\text { Metal and Lead } \\
\text { for Recycle }\end{array}$ & 155 & 155 & 155 & 155 & 155 \\
\hline $\begin{array}{l}\text { Total Solids } \\
\text { Exiting Facility }\end{array}$ & 5,126 & 5,067 & 4,919 & 4,115 & 4,271 \\
\hline
\end{tabular}

*Note: Because no treatment is assigned to this waste stream, it is assumed the entering mass is the same as the output mass. 
Table 6-8. Estimated number of shipments of reagent per year.

\begin{tabular}{l|c|c|c|c|c}
\hline Reagents & $\begin{array}{c}\text { Grout Debris } \\
\text { System 1 }\end{array}$ & $\begin{array}{c}\text { Desorption } \\
\text { System 2 }\end{array}$ & $\begin{array}{c}\text { Wash } \\
\text { System 3 }\end{array}$ & $\begin{array}{c}\text { Acid } \\
\text { Digestion } \\
\text { System 4 }\end{array}$ & $\begin{array}{c}\text { CWO } \\
\text { System 5 }\end{array}$ \\
\hline Sodium Hydroxide & 9 & 15 & 10 & 16 & 16 \\
\hline Hydrochloric Acid & 0 & $<1$ & 0 & 0 & $<1$ \\
\hline Ammonia & 1 & 0 & 1 & 1 & 0 \\
\hline Nitric Acid & 2 & 0 & 3 & 5 & 0 \\
\hline Hydrogen Peroxide & 4 & $<1$ & 7 & 0 & 0 \\
\hline Total & 16 & 16 & 21 & 22 & 17 \\
\hline
\end{tabular}

Note: This table provides an estimate of the number of 3500 gallon tanker trucks received annually at the site. 


\section{DEMONSTRATION, TESTING AND EVALUATION NEEDS}

Many of the technologies incorporated in the systems evaluated in this report have been developed within the last 10 to 15 years. In general, there is very limited experience (in some cases none) at a scale greater than laboratory or small engineering bench scale operation. Consequently, in developing this study, considerable engineering judgement regarding application of the technologies to new waste streams at larger scale had to be used. Thus, if any of these systems is to be considered for treatment of DOE MLLW, considerable engineering research will be needed to develop the design parameters and to verify the applicability and performance of the technologies comprising these systems.

This section identifies the minimum demonstration, testing, and evaluation (hereafter referred to as development) necessary to resolve technical uncertainties associated with the technologies evaluated in this study. Identification of the development needs in this report does not constitute endorsement of the technology, nor does it constitute a recommendation to fund the development activities. It simply represents the views of the authors as to key development needs or technical data voids that must be resolved prior to full scale implementation of the technology or system in treatment of DOE MLLW. This section is intentionally limited to the top level technical needs. It is not intended to provide a detailed technology development plan for nonthermal treatment of mixed waste.

This section is organized first to describe development needs common to all systems and/or technologies, and second, to describe the development needs for individual systems.

\subsection{Research Approach}

Prior to the launching of substantial development efforts on specific technologies, a system engineering plan (SEP) for deployment of a pilot scale integrated facility should be devised. This SEP would include additional trade studies and evaluations of options at a greater level of detail than included in this study. One of the products of such an effort would be the selection of an integrated system to support further deployment as the pilot project. An additional product would be a list of specific technology development needs.

In this section, a preliminary list of development needs has been identified, based on the information gaps identified in the course of this comparative evaluation study. This list should provide the reader with some understanding of the hurdles to be overcome prior to production scale implementation of these systems.

\subsection{Development Objective}

The objective of the development program is to provide the technical basis for design of a productionscale INTS for managing DOE MLLW. This includes selecting the front end handling and sorting process, organic destruction process, organic separation processes, APC, wastewater treatment, and associated support 
systems. The production scale facility, which the demonstration program represents, must (1) meet current and future regulatory criteria; (2) minimize public and worker health risks by minimizing releases to the environment; (3) produce a waste form that ensures long-term isolation from human contact once it is in a MLLW land disposal unit; and (4) optimize cost.

The demonstration program must (1) examine state-of-the-art technologies; (2) conduct necessary bench scale tests and treatability studies; (3) select one or more technologies for pilot scale and prototype demonstration for each required function of a nonthermal treatment system; (4) identify waste streams (including matrices and contaminants) most applicable to nonthermal treatment from a performance, cost, and risk perspective; and (5) provide all information needed for detailed design of the production facility.

\subsection{Suggested Program For Common Development Needs}

The development program consists of: (1) initial evaluation of technology options; (2) bench scale tests; and (3) selection of one or more parallel units for prototype demonstration. Most nonthermal technologies are at the bench or laboratory scale of development (e.g. catalytic wet oxidation, acid digestion, and mediated electrochemical oxidation). Some are at the small pilot scale level (e.g. GPCR, vacuum thermal desorption, and washing technologies applicable to the range of mixed wastes requiring treatment). Before a prototype system can be considered, these technologies must be developed and tested at a sufficiently large pilot scale to validate laboratory and bench scale results. Pilot scale testing of individual technologies must also prove applicability to mixed waste matrices and contaminants, and resolve issues of safety and resource recovery.

Upon satisfactory completion of the individual pilot scale tests, an integrated pilot plant should be demonstrated. The demonstration system should be designed, fabricated, installed, and operated for a minimum of one year. At the end of this period, key design features of the production scale facility can be defined and finalized. The demonstration facility may continue to operate beyond the initial year if it can help refine the design of the production facility, and can provide additional information regarding performance, safety, and applicability of nonthermal technologies and systems.

\subsubsection{Receiving and Preparation}

The receiving and preparation subsystem includes receiving, characterization, separation and sorting, and size reduction. This subsystem and disposal are the highest cost components of the nonthermal systems, and the front end handling area has the highest potential for worker exposure because it is currently labor intensive. Therefore, improvements in this area have the greatest potential for decreasing worker risk and system life-cycle cost.

7.3.1.1 Characterization. DOE facilities currently have requirements to characterize incoming mixed waste to determine the types and quantities of constituents regulated under RCRA and the Atomic Energy 
Act (AEA), as well as the physical characteristics of the waste that may affect the safety of the treatment operations. Additional or new characterization procedures and/or methods may be required for nonthermal systems to determine appropriate treatment methods, and to meet regulatory and safety requirements. For example, excess amounts of organic material injected into an organic oxidation process may result in a runaway reaction. Materials incompatible with the reagents used in the processes must not be fed to the reaction vessels. Thermal systems require knowledge of the waste contaminants to validate achievement of the required destruction and removal efficiency of the process. However, such requirements have not been defined for nonthermal systems. Typical development requirements would include the following:

- Development of regulatory and safety characterization requirements for the nonthermal systems in question, and development of a strategy for characterizing the waste.

- Development of new, or refinement of existing, technologies.

- $\quad$ Testing of the selected characterization concepts.

7.3.1.2 Sorting and Segregation. Sorting and segregation in the waste industry is typically a manual operation. For nonthermal systems, relatively extensive sorting and segregation is required due to the number of different processes required for the different waste matrices. Currently two methods are in general use. One involves drum-by-drum sorting and segregation in which materials are manually removed from the drum, inspected, and separated for subsequent processing such as size reduction, decontamination, or treatment. The other method uses a "Lazy Susan" rotating table top that allows more rapid sorting and segregation of a larger quantity of material. This table top concept is used in INTS.

Although workers wear PPE, the direct contact with the waste required for these operations increases the potential for worker exposure to radiation, toxic chemicals, and industrial hazards. This type of activity also generates relatively large volumes of secondary waste from the PPE including shoe covers, gloves, tape, plastics, respirator filter cartridges, body suits, hoods, etc. The PPE required for the workers also restricts mobility and visibility, which introduces potential safety hazards when manually handling heavy or awkward objects.

Development of sorting and segregation technologies to minimize worker exposure will emphasize remote handling while allowing operational flexibility to recognize, sort, and manipulate a wide variety of materials. Maintenance in this environment and contamination control are major issues to be resolved with remote handling systems. However, such systems may also decrease the labor content of this subsystem and therefore the life-cycle cost.

7.3.1.3 Material Handling. The current industry practice for general material handling is manual operation using forklifts, pallet jacks, and other standard drum and container handling equipment. Material handling generally includes the activities necessary to transfer a waste container from storage to a staging 
area for pretreatment (e.g., sorting and segregation followed by size reduction, decontamination, etc.), or from the processing unit to a post-treatment storage area.

One aspect of current manual operations that would affect the design of any remote handling system, is the variation in size, type, and condition of the drums, boxes, and other containers in which DOE waste is stored. Various types of equipment, manual and robotic, will be required to handle the various types and sizes of containers in a contaminated area. Another issue is the inherent danger associated with corroded drums and other containers of questionable physical integrity. These situations pose potential danger to the workers and equipment.

Safe, cost-effective methods for manipulating containers with unknown contents and/or questionable integrity are needed. Manual operations generate substantial secondary waste in the form of PPE, and maintenance of multiple contaminated pieces of equipment may lead to additional worker exposure and secondary waste streams. As with sorting and segregation, material handling technologies are needed to minimize worker exposure and to decrease the labor content of the front end handling subsystem in order to reduce system life cycle cost.

7.3.1.4 Size Reduction. Nonthermal systems require significant size reduction due to the surface area dependence of the reagents used to oxidize organic material, and due to the need to access internal surfaces of complex debris and solidified sludges to remove contaminants. For soft debris destruction, sizes must be reduced to $1 / 2$-inch or less. Organically contaminated complex debris and solidified sludges must be reduced in size to the extent that internal surfaces are exposed. All waste must be sufficiently reduced in size such that it can be introduced into the waste treatment vessels exposing sufficient surface area for treatment.

Current size reduction techniques include the use of shredders, ball or hammer mills, saws, etc. High speed units operating on combustible wastes produce dust that has a high potential for fire and explosion. Thus, for radioactive and mixed waste the expense of using an inert gas atmosphere in such systems is warranted. Fines generated in hammer mills necessitate costly controls and containment measures to prevent the spread of contamination and to reduce worker exposure.

Development of the size reduction components will involve testing existing equipment to determine if: (1) internal surfaces of complex debris can be adequately exposed for treatment, (2) solidified sludges can be sufficiently reduced in size to expose organic contaminants within the solidified mass, and (3) soft debris can be sufficiently reduced in size for treatment. It may be difficult to reduce the size of complex metal debris and expose its internal surfaces. Size reduction is affected by the malleability of metals and their propensity to bend and form new internal surfaces and cavities that trap contaminants. Thus, if nonthermal methods of decontamination and metal recovery are to be pursued, new methods of size reduction such as cryofracture which embrittles the metal debris items prior to the size reduction process may be required. 


\subsubsection{Air Pollution Control System}

The five nonthermal systems studied all use essentially the same offgas treatment process. The offgas from the nonthermal processes is relatively cool (as compared to the offgas from thermal processes), and condenser units are used to cool the gases further to condense out most organic contaminants, acids, mercury, and volatile radionuclides. However, trace levels of volatile organics, acid gases, mercury vapor, and radionuclides may remain. The GPCR has been used in all systems to oxidize trace organics after the offgas passes through the cooler and condenser units. Trace levels of acids are removed in an acid gas scrubber. Sulphur impregnated carbon is used to remove trace levels of mercury. Trace particulate, including radionuclides, are removed by HEPA filters.

Development of an APC subsystem for nonthermal treatment would involve developing portions of the subsystem that satisfy regulatory and system requirements and would include the following tasks:

- Define analytical and offgas characterization requirements for nonthermal systems.

- Evaluate various offgas treatment technologies for performance, reliability, maintainability, etc. Such technologies may include the GPCR, electron-beam systems, and possibly others. This would include tests to determine the following:

- organic destruction/oxidation efficiency and limitations, including the effects of temperature, acid gas content, and particulate loading;

- the effect of the technology on mixtures of organics in the offgas stream and the resulting products of the reactions; and

- The potential to produce dioxins from organic mixtures containing precursors.

-

Determine the level of organics, acid gases, mercury vapors, and volatile radionuclides remaining in the offgas stream after treatment by the condenser to understand the level of

- performance required of the APC system.

- Evaluate methods for removing mercury and its salts from the offgas stream. In the nonthermal systems studied, it was assumed that mercury could be removed from the offgas stream using fixed beds of sulfur-impregnated activated carbon which are commercially available. This treatment must be carried out at temperatures below $212^{\circ} \mathrm{F}\left(100^{\circ} \mathrm{C}\right)$ to prevent oxidation of the sulfur with an oxygen-containing offgas. This should not be a problem with a nonthermal system. However, this technology requires verification of effectiveness and identification of potential limitations. 


\subsubsection{Aqueous Waste Treatment}

The five nonthermal systems studied use standard aqueous waste treatment processes. Photooxidation is used to oxidize organic contaminants in the aqueous phase. Standard techniques of precipitation and filtration are used to remove dissolved and suspended solids. Prior to recycling or discharge, ion exchange is used to remove trace levels of dissolved solids including radioactive materials. Activated carbon filters are used to remove trace levels of organics that may remain after the oxidation process. These standard technologies are commercialized, have been tested, and are believed to be effective in treating wastewater. Treatability studies will be required on specific waste streams to validate compliance with regulatory requirements prior to system implementation and operation.

Not considered in the INTS study was the need for some wastewater treatment facilities in the DOE complex to meet new permitting requirements which mandate extremely low levels for some metals (e.g. $0.001 \mathrm{mg} / 1 \mathrm{cadmium}, 0.003 \mathrm{mg} / \mathrm{l}$ lead, and $0.004 \mathrm{mg} / \mathrm{l} \mathrm{silver).} \mathrm{Several} \mathrm{ion-specific} \mathrm{resins} \mathrm{are} \mathrm{available,} \mathrm{as}$ well as metal specific ligands that make separation by ultrafiltration possible. Sulfide precipitation of these metals is also possible. However, it is not known whether these technologies are sufficiently specific to avoid rapid depletion by other ions in the wastewater and the associated generation of secondary wastes. Improved ion specific techniques, effective at micrograms per liter level in complex wastewater, are needed to meet certain site specific permit requirements while minimizing generation of secondary wastes.

The use of an aqueous phase organic oxidation process eliminates the need for large quantities of activated carbon to remove organics from the wastewater, and the secondary waste associated with contaminated carbon. However, these technologies have not been fully tested and demonstrated on the variety of mixed aqueous wastes in the DOE complex, or on the aqueous wastes generated during the nonthermal treatment processes.

Development of a wastewater treatment subsystem for nonthermal systems would involve developing and evaluating portions of the subsystem (particularly the organic destruction/oxidation technology) to satisfy regulatory and system requirements. Development needs include the following:

- Define wastewater treatment and characterization requirements for recycling and discharge for nonthermal systems.

-

Determine the ability to remove suspended solids and immiscible organics to the level where photooxidation can be effective. Solids and organics suspended in the water attenuate the UV light, thereby decreasing the destruction efficiency. In addition, they coat the surface of the window through which the light must pass to reach the wastewater, and cause overheating of the UV lamps, leading to increased lamp failure and maintenance requirements. 
Evaluate the need and determine the ability to remove dissolved solids. Certain dissolved solids may scavenge hydroxyl radicals, which are the oxidizing species produced by UV light, electrons, etc., thereby decreasing the destruction efficiency. Under certain conditions, dissolved solids may also produce scale on the window, preventing UV light from reaching the wastewater.

Evaluate various aqueous phase organic destruction/oxidation technologies for performance, reliability, maintainability, etc. Such technologies may include various commercialized photooxidation systems, electron-beam systems, sonic or ultrasonic treatment, and possibly others. These or other technologies to oxidize organics in the presence of suspended solids and immiscible organics should be evaluated or developed. Tests should be performed to determine the following:

- organic destruction/oxidation efficiency and limitations, including the effects of suspended solids, immiscible organics, and dissolved solids; and

- the effect of the technology on mixtures of organics in the wastewater stream and the resulting products of the reactions and secondary waste produced.

\subsubsection{Mercury Removal}

All systems use thermal desorption or washing to remove organic contaminants from solid waste matrices. These processes also have a tendency to remove mercury and its compounds. However, this removal is not expected to be complete. Therefore, a process is added to complete the removal of mercury and its compounds from the solid matrices to ensure the final waste form passes the TCLP tests. In four systems, the potassium iodide/iodine $\left(\mathrm{KI} / \mathrm{I}_{2}\right)$ mercury removal process is used after thermal desorption. Development in this area should include the following:

- Test and evaluate the $\mathrm{KI} / \mathrm{I}_{2}$ process, and other processes, for its effectiveness in removing mercury and its compounds from various waste matrices such as soils, sludges, and debris. Evaluate the treated matrices for mercury content and their ability to pass the TCLP tests before and after stabilization.

- Identify and/or develop methods for stabilizing the removed mercury and its compounds. Evaluate on a pilot scale the effectiveness of the mercury amalgamation technique which is proposed following the $\mathrm{KI} / \mathrm{I}_{2}$ process.

- Develop a pilot scale system to validate bench scale mercury removal tests, and to validate the ability to stabilize the separated mercury and its compounds. 
Evaluate the effects of other heavy metal contaminants on the mercury removal and stabilization processes.

\subsubsection{Waste Stabilization}

All five nonthermal systems studied use grout or phosphate ceramic and polymer to stabilize various treated or untreated wastes. One system uses phosphate bonded ceramic to stabilize treated soils, process residues, and insoluble salts. Assumptions were made regarding waste loadings and the ability of resulting final waste forms to pass the TCLP tests. These assumptions require validation. All waste forms require testing for long term stability at the assumed waste loading levels.

Several systems studied use grout to stabilize untreated debris. Consequently, grout needs to be evaluated for long term stabilization of organically contaminated debris and of combustible debris. Organic material may decompose over time as a result of microbial action or from radiolysis producing hydrogen and other gases that can degrade the stability and durability of grout. The grouted waste must be evaluated for acceptability under the EPA debris rule. Polymers must be evaluated for stabilization of soluble salts, and phosphate bonded ceramic must be evaluated for stabilization of soils, process residues, and insoluble salts and oxides. Prior to implementation, all waste forms must be evaluated for long term stability and performance using actual contaminants, waste matrices, and waste loadings.

After receiving and preparation, disposal is the second most costly element of the nonthermal systems. This cost is largely due to the volume of final waste form sent for disposal. A significant portion of this final waste volume is due to the use of grout which is mixed in the ratio of two parts grout to one part waste. Thus, if nonthermal waste forms are to be used, waste forms that produce lower volumes than those produced with grout should be considered for development to decrease the life cycle cost of these systems. For example, it may be advantageous to develop various forms of polymer to replace grout because polymers are typically mixed in the ratio of one part polymer to one part waste. Because the densities of grout and polymer are so different $\left(127 \mathrm{lb} / \mathrm{ft}^{3}\right.$ and $80 \mathrm{lb} / \mathrm{ft}$, respectively), the increase in volume due to use of grout is less than expected by simply looking at the mass ratios. However, the final waste form volume from polymer stabilization would be less than that for grout, and land used for disposal would be decreased by about $15 \%$. However, polymer also requires testing of its efficiency for stabilizing organically contaminated debris and combustible debris, and capital and operating costs are typically higher for polymer based stabilization technologies.

Methods for stabilization and disposal of special materials should be developed. Particular attention should be paid to the problems associated with special wastes, lead, and mercury. The capability of polymer stabilization to immobilize mercury should be evaluated, and full scale mercury amalgamation systems should be developed and tested. 


\subsection{Development Needs for System 1: Grout Debris}

The three distinctive characteristics of System 1 are (1) untreated debris is grouted, (2) vacuum thermal desorption is used to remove organic contaminants from soils and process residue (inorganic sludges), and (3) silver based MEO is used to oxidize organic liquids and organic sludges. The requirement to evaluate grout for long term stabilization of organically contaminated debris was discussed in Section 7.3.5. Such debris may be contaminated with oils, PCBs, semivolatile and volatile organics. Development issues for the other two processes are discussed below.

\subsubsection{Vacuum Thermal Desorption}

Vacuum thermal desorption should be tested to evaluate the feasibility of vaporizing organics from noncombustible waste for subsequent liquid organic waste treatment. Typical development tasks include the following:

- $\quad$ Perform bench scale tests, as needed, to identify an appropriate desorber design for further testing, and to determine if different designs are required as a function of the solid matrix (soil or inorganic sludge) or organic contaminant.

- Determine the fate of mercury, radionuclides, and organics in the desorber system.

Process conditions such as operating temperatures and residence times need to be determined and optimized for various waste matrices and contaminants which represent a wide range of volatility. The ability to remove high boiling point (B.P.) organics, such as PCBs, from noncombustible matrices should be evaluated. The fate of volatile components in the DOE MLLW streams should be determined to evaluate the effectiveness of this approach. Vacuum thermal desorbers should be evaluated for (1) the volume of condensable and noncondensable organic gases leaving the desorber; (2) the potential for mercury and its compounds and radionuclides to volatilize and their fate in the desorber system; and (3) the ability of the offgas system condensers to remove the volatiles from the offgas stream.

A pilot scale system should be developed and tested to determine the following:

- The ability to remove organic contaminants from all relevant matrices down to the UTS established by EPA. Of particular concern is the ability to desorb inorganic sludges; this capability has not been demonstrated.

- The effectiveness of desorbing mercury and its compounds.

- The effectiveness of the offgas system. 
The optimum process conditions and throughput of the system for various inorganic matrices and organic contaminants.

\subsubsection{Mediated Electrochemical Oxidation}

As a new technology, MEO is at the bench scale level of development for hazardous waste destruction. Further bench scale testing is required. Pilot scale testing will be required to validate the bench scale tests. Typical tasks to develop and assess the MEO technology include the following:

- Evaluate the various MEO technologies currently under development to determine the most cost-effective option that produces the minimum secondary waste and uses the least resources. This includes MEO processes based on silver, cerium, and cobalt. Another process similar to MEO is under development and should be considered. This process uses peroxydisulfate as the oxidizing agent.

- Evaluate and validate at the pilot scale methods for removing $\mathrm{HCl}$ from processes that use nitric acid as the electrolyte. Chlorine is produced by the treatment of chlorinated hydrocarbons. In some $\mathrm{MEO}$ systems $\mathrm{HCl}$ is produced which, in solution with $\mathrm{HNO}_{3}$, produces aqua regia, a very corrosive reagent that would quickly destroy electrodes in the electrochemical cells.

- Perform tests to evaluate operating conditions, and optimize process design including (1) ratio of feed rate to electrolyte volume, (2) the rate of electrolyte circulation through the system, (3) the frequency of inert material removal, (4) the frequency of electrolyte replacement, and (5) the optimum temperature.

Test the destruction effectiveness of the processes on applicable organic liquids and organic sludges. The potential for volatile organics to vaporize before destruction can take place should be determined.

- Evaluate the fate of RCRA metals and radionuclides in the MEO process. These materials should remain in the electrolyte. This needs to be verified. The frequency at which these dissolved and/or suspended solids need to be removed to maintain effective operation needs to be determined.

- Validate or develop effective methods of recycling metals and acids from the electrolyte. The electrolyte (nitric acid and silver in the case of the process used in System 1) must periodically be purged of inert or inorganic materials. A side stream of electrolyte is typically bled from the process and treated to remove a percentage of metals, radionuclides, and particulates, and to recycle the electrolyte. 
Validate the silver recovery process in a pilot scale system. The silver based MEO process produces an insoluble silver chloride $(\mathrm{AgCl})$ salt when treating chlorinated hydrocarbons. Production of $\mathrm{AgCl}$ removes the chlorine from solution and prevents production of $\mathrm{HCl}$ which may corrode the electrodes. A disadvantage of this system has been that the silver as $\mathrm{AgCl}$ was discarded as waste. The developers have identified a method of recovering up to 99.9\% of the silver from the $\mathrm{AgCl}$ which is included in Systems 1 and 3. This recovery process has been only demonstrated at a laboratory scale and requires verification at the pilot scale.

- Demonstrate at the pilot scale the recovery of nitric acid from nitrous acid and $\mathrm{NO}_{\mathrm{x}}$ produced in the MEO processes, and determine the recovery efficiency.

Perform tests to determine the flexibility of the MEO process in destruction of a wide range of organic compounds from various feed types, and to determine the maximum acceptable particle size that can be processed.

- $\quad$ Determine the effect of the process on mixtures of organic components, the resulting reaction products, and the secondary wastes produced. The potential to produce dioxins from organic mixtures containing precursors must also be determined.

Determine the corrosive properties of reagents and reaction byproducts (such as $\mathrm{HCl}$ in the case of cerium based MEO) to identify materials of construction.

Determine how the process performs under upset conditions, or when a slug of organic material is introduced into the process. Determine what effect this has on the offgas system, and how the offgas system performs with off-normal quantities of gases and volatile organics.

\subsection{Development Needs for System 2 Desorption}

The distinctive characteristics of System 2 are: (1) all debris including combustible debris is treated by vacuum thermal desorption, and (2) CWO is used to oxidize organic liquids and organic sludges. Development issues for these processes are discussed below.

\subsubsection{Vacuum Thermal Desorption}

The development needs for vacuum thermal desorption for application to System 2 are the same as those identified in Section 7.3, with the addition of the need to resolve issues associated with desorbing debris. These issues are as follows: 
The vacuum desorption system being developed and used for the INTS study is limited to a maximum particle size of less than one inch. A desorber should be developed that will accept larger debris items.

Operating conditions for various feed types, including the volume and type of plastics and other combustible debris, should be determined. Operating temperatures for combustible debris need to be lower than for inorganic debris to prevent plastics melting. The feasibility of desorbing high boiling point contaminants, such as PCBs, from combustible debris should be determined.

- $\quad$ Feed sorting requirements to minimize process upsets due to plastics melting, and to maximize vaporization of organic contaminants, including high boiling point organics, should also be determined.

Desorption of combustible debris under an inert gas (nitrogen) atmosphere can generate large quantities of gaseous products, depending on the temperature, due to volatilization of organic materials that make up the combustible debris matrix. The effect of desorbing combustible debris on the offgas system design, and the operating conditions for non-plastic combustible debris, should be determined.

\subsubsection{Catalytic Wet Oxidation}

A development program to resolve issues associated with the CWO process will be similar to that identified for the MEO process. Many of the issues are the same, but present a higher degree of potential risk due to the more aggressive nature of the CWO reagents, and the higher temperatures and pressures associated with the process. As with other technologies that are at the bench scale level of development, pilot scale testing will be required to validate the bench scale test results. Typical technology development efforts are as follows:

- Evaluate the corrosive properties of the reagents to identify materials of construction and methods of joining different sections of the process equipment. Establish equipment lifetimes and maintenance requirements.

- Perform tests to evaluate operating conditions and optimize process design including ratio of feed rate to reagent volume, the rate of reagent circulation through the system, the frequency of inert material removal, the frequency of reagent replacement, and the optimum temperature and pressure. 
Test the destruction effectiveness of the processes on applicable organic liquids and organic sludges. The potential for volatile organics to vaporize before destruction can take place should be determined.

Evaluate the fate of RCRA metals and radionuclides in the CWO process. These materials should remain in the reagent. This needs to be verified, and the frequency at which these dissolved and/or suspended solids need to be removed to maintain effective operation needs to be determined.

Evaluate or develop effective methods of recycling metals, catalysts, and acids from the reagent. The reagent must periodically be purged of inert or inorganic materials that were fed to the system, or that result from the formation of soluble or insoluble salts. A side stream of reagent is typically bled from the process and treated to remove a percentage of metals, radionuclides and particulates, and to recycle the reagent. This process must be validated on a pilot scale level.

The CWO process uses $\mathrm{HCl}$ which is vaporized and enters the offgas stream. This acid gas is condensed and recovered. Acid recovery needs to be demonstrated at the pilot scale and the recovery efficiency determined.

Tests should be performed to determine the flexibility of the CWO process in destruction of a wide range of organic compounds from various feed types, and the maximum acceptable particle size that can be processed.

The effect of the process on mixtures of organic components and the resulting reaction products and secondary wastes must be determined. The potential to produce dioxins from organic mixtures containing precursors must also be determined.

Determine how the process performs under upset conditions or when a slug of organic material is introduced into the process. Determine what effect this has on the offgas system and how the offgas system performs with off-normal quantities of gases and volatile organics.

\subsection{Development Needs for System 3: Washing}

The distinctive characteristics of System 3 is that all solid matrices are treated by washing processes, and MEO is used to oxidize organic liquids and organic sludges. The washing processes includes lower pressure wash for soft debris, high pressure spray wash for open and complex debris, and a soil washing process for soils and inorganic sludges. The development needs of the MEO process were previously discussed in 
Section 7.4.2. Only the needs of the washing process will be discussed in this section. Development issues for the washing process are as follows:

Determine the extent of waste characterization required to select suitable surfactants to remove the organic contaminants.

- Evaluate removal efficiencies for soil, inorganic sludges, and debris at the pilot scale, and determine the ability of the process to meet the UTS for all matrices and contaminants.

- Determine the optimum operating conditions, and the residence time and throughput, for the system to meet the UTS for various inorganic matrices and organic contaminants.

At the pilot scale, validate surfactant selection for various contaminants and the ability to recover surfactants to minimize generation of a secondary waste stream and to decrease cost.

Evaluate the ability of the washing process to remove contaminants from crevices and cavities associated with size reduced complex debris.

Determine the extent to which heavy metals and radionuclides are removed from the various matrices during the washing process, and determine the fate of these species and organics in the washing system.

Determine the fate and rate of release of volatile components during the washing process.

\subsection{Development Needs for System 4: Acid Digestion}

System 4 uses several of the previously discussed technologies for various applications in combination with an acid digestion process for the destruction of organic matter, including soft debris. The distinctive characteristic of this system is the use of nitric/phosphoric acid digestion to oxidize organic liquids, organic sludges, and soft debris. Phosphate bonded ceramic, discussed in Section 7.3.5, are used to stabilize the treated soils, inorganic sludges, and insoluble salts. Issues regarding grouting debris, polymer stabilizing soluble salts, washing soils and open debris, and thermally desorbing sludges were discussed in previous sections. Of particular concern is the ability to adequately desorb sludges. This has not yet been fully demonstrated. Because development needs of most technologies used in this system have already been presented, only the needs for the acid digestion process will be discussed in this section.

A development program to resolve issues associated with the acid digestion process will be similar to that identified for the CWO process. As with other technologies that are at the bench scale level of development, 
pilot scale testing will be required to validate the bench scale test results. Typical technology development efforts are as follows:

- Evaluate the corrosive properties of the nitric/phosphoric acid solution to identify materials of construction and methods of joining different sections of the process equipment.

Perform tests to evaluate operating conditions and optimize process design including (1) ratio of feed rate to acid volume, (2) the rate of acid circulation through the system, (3) the frequency of inert material removal, (4) the frequency of acid replacement, and (5) the optimum temperature and pressure.

Test the destruction effectiveness of the processes on applicable organic liquids and organic sludges. The potential for volatile organics to vaporize before destruction can take place should be determined.

- Test the effectiveness of reducing the volume of combustible debris, the optimum conditions and throughput, and the level of size reduction required.

Evaluate the fate of RCRA metals and radionuclides in the acid digestion process. These materials should remain in the acid. This needs to be verified. The frequency at which these dissolved and/or suspended solids need to be removed to maintain effective operation needs to be determined.

Evaluate or develop effective methods of recycling metals and acids from the reagent. The acid must periodically be purged of inert or inorganic materials that were fed to the system, or that result from the formation of soluble or insoluble salts. A side stream of acid is typically bled from the process and treated to remove a percentage of metals, radionuclides, and particulates, and to recycle the acid. This process must be validated on a pilot scale level.

The acid digestion process uses nitric acid as the oxidizing agent. Products of oxidation include NOx and nitrous acid which are reacted with oxygen and recovered as nitric acid. Acid recovery needs to be demonstrated at the pilot scale and the recovery efficiency determined.

When treating chlorinated hydrocarbons $\mathrm{HCl}$ is produced and a portion is vaporized. If this $\mathrm{HCl}$ is not removed from the offgas it will return to the acid bath through the nitric acid recovery process. The mixture of $\mathrm{HCl}$ and $\mathrm{HNO}_{3}$ (aqua regia) is extremely corrosive and will present a serious problem for materials of construction. Thus, a method for separating $\mathrm{HCl}$ from the nitric acid must be developed and demonstrated at the pilot scale. 
Tests should be performed to determine the flexibility of the acid digestion process in destruction of a wide range of organic compounds from various feed types, and the maximum acceptable particle size that can be processed.

- The effect of the process on mixtures of organic components and the resulting reaction products and secondary wastes must be determined. The potential to produce dioxins from organic mixtures containing precursors must also be determined.

Determine how the process performs under upset conditions, or when a slug of organic material is introduced into the process. Determine what effect this has on the offgas system and how the offgas system performs with off-normal quantities of gases and volatile organics.

\subsection{Development Needs for System 5: Catalyzed Wet Oxidation}

System 5 is similar to System 4 except that the acid digestion of organic liquids, organic sludges, and soft debris is replaced with the CWO process, and grout and polymer replace phosphate bonded ceramic stabilization. As with the acid digestion process, CWO must be evaluated for its effectiveness in reducing the volume of combustible debris, the optimum conditions and throughput, and the level of size reduction required. Other development needs for the CWO process are discussed in Section 7.5.2. Development needs for the other technologies in this system have been discussed in previous sections and will not be repeated here. 


\section{CONCLUSIONS}

This study has developed conceptual mixed waste processing systems using nonthermal technologies for the unit operations. The criterion for defining nonthermal technologies was treatment temperatures below $660^{\circ} \mathrm{F}\left(350^{\circ} \mathrm{C}\right)$. The use of $660^{\circ} \mathrm{F}$ as the upper bound for the allowable temperature for process operations was somewhat arbitrary but correlates with the generally agreed lower bound temperature threshold for the formation of dioxin by the recombination of chemical species in a combustion offgas system.

The study was initiated by developing a set of functional requirements for systems to separate, decontaminate, oxidize, immobilize, and destroy the various physical matrices and contaminants associated with $\mathrm{DOE}$ mixed waste. In parallel, a survey was made to determine what technologies were either under development or in the commercial marketplace and could be used or adapted to support the functional requirements of the integrated flow sheets for processing the DOE mixed waste inventory. Lastly, and also in parallel, a panel of Tribal and Public Stakeholders was convened to help establish criteria for the selection of technologies. It established Stakeholder Principles against which the systems would be evaluated. Many of the stakeholder values translated directly into good engineering and environmental criteria that should guide the design of waste processing facilities.

\subsection{Technical Issues}

These activities resulted in the conceptualization of five discrete processing systems of nonthermal technologies ranging from relatively non-aggressive to chemically very aggressive. These five systems have been evaluated in this report with respect to stakeholder principles, environmental effectiveness, residue production, offgas generation, and costs. The systems were found to have very similar results in terms of life cycle costs, disposal volumes and offgas generation. Furthermore, since virtually all of the technologies are immature (only UV oxidation for organic contaminants in an aqueous waste stream is a commercial technology), the implementation risks for these systems were evaluated and shown to be very high. Thus, the standard contingency of $25 \%$, applied to the cost estimates and used in previous system studies, probably understates the real cost for deployment and operation.

From this study it can be concluded that it may be technically possible to treat the DOE mixed waste inventory using nonthermal technologies. However, due to the relative immaturity of nonthermal technologies, adoption of such a strategy would entail high costs, lengthy development time, high waste disposal volumes, and uncertain environmental performance and compliance with regulatory requirements. The immature technologies available for application to DOE mixed waste have only limited performance basis, and only under relatively ideal testing conditions. Therefore, performance in terms of destruction efficiency and process throughput under real waste stream conditions where multiple contaminants are present in very complex mixtures is unknown. 
The key aspects of nonthermal treatment systems are sorting and stabilization. They are the major drivers of cost, worker and public health, and land use, for both treatment and disposal. Since the nonthermal technologies will require a more exacting sorting process, the cost for this operation will be quite high. Additionally, because of the immaturity of the nonthermal technologies, the operation, utility, maintenance labor and material requirements can only be estimated. Although the estimates are based upon the requirements of similar unit operations, the estimates can have a high degree of uncertainty.

The most favorable aspect of the nonthermal systems is the low volume of estimated offgas. In a preconceptual design study such as INTS, the concentration of contaminants in the offgas cannot be accurately estimated. This would require an experimental program. However, in the judgement of the authors, for such small offgas volumes, it should be possible to engineer an APC system similar to that used in this study to ensure there are virtually no hazardous or radionuclide emissions.

\subsection{Future Evaluation}

The results of this study suggest that a hybrid system, employing both thermal and nonthermal treatments may provide a more effective approach. A more stable and lower volume waste form would be generated from a vitrification stabilization. Offgas would be increased somewhat, though it would still be within the treatment range of the technologies described in this study.

The next phase of the study, Enhanced Nonthermal Treatment Systems, will look at hybrid systems. 


\section{REFERENCES}

Aspen Technology, Inc, ASPEN PLUS, Version 9.13 for PCs, Cambridge, MA, 1994.

Barkley, Naomi P., and Michael L. Taylor, "Control Technology: Development and Demonstration of a Pilot Scale Debris Washing System", Journal of Air and Waste Management Association, Volume 41, No. 4, April, 1991.

Biagi, Charles, William Schwinkendorf, and Ben Teheranian, Enhanced Integrated Nonthermal Treatment System Study, First Interim Draft, prepared for Idaho National Engineering Laboratory, Idaho Falls, ID, February, 1997.

Chiba, Z., P.R. Lewis and L.C. Murguia, Mediated Electrochemical Oxidation Treatment for Rocky Flats Combustible Low-Level Mixed Waste, UCRL-ID-118679, Lawrence Livermore National Laboratory, Livermore, CA, September, 1994.

Chiba Z., B. Schumacher, P.R. Lewis and L.C. Murguira, "Mediated Electrochemical Oxidation as an Alternative to Incineration for Mixed Wastes", paper presented at the Waste Management ' 95 Symposium, Tucson, Arizona, March 1, 1995.

Chiba, Z., "Mediated Electrochemical Oxidation Technology in the Mixed Waste Management Facility at LLNL", part of paper titled "Demonstration of Waste Treatment Technologies in the Mixed Waste Management Facility at LLNL", paper presented at ASME International Mixed Waste III Symposium, Baltimore, Maryland, August, 1995.

Delphi Research, Inc, Continuing Development Studies of the DETOX Process, Final Report, prepared for the U. S. Department of Energy, Morgantown Energy Center, Albuquerque, New Mexico, December, 1992.

Dhooge, Patrick M. and Lena B. Hakim, Development Studies For a Novel Wet Oxidation Process, prepared for the U. S. Department of Energy, Morgantown Energy Center, Albuquerque, New Mexico, January, 1994.

Dosani, M.A., M.L. Taylor, J.A. Wentz, A.N. Patkar, and N.P. Barkley, "Results of Pilot Demonstration of a Newly Developed Pilot-Scale Debris Washing System", Environmental Progress, Vol. 11, No. 4, November, 1992.

Dosani, M.A., M.L. Taylor, and N.P. Barkley, "Field Experience with a Full-Scale Debris-Washing Process", paper presented at the 87th Annual Air and Water Management Association Meeting, June 24, 1994. 
Dosani, M., IT Corporation, personal communication to Kathy Nakazawa of Morrison Knudsen Corporation, August 8, 1995, San Francisco, CA.

EOSystems, Inc., Equipment \& Technology Introduction, Private Communication, San Jose, CA, July, 1995.

Feizollahi, F. and D. Shropshire, Waste Management Facility Cost Information Estimating Data, WTD-92046, prepared for Idaho National Engineering Laboratory, Idaho Falls, ID October, 1992.

Feizollahi, Fred, William J. Quapp, Hugh G. Hempill, and Frank J. Groffie, EG\&G, Inc., Integrated Thermal Treatment System Study - Phase 1 Results, EGG-MS-11211, prepared for Idaho National Engineering Laboratory, Idaho Falls, ID, July, 1994.

Feizollahi, Fred, D. Shropshire, and D. Burton, Waste Management Facilities Cost Information for Transportation of Radioactive and Hazardous Materials, INEL-95/0300, prepared for Idaho National Engineering Laboratory, Idaho Falls, ID, June, 1995a.

Feizollahi, Fred and William J. Quapp, Integrated Thermal Treatment System Study - Phase 2 Results, EGG-MS-11211, prepared for Idaho National Engineering Laboratory, Idaho Falls, ID, August, $1995 \mathrm{~b}$.

Gotlieb, Itzhak, Joseph Bozzelli, and Erez Gotlieb,"Soil and Water Decontamination by Extraction with Surfactants", Separation Science and Technology, 28 (1-3), pp. 793-804, New York, NY, 1993.

Heath, William O., Stephen Barlow, Theresa Bergsman, Delbert Lessor, Thomas Orlando, Anthony Peurrung, and Rahul Shah, Development and Analysis of High-Energy Corona Process For Air Purification, PNLSA24432, prepared for the U. S. Department of Energy, Office of Energy Research, and Office of Technology Development, Pacific Northwest Laboratory, Battelle Memorial Institute, Richland, Washington.

Heath, William O. and Joseph G. Birmingham, Nonthermal Plasma Technology for Organic Destruction, prepared for the U. S. Department of Energy, Pacific Northwest Laboratory, PNL-SA-25844, Battelle Memorial Institute, Richland, WA.

Huebner, T.L., J.J. Wilson, A.H. Ruhter, and S.J. Bonney, Quantities and Characteristics of the ContactHandled Low-Level Mixed Waste Streams for the DOE complex, EGG-MS-11303, prepared for Idaho National Engineering Laboratory, Idaho Falls, ID, August, 1994.

Kennedy, R. P., S.A. Short, J.R. McDonald, M.W. McCann, Jr., R.C. Murray, J.R. Hill and V. Gopinath, Natural Phenomena Hazards: Design and Evaluation Criteria for Department of Energy Facilities, Report UCRL-15910, Rev. 2, University of California Research Laboratories, 1992 (draft). 
Meta Berger, Inc, Programmatic Environmental Impact Statement, prepared for the U.S. Department of Energy, Washington D.C, 1995.

Morrison Knudsen Corporation/Chem Nuclear Services, Chapter 3: Illinois Low-Level Radioactive Waste Disposal Facility; Design, Construction and Capital Cost, from Illinois Low-Level Radioactive Waste Disposal Facility Application, prepared for the State of Illinois, Boise, ID, May, 1991.

Nuclear Waste News, "Mixed Waste: Rust's VAC*TRAX Technology On Track for Commercialization", Nuclear Waste News, Vol. 15, No. 445, pp. 447-448, November 16, 1995.

Palmer, Carl R., Mike McElwee, and Gregg Meyers, Demonstration of a Batch Vacuum Thermal Desorption Process on Hazardous and Mixed Waste, Rust Federal Services Inc., Clemson Technical Center, Anderson, $\mathrm{SC}$.

Pierce, R.A., J.R. Smith, and D.C. Poprik, Nitric-Phosphoric Acid Oxidation of Solid and Liquid Organic Materials, Doc No. WSRC-175--95-0009, prepared for the U. S. Department of Energy by the Westinghouse Savannah River Company, Aiken, SC.

Roesener, W.S., N.R. Soelberg, and A.L. Ayers, Jr., Identification of a Treatment Process for the Idaho Waste Processing Facility Project - A Preconceptual Design Study, WM-PD-92-009, prepared for the U. S. Department of Energy, Idaho National Engineering Laboratory, Internal Technical Report, Idaho Falls, ID, July, 1992.

Schlueter, R., and Schaefer, Low-Level and Transuranic Waste Transportation, Disposal, and Facility Decommissioning Cost Sensitivity Analysis, EGG-WTD-10092, prepared for the U. S. Department of Energy, Idaho National Engineering Laboratory, Idaho Falls, ID, 1992.

Science Application International Company (SAIC), Waste Management Technology Division, Integrated Thermal Treatment Study Quantities and Characteristics of the Contact-Handled Low-Level Mixed Waste Streams for the DOE Complex and its Sites, Vol. 1 \& 2, prepared for the U. S. Department of Energy, Idaho National Engineering Laboratory, Idaho Falls, ID, February, 1993.

Shropshire, D., M. Sherick, and C. Biagi, Waste Management Facilities Cost Information for Mixed LowLevel Waste, INEL-95/0014, prepared for the U. S. Department of Energy, Idaho National Engineering Laboratory, Idaho Falls, ID, June, 1995.

Surma, J.E., J. Geeting, D. Alexander, M. Hyatt, and R. Holeman, Catalyzed Electrochemical Oxidation (CEO) of Rocky Flats Contaminated Combustible Materials, PNL 9974/UC 210, prepared for the U. S. Department of Energy, Pacific Northwest Laboratory, Battelle Memorial Institute, Richland, Washington, August, 1994. 
Taylor, M.L., M.A. Dosani, and N.P. Barkley, "Field Experience With a Full-Scale Debris Washing Process", paper presented at the 87th AWMA Meeting, June 24, 1994.

Tyson, David R., Treatability Study for the amalgamation of radioactively contaminated elemental mercury waste at the Idaho National Engineering Laboratory, unpublished report, prepared for the U. S. Department of Energy, Idaho National Engineering Laboratory, Idaho Falls, ID, 1996.

U.S. Department of Energy Order 5400.5, Radiation Protection of the Public and the Environment, issued $1 / 7 / 93$ (change 2)

U.S. Department of Energy Order 5820.2A, Radioactive Waste Management, issued 9/26/88

U.S. Department of Energy Order 6430.1A, General Design Criteria, issued 4/6/89

U.S. Department of Energy, Overview of Nonthermal Mixed Waste Treatment Technologies (DOE/EM-0281), June, 1995a.

U.S. Department of Energy, Description of Recommended Nonthermal Mixed Waste Treatment Technologies (DOE/EM-0282), August, 1995b.

U.S. Department of Energy, Interim Mixed Waste Inventory Report, (DOE/NBM-1100) March, 1993.

U.S. Environmental Protection Agency, Polychlorinated Biphenyls Question \& Answer Manual, 1994a.

U.S. Environmental Protection Agency, Superfund Innovative Technology Evaluation (SITE) Emerging Technology Bulletin, New Jersey Institute of Technology, GHEA Associates Process, EPA 540/F-94/509 September, 1994b.

U.S. Environmental Protection Agency, Guidance Document, Quality Assurance and Quality Control for Waste Containment Facilities, September, 1993.

U.S. Environmental Protection Agency, Technology Evaluation Report: Design and Development of a PilotScale Debris Decontamination System, Volume 1, EPA/540/5-91/006a, August, 1991.

U.S. Environmental Protection Agency, Test Methods for Evaluating Solid Wastes, SW-846, Third Edition, Revision 1, November, 1990.

U.S. Department of Energy, Federal Facility Compliance Act (FFCA) Task Force, Treatment Selection Guides, Revision 0, March 14, 1994. 
U. S. Department of the Navy, Naval School, Civil Engineer Corp Officers, Environmental Law for NonLawyers, Port Hueneme, CA, January, 1995.

Wagh, Arun S., Dileep Singh, and J. Cunnane, Annual Report for FY 1994 on TTP 2-4-20-4, PhosphateBonded Ceramics for Stabilizing Problem Low-Level Mixed Waste, prepared for U.S. Department of Energy, Office of Technology Development, by Argonne National Laboratory, Argonne, IL, 1995.

Westem Governors Association, A Guide to Tribal and Community Involvement in Innovative Technology Assessment, Western Governors Association, Federal Advisory Committee to Develop On-Site Innovative Technologies, DOIT Tribal and Public Forum on Technology Acceptance, Reno, NV, May 4-5, 1995 (Prepared by the Colorado Center for Environmental Management, Denver, CO). 


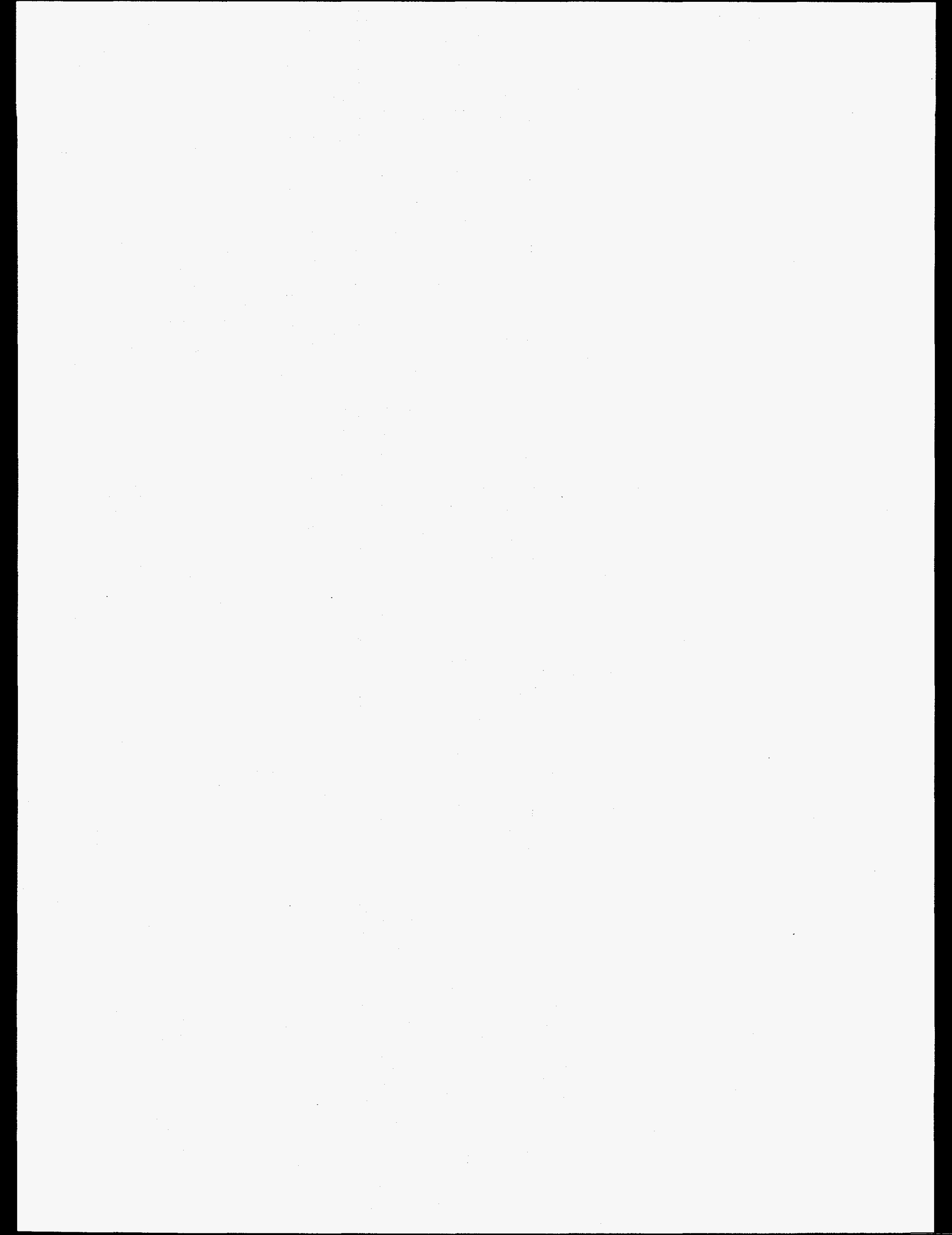




\section{APPENDIX A}

\section{System Requirements and Criteria}




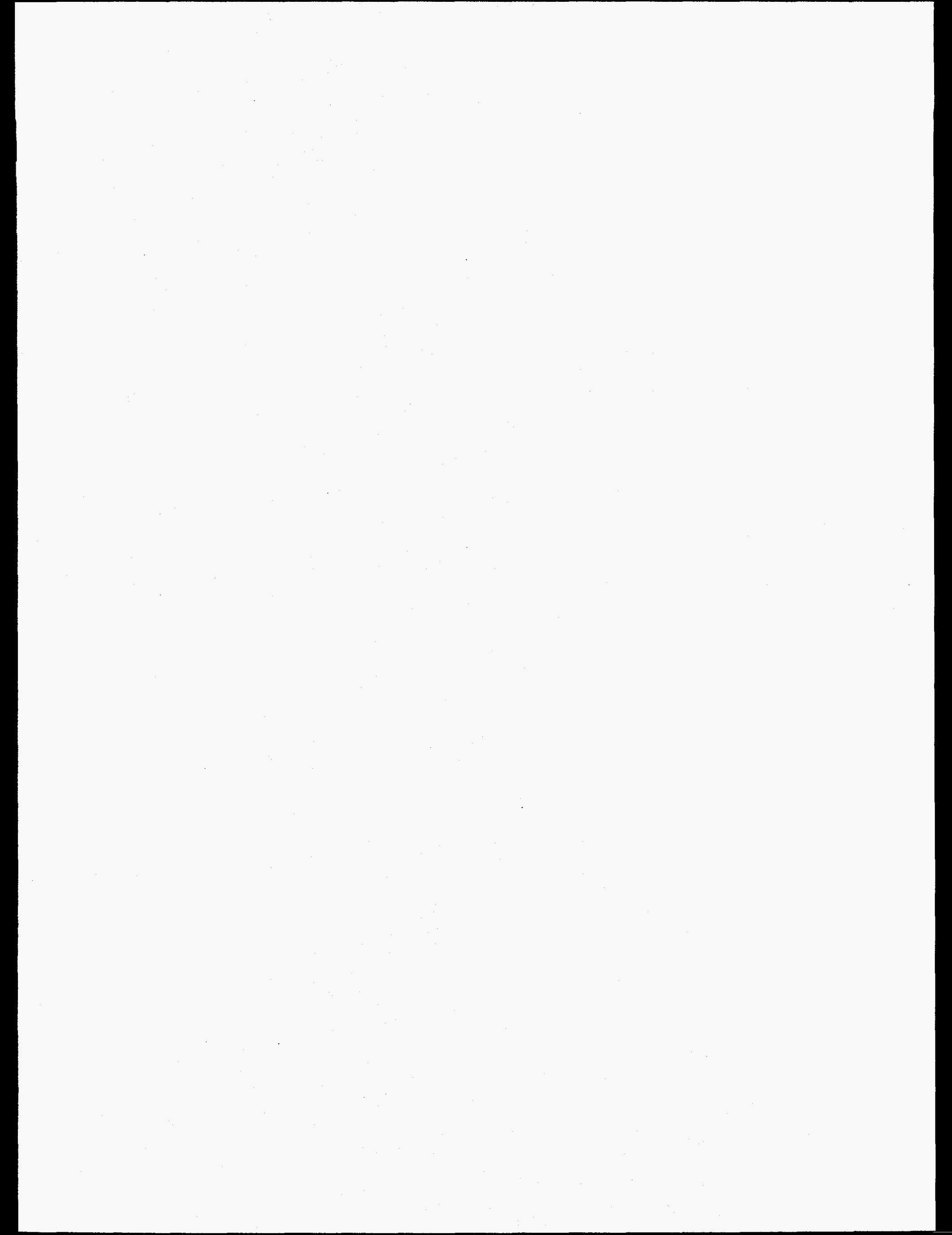




\section{CONTENTS}

A-1. REGULATORY AND PERMITTING REQUIREMENTS $\ldots \ldots \ldots \ldots \ldots \ldots \ldots$. . . . A

A-1.1 Nuclear Regulatory Commission Regulations . . . . . . . . . . . . . . A A-1

A-1.2 National Environmental Policy Act . . . . . . . . . . . . . A A-1

A-1.3 Toxic Substances Control Act . . . . . . . . . . . . . . . A

A-1.4 Comprehensive Environmental Response, Compensation, and Liability Act . . . . A-2

A-1.5 Resource Conservation and Recovery Act . . . . . . . . . . . A A-2

A-1.5.1 Characteristic and Listed Hazardous Wastes . . . . . . . . . A A-2

A-1.5.2 Derived-From Rule . . . . . . . . . . . . . . . A-2

A-1.5.3 Land Disposal Restrictions . . . . . . . . . . . . . A-3

A-1.5.4 Delisting . . . . . . . . . . . . . . . . . A-3

A-1.5.5 RCRA Permitting Requirements $\ldots \ldots \ldots \ldots \ldots \ldots \ldots \ldots \ldots$ A-5

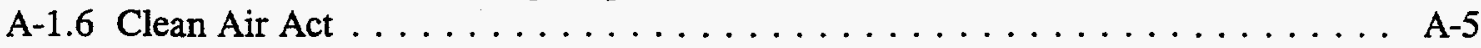

A-1.6.1 Hazardous Air Pollutants $\ldots \ldots \ldots \ldots \ldots \ldots \ldots \ldots \ldots \ldots$ A-5

A-1.6.2 Permits . . . . . . . . . . . . . . . . . . A A-6

A-1.6.3 National Ambient Air Quality Standards . . . . . . . . . . . . A-6

A-1.7 Clean Water Act . . . . . . . . . . . . . . . . . . . . . . A-6

A-1.8 Department of Energy Requirements . . . . . . . . . . . A-7

A-2. PROPOSED EMISSIONS LIMITS $\ldots \ldots \ldots \ldots \ldots \ldots \ldots \ldots \ldots \ldots$

A-3. PROPOSED CHARACTERIZATION REQUIREMENTS . . . . . . . . . . . . A-15

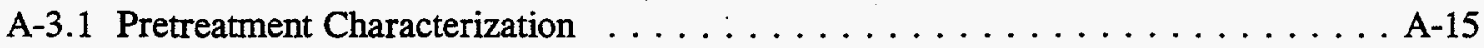

A-3.1.1 Non-Destructive Drum Assay Techniques . . . . . . . . . . . A-15

A-3.1.2 Chemical Analysis for RCRA Organics and Hazardous Metals . . . . A A-15

A-3.1.3 Analytical Methods . . . . . . . . . . . . . . . . A-16

A-3.2 Characterizing Residues from Treatment $\ldots \ldots \ldots \ldots \ldots \ldots \ldots$ A-16

A-3.2.1 Offgas . . . . . . . . . . . . . . . . . . . A-16

A-3.2.2 Treatment Residues $\ldots \ldots \ldots \ldots \ldots \ldots \ldots \ldots \ldots \ldots$ A $\ldots \ldots \ldots$

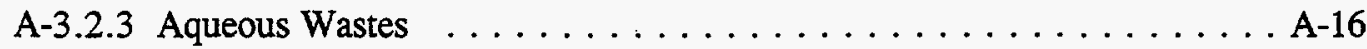

A-3.3 Characterizing Post-Treatment Wastes . . . . . . . . . . . A-16

A-3.3.1 Salts and Treatment Process Residues . . . . . . . . . . . A-16

A-3.3.2 Final Waste Form . . . . . . . . . . . . . . . A-17

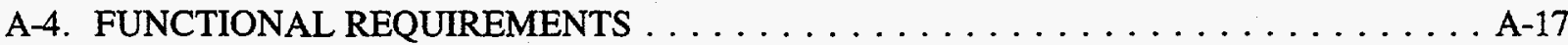

A-4.1 Input Waste . . . . . . . . . . . . . . . . . . . A $\ldots \ldots$

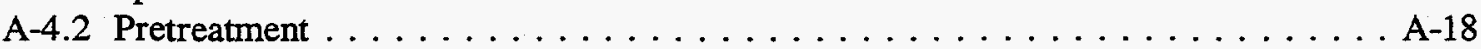

A-4.3 Primary Treatment . . . . . . . . . . . . . . . . . . A-21

A-4.3.1 Top-Level Requirements . . . . . . . . . . . . . . . . A-21

A-4.3.2 Derived Requirements $\ldots \ldots \ldots \ldots \ldots \ldots \ldots \ldots \ldots$ A-22

A-4.4 Output Waste Form . . . . . . . . . . . . . . . . . A-23

A-4.4.1 Top-Level Requirements . . . . . . . . . . . . . . A-23

A-4.4.2 Derived Requirements . . . . . . . . . . . . . . A-24

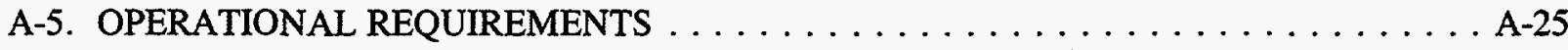

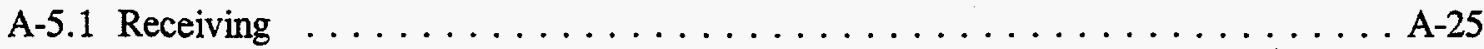

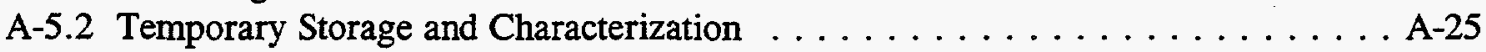




\section{CONTENTS (Continued)}

A-5.3 Preparation . . . . . . . . . . . . . . . . . . . A-25

A-5.4 Treatment of Organic Liquids and Sludges . . . . . . . . . . . . A-26

A-5.5 Treatment of Lead Contaminated Solids and Sludges . . . . . . . . . . . A-26

A-5.6 Treatment of Mercury Contaminated Solids and Sludges . . . . . . . . . . A-26

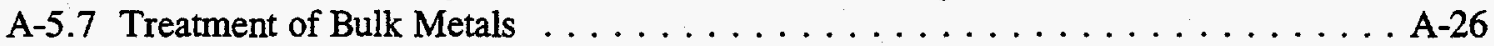

A-5.8 Treatment of Process Residue and Inorganic Sludges $\ldots \ldots \ldots \ldots \ldots \ldots$ A-27

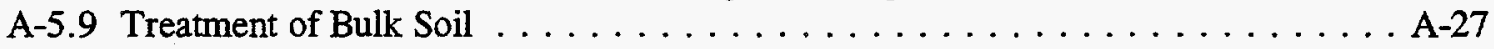

A-5.10 Treatment of Debris . . . . . . . . . . . . . . . . . A-27

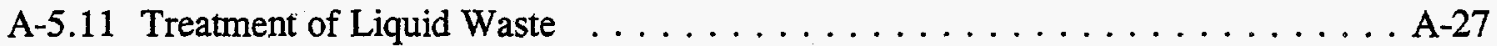

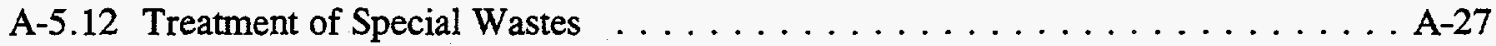

A-5.13 Stabilization . . . . . . . . . . . . . . . . . . A-27

A-5.14 Certification and Shipping of Packaged Waste $\ldots \ldots \ldots \ldots \ldots \ldots \ldots$ A-28

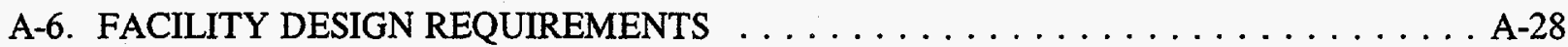

A-6.1 General Assumptions . . . . . . . . . . . . . . . . . . A-28

A-6.2 Treatment Facility Support Functions . . . . . . . . . . . . . . . . A-29

A-6.3 Support Functions of the Administration Unit of the Treatment Facility . . . . . . A-29

A-6.4 Facility Operation . . . . . . . . . . . . . . . . . . A-29

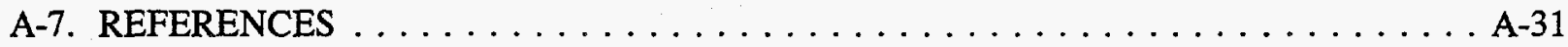

\section{TABLES}

A-1. National ambient air quality standards $\ldots \ldots \ldots \ldots \ldots \ldots \ldots \ldots \ldots$

A-2. Proposed emission limits for nonmetals . . . . . . . . . . . . . . . A-12

A-3. Proposed emission limits for metals for INTS . . . . . . . . . . . . . . . . A-14

A-4. Types of input waste for INTS and their first treatment step within the INTS . . . . . . . A-19

A-5. Input feed rates to INTS subsystems $\ldots \ldots \ldots \ldots \ldots \ldots \ldots \ldots \ldots \ldots \ldots$ 


\section{Appendix A.}

\section{System Evaluation and Comparison Methods}

Appendix A presents the methods used for system selection. Also included are system requirements, which are key elements of system evaluation and comparisons.

\section{A-1. REGULATORY AND PERMITTING REQUIREMENTS}

\section{A-1.1 Nuclear Regulatory Commission Regulations}

The Nuclear Regulatory Commission (NRC) authority under the AEA applies only to commercial facilities.

NRC's authority in regulating nuclear emissions is under the AEA of 1954, 42 United States Code USC 2011, and the Energy Organization Act of 1975, 42 USC 7101. NRC implements their regulations through 10 CFR Parts 20, 30, 40, 51, and 70.

\section{A-1.2 National Environmental Policy Act}

It is assumed that a full environmental impact statement will be needed for the integrated nonthermal treatment facility.

NEPA requirements are an integral part of any project planning process and are set forth in 42 USC 4330, 40 CFR 1500, and DOE Order 4700.1. NEPA is intended to ensure that every proposed action (in this case construction of a new nonthermal treatment facility) has been reviewed for significant effects to the quality of the human environment and to ensure that harmful effects on the environment have been minimized.

\section{A-1.3 Toxic Substances Control Act}

The nonthermal treatment facility must conform to TSCA requirements for the treatment of PCBs. PCB waste should be treated in accordance with operating requirements set forth in 40 CFR 761.60 through 761.70. This section requires demonstration of alternate treatment methods (to incineration or industrial boilers) for PCBs above 500 parts per million (ppm). The agreed upon requirements will be written into the permits required under RCRA and the CAA, or if necessary, under a supplementary TSCA permit. 


\section{A-1.4 Comprehensive Environmental Response, Compensation, and Liability Act}

It is assumed that the integrated nonthermal treatment facility will process only limited quantities of the waste covered by the Comprehensive Environmental Response, Compensation, and Liability Act of 1980 (CERCLA) but all of the RCRA waste.

CERCLA regulates most of the buried waste within DOE installations. Current plans call for the disinternment of most of this waste. All stored waste at DOE installations comes under the purview of RCRA.

\section{A-1.5 Resource Conservation and Recovery Act}

\section{A-1.5.1 Characteristic and Listed Hazardous Wastes}

In this study it is assumed that listed waste will be treated by the nonthermal treatment facility. Characteristic waste should be pretreated if necessary and should be treated by an appropriate subsystem.

Section 3001 of RCRA gave the EPA a statutory mandate to identify and regulate as hazardous those solid wastes that could pose a threat to human health and the environment. Solid wastes that exhibit a characteristic or are listed must also be properly managed as hazardous wastes under RCRA Subtitle C, unless excluded from regulation or excluded from the definition of hazardous waste.

Regardless of whether the waste is listed or not, the generator must determine if the waste exhibits any of the four characteristics of hazardous waste by either (1) testing the waste or (2) applying engineering/process knowledge of the hazardous characteristic based on the materials or the processes used $(\$ 262.11(c))$. The characteristics of hazardous waste are ignitability ( $\$ 261.21)$, corrosivity (\$261.22), reactivity (\$261.23), and toxicity ( $\$ 261.24)$.

\section{A-1.5.2 Derived-From Rule}

Management of secondary wastes shall be according to the EPA "derived from" rule.

The EPA has also implemented "mixture" and "derived from" rules to manage the residues from listed wastes. However, a recently successful court challenge to these "mixture" rules has prompted EPA to consider concentration-based exemption criteria for these residues. The "derived from" rules or some 
version of it will have significant impact on the management of secondary waste from the integrated nonthermal treatment facility.

\section{A-1.5.3 Land Disposal Restrictions}

In this study, it is assumed that residue from treatment of all waste is stabilized to meet the concentration-based extraction test (TCLP). This requirement is also a part of DOE Order 5820.1A.

On November 8, 1984, the Hazardous and Solid Waste Amendments (PL-98-616) became effective. EPA was required to establish treatment standards for all listed and hazardous waste destined for land disposal. Before these wastes can be disposed of (buried in a landfill) they must be treated to conform with applicable treatment standards.

Typically, treatment requirements are concentration based (40 CFR 268.41), according to which the hazardous constituents in question (in an extract of the waste) cannot exceed established threshold levels. In addition to concentration-based standards, technology-based standards are permitted for certain types of waste ( 40 CFR 268.42). These treatment standards were determined by assuming that wastes are treated using the best technology currently available for each waste type. These technologies are called the best demonstrated available technology (BDAT). Unlike listed wastes, characteristic wastes can be reclassified as nonhazardous if they can be treated to remove the characteristic hazard. These wastes may then be buried in any municipal waste landfill. For DOE waste, this means that characteristic waste after treatment can be disposed of in existing low-level waste burial sites.

Listed waste will remain hazardous even after treatment. It must be disposed of in an interim status or newly permitted RCRA-conforming Subtitle $C$ landfill. Such a landfill must have liners to prevent waste migration, a system for collecting and removing leachate, and stormwater run-on and run-off control as discussed in the design and operating requirements in 40 CFR $\$ \S 264 / 265.301$ and 265.302 .

Containerized ignitable wastes, however, may be placed in landfills provided that they are protected from any materials or conditions that could cause them to ignite (i.e. avoiding heat, providing a daily cover of soil or noncombustible material $[\$ \S 264 / 265.312(b)])$.

\section{A-1.5.4 Delisting}

Any waste stream that might be subject to delisting should be pursued according to the following requirements.

Delisting is used for listed wastes that have been treated and no longer pose a threat to human health or the environment. The intent behind delisting is to allow an exclusion pathway for the waste and for the 
waste to become regulated as nonhazardous (solid waste) if the final form of the listed waste does not exhibit characteristics of hazardous waste. In this study, some of the secondary waste streams are investigated for potential delisting.

If not delisted, the waste could pose several problems. Disposal construction costs could be higher, due to the need for special designs and complications associated with the long-term permitting process for RCRA Subtitle C landfills. Dual EPA/DOE oversight is required for mixed waste RCRA-type landfills. The long-term oversight (monitoring and maintenance) requirements for RCRA landfills are assumed to be the same as the DOE requirements for LLW facilities.

There are three major steps in the petition process: (1) submitting the petition, (2) technical review, and (3) promulgating the decision. Delisting is a full rulemaking process and typically takes about two years.

Anyone may submit a petition to delist a waste generated at a particular facility. Separate petitions must be submitted for waste generated at different facilities, even if the contributing processes and raw materials are similar. Upon receiving a petition, EPA will first confirm whether the petitioned waste is listed as a hazardous waste, and therefore, eligible for an exclusion.

During the technical review, EPA will first determine whether the waste meets all required criteria for delisting. Specifically, the waste (1) must not meet the criteria for which it was listed; (2) must not exhibit any of the hazardous waste characteristics; (3) must not exhibit any additional factors, including additional constituents, that would cause the waste to be a hazardous waste (unless EPA determines that such factors do not warrant characterizing the waste as hazardous); and (4) must equal or exceed the regulatory criteria for land disposal. Once the technical review is completed, EPA will decide whether to grant or deny the petition for delisting.

To reclassify a waste as nonhazardous and to delist it, EPA needs information from the petitioner assuring that several criteria are met. The waste (1) must not meet the criteria for which it was originally listed; (2) must not exhibit any of the hazardous waste characteristics; (3) must equal or exceed the regulatory criteria for land disposal; and (4) must not exhibit any additional properties of hazardous waste or otherwise pose a threat to human health or the environment.

Waste and waste management history information must be presented, including the type and volume of waste, waste management practices (past, present, and future), and the basis for waste listing. The physical form of the petitioned waste (e.g., sludge, liquid, solid, ash) must be described. It must be specified whether the waste has been generated in the past or whether it is presently being generated. It must be specified how the waste will be managed if the delisting petition is granted. The petitioner should provide an estimate of the waste volume petitioned for delisting. 
The petitioner should describe operations, process and materials contributions to the petitioned waste, and current waste management operations.

An analytical plan must be prepared that includes a complete list of the hazardous constituents of concern in the waste and a plan for waste testing. This section should precede the sampling plan.

Analytical data must be collected from a sufficient number of representative samples (no fewer than four). These data must be collected over a sufficient period of time to represent the variability or uniformity of the petitioned waste. EPA will review the information on waste sampling to determine if (1) the samples sufficiently represent the variability or uniformity of the petitioned waste; (2) the waste sampling was conducted by qualified personnel; and (3) quality control protocol was followed.

\section{A-1.5.5 RCRA Permitting Requirements}

Treatment units shall be permitted for RCRA regulations as described below.

\section{A-1.5.5.1 General.}

RCRA requires a facility permit for the treatment and storage of hazardous and radioactive waste (40 CFR 264). The debris rule will be used as a basis for debris treatment, as discussed in 40 CFR, parts 148 and 268.

\section{A-1 5.5.2 RCRA Operating Permits Needed for Treatment Units.}

The permit issued by EPA will specify a set of operating requirements. These are specified on a caseby-case basis and largely based on data from the permit application and any demonstration testing. Permitting requirements for miscellaneous units and facilities (40 CFR 264.600 and 270.23) and for tank systems (40 CFR 264.190) are applicable to all INTS systems.

\section{A-1.6 Clean Air Act}

\section{A-1.6.1 Hazardous Air Pollutants}

Section 112(c) of the CAA requires that all source categories, including waste treatment process, be identified and that technology-based emission standards be promulgated for each category.

RCRA metal emission standards for boilers and industrial furnaces are risk based. INTS organic destruction devices, including the GRCR of the APC subsystem, must comply with technology based standards. Demonstration to EPA of the effectiveness of the INTS technologies is required. The maximum 
achievable control standards are to be based on the best technology currently available for the source category. Stack rate emissions are limited to levels that provide an adequate margin of safety when dispersed by air currents between the stack and the nearest maximally exposed individual.

\section{A-1.6.2 Permits}

Previously, operating permits were not required by federal law, though many state laws provided for such permits. Permits are now required for all major new sources. This permit process requires determining whether the facility is to be located in an area where national ambient air quality standards are met. It also requires engineering justification of facility emission rates, new source air quality impacts, and assessment of other risks to the environment. As a result, specific operating limits might be imposed.

\section{A-1.6.3 National Ambient Air Quality Standards}

Section 109(a) of the Clean Air Act covers the National Ambient Air Quality Standards. 40 CFR 50 standards codified to date are shown in Table A-1.

Table A-1. National ambient air quality standards.

\begin{tabular}{lll}
\hline Pollutant & Averaging Period & $\begin{array}{l}\text { Primary standards } \\
(\text { micrograms per cubic meter } \\
\left.\left[\mu \mathrm{g} / \mathrm{m}^{3}\right]\right)\end{array}$ \\
\hline Carbon monoxide $(\mathrm{CO})$ & 8 hours & $10,000(9 \mathrm{ppm})$ \\
& 1 hour & $40,000(36 \mathrm{ppm})$ \\
Lead & 3 months & 1.5 \\
Nitrogen dioxide & 1 year $(\mathrm{yr})$ & $100(0.053 \mathrm{ppm})$ \\
Ozone & 1 hour & $235(0.12 \mathrm{ppm})$ \\
Particulate matter smaller than & 1 year & 50 \\
10 microns & 24 hours & 100 \\
Sulfur dioxide & 1 year & $80(0.03 \mathrm{ppm})$ \\
& 24 hours & $365(0.14 \mathrm{ppm})$ \\
Beryllium & 30 days & $0.01 \mu \mathrm{g} / \mathrm{m}^{3}$ (or $10 \mathrm{~g} / 24$ hours $)$ \\
\hline
\end{tabular}

\section{A-1.7 Clean Water Act}

The Clean Water Act requires permits for the discharge of liquids. Liquids discharged into waters of the United States must comply with a NPDES permit. Liquids discharged to an industrial sewer must comply with the pretreatment standards of the local POTW. 


\section{A-1.8 Department of Energy Requirements}

The following DOE orders and their referenced requirements appear applicable at this time to all systems:

1. Quality Assurance, DOE 5700.6C

Quality Assurance requirements have been established to ensure that risks and environmental impacts are minimized and that safety, reliability and performance criteria are adhered to. The DOE requires that management develop and implement a Quality Assurance program, maintain trained and qualified personnel and provide a program to assess performance. The program is to detect, correct, and prevent quality problems, and maintain documents and records to ensure compliance with DOE requirements. There are approximately seven additional DOE orders that affect the quality assurance requirements as well as three American Society of Mechanical Engineer (ASME) standards, one International Atomic Energy Agency (IAEA) standard, one International Organization for Standardization (ISO) Guideline, five EPA guidance documents, and an American Society of Testing and Materials (ASTM) standard.

2. Occurrence Reporting and Processing of Operations Information, DOE 5000.3B

An occurrence reporting system has been developed to keep the DOE and DOE contractor line management fully informed about events which adversely affect the environment, the intended purpose of the facility, the health and safety of the general public and facility workers. The system consists of identifying, analyzing, and reporting all "Reportable Occurrences," evaluating and implementing corrective actions, preparing reports to prevent similar occurrences, and maintaining a database of unclassified reports. There are approximately 13 additional DOE orders that affect the system.

3. Conduct of Operations Requirements for DOE Facilities, DOE 5480.19

Requirements exist that DOE facilities be organized, managed, and operated in a manner to assure an acceptable level of safety. Operators of facilities are required to have procedures in place to control the conduct of operations, which are to be consistently reviewed for effectiveness. These procedures include, but are not limited to, operations organization, shift routines and operating practices, control area activities, communications, control of on-shift training, investigation of abnormal events, notifications, control of equipment and systems status, lockouts and tagouts, independent verification, log keeping, operations turnover, operations aspects of facility chemistry and unique processes, operations procedures, and equipment and pipeline labeling. 
4. Hazardous Material Packaging for Transport - Administrative Procedures, DOE 1540.2

Procedures have been established by the DOE to ensure that all packaging approved for use in transporting radioactive and other hazardous materials meets all applicable safety requirements. These procedures cover DOE Certificates of Compliance, DOT exemptions, DOE alternatives, NRC Certificates of Compliance, IAEA Certificates of Competent Authority, DOT specification 7A Packaging Certification, DOT Type B Specification Packaging, Special form certification, other hazardous materials packaging, explosives classifications, and rule-making requests. This order also includes the procedures to review DOE safety evaluations and certificates of compliance. There are approximately 16 additional orders, regulations and procedures that affect the hazardous materials packaging from various agencies, including the DOE, DOT, DOD, the IAEA, and the International Air Transport Association (IATA).

5. Value Engineering, DOE 4010.1A

Value engineering programs have been established to reduce nonessential costs and to improve productivity. In creating a value engineering program, management must establish criteria and guidelines, provide funding for value engineering activities, train personnel in its use, and perform value engineering studies. There are approximately six additional orders and policies that affect value engineering from the DOE, Office of Management and Budget (OMB), Federal Acquisition Regulations (FAR), and the Office of Federal Procurement Policy.

6. Maintenance Management Program, DOE 4330.4A

Maintenance activities at DOE facilities are required to promote operational safety, worker health, environmental protection and compliance, property preservation, and cost-effectiveness. Safety-critical structures, components, and systems (active and passive) must have a maintenance program to ensure that they meet their design requirements. Periodic inspections must be conducted to assess safety. There are approximately 12 additional DOE orders that affect the Maintenance Management Program.

\section{Project Management System, DOE 4170.1}

The project management system is developed to provide a systematic and coordinated approach to project management resulting in efficient planning, management, review, and control of DOE projects. There are approximately 45 additional DOE orders, OMB circulars, FAR, and other public laws that affect the Project Management System. 
8. General Environmental Protection Program, DOE 5400.1

DOE policy is to conduct its operations in compliance with all applicable environmental statutes, regulations, and standards. The DOE actively oversees contractors' activities to assure compliance with this policy. There are approximately 13 additional DOE orders that affect the General Environmental Protection Program, as well as 22 pieces of Supreme Court legislation, 4 executive orders, various state and local legislative requirements, and DOE Amended Guidelines on NEPA.

9. Hazardous and Radioactive Mixed Waste Program, DOE 5400.3

This guidance is specific to the Oak Ridge Operations Office, and could apply, depending upon the proposed location(s), to the anticipated integrated nonthermal treatment system(s).

10. Environment, Safety \& Health Program (ES\&H) for DOE Operations, DOE 5480.13

The ES\&H Program has been adopted by the DOE to assure the protection of the environment and the health and safety of the public, to provide a safe workplace for employees, to protect government property, to assure compliance with applicable statutory requirements affecting federal facilities and operations, to assure quality, and to require line management to be responsible for effective ES\&H performance in their programs. There are approximately 16 additional DOE orders and several executive orders that affect the ES\&H Program.

11. Environmental Protection, Safety, and Health Protection Standards, DOE 5480.4

This order specifies requirements for the application of the mandatory ES\&H standards applicable to all DOE contractor operations. It also provides a listing of these standards and identifies their sources. There are approximately six additional DOE orders, one public law and eight primary Title 29 Occupational Safety and Health Administration (OSHA) regulation sections, as well as many additional executive orders and other agency requirements that affect the ES\&H Protection Standards.

12. Fire Protection, DOE 5480.7A

The DOE has enacted fire protection standards to minimize the potential for fire and harm due to fire. This order ensures that a fire will not cause a release of radiological or hazardous material that will threaten the environment or public health and safety; that DOE employees, contractor employees and the general public be protected from the effects of a fire at a DOE facility; that process control and safety systems are not damaged; that vital DOE programs will 
not suffer unacceptable delays as a result of fire; and that property damage from fire does not exceed an acceptable level. There are approximately eight mandatory fire protection regulations that must be followed which are published by the National Fire Protection Association (NFPA), the DOE, and the CFR. There are also 14 reference criteria that pertain to the DOE fire protection program. This order will dramatically affect any treatment system designs.

13. Radiation Protection for Occupational Workers, DOE 5480.11

It is DOE policy that facility operations are performed so that radiation exposures are maintained within the limits set by this order. The radiation protection standards are consistent with guidelines from the EPA, National Council on Radiation Protection and Measurements (NCRP), and the International Commission on Radiological Protection (ICPR). There are approximately 17 additional DOE orders that affect the Radiation Protection for Occupational Workers order, and numerous references by the Nuclear Energy Agency, the NCRP, the ICPR, the EPA, and the American National Standards Institute (ANSI). The radiation limits in the reference material are subject to change.

14. Planning and Preparedness for Operational Emergencies, DOE 5500.2A

The DOE policy regarding emergency planning would apply during both construction and operations. The selected design of the nonthermal treatment process and the location of the potential construction will require site specific considerations for these plans. References similar in magnitude to those required for the earlier identified Fire Protection Order will need to be applied to selected treatment systems and subsystems. SARA Title III and community notification procedures will apply. RCRA location standards, where applied, may reduce some regulatory burden.

15. Work Authorization System, DOE 5700.7C

It is DOE policy to establish a work authorization and control process for work performed by designated management and operating contractors. There are approximately nine additional DOE orders that affect the Work Authorization System.

16. Radioactive Waste Management, DOE 5820.2A

This DOE document establishes requirements for managing radioactive and mixed waste, and contaminated facilities. Over $50 \mathrm{DOE}$ requirements and laws are attached to determine compliance. This document alone will take extensive analysis to determine whether the designed facility will comply with all applicable federal, state, and local environmental, safety, and health 
laws and DOE requirements. Chapter 3, Management of Low-Level Waste, and Chapter 6, Waste Management Plan Outline, are relevant to construction design and operation requirements.

\section{General Design Criteria, DOE 6430.1A}

The DOE has adopted hundreds of pages of General Design Criteria to guide the acquisition of the department's facilities and to establish responsibility and authority for the development and maintenance of these facilities. The criteria require that architectural and engineering principles be applied to the planning, design, construction, and alteration of DOE facilities, including the incorporation of energy conservation. All planning, design, and construction of facilities will be performed in a manner that will satisfy all applicable Executive Orders, Federal laws, and regulations. All facilities will be designed and constructed to be reasonable and adequate for their intended purpose and consistent with health, safety, security, and environmental protection requirements. Numerous references will need to be reviewed for current applicability.

\section{A-2. PROPOSED EMISSIONS LIMITS}

According to 40 CFR 264.601 miscellaneous treatment units, operated in accordance with the permit requirements, must meet performance standards. In 40 CFR 264.1030-1033, air emissions standards for process vents are promulgated. These regulations apply to process vents from distillation, fractionation, thin-film evaporation, air or steam stripping operations that manage hazardous wastes with organic concentrations of at least $10 \mathrm{ppm}$. These requirements may apply to INTS systems. Incineration standards are not required for INTS.

According to 40 CFR 264.1032 , total facility organic emissions must be below $1.4 \mathrm{~kg} / \mathrm{hr}$ and 2,800 $\mathrm{kg} / \mathrm{yr}$ or total organic emissions from all affected process vents at the facility must be reduced by the use of a control device to 95 weight per cent. A control device is defined as an enclosed combustion device, vapor recovery system or flare. The GPCR may qualify as a control device.

Tables A-2 and A-3 show emission limits for nonmetals and metals, respectively, for INTS design. These limits were developed for ITTS work and are more stringent than the requirements for miscellaneous facilities or process vents. However, for consistency, these numbers have been retained as the standard for INTS. 
Table A-2. Proposed emission limits for nonmetals for INTS.

\begin{tabular}{|c|c|c|c|c|}
\hline Pollutant & Regulatory limit & $\begin{array}{l}\text { Averaging } \\
\text { period of } \\
\text { emission } \\
\text { limit }\end{array}$ & $\begin{array}{l}\text { Origin of } \\
\text { limit (see } \\
\text { notes) } \\
\end{array}$ & $\begin{array}{l}\text { Proposed level to be used by } \\
\text { INTS working group }\end{array}$ \\
\hline \multirow[t]{2}{*}{ Carbon monoxide } & $\begin{array}{l}100 \text { parts per million by } \\
\text { volume (ppmv) from } \\
\text { thermal treatment unit }\end{array}$ & & $a, b$ & $\begin{array}{l}100 \text { ppmv from thermal } \\
\text { treatment unit }\end{array}$ \\
\hline & $\begin{array}{l}35 \text { ppmv from stack } \\
9 \text { ppmv from stack }\end{array}$ & $\begin{array}{l}1 \text { hour } \\
8 \text { hour }\end{array}$ & $\mathrm{k}$ & $\begin{array}{l}35 \text { ppmv from stack } \\
\text { (1 hr period) } \\
9 \text { ppmv from stack } \\
\text { ( } 8 \mathrm{hr} \text { period) }\end{array}$ \\
\hline Total hydrocarbons & $20 \mathrm{ppmv}$ & & c & $20 \mathrm{ppmv}$ \\
\hline Ozone & $235 \mu \mathrm{g} / \mathrm{m}^{3}$ & 1 hour & $\mathrm{k}$ & $235 \mu \mathrm{g} / \mathrm{m}^{3}$ \\
\hline $\begin{array}{l}\text { Particles smaller than } \\
10 \text { microns }\end{array}$ & $34 \mathrm{mg} / \mathrm{m}^{3}$ & & d & $34 \mathrm{mg} / \mathrm{m}^{3}$ \\
\hline Sulfur dioxide & $\begin{array}{l}0.03 \mathrm{mg} / \mathrm{m}^{3} \\
0.5 \mathrm{mg} / \mathrm{m}^{3} \\
0.14 \mathrm{mg} / \mathrm{m}^{3}\end{array}$ & $\begin{array}{l}1 \text { year } \\
24 \text { hours } \\
3 \text { hours }\end{array}$ & $\mathrm{k}$ & $0.5 \mathrm{mg} / \mathrm{m}^{3}(24 \mathrm{hr}$ period $)$ \\
\hline PCB & DRE of $99.9999 \%$ & & f & DRE of $99.9999 \%$ \\
\hline Radionuclides & $10 \mathrm{mrem} / \mathrm{yr}$ & 1 year & j & $10 \mathrm{mrem} / \mathrm{yr}$ \\
\hline POHCs & DRE of $99.99 \%$ & & $e, f$ & DRE of $99.999 \%$ \\
\hline Dioxins & $\begin{array}{l}30 \text { nanogram per cubic } \\
\text { meter }\left(\mathrm{ng} / \mathrm{m}^{3}\right)\end{array}$ & & $\mathrm{g}$ & $0.1 \mathrm{ng} / \mathrm{m}^{3}$ \\
\hline $\mathrm{HCl}$ & $1.8 \mathrm{~kg} / \mathrm{hr}$ & 1 hour & $\mathrm{h}$ & $1.8 \mathrm{~kg} / \mathrm{hr}$ \\
\hline Chlorine $\left(\mathrm{Cl}_{2}\right)$ & $\begin{array}{l}0.001 \text { gram per second } \\
(\mathrm{g} / \mathrm{s})\end{array}$ & & $a, i$ & $.001 \mathrm{~g} / \mathrm{s}$ \\
\hline Radon 222 & $\begin{array}{l}3 \times 10^{-9} \text { microCurie per } \\
\text { milliliter }(\mu \mathrm{Ci} / \mathrm{ml})\end{array}$ & 1 year & 1 & $3 \times 10^{-9} \mu \mathrm{Ci} / \mathrm{ml}$ \\
\hline
\end{tabular}

Notes (a through 1) on origins of limits are listed on the next page. 
Table A-2. Proposed emission limits for nonmetals (Continued).

a. It is EPA's intent to extend the Boiler and Industrial Furnace (BIF), rule 56 FR 35 pg 7134-39, to hazardous waste thermal treatment units. This limit comes from the BIF rule.

b. 40 CFR $266.104, \mathrm{CO}$ is corrected to $7 \%$ oxygen, dry basis. If $\mathrm{CO}$ is above $100 \mathrm{ppmv}$, the level of total hydrocarbons may exceed 20 ppmv.

c. 40 CFR 266.104

d. 40 CFR 264.344 currently calls for 180 milligrams per dry standard cubic meters (mg/dscm). However, it is EPA's intent to lower this limit to at least $34 \mathrm{mg} / \mathrm{dscm}$. Some states have already taken this approach in granting permits.

e. 40 CFR 264.343

f. The Clean Air Act, as interpreted by some states, requires an aggregate risk for carcinogens of $1 \mathrm{x}$ $10^{-6}$. By requiring a destruction removal efficiency of $99.999 \%$ instead of $99.99 \%$, the spirit of these new requirements should easily be met.

g. The proposed European Union limit is $0.1 \mathrm{ng} / \mathrm{m}^{3}$. Germany and the Netherlands have already adopted this standard. This is a technology-based standard, and technology is available to meet this low level. EPA, after lifting its current moratorium on permitting of thermal treatment units, can be expected to move down to substantially lower levels in the issuance of future permits. Currently, there is no set limit for dioxins, except for municipal waste thermal treatment units, where it is 30 $\mathrm{ng} / \mathrm{m}^{3}$. The European Union goal appears to be reasonable for the ITTS and INTS.

h. $40 \mathrm{CFR} 264.343$ restricts $\mathrm{HCl}$ to $1.8 \mathrm{~kg} / \mathrm{hr}$ or $1 \%$ of the quantity of $\mathrm{HCl}$ in the stack before entering air-pollution-control devices, whichever is larger. As a conservative measure, the lower value was selected for the ITTS and INTS.

i. The EPA limits for chlorine and metals are risk-based emission limits in $\mathrm{g} / \mathrm{s}$. They were back calculated from maximum acceptable inhalation exposure doses (in $\mu \mathrm{g} / \mathrm{m}^{3}$ ) coupled to the expected dilution factor occurring between the stack outlet and the nearest maximally exposed individual. For the purpose of this study it was assumed that the thermal treatment unit would have a $24 \mathrm{~m}$-tall stack and would be located in a rural area having simple (little variation) terrain. These risk-based limits for carcinogens are based on a cancer risk of $1 \times 10^{-5}$ to the maximally exposed individual. Non-carcinogenic metals were based on the maximally exposed individual not being exposed to a demonstrable effect level times an arbitrary safety factor. For the purpose of the ITTS and INTS, risk-based limits for metals have been arbitrarily reduced by a factor of 10 to decrease the risk (for carcinogens) from $1 \times 10^{-5}$ to $1 \times 10^{-6}$. This is consistent with EPA's concerns that risk-based standards allow too much metal to leave the stack and with EPA's long-term preference for adopting morerigorous, technology-based standards.

j. 40 CFR 61.92 restricts exposure of the public to an effective equivalent dose of $10 \mathrm{mrem} / \mathrm{year}$.

k. National Primary and Secondary Ambient Air Quality Standards, 40 CFR 50.

1. 10 CFR 20, Appendix B. Limit applies at the boundary between the restricted and unrestricted areas. Concentration at stack may be higher depending on dilution, dispersion, and decay between stack and unrestricted area. 
Table A-3. Proposed emission limits for metals for INTS.

\begin{tabular}{|c|c|c|c|c|}
\hline Pollutant & EPA Limit & $\begin{array}{c}\text { Averaging } \\
\text { Period of } \\
\text { Emission Limit }\end{array}$ & $\begin{array}{c}\text { Origin of } \\
\text { Limit (See } \\
\text { Notes) }\end{array}$ & $\begin{array}{l}\text { Proposed Level to be } \\
\text { Used by ITTS Working } \\
\text { Group } \\
\text { (g/s averaged } \\
\text { over } 1 \text { hour) } \\
\end{array}$ \\
\hline Antimony & $0.061 \mathrm{~g} / \mathrm{s}$ & 1 hour & a & 0.0061 \\
\hline Barium & $10.0 \mathrm{~g} / \mathrm{s}$ & 1 hour & a & 1.0 \\
\hline Nickel & $0.0084 \mathrm{~g} / \mathrm{s}$ & 1 hour & a & 0.00084 \\
\hline Mercury & $0.016 \mathrm{~g} / \mathrm{s}$ & 1 hour & a & 0.0016 \\
\hline Selenium & $0.80 \mathrm{~g} / \mathrm{s}$ & 1 hour & a & 0.080 \\
\hline Silver & $0.61 \mathrm{~g} / \mathrm{s}$ & 1 hour & a & 0.061 \\
\hline Arsenic & $0.00047 \mathrm{~g} / \mathrm{s}$ & 1 hour & a & 0.0000471 \\
\hline Thallium chlorides & $0.061 \mathrm{~g} / \mathrm{s}$ & 1 hour & a & 0.0061 \\
\hline Cadmium & $0.00111 \mathrm{~g} / \mathrm{s}$ & 1 hour & a & 0.000111 \\
\hline Lead & $\begin{array}{l}0.0177 \mathrm{~g} / \mathrm{s} \\
1.5 \mu \mathrm{g} / \mathrm{m}^{3}\end{array}$ & $\begin{array}{l}1 \text { hour } \\
\text { annual }\end{array}$ & $a, c$ & 0.00177 \\
\hline Beryllium & $\begin{array}{l}0.000833 \mathrm{~g} / \mathrm{s} \\
0.0001155 \\
\mathrm{~g} / \mathrm{s}\end{array}$ & $\begin{array}{l}1 \text { hour } \\
24 \text { hours }\end{array}$ & $a, d$ & 0.00008333 \\
\hline Chromium & $\begin{array}{l}0.0001666 \\
g / s\end{array}$ & 1 hour & $a, b$ & 0.0000166 \\
\hline
\end{tabular}

a. The EPA limits for chlorine and metals are risk-based emission limits in $\mathrm{g} / \mathrm{s}$. They were back calculated from maximum acceptable inhalation exposure doses (in $\mu \mathrm{g} / \mathrm{m}^{3}$ ) coupled to the expected dilution factor occurring between the stack outlet and the nearest maximally exposed individual. For the purpose of this study it was assumed that the thermal treatment unit would have a $24 \mathrm{~m}$-tall stack and would be located in a rural area having simple (little variation) terrain. These risk-based limits for carcinogens are based on a cancer risk of $1 \times 10^{-5}$ to the maximally exposed individual. Noncarcinogenic metals were based on the maximally exposed individual not being exposed to a demonstrable effect level times an arbitrary safety factor. For the purpose of the ITTS, risk-based limits for metals have been arbitrarily reduced by a factor of 10 to decrease the risk (for carcinogens) from $1 \times 10^{-5}$ to $1 \times 10^{-6}$. This is consistent with EPA's concerns that risk-based standards allow too much metal to leave the stack and with EPA's long-term preference for adopting more rigorous, technology-based standards.

b. Based on hexavalent chromium.

c. 40 CFR 50.12 , national primary air standard for lead.

d. National emission standard for hazardous air pollutants, 40 CFR 61 . 


\section{A-3. PROPOSED CHARACTERIZATION REQUIREMENTS}

Each subsystem of the nonthermal treatment process will require characterization prior to and following treatment, starting with the individual waste containers and ending with the final waste forms, offgas, and aqueous discharges.

\section{A-3.1 Pretreatment Characterization}

\section{A-3.1.1 Non-Destructive Drum Assay Techniques}

Current drum assay procedures for radionuclides can have errors of $\pm 100 \%$ at the threshold level of $100 \mathrm{nCi} / \mathrm{g}$ between TRU waste and LLW. This accuracy should be adequate for characterizing input feed to a treatment unit. There are no EPA requirements for radionuclides related to nondestructive assaying.

$\mathrm{X}$-ray radiography can be useful in presorting waste before processing. X-ray radiography and nuclear scanning devices are available at several sites, including INEL.

\section{A-3.1.2 Chemical Analysis for RCRA Organics and Hazardous Metals}

40 CFR 264.31 (Waste Analysis) states that "throughout normal operation the owner operator (of a treatment unit) must conduct sufficient waste analysis to verify that waste feed to the treatment unit is within the physical and chemical limits specified in the permit.

40 CFR 265.13 requires a written analysis plan that provides for a detailed chemical or physical analysis of a representative sample.

40 CFR 264.13 (General Waste Analysis) requires a detailed chemical and physical analysis before treatment of a representative sample. Historical information from a facility's records of analysis performed before the effective data of the RCRA regulations, or studies conducted on hazardous wastes generated from processes similar to that which generated the waste to be managed (treated) at the facility, can be used to comply with this requirement.

For the INTS it is proposed that batches of waste sufficient for 8 hours of waste processing in each treatment subsystem be organized into campaigns of pre-analyzed waste. Following shredding (as required), a suite of EPA SW-846 analyses will be performed. 


\section{A-3.1.3 Analytical Methods}

EPA SW-846 should be adapted for analysis of alpha-contaminated mixed waste. Several DOE laboratories are in the process of improving analytical methods for this purpose.

Unless otherwise specified, chemical analysis should be done using Test Method for Evaluating Solid Waste, Physical/Chemical Methods, third edition, revision 1, SW-846, November 1990.

Methods for conventional stack gas sampling should comply with 40 CFR 60, Appendix A.

\section{A-3.2 Characterizing Residues from Treatment}

\section{A-3.2.1 Offgas}

Air emissions analytical methods in existing EPA SW-846 should be used for analyzing offgas.

\section{A-3.2.2 Treatment Residues}

Existing EPA SW-846 analytical methods should be used for analyzing scrubber solutions.

\section{A-3.2.3 Aqueous Wastes}

Existing EPA wastewater analytical methods should be used for analyzing aqueous waste and aqueous discharge.

\section{A-3.3 Characterizing Post-Treatment Wastes}

\section{A-3.3.1 Salts and Treatment Process Residues}

Radioassay of TRU content will be performed for residuals from organic destruction processes. Routine methods are available that should be readily adaptable to residue wastes.

Analysis for salt content may be needed to reach decision on how to formulate for conversion to the final waste form. Routine analysis methods should suffice.

Analysis for RCRA metals is needed for post-treatment wastes unless it can be assumed that the waste will have levels of regulatory concern. 
Analysis for total carbon may be required to show the absence of organics. If carbon is present in significant quantities, further analyses could be required (e.g., thermal desorption/GC-MS).

\section{A-3.3.2 Final Waste Form}

For non-debris waste, EPA will require analysis for toxics in accordance with the TCLP (40 CFR 261, Appendix II) to show that the treatment to create a final waste form is effective in reducing the toxicity level to that acceptable for burial in mixed waste facility (meets RCRA Subtitle C landfill requirements). Using this test, if the concentration of any contaminant does not exceed the concentration listed in the table, the waste is acceptable for disposal as LLW. DOE has not yet decided what tests are appropriate for showing the long-term stability of stabilized waste forms.

For debris waste, EPA requires specified treatment and/ or stabilization (40 CFR 268). TCLP is not required.

\section{A-4. FUNCTIONAL REQUIREMENTS}

\section{A-4.1 Input Waste}

INTS will treat the wastes types described in Table A-4. The nominal capacity of the system will be as shown in Table A-5.

The input waste assumed in the INTS study is mixed radioactive waste as defined in the 1993 mixed waste inventory report (MWIR) by the DOE, including alpha-contaminated MLLW which contains a variety of anticipated hazardous materials.

To ensure the safety of personnel, the system for treating alpha LLW and alpha MLLW should be designed to accommodate input waste with radioactivity levels of TRU waste, which is waste with radioactivity exceeding $100 \mathrm{nCi} / \mathrm{g}$. The primary design requirement stemming from this requirement is triple containment for control of alpha contamination. For nonalpha MLLW, standard design criteria for contact handled radioactive LLW should be sufficient.

All waste considered in the INTS study is contact-handled waste. If high-activity waste inside shielded containers is found, it will be rejected and returned to its point of origin. Consequently, the system will be designed with minimum shielding. Some shielding could be necessary in areas of the facility with concentrated TRU waste or fission-product radionuclides. However, shielding is not considered to be a factor in the performance of the different systems. 


\section{A-4.2 Pretreatment}

Waste pretreatment should be compatible with requirements of the waste treatment technologies. Pretreatment for the INTS systems is more extensive than for the I TTS systems; because of the inflexibility of these processes compared to incineration.

Bulk metals (e.g., ferrous metals and lead larger than about $10 \mathrm{~cm}$ ) should be removed from the waste stream and sent to the appropriate treatment subsystem. Wire wastes should not be considered bulk metals. Lead in any form, typically blankets and wool, should be removed from the waste stream as best as reasonably possible and sent to the appropriate treatment unit. Bulk mercury should be sent to the mercury treatment subsystem. Waste suspected of mercury contamination should be sorted, separated from debris waste, and treated by the mercury leaching process or a washing process.

Containers of potentially temperature sensitive materials, such as pressurized gas cylinders, should be set aside and safely depressurized, emptied, or otherwise made safe for the next step of treatment.

Waste containers should be decontaminated or processed along with bulk metals if not processed along with waste.

It would be desirable to monitor drums for plutonium content when technology is available. Drums should also be monitored for gross liquid content . The shredder discharges shredded waste to a sampling bin where vapor samples should be taken online with direct readout of the vapor composition to provide an indication of the VOC content. The shredding operation should be blanketed with carbon dioxide or another inert gas. 
Table A-4. Sources and types of input waste from the ten largest DOE installations and their first treatment step within the INTS.

\begin{tabular}{|c|c|c|}
\hline Waste categories & $\begin{array}{l}\text { MWIR } \\
\text { Waste } \\
\text { Matrix } \\
\text { Code }\end{array}$ & $\begin{array}{c}\text { INTS } \\
\text { Treatment } \\
\text { Subsystem }\end{array}$ \\
\hline Aqueous TC Metals & 1200 & Aqueous Waste \\
\hline Asbestos & 5125 & Complex Debris \\
\hline Firebrick & 5230 & Complex Debris \\
\hline Ceramics (molds \& crucibles) & 5230 & Complex Debris \\
\hline Glass Raschig Rings & 5220 & Complex Debris \\
\hline Drums & 5100 & Complex Debris/ Metal Decon. \\
\hline Leaded Rubber & 5120 & Lead Recovery \\
\hline Lead Shielding & 5120 & Lead Recovery \\
\hline Bulk Mercury & 7100 & Mercury Recovery \\
\hline Bulk (nontoxic) Metals & 5100 & Metal Decontamination \\
\hline Concrete & 5210 & Open Debris \\
\hline Misc. Glass & 5220 & Open Debris \\
\hline Aqueous Organics & 2100 & Organic Destruction \\
\hline Halogenated Org. Liquids & 2210 & Organic Destruction \\
\hline Organic Sludge & 3220 & Organic Destruction \\
\hline Non-halogenated Org. Liquids & 2120 & Organic Destruction \\
\hline Oil & 2220 & Organic Destruction \\
\hline Resins & 3100 & Organic Destruction \\
\hline Sulfate Salts & 3149 & Polymer stabilization \\
\hline Halide Salts & 3140 & Polymer stabilization \\
\hline Saits & 3149 & Polymer stabilization \\
\hline Nitrate Salts & 3143 & Polymer stabilization \\
\hline Cemented Inorganic Sludge & 3150 & Process Residue \\
\hline Absorbed Organics & 3114 & Process Residue \\
\hline Cemented Organic Sludge & 3200 & Process Residue \\
\hline Inorganic Sludge & 3120 & Process Residue \\
\hline Paint Residues & 3130 & Process Residue \\
\hline Absorbed Inorganics & 3113 & Process Residue \\
\hline Incinerator Ash & 3111 & Process Residue \\
\hline Cemented Resins & 3212 & Process Residue \\
\hline Sheetrock & 5200 & Soft Debris \\
\hline Metal Fines \& Turnings & 5100 & Soft Debris \\
\hline $\mathrm{PE} / \mathrm{PP}$ & 5310 & Soft Debris \\
\hline Flooring Materials & 5400 & Soft Debris \\
\hline Asphalts & 5300 & Soft Debris \\
\hline Paper & 5330 & Soft Debris \\
\hline Graphite & 5340 & Soft Debris \\
\hline Wood & 5320 & Soft Debris \\
\hline Cloth & 5330 & Soft Debris \\
\hline Cardboard & 5330 & Soft Debris \\
\hline TFE & 5312 & Soft Debris \\
\hline Rubber & 5310 & Soft Debris \\
\hline PVC & 5312 & Soft Debris \\
\hline FRP Boxes & 5120 & Soft Debris \\
\hline Plastic N.O.S. & 5319 & Soft Debris \\
\hline Diatomaceous Earth & 4000 & Soil Treatment \\
\hline Soil/Dirt & 4000 & Soil Treatment \\
\hline Bulk Barium & 5110 & Special \\
\hline Uranium Compounds & 7600 & Special \\
\hline Bulk Cadmium & 5110 & Special \\
\hline Tantalum (INEL) & 7000 & -Special \\
\hline Inorganic Labpacks & 6000 & Special \\
\hline Beryllium & 7300 & Special \\
\hline Batteries $(\mathrm{Pb}, \mathrm{Cd})$ & 7400 & Special \\
\hline Aqueous Cyanide & 1140 & Special \\
\hline Organic Labpacks & 6000 & Special \\
\hline
\end{tabular}

Note: MWIR (mixed waste inventory report) codes are commonly used by DOE for waste identification 
Table A-5. Nominal capacity of the INTS subsystems.

\begin{tabular}{l|r|r|r|r|r}
\hline Subsystem & $\begin{array}{r}\text { Grout Debris } \\
\text { System 1 }\end{array}$ & $\begin{array}{c}\text { Desorption } \\
\text { System 2 }\end{array}$ & $\begin{array}{c}\text { Wash } \\
\text { System 3 }\end{array}$ & $\begin{array}{c}\text { Acid Digestion } \\
\text { System 4 }\end{array}$ & $\begin{array}{c}\text { CWO } \\
\text { System 5 }\end{array}$ \\
\hline Administration & 2,927 & 2,927 & 2,927 & 2,927 & 2,927 \\
Receiving and preparation & 2,927 & 2,927 & 2,927 & 2,927 & 2,927 \\
Aqueous waste treatment & 1,192 & 1,256 & 1,627 & 1,983 & 1,950 \\
Organic destruction & 237 & 268 & 326 & 256 & 256 \\
Process residue and & & & & & \\
inorganic sludge treatment & 1,020 & 1,020 & 1,020 & 1,020 & 1,020 \\
Bulk soil treatment & 329 & 329 & 329 & 329 & 329 \\
Debris treatment & 0 & 693 & 693 & 693 & 693 \\
Metal decontamination & 462 & 462 & 462 & 462 & 462 \\
Mercury amalgamation & 3 & 5 & 3 & 3 & 3 \\
Lead recovery & 26 & 26 & 26 & 26 & 26 \\
Polymer stabilization & 1,069 & 1,110 & 1,246 & 7 & 1,126 \\
Phosphate bonded ceramic & & & & & \\
stabilization & 0 & 0 & 0 & 2,946 & 0 \\
Air pollution control & 695 & 823 & 827 & 1,396 & 1,433 \\
Grout stabilization & 1,082 & 1,228 & 958 & 265 & 650 \\
Special treatment & 155 & 155 & 155 & 155 & 155 \\
Certification and shipping & 5,126 & 5,067 & 4,919 & 4,115 & 4,270 \\
\hline
\end{tabular}

Note:

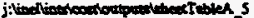

Input to all subsystems includes primary and secondary streams from other subsystems, but does not include additives. 


\section{A-4.3 Primary Treatment}

\section{A-4.3.1 Top-Level Requirements}

The key treatment subsystems should have a high likelihood of public acceptance and regulatory approval.

Acceptable technologies are those having successfully completed the proof-of-principle phase or those that have successful operating experience in other industrial applications. Technologies must be ready for pilot scale demonstration in 2 years, incorporation in a final design in 3 years, and construction in 5 years.

Liquid organic waste should be oxidized to achieve volume reduction and destruction of RCRAcontrolled materials.

Process residue, such as inorganic sludge contaminated with solvents, should be treated to destroy RCRA-controlled contaminants, if present, and to remove moisture prior to stabilization by polymer stabilization, if applicable.

Soil should be treated to remove RCRA contaminants prior to stabilization by grouting.

Debris should be treated and stabilized in accordance with the EPA debris rule.

Levels of substances in the offgas should meet requirements set forth in RCRA and TSCA.

Parts of the system that process alpha-contaminated MLLW should operate at a slightly negative pressure to avoid releasing contamination outside the units. Tolerable in-leakage should be provided for in the design requirements.

Treatment systems used for alpha LLW, alpha MLLW, and TRU waste should have a tertiary containment system for all process steps from waste sorting through waste stabilization. Only two levels of containment are required for other processes involving materials with a limited potential for becoming airborne. These requirements should be carefully assessed to avoid potential worker inhalation risks.

Process monitoring and control should be recorded and audited, and be sufficiently responsive to prevent upsets or excessive reactions.

Air pollution control systems must meet or exceed current and anticipated regulations. Performance specifications should be set to meet or exceed current regulations by one order of magnitude (10 times). 
Treatment and containment systems should be flexible enough to accommodate maximum process upsets and failures.

Bulk ferrous metals should be treated by decontamination to free-release standards of DOE Order 5400.5. Metal not successfully decontaminated will be stabilized. Metal meeting free-release standards should have a scrap value.

Bulk lead such as lead brick should be treated by surface decontamination if it is reasonably determined to have no activation products. Lead which cannot be decontaminated, such as blankets, wool, and activated lead, should be stabilized to EPA microencapsulation standards.

Mercury, if found to have radioactive contamination, should be treated by amalgamation. If not radioactive, elemental mercury should be recovered and sold as scrap.

Aqueous waste should be treated to destroy organics and remove inorganic and metal contaminants. The contaminants will be immobilized into final waste forms. Highly acidic or alkaline aqueous waste should be pretreated to return the $\mathrm{pH}$ to near neutral. Organic liquid waste should be treated by oxidation. If chloride salts are formed, these should be treated in a similar manner to salt residues from offgas treatment. Treated water will be used for recycle within the facility or discharged under local requirements or an NPDES permit.

The system should be designed for a 20-year operating life, with particular attention being paid to ease of maintenance and selection of materials to avoid undue failures due to corrosion.

\section{A-4.3.2 Derived Requirements}

Versatile technologies are desirable. A versatile technology is one that can handle variations in waste type and has a minimum number of separate operations.

Three organic destruction oxidation technologies meet the implementability requirements: (1) Mediated Electrochemical Oxidation, (2) Catalytic Wet Oxidation, (3) Acid Digestion.

The volume of effluent gases should be minimized to the extent possible. Ways to help achieve this include externally heating the CWO and Acid Digestion reactors.

The volatilization of metals should be kept to a minimum. Maximum temperature for all treatment processes is $660^{\circ} \mathrm{F}\left(350^{\circ} \mathrm{C}\right)$. Toxic secondary waste generated by the APC unit should be converted to nontoxic (non-RCRA-controlled) LLW if supported by economic considerations. The APC unit should offer the best decontamination factor for radioactive materials and the best removal efficiency for toxic 
materials. The APC unit should provide redundancy of selected components to allow limited maintenance while keeping the system on line. HEPA filtration should be used before the gas is discharged to the stack.

The solid effluents from the APC subsystem may be stabilized using a polymer process. Cement stabilization of wastes with a high salt content (percent salt to be determined) is not feasible. Process residue wastes will also be stabilized in polymer or by an equivalent process. Debris and treated soil waste may be stabilized by cement.

The final waste form should allow easy movement and disposal, as in standard 55-gallon drums.

Six low-temperature stabilization agents meet the implementability requirements: (1) Portland cement, (2) polymer made by Dow Chemical Company, (3) pozzolanic cement, (4) polyethylene, (5) sulfur cement and (6) phosphate bonded ceramic.

\section{A-4.4 Output Waste Form}

\section{A-4.4.1 Top-Level Requirements}

The characteristics of the system's primary and secondary waste output must meet current and anticipated disposal regulations, such as 40 CFR 268 for substances regulated by RCRA and DOE Order 5820.2A.

Shallow land burial is the assumed disposal option for LLW and treated MLLW. Engineered shallow land disposal is the assumed disposal option for alpha LLW and alpha MLLW. The report by Feizollahi and Shropshire (1992) contains preconceptual designs and PLCC estimates for the disposal facility.

For purposes of calculating transportation and disposal costs, it is assumed that the output LLW, MLLW, alpha LLW, and alpha MLLW will be shipped to and disposed of at the Nevada Test Site. However, for purposes of selecting an output waste form, it should be assumed that the Nevada Test Site might not be available. Therefore, the final waste form should be stable so that it can be accepted at surface disposal facilities at other major DOE installations, including Hanford Site, Washington; INEL, Idaho; Oak Ridge Reservation, Tennessee; Los Alamos National Laboratory, New Mexico; and Savannah River Site, South Carolina. All wastes from other sites will be transported to one of these sites for disposal.

The chosen final waste form should be able to satisfy performance assessment requirements for each site for alpha concentrations up to $99 \mathrm{nCi} / \mathrm{g}$ TRU materials. An average cost of disposal will be used for all shipments. It is assumed that processing sites for transportation purposes will be located at Oak Ridge Reservation and INEL (i.e. there will be no transportation costs for wastes at these sites). 
Disposal of TRU waste will be at WIPP.

\section{A-4.4.2 Derived Requirements}

The desired output waste form is a stable, leach resistant, high-integrity solid produced by solidification. Solidification is a low-temperature process that produces a stable solid by mixing the residue with such agents as cement, polymer, or epoxy. Bitumen should not be used.

Waste forms not destined for WIPP must meet EPA leaching criteria as determined by the toxicity characteristic leaching procedure (40 CFR 268). This requirement is known to exceed the WIPP waste acceptance criteria if EPA grants no migration variance. At this time, such variance is only granted for the demonstration phase. Therefore, if the variance were not to be granted, all waste would have to meet EPA land disposal restrictions. Another driving requirement regarding treatment standards for waste bound for WIPP, located New Mexico, is that the State of New Mexico has the authority to establish the characterization requirements for the RCRA-controlled components of the waste. These requirements, which are not yet established, could be so onerous as to make full treatment less expensive.

It is assumed that the input waste contains EPA listed hazardous organics or other RCRA-controlled substances and characteristic waste. To simplify permitting, the process for producing the output waste should be considered the BDAT by the EPA. Alternately, the waste stabilization process must be demonstrably equivalent to the EPA BDAT. The final waste form should be delistable to reduce the cost of disposal (Subtitle D versus Subtitle C) combined with the requirements of DOE 5820.2A for LLW.

If the waste qualifies as TRU waste, then the final waste forms should comply with WIPP waste acceptance criteria and TRUPACT II TRU packaging and shipping criteria. ${ }^{2}$ The potential for gas generation should be minimized. If this requirement results in a significant increase in the final waste volume, this requirement should be reconsidered.

It is desirable that the output waste be packaged in 55-gallon drums meeting DOT requirements.

Final waste forms must meet contact handling requirements with no additional shielding other than the shipping container.

a TRU waste can be shipped in other containers, such as TMI-2 fuel containers, if heat loads or fissile material limits of the final waste form exceed the TRUPACT II limits. 


\section{A-5. OPERATIONAL REQUIREMENTS}

\section{A-5.1 Receiving}

The treatment system will receive incoming solid waste and sludge in drums, metal bins, and wooden or fiberglass boxes shipped to the treatment facility by onsite or offsite transportation vehicles. Liquid waste will be shipped to the system either by a pipeline, in tank trucks, or in containers placed on wheeled vehicles.

\section{A-5.2 Temporary Storage and Characterization}

Incoming containers of solid waste or sludge will be unloaded from shipping vehicles, temporarily stored, characterized, logged, and recorded. Incoming liquid waste will be sampled, characterized, and temporarily stored. Characterization will identify the physical, chemical, and radiological properties of the waste to the extent needed by the treatment process. Characterization will identify containers with waste that will require sorting (SR waste), and waste that will not require sorting (SNR waste).

\section{A-5.3 Preparation}

When ready for processing, the solid waste and sludge will be prepared to the extent needed by the appropriate treatment unit. Depending on the type of treatment unit, preparation can include removing waste from containers, sorting, size reduction, and shredding. In general, it is undesirable for such materials as bulk lead and mercury to enter treatment units other than lead recovery or mercury amalgamation because they are inherently hazardous wastes. Mercury contaminated debris must be separated from other debris. If lead and mercury materials can be found using RTR performed on containers of intact waste, the containers will be sorted and placed in hoppers for the appropriate treatment subsystem. Other bulk metals (e.g., steel, aluminum) detected by the RTR will be similarly handled. This strategy minimizes challenges to the shredder system, as well as routing the waste appropriately.

Sorting requirements of solid and sludge wastes can result in several waste streams, including lead, organic liquids and sludges, process residues and inorganic sludges, bulk soil, open debris, soft debris, complex debris, bulk metals, elemental mercury, and waste requiring special treatment (e.g., chemically reactive waste or pressurized gas cylinders). Each waste stream will be conveyed to an appropriate treatment unit. Depending on the system configuration, more than one waste stream might go to a given treatment unit (e.g., vacuum desorption). Empty containers will be decontaminated for recycling or reuse, or undergo size reduction and be sent for stabilization. 
Liquid waste will be separated into two categories: aqueous waste and organic liquid waste. The limit of the organic concentration for organic liquid waste depends on the treatment technology employed. Each type of liquid waste will be sent to an appropriate treatment train.

\section{A-5.4 Treatment of Organic Liquids and Sludges}

Organic liquids and sludges will be prepared and then treated in an oxidation process where organics will be destroyed and discharged as offgas into the APC subsystem. The APC subsystem will treat the offgas to meet emission discharge limits and to ensure that minimal levels of contaminants are discharged to the environment. Solid residues from organic destruction treatment (inorganic sludge) will be treated in the process residue treatment subsystem and/or sent directly to the stabilization subsystem. Secondary aqueous waste from the organic destruction or APC subsystem will be sent to the aqueous waste treatment subsystem.

\section{A-5.5 Treatment of Lead Contaminated Solids and Sludges}

Bulk lead or lead encased in steel will be decontaminated and recycled for reuse. Depending on the development of de minimus criteria in the future, the clean lead will be sent for recycling or reuse in the DOE complex. Contaminated lead that cannot be cleaned will be encapsulated and sent for disposal.

\section{A-5.6 Treatment of Mercury Contaminated Solids and Sludges}

MLLW process residue and sludges contaminated with elemental mercury will be sent to the process residue treatment subsystem. Debris contaminated with elemental mercury will be treated for mercury removal either in the process residue treatment subsystem (System 1) or in the appropriate debris treatment subsystem (all other systems). The recovered mercury will be either chemically stabilized or will be amalgamated before disposal. Elemental mercury or highly contaminated waste will be sent to the mercury stabilization subsystem. The mercury will be stabilized by amalgamation if contaminated with radioactivity. Bulk elemental mercury will be recycled if not contaminated with radioactivity.

\section{A-5.7 Treatment of Bulk Metals}

Bulk metals are assigned to the open debris category. Following open debris treatment, bulk metals will be assayed, decontaminated if required, and released for recycling or reuse to the extent possible. Again depending on future de minimus contamination levels, the clean metals will be sent for recycling, and contaminated metals will be size reduced and stabilized for disposal. 


\section{A-5.8 Treatment of Process Residue and Inorganic Sludges}

Process residue and inorganic sludge will be treated to remove organic and mercury contaminants. Treatment which also removes RCRA metals and radionuclides is preferable. Process residue wastes will be sent to polymer stabilization or equivalent.

\section{A-5.9 Treatment of Bulk Soil}

Bulk soil will be treated to remove organic and mercury contaminants. Treatment which can also remove RCRA metals and radionuclides is preferable. Process residue wastes will be sent to grout stabilization or equivalent.

\section{A-5.10 Treatment of Debris}

Debris as defined by EPA will be sorted and kept strictly separate from soil and process residue. Debris will be treated and/or stabilized in accordance with EPA requirements. Treatment may be performed to remove organics, mercury, RCRA metals and radionuclide contaminants. Treatment is preferable to stabilization only. Treated or prepared debris will be sent to grout stabilization or equivalent.

Debris which is contaminated with mercury does not meet the EPA definition. Debris known to be contaminated with mercury will be separated and treated in campaigns by vacuum desorption or high pressure washing without mixing with other debris. Treatment will be performed to remove at least mercury and organics, and if possible, RCRA metals and radionuclides.

\section{A-5.11 Treatment of Liquid Waste}

Liquid waste includes aqueous waste and organic liquid waste. Incoming aqueous waste will be treated to remove dissolved solids, suspended solids, and organic materials. Treated waste water will be returned to the process for recycling or reuse or discharged. The treatment byproduct will be concentrated. Concentrated organic liquid waste and sludge will be sent to the organic destruction subsystem. Concentrated inorganic liquid waste will be sent for stabilization.

\section{A-5.12 Treatment of Special Wastes}

An area will be provided to house portable or specially designed units that can treat special wastes on a case-by-case basis.

\section{A-5.13 Stabilization}


Residues from the various treatment units will be stabilized and placed in a container suitable for disposal. The waste container will be inspected to ensure that surface radioactive contamination is below acceptable levels. Any loose contamination will be removed from the container and recycled to the stabilization process. After inspection, the container will be sent for assay, certification and shipping. The waste form produced by the stabilization process must meet transportation, storage, and disposal requirements imposed by the DOE, EPA, DOT, and the individual DOE disposal sites. It should also minimize release of toxic constituents to the environment.

\section{A-5.14 Certification and Shipping of Packaged Waste}

Filled waste containers will be inspected and assayed in accordance with transportation, storage, and disposal requirements. After inspection and assay, the containers will be certified, logged, recorded, and either sent to a temporary storage facility or loaded onto a transport vehicle for offsite or onsite shipping.

\section{A-6. FACILITY DESIGN REQUIREMENTS}

\section{A-6.1 General Assumptions}

Facilities handling MLLW are placed in seismic category 1. Buildings that handle MLLW are classified as moderate-hazard facilities (Kennedy et al. 1992). The facilities must be designed in accordance with applicable local, state, and federal regulations, including general design criteria for DOE facilities contained in DOE Order 6430.1. Specifically, RCRA places design constraints on storage and treatment facilities and places performance specifications on the waste stabilization.

All systems and critical operations related to handling alpha-contaminated MLLW are classified according to safety. They have high-quality, low-maintenance features to keep personnel exposure as low as reasonably achievable. Operations with alpha MLLW are confined, to the greatest extent practical, to remote cubicles.

The facilities' units should be designed to handle at least $125 \%$ of the expected mass flow rates, shown on PFDs for each system.

Public, employee, and environmental exposures to hazardous and radioactive material should be as low as reasonably achievable.

The SSL for each facility is based on two assumptions. Surge capacity for indoor storage of MLLW drums is 2-6 weeks. The preconceptual design of cubicles for areas for processing alpha-contaminated MLLW is based on a triple confinement system. 


\section{A-6.2 Treatment Facility Support Functions}

Support functions for all treatment facilities include, but are not limited to, (1) nonprocess heating, ventilation, and air conditioning; (2) fire protection; (3) electrical systems (including emergency); (4) process control and data logging; (5) radiation instrumentation; (6) bulk chemical and additive supply and storage; and (7) storm water runoff control.

Input to the facility includes utilities, service water, normal and emergency power, and communications.

Laboratory and personnel decontamination areas are designed to handle radioactive material.

Structures housing personnel and nonradioactive components are constructed in accordance with the appropriate building code. Laboratories and personnel decontamination areas are designed for handling radioactive material.

\section{A-6.3 Support Functions of the Administration Unit of the Treatment Facility}

Support functions for the administration facility include, but are not limited to, (1) security, (2) personnel decontamination (radioactive and hazardous), (3) noncontaminated maintenance, (4) health physics, (5) sanitary facilities, (6) work control and personnel support, (7) interior and external public relations and communications, (8) spill or emergency response provisions (hazardous and radioactive), (9) general analytical laboratory, (10) environmental field sampling, and (11) environmental regulatory reporting and records management.

The treatment facility should include the following interfaces: (1) facility communication; (2) alarm systems, including telephone, evacuation, fire, and security alarms; and (3) public address systems. All treatment processes are programmed for automatic shutdown if the fire system or the evacuation alarm is activated.

\section{A-6.4 Facility Operation}

The preconceptual designs are based on the assumption that MLLW inventories will be processed in treatment facilities, placed in storage facilities, and disposed of during a 20-year operating period. During this period, the treatment facility will operate 24 hours per day, 7 days per week, 40 weeks per year, at $60 \%$ of capacity during operation. This is equivalent to operating 168 days per year, or 4,032 hours per 
year of operation. In facilities with small capacities, where a three-shift operation is not justified, a single shift per day or part-time shifts are assumed.

\section{A-7. REFERENCES}

Feizollahi, Fred, and D.S. Shropshire, 1992, Waste Management Facilities Cost Information, Idaho National Engineering Laboratory, EG\&G, Idaho, Inc., October 1992, EGG-WTD-10443.

Kennedy, R. P., S.A. Short, J.R. McDonald, M.W. McCann, Jr., R.C. Murray, J.R. Hill and V. Gopinath, 1992 (draft). Natural Phenomena Hazards: Design and Evaluation Criteria for Department of Energy Facilities, Report UCRL-15910, Rev.,2, University of California Research Laboratories.

U.S. Code of Federal Regulations, 40 CFR 50, 190-299, 761, 1500, Protection of Environment, July $1,1989$.

U.S. Code of Federal Regulations, 40 CFR 264-270, Protection of Environment, August 27, 1993.

U.S. Department of Energy, DOE Order 5820.2A, Radioactive Waste Management, Office of Defense Waste and Transportation Management, September 26, 1988.

U.S. Department of Energy, DOE Order 6430.1A, General Design Criteria, Office of Project and Facilities Management (draft), December 25, 1987.

U.S. DOE Order 5000.3B, "Occurrence Reporting and Processing of Operations Information," issued 7/2/93 (change 1)

U.S. DOE Order 5400.1, "General Environmental Protection Program," issued 6/29/90 (change 1)

U.S. DOE Order 5480.19, "Conduct of Operations Requirements for DOE Facilities," issued 5/18/92 (change 1)

U.S. DOE Order 5700.6C, "Quality Assurance," issued 8/21/91

U.S. Environmental Protection Agency "Test Methods for Evaluating Solid Waste, SW-846" U.S. Third Edition, 1987 


\section{APPENDIX B}

Systems Preconceptual Design Figures 

These figures are provided in a separately bound document. 


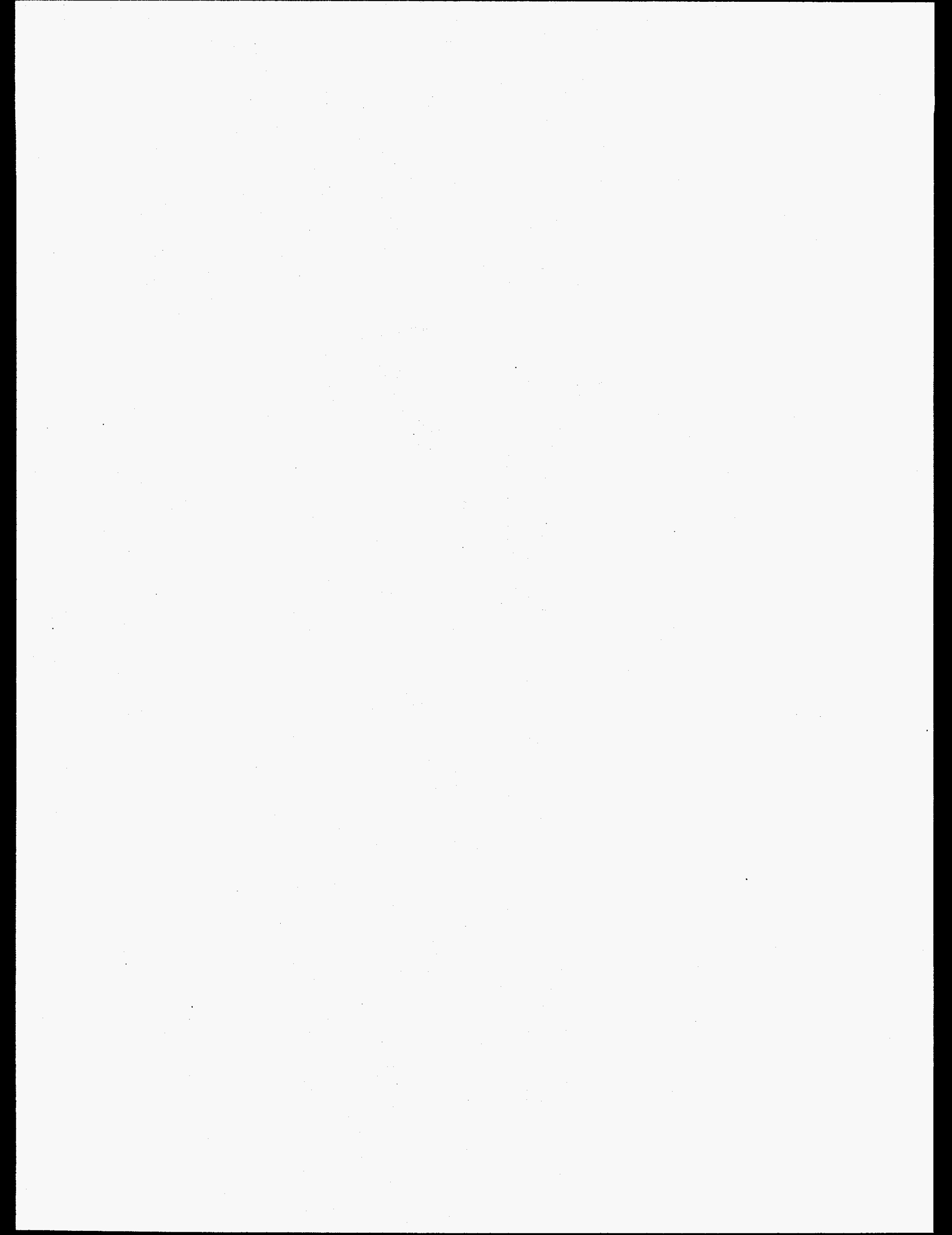




\section{APPENDIX C}

INTS Study Mass Balance Modeling 


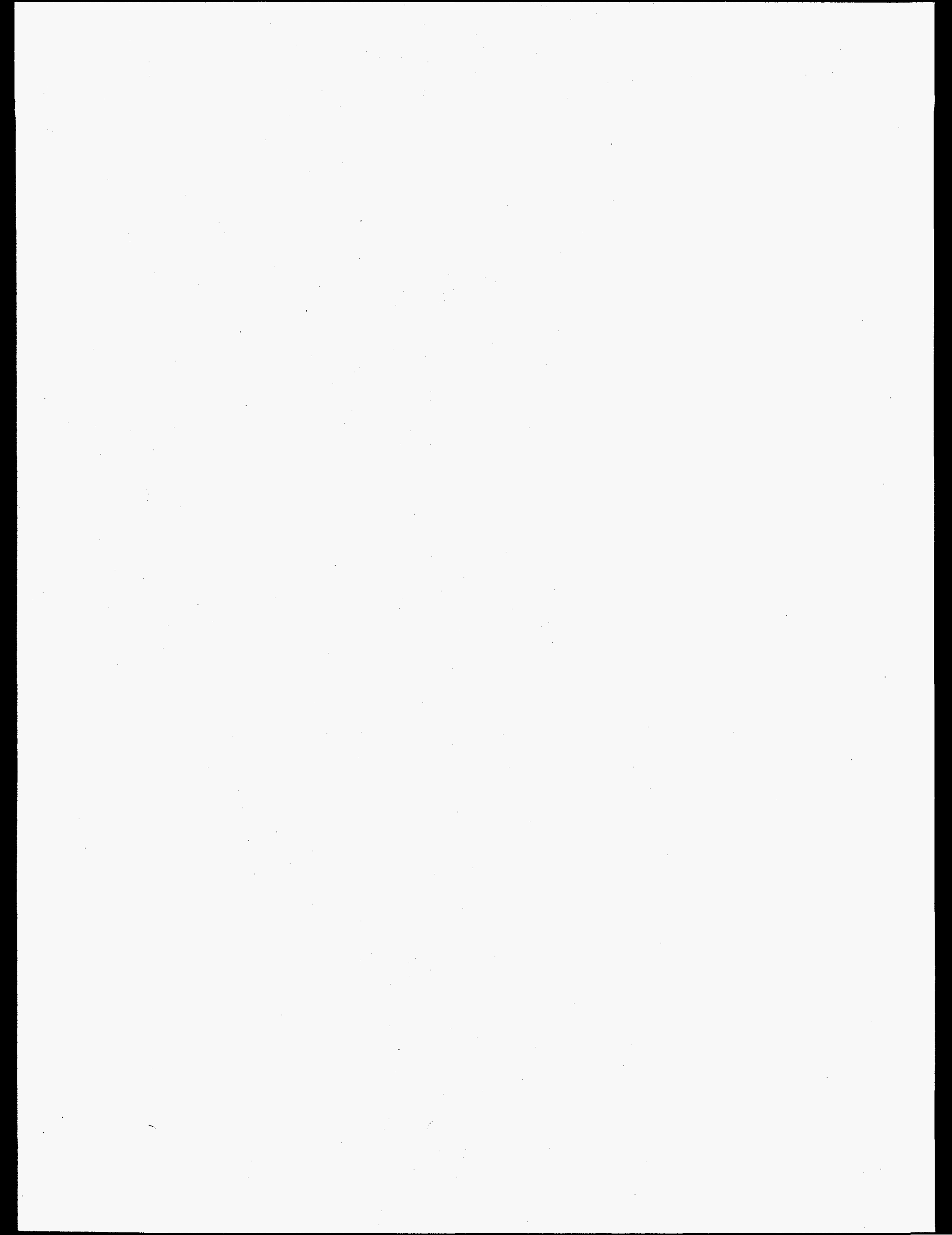




\section{APPENDIX C \\ KEY ASSUMPTIONS FOR MASS FLOW CALCULATIONS}

\section{C-1. Introduction}

This section describes the key assumptions used for the mass flow calculations. Process flowsheet modeling software (ASPEN) was used to perform the mass and energy balances. For unit operations that are similar to those of the ITTS study (e.g. gas scrubbers), the same assumptions were used as were used in the ITTS study.

Individual elements and compounds are tracked throughout the various unit operations. For modeling purposes, constituents such as hydrogen and oxygen are tracked as $\mathrm{H}_{2}$ and $\mathrm{O}_{2}$, respectively, although these terms are ordinarily used to describe gaseous constituents. Thus, the model uses $\mathrm{O}_{2}$ to describe the oxygen contained in a solid stream such as soft debris.

Likewise, $\mathrm{C}, \mathrm{H}_{2}, \mathrm{CH}_{4}$ or $\mathrm{C}_{2} \mathrm{H}_{4}$ are used to describe hydrocarbons in liquids, gases, or solids. Although most hydrocarbons contained in the waste matrices could not be described as simply $\mathrm{CH}_{4}$ or $\mathrm{C}_{2} \mathrm{H}_{4}$, these hydrocarbons were used instead of an exhaustive list of all hydrocarbons that might be present in the waste or breakdown products.

\section{C-2. Subsystems and Unit Operations Common to All INTS Options}

Mercury Amalgamation Subsystem. The mercury amalgamation subsystem combines mercury with copper to form an amalgam that is acceptable for land disposal. A Cu: $\mathrm{Hg}$ weight ratio of 0.7 is used and a conversion of $100 \%$ is assumed.

Air Pollution Control Subsystem. As modeled, the air pollution control subsystem is comprised of five different unit operations: the filter, GPCR, gas scrubber, salt separator, and mist eliminator.

The filter unit operation combines the functions of the baghouse and prefilter. It is assumed that $100 \%$ of the solid metals are trapped by the filter and that $100 \%$ of the aqueous and gaseous stream is allowed to pass through the filter. The constituents trapped by the filter are $\mathrm{As}, \mathrm{Ba}, \mathrm{Cd}, \mathrm{Cr}, \mathrm{Pb}, \mathrm{Ag}, \mathrm{Se}, \mathrm{SiO}_{2}, \mathrm{Al}_{2} \mathrm{O}_{3}, \mathrm{Fe}$, $\mathrm{Ag}$, and $\mathrm{Na}_{2} \mathrm{SO}_{4}$.

The reactions in the GPCR are assumed to occur at 1 atm pressure and with a power input of $56.49 \mathrm{~W} / \mathrm{cfm}$. The process temperature varies according to the power input, which was specified from vendor data. The reactions modeled are the following:

$$
\begin{gathered}
2 \mathrm{H}_{2}+\mathrm{O}_{2} \Rightarrow 2 \mathrm{H}_{2} \mathrm{O} \\
\mathrm{C}+\mathrm{O}_{2} \Rightarrow \mathrm{CO}_{2} \\
\mathrm{H}_{2}+\mathrm{Cl}_{2} \Rightarrow 2 \mathrm{HCl} \\
2 \mathrm{CO}+\mathrm{O}_{2} \Rightarrow 2 \mathrm{CO}_{2} \\
\mathrm{CH}_{4}+2 \mathrm{O}_{2} \Rightarrow \mathrm{CO}_{2}+2 \mathrm{H}_{2} \mathrm{O}
\end{gathered}
$$

A conversion of $95 \%$ is assumed for these reactions. Although other reactions could occur and conversion will vary with temperature, only these reactions were modeled. Additional air ( $100 \%$ excess) is introduced to the reactor, if necessary, to supplement the oxygen contained in the gas stream. 
The gas scrubber introduces caustic solution ( $\mathrm{NaOH}$ and water, $1 \%$ excess) to remove the acid gases that remain in the offgas. The following reactions are modeled:

$$
\begin{gathered}
\mathrm{HCl}+\mathrm{NaOH} \Rightarrow \mathrm{NaCl}+\mathrm{H}_{2} \mathrm{O} \\
2 \mathrm{Cl}_{2}+4 \mathrm{NaOH} \Rightarrow 4 \mathrm{NaCl}+2 \mathrm{H}_{2} \mathrm{O}+\mathrm{O}_{2} \\
\mathrm{H}_{2} \mathrm{SO}_{4}+2 \mathrm{NaOH} \Rightarrow \mathrm{Na}_{2} \mathrm{SO}_{4}+2 \mathrm{H}_{2} \mathrm{O}
\end{gathered}
$$

A conversion of $99 \%$ is assumed for these reactions.

The salt blowdown unit removes all of the $\mathrm{NaCl}, \mathrm{NaOH}$, and $\mathrm{Na}_{2} \mathrm{SO}_{4}$ from the offgas stream. Complete $(100 \%)$ removal of the salts is assumed. In addition, water is removed as a carrier of the salts. If sufficient water is available, it is removed to give a solids content of $20 \%$. The scrubber liquor is routed to the aqueous waste treatment subsystem.

The mist eliminator is modeled as a flashing operation at $100^{\circ} \mathrm{F}$ and $14.7 \mathrm{psi}(1 \mathrm{~atm})$ pressure. Vaporliquid equilibrium is assumed at these conditions. In this equilibrium condition, a small quantity of the gases is entrained in the liquid phase. The aqueous liquid is returned to the aqueous waste treatment subsystem and the gas phase is heated to $300^{\circ} \mathrm{F}$, passed through HEPA filters, and released.

Aqueous Waste Treatment Subsystem. The aqueous waste treatment subsystem is modeled by combining the aqueous wastes from the various other subsystems and reacting the residual organics in a UV reactor. Downstream of the reactor, the waste stream is separated into treated water (for recycling), offgas (routed to the APC subsystem), and salts and fines (to polymer stabilization). In addition, scrubber liquor is treated by the aqueous waste treatment subsystem. It undergoes concentration, with the aqueous phase being treated within the subsystem and the solids being routed to polymer stabilization.

One unit operation is the generation of ozone from air $\left(\mathrm{O}_{2}\right)$. The reactions proceeds at $100^{\circ} \mathrm{F}$ and 14.7 psi ( $1 \mathrm{~atm}$ ) pressure and with a conversion level of $99 \%$. An excess of $10 \%$ ozone is generated.

Hydroxyl radicals $(\mathrm{OH} \bullet)$ are generated from UV light, ozone, and hydrogen peroxide as follows:

$$
\begin{gathered}
\mathrm{H}_{2} \mathrm{O}_{2} \Rightarrow 2 \mathrm{OH} \cdot \\
\mathrm{O}_{3}+3 \mathrm{H}_{2} \mathrm{O} \Rightarrow 6 \mathrm{OH} \cdot
\end{gathered}
$$

A conversion of $99 \%$ is assumed for these reactions.

The photolytic reactor combines $\mathrm{OH} \cdot$ with organic constituents at $100^{\circ} \mathrm{F}$ and $14.7 \mathrm{psi}(1 \mathrm{~atm})$ pressure. An excess of $10 \% \mathrm{H}_{2} \mathrm{O}_{2}$ and air are used. The following reactions are modeled:

$$
\begin{gathered}
\mathrm{C}+4 \mathrm{OH} \bullet \Rightarrow \mathrm{CO}_{2}+2 \mathrm{H}_{2} \mathrm{O} \\
\mathrm{H}_{2}+2 \mathrm{OH} \Rightarrow 2 \mathrm{H}_{2} \mathrm{O} \\
\mathrm{H}_{2}+\mathrm{Cl}_{2} \Rightarrow 2 \mathrm{HCl}
\end{gathered}
$$

These reactions are assumed to have a conversion of $96 \%$.

After the reactions occur, the excess $\mathrm{O}_{3}$ is decomposed back to $\mathrm{O}_{2}\left(2 \mathrm{O}_{3} \Rightarrow 3 \mathrm{O}_{2}\right)$ at $100^{\circ} \mathrm{F}$ and 14.7 psi $(1$ atm) pressure, with a conversion of $99.9 \%$. 
Acids are neutralized with $\mathrm{NaOH}$ as follows:

$$
\begin{gathered}
\mathrm{HCl}+\mathrm{NaOH} \Rightarrow \mathrm{NaCl}+\mathrm{H}_{2} \mathrm{O} \\
2 \mathrm{Cl}_{2}+4 \mathrm{NaOH} \Rightarrow 4 \mathrm{NaCl}+2 \mathrm{H}_{2} \mathrm{O}+\mathrm{O}_{2} \\
\mathrm{H}_{2} \mathrm{SO}_{4}+2 \mathrm{NaOH} \Rightarrow \mathrm{Na}_{2} \mathrm{SO}_{4}+2 \mathrm{H}_{2} \mathrm{O} \\
2 \mathrm{Cl}_{2}+4 \mathrm{NaOH} \Rightarrow 4 \mathrm{NaCl}+2 \mathrm{H}_{2} \mathrm{O}+\mathrm{O}_{2}
\end{gathered}
$$

A conversion of $99 \%$ is assumed for these reactions.

The waste stream is next separated into three different substreams. The sludge phase consists of $\mathrm{Fe}, \mathrm{S}$, $\mathrm{SiO}_{2}, \mathrm{Al}_{2} \mathrm{O}_{3}, \mathrm{As}, \mathrm{Ba}, \mathrm{Cd}, \mathrm{Cr}, \mathrm{Pb}, \mathrm{Hg}, \mathrm{Se}, \mathrm{Ag}, \mathrm{NaOH}, \mathrm{NaCl}, \mathrm{Na}_{2} \mathrm{SO}_{4}$ and $\mathrm{AgCl}$. The offgas consists of $\mathrm{H}_{2}, \mathrm{O}_{2}$, $\mathrm{N}_{2}, \mathrm{Cl}_{2}, \mathrm{NO}, \mathrm{SO}_{2}, \mathrm{CO}_{2}$, and $\mathrm{O}_{3}$. The aqueous stream consists of $\mathrm{H}_{2} \mathrm{O}, \mathrm{HCl}, \mathrm{HNO}_{3}, \mathrm{HNO}_{2}, \mathrm{NO}_{3}-\mathrm{H}^{+}, \mathrm{Ag}^{+}$, $\mathrm{H}_{2} \mathrm{SO}_{4}, \mathrm{H}_{2} \mathrm{O}_{2}, \mathrm{OH} \cdot$, and $\mathrm{H}_{2} \mathrm{O}_{2}$. It is assumed that the separation of these streams is complete (100\%) except for $\mathrm{C}, \mathrm{SiO}_{2}$, and $\mathrm{Al}_{2} \mathrm{O}_{3} .1 \%$ of these constituents are assumed to be entrained in the aqueous stream.

Polymer Stabilization Subsystem. Four of the five INTS systems involve polymer stabilization as one means to solidify process residues. One INTS system (System 4) involves stabilization with phosphatebonded ceramic material instead of polymer.

The polymer stabilization subsystem is comprised of several unit operations: (1) mixing of the input substreams, (2) drying to remove excess moisture, and (3) polymer encapsulation of the solid residue. Moisture removed from the waste is routed to aqueous treatment, whereas the polymer stabilized waste is sent to disposal.

The waste is dried at $200^{\circ} \mathrm{F}$ to less than $5 \mathrm{wt} \%$ moisture content. In addition to moisture, gaseous constituents $\left(\mathrm{N}_{2}\right.$ and $\left.\mathrm{NO}\right)$ are separated from the waste streams before polymer stabilization. No waste dryer is used for System 4.

Polymerization of the waste material occurs by combining polyethylene $\left(\mathrm{C}_{2} \mathrm{H}_{4}\right)$ with the waste on a $1: 1$ weight ratio. It is assumed that the waste is completely stabilized by the polymer.

Grout Stabilization Subsystem. The grout stabilization subsystem combines grout material (cement, sand, and water) with waste residues to form a waste matrix that is suitable for disposal. A 2:1 weight ratio of grout to waste is used, and complete encapsulation of the waste is assumed. Dry grout material and water are added separately. If the waste contains sufficient moisture then the amount of water added is decreased accordingly. The ratio of dry grout to water is $0.7: 0.3$. To minimize water discharge and consumption, treated waste water is used in the grout formulation.

Special Waste Treatment Subsystem. Special waste is defined as that portion of the input waste stream that requires as yet unspecified special handling. The same assumptions were used in this study as those used in the ITTS study. Approximately $153 \mathrm{lb} / \mathrm{hr}$ of the incoming waste (total of $2927 \mathrm{lb} / \mathrm{hr}$ ) has been designated as special waste. No specific treatment of this waste has been proposed and no modeling of this subsystem was performed.

Metal Decontamination Subsystem. The metal decontamination subsystem accepts the metal portion of the incoming waste stream that can be treated for surface contamination and recycled. The subsystem uses a stream containing water to wash the metal. For modeling purposes, it was assumed that $100 \%$ of the contaminants on incoming metal debris was removed. The aqueous stream is routed to the aqueous waste treatment subsystem and the metal is recycled in the DOE complex. 
Lead Recovery Subsystem. The lead recovery subsystem treats lead-contaminated waste. Waste is first separated into leaded-gloves $(5 \mathrm{lb} / \mathrm{hr})$ and lead bricks $(21 \mathrm{lb} / \mathrm{hr})$. It is assumed that hydrogen, sulphur, carbon, silica, and alumina in the waste stream partition in the same proportion as does the lead.

\section{C-3. Subsystems Specific to Individual Treatment Systems}

Process Residue and Inorganic Sludge Treatment Subsystem - Vacuum Desorption. In Systems 1, 2,4 , and 5 , process residue and inorganic sludge are treated by vacuum desorption. Vacuum desorption of process residue and inorganic sludge was assumed to proceed at $1 \mathrm{psi}$ and $550^{\circ} \mathrm{F}$. For modeling purposes, no reaction of $\mathrm{O}_{2}$ is assumed to occur during this step. This prevents the formation of $\mathrm{CO}, \mathrm{CO}_{2}$, or $_{2} \mathrm{O}$ from organics in the debris.

A nitrogen sweep gas is used in the vacuum desorption units at a rate of $1.09 \mathrm{ft}^{3}$ per $\mathrm{lb}$ of waste.

Output streams from the vacuum desorption process and the partitioning of individual constituents include those found in Table C-1. The table shows the constituents contained in each of the output streams from the vacuum desorption process. For constituents that partition to more than one output stream, the percentage is given in the table. 
Table C-1. Partitioning of Constituents in Vacuum Desorption Process of Process Residue and Inorganic Sludge.

\begin{tabular}{|c|c|c|c|c|}
\hline Aqueous Waste & Treated Solids & Elemental Mercury & $\begin{array}{c}\text { Organic/Aqueous Liquid } \\
\text { Residual }\end{array}$ & Offgas \\
\hline \multirow[t]{16}{*}{$\mathrm{H}_{2} \mathrm{O}$} & $50 \%$ of $\mathrm{H}_{2}$ & $\mathrm{Hg}$ & $50 \%$ of $\mathrm{H}_{2}$ & $\mathrm{~N}_{2}$ \\
\hline & $50 \%$ of $\mathrm{Cl}_{2}$ & & $50 \%$ of $\mathrm{Cl}_{2}$ & \\
\hline & $50 \%$ of $\mathrm{C}$ & & $50 \%$ of C & \\
\hline & As & & $\mathrm{C}_{2} \mathrm{H}_{2}$ & \\
\hline & $\mathrm{Ba}$ & & $\mathrm{CH}_{4}$ & \\
\hline & $\mathrm{Cd}$ & & & \\
\hline & $\mathrm{Cr}$ & & & \\
\hline & $\mathrm{Pb}$ & & & \\
\hline & $\mathrm{Se}$ & & & \\
\hline & $\mathrm{Ag}$ & & & \\
\hline & $\mathrm{O}_{2}$ & & & \\
\hline & $\mathrm{SiO}_{2}$ & & & \\
\hline & $\mathrm{Al}_{2} \mathrm{O}_{3}$ & & & \\
\hline & $\mathrm{S}$ & & & \\
\hline & $\mathrm{Fe}$ & & & \\
\hline & $\begin{array}{l}\mathrm{H}_{2} \mathrm{O} \text { in leach } \\
\text { water }\end{array}$ & & & \\
\hline
\end{tabular}

Process Residue and Inorganic Sludge Treatment Subsystem - Aqueous Wash. For System 3, process residue and inorganic sludges are treated by aqueous wash. It is assumed that $95 \%$ of the organics are removed from the matrix and $1 \%$ of the silica and alumina are entrained in the organic stream. Make up water in the amount of $15 \%$ of the incoming solid waste stream is assumed. To simplify modeling calculations, no recycle of the wash water was assumed; only the makeup water was used to wash the waste, and two-thirds of the makeup water leaves the subsystem in the aqueous waste stream. The remainder of the water leaves in the solid waste stream. Table $\mathrm{C}-2$ describes the partitioning of the constituents in this process. 
Table C-2. Partitioning of Constituents in Aqueous Washing of Process Residue and Inorganic Sludges.

\begin{tabular}{|c|c|}
\hline Immiscible Organics & Treated Solids \\
\hline $95 \%$ of $\mathrm{Cl}_{2}$ & $5 \%$ of $\mathrm{Cl}_{2}$ \\
\hline $95 \%$ of $F_{2}$ & $5 \%$ of $\mathrm{F}_{2}$ \\
\hline $95 \%$ of $C$ & $5 \%$ of $C$ \\
\hline $1 \%$ of $\mathrm{SiO}_{2}$ & $99 \%$ of $\mathrm{SiO}_{2}$ \\
\hline $1 \%$ of $\mathrm{Al}_{2} \mathrm{O}_{3}$ & $99 \%$ of $\mathrm{Al}_{2} \mathrm{O}_{3}$ \\
\hline $95 \%$ of $\mathrm{H}_{2}$ & $5 \%$ of $\mathrm{H}_{2}$ \\
\hline \multirow[t]{14}{*}{$95 \%$ of $\mathrm{O}_{2}$} & $5 \%$ of $\mathrm{O}_{2}$ \\
\hline & $\mathrm{H}_{2} \mathrm{O}$ \\
\hline & $\mathrm{Fe}$ \\
\hline & As \\
\hline & $\mathrm{Cd}$ \\
\hline & $\mathrm{Cr}$ \\
\hline & $\mathrm{Pb}$ \\
\hline & $\mathrm{Hg}$ \\
\hline & $\mathrm{Se}$ \\
\hline & $\mathrm{Ag}$ \\
\hline & $\mathrm{H}_{2} \mathrm{SO}_{4}$ \\
\hline & $S$ \\
\hline & $\mathrm{HCl}$ \\
\hline & $\mathrm{N}_{2}$ \\
\hline
\end{tabular}

Organic Destruction Subsystem - Mediated Electrochemical Oxidation (MEO). For System 1 and 3 , organic liquids and sludges are treated using MEO. This reaction proceeds at $176^{\circ} \mathrm{F}$ and $35 \mathrm{psi}$. The MEO reactions are separated into anode reactions and cathode reactions as follows:

Anode reactions

$$
\begin{gathered}
\mathrm{C}+2 \mathrm{H}_{2} \mathrm{O} \Rightarrow \mathrm{CO}_{2}+4 \mathrm{H}^{+}+4 \mathrm{e}^{-} \\
\mathrm{H}_{2} \Rightarrow 2 \mathrm{H}^{+}+2 \mathrm{e}^{-} \\
\mathrm{Cl}_{2}+2 \mathrm{H}^{+}+2 \mathrm{e}^{-} \Rightarrow 2 \mathrm{HCl} \\
2 \mathrm{~S}+2 \mathrm{H}_{2} \mathrm{O}+3 \mathrm{O}_{2} \Rightarrow 2 \mathrm{H}_{2} \mathrm{SO}_{4} \\
2 \mathrm{~N}_{2}+2 \mathrm{H}_{2} \mathrm{O}+5 \mathrm{O}_{2} \Rightarrow 4 \mathrm{HNO}_{3}
\end{gathered}
$$




\section{Cathode reactions}

$$
\begin{gathered}
\mathrm{HNO}_{3}+2 \mathrm{H}^{+}+2 \mathrm{e}^{-} \Rightarrow \mathrm{H}_{2} \mathrm{O}+\mathrm{HNO}_{2} \\
\mathrm{AgNO}_{3}+\mathrm{HCl} \Rightarrow \mathrm{AgCl}+\mathrm{HNO}_{3}
\end{gathered}
$$

The reaction with nitric acid is assumed to be $100 \%$ complete and the reaction with silver nitrate is assumed to be $99 \%$ complete. Sufficient $\mathrm{HNO}_{3}$ is added to maintain the process solution at about $8 \mathrm{M} \mathrm{HNO}_{3}$. $\mathrm{AgNO}_{3}$ is added in stoichiometric amounts.

The reaction products are partitioned to various output streams according to Table C-3.

Table C-3 Partitioning of Constituents in Mediated Electrochemical Oxidation of Organic Liquids and Sludges.

\begin{tabular}{cccc}
\hline Liquid (to aqueous) & $\begin{array}{c}\text { Gases } \\
\text { (to } \mathrm{HNO}_{2} \text { recovery) }\end{array}$ & $\begin{array}{c}\text { Sludge (to process } \\
\text { aqueous treatment) }\end{array}$ & $\begin{array}{c}\text { Silver nitrate } \\
\text { (to Ag recovery) }\end{array}$ \\
\hline $\mathrm{H}_{2}$ & $\mathrm{O}_{2}$ & $\mathrm{~S}$ & $\mathrm{AgNO}_{3}$ \\
$\mathrm{Cl}_{2}$ & $\mathrm{CO}_{2}$ & $\mathrm{~F}_{2}$ & $\mathrm{HNO}_{3}$ \\
$\mathrm{H}_{2} \mathrm{O}$ & $\mathrm{HNO}_{2}$ & $\mathrm{Fe}$ & $\mathrm{NO}_{3}^{-}$ \\
$\mathrm{H}_{2} \mathrm{SO}_{4}$ & $\mathrm{CH}_{4}$ & $\mathrm{Ba}$ & $\mathrm{H}^{+}$ \\
$\mathrm{HCl}$ & & $\mathrm{Cd}$ & $\mathrm{Ag}^{+}$ \\
& & $\mathrm{Cr}$ & $\mathrm{AgCl}^{-}$ \\
& & $\mathrm{C}$ & $\mathrm{Cr}^{-}$ \\
& & $\mathrm{SiO}_{2}$ & \\
\end{tabular}

Recovery of the $\mathrm{HNO}_{2}$ is effected by the following reaction, which proceeds at $302^{\circ} \mathrm{F}\left(423^{\circ} \mathrm{K}\right)$ and 35 psi:

$$
2 \mathrm{HNO}_{2}+\mathrm{O}_{2} \rightarrow 2 \mathrm{HNO}_{3}
$$

The reaction is assumed to operate at a conversion of $99 \%$. Oxygen is added in $1 \%$ excess. After the reaction, the resulting nitric acid is separated and recycled and the remaining $\mathrm{O}_{2}, \mathrm{CO}_{2}, \mathrm{H}_{2} \mathrm{O}, \mathrm{NO}$, are sent to the APC system. 
The $1 \% \mathrm{HNO}_{2}$ not recovered is assumed to decompose as follows:

$$
4 \mathrm{HNO}_{2} \Rightarrow 2 \mathrm{H}_{2} \mathrm{O}+4 \mathrm{NO}+\mathrm{O}_{2}
$$

The NO produced is neutralized with ammonia as follows:

$$
6 \mathrm{NO}+4 \mathrm{NH}_{3} \Rightarrow 6 \mathrm{H}_{2} \mathrm{O}+5 \mathrm{~N}_{2}
$$

This reaction proceeds at a conversion of $99 \%$.

Silver is recovered from the MEO liquids by the following reactions:

$$
\begin{gathered}
4 \mathrm{AgCl}+4 \mathrm{NaOH}+4 \mathrm{H}_{2} \mathrm{O}_{2} \Rightarrow 4 \mathrm{Ag}+4 \mathrm{NaCl}+6 \mathrm{H}_{2} \mathrm{O}+3 \mathrm{O}_{2} \\
\mathrm{Ag}+\mathrm{HNO}_{3} \Rightarrow \mathrm{AgNO}_{3}+\mathrm{H}^{+}+\mathrm{e}^{-}
\end{gathered}
$$

The $\mathrm{AgNO}_{3}$ recovered from the process is recycled to the $\mathrm{MEO}$ unit. This process assumes a conversion of $99 \%$ and $1 \%$ excess $\mathrm{NaOH}$ and $\mathrm{H}_{2} \mathrm{O}_{2}$ are added. $\mathrm{HNO}_{3}$ is also added in $1 \%$ excess, if necessary. Offgases from the recovery process $\left(\mathrm{H}_{2}\right.$ and $\left.\mathrm{O}_{2}\right)$ are sent to the APC system. The remaining salt solution $\left(\mathrm{H}_{2} \mathrm{O}, \mathrm{Ag}\right.$, $\mathrm{NaOH}, \mathrm{NaCl}, \mathrm{HNO}_{3}, \mathrm{H}+, \mathrm{Ag}+, \mathrm{H}_{2} \mathrm{O}_{2}$, and $\mathrm{AgCl}$ ) is sent to the aqueous treatment system.

Organic Destruction Subsystem - Catalytic Wet Oxidation. For Systems 2 and 5, organic liquids and sludges are treated using catalytic wet oxidation. This subsystem consists of a wet oxidation reactor, a separation unit, an equilibration reactor, and a neutralization reactor.

The wet oxidation reaction proceeds at $302^{\circ} \mathrm{F}$ and $35 \mathrm{psi}$. The following reactions are used to model this process:

$$
\begin{gathered}
\mathrm{C}+\mathrm{O}_{2} \Rightarrow \mathrm{CO}_{2} \\
2 \mathrm{H}_{2}+\mathrm{O}_{2}=2 \mathrm{H}_{2} \mathrm{O} \\
\mathrm{H}_{2}+\mathrm{Cl}_{2} \Rightarrow 2 \mathrm{HCl} \\
\mathrm{S}+\mathrm{H}_{2}+2 \mathrm{O}_{2} \Rightarrow \mathrm{H}_{2} \mathrm{SO}_{4} \\
\mathrm{CH}_{4}+2 \mathrm{O}_{2} \Rightarrow \mathrm{CO}_{2}+2 \mathrm{H}_{2} \mathrm{O}
\end{gathered}
$$

These reactions do not show the interaction of the catalyst in the process; they only show the overall reaction of oxygen with the organics. Oxygen is added in 5\% excess. The catalyst used in this system consists of $60 \% \mathrm{FeCl}_{3}, 36 \% \mathrm{H}_{2} \mathrm{O}$, and $4 \% \mathrm{HCl}$. Catalyst is added in a ratio of $7 \mathrm{lb}$ per $200 \mathrm{lb}$ of incoming waste. A conversion level of $99 \%$ is assumed for these reactions.

After the wet oxidation reactor, the products are separated into gases, liquids, and sludges as listed in Table C-4. 
Table C-4 Partitioning of Constituents from Catalytic Wet Oxidation of Organic Liquids and Sludges.

\begin{tabular}{ccc}
\hline $\begin{array}{c}\text { Liquid } \\
\text { (to aqueous waste treatment) }\end{array}$ & $\begin{array}{c}\text { Gases } \\
\text { (to air pollution control) }\end{array}$ & $\begin{array}{c}\text { Sludge } \\
\text { (to polymer stabilization) }\end{array}$ \\
\hline $50 \%$ of $\mathrm{Fe}(\mathrm{OH})_{3}$ & $\mathrm{O}_{2}$ & $50 \%$ of $\mathrm{Fe}(\mathrm{OH})_{3}$ \\
$\mathrm{FeCl}_{3}$ & $\mathrm{CO}_{2}$ & $\mathrm{~S}$ \\
$\mathrm{H}_{2} \mathrm{O}$ & $\mathrm{Cl}_{2}$ & $\mathrm{Fe}$ \\
$\mathrm{H}_{2} \mathrm{SO}_{4}$ & $\mathrm{CH}_{4}$ & $\mathrm{Ba}$ \\
$\mathrm{HCl}$ & $\mathrm{F}_{2}$ & $\mathrm{Cd}$ \\
$\mathrm{NaCl}$ & & $\mathrm{Cr}$ \\
& & $\mathrm{C}$ \\
& & $\mathrm{SiO}_{2}$ \\
& & $\mathrm{Al}_{2} \mathrm{O}_{3}$ \\
\hline
\end{tabular}

Because the model used only stoichiometry to model the wet oxidation reaction, the products are not in equilibrium. An equilibrating reactor is used to convert the offgas to an equilibrium composition of $\mathrm{Cl}_{2}, \mathrm{CO}_{2}$, $\mathrm{CO}, \mathrm{H}_{2} \mathrm{O}$, and $\mathrm{HCl}$ at $302^{\circ} \mathrm{F}\left(423^{\circ} \mathrm{K}\right)$ and 35 psi.

The liquid stream from wet oxidation is neutralized before being sent to aqueous waste treatment. The following reactions are used:

$$
\begin{gathered}
\mathrm{HCl}+\mathrm{NaOH} \Rightarrow \mathrm{NaCl}+\mathrm{H}_{2} \mathrm{O} \\
2 \mathrm{Cl}_{2}+4 \mathrm{NaOH} \Rightarrow 4 \mathrm{NaCl}+2 \mathrm{H}_{2} \mathrm{O}+\mathrm{O}_{2} \\
\mathrm{H}_{2} \mathrm{SO}_{4}+2 \mathrm{NaOH} \Rightarrow \mathrm{Na}_{2} \mathrm{SO}_{4}+2 \mathrm{H}_{2} \mathrm{O} \\
\mathrm{FeCl}_{3}+3 \mathrm{NaOH} \Rightarrow \mathrm{Fe}(\mathrm{OH})_{3}+3 \mathrm{NaCl}
\end{gathered}
$$

Each of these reactions is assumed to have a conversion of $99 \%$. Excess $\mathrm{NaOH}(1 \%)$ is used.

Organic Destruction Subsystem - Phosphoric Acid Digestion. For System 4, organic liquids and sludges are treated using phosphoric acid digestion. This process uses a combination of nitric and phosphoric acids to oxidize the waste. Nitric acid is the oxidant used; phosphoric acid is present only to facilitate the reaction. Five different unit operations are used to model the acid digestion, $\mathrm{HNO}_{3}$ regeneration, gas separation, neutralization, and product separation. The following reactions are modeled at $392^{\circ} \mathrm{F}$ and $1 \mathrm{~atm}$. 


$$
\begin{gathered}
3 \mathrm{C}+4 \mathrm{HNO}_{3} \Rightarrow 3 \mathrm{CO}_{2}+4 \mathrm{NO}+2 \mathrm{H}_{2} \mathrm{O} \\
3 \mathrm{H}_{2}+2 \mathrm{HNO}_{3} \Rightarrow 4 \mathrm{H}_{2} \mathrm{O}+2 \mathrm{NO} \\
\mathrm{Cl}_{2}+\mathrm{H}_{2} \Rightarrow 2 \mathrm{HCl} \\
\mathrm{S}+\mathrm{H}_{2}+2 \mathrm{O}_{2} \Rightarrow \mathrm{H}_{2} \mathrm{SO}_{4}
\end{gathered}
$$

Each of these reactions is assumed to have a conversion of $95 \%$. Excess $\mathrm{HNO}_{3}(5 \%)$ is added to the reactor.

After the acid digestion reactor, $\mathrm{HNO}_{3}$ is regenerated by the following reaction at $392^{\circ} \mathrm{F}$ and $1 \mathrm{~atm}$ :

$$
4 \mathrm{NO}+3 \mathrm{O}_{2}+2 \mathrm{H}_{2} \mathrm{O} \Rightarrow 4 \mathrm{HNO}_{3}
$$

This reaction is assumed to go $99 \%$ to completion and the $\mathrm{HNO}_{3}$ is recycled to the acid digestion reactor.

Neutralization of the acidic liquid is modeled by the following reactions at $100^{\circ} \mathrm{F}$ and $1 \mathrm{~atm}$ :

$$
\begin{gathered}
\mathrm{HCl}+\mathrm{NaOH} \Rightarrow \mathrm{NaCl}+\mathrm{H}_{2} \mathrm{O} \\
2 \mathrm{Cl}_{2}+4 \mathrm{NaOH} \Rightarrow 4 \mathrm{NaCl}+2 \mathrm{H}_{2} \mathrm{O}+\mathrm{O}_{2} \\
\mathrm{H}_{2} \mathrm{SO}_{4}+2 \mathrm{NaOH} \Rightarrow \mathrm{Na}_{2} \mathrm{SO}_{4}+2 \mathrm{H}_{2} \mathrm{O} \\
\mathrm{HNO}_{3}+\mathrm{NaOH} \Rightarrow \mathrm{H}_{2} \mathrm{O}+\mathrm{NaNO}_{3} \\
6 \mathrm{NO}+4 \mathrm{NH}_{3} \Rightarrow 5 \mathrm{~N}_{2}+6 \mathrm{H}_{2} \mathrm{O}
\end{gathered}
$$

These reactions are assumed to go $99 \%$ to completion. Excess $\mathrm{NaOH}(1 \%)$ and $\mathrm{NH}_{3}(5 \%)$ are added in this process.

Partitioning of the various constituents from the phosphoric acid digestion process is shown in Table C-5. 
Table C-5. Partitioning of Constituents in the Phosphoric Acid Digestion of Organic Liquids and Sludges.

\begin{tabular}{ccc}
\hline Liquid & Sludge & Gas \\
\hline $\mathrm{H}_{2} \mathrm{O}$ & $\mathrm{Fe}$ & $\mathrm{O}_{2}$ \\
$\mathrm{NaOH}$ & $\mathrm{S}$ & $\mathrm{N}_{2}$ \\
$\mathrm{NaCl}$ & $\mathrm{C}$ & $\mathrm{Cl}_{2}$ \\
$\mathrm{HCl}$ & $\mathrm{SiO}_{2}$ & $\mathrm{NO}$ \\
$\mathrm{H}_{2} \mathrm{SO}_{4}$ & $\mathrm{Al}_{2} \mathrm{O}_{3}$ & $\mathrm{CO}_{2}$ \\
$\mathrm{Na}_{2} \mathrm{SO}_{4}$ & $\mathrm{Ba}$ & $\mathrm{CH}_{4}$ \\
$\mathrm{NH}_{3}$ & $\mathrm{Cd}$ & \\
$\mathrm{HNO}_{3}$ & $\mathrm{Cr}$ & \\
$\mathrm{NaNO}_{3}$ & & \\
\hline
\end{tabular}

Bulk Soil Treatment Subsystem - Vacuum Desorption. In Systems 1 and 2, bulk soils are treated by vacuum desorption. Vacuum desorption is described in Section C-3. The process for sludge is very similar to the process for soil. Partitioning of the constituents from this subsystem is shown in Table C-6.

Table C-6 Partitioning of Constituents in Vacuum Desorption of Bulk Soil.

\begin{tabular}{cccc}
\hline Liquids & Gases & Solids & Organics \\
\hline $\mathrm{H}_{2} \mathrm{O}$ & $\mathrm{N}_{2}$ & $\mathrm{H}_{2} \mathrm{O}$ in leach water & $\mathrm{CH}_{4}$ \\
$\mathrm{HCl}$ & & $50 \%$ of $\mathrm{H}_{2}$ & $50 \%$ of $\mathrm{H}_{2}$ \\
& $50 \%$ of C & $50 \%$ of C \\
& $\mathrm{SiO}_{2}$ & \\
& $\mathrm{Al}_{2} \mathrm{O}_{3}$ & \\
\hline
\end{tabular}

Bulk Soil Treatment Subsystem - Aqueous Wash. In Systems 3, 4, and 5, bulk soils are treated by aqueous wash. Aqueous wash for sludge, described Section C-3, is essentially same as aqueous wash for soil. Partitioning of the constituents from this subsystem is shown in Table C-7. A dryer is used to remove the moisture from the soil before the soil is sent to grout stabilization. The dryer operates at $200^{\circ} \mathrm{F}$ and $1 \mathrm{~atm}$ pressure. It is assumed that $100 \%$ of the moisture is removed. The moisture is sent to aqueous waste treatment. In addition, $1 \%$ of the $\mathrm{SiO}_{2}$ and $\mathrm{Al}_{2} \mathrm{O}_{3}$ is assumed to be entrained with the moisture. 
Table C-7 Partitioning of Constituents in Washing of Bulk Soil, Systems 3, 4, and 5

\begin{tabular}{ccc}
\hline Organics & Treated Soil & Aqueous \\
\hline $95 \%$ of $\mathrm{H}_{2}$ & $5 \%$ of $\mathrm{H}_{2}$ & $\mathrm{H}_{2} \mathrm{O}$ \\
$95 \%$ of $\mathrm{C}$ & $5 \%$ of $\mathrm{C}$ & \\
$95 \%$ of $\mathrm{Cl}_{2}$ & $5 \%$ of $\mathrm{Cl}_{2}$ & \\
& $\mathrm{SiO}_{2}$ & \\
& $\mathrm{Al}_{2} \mathrm{O}_{3}$ & \\
\hline
\end{tabular}

Soft Debris Treatment Subsystem - Grout Stabilization. In System 1, soft debris is treated by grout stabilization, described in Section C-2.

Soft Debris Treatment Subsystem - Vacuum Thermal Desorption. In System 2, soft debris is treated by vacuum thermal desorption, described in Section C-3. Partitioning of the various constituents is as shown in Table C-8.

Table C-8. Partitioning of Constituents in Vacuum Thermal Desorption of Soft, Complex, and Open Debris (System 2).

\begin{tabular}{|c|c|c|c|c|}
\hline Mercury & Solids & Liquids & Gases & Organics \\
\hline \multirow[t]{9}{*}{$\mathrm{Hg}$} & $90 \%$ of $\mathrm{H}_{2}$ & balance of $\mathrm{H}_{2} \mathrm{O}$ & $\mathrm{N}_{2}$ & $10 \%$ of $\mathrm{H}_{2}$ \\
\hline & $90 \%$ of $\mathrm{F}_{2}$ & $\mathrm{HCl}$ & & $10 \%$ of $F_{2}$ \\
\hline & $90 \%$ of $\mathrm{C}$ & & & $90 \%$ of $\mathrm{C}$ \\
\hline & $\mathrm{SiO}_{2}$ & & & $\mathrm{CH}_{4}$ \\
\hline & $\mathrm{Al}_{2} \mathrm{O}_{3}$ & & & \\
\hline & $\mathrm{H}_{2} \mathrm{O}$ in leach water & & & \\
\hline & $S$ & & & \\
\hline & $\mathrm{O}_{2}$ & & & \\
\hline & $\mathrm{Fe}$ & & & \\
\hline
\end{tabular}

Soft Debris Treatment Subsystem - Low Pressure Wash. In System 3, soft debris is treated by low pressure wash. Modelling assumptions are similar to the aqueous wash described earlier in Section C-3. It is assumed that only a small fraction of the organic material in the soft debris is organic liquid. Washing is assumed to remove only $10 \%$ of the total organics from the soft debris. Table C-9 describes the partitioning of constituents in the agitation wash of soft debris. 
Table C-9. Partitioning of Constituents in Low Pressure Washing of Soft Debris

\begin{tabular}{cc}
\hline Aqueous Waste & Washed Debris \\
\hline $10 \% \mathrm{Cl}_{2}$ & $90 \% \mathrm{Cl}_{2}$ \\
$10 \% \mathrm{~F}_{2}$ & $90 \% \mathrm{~F}_{2}$ \\
$10 \% \mathrm{C}$ & $90 \% \mathrm{C}$ \\
$10 \% \mathrm{O}_{2}$ & $90 \% \mathrm{O}_{2}$ \\
$1 \% \mathrm{SiO}_{2}$ & $99 \% \mathrm{SiO}_{2}$ \\
$1 \% \mathrm{Al}_{2} \mathrm{O}_{3}$ & $99 \% \mathrm{Al}_{2} \mathrm{O}_{3}$ \\
$10 \%$ of $\mathrm{H}_{2}$ & $90 \%$ of $\mathrm{H}_{2}$ \\
$5 \%$ of $\mathrm{Hg}$ & $95 \% \mathrm{Hg}$ \\
$2 / 3$ of wash $\mathrm{H}_{2} \mathrm{O}$ & balance of $\mathrm{H}_{2} \mathrm{O}$ \\
& $\mathrm{N}$ \\
& Fe \\
\hline
\end{tabular}

Soft Debris Treatment Subsystem - Phosphoric Acid Digestion. In System 4, soft debris is treated by phosphoric acid digestion, described above. Table $\mathrm{C}-10$ describes the partitioning of constituents in this process.

Table C-10. Partitioning of Constituents from the Phosphoric Acid Digestion Process of Soft Debris

\begin{tabular}{ccc}
\hline Liquid & Sludge & Gas \\
\hline $5 \% \mathrm{Hg}$ & $95 \%$ of $\mathrm{Hg}$ & $\mathrm{H}_{2}$ \\
$\mathrm{H}_{2} \mathrm{O}$ & $\mathrm{Fe}$ & $\mathrm{N}_{2}$ \\
$\mathrm{NaOH}$ & $\mathrm{S}$ & $\mathrm{Cl}_{2}$ \\
$\mathrm{NaCl}$ & $\mathrm{C}$ & $\mathrm{F}_{2}$ \\
$\mathrm{HCl}$ & $\mathrm{SiO}_{2}$ & $\mathrm{NO}$ \\
$\mathrm{H}_{2} \mathrm{SO}_{4}$ & $\mathrm{Al}_{2} \mathrm{O}_{3}$ & $\mathrm{CO}_{2}$ \\
$\mathrm{Na}_{2} \mathrm{SO}_{4}$ & $\mathrm{O}_{2}$ & \\
$\mathrm{NH}_{3}$ & & \\
$\mathrm{HNO}_{3}$ & & \\
$\mathrm{NaNO}_{3}$ & & \\
\hline
\end{tabular}

Soft Debris Treatment Subsystem - Catalytic Wet Oxidation. In System 5, soft debris is treated by catalytic wet oxidation, described above. After the wet oxidation reactor, the products are separated into gases, liquids, and sludges as shown in Table C-11. 
Table C-11. Partitioning of Constituents in Catalytic Wet Oxidation of Soft Debris.

\begin{tabular}{ccc}
\hline Liquids & Solids (Sludges) & Gases \\
\hline $\mathrm{H}_{2} \mathrm{O}$ & $\mathrm{S}$ & $\mathrm{N}_{2}$ \\
$\mathrm{HCl}$ & $\mathrm{Fe}$ & $\mathrm{Cl}_{2}$ \\
$5 \%$ of $\mathrm{Hg}$ & $95 \%$ of $\mathrm{Hg}$ & $\mathrm{F}_{2}$ \\
$\mathrm{H}_{2} \mathrm{SO}_{4}$ & $\mathrm{O}_{2}$ & $\mathrm{CO}_{2}$ \\
$\mathrm{FeCl}_{3}$ & $\mathrm{Fe}(\mathrm{OH})_{3}$ & \\
$\mathrm{NaCl}$ & $\mathrm{C}$ & \\
$\mathrm{NaOH}$ & $\mathrm{SiO}_{2}$ & \\
$\mathrm{Na}_{2} \mathrm{SO}_{4}$ & $\mathrm{Al}_{2} \mathrm{O}_{3}$ & \\
\hline
\end{tabular}

As described above for catalytic wet oxidation, an equilibration reactor and acid neutralization reactor are used downstream of the wet oxidation reactor. The operating conditions for these reactors are identical to those described above.

Complex Debris Treatment Subsystem - Grout Stabilization. In System 1, complex debris is treated by grout stabilization, described in Section C-2 above.

Complex Debris Treatment Subsystem - Vacuum Thermal Desorption. In System 2, complex debris is treated by vacuum thermal desorption, described in Section C-3 above. Partitioning of the various constituents is shown in Table C-8.

Complex Debris Treatment Subsystem - High Pressure Wash. In System 3, complex debris is treated by high pressure wash. Modelling assumptions are similar to the aqueous wash described in Section C-3 above. Table C-12 describes the partitioning of constituents in the high pressure wash of complex debris. 
Table C-12. Partitioning of Constituents in High Pressure Wash of Complex Debris.

\begin{tabular}{cc}
\hline Aqueous Organics & Treated Solids \\
\hline $98 \%$ of $\mathrm{H}_{2}$ & $2 \%$ of $\mathrm{H}_{2}$ \\
$98 \%$ of C & $2 \%$ of C \\
$1 \%$ of $\mathrm{SiO}_{2}$ & $99 \%$ of $\mathrm{SiO}_{2}$ \\
$1 \%$ of $\mathrm{Al}_{2} \mathrm{O}_{3}$ & $99 \%$ of $\mathrm{Al}_{2} \mathrm{O}_{3}$ \\
$2 / 3$ of wash $\mathrm{H}_{2} \mathrm{O}$ & balance of $\mathrm{H}_{2} \mathrm{O}$ \\
& $\mathrm{Fe}$ \\
& $\mathrm{Hg}$ \\
\hline
\end{tabular}

Complex Debris Treatment Subsystem - Grout Stabilization. In Systems 4 and 5, complex debris is treated by grout stabilization, described in Section C-2 above.

Open Debris Treatment Subsystem - Grout Stabilization. In System 1, open debris is treated by grout stabilization, described in Section C-2 above.

Open Debris Treatment Subsystem -Vacuum Thermal Desorption. In System 2, complex debris is treated by vacuum thermal desorption, described in Section $\mathrm{C}-3$ above. Partitioning of the various constituents is shown in Table C-8.

Open Debris Treatment Subsystem - High Pressure Wash. In System 3, open debris is treated by high pressure spray, described in Section C-3 above. Table C-13 describes the partitioning of constituents in the high pressure wash of open debris. As with other washing processes modeled, the amount of makeup water used is $15 \%$ of the mass of the open debris being washed. Two-thirds of the wash water is routed to aqueous waste and the remainder of the water leaves with the treated solids.

Table C-13. Partitioning of Constituents in High Pressure Spray of Open Debris.

\begin{tabular}{cc}
\hline Aqueous Organics & Treated Solids \\
\hline $98 \%$ of $\mathrm{C}$ & $2 \% \mathrm{C}$ \\
$1 \%$ of $\mathrm{SiO}_{2}$ & $99 \% \mathrm{SiO}_{2}$ \\
$1 \%$ of $\mathrm{Al}_{2} \mathrm{O}_{3}$ & $99 \% \mathrm{Al}_{2} \mathrm{O}_{3}$ \\
$98 \%$ of $\mathrm{H}_{2}$ & $2 \%$ of $\mathrm{H}_{2}$ \\
$98 \%$ of $\mathrm{O}_{2}$ & $2 \%$ of $\mathrm{O}_{2}$ \\
$2 / 3$ of wash $\mathrm{H}_{2} \mathrm{O}$ & balance of $\mathrm{H}_{2} \mathrm{O}$ \\
\hline
\end{tabular}


Open Debris Treatment Subsystem - High Pressure Spray. In Systems 4 and 5, open debris is treated by high pressure spray, described above in Section C-3. Table C-14 describes the partitioning of constituents in the high pressure spray of open debris.

Table C-14. Partitioning of Constituents in High Pressure Spray of Open Debris.

\begin{tabular}{cc}
\hline Washed Debris & Aqueous Waste \\
\hline $2 \% \mathrm{C}$ & $98 \%$ of C \\
$99 \% \mathrm{SiO}_{2}$ & $1 \%$ of $\mathrm{SiO}_{2}$ \\
$99 \% \mathrm{Al}_{2} \mathrm{O}_{3}$ & $1 \% \mathrm{Al}_{2} \mathrm{O}_{3}$ \\
$1 / 3$ of wash $\mathrm{H}_{2} \mathrm{O}$ & balance of $\mathrm{H}_{2} \mathrm{O}$ \\
$2 \%$ of $\mathrm{O}_{2}$ & $98 \%$ of $\mathrm{O}_{2}$ \\
$2 \%$ of $\mathrm{H}_{2}$ & $98 \%$ of $\mathrm{H}_{2}$ \\
$\mathrm{Fe}$ & \\
\hline
\end{tabular}

\section{C-4. Summary}

An overall summary mass balance is provided as Table C- 15 . 
Table C-15. INTS Mass Balance Summary (Sheet 1 of 10).

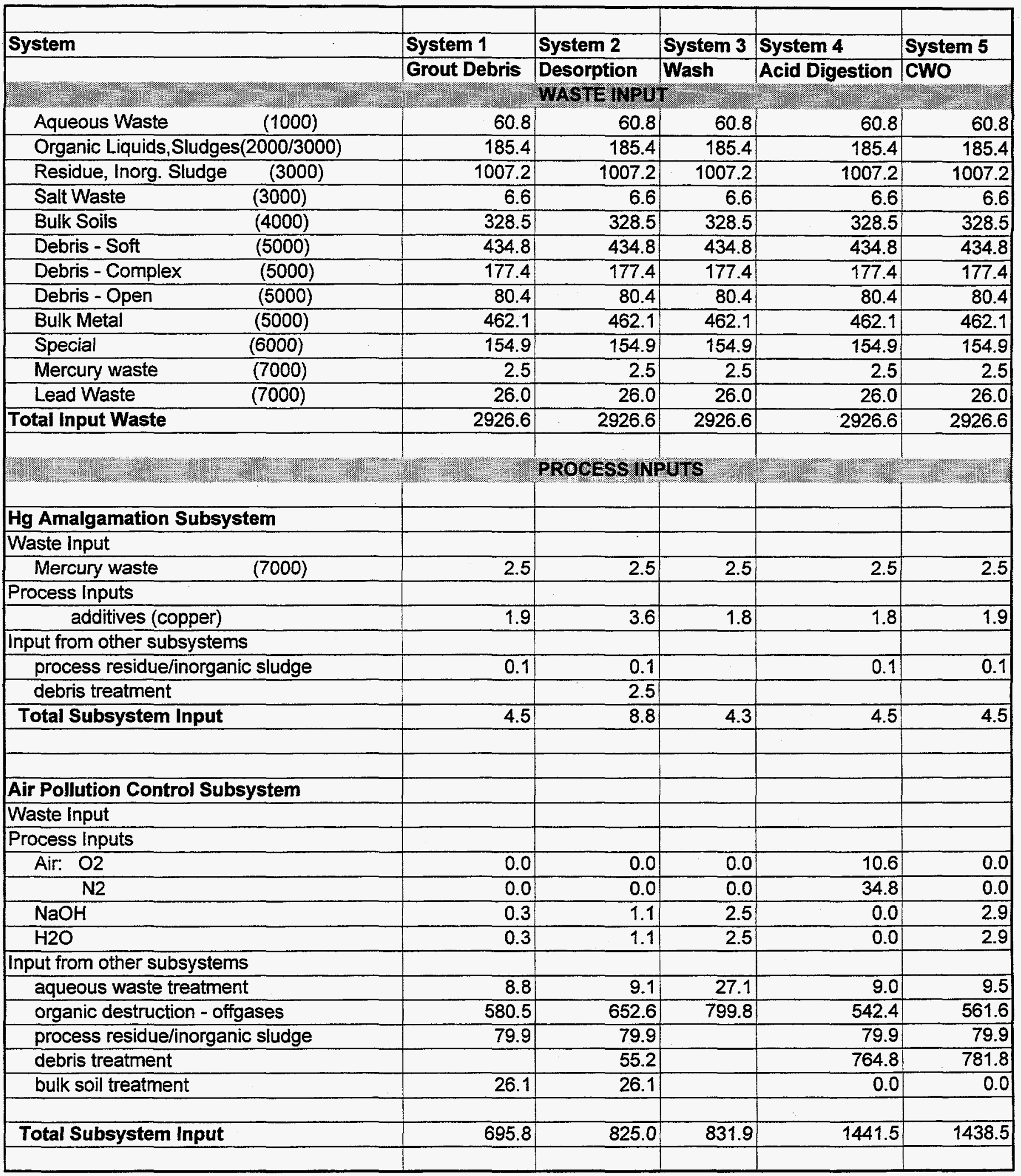


Table C-15. INTS Mass Balance Summary (Sheet 2 of 10).

\begin{tabular}{|c|c|c|c|c|c|}
\hline System & System 1 & System 2 & System 3 & System 4 & System 5 \\
\hline & Grout Debris & Desorption & Wash & Acid Digestion & CWO \\
\hline \multicolumn{6}{|l|}{ Aqueous Waste Treatment Subsystem } \\
\hline \multicolumn{6}{|l|}{ Waste Input } \\
\hline Aqueous Waste & 60.8 & 60.8 & 60.8 & 60.8 & 60.8 \\
\hline \multicolumn{6}{|l|}{ Process Inputs } \\
\hline Air (for ozone generation) & 3.5 & 3.8 & 8.6 & 3.8 & 3.9 \\
\hline $\mathrm{H} 2 \mathrm{O} 2$ & 0.6 & 0.6 & 1.4 & 0.6 & 0.6 \\
\hline $\mathrm{H} 2 \mathrm{O}$ & 24.1 & 45.8 & 0.5 & 7.6 & 9.0 \\
\hline $\mathrm{NaOH}$ & 24.1 & 45.8 & 0.5 & 7.6 & 9.0 \\
\hline \multicolumn{6}{|l|}{ Input from other subsystems } \\
\hline APC - moisture & 0.0 & 0.0 & 0.0 & 0.0 & 0.0 \\
\hline APC - concentrate & 0.3 & 2.0 & 5.0 & 0.1 & 5.2 \\
\hline process residue/inorganic sludge & 349.6 & 349.6 & 0.0 & 349.6 & 349.6 \\
\hline debris treatment & & 32.4 & 129.3 & 432.6 & 406.9 \\
\hline bulk soil treatment & 40.7 & 40.7 & 355.4 & 355.4 & 355.4 \\
\hline polymer stabilization & 267.5 & 265.7 & 456.7 & 238.3 & 265.5 \\
\hline metal decontamination & 126.2 & 126.2 & 126.2 & 126.2 & 126.2 \\
\hline organic destruction - liquids & 238.9 & 380.3 & 320.3 & 420.3 & 386.1 \\
\hline organic destruction - salts & 108.6 & & 178.7 & & \\
\hline Total Subsystem Input & 1244.8 & 1353.8 & 1643.3 & 2002.9 & 1978.1 \\
\hline \multicolumn{6}{|c|}{ Process Residue and Inorganic Sludge Treatment Subsystem } \\
\hline \multicolumn{6}{|c|}{\begin{tabular}{|l|l|l|} 
Waste Input & & \\
\end{tabular}} \\
\hline Residue, Inorg. Sludge & 1007.2 & 1007.2 & 1007.2 & 1007.2 & 1007.2 \\
\hline \multicolumn{6}{|l|}{ Process Inputs } \\
\hline \multicolumn{6}{|l|}{ oxygen } \\
\hline N2 sweep gas & 80 & 80 & & 80 & 80 \\
\hline $\mathrm{H} 2 \mathrm{O}$ & 306 & 306 & 151.05 & 306 & 306 \\
\hline \multicolumn{6}{|l|}{ NH3 } \\
\hline \multicolumn{6}{|l|}{$\mathrm{NaOH}$} \\
\hline \multicolumn{6}{|l|}{ Input from other subsystems } \\
\hline Pb recovery & 13.0 & 13.0 & 13.0 & 13.0 & 13.0 \\
\hline \multicolumn{6}{|l|}{ organic destruction } \\
\hline Total Subsystem Input & 1406.1 & 1406.1 & 1171.3 & 1406.1 & 1406.1 \\
\hline
\end{tabular}


Table C-15. INTS Mass Balance Summary (Sheet 3 of 10).

\begin{tabular}{|c|c|c|c|c|c|}
\hline System & System 1 & System 2 & System 3 & System 4 & System 5 \\
\hline & Grout Debris & Desorption & Wash & Acid Digestion & CWO \\
\hline \multicolumn{6}{|l|}{ Debris Treatment Subsystem } \\
\hline \multicolumn{6}{|l|}{ Waste Input } \\
\hline Debris - Soft & & 434.76 & 434.76 & 434.76 & 434.76 \\
\hline Debris - Complex & & 177.411 & 177.411 & & \\
\hline Debris - Open & & 80.4 & 80.4 & 80.4 & 80.4 \\
\hline \multicolumn{6}{|l|}{ Process Inputs } \\
\hline Acid-in ( $\mathrm{FeCl} 3, \mathrm{H} 2 \mathrm{O}, \mathrm{HCl})$ & & & & & 15.2 \\
\hline $\mathrm{HNO} 3$ & & & & 42.8 & \\
\hline oxygen & & & & 791.7 & 766.7 \\
\hline N2 sweep gas & & 54.9 & & & \\
\hline $\mathrm{H} 2 \mathrm{O}$ & & 207.9 & 103.8 & 49.7 & 42.9 \\
\hline $\mathrm{NH3}$ & & & & 3.9 & \\
\hline $\mathrm{NaOH}$ & & & & 37.7 & 30.9 \\
\hline \multicolumn{6}{|l|}{ Input from other subsystems } \\
\hline \multicolumn{6}{|l|}{$\mathrm{Pb}$ recovery } \\
\hline \multicolumn{6}{|l|}{ organic destruction } \\
\hline Total Subsystem Input & 0.0 & 955.4 & 796.4 & 1441.0 & 1370.9 \\
\hline \multicolumn{6}{|l|}{ Grout Stabilization Subsystem } \\
\hline \multicolumn{6}{|l|}{ Waste Input } \\
\hline Debris - Soft & 434.8 & & & & \\
\hline Debris - Complex & 177.4 & & & 177.4 & 177.4 \\
\hline Debris - Open & 80.4 & & & & \\
\hline \multicolumn{6}{|l|}{ Process Inputs } \\
\hline dry grout material (cement \& sand) & 1359.6 & 1290.0 & 1274.4 & 365.4 & 904.3 \\
\hline water required for grout & 471.5 & 246.4 & 498.9 & 152.4 & $\overline{383.4}$ \\
\hline \multicolumn{6}{|l|}{ Input from other subsystems } \\
\hline bulk soil treatment & 384.8 & 384.8 & 285.5 & & 285.5 \\
\hline $\mathrm{Pb}$ recovery & 5.0 & 5.0 & 5.0 & 5.0 & 5.0 \\
\hline \multicolumn{6}{|l|}{ process residue/inorganic sludge } \\
\hline debris treatment & & 838.2 & 667.1 & 82.8 & 182.2 \\
\hline Total Subsystem Input & 2913.3 & 2764.3 & 2731.0 & 783.1 & 1937.8 \\
\hline \multicolumn{6}{|l|}{ Metal Decontamination Subsystem } \\
\hline \multicolumn{6}{|l|}{ Waste Input } \\
\hline Bulk Metal & 462.1 & 462.1 & 462.1 & 462.1 & 462.1 \\
\hline \multicolumn{6}{|l|}{ Process Inputs } \\
\hline $\mathrm{H} 2 \mathrm{O}$ & 94.3 & 94.3 & 94.3 & 94.3 & 94.3 \\
\hline \multicolumn{6}{|l|}{ Input from other subsystems } \\
\hline Total Subsystem Input & 556.4 & 556.4 & 556.4 & 556.4 & 556.4 \\
\hline & & & & & \\
\hline
\end{tabular}


Table C-15. INTS Mass Balance Summary (Sheet 4 of 10).

\begin{tabular}{|c|c|c|c|c|c|}
\hline System & System 1 & System 2 & System 3 & System 4 & System 5 \\
\hline & Grout Debris & Desorption & Wash & Acid Digestion & CWO \\
\hline \multicolumn{6}{|l|}{ Organic Destruction Subsystem } \\
\hline \multicolumn{6}{|l|}{ Waste Input } \\
\hline Organic Liquids,Sludges $(2000 / 3000)$ & 185.4 & 185.4 & 185.4 & 185.4 & 185.4 \\
\hline \multicolumn{6}{|l|}{ Process Inputs } \\
\hline $\mathrm{O} 2$ & 566.7 & 701.0 & 775.6 & 575.7 & 604.6 \\
\hline HNO3 & 21.4 & & 28.8 & 30.9 & \\
\hline $\mathrm{H} 2 \mathrm{O}$ & 33.7 & 37.8 & 55.5 & 58.6 & 50.7 \\
\hline $\mathrm{NaOH}$ - gas scrubbing & 0.0 & 37.8 & 0.0 & 58.6 & 50.7 \\
\hline $\mathrm{Ag}$ recovery & 33.7 & & 55.5 & & \\
\hline $\mathrm{H} 2 \mathrm{O} 2$ & 28.7 & & 47.2 & & \\
\hline AgNO3 & 2.9 & & 4.7 & & \\
\hline Acid-in $(\mathrm{FeCl} 3, \mathrm{H} 2 \mathrm{O}, \mathrm{HCl})$ & & 9.7 & & & 9.3 \\
\hline $\mathrm{HCl}-\mathrm{Ag}$ precipitation & 0.0 & & 0.0 & & \\
\hline $\mathrm{NH3}$ & 4.0 & & 5.5 & 2.8 & \\
\hline \multicolumn{6}{|l|}{ Input from other subsystems } \\
\hline aqueous waste treatment - decanted orgs & 32.9 & 31.5 & 79.1 & 31.9 & 31.9 \\
\hline process residue/inorganic sludge & 17.0 & 22.6 & 45.4 & 22.6 & 22.6 \\
\hline debris treatment & & 27.1 & 0.0 & & \\
\hline bulk soil treatment & 1.6 & 1.6 & 16.1 & 16.1 & 16.1 \\
\hline Total Subsystem Input & 928.1 & 1054.5 & 1298.7 & 982.7 & 971.3 \\
\hline \multicolumn{6}{|l|}{ Lead Recovery Subsystem } \\
\hline \multicolumn{6}{|l|}{ Waste Input } \\
\hline Lead Waste & 26.0 & 26.0 & 26.0 & 26.0 & 26.0 \\
\hline \multicolumn{6}{|l|}{ Process Inputs } \\
\hline $\mathrm{H} 2 \mathrm{O}$ & 13 & 13 & 13 & 13 & 13 \\
\hline \multicolumn{6}{|l|}{ Input from other subsystems } \\
\hline Total Subsystem Input & 39.0 & 39.0 & 39.0 & 39.0 & 39.0 \\
\hline \multicolumn{6}{|l|}{ Polymer Stabilization Subsystem } \\
\hline \multicolumn{6}{|l|}{ Waste Input } \\
\hline Salt Waste & 6.6 & 6.6 & 6.6 & 6.6 & 6.6 \\
\hline \multicolumn{6}{|l|}{ Process Inputs } \\
\hline polyethylene or phosphate additive & 801.1 & 843.8 & 788.9 & 6.6 & 861.0 \\
\hline \multicolumn{6}{|l|}{ Input from other subsystems } \\
\hline process residue/inorganic sludge & 959.5 & 953.9 & 1125.9 & & 953.9 \\
\hline aqueous waste treatment - sludge & 102.2 & 125.9 & 108.1 & & 137.7 \\
\hline aqueous waste treatment - concentrate & 0.3 & 2.0 & 5.0 & & 5.2 \\
\hline organic destruction & & 21.2 & & & 23.0 \\
\hline \multicolumn{6}{|l|}{ bulk soil treatment } \\
\hline \multicolumn{6}{|l|}{ debris treatment } \\
\hline $\mathrm{APC}$ & 0.0 & 0.0 & 0.0 & & 0.0 \\
\hline Total Subsystem Input & 1869.7 & 1953.3 & 2034.5 & 13.2 & 1987.5 \\
\hline & & & & & \\
\hline
\end{tabular}


Table C-15. INTS Mass Balance Summary (Sheet 5 of 10).

\begin{tabular}{|c|c|c|c|c|c|}
\hline System & System 1 & System 2 & System 3 & System 4 & System 5 \\
\hline & Grout Debris & Desorption & Wash & Acid Digestion & CWO \\
\hline \multicolumn{6}{|c|}{ Phosphate Bonded Ceramic Stabilization Subsystem } \\
\hline \multicolumn{6}{|c|}{\begin{tabular}{|l|l|} 
Waste Input & \\
\end{tabular}} \\
\hline \multicolumn{6}{|l|}{ Process Inputs } \\
\hline phosphate additive & & & & 1354.0 & \\
\hline \multicolumn{6}{|l|}{ Input from other subsystems } \\
\hline process residue/inorganic sludge & & & & 953.9 & \\
\hline aqueous waste treatment - sludge & & & & 172.5 & \\
\hline aqueous waste treatment - concentrate & & & & 0.1 & \\
\hline organic destruction & & & & 19.4 & \\
\hline bulk soil treatment & & & & 285.5 & \\
\hline debris treatment & & & & 160.9 & \\
\hline APC & & & & 0.0 & \\
\hline Total Subsystem Input & & & & 2946.3 & \\
\hline \multicolumn{6}{|l|}{ Bulk Soil Treatment Subsystem } \\
\hline \multicolumn{6}{|l|}{ Waste Input } \\
\hline Bulk Soils & 328.5 & 328.5 & 328.5 & 328.5 & 328.5 \\
\hline \multicolumn{6}{|l|}{ Process Inputs } \\
\hline $\mathrm{H} 2 \mathrm{O}$ & 98.55 & 98.55 & 328.5 & 328.5 & 328.5 \\
\hline N2 sweep gas & 26 & 26 & & & \\
\hline \multicolumn{6}{|l|}{ Input from other subsystems } \\
\hline Total Subsystem Input & 453.1 & 453.1 & 657.0 & 657.0 & 657.0 \\
\hline \multicolumn{6}{|l|}{ Special Waste Treatment Subsystem } \\
\hline \multicolumn{6}{|l|}{ Waste Input } \\
\hline Special & 154.9 & 154.9 & 154.9 & 154.9 & 154.9 \\
\hline \multicolumn{6}{|l|}{ Process Inputs } \\
\hline \multicolumn{6}{|l|}{ Input from other subsystems } \\
\hline Total Subsystem Input & 154.9 & 154.9 & 154.9 & 154.9 & 154.9 \\
\hline & & & & & \\
\hline
\end{tabular}


Table C-15. INTS Mass Balance Summary (Sheet 6 of 10).

\begin{tabular}{|c|c|c|c|c|c|}
\hline System & System 1 & System 2 & System 3 & System 4 & System 5 \\
\hline & Grout Debris & Desorption & Wash & Acid Digestion & CWO \\
\hline 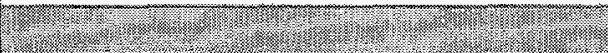 & 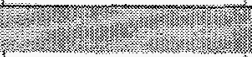 & \multicolumn{2}{|c|}{ PROCESS OUTRUTS } & 6 & \\
\hline \multicolumn{6}{|l|}{ Hg Amalgamation Subsystem } \\
\hline Process Outputs & 4.5 & 8.8 & 4.3 & 4.5 & 4.5 \\
\hline \multicolumn{6}{|l|}{ Output to other subsystems } \\
\hline Total Subsystem Output & 4.5 & 8.8 & 4.3 & 4.5 & 4.5 \\
\hline \multicolumn{6}{|l|}{ Air Pollution Control Subsystem } \\
\hline \multicolumn{6}{|l|}{ Process Outputs } \\
\hline \multicolumn{6}{|l|}{ Offgas } \\
\hline $\mathrm{O} 2$ & 29.1 & 35.6 & 55.1 & 10.4 & 30.9 \\
\hline N2 & 117.9 & 165.1 & 18.9 & 132.1 & 84.2 \\
\hline NO & 0.8 & 0.7 & 0.8 & 0.2 & 0.7 \\
\hline $\mathrm{H} 2 \mathrm{O}$ & 9.8 & 1.8 & 15.2 & 10.5 & 3.8 \\
\hline $\mathrm{CO} 2$ & 537.9 & 619.8 & 737.0 & 1288.0 & 1313.6 \\
\hline $\mathrm{HNO} 2$ & 0.0 & & 0.0 & & \\
\hline total offgas & 695.5 & 823.0 & 827.1 & 1441.4 & 1433.3 \\
\hline \multicolumn{6}{|l|}{ Output to other subsystems } \\
\hline aqueous waste treatment - salts & 0.3 & 2.0 & 5.0 & 0.1 & 5.2 \\
\hline aqueous waste treatment - moisture & 0.0 & 0.0 & 0.0 & 0.0 & 0.0 \\
\hline polymer stabilization - metals & 0.0 & 0.0 & 0.0 & 0.0 & 0.0 \\
\hline Total Subsystem Output & 695.8 & 825.0 & 832.1 & 1441.5 & 1438.5 \\
\hline \multicolumn{6}{|l|}{ Aqueous Waste Treatment Subsystem } \\
\hline \multicolumn{6}{|l|}{ Process Outputs } \\
\hline aqueous recycle & 1100.8 & 1186.1 & 1423.9 & 1789.9 & 1794.5 \\
\hline \multicolumn{6}{|l|}{ Output to other subsystems } \\
\hline organic destruction - decanted orgs & 32.9 & 31.5 & 79.1 & 31.9 & 31.9 \\
\hline polymer stabilization & 102.2 & 125.9 & 108.1 & 172.5 & 137.7 \\
\hline polymer stabilization - concentrate & 0.3 & 2.0 & 5.0 & 0.0 & 5.2 \\
\hline APC & 8.8 & 9.1 & 27.1 & 9.0 & 9.5 \\
\hline Total Subsystem Output & 1245.1 & 1354.5 & 1643.2 & 2003.4 & 1978.9 \\
\hline \multicolumn{6}{|c|}{ Process Residue and Inorganic Sludge Treatment Subsystem } \\
\hline \multicolumn{6}{|l|}{ Process Outputs } \\
\hline \multicolumn{6}{|l|}{ Output to other subsystems } \\
\hline polymer stabilization & 959.5 & 953.9 & 1125.9 & 953.9 & 953.9 \\
\hline organic destruction & 17.0 & 22.6 & 45.4 & 22.6 & 22.6 \\
\hline aqueous waste treatment & 349.6 & 349.6 & 0.0 & 349.6 & 349.6 \\
\hline APC & 79.9 & 79.9 & 0.0 & 79.9 & 79.9 \\
\hline grout stabilization & 0.0 & 0.0 & 0.0 & 0.0 & 0.0 \\
\hline $\mathrm{Hg}$ amalgamation & 0.1 & 0.1 & 0.0 & 0.1 & 0.1 \\
\hline Total Subsystem Output & 1406.1 & 1406.1 & 1171.3 & 1406.1 & 1406.1 \\
\hline & & & & & \\
\hline
\end{tabular}


Table C-15. INTS Mass Balance Summary (Sheet 7 of 10).

\begin{tabular}{|c|c|c|c|c|c|}
\hline System & System 1 & System 2 & System 3 & System 4 & System 5 \\
\hline & Grout Debris & Desorption & Wash & Acid Digestion & CWO \\
\hline \multicolumn{6}{|l|}{ Debris Treatment Subsystem } \\
\hline \multicolumn{6}{|l|}{ Process Outputs } \\
\hline \multicolumn{6}{|l|}{ Output to other subsystems } \\
\hline polymer stabilization & 0.0 & & & 160.9 & 0.0 \\
\hline organic destruction & 0.0 & 27.1 & 0.0 & 0.0 & 0.0 \\
\hline aqueous waste treatment & 0.0 & 32.4 & 129.3 & 432.6 & 406.9 \\
\hline APC & 0.0 & 55.2 & 0.0 & 764.8 & 781.8 \\
\hline grout stabilization & 0.0 & 838.2 & 667.1 & 82.8 & 182.2 \\
\hline $\mathrm{Hg}$ amalgamation & & 2.5 & & & \\
\hline Total Subsystem Output & 0.0 & 955.4 & 796.4 & 1441.0 & 1370.9 \\
\hline \multicolumn{6}{|l|}{ Grout Stabilization Subsystem } \\
\hline \multicolumn{6}{|l|}{ Process Outputs } \\
\hline grouted product & 2913.3 & 2764.3 & 2731.0 & 783.1 & 1937.8 \\
\hline \multicolumn{6}{|l|}{ Output to other subsystems } \\
\hline Total Subsystem Output & 2913.3 & 2764.3 & 2731.0 & 783.1 & 1937.8 \\
\hline \multicolumn{6}{|l|}{ Metal Decontamination Subsystem } \\
\hline \multicolumn{6}{|l|}{ Process Outputs } \\
\hline decontaminated metal & 430.3 & 430.3 & 430.3 & 430.3 & 430.3 \\
\hline \multicolumn{6}{|l|}{ Output to other subsystems } \\
\hline aqueous waste treatment & 126.2 & 126.2 & 126.2 & 126.2 & 126.2 \\
\hline Total Subsystem Output & 556.4 & 556.4 & 556.4 & 556.4 & 556.4 \\
\hline \multicolumn{6}{|l|}{ Organic Destruction Subsystem } \\
\hline \multicolumn{6}{|l|}{ Process Outputs } \\
\hline \multicolumn{6}{|l|}{ Output to other subsystems } \\
\hline process residue/inorganic sludge & 0.0 & 0.0 & 0.0 & 0.0 & 0.0 \\
\hline aqueous waste treatment - water & 238.9 & 380.3 & 320.3 & 420.3 & 386.1 \\
\hline aqueous waste treatment - salts & 108.6 & 0.0 & 178.7 & 0.0 & 0.0 \\
\hline APC - gases & 580.5 & 652.6 & 799.8 & 542.4 & 561.6 \\
\hline polymer stabilization & 0.0 & 21.2 & 0.0 & 19.4 & 23.0 \\
\hline Total Subsystem Output & 928.1 & 1054.1 & 1298.8 & 982.1 & 970.8 \\
\hline \multicolumn{6}{|l|}{ Lead Recovery Subsystem } \\
\hline \multicolumn{6}{|l|}{ Process Outputs } \\
\hline lead out & 21 & 21 & 21 & 21 & 21 \\
\hline \multicolumn{6}{|l|}{ Output to other subsystems } \\
\hline process residue/inorganic sludge & 13.0 & 13.0 & 13.0 & 13.0 & 13.0 \\
\hline grout - gloves & 5.0 & 5.0 & 5.0 & 5.0 & 5.0 \\
\hline Total Subsystem Output & 39.0 & 39.0 & 39.0 & 39.0 & 39.0 \\
\hline \multirow{2}{*}{\multicolumn{6}{|c|}{ Polymer Stabilization Subsystem }} \\
\hline & & & & & \\
\hline \multicolumn{6}{|l|}{ Process Outputs } \\
\hline polymer stabilized waste & 1602.2 & 1687.6 & 1577.8 & 13.2 & 1722.0 \\
\hline \multicolumn{6}{|l|}{ Output to other subsystems } \\
\hline aqueous waste treatment & 267.5 & 265.7 & 456.7 & & 265.5 \\
\hline Total Subsystem Output & 1869.7 & 1953.3 & 2034.5 & 13.2 & 1987.5 \\
\hline
\end{tabular}


Table C-15. INTS Mass Balance Summary (Sheet 8 of 10).

\begin{tabular}{|c|c|c|c|c|c|}
\hline System & System 1 & System 2 & System 3 & System 4 & System 5 \\
\hline & Grout Debris & Desorption & Wash & Acid Digestion & CWO \\
\hline \multicolumn{6}{|c|}{ Phosphate Bonded Ceramic Stabilization Subsystem } \\
\hline \multicolumn{6}{|c|}{\begin{tabular}{|l|l|} 
Process Outputs & \\
\end{tabular}} \\
\hline \multicolumn{2}{|c|}{ phosphate bonded ceramic stabilized waste } & & & 2707.9 & \\
\hline \multicolumn{6}{|c|}{ Output to other subsystems } \\
\hline \multirow{2}{*}{\begin{tabular}{l|} 
aqueous waste treatment \\
Total Subsystem Output
\end{tabular}} & & & & 238.3 & \\
\hline & & & & 2946.3 & \\
\hline & & & & & \\
\hline \multicolumn{6}{|l|}{ Bulk Soil Treatment Subsystem } \\
\hline \multicolumn{6}{|l|}{ Process Outputs } \\
\hline \multicolumn{6}{|l|}{ Output to other subsystems } \\
\hline APC & 26.1 & 26.1 & 0.0 & 0.0 & 0.0 \\
\hline aqueous waste treatment & 40.7 & 40.7 & 355.4 & 355.4 & 355.4 \\
\hline grout & 384.8 & 384.8 & 285.5 & 0.0 & 285.5 \\
\hline organic destruction & 1.6 & 1.6 & 16.1 & 16.1 & 16.1 \\
\hline polymer stabilization & 0.0 & 0.0 & 0.0 & 285.5 & 0.0 \\
\hline Total Subsystem Output & 453.1 & 453.1 & 657.0 & 657.0 & 657.0 \\
\hline \multicolumn{6}{|l|}{ Special Waste Treatment Subsystem } \\
\hline Process Outputs & 154.9 & 154.9 & 154.9 & 154.9 & 154.9 \\
\hline \multicolumn{6}{|l|}{ Output to other subsystems } \\
\hline Total Subsystem Output & 154.9 & 154.9 & 154.9 & 154.9 & 154.9 \\
\hline \multicolumn{6}{|l|}{ Reagents Input to Process (density, Ib/ft3) } \\
\hline $\mathrm{Cu}$ for $\mathrm{Hg}$ amalgamation & 1.9 & 3.6 & 1.8 & 1.8 & 1.9 \\
\hline $\mathrm{NaOH}$ & 58.0 & 84.7 & 58.5 & 104.0 & 93.4 \\
\hline acid solution $(\mathrm{FeCl} 3, \mathrm{H} 2 \mathrm{O}, \mathrm{HCl})(\sim 62)$ & 0.0 & 9.7 & 0.0 & 0.0 & 24.5 \\
\hline $\begin{array}{ll}\mathrm{NH} 3 & (45) \\
\end{array}$ & 4.0 & 0.0 & 5.5 & 6.8 & 0.0 \\
\hline HNO3 & 21.4 & 0.0 & 28.8 & 73.7 & 0.0 \\
\hline $\mathrm{AgNO3}$ & 2.9 & 0.0 & 4.7 & 0.0 & 0.0 \\
\hline $\mathrm{H} 2 \mathrm{O} 2$ & 29.2 & 0.6 & 48.6 & 0.6 & 0.6 \\
\hline grout material & 1359.6 & 1290.0 & 1274.4 & 365.4 & 904.3 \\
\hline water required for grout & 471.5 & 246.4 & 498.9 & 152.4 & 383.4 \\
\hline polyethylene/phosphate ceramic $(\sim 80)$ & 801.1 & 843.8 & 788.9 & 1360.6 & 861.0 \\
\hline
\end{tabular}


Table C-15. INTS Mass Balance Summary (Sheet 9 of 10).

\begin{tabular}{|c|c|c|c|c|c|}
\hline System & System 1 & System 2 & System 3 & System 4 & System 5 \\
\hline & Grout Debris & Desorption & Wash & Acid Digestion & CWO \\
\hline 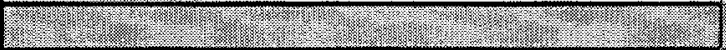 & \multicolumn{5}{|c|}{ MASS AMB VOLUMEREDUCTION CALCULATION } \\
\hline \multicolumn{6}{|l|}{ Densities $(\mathrm{lb} / \mathrm{ft} 3)$} \\
\hline Input Waste & 64 & 64 & 64 & 64 & 64 \\
\hline Soil & 67 & 67 & 67 & 67 & 67 \\
\hline Metal (Input) & 480 & 480 & 480 & 480 & 480 \\
\hline Metal (Output) & 480 & 480 & 480 & 480 & 480 \\
\hline Aqueous Liquid & 62 & 62 & 62 & 62 & 62 \\
\hline Slag & 187 & 187 & 187 & 187 & 187 \\
\hline Polymerized Salt & 80 & 80 & 80 & 80 & 80 \\
\hline Polymerized Ash/Metal/Salt & 94 & 94 & 94 & 94 & 94 \\
\hline Polymerized Lime & 96.5 & 96.5 & 96.5 & 96.5 & 96.5 \\
\hline Grouted Debris & 126.6 & 126.6 & 126.6 & 126.6 & 126.6 \\
\hline Stabilized Hg Amalgam & 733.0 & 733.0 & 733.0 & 733.0 & 733.0 \\
\hline \multicolumn{6}{|l|}{ Mass Flow Rate Calculation } \\
\hline Mass Solids In (lb/hr, no aqueous) & 2865.8 & 2865.8 & 2865.8 & 2865.8 & 2865.8 \\
\hline Mass Grouted Waste Out (lb/hr) & 2913.3 & 2764.3 & 2731.0 & 783.1 & 1937.8 \\
\hline Mass Special Waste (not stabilized) & 154.9 & 154.9 & 154.9 & 154.9 & 154.9 \\
\hline Mass Metal/Pb Out (lb/hr)clean & 451.3 & 451.3 & 451.3 & 451.3 & 451.3 \\
\hline Mass Polymerized Waste Out (lb/hr) & 1602.2 & 1687.6 & 1577.8 & 13.2 & 1722.0 \\
\hline Mass Phosphate Bonded Waste Out (lb/hr) & & & & 2707.9 & \\
\hline Mass Stabilized Hg Amalgam Out & 4.5 & 8.8 & 4.3 & 4.5 & 4.5 \\
\hline $\begin{array}{l}\text { Total Mass Processed Wastes Out (lb/hr, no } \\
\text { metals) }\end{array}$ & 4675 & 4616 & 4468 & 3664 & 3819 \\
\hline Waste Mass Reduction Ratio (In/Out) & 0.61 & 0.62 & 0.64 & 0.78 & 0.75 \\
\hline \multicolumn{6}{|l|}{ Volumetric Flow Rate Calculation } \\
\hline Volume Solids In (ft3/hr) & 45 & 45 & 45 & 45 & 45 \\
\hline Volume Grouted Waste Out (ft3/hr) & 23.0 & 21.8 & 21.6 & 6.2 & 15.3 \\
\hline Volume Special Wastes (not stabilized) (ft3/hr) & 2.4 & 2.4 & 2.4 & 2.4 & 2.4 \\
\hline Volume Metal/Pb Out (ft $3 / \mathrm{hr})$ & 0.9 & 0.9 & 0.9 & 0.9 & 0.9 \\
\hline Volume Polymerized Waste Out (ft3/hr) & 17.0 & 18.0 & 16.8 & 0.1 & 18.3 \\
\hline Volume Phosphate Waste Out (ft3/hr) & & & & 28.8 & \\
\hline Vol Stabilized Hg Amalgam Out & 0.0 & 0.0 & 0.0 & 0.0 & 0.0 \\
\hline $\begin{array}{l}\text { Total Volume Solids Out to Disposal (ft3/hr, } \\
\text { no metals) }\end{array}$ & 42.5 & 42.2 & 40.8 & 37.6 & 36.1 \\
\hline Volume Metal to Recycle (ft3/hr) & 0.9 & 0.9 & 0.9 & 0.9 & 0.9 \\
\hline Waste Volume Reduction Ratio (In/Out) & 1.05 & 1.06 & 1.10 & 1.19 & 1.24 \\
\hline & & & & & \\
\hline
\end{tabular}


Table C-15. INTS Mass Balance Summary (Sheet 10 of 10).

\begin{tabular}{|c|c|c|c|c|c|}
\hline System & System 1 & System 2 & System 3 & System 4 & System 5 \\
\hline & Grout Debris & Desorption & Wash & Acid Digestion & CWO \\
\hline \multicolumn{6}{|l|}{ Total Water Requirements } \\
\hline Aqueous Waste Treatment & 24 & 46 & 1 & 8 & 9 \\
\hline Inorganic Sludge Washing / Hg Leaching & 306 & 306 & 151 & 306 & 306 \\
\hline Lead Recovery & 13 & 13 & 13 & 13 & 13 \\
\hline Debris Treatment $/ \mathrm{Hg}$ Leaching & 0 & 208 & 104 & 50 & 43 \\
\hline Metal Decontamination & 94 & 94 & 94 & 94 & 94 \\
\hline Organic Destruction & 34 & 38 & 55 & 59 & 51 \\
\hline Soil Washing / Hg Leaching & 99 & 99 & 329 & 329 & 329 \\
\hline Air Pollution Control - scrubber water & 0 & 1 & 3 & 0 & 3 \\
\hline Water for grout ( 30 weight $\%$ ) & 471 & 246 & 499 & 152 & 383 \\
\hline Total & 1041 & 1051 & 1248 & 1010 & 1231 \\
\hline Total Water Available for Recycle & 1101 & 1186 & 1424 & 1790 & 1795 \\
\hline Net Water Generation Rate (recycle - required) & 59 & 135 & 176 & 780 & 564 \\
\hline
\end{tabular}




\section{APPENDIX D \\ TECHNOLOGY AND SYSTEM SELECTION PROCESS AND RESULTS}

\section{D-1. INTRODUCTION}

Technology selection for the INTS study began with a search for and identification and summary of nonthermal technologies that might be included in a system for the treatment of DOE's MLLW. This research resulted in the definition of over 210 potential technologies that are described in Reference 1. The identified technologies perform the major functions of organic destruction, separation of organic and inorganic contaminants from the waste matrix (either solid or aqueous), stabilization, decontamination, and air pollution control.

The first meeting of the TSG was held in Denver, Colorado on June 27-28, 1995 to review the functions required of a nonthermal treatment system, and recommend technologies for further consideration as part of a nonthermal treatment system. Because one of the assumptions for the INTS study is that all treated solid matrices (i.e., debris, soils, and inorganic sludges) will be stabilized, the TSG recommended that inorganic contaminants not be removed from these matrices, except for elemental mercury. Because elemental mercury is a liquid, stabilized waste containing mercury is not expected to pass the TCLP test for mercury. Thus, no special technologies were included in the nonthermal systems to separate and remove heavy metals or radionuclides from the solid wastes except for the removal of mercury. These inorganic contaminants will be stabilized with the solid wastes to produce a nonleachable final waste form for disposal.

However, the TSG identified a need to remove organic contaminants from the solid matrices such that the Universal Treatment Standards are met, and to ensure that the final waste form used to stabilize the treated waste would have long term stability and durability. These organic contaminants would require destruction (usually by oxidation) by converting them to nonhazardous compounds such as carbon dioxide and water. Two methods for treating the organics on the solid matrices were suggested: separation of the organic contaminants from the solids and subsequent destruction of the organics in an independent process, or "in-matrix" destruction of the organics adhering to the soil and sludge particulates without first separating the organic contaminants from the solids.

Two generic methods of separating organic contaminants from solid waste matrices were identified: liquid extraction including aqueous washing, organic solvent extraction, and supercritical fluid extraction; and thermal desorption. Two generic methods of organic destruction were also identified: chemical destruction and biological destruction.

Aqueous waste and offgas treatment were also considered. In both cases the typical treatment method for organic contaminants is to remove the organics from the water or offgas by using beds of activated carbon. However, this simply moves the contaminants from one medium (water or gas) to another medium (solid carbon) that must be subsequently treated. The TSG recommended that technologies be considered that would destroy organics in the aqueous or gas phase. Additional technologies are required to remove inorganic materials from the waste water and offgas prior to recycling or discharge. Offgas treatment requires removal of particulates, acid gases, and mercury vapor prior to discharge. Waste water requires removal of suspended solids and hydroxyl radical scavengers prior to treatment for organics and removal of dissolved solids composed of heavy metals and radionuclides prior to discharge. 


\section{D-2. FIRST TECHNICAL SUPPORT GROUP EVALUATION}

Results from this first TSG meeting are presented in Reference 2 and summarized here. Because this study is at the preconceptual design stage, and information on nonthermal technologies is preliminary and incomplete, many of the technical criteria discussed in Section 6 could not be quantitatively applied. The TSG considered these criteria and used their judgment and knowledge of the technologies to develop recommendations for further consideration. Following are the TSG recommendations for technologies to perform the indicated functions.

\section{D-2.1 Organic Destruction}

Two generic methods of organic destruction were identified: biological and chemical destruction. Biological treatment methods to destroy organic contaminants were not recommended because the microorganisms are very sensitive to the type of compounds in the waste and their concentrations. Although biological treatment may be appropriate for a single organic compound within a specified concentration range, DOE mixed waste is highly variable with many different organic and inorganic compounds with varying concentrations. Different organisms will be required to treat the different organic compounds. Some compounds are lethal to microorganisms used to treat other compounds, and variations in concentration may also be lethal. Thus, reliability would be compromised, maintenance requirements to re-start the reactions would be high, and a significant amount of secondary waste would be generated in the form of biomass containing radionuclides and heavy metals.

Chemical destruction technologies were recommended as having the best potential for destroying most of the organic contaminants and combustible waste. These technologies were considered capable of treating a wide variety of waste compositions and organic compounds. The technologies recommended for further evaluation were acid digestion, CWO, MEO, and wet oxidation. A silicate based technology was initially suggested but further investigation found that it was a stabilization process rather than an organic destruction process, and was applicable only to stabilizing soils.

\section{D-2.2 Organic Separation}

Two generic methods of separating organic contaminants from solid waste matrices were identified: liquid extraction including aqueous washing, organic solvent extraction, and supercritical fluid extraction; and thermal desorption. No specific technologies were suggested, but the TSG recommended further investigation of technologies in these categories.

\section{D-2.3 Aqueous Waste Treatment}

Conventional methods for aqueous waste treatment were recommended. These include filtration to remove suspended solids and immiscible organic liquids, precipitation followed by filtration to remove dissolved solids, evaporation to concentrate solids prior to stabilization, and ion exchange and activated carbon filtration to remove traces of dissolved solids and organics prior to discharge or recycle.

Several technologies were recommended for further consideration to destroy organic contaminants in the waste water. These included the following:

- Electrochemical Destruction

- Wet Oxidation 
- Mixed Oxidation (MIOX) Process

- Electron Beam

- Photo-oxidation

- Photo-Catalytic Oxidation

- Ultrasonic Oxidation

\section{D-2.4 Offgas Treatment}

The TSG recommended the air pollution control system consist of essentially the same treatment technologies as used in the ITTS study. All offgas streams would first pass through a cooler/condenser combination to condense organics, mercury, water, and $\mathrm{HCl}$ out of the offgas. The resulting liquid would then be sent to organic destruction. Particulates would be removed in a sintered metal filter. Several technologies were recommended for consideration for the destruction of remaining trace organics. These include the GPCR, an electron beam technology, and ultraviolet photo-oxidation. The remaining inorganic materials in the offgas are removed by liquid scrubbing. The final stage of treatment to remove any material passing through the earlier steps includes HEPA filters to remove particulates and radionuclides, activated carbon filters to remove trace organics, and sulfur impregnated carbon filters to remove trace mercury.

\section{D-2.5 Stabilization}

The recommended nonthermal stabilization methods included incorporation of the residues from the treatment steps in grout, polymer (polyethylene), or a chemical bonding agent. Grout is a cement based material and is included as a baseline reference since it has been widely used even though its limitations are recognized. Polymer has been established through testing to be an inert plastic material that can be mixed with salts and other primary or secondary wastes. It microencapsulates the material particles by surrounding them with an inert substance to prevent leaching. It can also be used to macroencapsulate by using it as a cocoon around larger objects. Chemical bonding uses the characteristics of chemical compounds to set up as a solid. While not well developed, this option provides an advanced method that may offer use of some of the chemicals in the residue to combine with other chemicals to form a relatively inert solid. These recommendations provide a range of known, developing, and future stabilization technologies.

\section{D-2.6 System Recommendations}

The TSG recommended three generic systems to be included in the INTS study. These were:

- Sludge, soil, and debris washing followed by chemical organic destruction of the separated organic contaminants.

- Thermal desorption of sludge, soil, and debris with organic vapor condensation followed by chemical organic destruction, and removal of remaining mercury from the desorbed solids.

- In-matrix chemical destruction of organic contaminants on the soils, sludges, and debris. 
All systems would treat the aqueous waste and offgas with organic destruction, and would stabilize the treated and secondary wastes that may retain residual organic material. A more detailed description of the technologies included in the selected systems can be found in Reference 3.

\section{D-2.7 Summary}

As discussed in Section D-2 above, few of the 210 plus technologies described in Reference 1 were recommended by the TSG for further consideration. Many of these technologies are variations to a general physical process that is either being commercialized or investigated by a national laboratory or university. For example, there are six photo-oxidation processes identified for destroying organics in water, and four photolytic destruction processes for destroying organics in the offgas. Rather than evaluating all these specific technologies, the TSG selected a technology that represents that category. Similarly, there were 24 washing or organic extraction processes identified, and 11 thermal desorbers. Of these, only one washing process claimed to be able to treat sludge, one washing process was developed to treat large debris items, and only one desorber operated under a vacuum (and therefore at low temperature) and could treat the variety of DOE wastes. Also a large number of separation technologies were not directly applicable to the generic or average waste stream used for the study.

Many of the separation technologies, of which there are 108 , have very specific and narrow applications and were not identified as necessary for treating the waste stream used for the INTS analysis. Other separation technologies were standard technologies (e.g., ultrafiltration, ion exchange, activated carbon beds, etc.) that were chosen for the conventional waste water treatment subsystem. Similarly, conventional air pollution control technologies, out of the 44 APC technologies identified, were chosen for the APC subsystem design. The judgment of the TSG was that the performance of these conventional technologies was sufficient for the application at this preconceptual level of design to develop representative system costs. Finally, complex technologies used to separate organics from water or gases were not considered necessary because the organic contaminants will be oxidized within the aqueous or gaseous phase.

Fifty-one destruction technologies were identified and categorized as follows:

- Nine biological destruction technologies that were not recommended for the reasons discussed previously.

- Six dehalogenation technologies that the TSG did not consider necessary because the chemical oxidation technologies that were recommended could oxidize halogenated hydrocarbons, including PCBs, without first dehalogenating the hydrocarbons.

- Eight chemical destruction technologies were identified and seven were recommended for further consideration by the TSG. The eighth technology, wet air oxidation, was not recommended because of the high pressure required (approximately $3000 \mathrm{psi}$ ) and the concern for worker safety when treating radioactive wastes.

- Ten gas phase destruction technologies were identified and divided into three categories. A representative technology from each category was recommended.

- Five technologies were identified for the decomposition of inorganic contaminants (nitrates and cyanides). Cyanides are not prevalent throughout the DOE complex, and nitrates are not a major concern because the Hanford tank wastes were excluded from the waste to be treated so 
that nitrates can be treated by the conventional aqueous waste treatment subsystem. Thus, these technologies were not recommended for inclusion in the study.

- Thirteen technologies were identified for aqueous phase organic destruction by radiation induced oxidation and divided into four categories. Representative technologies from three categories were recommended; use of $\mathrm{x}$-rays was considered too complex and there was concern for worker safety.

Twenty-two stabilization technologies were identified and divided into four categories. The TSG recommended four representative technologies from these categories be further evaluated.

\section{D-3. TECHNOLOGY SELECTION AND RATIONALE}

These initial recommendations by the TSG were presented to the TSWG. During this presentation meeting, criteria for evaluation of the technologies was established by the TSWG which were then evaluated in detail to determine which technologies should be included for further study in the design of nonthermal systems. These evaluations were presented to the TSG and a group of chemical experts at a second meeting in Salt Lake City, Utah on October 31 to November 2, 1995. The results of this evaluation and the recommendations from this second TSG meeting follow.

\section{D-3.1 Soil \& Sludge Washing}

There was a choice between aqueous wash processes (using various surfactants), organic solvents, or supercritical fluids $\left(\mathrm{CO}_{2}\right.$ or propane) for extraction of organics. Following is a discussion of these alternatives.

D-3.1.1 Solvent Extraction. Solvent extraction processes were rejected because they are complex and in some cases unreliable, several commercial processes used toxic solvents, and it was felt that it would be too difficult to remove organic solvents from the waste, or remove contaminants from the solvent for recovery of the solvent. Compared to aqueous wash processes, organic solvent extraction processes were considered too complex and potentially hazardous to workers and the environment.

D-3.1.2 Supercritical Fluids. Two supercritical fluids were considered for extraction of organics from solid waste: carbon dioxide $\left(\mathrm{CO}_{2}\right)$ and propane. Both fluids require very high pressures (approximately $900 \mathrm{psi}$ ) and were rejected because of safety issues (high pressures involved in a radioactive environment and flammability of propane). Without additives, supercritical $\mathrm{CO}_{2}$ is limited to extraction of non-polar organic molecules. The use of additives to extend the application of supercritical $\mathrm{CO}_{2}$ was considered unproven.

D-3.1.3 Aqueous Washing. Aqueous wash processes are relatively simple, well known, and introduce no problem chemicals that require treatment. Surfactants are used to combine with and remove organic molecules from the solid waste matrices, and surfactants with the contaminants are separated from the wash water so the water can be recycled to limit consumption. A commercial process has been identified that is reported to treat both soils and sludges for the removal of organic contaminants. The process uses a high loading of surfactant for organic removal, and the developer has available a variety of surfactants and additives to remove various organics and metals. Water and surfactants are separated through an ultrafiltration process, the surfactant and contaminants are separated, and the surfactant recovered for reuse, while the organics are sent to the destruction process. 


\section{D-3.2 Soil Washing}

Standard soil washing techniques separate small sludge particulates from the larger soil particles. Sludge particulates contain most of the organic contaminants which are more difficult to remove from the sludge. The same commercial process described above to wash sludge is used to wash soils because of the availability of a variety of surfactants to remove the various organic contaminants in the DOE complex. The washing process would also be more costly than other separation approaches if two different washing processes were used, one for soil and one for sludge.

\section{D-3.3 Debris Decontamination}

\section{D-3.3.1 Soft Debris.}

D-3.3.1.1 High Pressure Spray Wash. This process is not applicable to soft debris because under the high pressure spray paper and rags would have a tendency to stick together thereby preventing access to all surfaces by the wash solution.

D-3.3.1.2 Low Pressure Spray Wash. An operation using the high pressure spray wash equipment modified for a low pressure spray was selected. Paper, rags, etc. are agitated to prevent them from sticking together and to ensure all surfaces are accessed by the wash solution. As with the soil and sludge washing process, surfactants are used to remove organic contaminants.

\section{D-3.3.2 Open Debris.}

D-3.3.2.1 Low Pressure Spray Wash. This process is being developed by a commercial entity for the EPA, has been extensively tested, and appears applicable to decontaminating debris in which all surfaces are accessible. A crane and large washing and rinsing chambers are available for large debris items, and baskets are designed for smaller debris items. As currently configured, this is an open system which must be enclosed for treatment of debris contaminated with radionuclides. A similar spray wash process was also used to decontaminate lead for recycling without generating large quantities of lead fines from surface abrasion.

D-3.3.2.2 $\mathrm{CO}_{2}$ or Sand Blasting. Blasting processes are applicable to removal of contaminants and scale from metal debris to allow the metal to be recycled. Grit blasting has been used in the previously studied thermal systems, and the nonthermal systems studied in the INTS study, for decontamination of bulk metal for recycling. This process was also not recommended for general DOE debris wastes which consists of many different materials, shapes, and sizes. Blasting methods were rejected for general debris decontamination because these methods would also generate a secondary waste from surface fines eroded from the debris surface (e.g., particulates from concrete, bricks, etc.).

\section{D-3.3.3 Complex Debris.}

D-3.3.3.1 Grout Stabilization. Preliminary evaluation indicated that complex debris items (e.g., cast iron pumps, valves, motors, etc.) would be difficult to shred or size reduce such that internal surfaces and cavities would be exposed for decontamination. Thus, it would be difficult to decontaminate these items because there would be no guaranteed access to many of the internal cavities. Therefore, direct stabilization may be the most appropriate nonthermal treatment. 
D-3.3.3.2 Size Reduction and Decontamination. Remote operations could be used to saw these items apart to expose the internal cavities. Therefore, it was recommended that both alternatives be compared. Two systems will evaluate size reduction of these metal debris items: decontamination by thermal desorption or washing, and stabilization of the metal in grout. Three systems will evaluate direct stabilization and disposal in grout. Complex debris is a relatively small part $(6 \%)$ of the total waste stream, and making this comparison would shed light on the cost effectiveness of developing and demonstrating methods to "open" complex debris and provide access to internal surfaces. Shreddable items (e.g., PVC pipe, pumps, etc.) would fall into the category of open debris after shredding.

\section{D-3.4 Thermal Desorption}

\section{D-3.4.1 Inorganic Sludge, Soil, and Debris.}

D-3.4.1.1 Vacuum Batch Desorption. A low temperature vacuum operation that is a batch operation is being commercialized for treating soils and sludges, and only a batch operation will accept large debris. Batch operations generally take more time and labor than a continuous operation, thereby limiting the throughput. However, advantages over continuous operations include improved containment of contaminants and ability to handle variations in the input waste stream. Vacuum operation also allows desorption of high boiling point organics (such as PCBs) at low temperatures. An inert gas such as nitrogen is used to prevent oxidation and to carry off the desorbed organics.

\section{D-3.4.2 Soils.}

D-3.4.2.1 Continuous Desorption. Continuous operation desorbers are designed to accept soil and have been commercialized. These are open operations so an enclosure must be designed around these systems when treating radioactively contaminated soils. These desorbers are indirectly heated so the temperature can be controlled to below $660^{\circ} \mathrm{F}\left(350^{\circ} \mathrm{C}\right)$, and the continuous operation allows greater throughput than batch operations. However, the low temperature operation at atmospheric pressures may prevent removal of high B.P. organics and limit the usefulness of these types of desorbers.

The presence of high B.P. organics, the need for a large enclosure for containment with the continuous system operation, the variations in the type of organic contaminants in the feed, and the relatively small amount of soil that requires desorption, indicate that vacuum batch desorption is the most appropriate for soils. Thus, continuous desorption systems are not recommended for nonthermal (low temperature) mixed waste applications.

\section{D-3.5 Organic Destruction}

The volume of residuals for these chemical destruction processes depends on the volume of waste input. However, for a given waste input to all processes the volume of residuals from each process were expected to be about the same. This was validated by the results of the study provided in Section 3 of this report. It should also be noted that all these processes use corrosive chemicals to destroy organic contaminants. This presents a worker safety issue for all systems and must be dealt with in the design and operation of the facility. 
D-3.5.1 Catalyzed Wet Oxidation. This process uses the most aggressive reagent, under specified high temperature and pressure operating conditions, and is the most likely to be able to treat the widest range of organic wastes. The reagent consists of hydrochloric acid and ferric chloride with trace amounts of catalysts. This process is being commercialized but is still at the bench scale. However, a significant amount of data are available regarding reaction rates, residue (consisting of chlorides and oxides of trace heavy metals and radionuclides in the waste, and sodium chloride resulting from neutralization of spent acid), and process requirements. The process can treat all organic liquids, organic sludges (except perfluorocarbons), and shredded organic debris.

It was initially thought that this process could also treat inorganic sludges and soils by destroying the organic contaminants attached to the sludge and soil particulates. Subsequent discussions with the developer indicated that this is not a cost effective application for large quantities of soils or inorganic sludges. There would be excess buildup of solids in the CWO solution due to dissolution of both hazardous and non-hazardous inorganic material, and due to both suspended solids and dissolved solids which would precipitate upon reaching saturation. This would place an excessive load on the filtration system and require frequent disruption of operations to remove solids. Additional residue would also be produced by precipitation of the dissolved solids, both hazardous and non-hazardous.

Thus, this technology is recommended to treat organic liquids, organic sludges, and combustible debris. However, the third system recommended by the TSG (in-matrix destruction of organics) cannot be accommodated by this technology.

D-3.5.2 Mediated Electrochemical Oxidation. This technology is less aggressive than CWO but likely to treat most of the organic liquids and organic sludges. However, some organic debris would be difficult to treat by this process (e.g., some plastics). This technology is retained to determine the performance and costs associated with a less aggressive, and therefore less risky, process than CWO. This also allows comparison of a technology based on electrical regeneration of oxidant versus a system (CWO) that uses chemical regeneration.

Two MEO processes were evaluated for inclusion in the INTS study: one based on $\mathrm{Ag}(\mathrm{II})$ (silver) as the oxidant, and the other based on Ce(IV) (cerium), both in nitric acid. The silver based process was recommended by the TSG because (1) it is more aggressive, (2) precipitation of $\mathrm{AgCl}$ prevents build-up of aqua regia when treating halogenated hydrocarbons, and (3) a method that may recover up to $99.9 \%$ of the silver from $\mathrm{AgCl}$ has been identified.

D-3.5.3 Acid Digestion. This process was initially rejected because of (1) its similarity in cost, performance, and risk to CWO and MEO, (2) potentially high salt (sulfate) production and secondary waste for the sulfuric acid process, and (3) absence of data for the phosphoric acid process relative to the other processes. Subsequent evaluation of the sulfuric acid and phosphoric acid processes to determine the chemistry and residue from the processes found that the quantity of residue from the acid digestion processes is similar to the residue from the MEO and CWO processes.

At the recommendation of the TSG, the phosphoric/nitric acid digestion process was included in the study. The process is almost as aggressive as the CWO process but at a somewhat lower, thus safer, pressure. The residual solids are insoluble phosphates or oxides which are compatible with phosphate bonded ceramics and should form highly stable waste forms. This process can treat organic liquids, organic sludges, and shredded organic debris. 
As with CWO, the developer of the acid digestion process did not recommend that it be used for inmatrix destruction of organic contaminants. Excess solids and secondary wastes would be generated in the system, the system would need to be shut down often to remove the solids, and it would be very difficult to wash the very viscous acid solution from the solids. Thus, the third system recommended by the TSG (in-matrix destruction of organics) cannot be accommodated by this technology.

D-3.5.4 Wet Oxidation. This is the least aggressive of the chemical destruction processes and would be applicable only to organic liquids and some organic sludges. This technology has difficulty treating several organic contaminants such as PCBs, trichloroethane, and other insoluble organics. Also, slow reacting intermediate products of partial oxidation which require further treatment may be produced. This process has been evaluated to determine the chemistry and residuals. The quantity of residuals is approximately the same as for the other organic destruction processes. However, because of its limited applicability it was not recommended for the INTS study.

D-3.5.5 Silicate Technology. This technology was rejected because it is primarily applicable to contaminated soils and to selected organics and is primarily a stabilization process. The developers are working on a hybrid soil washing and stabilization process for dioxin contaminated soils. However, destruction data on a range of organic contaminants are not available. Similar to soil washing, it may have limitations with respect to the number of different organic contaminants it can treat.

\section{D-3.6 Aqueous Waste Treatment}

Several technologies were rejected because of complexity which might cause difficulties in control, increase maintenance requirements, increase the number of valves and fittings and, therefore, increase the potential for release and/or leaks.

D-3.6.1 Electrochemical Destruction. This technology was rejected because a caustic chemical is added to the reactor, and heavy metals will deposit on the electrodes, producing excessive secondary wastes. This process was also considered more complex than other available organic destruction processes described below.

D-3.6.2 Wet Oxidation. This technology was rejected because it is a complex chemical process and system relative to other available processes. The process is typically applicable to higher concentrations of organics with a low organic concentration distillate going to further aqueous treatment.

D-3.6.3 MIOX Process. This technology was rejected because it is a complex process and produces more secondary waste relative to other available processes. A mixed oxidant solution (chlorine, hypochlorite, and ozone) is generated electrolytically from an $\mathrm{NaCl}$ brine, and injected into waste water for organic destruction. Hydrogen and sodium hydroxide, a caustic residue requiring disposal, are produced.

D-3.6.4 Electron Beam. This technology was retained for potential future evaluation because of its ability to distance itself from the waste stream, thus preventing equipment contamination. No chemicals that would produce a secondary waste are added. Water with up to $5 \%$ suspended solids can be treated. High energy electrons produced in an accelerator react with the water molecules of the aqueous waste to produce oxidizing radicals that attack and destroy the organic contaminants. This technology has been tested and is being commercialized. Sufficient data are available. 
Due to time and resource limitations, and the addition of the phosphoric acid digestion process, only one aqueous waste treatment technology was chosen to be evaluated for all five systems. The technology recommended by the TSG was photo-oxidation because more is known about this technology.

D-3.6.5 Photo-oxidation. This technology was retained because no chemicals that would produce a secondary waste are added. Ultraviolet radiation reacts with oxidizing agents (hydrogen peroxide and ozone) added to the aqueous waste to produce oxidizing radicals that attack and destroy the organic contaminants. The use of both hydrogen peroxide and ozone allows treatment of a wide variety of organic contaminants. This technology requires significant pretreatment to remove dissolved or suspended solids and immiscible organic material that may cloud the water or produce scale on the UV lamps or windows. This technology has been tested, is commercialized, and more is known about this technology than others, such as CWO. Sufficient data are available for analysis.

D-3.6.6 Photo-catalytic Oxidation. This technology was rejected because it is similar to photooxidation, but with the addition of a solid catalyst. Cost and performance are not expected to be significantly different. Cost may be higher due to use of the titanium dioxide catalyst and the eventual need for disposal of the catalyst as a secondary waste.

D-3.6.7 Ultrasonics. This technology was rejected because it is early in the development phase with little data on a variety of compounds. However, if new and additional data become available, this technology might be considered in a subsequent study.

\section{D-3.7 Air Pollution Control}

D-3.7.1 Electron Beam. This technology was rejected because the system cannot handle particulates in the offgas stream, and the offgas must be cooled to $400^{\circ} \mathrm{F}$ or below although the offgas is expected to be significantly cooler than this after it passes through the condenser. This technology is being commercialized. Medium energy electrons produced in an accelerator react with the oxygen molecules of the air to produce oxidizing radicals that attack and destroy the organic contaminants. Organic destruction would simulate the use of a secondary combustion chamber in the Integrated Thermal Treatment Systems (ITTS) study. The performance and cost of this technology is not expected to be significantly different from the GPCR selected for inclusion in the INTS study.

D-3.7.2 Gas Phase Corona Reactor. This technology was retained as a typical cold plasma technology and is relatively well developed. Particulates in the offgas stream and offgas temperatures of $600^{\circ} \mathrm{F}$ are not a problem. Low energy electrons produced in the electric discharge (or corona) react with the oxygen molecules of the air to produce oxidizing radicals that attack and destroy the organic contaminants. Initial data also indicate relatively good performance. Organic destruction would simulate the use of a secondary combustion chamber in the ITTS study. Also, due to time and resource limitations, and the addition of the phosphoric acid digestion process, only one gas phase organic destruction technology was chosen to be evaluated for all five Systems.

D-3.7.3 Photolysis. This technology was rejected due to poor performance and formation of organic products of incomplete reaction. 


\section{D-3.8 Stabilization}

D-3.8.1 Grout. Grout will be used to stabilize debris, as was done in the ITTS study, and to stabilize soils. Grout is also suggested as a standard stabilization agent throughout the DOE complex. Grout is representative of the chemical fixation category of stabilization technologies. However, grout is not appropriate for salts and sludges.

D-3.8.2 Polyethylene. Polymer encapsulation of salts and sludges will be used as was done in the ITTS study. Grout is not applicable to stabilizing these materials. Polymers are particularly suitable for soluble salts which can be microencapsulated in the polymer matrix and protected from contact with water in the environment.

D-3.8.3 Asphalt/Bitumen Solidification. This technology was rejected because of safety issues associated with potential fire and explosion. Long term durability is an issue with some salts and there is a potential for biodegradation and loss of structural integrity over time.

D-3.8.4 Phosphate Bonded Ceramics. The TSG and chemical experts recommended retaining this stabilization agent for the system using phosphoric/nitric acid digestion because of the synergy between the phosphoric acid and precipitated phosphates and oxides, and the formation of the ceramics. It will also be used to stabilize treated soils and sludges. However, this waste form is specific to waste stream composition, depends on the chemistry of the final waste form, and is inefficient for waste streams of diverse composition. Small particulate sizes are required for chemical reactions to take place. Polymer encapsulation will still be required for highly soluble salts (e.g., $\mathrm{NaCl})$.

D-3.8.5 Vitrification. This technology was rejected because it is a high temperature operation requiring an offgas system to capture volatile metals, metal compounds, and possibly trace organics.

\section{D-4. SYSTEM SELECTION AND RATIONALE}

Based on the technologies recommended by the TSG, five systems were selected and approved by the TSG for analysis as part of the INTS study. These systems were described in Section 2 of the INTS Report. Following is the rationale for selecting these systems.

\section{D-4.1 System 1: Base Case - Grout Debris}

This system was initially perceived to be the least complex, least expensive, and with the lowest operator risk because of minimum handling of debris and use of an organic destruction process that operates at near room temperature and pressure. It complies with regulatory requirements for debris and process waste. No contaminants will be separated from debris before stabilization in grout and disposal. This is similar to what is being proposed in several Site Treatment Plans so that this system allows disposal of debris without treatment to be compared to other systems in which debris is treated before disposal. Information will be developed for photo-oxidation, MEO for small organic waste streams (organic liquids and organic sludges), and for the APC system with GPCR technology used with thermal desorption. Treated soils will be stabilized in grout, and treated sludge and secondary wastes (salts) will be stabilized in polymer similar to the ITTS. This was considered the simplest and safest system expected to meet regulatory requirements. However, there is a long term environmental risk associated with potential failure of grout containing organically contaminated debris. 


\section{D-4.2 System 2: Desorption Case}

This system uses thermal desorption for all solid waste streams, including size reduced complex debris, and exceeds regulatory requirements for debris. However, operating temperatures are limited to less than $250^{\circ} \mathrm{F}$ for combustible debris to prevent melting plastics. This appears to be only slightly more complex than System 1. Information will be developed for photo-oxidation, CWO for small organic waste streams (organic liquids and organic sludges), and for the APC system with GPCR technology used with thermal desorption and a relatively large offgas stream. Analysis of this System will allow (1) evaluation of enhanced debris treatment's (e.g., decontamination by thermal desorption) compliance with the debris rule, and (2) comparison between MEO and CWO. The CWO process is considered more risky than MEO because of the higher temperature and pressure operation. There is less long term environmental risk with this system because organic contaminants are removed from debris before stabilization.

\section{D-4.3 System 3: Wash Case}

This system uses washing for all solid waste streams, including size reduced complex debris, and exceeds regulatory requirements for debris. Information will be developed for photo-oxidation, for MEO for organic liquids and sludges from external and internal sources (e.g., organics separated from the wash water), and for GPCR in the APC system with a very small offgas stream. This will compare washing with thermal desorption. Also, this system provides an alternative treatment of solid wastes. As with System 2, the long term environmental risk is decreased because organic contaminants are removed from debris before stabilization.

\section{D-4.4 System 4: Combination Case with Acid Digestion}

This system uses a combination of technologies that are considered most applicable to the specific waste matrices. An aggressive technology (phosphoric/nitric acid digestion) is used to treat organic liquids and organic sludges, and to destroy soft (combustible) debris to reduce the volume of waste going to disposal. Inorganic sludges are thermally desorbed because they may be difficult to treat by washing. Soil washing is used to remove organic contaminants from the soil matrix. Sludge from the acid digestion process, which consists of insoluble phosphates and oxides, treated soils, and treated inorganic sludge will be stabilized in phosphate bonded ceramics. Soluble salts (e.g., sodium chloride) will require polymer stabilization. Treated open debris and untreated complex debris are grout stabilized. This system allows an evaluation of an aggressive technology that provides more complete destruction of combustible debris and other organic contaminants, and provides an analysis of the impact of volume reduction.

\section{D-4.5 System 5: Combination Case with CWO}

This system is similar to System 4 except CWO replaces acid digestion. CWO is somewhat more risky than acid digestion because it operates at a higher pressure and requires more corrosion resistant construction materials. However, it appears more aggressive and may provide more complete destruction of more materials with a shorter residence time. Treated open debris, untreated complex debris, and soils are stabilized in grout. Sludges and secondary wastes (salts) are stabilized in polymer. This provides a comparison between CWO and acid digestion. 


\section{D-5. REFERENCES}

1. U. S. Department of Energy, Overview of Nonthermal Mixed Waste Treatment Technologies, Office of Technology Development (EM-50), June 20, 1995, DOE/EM-0281.

2. U.S. Department of Energy, Description of Recommended Nonthermal Mixed Waste Treatment Technologies, Office of Technology Development (EM-30), August 1995, DOE/EM-0282. 



\section{APPENDIX E}

Tribal and Stakeholders Principles

for Use in Evaluating Technology System for

Mixed Low Level Waste Treatment 


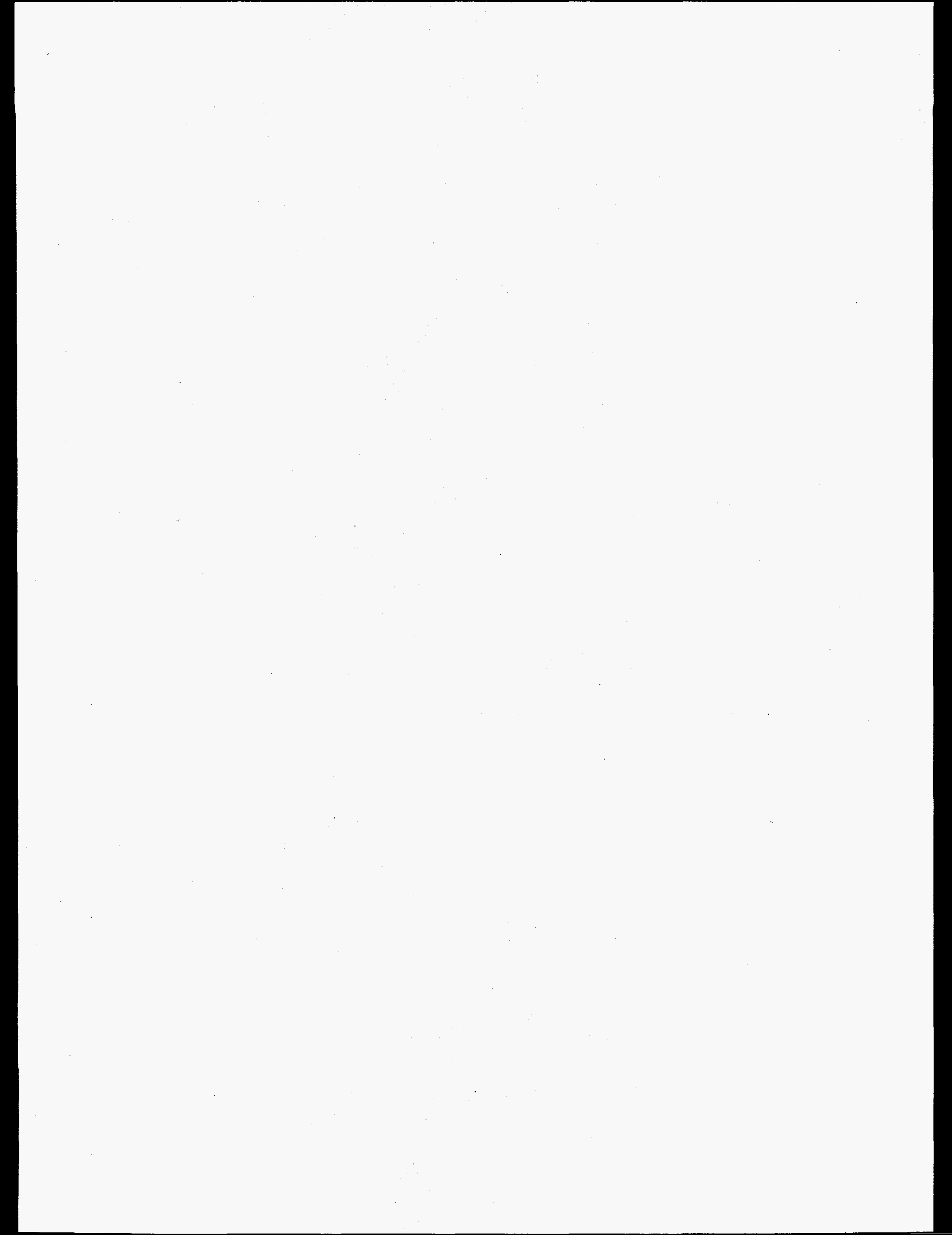




\title{
Tribal and Stakeholders Principles for use in Evaluating Technology Systems for Mixed Low Level Waste Treatment
}

\author{
Developed Jointly by the Office of Science and Technology and \\ Tribal and Stakeholder Working Group (TSWG) \\ Mixed Low Level Waste Treatment Systems Studies
}

\author{
January 1997 \\ Sponsored by \\ U.S. Department of Energy \\ Assistant Secretary for Environmental Management \\ Office of Science and Technology (EM-50)
}





\section{FOREWORD}

As a complement to the Integrated Thermal Treatment Systems Study (ITTS) completed in August 1995 by the U.S. Department of Energy's (DOE) Office of Science and Technology (OST) of the Office of Environmental Management (EM), OST commissioned an Integrated Nonthermal Treatment Systems Study (INTS). Each study compared the costs and performances of various thermal or nonthermal treatment systems for mixed low level waste. To assist in evaluating the systems, a tribal and stakeholder working group (TSWG) was formed to provide their perspectives. This was the first time that tribal and stakeholder representatives were involved so early in the technology system evaluation process.

The TSWG members represented a broad cross-section of American society. Each person represented an organization (i.e., a local or national environmental group, a state government, a municipality or county, a regional body, a commercial company, an Indian tribe, a citizen's group); however, the views expressed during the course of the INTS study were those of the individuals themselves.

This document is an expression of principles developed by the TSWG, in conjunction with a DOE technical group, to offer guidance to DOE headquarters, field managers, and other tribal and stakeholder groups when evaluating technology systems or making technology selections for waste treatment systems. The principles document is the result of four meetings of the TSWG to consider technology systems and reach agreement on the guiding principles to be used. 



\section{INTRODUCTION}

\section{Background}

The Tribal and Stakeholder Working Group (TSWG) ${ }^{1}$ assisted the U.S. Department of Energy (DOE), Office of Science and Technology (OST, EM-50) with its study and evaluation of treatment systems for Mixed Low Level Waste (MLLW). To supplement the technical criteria of the Technical Support Group (TSG), representatives of the TSWG and the TSG identified their social, cultural, and spiritual concerns in the form of six tribal and stakeholder principles. These principles were used concurrently with the technical criteria to study and evaluate treatment systems and are intended for that purpose.

Earlier versions of these principles were developed, discussed, and reviewed by the tribal and stakeholder representatives. Their comments have resulted mainly in the addition of clarifying explanations to most of the principles.

The principles are intended to be a continuing reference that can be used by tribal and stakeholder representatives, technical staffs, and by state, tribal, and Federal officials in the study and evaluation of a broad scope of systems.

\section{Objective}

Involvement of stakeholders and tribal representatives at every stage of the evaluation and selection of treatment systems and vendors will lead to better, more defensible selections and expedite clean up of the contaminated sites. Within the restrictions set by Federal regulations, DOE's objective should be to integrate stakeholder involvement into all of its processes, including procurement and performance evaluation.

\section{The Six Principles}

The six tribal and stakeholder principles are:
1. Minimize Effluents
2. Minimize Effects on Human Health and the Environment
3. Minimize Waste Generation
4. Address Social, Cultural, and Spiritual Considerations

1 The term "Tribal and stakeholder" is used throughout the text to represent
interested individuals from any ethnic, cultural, religious, or other special
background who want to be involved in assuring that system analysis adequately
considers and evaluates their issues related to future research and development. 
5. Provide Adequate and Understandable Information

6. Incorporate Tribal and Stakeholder Involvement into the DOE Procurement Process and the Performance Evaluation of Contractors on Stakeholder Participation.

These principles and their associated subprinciples are identified and explained in the following main sections.

In some cases the tribal and stakeholder representatives have suggested considerations to satisfy a particular principle or subprinciple. If there are suggested considerations, they follow the explanations that are applied to each principle. A list of TSWG and TSG members is attached. 


\section{TRIBAL AND STAKEHOLDER PRINCIPLES}

\section{General System Concepts}

Because some concepts apply to more than one principle, these concepts are explained here. They apply, as applicable, throughout the principles and subprinciples.

\section{- Minimization ${ }^{2}$}

Minimization is viewed by the tribal and stakeholder representatives as a two part process:

1. Avoid adverse conditions (effluents, effects, waste generation, etc.), if practicable.

2. When avoidance is not practicable, reduce remaining adverse conditions to their lowest practicable levels.

For each principle or subprinciple where minimization is addressed, both avoidance and reduction of adverse conditions should be considered. Research should be expedited to minimize adverse health and environmental impacts from effluents and waste streams. Releases and impacts should be minimized.

\section{- Reliance on Regulatory Standards}

Several principles and subprinciples deal with conditions limited by regulatory standards (e.g., operational releases of radioactive and hazardous materials, worker exposures, releases from disposed final waste forms). From the tribal and stakeholder perspective, mere conformance with these standards may provide less than adequate human health and environmental protection. Indeed, mere conformance with regulatory standards does not mean that a proposed system will gain widespread public acceptance. The regulatory standards represent minimum protective values. Actual exposures and releases should be reduced to their lowest possible ${ }^{3}$ levels, i.e., well below regulatory standards, in order to increase the level of public and environmental protection and enhance public acceptance. Requirements for monitoring over exposure to human population or flora and fauna should be identified and included in Total Life Cycle Costs (TLCC).

\section{- System Selections}

A general way of satisfying tribal and stakeholder principles is to select system elements and

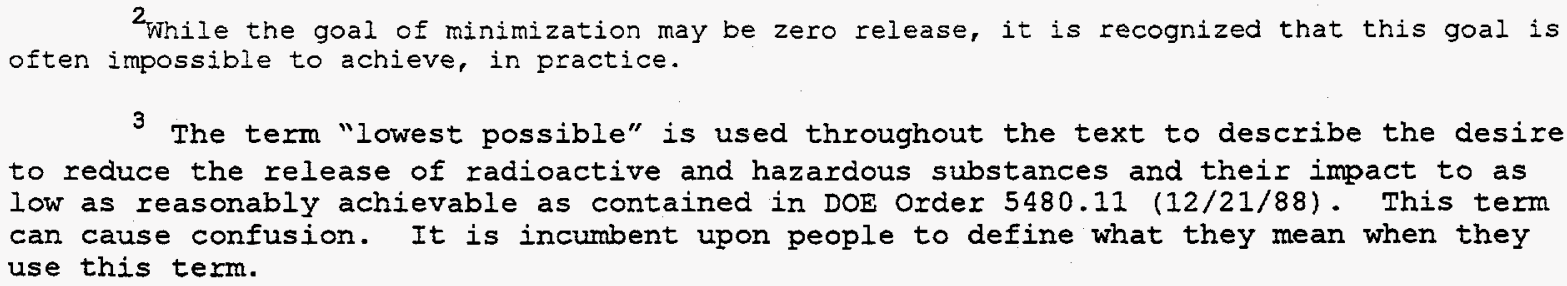


designs that best comply with these principles. This includes appropriate choices of (a) equipment, technologies and processes, (b) system design configurations, (c) construction and process materials, (d) operating practices (construction, operations, transportation, storage/disposal, facility closure and environmental restoration), and (e) continuous monitoring of effluents. In some cases the selections may contribute directly to satisfying a particular principle. In other cases, the selections may facilitate the use of specific methods needed to satisfy a particular principle.

\section{- Procurement Process}

Stakeholder and tribal representatives should be involved in procurements and the selection process. Tribal and stakeholder representatives should participate in the performance evaluation of agencies and contractors.

\section{Principles and Subprinciples}

\section{Minimize Effluents}

1a. Minimize airborne and wastewater effluents that can carry hazardous materials or substances capable of causing adverse human health and environmental consequences.

Operational releases of radioactive and hazardous pollutants contained in airborne and wastewater effluents should be kept to minimum levels, and below regulatory standards, from construction through facility closure and decommissioning. Release of pollutants should be minimized by avoidance and reduction methods. Expected pollutant and effluent releases should be publicly specified. By minimizing these releases, exposures to the general population, the ecology, and the environment should be minimized. Both the number of pollutants and the pollutant concentrations in effluents should be minimized.

Among the specific measures taken to minimize pollutant and effluent releases, consideration should be given to (a) applying newly developed pollution prevention and waste reduction methods, (b) recovering hazardous materials and byproducts, (c) built-in recycling or reuse of potential effluent materials, (d) posttreating internal effluents before they are released, (e) monitoring and controlling airborne and wastewater effluents, (f) diverting pollutants from airborne and waste water effluents into the solidified final waste form, and (g) their possible retention to analyze and validate effluent pollutant levels before they are released.

\section{Minimize Effects on Human Health and the Environment}




\section{2a. Minimize worker exposures now and in the future.}

Worker exposures should be minimized during construction, operation, shutdown, maintenance, facility closure, and environmental restoration periods. Tribal and stakeholder representatives are concerned with exposures associated with ionizing radiation and the intake of, or contact with hazardous materials. These exposures should be limited to operating areas where access is restricted to workers only and the general public is not affected.

Among the specific measures taken to minimize worker exposures beginning with the highest risks, consideration should be given to (a) minimizing the use of hazardous reagents and additives; (b) using confinement, containment, and shielding systems to reduce worker exposures; (c) making appropriate choices of operating and maintenance procedures to avoid or reduce worker exposures; (d) maximizing reliance on automated processes where hazardous equipment, reagents, and additives are involved; (e) using protective clothing and equipment appropriately, (f) using monitoring and alarm systems and emergency procedures to protect against abnormal exposures and environmental and public health hazards, and (g) involving worker representatives at each stage of planning.

\section{2b. Minimize the potential for release of hazardous and radioactive materials from final storage/disposal products.}

Future exposure of humans and the environment to ionizing radiation and hazardous and radioactive material released from final waste forms, whether in an interim storage or disposal configuration, should be minimized. By minimizing these releases, future effects on human health and the environment may be minimized.

Among the specific measures taken to increase waste form stability and minimize releases from disposed or stored final waste forms, consideration should be given to the following: (a) optimum combinations of final waste forms, the hazardous and radioactive materials and other waste that are stabilized in the final waste forms, and the selection, design and construction of storage/disposal facilities; (b) use of natural and engineered protective barriers at storage/disposal facilities to provide additional protection against releases; (c) use of personnel access control, physical security, and environmental monitoring systems at storage and disposal facilities; and (d) availability of a retrieval strategy to recover stored/disposed final waste forms, if adverse releases occur.

\section{2c. Minimize the potential for accidents and incidents in the system.}

The probability that an accident or incident associated with physical, chemical, radiological, and electrical hazards will occur should be minimized. Should an accident or incident occur, appropriate safety features to limit and mitigate accident/incident consequences should be provided or readily available. Accident/incident risks, the combination of probabilities and consequences, should be minimized when both accident/incident probabilities and accident/incident consequences are minimized.

Among the specific measures that can be taken to minimize accident/incident 
probabilities and limit and mitigate the consequences should they occur, the following should be given consideration: (a) appropriate choices of operating practices (transportation, operations, storage/disposal and facility closure); (b) minimization of physical, chemical, electrical, and radiological hazards; (c) appropriate reliance on automated process control equipment to reduce accident probabilities; (d) specification of adequate safety margins for system operations; (e) use and availability of protective clothing and equipment; and (f) use of emergency shutdown, confinement, containment, shielding and recovery systems, as appropriate, to limit and mitigate accident/incident consequences.

\section{Minimize Waste Generation}

3a. Minimize creation of new hazardous material by treatments.

The generation of new hazardous and/or mixed hazardous and radioactive wastes as byproducts of the treatment process should be kept to the minimum.

Among the specific measures taken to avoid or minimize waste generation, consideration should be given to the following: (a) application of newly developed pollution prevention and waste minimization methods, (b) implementation of waste segregation techniques, (c) recycle and reuse of appropriate waste materials, (d) use of techniques not requiring utilization of hazardous reagents, and (e) cost credits for recycle, reuse, and recovery of resources and materials.

\section{3b. Minimize the volume of final waste forms for storage/disposal.}

The volume of final waste compared to the initial waste volume should be minimized. Among the measures taken to minimize the volume of final waste forms for storage/disposal, consideration should be given to: (a) making appropriate choices of waste pre-treatment, treatment, post-treatment, and stabilization processes that minimize the volume of final waste forms compared to the volume of initial wastes, (b) maximizing waste loading with respect to final waste form options, (c) minimizing the volume of non-hazardous waste matrix materials that is solidified in the final waste form, and (d) maximizing recycle giving cost credit for recycling and reuse (including the credit in the TLCC).

\section{Address Social/Cultural/Spiritual Considerations}

Social, cultural, and spiritual concerns, values and perceptions must in some way be factored into the entire technology system assessment process. Although many cultural concerns will generally be addressed on a site-specific basis, it is important that the consideration of cultural concerns be done on a global basis, as well. A relevant generic set of social concerns should be identified and addressed for 
systems studies. Most importantly, the site-specific concerns can be linked to those identified from the system studies. There is a need to develop cultural protocols for both $R \& D$ decisions and also for use at specific sites.

A major consideration relates to the environment. Everything revolves around the environment and encouragement of practices that might benefit the whole of humanity and future generations. Continuing damage to the environment should be minimized. To fully address environmental considerations, facility design, development, operations, and closure processes must be conducted with careful sensitivity to protecting environmental health.

Regulatory realities will need to be considered. Examples are: regulatory barriers, insufficient regulatory resources (manpower, equipment, training), and undeveloped or underdeveloped regulatory standards.

\section{4a. Minimize land use for storage /disposal}

Land use commitments, in terms of acreage and time, should be minimized. This includes land use for disposal and storage facilities and for the treatment facility itself. Contamination of soil and water (both surface and ground water) that occurs while the land is in use should be kept to the lowest possible levels. Commitments should be made regarding land cleanup and reuse, e.g, to restore the land to its original state upon completion of its use where feasible. When not restoring the land to its original state, institutional controls should be used to ensure the protection of future generations.

Among the measures taken to minimize land use requirements and soil and water contamination while in use, consideration should be given to: (a) avoiding natural habitats and culturally important locations; (b) using pollutant confinement, containment, and retention systems to avoid soil and water contamination; (c) limiting the type and amount of soil contaminants and water pollutants to those that are amenable to cleanup with available remediation technologies; and (d) initiating an environmental restoration program upon completion of facility closure operations (including pollution prevention).

\section{4b. Minimize transportation from one site to another.}

Added traffic along public roads in the vicinity of the treatment facility due to plant operations should be minimized. Tribal and stakeholder representatives are concerned that all sources of traffic be addressed and impacts identified. This includes traffic related to construction and closure activities, employee commuting, delivery of goods and services, delivery of process materials and reagents, delivery of wastes to be treated, and shipment of storage and storage/disposal products.

Among the measures taken to minimize transportation requirements, consideration should be given to work force minimization, reduced reliance on process additives and reagents, minimization of final waste form volume, and access roads that avoid populated areas.

\section{4c. Reflect special considerations for specific sites.}


Identification and response to special considerations at specific sites is critically important to local acceptance of treatment technologies. The representatives strongly encourage the Department of Energy to include site specific considerations as an integral part of future treatment system studies.

\section{4d. Waste should be disposed in a retrievable manner.}

Final waste forms must be disposed in a manner that allows the waste to be retrieved, if pollutant releases prove to be unacceptable. Retrieval may also be required if future land use considerations dictate its relocation. In line with this position, contingency plans for waste retrieval should be addressed as part of the waste storage/disposal process. Furthermore, a solid final waste form, with long-term stability, should be used to facilitate retrieval, if retrieval is required.

\section{4e. Respect cultural values.}

At least two approaches are available to assure that cultural values are properly respected in studying, siting, constructing, operating, and closing treatment facilities:

1. Assignment of scientists and engineers from diverse cultural backgrounds to the technical staff supporting the study to assist in identifying and addressing cultural value issues, e.g., Native Americans.

2. Integration of a meaningful tribal and public participation process into site systems management and planning models for site specific studies. This includes participation that provides identification of issues and timely responses regarding estimated impacts.

In both cases, it is important to the tribal and stakeholder representatives that early and meaningful involvement be achieved. Provisions should be made for tribal and stakeholder involvement in studies, assessments of treatment technologies and systems, and for planned treatment system demonstrations or implementations at DOE sites. DOE management should take appropriate action to ensure that both approaches are implemented.

Involvement, e.g., Indian Tribal representation, allows appropriate consideration of the impact of technologies on food, medicine, religious ceremonies, and other features essential to Native cultures. Similar issues have been addressed by the Development of On-site Innovative Technologies (DOIT) program in issuing "A Guide to Tribal and Community Involvement in Innovative Technology Assessment."

\section{4f. Minimize noise.}

Noise due to traffic is addressed in subprinciple $4 \mathrm{~b}$ above. Additional sources of noise and vibrations, whether in facility construction, operations, or closure activities must be identified and addressed.

Among the measures taken to minimize these sources of noise, consideration should be 
given to elimination of noise sources if practicable, installation of sound-barrier walls or other mitigating measures where appropriate, and selection of remote locations for proposed systems.

\section{4g. Protect local vistas.}

Some sites have intrinsic value because of their panoramic views of distant mountains, bodies of water, and scenic landscape. These vistas need to be protected. A particular concern is the obstruction of vistas and visual impact upon the horizon by "dotting the landscape" with assorted treatment facilities, power lines, effluent stacks, and other supporting requirements.

Among the measures than can be taken to protect local vistas, consideration should be given to minimizing above-ground structures, using facilities that can be powered by underground electrical systems, and carefully siting facilities so that existing vistas are not disturbed. Permanent massive structures that dominate an otherwise attractive vista are to be avoided, as they may compromise religious use of such sites. All should be considered as part of the evaluation of systems.

\section{4h. Include Costs of Complying with Intergovernmental Agreements}

Compliance agreement and MOU-related costs such as monitoring, technical assistance, and third party review should be included in Total Life-Cycle Costs.

\section{Provide Adequate and Understandable Information}

Detailed, timely, and accurate information should be provided to stakeholders in accessible formats and locations. The written language should be understandable to a general audience, and examples or analogies should be used for explanation.

5a. Provide technology screening and down-selection, and system description and cost information, including details of the process and how unsuccessful systems could become viable.

5b. Provide information about systems hazards and risks, and the safety features provided to avoid or reduce these hazards and risks.

5c. Describe the processes that govern the conduct of the study:

- Treatment study decision-making

- DOE decision-making 
- Tribal and stakeholder involvement

- Planned documentation and outcomes

- Likely pollutants in gas or water effluents

5d. Provide system and technology information within the framework of Tribal and Stakeholder Principles and Technical Criteria.

5e. Integrate or "bridge" thermal, nonthermal and other applicable treatment systems studies for overall systems comparisons.

5f. Focus on existing stored waste and then add projected impact of future waste generation.

5g. Keep the Tribal and stakeholder representatives involved and informed throughout the evolution of systems studies.

5h. Provide information on qualitative and quantitative analysis and monitoring of hazardous and radioactive components in effluents.

5i. Independent advisory resources should be made available to review the information and respond to those supplying the information. 


\section{Incorporate Tribal and Stakeholder Involvement into the DOE Procurement Process and the Performance Evaluation of Contractors on Stakeholder Participation}

Stakeholder and tribal representatives should be involved in procurements and the selection process. Tribal and stakeholder representatives should participate in the performance evaluation of agencies and contractors.

\section{CONCLUSIONS AND PROPOSALS}

\section{Conclusions}

These principles, reflecting the concerns of Tribal and stakeholder representatives, are applicable to evaluations of mixed low level waste treatment systems and may be applicable to selection of other systems. They augment conventional technical criteria by introducing additional considerations that technical staffs, possibly assisted by technically trained tribal and stakeholder representatives, should address in order to make treatment systems more acceptable to the general public. The principles are generic and can be applied to a broad range of systems studies in planning future research and development.

The principles are qualitative in nature. They provide no quantitative limits that can be used to delineate between acceptable and unacceptable systems. They are goals toward which technical staffs should strive in studying treatment systems including siting, design, development, implementation, and closure processes. They are goals which, when better satisfied, should enhance public acceptance.

Uncertainties regarding public acceptance of mixed waste treatment systems will always exist. These uncertainties can be reduced, preferably on a site-specific basis, by timely and meaningful involvement of tribal and stakeholder representatives from the beginning of technology development processes. Doing so will promote early identification of significant issues, joint resolution of these issues, and "real time" concurrence about public acceptance of the proposed treatment system.

Tribal and stakeholder representatives place great importance on being kept informed whenever a treatment system is planned or proposed for implementation. Means to identify appropriate representatives and keep them informed are recommended.

\section{Proposals}

It is proposed that tribal and stakeholder principles be applied in future mixed waste treatment systems studies and implementation activities. There should be community involvement in the analysis and selection of storage and disposal technologies. Based on the favorable results of discussion of these principles, affected communities should be involved in analysis of storage and disposal technologies intended for use at specific sites. 
It is proposed that technically trained tribal and stakeholder representatives be available to technical staffs and Tribal and stakeholder representatives responsible for future systems studies to assist in evaluation of treatment system options with respect to tribal and stakeholder principles. 


\section{BIBLIOGRAPHY}

Meeting Report, Tribal and Stakeholder Working Group Meeting, Stouffer Concourse Hotel, Denver, Colorado, U.S. Department of Energy, Office of Technology Development, Integrated Nonthermal Treatment Systems Study, Washington, D.C., August 8-9, 1995

Meeting Report. Tribal and Stakeholder Working Group Meeting, Radisson Midtown Hotel, Phoenix, Arizona, U.S. Department of Energy, Office of Science and Technology, Integrated Nonthermal Treatment Systems Study, Washington, D.C., January 17-18, 1996

Tribal and Stakeholder Involvement in the DOE INTS Review: History, Process and Products, Swartz and Associates, Boulder City, Nevada, 11 July 1996 (DRAFT)

Meeting Report, Joint meeting of the Tribal and Stakeholder Working Group with the Technical Support Group of the Integrated Nonthermal Treatment Systems Study, Idaho Falls, Idaho, August 6-8, 1996

A Guide to Tribal and Community Involvement in Innovative Technology Assessment. Western Governors Association, Federal Advisory Committee to Develop On-Site Innovative Technologies, DOIT Tribal and Public Forum on Technology Acceptance, Reno, NV, May 4-5, 1995 (Prepared by the Colorado Center for Environmental Management, Denver, $\mathrm{CO}$ )

Meeting Report. Tribal and Stakeholder Working Group Meeting, Waste Policy Institute, Gaithersburg, MD, U.S. Department of Energy, Office of Science and Technology, Integrated Nonthermal Treatment Systems Study, Washington, D.C., November 7-8, 1996

DOE Order 5480.11 (accessible by Internet: www.explorer.doe.gov:1776/htmls/alldirectives.html) 


\section{TRIBAL AND STAKEHOLDER WORKING GROUP (TSWG) MEMBERS}

1. Richard Brusuelas, Bernalillo County, New Mexico

2. Anne Callison, Lowry AFB Restoration Advisory Board, Denver, Colorado

3. David Chambers, Sierra Club Legal Defense Fund, Bozeman, Montana

4. Terry Escarda, Department of Toxic Substances Control, Sacramento, California

5. Karen Fagg, MSE-HKM, Inc., Billings, Montana

6. Critz George, Brown \& Root Environmental, Albuquerque, New Mexico

7. Lorna Harrell, Butler County, Ohio Soil \&Water Conservation District

8. Steve Juanico, Pueblos of Acoma, New Mexico

9. Dr. William F. Lawless, Savannah River Citizens Advisory Board, Augusta, Georgia

10. Momi Lovell, Kaho'olawe Island Res.Commission, Honolulu, Hawaii

11. Tom Marshall, Rocky Mountain Peace Center, Boulder, Colorado

12. Betsy McBride, League of Women Voters of the U.S., Chesapeake, Virginia

13. Toby Michelena, Washington State Department of Ecology

14. Roy Mink, University of Idaho, Moscow, Idaho

15. Laura Olah, Citizens for Safe Water, Merrimac, Wisconsin

16. Terry Smith, Idaho Division of Environmental Quality, Boise, Idaho

17. Stan Sobczyk, Nez Perce Tribe, Lapwai, Idaho

18. Edwin Tafoya, Eight No. Ind. Pueblos, San Juan Pueblo, New Mexico

TSG Technical Stakeholders:

1. Gary King, New Mexico State Legislature, Albuquerque, New Mexico

2. Ross Vincent, Sierra Club, Pueblo, Colorado

Native American Technical Consultant:

Mary Jo Ondrechen, Northeastern University, Boston, Massachusetts

\section{TECHNICAL SUPPORT GROUP (TSG)}

1. Leon Borduin, Los Alamos National Laboratory

2. Carl Cooley, U.S. DOE, EM-50, Chair

3. Floyd DesChamps, U.S. DOE, EM-53

4. Jim Cummings, U.S. Environmental Protection Agency

5. Terry Dyches, DOE-Savannah River

6. David L. Eaton, LMTCO

7. Steve Priebe, LMTCO

8. Dave A. Hutchins, DOE-Oak Ridge

9. Don Musgrave, BCM Inc.

10. Jack Watson, Oak Ridge National Laboratory

11. Michael Torbert, U.S. DOE, EM-34

12. Paul Strider, U.S. DOE, EM-44 


\section{APPENDIX F}

\section{Glossary of Terms}





\section{Glossary of Technical Terms}

Absorption

Acceptability

Acid digestion

Acid gas scrubbing

Activated carbon

Additives

Adsorption

Advisory Groups

Aggressive chemical treatment

Agitation wash

Air pollution control (APC)

Airborne releases
The dissolving of one compound, usually a gas, in another, usually a liquid.

Satisfactory performance to achieve overall goals; feasibility in the real world.

The use of strong acids such as nitric acid and phosphoric acid to dissolve organic and inorganic compounds in an acid medium.

The removal of by-product gases such as sulfur dioxide $\left(\mathrm{SO}_{2}\right)$ or hydrogen chloride $(\mathrm{HCl})$ by bringing the gases into contact with water or a mildly alkaline solution.

Fine granular carbon specifically formulated to adsorb organic compounds.

Chemicals added to waste as part of treatment. Examples are sodium hydroxide added to neutralize wastewater or surfactants (detergents) added to water to wash soil.

The physical attachment of one chemical compound to another, such as benzene to carbon.

Individuals selected to provide input to and review of the development of a project.

A process using strongly acid or strongly basic chemicals to quickly attack other materials, including organics and metals.

A washing system which uses the same principles as the common household washing machine.

Equipment designed to treat air, vapors and gases exiting a process before releasing the gas stream to the atmosphere.

The volume of gas (including air, carbon dioxide, and contaminants) released to the atmosphere from a treatment facility. 


\section{Alpha particle}

Alpha waste

Amalgam

Amalgamation

Ambient

Analysis

Anolyte

Applicability

Application

Aqueous waste

Aqueous wash

ASPEN PLUS (ASPEN)

Assess

Assumptions
A positively charged particle consisting of two protons and two neutrons that is emitted from the nucleus of certain nuclides during radioactive decay. It is the least penetrating of the four common types of radiation (alpha, beta, gamma and neutron).

Waste contaminated with alpha radioactivity measuring 10 to 100 nanoCuries per gram of waste.

An alloy of mercury with another metal that is solid or liquid at room temperature according to the amount of mercury present.

A process of stabilizing a mixture of diverse elements together; as described in this report, mercury and other metals are stabilized as an amalgam.

Environmental/weather conditions that exist at a given location, including, temperature, pressure, rainfall, etc.

Chemical analysis: the identification of ingredients of a substance by performance of laboratory tests. Engineering analysis: an examination of a process or problem, its elements and their relationships.

Liquid solution containing positively charged ions produced in contact with electrodes, used for destruction of organic compounds.

Capable of being put into practice.

An act of putting to use new techniques.

Wastes that are combined with water and have an organic content of $1 \%$ or less.

A washing technique which uses water in a combination with various surfactants.

A computer program which applies chemical engineering principles to generate information relating material flowing into a system to material flowing out of the system.

To determine the importance, size or value of; to evaluate.

A fact or statement taken for granted. 


\author{
Atmospheric pressure \\ Atomic Energy Commission (AEC)
}

Availability

Bench scale testing

Beta particle

Biota

\section{Blowdown}

Bulk soil

Byproducts and residuals

Canister
Normal barometric pressure.

A five-member commission established after World War II to supervise the use of nuclear energy. The AEC was dissolved in 1975 and its functions transferred to the Nuclear Regulatory Commission (NRC) and the Energy Research and Development Administration (ERDA). The latter became the Department of Energy (DOE).

This criterion identifies the amount of time the technology or system is available for operation and treatment of waste.

Chemical or physical testing that occurs as a first step in a laboratory. This test equipment is usually very small in scale (the size of household blenders and 2 or 4 cup measures). Bench scale testing is an initial evaluation to determine if a process is a good idea (Does it work well? Would anybody be interested in it?).

An elementary particle emitted from a nucleus during radioactive decay. It is negatively charged, is identical to an electron, and is easily stopped by a thin sheet of metal.

The plant and animal life of a region.

The withdrawal of water from an evaporating process to maintain a solid balance within specified limits of concentrations of those solids.

Bulk quantities of soil removed from the ground for treatment.

During treatment, components of wastes are separated to isolate hazardous compounds and remove them from the waste. These separation processes may generate byproducts. As an example, the neutralization of acids generates insoluble salts:

$\mathrm{HCl}+\mathrm{NaOH} \Rightarrow \mathrm{NaCl}+\mathrm{H}_{2} \mathrm{O}$

(Hydrochloric acid + sodium hydroxide $\Rightarrow$ table salt + water)

Residuals are the leftovers, such as the solids remaining at the bottom of the evaporator.

A stainless-steel container in which immobilized radioactive waste is sealed. 
Canyon

Capping

Catalytic wet oxidation (CWO)

Catholyte

Centigrade (Celsius)

Characterization

Chemical oxidation

Chemical Composition

Complex Debris

Complexity

Concentration
A heavily shielded building used in the chemical processing of radioactive materials to recover special isotopes for national defense or other programmatic purposes. Operation and maintenance are by remote control.

The process of sealing or covering a waste unit with a low permeability medium.

A chemical process which utilizes a strong acid medium in the presence of a catalyst to convert organic compounds to carbon dioxide, water and acid gases.

Liquid solution containing negatively charged ions produced in contact with electrodes in conjunction with the positively charged anolyte.

A temperature scale that registers the freezing point of water as $0^{\circ} \mathrm{C}$ and the boiling point as $100^{\circ} \mathrm{C}$ under normal atmospheric pressure.

The description of the chemical nature of waste. Laboratory analysis is performed on waste to determine generically what it is and whether it has chemical such as hazardous organics that must be treated. Characterization may be necessary for waste as it enters the facility to determine the nature of treatment required. Also characterization may be needed before shipment, to determine that the hazardous substances in the final waste form are below regulatory limits.

The reaction of a compound with oxygen or an oxygen donor to convert the compound to an oxidized state.

A list of the chemicals in a sample, and the percentages of each chemical or element.

Debris that has crevices or hard to access areas, such as pipe, valves, pumps, complex machinery, etc.

The state of intricacy, complication, multiple parts and processes and the interrelatedness of these parts. Mechanical, chemical, and operational complexity as well as the number of processes required are considered part of this aspect.

The quantity of a substance contained in a unit quantity of a medium (e.g., micrograms of aluminum per liter of water). 


\section{Condensate}

Confidence level

Constituents

Contaminant

Contaminant loading

Criteria pollutant

Curie (Ci)

Decay, radioactive

Decommissioning

\section{Decontamination}

Liquid water obtained by cooling the steam produced in an evaporator system.

The certainty of a particular point (measurement, amount, value) being within a statistically determined range.

Parts or components of a chemical system.

Hazardous or radioactive constituents, as defined by EPA and other regulatory agencies.

The concentration of contaminants in the waste.

Air pollutants for which the EPA has established concentrations below which the pollutants do not pose a threat to public health and welfare.

A unit of measure of radioactivity equal to $37,000,000,000$ decays per second. A curie is also a quantity of any nuclide or mixture of nuclides having one curie of radioactivity.

The spontaneous transformation of one nuclide into a different nuclide or into a different energy state of the same nuclide. The process results in the emission of nuclear radiation (alpha, beta, gamma, or neutron radiation).

The removal from service of facilities such as processing plants, waste tanks, and shallow land disposal units, and the reduction or stabilization of radioactive contamination. Decommissioning concepts include:

- Decontaminate, dismantle, and return area to original condition without restrictions.

- Partially decontaminate, isolate remaining residues, and continue surveillance and restrictions.

The act of removing a chemical, biological, or radiological contaminant from, or neutralizing its potential effect on, a person, object, or environment by washing, chemical action, mechanical cleaning, or other techniques. 


\section{Decontamination and decommissioning} (D\&D)

\section{Desorption}

\section{Dioxin}

DOE

DOE Complex

Ecological effects

Ecology

Effectiveness

Effluent

Engineered trench

Engineering judgement

Environmental restoration

Evaporation
This criterion assesses D\&D requirements including hazards, expected effectiveness, residual contamination levels, and the potential for turning over the facility for alternative uses. All five nonthermal systems will require approximately the same amount of D\&D. At this stage of design this criterion cannot discriminate between systems. Specific requirements and results of D\&D efforts have not been determined.

The process whereby a liquid leaves a solid by diffusing into a gas phase.

Highly toxic chlorinated organic compound present in Agent Orange. Probably a carcinogen.

U.S. Department of Energy.

The $50+$ sites owned by the DOE.

Damage or benefits to soil or groundwater as a result of the construction, operation, or D\&D of the treatment facility.

The study of the relationships between living things and their environments.

How well the technology or a system performs its required function. Effectiveness answers the question "How well does this work?"

Any material which leaves a system.

Reinforced, concrete-formed, walled disposal trench with steel covers over each area to minimize rainwater intrusion and direct drainage away from the trench. A leachate collection system installed below the floor of the trench monitors the performance of the disposal cells.

Qualitative judgment based on experience and familiarity with the subject.

Required activity to return soil, groundwater and vegetation to conditions before facility operation.

The conversion of a volatile compound to a gaseous phase, such as the boiling of water. 
Evaporator bottoms

Exothermic

Exposure to radiation

Filtration

Final waste form performance

Final waste form volume

Flexibility

Flow rates

Full-time equivalent worker (FTE)

Gamma rays

Gas phase corona reactor (GPCR)

Groundwater
The sludge that remains in the bottom of an evaporation apparatus after the desired product has evaporated and been removed.

A chemical change accompanied by a release of heat.

The incidence of radiation on living or inanimate material by accident or intent. Background exposure is the exposure to natural background ionizing radiation. Occupational exposure is the exposure to ionizing radiation that occurs during a person's working hours. Population exposure is the exposure of a number of persons who inhabit an area.

The separation of solids from a liquid or gas by passing the liquid or gas through a porous media which will pass the liquid or gas and retain the solids.

The ability of the disposed waste to remain stable or to release contaminants such as radionuclides. Waste form performance is estimated by performing leaching tests (see TCLP).

The volume of stabilized waste shipped out of a treatment facility.

Ability of a system or process to tolerate a range of waste compositions

The amount of material per hour that passes through a process; e.g. a typical shower has a water flow rate of 1 to 7 gallons per minute.

For cost estimates, $40 \mathrm{hrs} / \mathrm{wk}$ of labor. Since labor may be used flexibly, FTEs are calculated rather than assigning a head count.

High-energy short-wavelength electromagnetic radiation accompanying fission, radioactive decay, or nuclear reactions. Gamma rays are very penetrating and require relatively thick metal/lead shields to absorb the rays effectively.

A device which uses electricity to break down organic compounds in a gas stream.

The supply of fresh water in an aquifer under the Earth's surface. 
Grout

Grout stabilization

Hazardous materials

Hazardous operating conditions

Hazardous process equipment

Hazardous reagents

Hazardous waste generation

Hazardous waste storage facility

Heavy metals

HEPA filter

High pressure wash

Hydrolysis

Hydroxyl radicals
A material composed of ingredients such as cement, sand, ash and water.

The use of combination of cement, sand, ash and water with waste to form solid concrete-like mass which is resistant to leaching.

Chemicals defined by EPA as hazardous based on toxicity, reactivity, flammability or other characteristics.

Conditions in the workplace which may pose a hazard to operations personnel. Typical hazardous conditions include moving machinery, temperatures and pressures above ambient, and chemicals and radionuclides which can cause illness.

Equipment which under normal operation presents hazards to workers. Examples of hazardous process equipment are reactors under pressure, electrical transformers, and metal melting equipment.

A hazardous substance used in chemical reactions.

Creation of new hazardous waste during the treatment of existing DOE waste.

Resource Conservation and Recovery Act (RCRA) permitted temporary holding area for hazardous waste prior to treatment or disposal.

Metallic elements of high atomic mass such as mercury, chromium, cadmium, lead, or arsenic, that are toxic at known concentrations to plants and animals.

High-Efficiency Particulate Air filter designed to remove from a flowing air stream $99.95 \%$ of the particles as small as 0.3 micrometer.

A process which uses a liquid spray, usually water, at high pressure to remove surface adhered particles.

A process of decomposition in which a compound is broken down and changed into other compounds by taking up the elements of water.

A negatively charged ion which consists of one oxygen atom and one hydrogen atom. 
Immiscible organics

Immobilization

Implementability

\section{Incineration}

INEL

Influent

Inorganic

Input

INTS

Ion

Ion exchange

ITTS

Kilo

Leach resistant waste form
Organic compounds that are not soluble in another liquid, such as oil which would float on the top of water.

Conversion of a material into a form that will resist environmental dispersion.

Potential to actually be put into service; a combination of practicality and acceptability.

The burning of waste.

Idaho National Engineering Laboratory near Idaho Falls, which is one site in the DOE complex.

Any material that flows into a system.

Any chemical compound which does not contain carbon in its structure.

Something that is put in, such as labor, raw material, waste, additives. In this report, input often refers to a waste or additives put into a process.

Integrated Nonthermal Treatment Systems- a series of conceptual designs using nonthermal processes to treat and stabilize radioactive mixed waste.

An atom or molecule that has gained or lost one or more electrons and has become electrically charged.

Process in which a solution containing soluble ions to be removed is passed through a column of material that removes the soluble ions by exchanging them with ions from the material in the column. The process is usually reversible so that the trapped ions can be collected (eluted) and the column regenerated.

Integrated Thermal Treatment Systems- a series of conceptual designs using thermal processes to treat and stabilize radioactive mixed waste.

A prefix meaning one thousand $\left(10^{3}\right)$ of any measurement

A waste form that is stable enough to resist outside materials from removing any trapped components from its structure. 
Leaching

\section{Macroencapsulate}

Magnesium phosphate

Maintainability

Maintenance worker exposure

Mass

Material mass balances

Material

Matrix

Mediated electrochemical oxidation (MEO)

Mercury Waste

Micro

Milli
The removal of compounds by the percolation of liquids; e.g. drip coffee is a leaching into water of compounds in ground coffee beans.

To seal (e.g., in a box or polymer) a contaminated component so that the contamination is contained.

A nontoxic inorganic compound.

The ability of equipment, technologies or systems to be kept in working condition.

Exposure of maintenance workers to hazardous or radioactive waste. Workers repair equipment which may have been in contact with radioactive and/or hazardous waste.

A measure of the amount of material in a given body; most commonly in this report, the weight of the body.

An analysis showing the mass movement of materials or constituents through a process. For example, a mass balance of cooking vegetables in hot water would list the weight of water and vegetables added to the pot, the mass of water vaporized, the water remaining in the pot following cooking and the final weight of the cooked vegetables. The total weight of cold water and uncooked vegetables in the pot must equal (balance) the weight of the water lost in evaporation, the water remaining in the pot, and the weight of the cooked vegetables.

Matter that has individual qualities that define it (hazardous material, waste material).

A material in which something is enclosed or embedded; eg, radionuclides in soil, solvents in wastewater.

MEO is an aqueous process which uses electric energy to react with organics and break them down to carbon dioxide and water.

Waste material such as debris which contains concentrations of mercury above regulatory limits.

A prefix meaning one millionth $\left(10^{-6}\right)$ of any measurement.

A prefix meaning one thousandth $\left(10^{-3}\right)$ of any measurement. 
Nano

NEPA

Nitric acid

Non-alpha waste

Nonthermal

Nonthermal systems

$\mathrm{NO}_{\mathrm{x}}$

NRC

Nuclear radiation

Offgas

Off-normal conditions

Open Debris

Organic
A prefix meaning one billionth $\left(10^{-9}\right)$ of any measurement.

National Environment Policy Act of 1969; it requires the preparation of an Environmental Impact Statement (EIS) for Federal projects that could significantly impact the environment.

A common acid composed of nitrogen, hydrogen and oxygen $\left(\mathrm{HNO}_{3}\right)$.

Waste contaminated with alpha radioactivity measuring less than 10 nanoCuries per gram of waste.

Processes with maximum temperatures below $660^{\circ} \mathrm{F}$ $\left(350^{\circ} \mathrm{C}\right)$.

Systems composed of physical and chemical processes which occur at temperatures below $350^{\circ} \mathrm{C}\left(660^{\circ} \mathrm{F}\right)$.

Oxides of nitrogen ( $\mathrm{NO}, \mathrm{NO}_{2}, \mathrm{NO}_{3}$ ) which form at high temperatures (as in a car engine) or as breakdown products of nitric acid.

Nuclear Regulatory Commission; the independent Federal commission that licenses and regulates commercial nuclear facilities.

Radiation, usually alpha, beta, gamma, or neutron, which emanates from an unstable atomic nucleus.

Vapors and gases (including air) which are given off from a process; cooking odors are a form of offgas.

Conditions outside of normal processing range, for example: elevated temperature or pressure, $\mathrm{pH}$ too high (basic) or too low (acid), excessive electricity usage, jammed equipment.

A classification of debris which includes concrete, bricks, drums, glass, metals.

Chemical compounds which contain carbon and hydrogen; these chemicals are associated with living entities. 
Output

\section{Oxidation}

Parameter

Particulates

Peer review

pH

Phosphate bonded ceramic

Photo-oxidation

Pilot scale systems

\section{Pollution}

Polymer

Polymer stabilization

Precipitate
Something that is produced; a yield. Typically in this report we describe as output the materials resulting at the end of a process, e.g. treated stabilized waste, evaporated water, etc.

In this report, the reaction of a compound with oxygen, either directly or indirectly.

A characteristic element; any of a set of physical properties whose values determine the characteristics or behavior of something, e.g. volume, temperature, etc.

Solid particles small enough to become airborne.

Scientific review of documents by qualified outsiders.

A measure of the hydrogen ion concentration in aqueous solution. Pure water has a $\mathrm{pH}$ of 7 , acidic solutions have a $\mathrm{pH}$ of less than 7, and basic solutions have a $\mathrm{pH}$ greater than 7 .

A form of cement which uses phosphates rather than the more common calcium based ingredients used in concrete.

A process that uses light to cause a reaction of organic chemicals in water yielding harmless substances (carbon dioxide and water).

Chemical process equipment at a small scale (fits in a large laboratory) designed to test the practical aspects of a new process. These systems often process volumes of 20 to 50 gallons.

The addition of any undesirable agent to an ecosystem in excess of the rate at which natural processes can degrade, assimilate, or disperse it.

Long chain chemicals with repeating chemical units; for example, nylon and plastics.

Mixing of waste residues together with melted plastics which cool to form a stable solid.

A solid (used as a noun).

To form a solid substance in a solution by a chemical reaction (used as a verb). 
Preconceptual design

Precursors

Process flow diagrams (PFD)

Process controls

Process containment

Promulgated

Public acceptability

Qualitative

Quantitative

Radioactive materials

Radioactive waste
Engineering design analysis performed to develop basic concepts. This design evaluates the feasibility of a project. Specific details, such as exact location, supporting equipment and instrumentation, are not included.

Chemicals that are the building material for the formation of other chemicals.

Box diagrams with arrows indicating material flow through a process. In this report, the PFDs show the type of waste treated, the treatment process used, and the products of the process.

Instrumentation which is provided to control and operate equipment. Sensors receive electrical and air pressure signals. The signals are processed by the instrumentation. For example, a device is installed in a water-jacketed reactor to monitor the temperature. If the temperature gets above an instrument set point, flow of cooling water through the jacket is increased until the temperature in the reactor is in the proper range.

Engineered safety features such as instrumentation that shuts down malfunctioning equipment, concrete barriers that collect spilled liquid, and warning devices that notify staff of off-normal conditions.

A law put into action or force.

Concepts (or designs, facilities) found to be satisfactory or adequate by the general public.

Related to or involving the general qualities and characteristics; not involving numerical analysis.

Related to or involving the measurement of amounts, quantities, and parameters such as temperature, pressure, $\mathrm{pH}$, chemical concentration.

Materials which contain artificially produced radioactive metals which spontaneously emit radiation (atomic particles).

Materials from nuclear operation that are radioactive or are contaminated with radioactive materials for which there is no practical use or for which recovery is impractical. 
Radiolysis

Radionuclides

RCRA metals

\section{Reagents}

Recycled metals

Reliability

Research and development (R\&D)

\section{Residues}

\section{Resource utilization}

Salts

Schedule
The decomposition of a material (usually water) into different molecules due to ionizing radiation. In water, radiolysis results in the production of hydrogen gas and oxygen.

Elements which emit radiation.

Metals whose handling and disposal are regulated by the EPA. RCRA requires that metals disposed of pass a TCLP test demonstrating that they will remain in the stabilized waste and will not migrate at any appreciable rate.

Pure form of chemicals used in the laboratory.

Metals from which radionuclides or hazardous compounds have been removed that can be reused within the DOE complex

Dependability; the expected stability of system operations.

Work performed in laboratories and research facilities. Research looks at which processes are the most promising and considers whether a process is effective. Development takes the best processes from research, evaluates the key parameters, and tests the process pilot scale equipment.

Remainder of waste left after treatment, often sludges and dried solids (e.g., coffee grounds).

In a proposed design or project, the requirements for resources to support the project. These include energy (electrical or thermal in the form of hydrocarbons), chemical reagents, and personnel.

Any of numerous compounds that result from the replacement of part or all of the acid hydrogen of an acid by a metal or a group acting like a metal; an ionic crystalline compound. For example, when hydrochloric acid is mixed with sodium hydroxide (caustic) the result is table salt and water:

$\mathrm{HCl}+\mathrm{NaOH}=>\mathrm{NaCl}+\mathrm{H}_{2} \mathrm{O}$.

A planned sequence of operations. Key schedule aspects for technologies include required research, development, demonstrations, engineering design, permitting, construction and installation and start-up. 
Scrubber

Silver chloride

Simplicity

Site expanse

Sizing

Sludge

Soft Debris

Solvent

Special Waste

Stabilization

Storage

Surfactants

Suspended solids

TCLP tests
Engineered equipment used to remove constituents from a gas stream by absorption and/or chemical reaction.

A common chemical used in photography.

Easy to operate, not complicated.

The fenced area required to accommodate operating facilities.

Reduction of size by cutting, shredding, grinding or other methods.

The precipitated solids (primarily oxides and hydroxides) that settle to the bottom of the storage tanks containing liquid high-level waste.

A category of debris which includes cardboard, plastic, wood.

A substance, usually liquid, that can dissolve other substances.

Waste requiring unusual treatment.

Process designed to limit the mobility of chemicals and prevent toxic chemicals from escaping into the environment.

Retention of radioactive waste in man-made containers, such as tanks or vaults, in a manner permitting retrieval (as opposed to disposal, which implies no retrieval).

Chemicals used to bring together oily and water-based solutions; soaps and detergents are examples.

Tiny solid particles that remain in a liquid; for example, mud which stays suspended in flowing water.

Toxicity Characteristic Leaching Procedure - a chemical test required by the EPA for certain wastes at the time of disposal; the test imitates nature's ability to react with metals in the ground and demonstrates how much metal could be leached out of the waste over time. The EPA requires the tested wastes disposed of to be below a legal limit as described in 40 CFR 268. 
Technology development status

Thermal desorption

Thermal system

Total life cycle cost

Toxic

TRUPACT II

Upset and accident conditions

Vacuum thermal desorption

Versatility

Vitrification

Volatile organic compounds
The current level of development of the technologies, i.e., laboratory or bench scale (about 1 quart process or less), pilot scale (about a 5 gallon to 50 gallon process), production or full scale (size required, usually over 200 gallons), commercial (available and proven), etc.

A process which uses heat to convert volatile liquids into a gas phase thus allowing them to be removed from solids.

System that employs enough heat that raises the temperature above $350^{\circ} \mathrm{C}\left(660^{\circ} \mathrm{F}\right)$.

Total life cycle cost includes the cost of all facets of treatment and disposal of the waste, from research of treatment processes through disposal of the last of the waste. Facets include R\&D, pre-operational activities, installation of production facilities and equipment, O\&M activities, D\&D, and disposal. TLCC costs which do not include disposal are described as "TLCC without disposal".

Chemicals known to cause harm to humans or animals through exposure routes such as ingestion (eating), inhalation (breathing), dermal contact (through the skin).

Trademark name for a DOE approved transportation container for contact-handled transuranic waste. The container is capable of holding 14 55-gallon drums.

Processing conditions outside the normal range and/or mechanical failures. Examples include overflowing tanks, runaway reactions, unexpected sudden loss of pressure.

A process similar to thermal desorption but additionally employs the use of vacuum to enhance the transport of volatile liquids into the gas phase for removal.

Capable of varied uses or functions; as an evaluation criterion, considers the fraction of the waste inventory that can be treated by the technology or system.

The conversion of materials through the use of high temperature and additives to a glass-like form.

An organic compound with a vapor pressure greater than 0.44 pounds per square inch at standard temperature and pressure. 
Volatilized

Volume reduction

Waste acceptance criteria

Waste certification criteria

Waste Isolation Pilot Plant

Wastewater

Wastewater releases

${ }^{\circ} \mathbf{C}$

${ }^{\circ} \mathbf{F}$

${ }^{\circ} \mathbf{K}$
Evaporated; passed off as a vapor.

The relationship of the volumes of output waste to input waste. If less waste volume is left after treatment and stabilization than the volume of waste that entered, then waste is reduced.

Criteria established by a waste management facility which define the waste it will accept.

Criteria that must be met for transport, treatment, and disposal of waste.

DOE facility located near Carlsbad, New Mexico, built to demonstrate the safe underground disposal of transuranic waste from numerous facilities owned by DOE.

Water, usually used in some process, that contains organic or inorganic contaminants that are considered to be undesirable.

The volume of treated wastewater (including contaminants) discharged from the treatment facility.

Degree Centigrade (Celsius). ${ }^{\circ} \mathrm{C}=5 / 9 \times\left({ }^{\circ} \mathrm{F}-32\right)$.

Degree Fahrenheit. ${ }^{\circ} \mathrm{F}={ }^{\circ} \mathrm{C} \times 9 / 5+32$.

Degree Kelvin. ${ }^{\circ} \mathrm{K}={ }^{\circ} \mathrm{C}+273$. 


\section{APPENDIX G}

\section{Mercury Vapor Pressure Data}


Vapor Pressure (atm.) Data as a Function of Temperature

\begin{tabular}{|c|c|c|c|c|c|c|c|c|c|c|c|}
\hline Temp (C) & Temp (F) & $\mathrm{Hg}$ & $\mathrm{HgCl}$ & $\mathrm{HgCl} 2$ & $\mathrm{HgO}$ & $\mathrm{HgF}$ & $\mathrm{HgF} 2$ & $\mathrm{HgBr}$ & $\mathrm{HgBr} 2$ & $\mathrm{Hgl}$ & $\mathrm{Hgl} 2$ \\
\hline 0 & 32 & $2.788 \mathrm{E}-07$ & $1.705 \mathrm{E}-32$ & 6.558E-09 & $2.667 \mathrm{E}-17$ & 7.470E-40 & $1.475 \mathrm{E}-17$ & $1.038 \mathrm{E}-31$ & 5.810E-09 & 1.883E-29 & $1.019 \mathrm{E}-09$ \\
\hline 10 & 50 & 7.240 E-07 & $4.506 \mathrm{E}-31$ & 2.427E-08 & $2.106 \mathrm{E}-16$ & $3.582 \mathrm{E}-38$ & $1.101 \mathrm{E}-16$ & $2.581 E-30$ & $2.155 \mathrm{E}-08$ & $3.849 E-28$ & $4.106 \mathrm{E}-09$ \\
\hline 20 & 68 & 1.763E-06 & $9.503 E-30$ & $8.197 \mathrm{E}-08$ & $1.441 \mathrm{E}-15$ & 1.316E-36 & $7.151 \mathrm{E}-16$ & $5.141 E-29$ & 7.296 E-08 & $6.389 E-27$ & $1.501 \mathrm{E}-08$ \\
\hline 30 & 86 & $4.042 E-06$ & $1.636 \mathrm{E}-28$ & 2.549E-07 & $8.678 \mathrm{E}-15$ & 3.803E-35 & $4.091 \mathrm{E}-15$ & $8.387 E-28$ & 2.274E-07 & $8.794 \mathrm{E}-26$ & $5.029 E-08$ \\
\hline 40 & 104 & $8.781 E-06$ & $2.344 \mathrm{E}-27$ & 7.359E-07 & $4.652 \mathrm{E}-14$ & $8.849 E-34$ & $2.089 E-14$ & $1.142 E-26$ & $6.581 \mathrm{E}-07$ & $1.022 E-24$ & $1.556 \mathrm{E}-07$ \\
\hline 50 & 122 & $1.816 \mathrm{E}-05$ & $2.843 \mathrm{E}-26$ & $1.986 \mathrm{E}-06$ & $2.245 \mathrm{E}-13$ & $1.691 \mathrm{E}-32$ & $9.615 \mathrm{E}-14$ & 1.321E-25 & 1.780E-06 & $1.018 \mathrm{E}-23$ & $4.481 \mathrm{E}-07$ \\
\hline 60 & 140 & $3.590 \mathrm{E}-05$ & $2.963 \mathrm{E}-25$ & 5.039E-06 & $9.840 \mathrm{E}-13$ & $2.704 \mathrm{E}-31$ & $4.030 \mathrm{E}-13$ & 1.317E-24 & $4.526 \mathrm{E}-06$ & $8.820 \mathrm{E}-23$ & $1.209 \mathrm{E}-06$ \\
\hline 70 & 158 & 6.830 E-05 & $2.691 \mathrm{E}-24$ & 1.209E-05 & 3.953E-12 & $3.671 \mathrm{E}-30$ & $1.550 \mathrm{E}-12$ & 1.146E-23 & $1.088 \mathrm{E}-05$ & $6.726 \mathrm{E}-22$ & $3.071 \mathrm{E}-06$ \\
\hline 80 & 176 & $1.251 \mathrm{E}-04$ & $2.153 E-23$ & 2.756E-05 & $1.466 \mathrm{E}-11$ & $4.293 \mathrm{E}-29$ & $5.514 \mathrm{E}-12$ & $8.811 E-23$ & $2.486 \mathrm{E}-05$ & $4.565 E-21$ & $7.390 \mathrm{E}-06$ \\
\hline 90 & 194 & $2.214 \mathrm{E}-04$ & $1.543 E-22$ & 5.996E-05 & $5.051 \mathrm{E}-11$ & $4.378 E-28$ & $1.826 \mathrm{E}-11$ & $6.044 \mathrm{E}-22$ & 5.418E-05 & $2.784 \mathrm{E}-20$ & $1.691 \mathrm{E}-05$ \\
\hline 100 & 212 & $3.798 \mathrm{E}-04$ & $9.826 \mathrm{E}-22$ & 1.249E-04 & $1.627 \mathrm{E}-10$ & $3.397 \mathrm{E}-27$ & $5.659 \mathrm{E}-11$ & $3.734 \mathrm{E}-21$ & $1.131 \mathrm{E}-04$ & $1.538 \mathrm{E}-19$ & 3.696E-05 \\
\hline 110 & 230 & $6.330 \mathrm{E}-04$ & $5.705 E-21$ & 2.501E-04 & $4.926 \mathrm{E}-10$ & $3.153 E-26$ & $1.651 \mathrm{E}-10$ & $2.094 E-20$ & $2.268 E-04$ & $7.766 \mathrm{E}-19$ & 7.743E-05 \\
\hline 120 & 248 & $1.028 \mathrm{E}-03$ & $3.025 \mathrm{E}-20$ & $4.829 \mathrm{E}-04$ & $1.408 \mathrm{E}-09$ & $2.268 \mathrm{E}-25$ & $4.553 \mathrm{E}-10$ & 1.075E-19 & $4.386 \mathrm{E}-04$ & $3.605 \mathrm{E}-18$ & $1.560 \mathrm{E}-04$ \\
\hline 130 & 266 & $1.628 \mathrm{E}-03$ & $1.475 \mathrm{E}-19$ & $9.010 \mathrm{E}-04$ & 3.816E-09 & $1.478 \mathrm{E}-24$ & 1.192E-09 & $5.079 \mathrm{E}-19$ & $8.196 \mathrm{E}-04$ & $1.549 \mathrm{E}-17$ & 3.023E-04 \\
\hline 140 & 284 & $2.520 \mathrm{E}-03$ & $6.653 \mathrm{E}-19$ & 1.629E-03 & 9.845E-09 & $8.785 E-24$ & 2.976E-09 & $2.224 \mathrm{E}-18$ & 1.484E-03 & $6.194 \mathrm{E}-17$ & 5.577E-04 \\
\hline 150 & 302 & 3.821E-03 & $2.792 \mathrm{E}-18$ & $2.861 \mathrm{E}-03$ & 2.427E-08 & $4.795 \mathrm{E}-23$ & $7.104 \mathrm{E}-09$ & $9.069 \mathrm{E}-18$ & $2.610 \mathrm{E}-03$ & $2.317 \mathrm{E}-16$ & $9.977 \mathrm{E}-04$ \\
\hline 160 & 320 & $5.680 \mathrm{E}-03$ & $1.095 \mathrm{E}-17$ & 4.890E-03 & 5.732E-08 & $2.417 E-22$ & 1.627E-08 & $3.462 E-17$ & 4.467E-03 & $8.145 E-16$ & 1.735E-03 \\
\hline 170 & 338 & $8.291 E-03$ & 4.037E-17 & $8.149 E-03$ & 1.301E-07 & $1.132 E-21$ & $2.585 E-08$ & 1.243E-16 & $7.454 \mathrm{E}-03$ & $2.702 \mathrm{E}-15$ & $2.939 \mathrm{E}-03$ \\
\hline 180 & 356 & $1.190 \mathrm{E}-02$ & $1.403 \mathrm{E}-16$ & $1.326 \mathrm{E}-02$ & $2.846 \mathrm{E}-07$ & $4.944 \mathrm{E}-21$ & $7.619 \mathrm{E}-08$ & $4.212 \mathrm{E}-16$ & $1.215 \mathrm{E}-02$ & $8.495 E-15$ & E-03 \\
\hline 190 & 374 & $1.680 \mathrm{E}-02$ & $4.616 \mathrm{E}-16$ & $2.112 \mathrm{E}-02$ & $3 E-07$ & $2.025 E-20$ & $1.566 \mathrm{E}-07$ & $1.353 \mathrm{E}-15$ & $1.936 \mathrm{E}-02$ & $2.539 E-14$ & 7.845E-03 \\
\hline 200 & 392 & $2.337 \mathrm{E}-02$ & 1.443E-15 & 3.293E-02 & $1.230 \mathrm{E}-06$ & $7.806 \mathrm{E}-20$ & 3.117E-07 & $4.133 E-15$ & $3.022 \mathrm{E}-02$ & $7.238 \mathrm{E}-14$ & 1.240E-02 \\
\hline 210 & 410 & 3.206E-02 & $4.300 \mathrm{E}-15$ & $5.038 \mathrm{E}-02$ & $2.440 \mathrm{E}-06$ & $2.844 \mathrm{E}-19$ & $6.026 \mathrm{E}-07$ & $1.204 \mathrm{E}-14$ & $4.627 \mathrm{E}-02$ & $1.974 \mathrm{E}-13$ & $1.921 \mathrm{E}-02$ \\
\hline 220 & 428 & 4.341E-02 & $1.225 E-14$ & 7.567E-02 & $4.706 \mathrm{E}-06$ & $9.822 \mathrm{E}-19$ & 1.133E-06 & $3.357 \mathrm{E}-14$ & $6.956 \mathrm{E}-02$ & $5.163 \mathrm{E}-13$ & $2.921 \mathrm{E}-02$ \\
\hline 230 & 446 & 5.805E-02 & $3.343 E-14$ & $1.117 \mathrm{E}-01$ & 8.833E-06 & $3.227 \mathrm{E}-18$ & 2.076E-06 & 8.977E-14 & $1.028 \mathrm{E}-01$ & 1.299E-12 & 4.363E-02 \\
\hline 240 & 464 & $7.673 \mathrm{E}-02$ & $8.770 E-14$ & 1.624E-01 & 1.617E-05 & $1.011 \mathrm{E}-17$ & 3.710E-06 & $2.308 \mathrm{E}-13$ & 1.495E-01 & $3.149 \mathrm{E}-12$ & 6.409E-02 \\
\hline 250 & 482 & $1.003 E-01$ & $2.216 \mathrm{E}-13$ & 2.324E-01 & 2.889E-05 & $3.031 \mathrm{E}-17$ & $6.480 \mathrm{E}-06$ & $5.721 \mathrm{E}-13$ & $1.989 \mathrm{E}-01$ & $7.375 E-12$ & $9.268 \mathrm{E}-02$ \\
\hline 260 & 500 & 1.298E-01 & $5.402 E-13$ & 3.280E-01 & 5.049E-05 & $8.714 \mathrm{E}-17$ & 1.107E-05 & $1.369 \mathrm{E}-12$ & $2.596 E-01$ & $1.671 \mathrm{E}-11$ & 1.287E-01 \\
\hline 270 & 518 & $1.664 \mathrm{E}-01$ & $1.274 E-12$ & $4.566 \mathrm{E}-01$ & 8.637E-05 & $2.408 \mathrm{E}-16$ & 1.854E-05 & $3.171 \mathrm{E}-12$ & 3.350E-01 & $3.673 E-11$ & 1.671E-01 \\
\hline 280 & 536 & $2.112 \mathrm{E}-01$ & $2.909 \mathrm{E}-12$ & $6.126 \mathrm{E}-01$ & $1.448 \mathrm{E}-04$ & $6.409 \mathrm{E}-16$ & 3.044E-05 & $7.119 \mathrm{E}-12$ & 4.276E-01 & $7.838 \mathrm{E}-11$ & $2.144 \mathrm{E}-01$ \\
\hline 290 & 554 & 2.659E-01 & $6.449 E-12$ & 7.714E-01 & 2.382E-04 & $1.646 \mathrm{E}-15$ & 4.907E-05 & $1.552 \mathrm{E}-11$ & $5.403 E-01$ & $1.627 \mathrm{E}-10$ & $2.724 \mathrm{E}-01$ \\
\hline 300 & 572 & $3.319 E-01$ & $1.389 E-11$ & $9.620 \mathrm{E}-01$ & 3.849E-04 & $4.090 \mathrm{E}-15$ & 7.772E-05 & $3.209 \mathrm{E}-11$ & 6.760 E-01 & $3.290 \mathrm{E}-10$ & 3.426E-01 \\
\hline 310 & 590 & $4.110 \mathrm{E}-01$ & $2.914 \mathrm{E}-11$ & $1.189 E+00$ & $6.113 \mathrm{E}-04$ & $9.843 E-15$ & $1.211 \mathrm{E}-04$ & $6.793 E-11$ & 8.382E-01 & $6.488 \mathrm{E}-10$ & $4.269 \mathrm{E}-01$ \\
\hline 320 & 608 & 5.053E-01 & $5.957 \mathrm{E}-11$ & $1.475 E+00$ & 9.553E-04 & $2.298 \mathrm{E}-14$ & 1.857E-04 & $1.368 \mathrm{E}-10$ & $1.030 E+00$ & $1.250 \mathrm{E}-09$ & $5.274 \mathrm{E}-01$ \\
\hline 330 & 626 & $6.169 \mathrm{E}-01$ & $1.188 E-10$ & $1.771 E+00$ & $1.470 \mathrm{E}-03$ & $5.214 \mathrm{E}-14$ & 2.807E-04 & $2.689 E-10$ & $1.256 E+00$ & $2.354 \mathrm{E}-09$ & $6.460 E-01$ \\
\hline 340 & 644 & $7.480 \mathrm{E}-01$ & $2.317 \mathrm{E}-10$ & $2.136 E+00$ & 2.229E-03 & $1.151 \mathrm{E}-13$ & $4.182 \mathrm{E}-04$ & $5.168 \mathrm{E}-10$ & $1.520 E+00$ & 4.341E-09 & $7.850 E-01$ \\
\hline 350 & 662 & $9.012 \mathrm{E}-01$ & $4.419 E-10$ & $2.558 \mathrm{E}+00$ & 3.334E-03 & $2.476 \mathrm{E}-13$ & $6.147 E-04$ & $9.722 \mathrm{E}-10$ & $1.825 E+00$ & 7.943E-09 & $9.469 \mathrm{E}-01$ \\
\hline
\end{tabular}




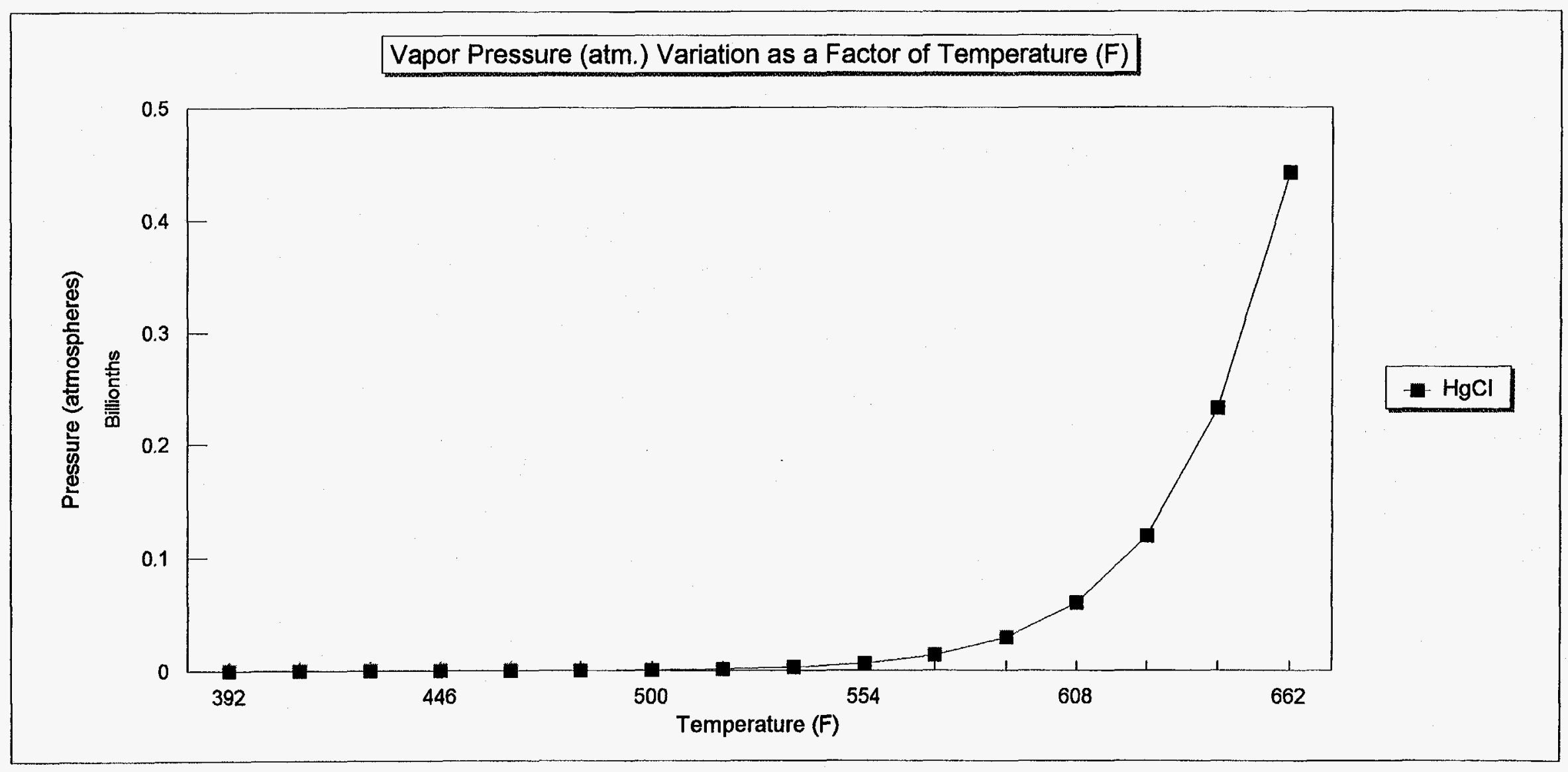




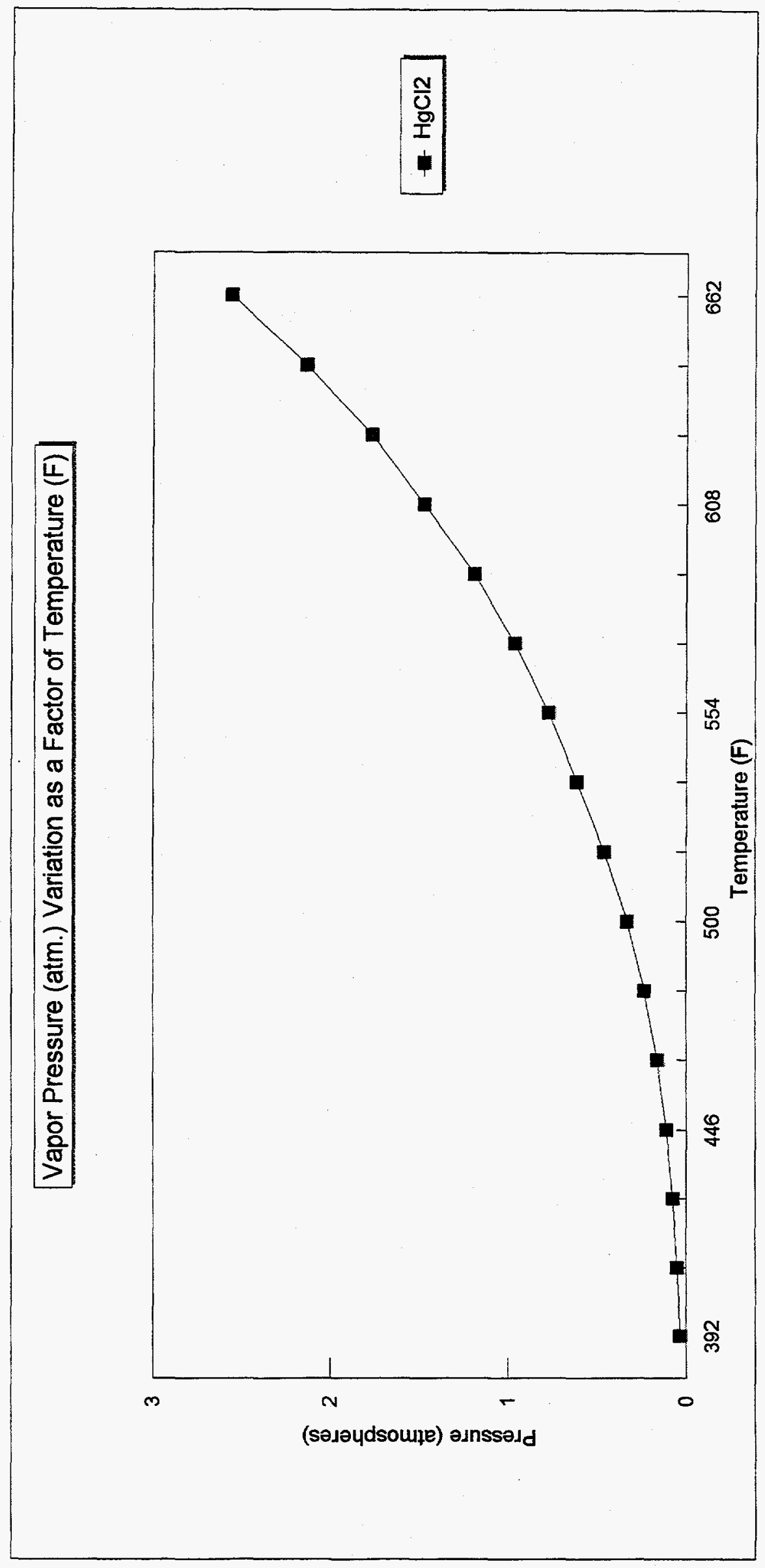




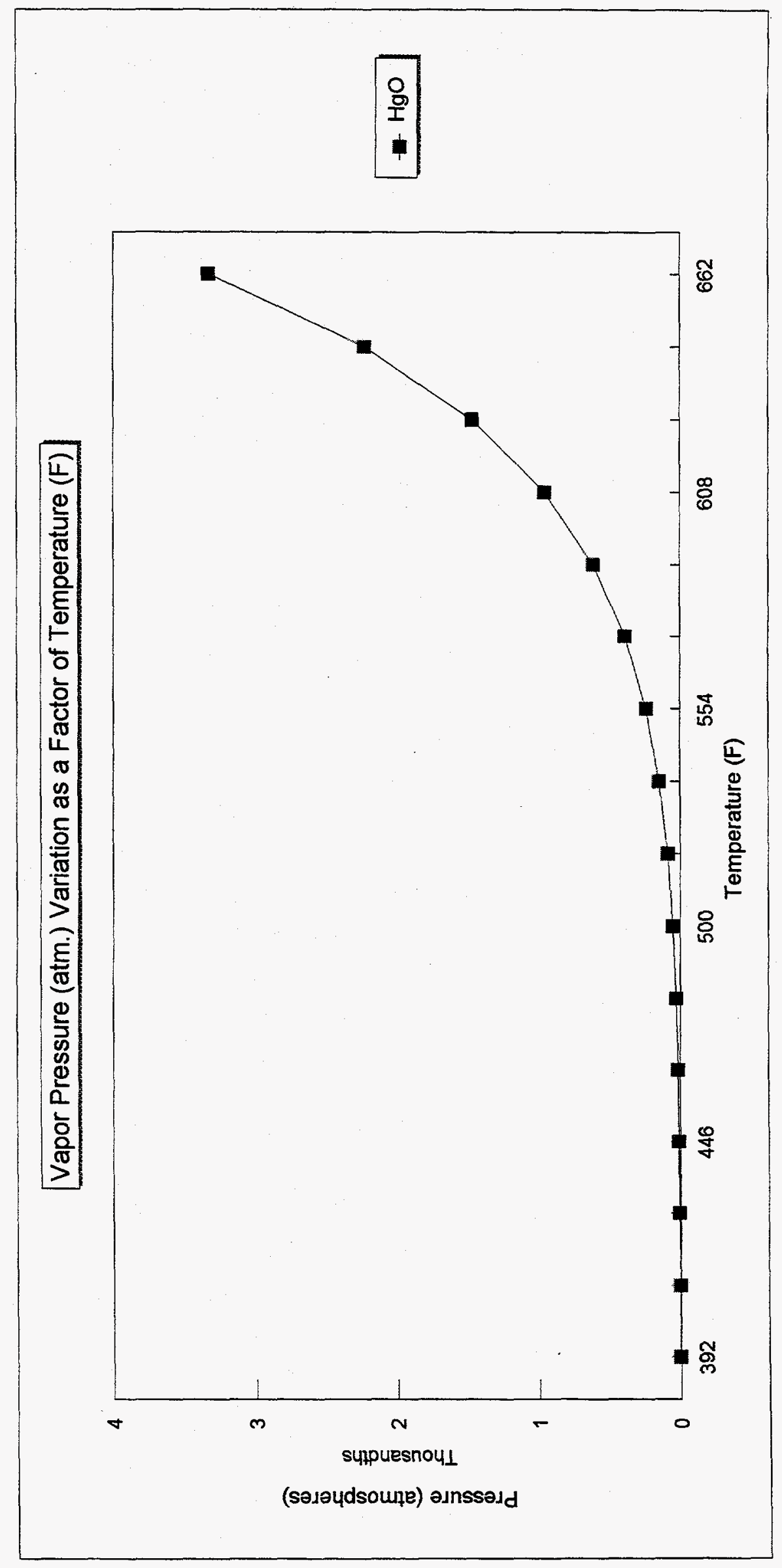




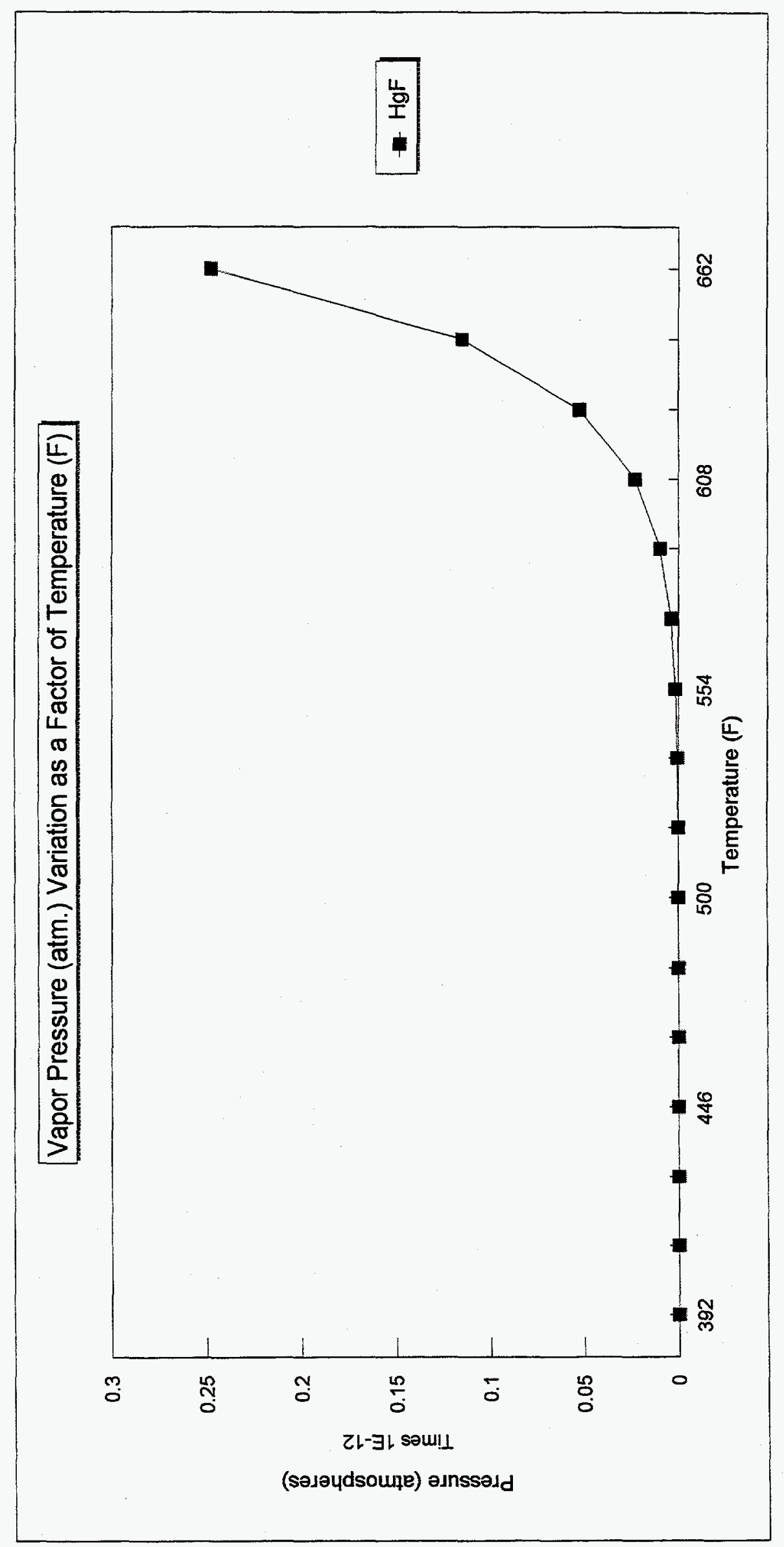




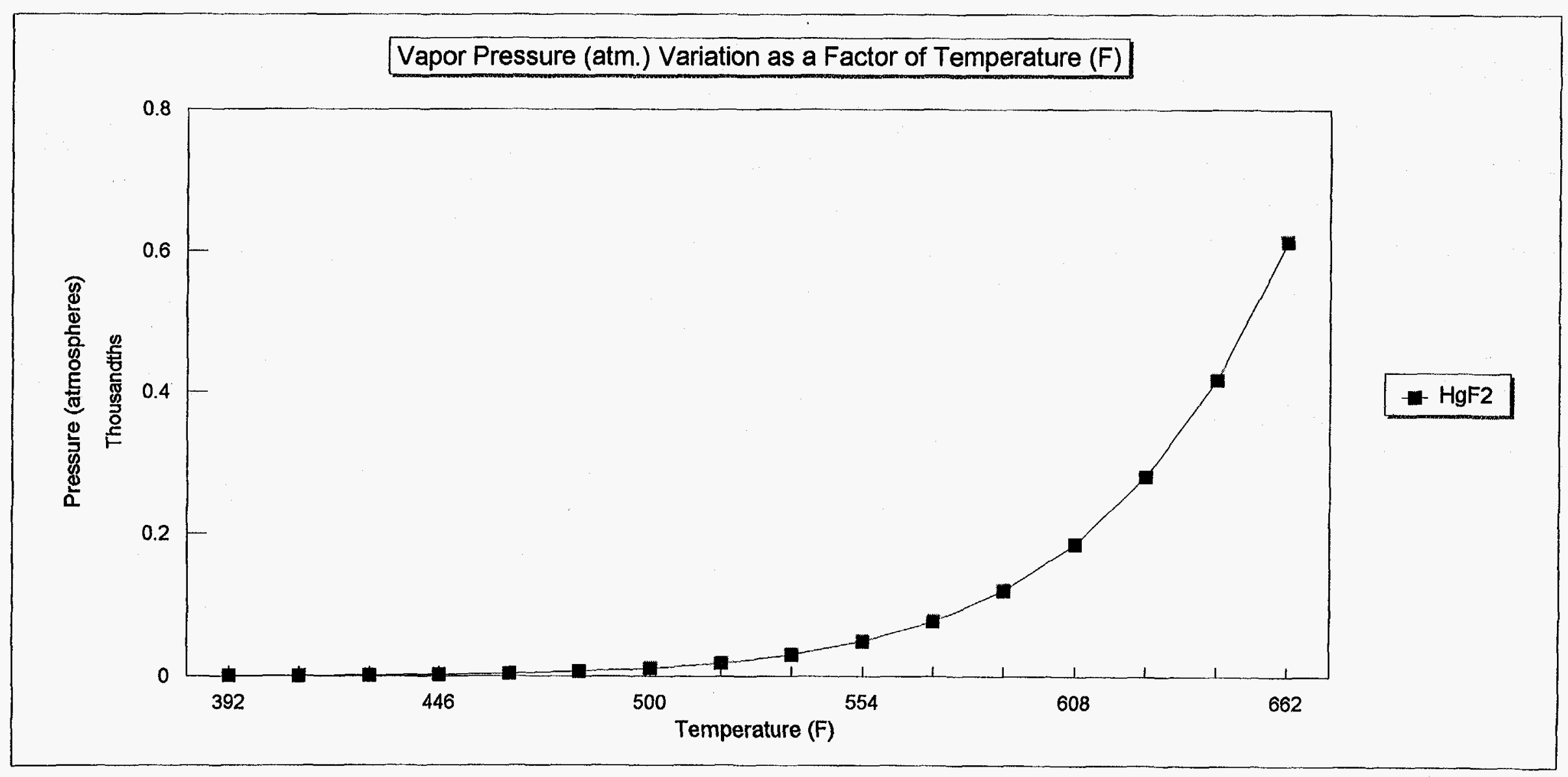




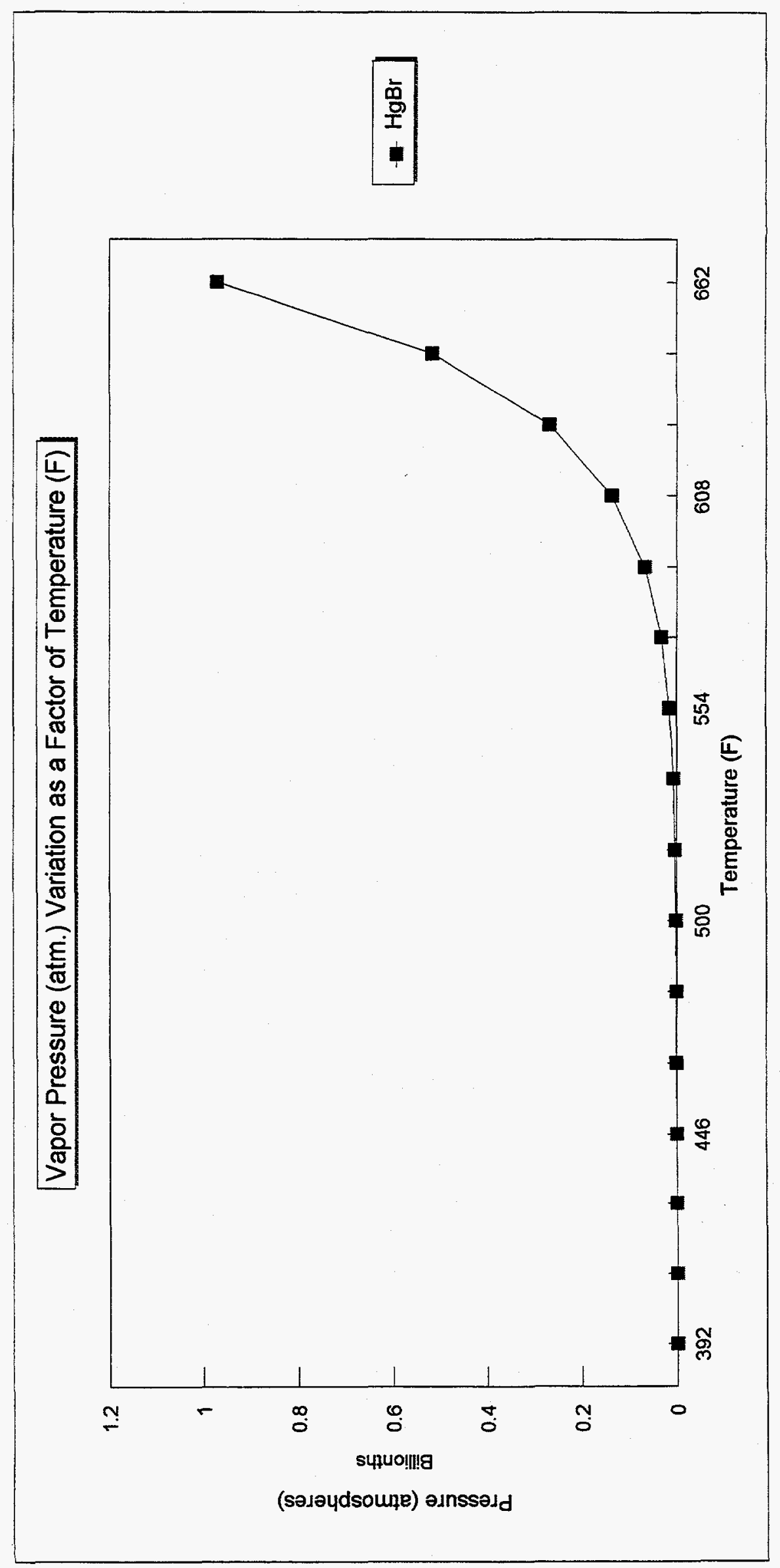




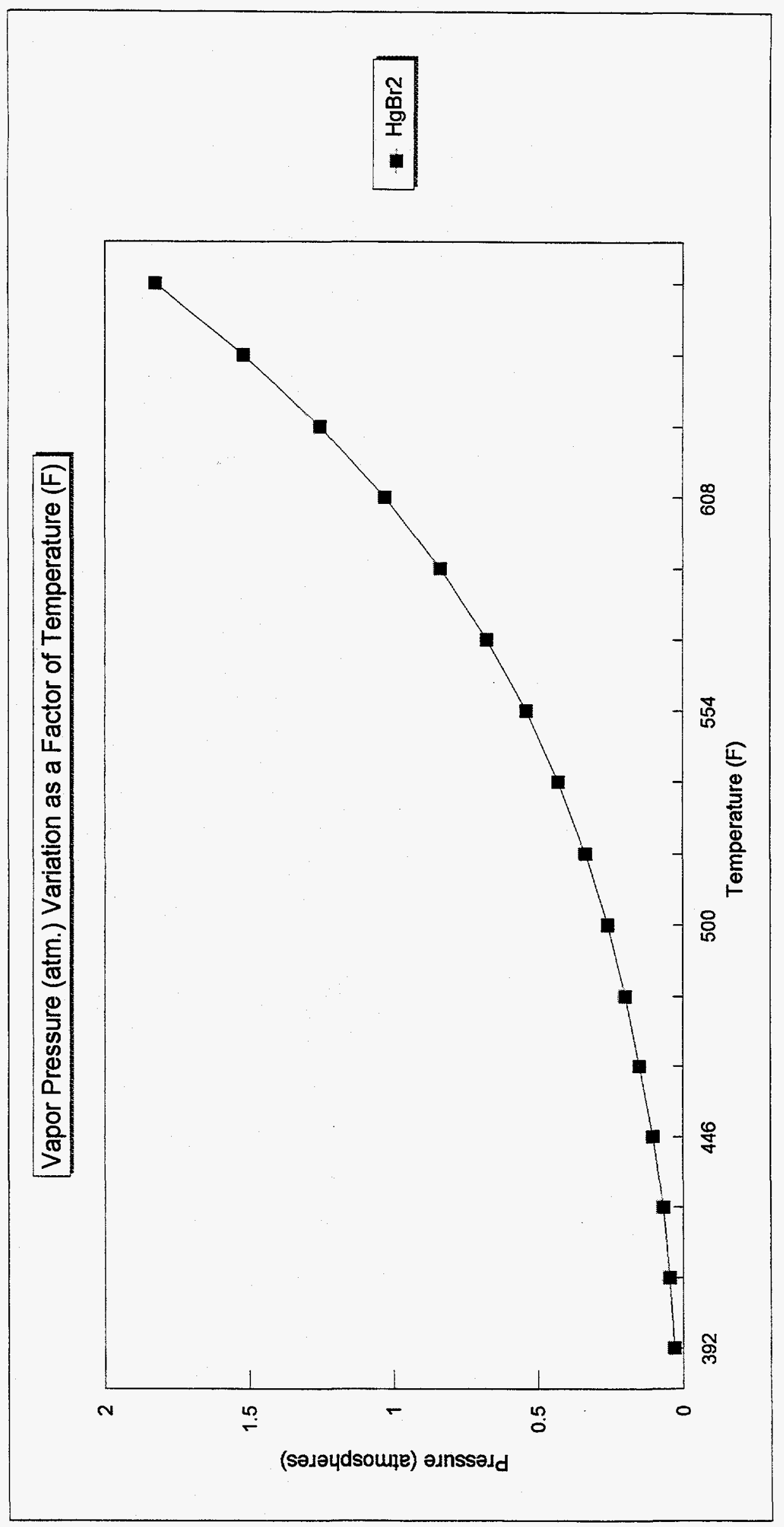




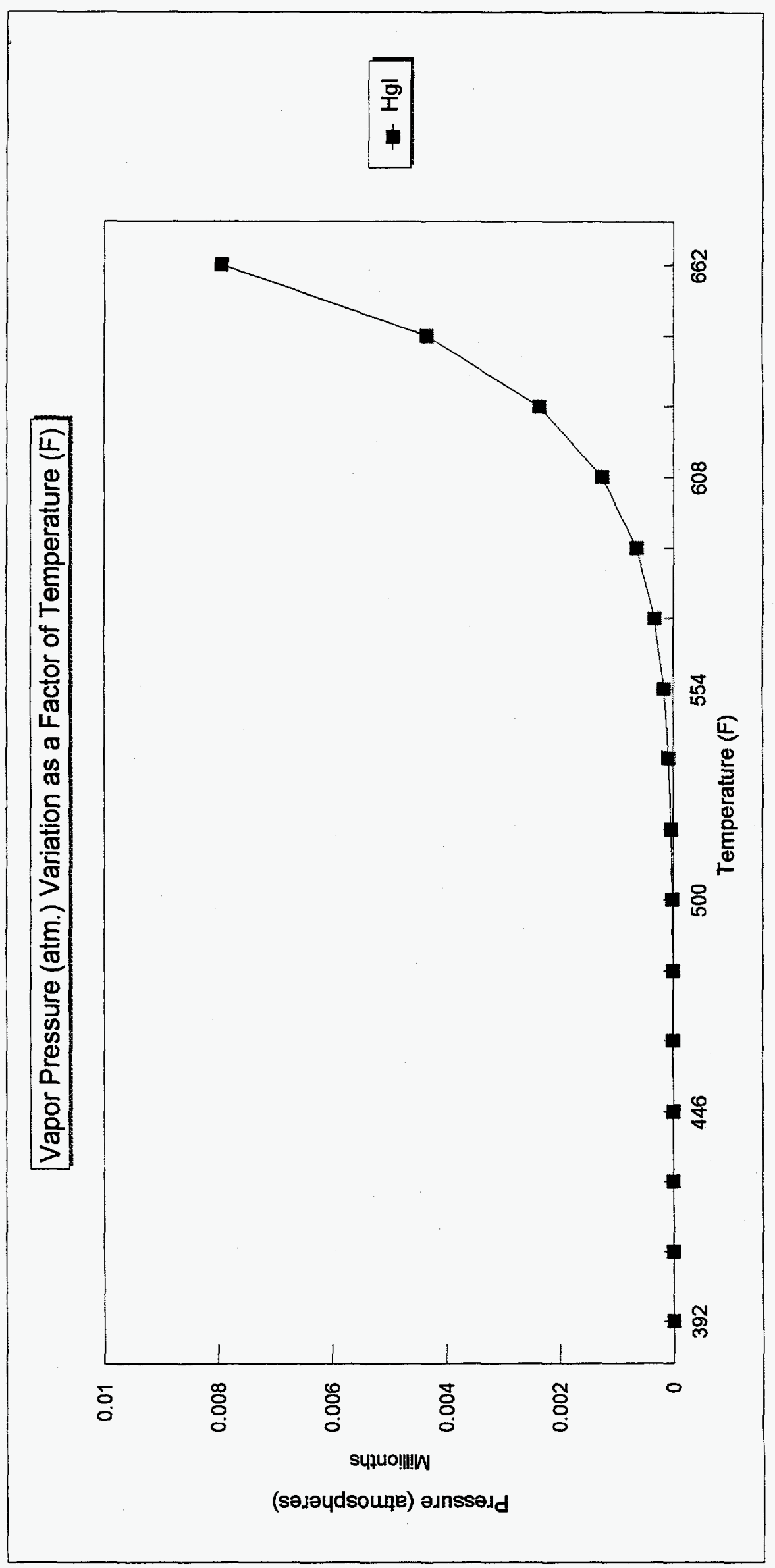




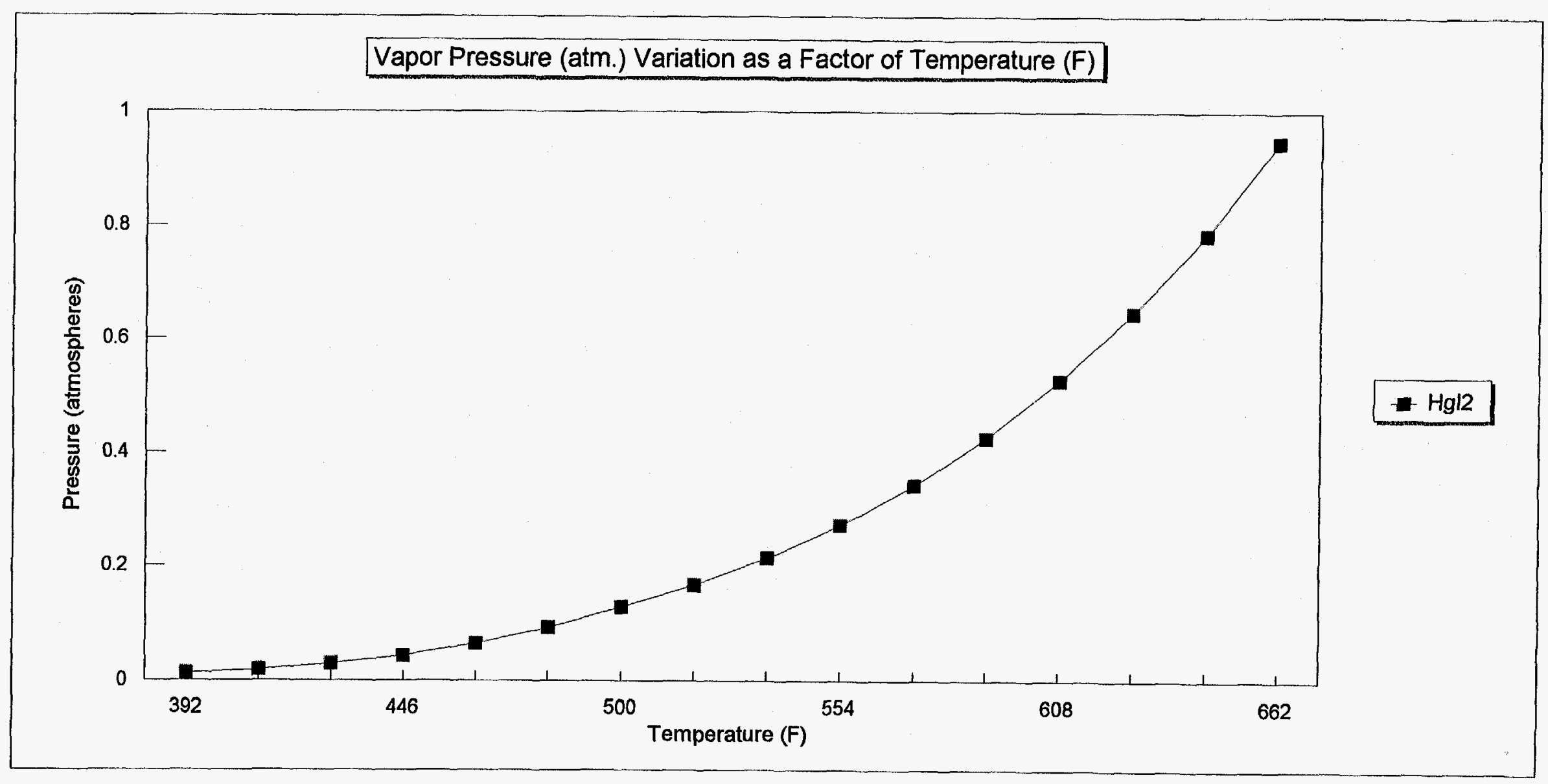

